


5.981. 


\section{BULLETIN}

GÉNËRAL ET UNIVERSEL

DES ANNONGES ET DES NOUVELLES SCIENTIFIQUES;

DÉDIÉ AUX SAVANS DE TOUS LES PAYS

ET A LA LIBRAIRIE NATIONALE ET ÉTRANGÈRE :

PUBLIÉ

SOUS LA DIRECTION DE M. LE B BN. DE FÉRUSSAC,

OFFICIER SUPÉRIEUR AU CORPS ROYAL D'ÉTAT-MaJOR,

CHEVALIER DE SAINT-LOUIS ET DE LA LEGION-D'HONNEUR,

MEMBRE DE PLUSIEURS SOCIÉTÉS SAVANTES NATIONALES OU ETTRANGÉFES,

\section{TOME PREMIER.}
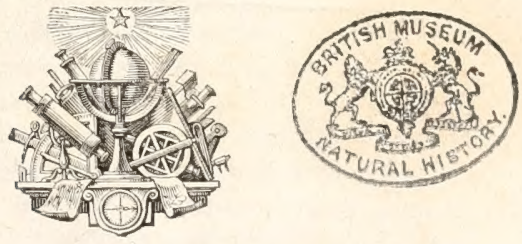

\section{A PARIS,}

AU BUREAU DU BULIETIN,

RUE DE L'ABEAXE, N. 3. 1823. 



\section{AVIS.}

0

N connaît les difficultés imprévues et inévitables qui arrêtent toujours la marche des choses dans le commencement d'une entreprise un peu considérable; on ne sera donc point étonné du retard qu'a éprouvé la publication du premier $n^{\circ}$. du Bulletin. Il fallait mettre en mouvement les rouages d'une machine vaste et compliquée; ce premier pas est fait, et l'on peut espérer dorénavant que ce mouvement sera de plus en plus régulier et uniforme. Ces observations peuvent s'appliquer avec plus de raison encore à la composition de ce premier $\mathbf{n}^{\circ}$. : c'est, dans son ensemble, une ébauche de ce que peut et doit devenir notre journal, sous le rapport de la généralité, de la variété et de la nouveauté des annonces, et sous celui de l'accord qui doit régner dans chaque partie, quant à sa rédaction et à l'uniformité de composition typographique. Mais tel qu'il est, ce premier no. suffira pour faire apprécier l'influence immense que peut avoir ce journal sur les progrès des sciences; pour prouver l'avantage qu'il doit offrir à tous les auteurs, en leur fournissant un moyen prompt et assuré de faire rapidement parvenir la connaissance de leurs ouvrages à ceux même qui sont le plus intéressés à savoir qu'ils existent, et par conséquent d'en déterminer le plus prompt débit. Ce premier no. montrera aussi à toutes les personnes qui cultivent, par goût ou par état, une branche quelconque des sciences, ce qu'elles peuvent espérer du Bulletin, en y trouvant réunies les annonces d'une foule d'ouvrages, d'expériences, de faits et d'inventions dont elles n'auraient peut-ètre pas eu l'idée ; et qu'il peut leur être si important de connaitre pour leurs propres travaux. Le Bulletin, destiné à rassembler tout ce qui se trouve en ce genre, dans les 400 a 500 journaux ou recueils qui se publient dans le monde, doit, par cela seul, devenir indispensable, nous ne dirons pas seulement aux savans par goût, toujours empressés d'être au courant des sciences qu'ils cultivent, mais même, à tous les agriculteurs, médecins, chirurgiens, pharmaciens, mécaniciens ; aux mili- 
taires instruits et aux marins qui, éloignés des grands centres scientifiques, ne pourraient être au fait du progrès des arts auxquels ils sont voués par état, qu'en s'abonnant à une quantité de journaux des différens pays. Ce journal n'offrira pas moins d'intérêt aux professeurs des diverses sciences à qui il convient de saisir, pour ainsi dire, à leur naissance, les progrès qu'elles font; aux établissemens publics ou privés, destinés aux sciences ou à l'industrie, et enfinà tous les libraires même, qui, n'ayant point, pour la plupart, les journaux d'annonces des diverses contrées, sont si souvent dans l'embarras pour découvrir le lieu de publication d'un ouvrage dont on ne connait que le titre, et le libraire auquel il faut s'adresser pour l'obtenir.

Il est d'ailleurs une vérité incontestable, c'est qu'en répandant partout, et plus généralement que cela ne se fait aujourd'hui, puisqu'il n'existe aucune entreprise qui ait ce but, la connaissance des divers travaux publiés, ou celle des faits observés, on multiplie, d'une part, les chances de débit pour les ouvrages, et nous ne craignons pas de le dire, de manière à en doubler peut-être la vente; car qui n'a pas regretté de n'avoir point été instruit à temps de la publication de tel ou tel livre? et, d'un autre côté, cette connaissance plus générale des faits augmente, dans une progression indéfinie, l'impulsion donnée aux esprits occupés des sciences; régularise la marche de leurs travaux ; évite une foule d'essais, de tâtonnemens, d'écrits inutiles, fruits naturels de l'isolement où sont en général les savans. On peut présumer ce que produirait en résultats utiles le temps ordinairement perdu par cette absence d'un lien commun et d'une correspondance active, qui montrerait aux savans des parties les plus reculées, l'état de la branche des sciences qu'ils cultivent, ce qu'il reste à faire, et le point d'où ils doivent partir s'ils veulent lui faire faire des progrès.

Nous croyons qu'il nous est permis de parler nous-mêmes de l'intérêt d'une semblable entreprise sans manquer aux convenances; nous n'avons ni gloire ni profit à en espérer : peint de gloire, parce que c'est une opération purement mécanique, et qu'elle appartient tout entière à nos savans collaborateurs; point de profit présumable, parce que chacun peut aisément calculer que nous serions fort heureux de cou- 


$$
\text { Avis. }
$$

vrir les frais considérables qu'elle exige. Le zèle seul pour la science a pu nous déterminer à la tenter, et à ralentir, pour quelques momens, les travaux que nous avons entrepris sur l'histoire naturelle et la géologie. Il appartenait à la France d'essayer un aussi grand moyen de favoriser les progrès et les applications multipliées des connaissances utiles, des connaissances qui ne portent avec elles aucun germe dangereux, et qui sont les bases de la prospérité des peuples, peut-être même, de leur repos, et, par cela, du bonheur des rois. Tout ce qui tient à la politique est absolument étranger à notre journal : tout ce qui touche anx intérêts de la religion et de la morale, y sera scrupuleusement respecté; nos principes personriels et lintérêt même des sciences nous en font une loi. Si, malgré nos soins et nos efforts, nous n'étions pas assez heureux pour que notre Bulletin s'exécutât tel que nous l'avons conçu, pour qu'il remplisse son importante destination, nous aimons à penser que les hommes éclairés de tous les pays nous sauront toujours quelque gré de cet essai, que des personnes, placées dans des circonstances plus favorables, pourront peut-être continuer avec plus de succès.

Le Bon de Férussac. 


\section{TABLE DES MATIËRES.}

PREMIERE SECTION.

Annonce des Ouvrages, Extraits des Journaux et Nouvelles Scientifiques.

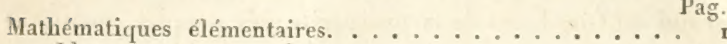

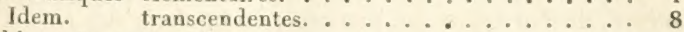

Machines et construction. . . . . . . . . . . . 12

Topographie, géodosie.................. 22

Astronomie, cosmographie................ 24

Physique. .......................... 3o

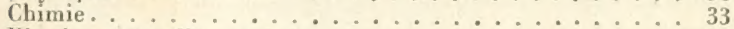

Histoire naturelle générale. . . . . . . . . . 39

Minéralogie.......................... 43

Géologie. ....................... 43

Zoologie........................... 54

Botanique. ..................... 67

Palontographie. ............................ So

Physiologie........................ 8 I

Anatomie. . . . . . . . . . . . . . . . 85

Médecine. . . . . . . . . . . . . . . . . . 90

Chirurgie .......................... 98

Art vétérinaire ............................. $9^{8}$

Agriculture, économie rurale et domestique ........ . . 102

Géographie ........................ III

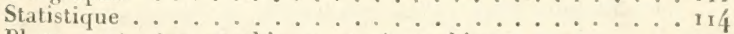

Plans, cartes topographiques et géographiques ........ 122

Art nautique .......................... 139

Art militaire .................................. 30

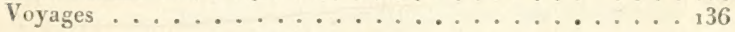

DEUXIEME SECTION.

Revue.

Progrès des Sciences................ 139

Journaux..............................

\section{TROISIEME SECT I ON. \\ Travaux des Sociétés savantes.}

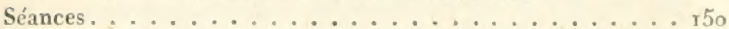

Prix proposés. . . . . . . . . . . . . 154

Nominations, éloges. ............... I 59

QUATRIEME SECTION.

Annonces diverses.

Entreprises scientifiques................ I6 6

Indications cientifiques . . . . . . . . . . . . 163

Ventes d'objets scientifiques................ 164

CINQUIEME SECTION.

IVécrologie.

Nécrologie. 166 


\section{BULLETIN}

GÉNÉRAL ET UMIVERSEL

DES ANNONCES ET DES NOUVELLES SCIENTIFIQUES.

meงเอง

\section{PREMTERE SECTION.}

ANNONCFS DES OUVR AGES, EXTRAITS DES JOURNAUX ET NOUVELLES SCIENTIFIQUES.

\section{MATHÉNATIQUES ÉLÉMENTAIRES.}

I. Nouveau traité D'arithuétique; par L.-M.-D. de Vericia, ancien élève del'école Polytechnique. In-8 de $\gamma$ feuilles et demie, et une planche gravée. A Besançon, chez Victor Cabuchet.

Malgré le peu d'étendue de re livre élémentaire, on y trouve les articles suivans: $\Lambda$ pplications usuelles de l'arithmétique aux mesures anciennes et aux mesures nouvelles; règle de trois; règle de sociélé; équations numériques; règle d'intćrêt; règle d'alliage; mesure des surfaces rectangulaires; mesure des volumes des parallélipipèdes rectangles. B. Y.

2. Traité complet d'atithmétiQue, contenant, etc. Ouvrage adopté par l'académie des sciences, et reconnu propre à l'enseignement des premiers élémens des sciences mathémati iues; par L.-A. Бонцот. In- 8 de 18 feuilles et demie. Prix, 4 fr. A Lyon, chez Victor Lagier; à Louhaus, chez l'auteur.

L'académie des sciences n'adoptant pas d'ourrages, l'auteur a été invité à retirer cette phrase du titre de son livre.

TOME I. 
Outre les choses qu'on trouve dans les Iratite ord naire de ce genre, celui-ci contient:

$1^{\circ}$. La thérie des diffirens systimes de mumeration;

$2^{\circ}$. Celle de la divisibilité des nombres, avee 'quelgyues app. plications curieuses et utiles;

$3^{\circ}$. Notions et application des fractions continues. B. Y.

3. Tarif géxéral de toutes les nouvelles mesures el poids métriques réunis pour le solivage ; fait par J. Mitime en 1815 , in-Is de 9 feuilles un quart. 1 s's. A Givet, chez Gamaches-Colson.

4. Biblotheque nécessare, ou répeitoiredelout ce: que le systeme métrique offre de plus intéressant, par J.-J. Fenntox, in-18 de 5 feuilles. A Dijon. chez Noellat; et a Neuglet (Cóte d'or), rhez l'auteur.

5. Description d'un titalon metriele, omé dhiciroglyphes, découvert dans les ruines de Memphis, par les soins de M. le chevalier Drovett, consul général de France, en Égypte. In-\{́ de a firille's et demie, et une planche gravée. Imp. d'Eberhart, i Paris; par MI. Jomınn, de l'institut.

Le savant auteur de ce Mémoire a jeté un grand jour sur la recherche des mesures indiquées par ce précienx étalon. Iar longueur totale de l'instrument est de 520 millimetres; if porte vingt-lauit divisions principales, formant denx siriss placées bout à bout, la longucur moyerne des quatre divisions dont la première série se compose est de $0^{m}$, o r! $)^{n}$; celle des vingt-quatre divisions de la deuxieme série est de $0^{\text {m }}$, o 185 . Les signes hitruglyphiques qui comvent litalon indiguent, suivint M. Jomard, que les quatre pre mieres divisions sont autint de doigh, ou le prolme de lit

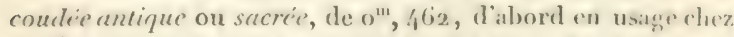
les Eggptiens, laquelle étant de 2.q doigts, a dome subu une augmentation de 3 doigis pour former la roudice de $\mathrm{Mem}-$ phis. L'auteur regarde cotte derniere coutée, contenue 400 fois dans l'étendue de lat face nord du chepleren, mesuree sur 
le socle, comme identique avec la condée noire ou d'al-Mimoun, qui itvait 27 doigts, et avec celle du nilométre d'Eléphantime, découverte par M. Girard. La coudíe nilométrique? des Arabes, gravéc sur la colonne du meqyâs, surpasse d'un doigt antirue la coudéc de Memphis, et d'un palme la coudée sacrée; et comme le pyk-belady ou coudée dupays vaut 30 doigts antiques, les rapports de longueur des coudées mentiomées sont $24,27,28$ et 30 , rapports qui se retrouvent dans les dimensions comparées des principales pyramides de Memplis.

Nous croyons devoir faire ici une remarque qui ne se trouve pas dans le Mémoire de M. Jomard: il est curieux en effet que la longueur totale des 24 divisions de la $2^{\circ}$. série, tracées sur la condée de Memphis, soit de $\mathrm{o}^{\mathrm{m}}, 443$, valeur de la dix-millième partie du pharsang ou parasange persane, et de la coudée romaine dont l'instrument porte ainsi l'étalon, et découvre peut-être l’origine.

B.

6. Tarifs servant à convertir les mesures générales de superficie, de longueur, de capacité et de pesanteur, anciennement en usage dans le départenent de la Charente, en mesures nouvelles analogues, suivis des prix cornparatifs entre ces diflérentes mesure's, redigés sur un nourcau plan, et mis à la portée de toutes les personmes quj, peu rersées dans le systime décimal, désirent connaítre sur-le-cliamp et sans le moindre uavail le rapport qui existe cntre les mesnres et poids. On y a joint un tableau comparatif des picees de 3 liv., 6 liv., 2/ et 48 liv., en fr.; par Fr. Trémau, imp. du Roi, ancicn chef du burcau de l'intérieur, à la préfecture de la Charente, chargé à cette époque du travail relatif à la mise en activité dus système métrique. In-8 d'une feuille et demie: I822. A Angouléme, chez Trémau.

7. Fińmens de Goumétrie, à l'usage de l'école centrale des Quatre-Nations: par S.F. LAcrorx. Dou- 
zienc edition, revue et rorrigáe. In-8 de a 6 feralles et denie: plus, 9 planches. Imp. de IItzard-Comprier, a Paris. A Paris, rhez Barhelier. Prix, fo fo.

Le zrrand nombre d'editions de ce traité et de tous cens qui composint fe cours complet de mathemaliques dont if fait partie, pourrait inous dispenser de toute analyse. Cependint, en nous aidant de celle que l'auteur lui-méme a faite dece cours, dans sen Eisctis sur l'enseignement, nous rappellerons que le but qüil s'est proposé dans la ridaction de ces élémens a cté d'írablir dans les propositions de son ouvrage un ordre conforme à celui des abstractions, en considérant d'abord les propriétes des liznes, puis celles des surfaces, et enfin celle des corps; et cela en conservant aux démonstrations toute leur rigueur. La premiere édition de cet ourrage est de lan $7\left(179^{8}\right)$.

Deflers.

8. Essurs de: GÉonćtnre sur les plans et les surfaces courbes (Élémeus de géométrie descriptive); par S.-F. Lichors. Cinquième édition, revue et corrigéc. In-S de 8 feuilles un quart, et" ro planches. Imp. de Inzard-Courcier, à Paris. A Paris, chez Bachelier Prix, $3 \mathrm{fr}$.

Cet ouvrage, dont la première édition a paru en $179^{5}$, complète le précédent, en offrant la solution des principales questions auxquelles donnent lieu les relations des plans, des lignes et de la sphère. Afin de préparer ses lecteurs à l'etude de la coupe des pierres, de la charpente et des autres applications de cette branche de la grémitrie, l'auteur s'est occupé ensuite de la génération et de l'intersection dessurfaces courbes, et a lerminé son ouvrage par l'exposition des principes fomdamentaux de lit perspective.

Deflers.

9. Traltí des propratétes phojectives des figures, ouvrage utile it ceux qui s'occupent des applications de la géométriedescriptive, et d'opérations géométriquessur le terrain; par J. V. Poncelet, ancien élève delicole polythernique, capitaine au Corpsroyal du genic. 1823 . In-f. xlvj, rt 426 pages; plus, 12 pl. 
en taille-douce. De l'imprimerie de Lamort, a Metz. Prix, $16 \mathrm{fr}$. Chez Barhelier, libraire, à Paris.

Le but de l'ouvrage que nous annonçons est indiçué dans la préface (v-vj) en ces termes: " D’après la facilité avec la"quelle les théories que j'expose conduisent à la plupart des "propriétés générales et particulières des figures, on de" meurcra persuadé que le but de ce livre, quelque volumi2) neux qu'il paraisse être, est moins de multiplier le nombre v de ces propriétés, que dindicquer la route que l'on doit "suivre. En un mot, j'ai cherché, avant tont, à perfection" ner la méthode de démontrer et de découvrir en simple " géométrie. C'est cette geométrie particulière (pag. xIx) qu'il v faut chercher actuellement à perfectionner, à généraliser, à " rendre enfin indépendante de l'analyse al cébricque. " L'auteur croit qu'on pent fonder cet état d'indépendance à l'aide de la doctrine des projections et du principe de continuité, ouvertement admis, et consenablement appliquú. La doctrine des projections consiste à considérer la figure dont on veut étudier les propriétés, comme dérivée par un mode donné de transformation, comme projection prise dans le sens gé néral, d'une autre figure dont les propriétés sont commues. Le principe de continuité consiste à admettré que ioufes les propriétés de la figure se reproduisent dans la figure transformée. Le tout est de sawir les reconnaitre; à eet effet, supposons qu'un plan tournant aulour d'une droite fixe aille rencontrer un cône du second degré. $\Lambda$ chaque position du plan correspond une ligne d'intersection. Malgré la diversité de formes 'qu'affectent les courbes ainsi obtenues, on peut les regarder toutes comme des transformées (projections centrales de l'inteur) d'une quelconque d'entre clles. Il suffit doncrle bien examiner une deces lignes, cl, au moyen du prineipe de continuité, dadapter au système cnticr les résultats de cet examen. C'est ce que MI. Poncelet a cxécuté avec beaucoup de sagacité; il est parrenu à démontrer les propriétés les plus compliquées des sections coniques, et à résoudre les problèmes les plus difficiles qu'elles présentent, en choisissant pour ligne de comparaison, la section la plus simple el la mieux connue, le cercle.

L'ouvrage est divisé en quatre sections, ct claque section 
en trois chapitres: la $x^{r e}$. section $(2-23)$ renferme le's principes généraux, la théorie de la projection centrale (perspective) et des faisceanx harmoniques; les propriétés fondamentales des sections coniques ef des cordes, on sécantes idéales. L'auteur appelle corde on sécante idéale la droite fixe dont nous avons parlé ci-dessus, dans le cas où elle est entierement liors du cone, et lorsqu'on ramene toutes les

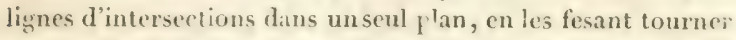
autour de la droite comme charniere. M. Poncelet tire un parti trís-avantageux de ces sécantes idéales, qui sont les axes radicaux de M. Gauthier. (Journal de l'Ébole poly teithnique, Tome IX.) I.e troisieme chapitre est d'un grand interèt; l'auteur y diecute les cas où plusienrs contes du secont degré peuvent être considérées simultanément comme les projections centrales (perspectives) de plusieurs cerrles. Ia seconde section $(-6-193)$ est consacrie a la grénmetrie dite de lat règle, et contient lat theorie des transversales, des póles et polaires, dessections coniques semblables, et semblablement placées. On y trouve des idées ingrinieunes sur les centres de similitude; c'est ainsi que l'auteur désigne les points de rencontre destangentes communes a deux lignes du s- eond ordre, semblables et s.p.; l'auteur donne la solution complete de tous les probiènes relatifs anx contacts des cereles. La troisième section ( $156-25.4)$ traite des systèmes des sections coniques, des centres d'homolosir; rest ainsi que l'auleur nomme le point de renentre des tangentes communes a deux lignes du second ordre. l.es théories des tangentes communes, des donbles contacts, et les problemes qui s'y rapportent; les constructions des sections conirgues assujetties a certaines conditions, sont exposés avee les développemens désirables. Lat quatrieme et demière section $(257-366)$ traite des angles constans on variables, suivant certaines lois; des polygones incrits ou circonscrits a des sections coniques, et dont les cottés sont assujettis a passer par des points lixes; ou dont les sommets sont assujettis a se trowver sur des droites ou des courbes d'un ordre queleonque. Celle parlie de louvrage renferme des propriétís neuves, tres-eurieuses, dirnes de lattention des géomitres; la question des polygones est tratic avec une admirable intelligence du sujet et une 
grande: supériorité de moyens. Datns le supplément (36gfig) placé a la fin de l'ouvrage, on trouve des considérations sur les fiçures considérées dans l'espace et sur les surfaces du second degré, et une application très-utile au tracé des basreliefs.

T.I. Poncelet a rendu un grand service aux savans, en ratfachant an seul principe de projection les nombreux problemes 't theoremes dispersés dins beaucoup d'ouvrages. Il a enrichi la science de beaucoup de fitits nouveaux et utiles. Dans l'introduction ( $\mathrm{xv}-\mathrm{xLvj}$ ) on lit l'histoire trés-instructive et trés-impartiale des iravaux qui nnt précédé ceux de l'auteur; et partout MI. Poncelet rend justice complète à ses devanciers daus une carrière qu'il parcourt avec honneur et distinction.

J. Terquen, professeur.

IO. ON ANNONCE comme devant paraître prochainement, nu Ménoire qui présentera beaucoup de faits nonveaus sur los fractions périodiques en général, quelle que soit la base de numération : ce!te théoric parait devoir faciicier les recherclies de l'analyse indéterminée. Voici l'énoncé de quelques théorèmes sur les fractions décimales.

Aucun nombre qui affecte les formes suivantes:

$p=40 n \pm \mathrm{I}, p=40 n \pm 3, p=40 n \pm 9, p=40 n \pm 13$, ne peut avoir $p$ - 1 de chiffres décimaux à sa période, ou, ce qui est la même chose, io est une racine non primitive de tous les nombres de cette forme. Donc io n'est racine primitive que dans les seuls cas ou $P$ a l'une des formes $40 n \pm 7,40 n \pm 1 \mathrm{I}, 40 n \pm 17,40 n=19$. Des théorènes ont fait connaitre les cas où, dans ces dernières formes, la va. leur de $n$ rend o, racine primitive. Ces diverses reclierches ont fait apercevoir quelques crreurs dans Ja table des grandeurs de la période décimale de MI. Burchardt, placéc à la suite de sa Table des nombres premiers du premier million.

Si $n$ est la base de numération, on aura cette propriéć : $n$ ne peut jamais être racine primitive d'un nombre qui a la forme $4 n \pm \mathbf{I}$, et plus généralement $4 n \pm r^{\mathrm{a}} \pm r^{b \pm r^{r}}$ $a, b, r$, étant les diviseur's de $p-1$.

Bertievin.

1. Ie Cambiste univinsel, ou Trailé complet des changes, banques, monnaies, poids, mesures et. 
règlemens de commerce de toutes les nations. In 18 d'une demie feuille. Imprim. de Rignoux, à Paris. L'ourrage, composé de a vol. inblić dans le conrant de janvier 1823. Pris $1_{2} \mathrm{fr}$. A Paris, chez bossange freres. (Prospectus.)

Dès que cet ourrage paraitra, il en se ra rendu compte.

MATIEMATIQUES TRANSCENDANTES.

12. Múmones str l'ixtégrition des équations anx différences particlles, et sur la distribution de la chaleur dans les corps solides; par M. Porsson.

L'un de ces Mémoires, qui paraitront dans le tome $12^{\circ}$. du Journal de l'École polytechnique, at été lu à l'Académie en mai I $8_{2}$ I, et les denx autres en décembre de la mème année.

Le premier contient une démonstration des éfuations du mouvement de la chaleur, établie sur les principes phrsigues adoptés par MI. Fourier, mais présentée sous une forme diffírente. On y trouve les conditions relatives an passage de la chaleur d'un corps solide à un autre qui est en contact isec lui. Les équations sont appliquées à diverses questions, telles que le mouvement de la chaleur dans une barre prismatique, dans un anneau, dans une sphère homogène, dans une syhère composíe de deux parties de matières différentes, dans un parallélipiperede rectingle. L'analyse que l'tuteur emploie pour la solution de ces questions, consiste à appliquer à des corps compris entre des limites données, l'evpression. sous formedintegrale difinie, qui convient sprécialement aux corps d'une itendue illimitie; puis, à faire servir les conditions relatives anc limites, a tramformer l'expression dont il s'agit, en detrminant convenallement la fonction arbitraire qui s'y tronve contenue. Lanteur retrouse, dans les eas déja traites par II. Fonnier, les formules auxquelles ce dernier greometre étair parvenu par la distinction et la composition des mouvemens simples.

Le second Mémoire contient l'inteyration de diverses équantions aux différences partielles, relatives au mouvement de lit chaleur. Coctte integration est effectuce en développant les in- 
tégrales cherchées en séries, et sommant les séries par des inlégrales définies.

On trouve dans le troisième Mrémoire uno nouvelle démonstration de l'équation du mouvement de la clsaleur, qui convient aux points de la surface des corps, et une nouvelle énonciation des conditions relatives au passage de la chalcur d'un corps dans un autre. Il contient ensuite les solutions de diverses questions, telles que le mouvement de la chaleur dans une splière et dans un cylindre échauffés d'une manière entièrement arbitraire, dans un corps dont quelques dimensions sont infinies, dans une sphère ou une barre composées de deux matières différentes. La solution relative à la sphère s'accorde, pour le résultat, avec celle que M. de Laplace avait donnée dans la Connaissance des Temps pour i 823. L'auteur emploie pour quelques-unes de ces questions l'analyse dont il avait fait usage dans le premier Mémoire, et qui a été indiquéc ci-dessus: il revient dans d'autres cas à une analyse semblable à celle qu'a adoptée M. Fourier, et qui consiste à développer l'intégrale cherchée en une série de termes affectés de coefficients coustans. On détermine ces coefficients de manière à satisfaire aux conditions relatives aux extrénités des corps, et à l'état initial représenté par une fonction arbitraire.

Ces mémoires offrent de nouveaux exemples de l'habileté de l'auteur dans l'art difficile de manier le calcul analytique.

$\mathrm{R}$.

I3. Hrdmatlisk ғoеnsoek anstoellda vid Fahlu grufva, etc., c’est-i-dire, Expérienceshydraulicques faites aux mines de Fahlun ( cn Suede), par MIM. LAgramelix, Fonselfas et Kallstenus, anx dépens de la société des maitres de forges (bruks socictet). Stochlolm, 2 vol. in-8, l'un de 3 . fo pages, avec 9 planches; l'autre de 35 pages, avec 6 planches. Stockholm, i $818-$ i 822 .

D'après le compte rendu de cet ouvrage à l'Académic royale des sciences de l'Institut, et l'opinion manifestéc par M. Girard, membre de cette compagnie, il serait à désirer qu'il füt traduit dans notre langue. Voici les titres des cha- 
pitres rlont il est conposí. Ces titres suffiront pour finir juger de son importance.

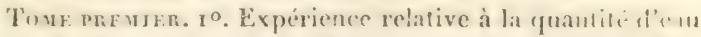
qui sicenle par des orifices a minces parois, an fond d'un reservoir dans lequel la surface de l'eau garde constamment la mème hauteur.

$2^{0}$. Sur la quantité d'ean qui s'écomle par de cemblabla orifices, au fomi d'un bassin donl la grrmeleur pront dire supposéc sans influence sur la quantité d'eau.

30. De la vitesse de l'ean dans la moindre section de: la veine fluide contractée.

"4. Sur la difference qui se: Irouve entre la quantiti d"an qui s'écoule, d'une part duus l'eau, et de l'autre dans l'aiy libre, par une ouverture pratiquee an fond d'un bissine

50. Sur la hauteur de pression correspondante à la vitesse" de l'ean, a ume distance inegale du plan de l'onverture; cente liautcur de gression étant rapporté a une ligne verticale, aboutissant au centre de l'orifice pratiqué au fond du bal nius.

6o. Sur la pression qu'une colonue d'ean trantulthe iprouve de la part diune colome d'ean courante, dias leass. plan de contact.

$7^{\circ}$. Exprérience relative a la qliantité d'eau qui seronle par-dessus un deversoir, lorsque la veine fluide commenes: à la surface de l'eau.

8\%. De la quantité d'air, qui, sous des pressions inigales, s'échapje par des orifices a minces parois, et sur la sucliou qui a lieu lorsque l'air est conduit par des tuyaux courts. (Un rapport a eté fait a l'Acadénie sur cetle partie do louvrage, par MIT, Girsed et Ampère, d'aprés une lraduc: tion envoyée par M. Ollivier.)

$9^{\circ}$. Exprerience relative a la resistane qu'éprouvent les corps qui se meuvent dans des eaux tranquilles.

TouE nfexneur. 1\%. Theorie des roues hydrauliques ¡ui recoivent l'eau par-dessus.

$2^{\circ}$. Sur les rones livinauliques qui recoivent l'ean fia dessous et qui sont mut's par le seul poids de l'eau.

3o. Expériences à ce sujet.

C. M1.

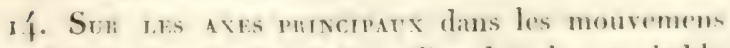
de rotation, or utr le milien le plus probable: 
a prendre entre des observatious données; par M. Svanberg. ( Mím. de l' Acad. des Se. de blochholm, х82x , p.388. )

L'auteur appliquant sa méthode au changement anıuel dans la déclinaison de létoile polaire, en conclut, d'après des observations faites dans le's années $\times 79^{3}-1799$, que cette variation doit ètre évaluée à $19 "$ ", 34.,1l emploie aussi sa méthode pour trouver le milicu probable entre des observations de distances angulaires, faites au moyen du cercle répétiteur de Borda.

C. M.

15. Traité élémentarne du calcul des probabilités; par S.-F'. Lacrorx. Denxième édition, revue et augmentée; avec cette épigraphe: " 11 ne faut pas receroir les opinions de nos pères comme drs cnfans, par la seule raison que nos pères les ont cues." (Pensées de Marc-Aurile, trad. de Joly, chap, ig, 9.9.) In-8 de 22 feuilles; r planche. Imp. de Inzard-Courcier, à Paris. A Paris, chez Bachelier. Prix, $5 \mathrm{fr}$.

Dans cet ouvrage, l'auteur s'est proposé de démontrer les principes fondamentaux du calcul des problabilités, en ne s'appuyant que sur les dimens d'algèbre. Il en a offert ensuite des applications variées, soit aux jeux, aux probabilités de la vie humaine, aux rentes viagères, aux assurances, atux témoignages et aux observations. Il a multiplié les résumés de manière a présenter une exposition du sujet qui peut être lue en passant, si l'on veut, les calculs. Dans la vue de prépares à la lecture des ouvrages les plus élevés, il a joint des notes qui montrent comment on peut appliquer aux questions de ce genre l'analyse transcendante.

La première édition est de $18 \times 6$.

Deflets.

16. Letro à messicurs les anciens élèves de l'b́cole Polytechnique et les membres de plusieurs neadémies des sciences, in-s d'un quart de feuille. Inpriserie de Momnoyer, au Mans, Par Dutertre, ex- 
rapitaine d'artillerie et ancien élève de l'école Pulitechnique.

C.ct ouvrage dtant inconnu a Paris, et n'itant annonce chez aucun libraire de la capitale, on n'a pu se le procurer pour en donner l'analyse.

\section{MACIIINES ET CONSTRUCTIONS.}

I7. Collfatiox ne michines, diustrumens, ustenciles, constructions, appareils, etc., emplorés dans léconomie rurale, domestifue et industrielle, d'apres les desnins faits dans les diverses parties de lFurope; par M. le comte de Listexre. Deuxiéme

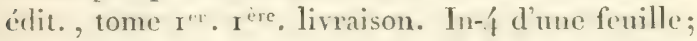
plus, de dix planches et un froutispice gravé. Imp. de Lebel, à Paris. A Paris, chez Árthus Bertrand.

Les souscripteurs qui paient d'avance la totaliti de l'ouvage, lauront a 70 fr. Le prospectus de cette nouvelle dition a paru quelques temps avant, en un quart de feuille in-f. Elle formera 2 vol. in-'t, en 20 livraisons, comtine lit première, du prix de $4 \mathrm{fr}$. chaque.

18. IXY EXTIONS, MODIFICATIONS ET PERFCCTIONNEMENT

de plusieurs instrumens conecruant la gnomonique (l'art de tracer les cadrans solaires), la phrsigue: et lhorlogerie; à publier ou ì exéruter sucessirement sur la demande gui pourra itre fitite de dracm de ces instrumens, an choix, par écrit, adressé frame de port is la librairie de P. II. Taurquelin. ruc Cit-le-cour, n. 4, a Paris. In-S. dune demi-linill(. Imp) de Latureux, ainé, ì Paris.

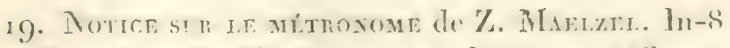
d'unedemi-fenille. Imprimerie de Constant Chantpic, à Paris.

Ie metronome ent un pendule a deur lentilles, sitnes de prart et d'autse du point de suspension qui me fixe. La lenville supérieure peut ète placéc a un endroit queleonrpe 
de la verge du pendule, le long de laquelle sont gravées des échelles propres à indiquer le lieu que doit y occuper cette lentille, pour que les battemens du pendule aient des durées relatives aux mouvemens des diverses espèces de mesures.

B.

30. Exrosé d'un nouveau système de fabrication des montres; par Z. Vicentr. In-f́ d'une feuille et demie. Imp. de Rougeron, à Paris.

2 j. Mécanique industrielle, ou Exposé de la sciencr de la mécanique déduite de l'expérience et de l'observation, principalement à l'usage des manuficturiers et des artistes; par M. Cnnistran, directeur du conservatoire royal des arts et métiers à Paris. Cet onvrage sera composé de 3 volumes in-f et d'un atlas de 60 planclues. Prix, $75 \mathrm{fr}$. Se vend à Paris, chez Bachelier, libraire, quai des Augustins; et chez Gocury, libraire des ponts et chaussćes, quai des Augustins. Tout l'ouvrage aura paru en juin 1823 . Le premier volume contient $49^{6}$ pages, neuf planches doubles de dessins de machines, formant une première partic de l'atlas de Go planches; et deux planches simples de figures relatives aux expériences et aux notes qui sont à lia fin du volume.

L'auteur divise l'étude des machines en trois partics: I ${ }^{\circ}$ Les moteurs. $2^{\circ}$. Le systène de transmission qui met le mouvement à la disposition du manufacturier. $3^{\circ}$. Les parties de la machine spécialement destinées à un travail particulier.

Le premicr volume, entièrement consacré à la première division, traite d'abord des moteurs en générit, et de leurs modes d'application. L'auteur essaie de mettre à la portée des personnes qui ont peu de connaissances mathématienes les principes mécaniques qui servent de base à la théorie des. machines, et qui doivent guider dins la recherelie de lat mesure de leur effet. Il annonce (qu'il traitera des six moteurs 
suivans: Thomme, les animan, l'cau, lo vent, lat wapeures la dilatation des solides. Le reste du premier volume est consacre aux trois premiers moteurs. A larticle de l'homme, il donne des extrats des differens anteurs qui ont traté cetle matière. L'article des animanx est fort court. In tratiant de l'eau, l'auteur rapporte les experiences de Morosi sur te choc des fluides. Il donne les résultals de celles qu'il a faites sur le même sujet. Il rapporte ausii lés expériences de Bossut et de Sincaton sur les roues a aubes et les roues a augets. If donne les résultats de quelques expériences qu'il at faites avec des roues de $2^{\mathrm{mi}}$ a $10^{\mathrm{m}}$ de circonférence; ces expériences, quoique de trì-peu de duriée, ont donné des résultats assez approchés de ceux de Smeaton. L'autcur expose ensuite les régrles qui doisent gruider dans le choix des roues hydrauliques, et dans la manière de les établir suivant les circonstances.

I'auteur donne en outre des expériences sur les écoulemens de superficie arec des orifices de 2 a 8 décimètres do latgeur, et de 1 à 8 centimitres de hauteur. Il a joint $\mathbf{S}_{2}$ pasces de notes d'arithmétique, le mécanique et de pliysique, qu'il a crues nécessaires à lintelligence de louvrage. Le volume est terminé par une légende explicative des 36 machines que contient l'atlis de ce volume. Les planches ont ¿té dessinées et gravées avec beancoup de soin par M. Leblanc.

G. C:

23. Croux des moyens propres à soulever les navires submergés dans les lieux sujets aux flux et reflux, recueillis dans les mémoires envoy és au concours ouvert par la Société des sciences, belles-lettres et arts de Bordeaux; seconde édition, I vol. in-í, 5 planches. Prix, 7 fr. 50 a. A Bordeaux; et à Paris, chez Barrois l'aîné.

La société des sciences, belles-lettres et arts de Bordeaux, frappée des inconvéniens qui résultent pour la navigation dans les ports de mer et les fleuves, de la présence des navires submergés qui y forment des ecueils diantam jlia dangereux que les courans sont plus rapides, avait mis an concours, il y a quelques années, la question suivante: 
"Quel est le moyen le plus sûr de soulever lés corps submerurés a une profondeur déterminie, quelle gue soit leur "pesanteur, dans un lieu où le flux et le reflux se font "sentir?"

La société prévint dans son programme que l'attention des concurrens devait principalement se porter sur les navires snbmergés, parce qu'ils présentent pour les soulever et les saisir des obstacles bien plus grands et bien plus nombreux que ceux quoffrirait dans le même cas tout autre corps.

Plusieurs mémoires remarquables lui furent adressés, mais aucun n'ayant pu atteindre complétement le but que la soriété s'était proposé, la question fut remise an concours pendant quatre années consécutives. Il en est cependant résulté des vues utiles, propres à ètre mises en pratique, et que la société a jugé avantageux de faire connaitre. On Irouvera dans ce volume l'indication des moyens proposés par M. Georget, ingénieur en chef des ponts-et-chaussées, que la société a couronné, et à qui elle a accordé unte médaille de la valeur de la moitié du prix proposé. Elle y a joint l'analyse du mémoire de M. Roland, ancien officier de marine a Brest. Les moyens qu'il propose sont ingrénieux, mais ils ne peuvent ètre employés qu'autant que le fond serait de vase ou de sable. Afin de compléter la série des renseinnemens recueillis sur cet important objet, la socicté a jublié les moyens puisés dans ses archives, et qui ont été 'prouvés lors du soulivement d'un navire submergé dans le port de Bordeanx, ayant i bord deux cents tonneaux ou quatre cent mille livres de roche. Louvrage se termine par la relation des traviux faits pour relever le navire le Tojo, coulé bas le 10 octobre 1702 , dans la baie de ij, et mis a trre le 6 férrier 17,42 , par H. Gaubert, ancien aficier de maxine.

Desplanclies graven avee soin sont jointes a cet ourrage, et facilitent l'intelligenec des divers moyens qui sont proposés.

B.

23. Le petit anchitecte, ou Tracé linéaire appliqué a l'art de representer toutes sortes d'oljets en papier et en cartom. tols que des maisons, palais, temples; 
monumens, jonts, meubles, ctc., à l'usage de l'enfinece et de la jeunesse; par D).-II. Ronekstron et F.-fx. Brigar rin. In-12 de 5 fenilles et demie; plus 22 planches. Imprimerie de Darid, à Paris. A Paris, chez Eymery. Prix, $5 \mathrm{fr}$.

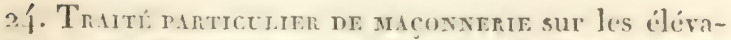
tions des légers ourrages, par Alexandre Dr.u 1.f.r, architecte expert, autur du traté simple ct concis descionctric pratique, applicable au mesurage de toute esprece d'our rages de bitiment: deuxiemédition. In-s de iz feuilles. A Paris, chez CarillanGoeury. Prix, 3 fr.

25. Étunes relatives a l'art des constructions, recucillies par L. Bra vine, oflicier de la Iérgion-dlHonneur, inspecteur général des ponts et chaussces, maitre des requetes, et ancien directeur des tratvaux de Paris. (Mutrieme recueir.tusfol. de a feuil.; plus, r.́ pl. Imp. royale, à Paris. A Paris, chez Bance aîné.

Il y aura 12 recueils, qui contiendront :

I. ponts en pierre.

II. Greniers publjes et halles aux grains.

III. Ponts en fer.

IV. Foires et marchés (le $\mathrm{I}^{\mathrm{er}}$. dans lordre de publication, et le seul qui paraisse encore).

V. Navigation.

VI. Abattoirs et boucheries.

VII. Details relatifs aux portes d'écluses, pont en bois et antres constructions.

VIII. Petites maisons de ville et de campagne.

1X. J)es tuiles antiques et modernes, et, en général, des couvertures.

$\mathrm{X}$. Esquisse d'une petite ville maritime.

XI. Projet de diverses constructions indiquées sur le plan des villages de...

XII. Mélanges. 
Le $4^{\circ}$. recueil, le seul qui ait enrore para, se compose de 8 pages gr. in-fol. de texte, et de 13 planches gravées au trait.

Le terte contient : I $^{0}$ quelques notions historiques sur les foires qui ont existé à diverses époques dans la ville de Paris; $2^{\circ}$. des considérations générales sur les marchés de la méme ville; $3^{\circ}$. le programme d'un marché pour la vente ess détail des comestibles, qui avait été étudié avec le plus grand soin sous le précédent gouvernement, et qui a servi de base à la disposition des marchés construits sous la direction de IM. Bruyère; $4^{\circ}$. des notices particulières sur les divers marchés de France et d'Italie, et sur les marchés construits ou projetés à Paris.

Les planches offrent les dessins des édifices dont il est fait mention dans le texte. On remarque principalement ceux des marchés Saint-Martin, des Cirmes, Saint-Gervais, SaintGermain; de l'entrepôt général des vins et eaux-de-vie, et d'un marché projeté par M. Alavoine, près de la place de la Bastille.

La composition de cet ouvrage est très-soignéc, et les planches sont gravées avec beaucoup de précision et d'élégance.

R.

26. Recueil o'expériences et d'observations faites sur dilférens travaux exécutés pour la construction du pont de Nemours, pour celle de l'arsenal et d $n$ port militaire d'Anvers, et pour la reconstruction du port de Flessingue, dans lequel on a traité la thé:rie de l'équilibre des voûtes; par L.-C. Borstanı, ingénieur en chef, directeur au corps rojal des ponts et chaussées. I vol. in- $\{$. avec i 7 planches. Prix, 12 fr. A Paris, chez J.-\$. Merlin, libraire, quai des Augustins, n. 7 .

Ce recucil d'experiences et d'observations est divisé en trois partics; la premiere comprend:

$\mathbf{1}^{0}$. La description abrégéc du pont de Nemonrs en tro: arches, et particulierement remarquable par siz hardics.e.

2o. Lextrait du jonenal des travaux eseculés, dans chaque campagne, pour la construction de ce pont.

'оме I. 
3०. Le calcul des ordonnées nécessaires ponr tracer les arches de $9^{3}$ pieds de mayon ( $30 \mathrm{~m}$. 2x c.); ceux nécessaires au tracé de l'épure, a la distribution des joints entre les voussoirs, et à la fixation de leur largeur différente, eu égard au tassement; cenx relatifs an surhaussement des arches pour prérenir l'effet du tassement, etc.

$4^{n}$. Des expériences sur la main d'ouvre des differens ouvrages excecutés pour la construction de ce pont.

5 . Le rourcau moyen qui a ćé emplopé pour décintrer les trois arches en même temps.

6o. Des expériences sur le produit des machines à épuiser, employies a la construction du mème pont.

La seconde partie comprend :

$I^{\circ}$. Des expériences pour la siabilité des voûtes, y compris celles en plate-bande.

$2^{\circ}$. Des remarrques sur ces expériences, et l'application de calcul.

$3^{\circ}$. Des expériences sur la force d'adhérence des mortiers de chaux et sable et de chaux et ciment, employés a l'air et sous l'eau, et pour combien ils entrent dans la résistance de la puissance.

$4^{\circ}$. Des expériences sur le frottement des pierres.

$5^{\circ}$. L'application des expériences sur la stabilité des voûtes à celles du pont de Nemours.

La troisième partic comprend:

$1^{\circ}$. L'extrait des précis historiques des travaux de fondation d'une forme, au port de la marine militaire a Anvers

2 ". Une notice sur la reconstruction de la grande écluse du bassin du port militaire de Flessingue, détrnite en $180 \%$ par les Anglais.

30. Différens systemes de charpente employés dans la construction de l'arsenal maritime d'Anvers et dans le port dc Flessingue.

$4^{n}$. Enfin, lindialion des mesures et précautions que doivent prendre les ingénicurs, lorsqu'ils sont claregés de projeter et diriger des travaux d'art.

Le seul énoncé des matieres qui sont traités dans cet nuvrage, le nom et les fonctions de son autcur, suffisent pour en faire apprécier toute l'importance. 
27. Traité sur l'art de ia chanpente théorique et pratique, publié par J.-Ch. KnafFt, architecte, et rédigé par M. le colonel A.-F. Lonet, ancien ingénieur en chef des ponts et chaussées, l'un des professeurs del'ancienne école polytechnique, etc. Sixième partie, construction des théàtres, première section. In-folio de 8 feuilles et demie, plus 30 planches. Imprimerie royale, à Paris. Prix, $3 \circ$ f. A Paris, chez l'anteur, rue de Bourgogne, $\mathbf{u}^{\circ} .25$; chez Firmin Didot; chez Rey et Gravier; chez Treuttel et Wurtz; chez Goeury : à Manheim, chez Artaria. Le texte est imprimé sür trois colonnes, (français, allemand, anglais.)

Cette sixiè me partie, la dernière du traité de charpente publié par M.Krafft, comprendra deux sections; la première, qui vient de paraitre, contient la description de quelques théâtres, où I'on a mis beaucoup de soins et d'intelligence dans le choix des dispositions générales et dans la distribution des machines. On y a réuni plusieurs exemples de la construction des combles de divers théutres existans, ou seułement projetie tant en France que dans les pays étrangers, ce qui comprend la description des planchers supérieurs, celle des galeries de service ei de toutes les combinaisons adoptées jusquàa prísent, tant pour la répartition que pour l'établissement des machines. M. le baron Lomet qui, par zele pour la propagation des aris, et par amitié pour M. Kraft, a bien voulu enrichir ses dessins d'un texte technologirque, enscigne tout ce qu'il faut savoir, tout ce qu'il faut faire pour l'établissement d'un théâtre, et signale les fautes qu'il importe d'éviter dans ces sortes de constructions, les plus compliquées et les plus difficiles de toutes celles: qu'il est possible d'entreprendre. Le texte de II. Lomet, remarquable par la facilité avec larpuelle viennent se groupes autour du sujet principal tous les objets accessoires plus on moins éloignés, contient des idées originales, toujours des iugemens justes, mas parfois sévères, et qu'on ne peut bli mer, parce que c'est surtout dans la pratique des arts de 
construction que les recherches et l'examen appiofondi des fantes materielles hien caraclérisées instruisent micux gue des préceptes. I.es matières embrassées par cet ouvraire sont distribuées en trois paragraphes, de l'Arehitecture des theatres, des Décorations et des ornemens, des Muchines de thécitre, suivis de légendes instructires et trés-dévelopdées sur les détails de construction représentés dins les trente planches.

Nous eroyons faire plaisir à nos lecteurs on rappelant ici que les cinc premieres parties publices par M. Krafft, de I 81 y a i $8_{22}$, traitent des matières suivintes. I'remiète partic; des assemblages, des armatures des poutres, des voutes et. des combles courbes construits en planches, des soffites, des parois, des planchers; dix feuilles de texte et trente planches; prix, 25 f. Secunle partie: des escaliers de toute forme et de toule espece, dix feuilles de texte et trente planches; prix, $26 \mathrm{f}$. Troisiéme purtic : du tracé et des principales propriétés des courbes usuelles en architecture, de l'édrographic, du dive loppement des surfaces des corps usités diuns l'art du trait; système des projections, des angles plans, des angुles solides, ctc., 3 I feuilles de texte et 30 planches; prir, 30 f. Quttricme partic: des combles de toute forme et de toute esperee, 7 feuilles de texte et 30 planches, prix, 26 f. Cinquieme purtie': combles d'église, dòmes, coupoles, clochers, beffrois, cte., 4. feuilles de texte et 30 planches; prix, 26 f. Les recueils de M. Krafft sont très-utiles aux personnes qui s'occupent des arts de construction; elles n'auront que l'embarras du choix, ee? qui vaux mieux que d'être dépourvu de modsles, et l'on en chercherait vainement ailleurs une plus ample collection. H.S.

28. Mivorne, plan et observation sur les ouvrases à exécuter au port de Cette, présentés à son Exe. le: Ministre de l'intéricur, le 6 fructidor, an X; par J. Rours, ex-capitaine d'infanterie, citoyen de la ville: de Cette. Imprimerie de J. Martel le jeune, à Montpellier.

y. Onserratrons sur lesourrages a cxéculer an pout

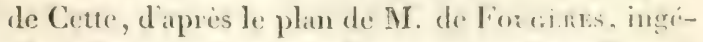
nieur des ponts ct chitussées, envoyées a sun Fre. le: 


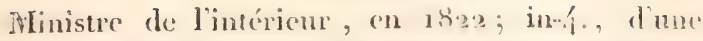
fruille. Imprimerie de J. Martel le jeune, a MTontpellier.

Ces deux ouvrages inconnus ì Paris n'ont pu être analysés. F.

3.). Prospectus du canal projeté, de Dieppe à la wiviere d'Oise. In-8 de a feuilles et demie. Imprimerie de Boucher, à Paris.

On s'est beaucoup oceupé des moyens de rendre Paris nu port de mere, ou plutôt de lui en procurer les avanta¿̂s; la navigation directe n'a pas toujours été regardée ‘omme une chimère; nous possédons un mémoire de Forfait, dans lequel eet habile ingénieur discute les avantages de plusienrs projets de communication. Mais Dieppe étant le port de mer le plus rapproché de Paris. On a dù souvent s'occuper de la pensée de créer un canal pour joindre les caux de la Seine a celles de la mer par une voie plus prompte et surtout praticable en tout temps. La difficulté la plus grande qui se présentait était de tronver un point de départ pour les caux. Les obstacles du terrain, ceux qui résultent de la nécessité de faire passer le canal par les villes les plus jopuleuses qui se rencontrent dans la route, ont fait naitre des projets plus on moins ingénieux; mais comme tontes ces entreprises roulitent sur un devis d'environ cinfuante millions, l'exícution n'a pas tenté les capitalistes. Le prospectus que nous amoneons admet des bases nouvelles, et nous formons des voux pour qu'elles paraissent, autant aux f:'ns de l'art qu'aux actionnaire's, susceptibles d'une exécutioir ficile et d'un produit assuré.

Bentuevin.

Sr. Récue mes eatr, des rivieres navigables, flotiables ou non, et de tous les autres cours d'eau; des obligations, droits et actions qui en résultent pour lítat et pour les particuliers, et de la compétence des ántorités atministratives et judiciaires, meme des justices de pais en matiere possessoire suivant la jurispredenee du conseil d'état et de la cour de:

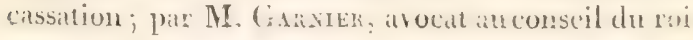


et a la eour de cassation. I volume in-s de 356 pages; se vend a Paris chez l'auteur, rue de líperon, n. Io. Prix $5 \mathrm{fr}$.

Qnoique cet ouvrage ne soit qu'un recueil de jurisprudence franciase, ecpendant il sera fort utile amx ingenieurs, aux manfacturiers et aux mécaniciens qui ont besoin, pour l'établissement des usines sur des cours d'ean de France, de connatre la jurisprudence qui regle le droit des particuliers entr'eux et envers l'ćtat.

G. C.

32. Lf Gorvenximext de la rípublique de la Platia a fait des offres avantagreuses a plusieurs savans anţlais. M. Béran, habile ingenieur civil de Londres, s'est embarqué avec sa famille pour Juenos-Ayres, ou il doit surveiller les travaux des chanssées qu'on éleve sur les rives de la Plata, et introduire dans le pays celles des sciences européennes qui influent sur la prospérité publique. (Revuc encyclop. déc. I322. p. $6 \times 8$.)

TOPOGRAPHIE, GÉODÉSIE.

33. Mémonesur le figuré du terrain dans les cartes topographiques. In-8 de 3 feuilles et un quart, et une planche gravée. Imp. de J. Didot, ainé, à Paris. Signé, le général II. ( Ve se reud pas.)

MI. le général II. divise les pentes des terrains en trois classes: $1^{\circ}$. les pentes douces, comprenant toutes celle's dont la hase est plus grande que cinq fois lit hauteur; 2 ". les pente's reides, dont la base est plus petite que ciur fois lat hauterur; 3". les escarpemens, comprenant toutes les pentes qui ont plus de hauteur que de base. Pour rendre les deux premieres classes, l'anteur se sert de hurlures ou projections des lignes de plus grande pente, et recommande l'usage des trunches ou projections des combes horizontales, pour exprimer les escarpemens. (Duand les pentes sont douces, Mr. le erénéral 11. adople la bi de leeplacement des hachures propurtionnel a la lonsurne de celles-ci, entre des tranches vertiealement Equilistantes, proposie par H. Fomoit, dans ses lecons à lit: cole d'anplication du Corperoyal d'état-major. Cette loi a ete 
modiliéc sur les pentes raides; car M. le général II. y fait l'espacement des hachures égal au seizième de l'excès de un centimetre sur leur propre longueur, et grossit alors ces hachures à mesure qu'elles deviennent plus courtes, suivant une loi qui n'est pas énoncée. Ce qui caractérise les méthodes de M. le général II., c'est l'exclusion des tranches horizontates continues, verticalement équidistantes, pour limiter les longueurs des hachures; de sorte que l'écartement de cellesci devient l'indice principal des inclinaisons des pentes, leur longueur élant arbitraire. D'ailleurs, pour olstenir l'écartement voulu, H. le général H. suppose, comme cela doit ètre, que l'on a exécuté sur le terrain des opérations telles que la forme en soit parfaitement déterminée, et desquelles on pourrait évidemment alors conclure les tranches continues généralement employées. La raison qui, sans doute, a porté l'auteur à modifier ainsi la méthode de M. Benoit, est la difficulté que l'on éprouve dans le tracé des hachures voisines des lignes, soit de partage, soit de réunion des eaux, quand on se conforme à l'usage, et que l'on ne trace pas, d'une manière particuliere, ces lignes caractéristiques des formes du terrain, pour lcur faire aboutir tangentiellement les hachures voisines, comme cela est dats la nature. La résistance des terrains, avoisinant les lignes de partage des eaux, à laction destructive des pluies, et la formation des ravins le long des lignes de réunion des eaux, par les torrens, résultent en effet de la disposition indiquée des lignes de plus grande pente. Le mémoire de M. Ie général II. est terminé par une table pour réduire, en degrés et grades, les inclinaisons des pentes, représentées par les rapports des bases aux hauteurs, et réciproquement; et par une carte gravée avec soin, d'après les principes développés dans le texte.

$B$.

34. Ile de l'Ascension. Le capitaine Sabine a fait voile pour cette ile, dans lintention d'y répéter les expériences sur le pendule, ctc. qu'il a faites dernierement sous le cercle polaire, pour déterminer la figure de la terre. (Resue enc)clop. déc, $1822, p .618$.)

I. 


\section{ASTRONOMIE, COSMOGRAPHIE.}

35. Notvel essir sur lorganisation des mondes at le mécanisne de: l'unis ers: par. M. D. seconde ćlition, augmenter. In-s de 6 feruilles et demir. Imp. de David, a l'aris, prix, : fr. 5o c. A l'aris, ahez Locard, et Delaunay.

36. Commatine of Thón d'Alexandrie sur lestables manuelles astronomigues de Polomée, justu ia prescut incides, traduites pour lat premiere fois dal srec culrancais, sur les manuscrits de la bibliothéfue du roi, par MI. labbé llisma, première partie, contenant lei prolégomines de Ptolomie, les commentaires de Théon, et les tables préliminaire's, terninées par les ascensions des signe's du zodianue: dans la sphere droite; précédées d'un Vlémoire traduit de l'allemand de M. Idrler sur l'annó de la mort d 1 lexandre le (irand. (Troisieme livaison.)

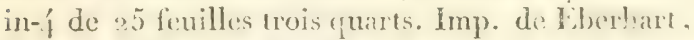
a Paris. A Paris, chez Merlin.

37. Míthode géterale pour oltenir le résultat moyen d'une série d'ubservations astronomiques faites arece

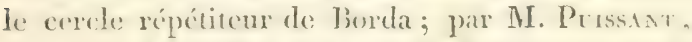
chevalier des ordres royan de Saint-Lonis et de

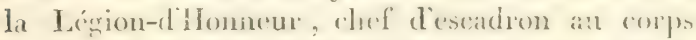

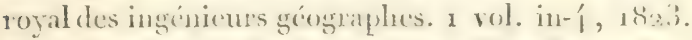
Chez bachelier, libatise, guai des Augustins. Paris.

Les observations astronomigues gue l'oa prut fater avec

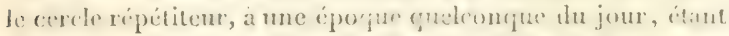

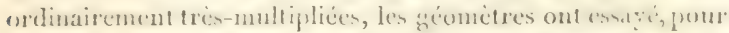
citer de calculer isolinent dacune de res observations, de les gromper au nombre de: viugl el plus, de maniere it parvenir le plus simplement possible, par une opération unicpue, : n césultat mène que l'on obliendèit on pronant lik movenne 
arithmétique de tous les résultats particls. Jál méthode qui frrésente, a cet égard, le plus d'élegance et de simplicité est celle par laquelle on rapporte toutes les olsservations an miliru de leur durée, ainsi que l'a proposé M. Solther dans le; Fiphémérides de Berlin (année $\mathbf{1} 8 \mathbf{1 8}$ ), pour la détermination du temps vai par des hauteurs absolues du soleil; mais linf.mr du mémoire que nous annonçons a non-seulement repris celte question, qu'il a résolue plus complétenent qu'on ne l'avait fait jusqu'ì présent, mais même a étendu aux ob. servations de latitude et d'azimut les principes qui servent de base à la méthode de M. Soldner.

Le premier paragraphe a pour objet la détermination exacte de lit correction d'une pendule astronomique, c'est-i-dire son avance ou son retard sur le temps vai, le temps moyen an le temps sidéral. On y examine l'influence que la variation de déclinaison et le changement de réfraction, pendant lit durée des observations, exercent sur le résultat que l'on cherche, ct l'on fait a ce sujet une utile application du thóorime de Taylor.

Le deuxiène paragraphe a principalement rapport aux obscrvations de latitude, par l'éloile polaire prise at un point unuconque de son parallicle, et à un mode de calcul gui simflifie considérablement le procédé employé en pareil cas par M. Littrow.

Ie troisieme parasraphe traite d'une manière tout-à-fait now clle des observations azimutales fitites avec le théololite ou le cercle répétitcur ordinaire.

Enfin des notes renfermant beaucoup d'applications numériques doment la preuve que la nouvelle métlode de calcul, proposée par M. Puissant, a beaucoup d'avaintages sur celle nsite. C'est surtout dans le moment oì l'on firit fie lat science greolésicque, soit en France, soit dans d'antres ciats de lYurope, les applications lés plus utiles à la géngrat. phice, que cette méthode mérite do recevoir de la publicité.

DEN.

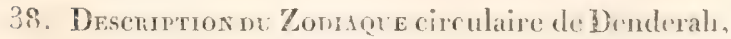
confenant l'explication dessignase etes figures retrat-

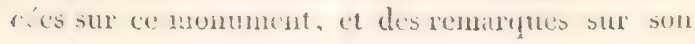


anticiuté, sur le temple dont il faisait partic, et sur la manière dont il a eté rapporté en France; par B. I. C.... homme de letters, in-s d'une fenille et demie, et une planche gravér. Imp. de: Testu, at Paris. A Paris, chez Bulla, rue Saint-Jacques, n. 38. 39. Essa ninterphetation du Zonhece circulaire de Denderah, par S. II. Leprence, sous-hibliothécaire de lit ville de Versailles, auteur d'une Votice sur l'Auroreloréale, etc., in-s défeuilles et demir. Imp. de Dupont, à Paris. A Paris, chez Ponthicu et Delaunay.

I 'auteur, rejetant toute idée de projection dans ce monument, n'y voit qu'une espéce de tableau des constellations dins l'ordre de leurs levers ou de leurs concliers, en raison de lit coüncidence de cetux-ci avec certaines époques, telles que les phases du Xil. Ainsi ce zodiaque ne serait plus qu'une espéce de catlendrier agronomique, et H. Leprince croit y reconnatre une exactitude conforme aux principes qui présiderent à son origrine. C'est lá ce qu'il cherche à démontrer par l'explisation symbolique et allegorique des figures diverses du zodiatque; explication où l'art de conjecturer jouc nécessairement un grand role. L'auteur, qui s'attache particulierement à contredire un des ouvrages de Dupuis, assez gémíralement consideré comme bon, son Mémoire sur l'origine de's eonstellations insiré à la suite de l'Astronomie de Lalancie, donne. aussi son opinion sur l'origgine du zodiaque en grintral, et il lat rapporte a l'an 82 i avant notre ère; tontefois il n'est pas sirr que l'auleur n'ait pas aussi entendu domner cette date au zodiarque de Denderah; lampluhologie de la conclusion de son Mémoire laisse subister ce doute. Du reste on doit faire remarquer que l'auteur annonee que la Disserlation de Innpuis sur le zodiaque de Denderale est extrate de son Origine de tous les cultes; mais cet ouvrage était publit bien des anneres avant gue l'on somperonnà l'existence de ces zodliaçues. ( $\left.170^{3}\right)$; et la Jlissertution de: Dupuis n'a éle lue a l'Intitut, et ria paru dans la Dickate philosophique, quen 1 solo.

W. 
40. Dissentation sur le Zodiage de Denderah; par

Depurs, membre de l'institut national; précédée d'une description du Zodiaque Circulaire, maintenant à Paris, par un membre de l'académie des inseriptions et belles-lettres. In-18 d'une feuille trois quarts, plus, deux planches. Imp. de Dupont, à Paris. A Paris, chez Chasseriau.

Cette dissertation a été publicée du vivant de l'auteur dans l'ancienne Dicade philosophique; elle est plus particulierement relative au Zodiuque rectungulaire du portique de Denderah, cependant comme le zodiaque circulaire, rui est it Paris, est identique par sa composition avec le premier, la discertation peut, à certains égards, s'appliquer au second, dont une gravure est jointe au mémoire. La description de ce 'dernier, at tribuée à un membre de l'académie des inscriptions, doit intéresser par sa fidélité, et c'est un bon document de plus dans les grandes questions qu'a fait naitre l'étude des monumens astronomiques des anciens ligyptiens. W.

41. Essir ser le Zodiege circulaire de Denderah, maintenaut au musée du roi ; par Alex. Lexori, in- 8 de 6 feuilles un quart. Imp. de Richomme, a Paris, et ì la librairic des annales francaises, boulevart du Temple, n. 45 .

L'auteur de cet Essai, connu par des recherches utiles sue les arts en France dans le moyen àge, pense que le planisplière de Denderah " est une espèce de calendrier sur lequel on a tracé au complet les signes du zodiaque et quelques-unes des constellations extra-zodiacales de la partie supérienre en remout ant vers le póle. Les figures qui sont tracées au-dessous de la ligne du zodiaque, lui paraissent indiquer le complément del'année rurale dont il croit que l'ouverture se faisait sous le signedu taurean, et dont le lion était censé le précurscur. Enfin la bande de figures cqui forme lia circonférence du médlaillon du monument serait une peinture de l'annće civile et des fètes que les Egyptiens célébraicnt. "( Quant a l'éporue ou le monument a cté exécute, M. Lenoir, à l'aide de l'histoire et du goùt de la sculpture, comme il le dit, considerant ce plia- 
uspluce comme une des belter productions du seenal sty ix

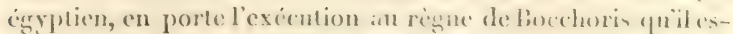
time à l'an 7:1 atsant l'ere chrélienme. Vuilit toutes ses opi nions sur ce momment, développées dans son Essai ou se trouvent ausi diverses considérations sur d'atutres points des antiquités égyptiennes.

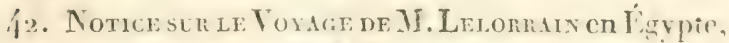
et observations sur le Zodianue cirenlaire do Denderah : avec un dessin lithographié dı Zodiacjur, sur pap. grand aigle, par M. Gau, anteur des Antipuités de la Nubie; par M. Saulnier fils, (annmere el extrat), in-8 d'un quart de fenille. Imp. de sectice, à Paris. A Paris, ehez lussinge, pre et fils.

Cette notice contient lhistoire de la translation du zodiaqquede Denderahen France. On ne peut lire quavec bean(oup) d'interét lat relation des circonstances qui ont fitit conrevoir a M. Satulnier fils le projet d'enlever a l'ksppte ce cilibre monument; et celle des noyens d'exícution emplovijar MI. Lelorain dans une aussi dificile entreprise, at milien de tous les hasards et dies contrariétés de tout sinte quil a cu a escuyer. A cette relation est joiste une planche lithographiée, copie fidele du zodliaçue : la notice et h gravure réunies sont des documens indispensables dans l'itule du cellure planisphere dont la France est redevable a la munificence royale, et pour l'eximen des nombreuses opinion: que son explication a fait naître.

W.

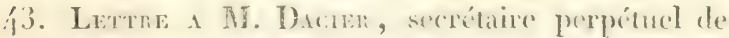

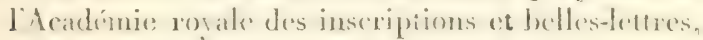

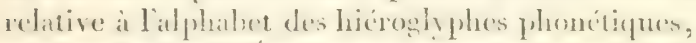
cmployes par les ligytions pour inserine sur lours mommone les titres, les noms et le's surmoms de's

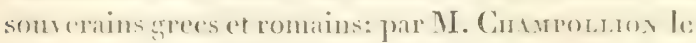

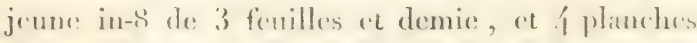

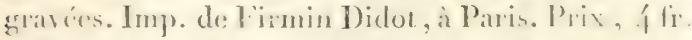
凡 Paris, chez Didot père et fils, rue Jacol.

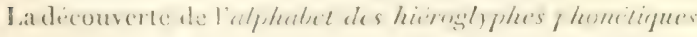
ou rendant un son, due aux longues reclierches de iH. Chan- 
pollion le jeune, exposée dlans la lettre que nous amnoncons, :t vivement excitél'attention du mondesavant, a été annumcée par les journaux français et étrangers, et a été déjit en Angleterre l'objet de plusicurs publications. Ses résultats sont en effet précicux pour l'histoire, puisqu'avec cet al phabet on a reconnu sur des monumens de l'Egypte les noms de presque tous les P'tolémées, et ceux de plusieurs empereurs romains, qui avaient fait élever, agrandir ou restaurer ces monumens; on a reconuu que le zodiaque de Denderah, qui est à Paris, portait les titres de l'empereur Néron, et c'est un point essentiel qui, en dérangeant quelques opinions relatives à l'antiquité exagérée de ce monument, ne peut pas ètre négligé dans les recherches multipliées dont il est l'oljet. Au reste, on sait dijà que l'alphatuet de M. Champollion s'applique aussi aux monnmens antérieurs aux Grecs, à ceux des Pharaons, comme aux divers manuscrits épyptiens en différenscaractères, qui sont connus. Il était done difficile de faire une découverte plus neuve et plus importante pour l'histoire. Elle n'est qu'une portion des travaux que l'autcur a communiçués à l'Institut et dont nous apprenons que le roi a bien voulu ordonner l'impression à l'imprimerie royale.

IV.

4\}. Note ne M. Cronstrand, sur la différence cu. longitude, entre lobservatoire de Stockliolm et treize autres observatoires d'Europe, déduite de: lobservation de l'éclipse de soleil, du r9 novenbre I 8 i6. ( Mém. de l'Acad. des Sc. de Stochitiolm.

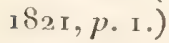

L'auteur, n'ayant pas connaissance d'observations de cette éclipse faites à Paris, se sert de la détermination relative à Vienne et à Berlin, lieux dont la position paraî bien connue, et il trouve pour la différence en temps cntre l'olsservatoire de Paris et celui de Stockholm, I h. 2 m. 33 s., résultat semblable à celui qu'a donné l'occultation d'Alelcharan, en 1810 , suivint les imémoires de l'Académie de Sucde', 18 5 , p. 3/.

C. M. 


\section{P II Y S I Q U F.}

\{5. Thione dxiryteete de La Chaleur; par M. Forrier, I vol. in-f de (i3- pageset 2 pl. Paris, 1822 , chez Firmin Didot père et fils.

Les recherches de 21 . Fourier sur la theorie de la chaleur sont contenues dans deux mémoires manuscrits, présentés a l'Institut en 180 ; et I $8 \mathrm{r}$, et dans plusieurs antresmemoires, également manuscrits, et communiqués dans ces dernieres annies a l'Académie des seiences. L'ouvrage que nous annoncous conticnt une grande partie de ces recherchics. On y trouve les bases les plus essentielles de la théorie que M. Fourier a fondée.

L'objet principal de cette théorie est la connaissance des lois suivant lesquelles la chaleur se transporte de la partie la plus ćchanffée d'un corps dans celle qui l'est moins, et tend a parrenir à une sorte d'équilibre dans lequel toutes les parties du corps ont une égale température.

L'onvrage, précédé d'un discours préliminaire, est partagé en neuf chapitres.

Je premier offre lexposition des propriétés physiques des corps dont dépend le mouvement de la chalcur, le principe de la communication de la chaleur, et les premieres applications de la théorie, qui ont pour objet l'échauffement des espaces clos.

Le deuxiume contient les áquations générales du monvement de la vhalcur dans l'intirieur des corps solides. Considerant la température de chaque point d'un corps comme une fonction des coordonnés de ce point et du temps, ees équattions consistent dans des relations entre les coeflicients differentiels partiels de cette fonction. Il existe unéejuation pour tous les points du corps, et une autre pour les points de la surface seulement.

On trome dans leschapitres enivans l'application des ćpuntions finerales a des corps de diverses figures, tels guiun solide rectinşulaire, un anneas, une sphere, un cylindre, un prisme rectangulaire d'une longucur infinie, un cube. On smpose un foyer de chalcur ichauffant constamment une 
partic d'un de ces corps, et on détermine la manière dont la chaleur s'y propage; ou bien, supposant le corps primitivement échauffé d'une manière arbitraire, on détermine la manière dont les températures de ses points varient avec le temps, à mesure que ce corps se refroidit

La solution de ces questions exigeait l'intégration des équations différentielles du mouvement de la chaleur. On ne pouvait parvenir à effectuer cette intégration en suivant les méthodes connues. MI. Fourier a résolu cette difficulté au moyen d'une méthode spéciale, qui consiste principalement à composer l'expression cherchée d'un nombre indéfini de iermes, dont la somme satisfait aux équations différentielles, et à déterminer les constantes arbitraires qui se trouvent dans chacun de ces termes, d'après les conditions particulieres de chaque question. L'application de cette méthode supposait la détermination de ces constantes, de maniere à représenter une fonction entièrement arbitraire au moyen d'une série de termes, dont la forme est donnée par la nature même de la question.

Le $9^{\text {e }}$ et dernier chapitre a pour objet la propagation de la chaleur dans une masse solide d'une étendue indéfinie. Les intégrales prennent ici une forme différente, les sommes formées d'un nombre indéfini de termes se changeant en une intégrale définie. Ce chapitre est terminé par un morceau d'analyse, dans lequel l'auteur rapproche et présente sous divers points de vue les intégrales des équations aux différences partielles, et les diverses méshodes d'intégration.

La publication de cet ouvrage, dans lequel brillent au plus haut degré l'art de l'analyse et l'esprit d'invention, formera dans les sciences une époque remarquable, soir parce qu'il offre la première application du calcul a une classe importante de phénomènes, soit it raison des nouvelles méthodes que l'auteur a créées, et qui servent à résoudre un grand nombre de questions naturelles de divers genres, dont on n'avitit pu encore découvrir la solution. R.

\{6. Des Aurones borélies el de quelques autres Météores, in-8 d'une demi-feuille, par Dutentne, capitaine diartilleric. Imp. de Monnoyer, au Mans. 
i-. Drserexcipss ne voxne, nil lon traitedu nonvement primilil, de l'air principe, dufeu, dre lat lumiere, dusoleil, de l'allraction, de l'ean, de laite

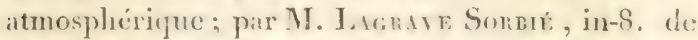
I6 feuilles, imprimerie de Moreau, à Bordeaus.

Cet ourrage etant inconnu it Paris, on ne pent en donner l'analyse.

你. Strpetrufre à la Théorie des verresoptiques simplés; par M. A. G. de Scheltix. (Mém. de l'Aien. des Sc. de Stochlolm, $182 \mathrm{I}, \mathrm{p} .265$.$) C. M.$

\section{Effet remarquable de la foudre.}

Le 12 novembre dernier, vers une heure après milli, is l'entrée du canal de Céplıalonic, au moment oi la frégille: autrichienne la Lijsia, qui revenait de Zante, luttait contre une tempette furieuse, et que le contre-amiral et commin:dant de la flotsille autrichienne de la Méditcranéc, le matquis de Paulneci se trouvait sur le pont, la foudre tumbia sur le grand mat, le fendit dans tonte sa longueur avee un sifflement et un fracas épouvantalle's, et en dispersit les deibris à une distance prodigieuse. La foutre atteignit ensuito le pont, se divisa, en partie, en deux langues de feu qui s'itendirent dés deux côtés du battiment, tandis que le corph principal de ce terrible météore prit avec une étonnante velociti sal direction vers le bas du vaisseau, ou il reneontra un grand panier de boulcts couverts de roulle, et séteisnit aussitòt. La matiere dectrique agit aree lant de force sur it. boulets, que lat rouille disparut et fit place a un puli aussi lu. sant que reluidel'acier le plus brillant, travaille a facettes. I'as un grand bonheur la foulre, arrivic an pied du mat, n’atleignst pas plusicurs caisses de fusées a la Congrevequi étaient tom pres de lit, sans quoi la frégate ent ite perdue sins re. source. ( Observateur de Trieste, d'apres le Moniteur d! : janvier, 1823. )

F. 


\section{CHIMIE.}

50. Dingramaes cumpues, ou Recueil de 36 fignres(sur i a 2 planches), qui expliquentsuccinctement les expérienees, par l'indication des agens et des produits à còté de l'appareil, et qui rendent sensible la théorie des phénomènes, en représentant le jeu des attractions par la convergence des lignes. Ouvrage élémentaire auquel on a ajouté, pour les étrangers, un essai de nomenclature chimique en six langues; et pour les commençans, $\mathrm{I}^{\circ}$. un vocabulaire contenant l'étymologie et la définition des mots techniques; $\mathbf{2}^{\circ}$. une série de tableanx synoptiques qui représentent la préparation et les parties proportionnelles des produits; par M. Decremps. In- 4 de 16 feuilles; plus, 1 i 2 planches. Imprimeric de Didot jeune, à Paris. A Paris, clez Carilian Goury, chez veuve Desray, chez Treuttel et Wurtz, chez Rey et Gravier, prix, $40 \mathrm{fr}$.

L'épigraphe choisie par l'auteur :

Segnius irrizant animos demissa per auren

Quam qux sunt oculis subjecta fidelibus.

indique le but qu'il se propose; c'est surtout aux yenx qu'il veut parler.

Par un assemblage de lignes et à l'aide de quelque mots, il exprime des faits et des théories dont l'explication exigerait des pages entieres; au moyen de ces lignnes et de ces mets, il construit des firures atuxquelles il a donné le non de Diagrammes. Ces figures sont au nombre de 360 , comprises dans 1 iz planches. Chacune d'elles représente une opération chimique souvent très-compliquée, agens, produits de l'opiration, théorie des phénomènes, jeu des attractions, tout sy trouve mis en action.

Chaque corps élémentaire est représenté par une bande dont l'interligne est remplie soit par des points, soit par dehachures horizontales, obliques ou verticales, differrems TOME I. 


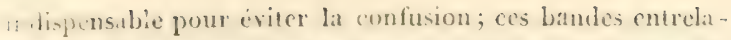
$\therefore$ de diverses manieres ont une direction determine par is bul: que jout dans lopuration l'áment qu'elles représen-

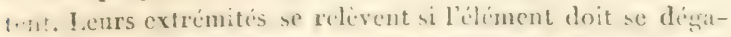
..r, elles se baissent an contraire si lidement doit se prece-

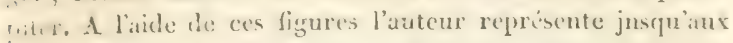
tones dincutés par Dalton pour donner nne idée de la comfortima des corps. Il amigne une forme at ces atomes, il les (w) fuce, il les suppese groupis daus un disolvant sans actimn sir cus, et il indique le changement qui doit s'operes Si! nurvent un atome d'un antre corps doue d'une afinite - spirieure, ch vertu de laquelle il se substitue a l'atome qu'il a déplacé.

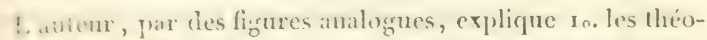
ices las laffnite, du calorique, de l'action mutuelle des wices et des alcalis, di la décomposition réciprorue des w-, de lifhérification, du blancliment, de la congelation artilicielle, de lu pile galvanique, efe.; 2 ". les procétés d'extraction de la potasse, du potassium, des metaux, du soufre, du nitre, du sel marin, du sel d'ipsum, des acides nitrique te iyylrochlorique, de l'ulcool, du sucre de canne, ele.; 3". les unyens de fibrieation du sel ammoniac, de l'alun, dusulfiate. de fer, du charbon, de la poudre de chasse, du blen de Prusse, elc.

Les planches renfermant les diagrammes sont précélées pat une strie de tableaux synoptiques qui, dans un pelit copace, presenten la préparation et les parties proportionnclles des produits chimiques les plus intéressans. Cers tableaux sont distribues en sept chapitres sur les malieres suivantes: $x^{\prime \prime}$. affinte; $2^{\prime \prime}$. calorique; 30. corps composés gatzenx, et corps simples conerets non métalliques; $f^{\prime \prime}$. batses sitifiables; $j^{\prime \prime}$. acieles et sels; $6{ }^{\circ}$. mctaux; $g^{\prime \prime}$. corps orsaniques. Lorspue ces tableaux traitent dobjets dicrits dans les diansammes, l'anieue a le soin d'y indiquer la planche: correpondante; ainsi ils peuvent supplécer a ce que les diatgrammes n'auraicut point représenté.

On trouve encore dan cet ourage, 1". 1m Vocabulaire rontenant letymologre: of fa defintion de 153 mots terhm- 


\section{Chimie.}

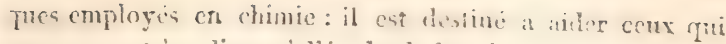
commencent à se livrer à l'éludo de la science.

$2^{n}$. Un Abrigi de nomenelature en langues franraise, an-

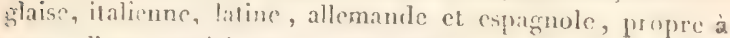
mettre l'ouvrage à la portée des étrangers.

3". Ine Table des matières qui précide tous les objets enoncés plus laut; clle divise louvrage en trois parties: l: fremiere comprend lit Nonenclature et le Vocabulaire chimirpues; la denviène les planches; la troisieme lins tibleanix synoptiçnes; ordre cque l'autcur n'a point suivi, car Ies intanclirs indiquées à la seconde partie se trouvent it la fin du volume.

Laug.

it. So it a composition des sulíures alcalins, par

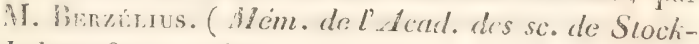
holm, i82 i, p. 8o.)

Ce mémoire a été inséré en français dans los $\Lambda$ nnales de Chimie et de Pliysique de MMT. Gay-Lussac et A ragro. Thai, juin et juillet, 1822 .

C. $M$.

i.. St n mal matron qui existe entre la composilion chimique et la forme des cristaur, en ce qui concorne les arseniates et les phosplates; par M. W.

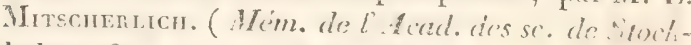
holm, I821, page 4.)

La traduction de ce mémoire, filte par l'menten lui-mine ic trouve dans les Annales de Mhyirna et de Climin to

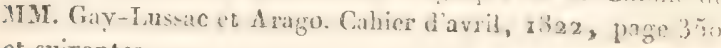
et suivantes.

73. Fssir sur la composition chiruigue des minérau: qui cristallisent à la manière de l'amphibole, par M: P. A. de Bonsmonff. (Meme de l'sead. de sc. de Stochliolm, 182 r, p. 197.)

Ce mémoire se troute ort francais a lat the din tome $x y$

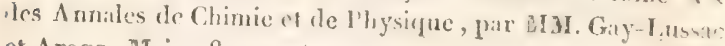
et Arago. Mai, 1822 .

C. $M$.

if. Notgelaes arentuches sur la composition chimique de larent antimonić sulfuré (roll cuilden): 
par MI. P. A. de Bonsmonre. (Mime de l' Icall des sc. de Stockholm, I82 I, p. 338.)

Voyez les Annales de Chimie et de I'hysique de Mu. cayLussac et Arago, janvicr i $S_{22}$, ou ce mémoire est traduit.

C. II.

55. Supplévext à ce qui a été dit du Lithion, dans les Mémoires de l'icadémie des Scinnces de Sto. chlohn, amméc ISI8, par M. Augr. Ariwroson. (Mem. de l'Acul, des sc. de Stochholm, r 82 I, p. 156.)

L'auteur fait connaitre d'abord les procédés par lesquels il retire le lithion de la petalite et du spodumene; puis il traite de la combinaison du nouvel alcali avec lacide sulphurique, et trouve pour le lithion crystallist: la composition suivante, sur eent partics: acide sulfurique 58,86 , lithion 26,87 , eau 14,27 .

Il ajoute que c'est à tort qu'il avait admis l'existence d'un sel à double base d'alumine et de lithion, qu'il nommat alun de lithion. Son crreur venait de ce que l'alumine employée dans les expériences, ayant été retirée de l'ahun, contenait une portion, quoique très-faible, de potasse.

\section{BI.}

56. Description et analyse chimique du grenat calcarifère de Lindbo, par M. W. Hisinger. (Mem. de l'Acad, des sc. de Stochholm, 1821,1$) .365)$.

Cutre espece de grenat se trouve parseméc dans un bane de pierre calcaire spathique, accompagné de micaschiste an milicu du gneis; il est noir; sa forme est celle du grenal rhomboïdal primitif. L'analyse a donné :

Silice. . . . . 3 37,55 contenant 18,78 d'oxygène. Fer oxidé. ..... 31,35— 9,6 id.

Chaux. . . . . 26,7:- oxycrine 7,52 ensemble Manganese oxidule. . 4, 4, 8- oxysene $\mathrm{r}, \mathrm{r} 3\} \quad 8,6 \mathrm{i}$ )

Si l'on additionne ce qu'il y a d'oxygrine dans la chanx of dans le manganése oxilule, on trouve une quantite $i$ gite at celle que contient le fer oxidé; el si l'on additionne de mime ce qu'il y a d'oxygene dans ces trois bases, fer oxidé, chans 
\&-t manganèse oxidulé, on aura une quantité égale à celle qu'en contient la silice.

C. DI.

57. Sur les conernarsons du titane avec les acides et avec le soufre ; par M. Henri Rose. (Mém. de I'Acad. des sc. de S'tockholm, 182 r , p. 23r .)

IL n'y a point de métal, dit l'auteur, dont les propriétés chimiques soient moins bien connues que celles du titane, ce qu'il attribue à son peu d'affinité soit avec les alcalis, soit avec les acides. M. Grégor, qui a découvert le titane, et Klaproth, qui obtint le mème résultat sans connaitre le travail du premier, ont opéré sur des oxides de titane qu'ils croyaient être purs et qui ne l'étaient pas.

Suivant M. Mitscherlich, l'oxide d'étain natif, et le rutile qui n’est qu'un acide de titane, affectent les mêmes formes; ce qui lui parait indiquer, beaucoup d'analogie dans les propriétés chimiques. Ces deux substances sont en effet, l'une comme l'autre, des acides faibles, qui par la roie sèche digagent avec effervescence l'acide carbonique des alcalis carbonatés, et se combinent arec lesalcalis; tous deux, après avoir été chauffés à rouge, sont absolument indissolu. bles dans les acides les plus forts, etc. $\quad$ C. M.

5i. Sur erelouts spits contenus dans l'eau de la ner; par M. A. Minticet, membre de la Société royale de Londres. (Tilloch. Plillus. Magasasine, déc. 1822, p. 434.)

On pouvait soupeonner que l'eau de la mer contenait de petites quantitís au moins de tontes les substances que l'eau peut dissoudre; Rouelle et M. Proust, avaient mème annoncé quill s-y trouvait du mercure, et ce dernier en avait apercu quelques traces dans les échantillons d'acide muriatique qu'il avait examinés.

MI. Marcet n'a trouvé ni mercure ni sel mercuriel dans les caux de l'Océan, et il se croit foudé à penser qu'il n'y cn a absolument point.

Il n'y a pas trouvé non phiun de nitrates ni de sel ammoniac, ni de muriate de chalux; mais il y a recomnu la présence du 


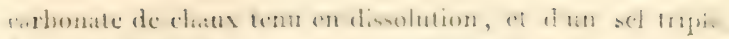

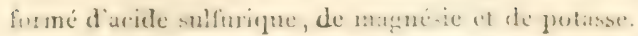

\section{II.}

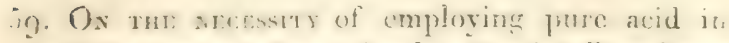
-echinz roppor-pliaic.s. Sur ha meresité demployer

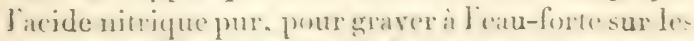

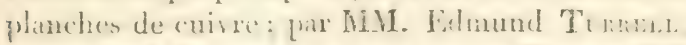

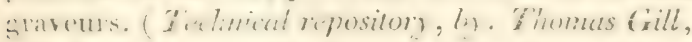
vol. $3,1^{\circ}=1$, pag. 55.$)$

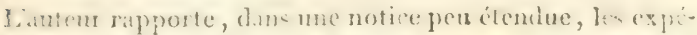

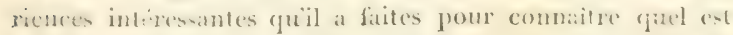
l'uble qui donne les meilleurs résultats dans l'opiration de

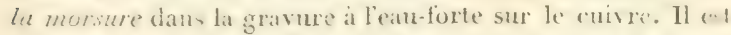

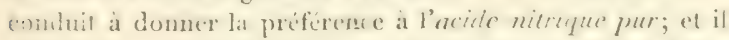

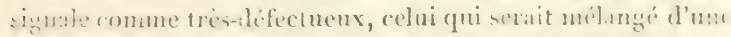
petite portion d'acide sulfurique.

L. F.

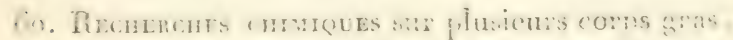

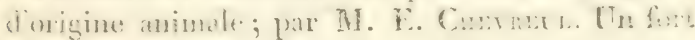
vol. in-8. A Paris, chez Levrault.

in amence comme levant paraire dins cutques mo: cet ouvrage attendu de tous les chimistes.

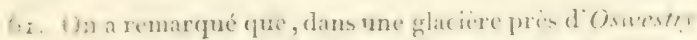

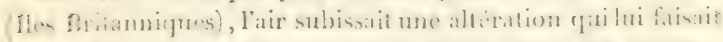

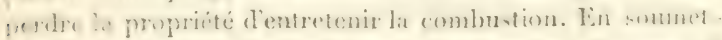

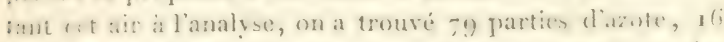

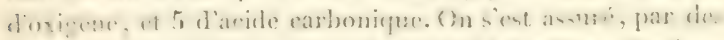

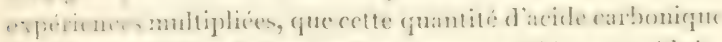

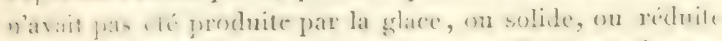

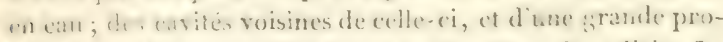

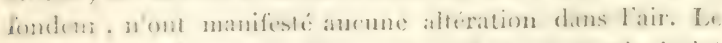

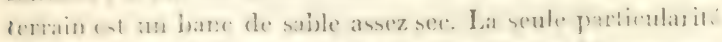

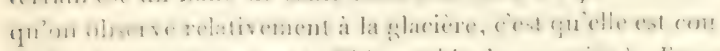

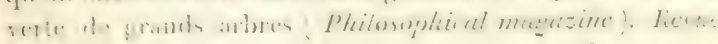
encyclop. dic., 8822.7 . 622 . 


\section{IISTOIRE NATURELLE GENERALE.}

6.2. Oruves compites ne Beffox; nouvelle édition publice par G. R. Di rmiloeur. Tomes r, 2, 3, 4, 5 ct 6 . Six volumes in-8, ensemble de 185 fenilles et demie; plus les planches. Imprimerie de Wagrez ainé, à Donai. A Douai, chez Tarlier.

Il y aura i 2 volumes qui sont du prix de $60 \mathrm{fr}$.

63. OFunes completes ne BufFon, mises en ordre par ML. le eomute de LAcépère, seconde édition. TouE X VV, in-\$. de 3. feuilles; plus la r fivraisnn des plinches, contenant i r planches, lo portiait compris. Inp. de Plassan. Prix, papier tues-fin, en noir, io fr. le vol.; papier vélin, fig. color.,

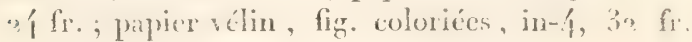
A Paris, chez lapet.

Cette édition devait avoir $24 \mathrm{rol}$. in-80.

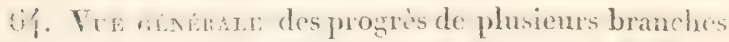
des sciences naturelles, depuis la mort de Bufiom.

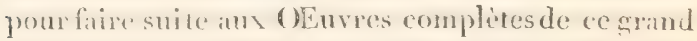

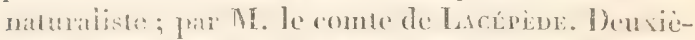
me édison, in-s de dix fenilles. Inp. de Plassan. Prix, 3 fr. 50 c. A Paris, chez Rapet.

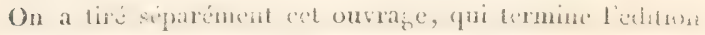
de Buffon entreprise par Rapet.

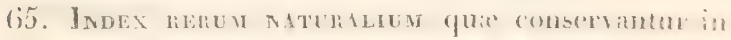

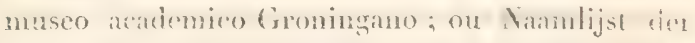

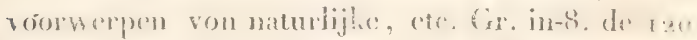
pages, r 22. $_{2} \Lambda$ Groningue, chez J. Oomkens.

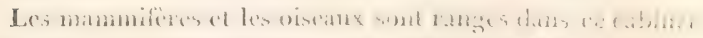

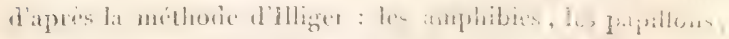

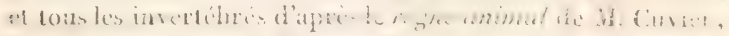

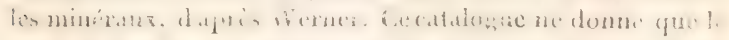

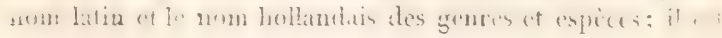


comine par nu desiderate, et a éte public par Mi. TH. VAN

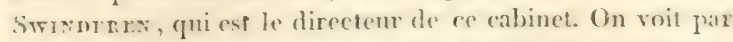
l'énumération des especes que le musée de Groningne est assez riche; ot, fiar lenr classification, que $M$ le directeur s'occupe aroe zibe et talent te fare prospérer cet établissement. On renarque dans tontes le's classes des noms spécifiques inconmus of numverux; il serait à désirer que si ces especes sont nouvellon, ollen fu-sent décrites et figurées dans un ouvrage qui confribuerait a illustrer encore le musém dont le catalogne que nous amnourons donne déja une idéc trés-avantageuse.

T.

66. Fos ace autour du monde, fait par ortre du roi, sur les corvettes de S. M. IT ianie et la Ily sivienne, commandées par .I. Louis de Freycrist, capilane de vaisseau, correspondant de l'Institut de Irance,

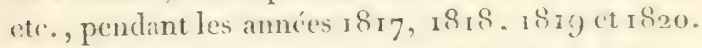

La priblication du Voyage, elont nous ne domons ici que le titre abrégé, se poursuit par ondre du gonvernement sous la direction du capitaine Louis de Freycinet, et bientôt le public pourra jouir des differens onvrages dont cette Relation se compose.

Elle sera partagée en sepi prattirs dintinctes, siroir: 10. Whistonire de la navigation; 2". la zoologice; 30. La hotanique; $4^{\circ}$. 1'hyalrographie; 50. le magnétisme ot la figrure de la terre; $6^{\circ}$. la méterologie; $7^{\circ}$. enfin les recherehes sur les langues des sauvages. Nous reviendrons ailleurs sur ces diverses annonces, notre objet n'étant aujourd'hui que de parler de la partie zoologique qui est sur le point de paraitre.

Les médecins de la marine, appartenant à l'Expédition, étaient clargís 'n mème temps des travaux d'histoire naturelle pemiant le royage, et c'est cux qui se sont occupés au retonr de lat ridaction de ecte prartie. Nous ne ferons point ici l'énumération de'; contrées qui ont été visitées par nos voyágene; nous dirons seulement que l'expédition a sëjourné lone-trmps atu Brésil; 'qu'elle a touché deux fois à la Irouvelle Hollande, et sur der points différens; qu'clle a reliche a Timor, traversé les Molugures, visité la terre des Papoux, et sit un long sijonr aux iles Bariannes. Dans la plupart de ces 
licux, remarquables par l'admirable fécondité du règne organiqque, nos voyageur's n'ont rien négligé pour récolter le plus d'échantillons possible des trois rèsnes. Ceux qui, par leur nature, se refusaient aux moyens connus de conservation, étaient aussitòt dessinés, avec toutes leurs couleurs, prar MIM. Arago et Taunay.

MM. Quoy et Gaimard s'occupaient spécialement de la zoologie, leur zèle à cet égard est digne des plus grands éloges; nous allons donner un très-léger aperçu des choses nouvelles que leur ouvrage doit faire connaitre.

L'ensemble sera renfermé dans un volume in- $4^{\circ}$, et un atlas séparé composé de $9^{3}$ planches in-fo., la plupart coloriées : toutes ont été gravées par M. Coutant; elles représentent iu delà de deux cents espèces nouvelles d'animaux. Quoiqu’il ne faille pas considérer ce recueil comme un ouvrage systématique, cependant les auteur's se sont astreints, autant qu'ils l'ont pu, à suivre l'ordre méthodique du régne animal de M. Cuvier.

En visitant la terre des Papoux, ces messieurs ont pu se procurer des crìnes de ces peuples encore peu commus. Hls in ont fait dessiner deux, et y ont joint les remaryues de MII. Gall et Spurzheim, qui concordent parfaitement avec ce qu'on a été à même d'observer du moral de ces insulaires.

Six planches sont destinées aux manmifères. Elles représentent une grande roussrite, espece nouvelle des îles Mariannes; le Dissure maugré, qui n'avait point encore été figuré, de mème que le peramète denté; une variété du pmalaticen maculé, dessiné vivant; plusieurs crânes de ces animaux; enfin un grand naxgurvo rouge laineux, nouvellement découvert au Port-Jackson.

MIN. Quoy et Gaimard ne voulant répondre que des choses quills ont vues et parfaitement examinées, ont cru devoir reléguer à la fin de leur article sur les animaux vertébrés, et comme formant un $\Lambda$ ppendice, la figure d'un cachalot qui leur a été communiquée dans les Moluques par un pécheur auglais, ainsi que celles de plusieurs dauphins vus à la mer, dout un, très-singulier, porte sur le nez une protubérance semblable à la nagreoire dorsale, d'où le nom de Daupher rhinocéros qui lui a été donné. 
I.es oiscaux, au nomber de 27 , sont contenus daus 21, planclies. Presque foris sont des epreces nouvelles. (guelquesuns déjà connus n'itairent pas fignures, on l'étaicut mat. It confenait d'en donner de meilleures gramures.

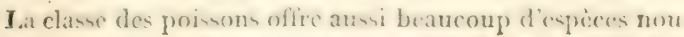
velles, contenue dian á planches. La plupart ont éti des. sinées au sortir de l'cau aree tout l'éclat de leurs contener.

Dis planches ont ite reserves ponr representer an dele. de 60 mollaspues de disers genres, parmi les fucts, dans lis.

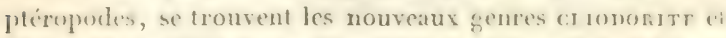

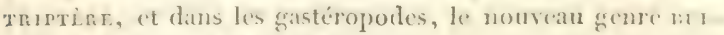
s.sт, etabli far M. de Firussar, auquel on doit ausi nu. Catalnone descriptif des mollungues terrestres et flasiatidrapportés par l'expédition.

J.es cruslacés sont contenus dans six plinclies. (On ?

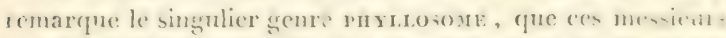

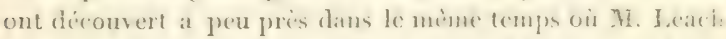
te nommait en Angleterre. Cel animal pedagien, qui na

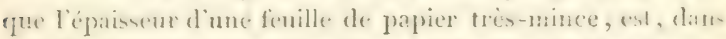
son cetat naturel, tramsparent comme le cristil le phus pus.

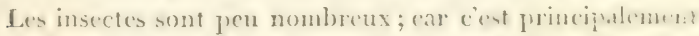
dans celte parlie de ses collections que l'l iamir at ciprous. le plus de perten lors de son naufrage anx iles Malsumen Cour recueillis a la tere des Papous et an dela des mom.

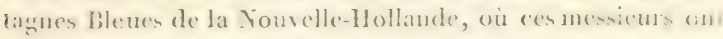
fait une incursion, étaient presque tous nouvcaux.

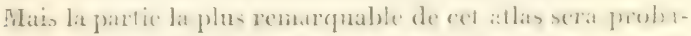
blement celle gui contient, dans 13 planehes, une growe

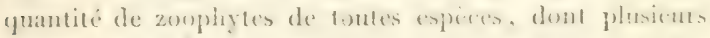

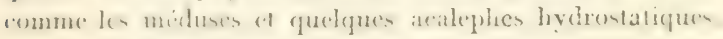
sont ornés des plus belles coulcurs.

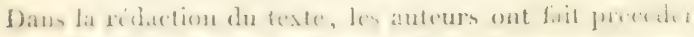

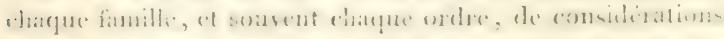

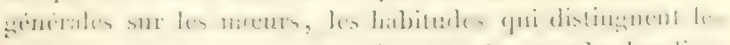

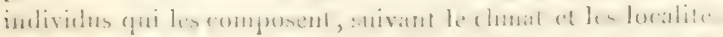

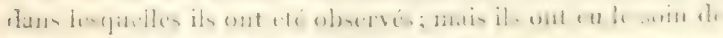

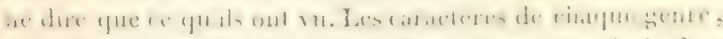

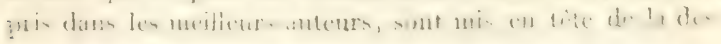




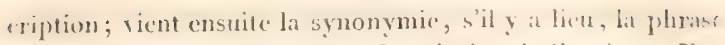
sprecificgue en latin; et enfin la description de l'esprece. J'.

MINERALOGIE.

(i- Deschirtios et Analyse diune nouvelle substance minérale; par M. P. Strom avec des olsurvations supplémentaires, par M. BẼzét.us. (Hcim. de l'Acad des sc. de Stochlolm, isar. P. 160 et 163).

Le minéral cristallisé dont il s'agit dans ce mémoire provient de lat paroisse de Riger, dans la partic méridionale de lit Norwigge. Il s'est trouvé engagé dans un rognon quartzenx . au milieu de l'espèce de grranite que II:ussmann et de Buct ont reszardé, peut-ètre sans motifs suffisans, dit M. Strocn comme appartenant aux terrains de transition.

J'sumalyse a donné à Mí. Berzélins pour lit composition de ce minéral :

Silice. . . . . 56,64

Jer oxidé. .... 3 I, 60

Soude. . . . . 12,36

Outre un peu de chaux.

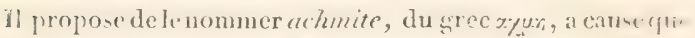

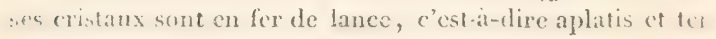
minés en pointe.

C. MI.

GËOLOGIE.

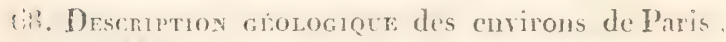

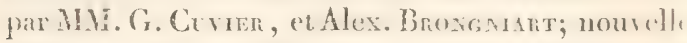
chition, dans larguelle on a insécé lat deseription d'un warad nombe de liens de J Allemagne, de lis Suisse, de lifalie, ete., qui présentent desterrain:

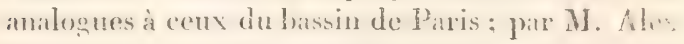

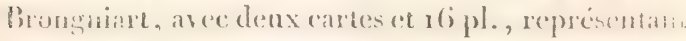

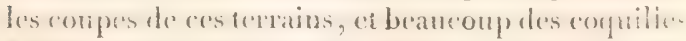

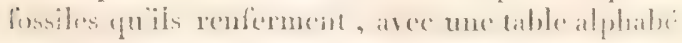

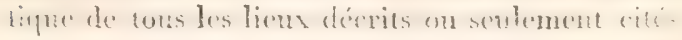


Paris, chez G. Dufour, at E. d'Ocagne, libr., yutsi Foltaire, 11.13 ; et à $\Lambda$ msterdam, chez les nimes. I822. I vol. in- 4 . de 428 pag.

Ce volnme, qui est une seconde idition, considérablement augmentée, de leosai sur la groographie minéralogique dé cuvirons de laris publié par les mémes anteurs en $181 x$, fait partie du grand ouvrage de MI. G. Cuvier sur les ossemens fussiles, bien qu'il puisse en ètre entièrement détaclié.

Les auteurs ont dirisé leur travail en trois sections. Dans lit premiere section, après avoir jeté un coup d'œil rapicte sur la configuration extérieure du sol des environs de Paris, et avoir circonscrit d'après la nature méme de celui-ci, et non d'aprés les considérations géographiques, la contrée soumise a leurs observations spéciales, ils énumerent les différentes formations dont ce sol se compose; ot dans autant de chapitres séparés ils doment pour chacune d'elles ses caracteres minéraluniques et gíologiques, ainsi que ceux fournis par les corpss organisés fossiles.

Les formations sont ainsi successivement ibtedices dans f'ordrede leurancienneté relative et d'aprésle talblean suivant.

\section{$x^{\circ}$. Terrain de sédiment moyen.}

Formations. Sous-formations et roches principales qui les composent.

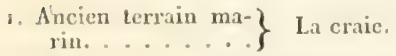

$2^{\circ}$. Terrain de sédiment supérieur.

2. P'remier terrain d'eau $\begin{aligned} & \text { Argile plasticaue. } \\ & \text { Lignites. } \\ & \text { doucc.. . . . }\end{aligned}$

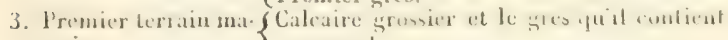
rin. ....... souvent.

4. Deuxième terrain falcaire siliceux. d'eau douce. . . Harnes d'ear douce.

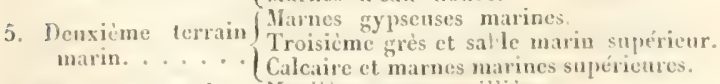

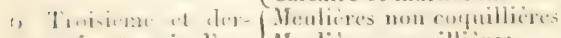
nies tersain d'cau Menliéres coquilliceses. donce....... (Harnes d'ean douce supérieures.

7. Terrain de trans- Cailloux roulés et pondingue ancicn. port et d'allu- $\{$ Limon d'alférissement ancien et moderne. viun. ..... Marnes argileuses noires, et tourbes. 


\section{Géologie.}

Dans ja deruxirme section les faits desfuels ont puêtre déduits les principes et les généralités exposés dans la première sont énoncés et développés; les auteurs reprennent chaque formation séparément; et passant en revue toutes les localités des environs de Paris ou elle peut être observée, ils sont conduits à indiquer les modifieations locales qu'elle peut présenter. Chacun des chapitres consacrés à l'histoire de chaque formation des terrains parisiens, est suivi dans cette nouvelle édition d'olsservations du plus grand intérèt sur les formations de méme nature qui ont été reconnues hors du bassin de Paris, soit en France, soit en Angleterre, cn Allemagne, en Suisse, en Fspagne, en Italie, elc. Cette addition importante est due à M. Brongniart.

La troisieme section est consacrée aux nivellemens et coupes du sol, aux rapports des divers terrains entr'eux et à des considérations générales.

M. Brongniart fils a donné, comme appendice au travail de son père, la description des végétaux fossiles observés dans les terrains des environs de Paris.

Une grande carte coloriée, plusicurs coupes générales, et seize planches qui contiennent un grand nombre de coupes de détail, et. les figures de beaucoup de fossiles caractéristiques de chaque terrain, accompagnent la description géologrique des environs de Paris; la plupart des planches sont lithographices, et leur exécution atteste les progrès rapides d'un art si important pour les travaux d'histoire nitturelle.

C. P.

69. Simti's grologichl $\Lambda$ tias, parts $\mathrm{x}-5$, ou

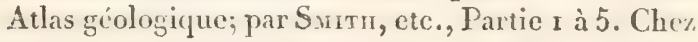
James Cary, St.-James Street, no. 86, near this palace, London, r819, ז82 I.

Ces belles cartes geologiques des comtés d'Angleterre, tracées à une tris-grande échelle, font partie d'un atlas dont la publication a commencé en 18 rg, et qui se continu. L'auteur, MI. William Smith, ingénieur des mines, est déja connu de toute l'Europe par sa grande carte géologique de l'Angleterre et d'une partic de l'Écosse, publice en 15 feuilles, en 1815 . 


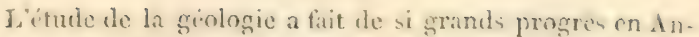
ileterre depuis ruelpues annés, que mous croyons fire une: chose agréable aursatuns en leur rappelant ici les priuci. pithes cartes of conpes géologriques ditachées qui ont ite publiées dans ce pays.

f. Lit premicre fut la carte de Smith, en r 5 fenilles, dont nous venons de parler; elle porte le titre suivant:

I delincation of the strata of England and Wiales, and part of Solland, che (Carte de's couches de l'Angleterre, du pays de Gralles, ot dinne partie de l'ĺcosse, en i j feuilles colorices; par 11 . Smith. Londres, aout $18 \mathbf{8 5}$. ) I'rix, 5 lives 5 schellings sterling ou $\mathbf{x} 3 \mathbf{x}$ francs environs.

Le meme auteur a donné ensuite une série de coupes colorices pour correspondre avec ectre mème carte, savoir :

1 . I gee losical section from London to Snowden, ete.; fonpe géologrigue depuis Londres a Snowden. (dans le pays de (ralles), montrant la succession des conches et l'ilevation "xacte des montagnes, publice le is juillet is I 7 ; une finille-

2". I ertical wertion of stratu in Surrey, etc., compe verlieale des conches dit conté de Surrey inclimés vers le nord. ef de celles che comté de Sussex inclinces verd ke sud; publiéc le $\mathrm{I}^{\mathrm{er}}$. mai $\mathrm{x} 8 \mathrm{I} 9$, une feuille.

3. Section of the strate throush Hompshire, etc.; Coupe. a trasers le II:mpshire et le Wiltshire, jusqu it Bath, sme lit route de: Batle a Salisbury; ter. mai sorg; me fenille.

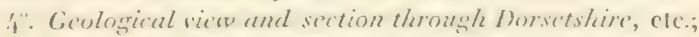
Fue el coupe grologigne a travers le Dorsethlire et le Sommersetshire jusca'ia Taunton, sur la route de Wimbormmin ier, par Yeovil, ete., rer. mai 18 r 9 , une feuille.

5. Geolonical vien and sertion in Lissex ot llartfordshire. Vue et conje gúoblogirpue prise dans le comtéd'knsex el dans le?

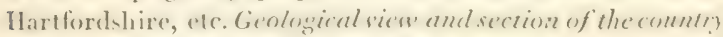
bepasen fonden and Cambrielge. Vue et coupe gुcologrique du fays entre Londres et Cambrilge; une fenille, sans dalle.

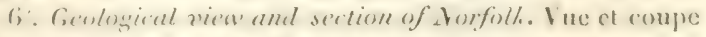

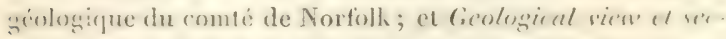

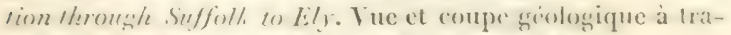
vers le sulfoll jusrua lity ; me ferille, silss dinte.

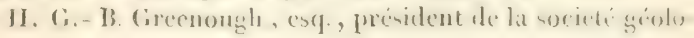


ziriue, elc, a publié une trés-belle carte géologique de l'Andeterre et du pays de Galles, en 6 feuilles, qui a paru le $\mathbf{I}^{\mathrm{er}}$. novembre 1810 .

III. Sinfin, en $r 82.0$, M. Smith a publié une carte gćolngifuo de l'Angleterre et du pays de Galles, on une feuille, qui n'est qu'une réduction de sa grande carte de 18 i5.

Les ouvrages de M. Smilh se trouvent chez H. Cary, marchand de cartes, n". 86 , Saint-James strect, près le palais, a Londres. On peul se procurer les coupes et les cartes des comtés séparément.

La carte de M. Grecnough, se vend chez Longman, IIurst, Rees anil comj). Paternoster rorr. Londres. Prix, 6 livres, 6 shellings sterling ou 157 francs environ.

DE BASTEROT.

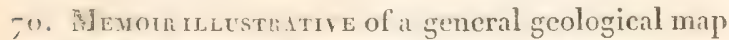
of the principal mountain chains of Furope, ou Mémoire pour l'iutelligence d'une carte géologicque: des principales dhaines de montagnes de l'Europe; par le Rer. M. D. Conventre, membre de la snciété royale de Londres. (Annals of philosoplyy, janvier 1823 .)

Iestravaux de MiI. Buckland, Ebel, Raumer, Treferstein, et particulierement l'imporlant ouvrage de MI. Beudant sur lat Liongrie, ct le Mémoire de M. Strangways sur la liussie, dans les Transactions géoloniques, ont déja préparé des matériaux nombreux pour un ouvrage de la nature de celni-ci. MI. Conybeare cite aussi, commederant paraitre incessamment, une: carte géographique de Jrance et une d'\$llemagne. Celle de France est le fruit du travail commun de MM. Coquelert Hontbret et Omalius de Iialloy; et le premier en a dijà fait hommagne, il y a un an, à l'Académie des sciences; la seconde sera l'oúvage du célèbre géoloz̧ue prussien, M. de Buch. On atiend également avec inpatience la Deseription des Pyrcúes, par M. de Charpentier. Lauteur est dans re monent a Paris pour faire imprimer cet ouvragse, courome l’annéc dernière par l'Institut.

La carle de l'Europe, juinte an Mémoire de Mr. Conybeare, -t sur lacuelle il a dislingui par des teintes pales les diffé- 
rentes formations, est au fond, d peu de chose prés, celle de? l'ourrage d'Ebel sur les Alpes, mais rectifies a plusienr. cugards. On n'y troure ni liz píninsule des Espagnes, ni l'Italic méridionale, parep que l'autcur a jugé que ces pays étaient encore trop imparfaitement connus sous le rapport géologique. Les contrées situces an nord de la mer Ballique: manquent également; ce qui est peu à regretter, dit M. Conybeare, parce que ces pays n'offrent que des roches primitives et de transition.

L'impossibilité de multiplier les distinctions de terrains, dans un cadre aussi restreint, a forcé l'auteur à n'indiquer, pardes couleurs différentes; que huit formations principales, savoir :

10. Les roches primitives et de transition;

$2^{0}$. Les séries qu'il appelle carboniferes, comprenant le gris rouge, le mountain lime-stone et les terrains houilliers;

3o. Les terrans de grès postérieur au grès rouge et calcareo-magnésiens.

4. Le calcaire du Jura, qui est le lias des $\Lambda$ nglais et la séric oolitique.

5. Le sable vert (green sand);

6. La craie.

7 . Les formations tertiaires ou terrains supérieurs à la craie;

8. Enfin le trapp supérieur et les terrains volcaniques.

Après avoir indiqué rapidement, sous les lettres $\Lambda$ à $P$, les principales chaines de montagnes de l'Europe, l'auteur interrompt, à la page $x 6$, ce Mémoire, dont il promet la suite dans un autre cahier du mème journal. C. 11 .

7 r. Str ia tempiratire des mines; par M. Movle. (Amals of plitosophy, janvier, 1823. )

La question de la chaleur propre attribuée a la terre par plusieurs physiciens, et contestée par d'autres, parait excile: un grand intérèt, surtout en Angleterre. MII. Fox et Forlen se sont accordés pour l'affirmative, dans les mémoires de liz société géologique de Cornouailles; et le premier, cn particulier, dans le même journal d'ou cet article eat tire.

Cette opinion a été combattue dans les mémes collections 


\section{Géologie.}

par M. Moyle; et il cite, dans le mémoire que nous avons sous les yeux, plusieurs faits qui semblent en effet prouver que dans les mines abandonnées depuis long-temps et remplies d'eau, la tempéruture est la mème à différentes profondeurs, et inférieure à ce qu’on supposerait être la température moyenne du climat.

Un fait remarquable qui résulte des expériences, c'est qu'on trouve a pcine deux points également éloignés de la surface et placés dans des circonstances semblables (en ap)parence?) qui offrent la mème température.

Comme ces anomalies ont toujours lieu dans des mines en exploitation, il y a lieu de les attribuer à des causes accidentelles; je suis persuadé, en conséquence, dit M. Moyle, ¿iu'il est très-difficile de déterminer la véritalile température dans quelque partie que ce soit d'une mine actuellement exploitée, et que, pour obtenir ce qu'on peut appeler une donnée certaine, il faut avoir recours à des mines abandonnées depuis long-temps.

C. MI.

72. Cenni geologici, etc. Aperçus géologiques sur le

territoire de Nassa Lubrense, par le comte Micifir.

Milano. In-4. avec une carte geograp. 1820, Naples.

Massa Lubrense est la partie méridionale du territoire de Sorrento, dans le royaume de Naples. L'auteur en recherche la formation, décrit son état actuel, ses produits volcaniques; il hasarde quelques conjectures sur l'époque ou l'ile de Caprifut séparée du continent; il attribue quelgues inondations extraorlinaires an dégagement sous-marin de l'hydrogene; il s'attache à expliquer les anciennes révolutions des continens, par l'état où nous les voyons aujourd'hui. Resue Encyclop., décembre 1822 , pag. 579 .

F

73. Transactions of the geological Societr, established nov. 13, r 807 , ou Transactions de la sociélé géologique deLondres, établie le 13 novembre r 807. Seconde série, $I^{\prime r}$. volume, première partic. ln-f de 184 pages, avec 24 planches. Londres, 1822.

La société géologique a entrepris de publier clle-méme ses Transactions, afin d'en réduire le prix qui éliai peru accessible TOME I. 


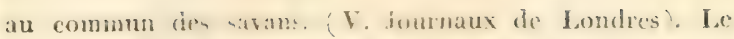
nombre de, mémoires contents dians cette premiere partic est de 12 , sani y comprentre dins extraits des procés verbaux des séa nces de cette société.

Voici la liste de ces mémoircs.

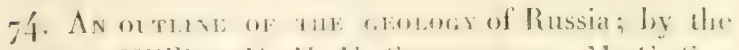

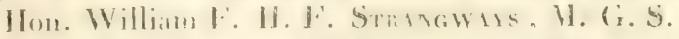

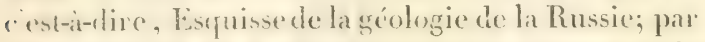
thomorable I'. II. F. TV. Strangways, membere de la socićté géologicper avee une planche et une carle géologrieque de la Russie européenne. (T'rans.geolog., $2^{\text {e }}$ séric, vol. I, pl. première.)

75. Remiris on tur geology of the south coast of Fingland from Bredport Ilasbour, Dorset to Biabbacombe Bay, Deron; by II. T. de la Bicue. eserr. M. G. S. Observations sur la géologie de la cote méridionale d'Angleterre, de Bridport dans le comté de Dorset, à Bab)acombe daus le comlé de Devon; par H. T. de la Bèche; avec planches.

Les terrains décrits dans ce mimoire sont la crive, te sable vert (green sand), le lias et le nouveau grès rouge.

76. On the Bagshot sand by H. Warditon. Sur le sable de Bagshot; par H. Warburton.

Ce nom est donné à la formation de sable cui est immédiatement sur l'argile de Londres, et qui s'étent de l'est a l'ouest, sur l'ase du bassin de Londres. Le test des corpuilles qu'on y tronve étant décompasé, il est inpossible de diterminer les espèces; mais le fossile le plus abondant est un moule d'une crassatelle.

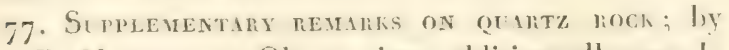
1). Ilecurocn. Observations additionnelles sur Ie quartzite.

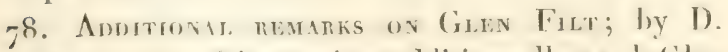

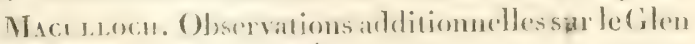

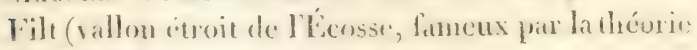
d'Ilutton.) 
79. ON TIE: Geolocis of the coast of France, and the inland comtry atjoining from Fecamp to SaintVaast; by H.T. de la Bècrie. Sur la geologie de la còte de France, depuis lécamp jusqqu ì Saint-Taast avec deux coupes de la còte et une carte géologique.

8o. Ov the freshwater formation in Hardwell cleff Iamsphire, and on the subjacent bed of Hardwell to Muddiford; by F. Webster, secretary to the scological socicty. Sur la formation d'eau donce de la falaise de Hardwell dans le Hamsphire, et sur les couches adjacentes de Ifardwell a Muddiford; par F. Webster, secrétaire de la société géologique, avec une planche.

Cente localité est célébre par l'ouvrage de Brander sur les fossiles qu'il y a trouvés, intitulé Fossilia huntoniensiut, dans lequel il a fait une errenr, en plaçant la formation marine is Hardvell, tandis que c'est dans la falaise adjacente de Barton que se trouvent les fossiles qui sont gravés dans ce bel ouvrage. C'est à M. Webster que nous devons la rectification de cette erreur importante, et la description exacte des dem formations.

Si. On tue exchutron of valleys by diluvian action as illustrated by a succession of valleys which intersect the south coast of Dorsetshire and Deromshire; by the rev. William Buckisom, R. R.S. Fi. L. S. S. P. G. S. and professor of gecology in the university of Oxford. Sur l'exeavation des vallone parl'action des débàcles diluvieunes, démontrice par üne suite de vallons sur les côtes din Dorsetshire. etdu Devonsine, arec dens planches; jar le R. W. Buclland, professenr de géologice it liuniversist d'Oxford.

Ce mémoire, (quoique conte, est trio-intersisant pour li

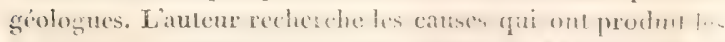

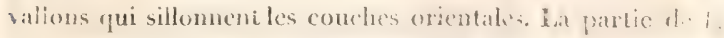
côte d'ingleterie quil décrit en détail ut quil tst con 
entre les villes de Lyme et Sidmouth, dans les comtés de Devon et Dorset, offre les preuves de ses opinions à ce sujet. Son système cst celui d'un cataclysme, ou de ce qu'il nomme les eaux diluviennes, qui a prodnit des changemens trèsétendus sur la surface de la terre, qui fut antérieurement modifiée par d'autres catastrophes semblables, lesquelles ont produit les formations du nouveau grès rouge et celle de l'argile plastique.

8.. Annitronil Notices on the fossil genera Ichthyosaurus et Plesiosaurus; by the rev. William Conybeane. Additions au mémoire sur les genres fossiles Iclithyosame et Plésiosaure; par le rev. W. D. Conybeare; avec 8 planches.

83. On the Valley of the Sctudy river in the Limalaya mountains; by H. F. Colebnooke, esq. Sur le vallon de la riviere Suluj, dans les montagnes de l'Himalaya; par I1. I'. Colchrooke; avec une planche.

Ces observations furent faites par le lieutenant A. Gírard, en 1818 . Les pies les plus élevés sont de 16,921 , à $18: 49^{3}$, et 19, fir pieds anglais au-dessus du niveau de la mer.

Les bords de la Sutluj, à la hauteur de 1500 à 2000 pieds, sont calcaires. L'inclinaison des couches de $10^{\circ}$. à $15^{\circ}$. On voit à Janse 8 ou 10 sources thermales près la rivière. Elles ont la température $130^{\circ} \frac{x}{2}$ Fahrenheit, la rivière étant $61^{\circ}$. A 6,000 pieds est du gneiss; à 15, roo pieds du mica-schiste et du granite. M. Gérard a traversé l'Himalaya par un col, à l'élivation de 15,00o pieds; mais les roches étant couvertes de neigre, leur nature ne pouvait pas être observée. Apris avoir jathé ce col, on trouve la jonction des rivières Sutluj et Bespa 6,300 pieds au-dessus du nivean de la mer. Les couches plongent vers l'orient sur un angle de $30^{\circ}$, et sont de de gneiss, granite et quartzite.

La ville de Marang est dominée par une montagne de schiste arcilenx, sur laquelle, à la hautcur de 12,000 pieds, on trouve les éricas, les genievres et les groseilles à maqueıcau. Dans le reste de la rourse des voyageurs, ils ont trouvé 
Ie granite avec et sans la tourmaline, le gneiss, le schiste micacé et l'argile schisteux.

Entre Namgia et Shipké, situées sur la Sutluj, d̀ 9,00o pieds au-dessus de la mer, on voit le granite avec et sans tourmaline et grenats, gneiss, mica-schiste, quartzite, quaryz et mica, avec cyanite et steatite. Ceci se voit aux limites de la Tartarie chinoise, au nord de laquelle M. Gérard a gravi la montagne de Farhigang, élevée de $\mathbf{9}, 4$ II pieds anglais au-dessus de la mer. Cette montagne est de calcaire granulaire, et le sommet est probablement élevé de 22,00o pieds anglais; il parait que le granite, le gneiss, le schiste micacé et le calcaire, alternent l'un avec l'autre partout ou M. Gérard a été dans ce voyage; et dans la latitude $32^{\circ}$ nord, à l'élévation de 16,800 pieds, il a trouvé une espéce de campanula et des arbrisseaux encore plus laut.

84. On the Geology of the north easter border of Bengal; by H. T. Colemnook . Sur la Géologie de la frontière nord-est du Bengale; par H. T. Colebrooke.

Les observations qui ont donné lieu à ce mémeire furent faites par MI. David Scott, commissaire de la compagnie des Indes, à Cooch Behar, dans le Bengale. Les échantillons furent recueillis dans la partie N.E. du Bengale, sur la rive gauche de la rivière Brahın-Putra, et dans les montagnes Caribari, au confluent de la rivière Caln, à 150 pieds atdessus de la mer. Ces montagnes ou collines sont de schiste argileux, en couches horizontales, surmontées de sable rouge ou blanc. Les fossiles qu'on trouve dans ces couches sont analogues à ceux des bassins de Paris et de Londres supérieurs à la craie, sinon en espéces, du moins certaines en caraclère générique : on y trouve du bois fossile, des dents de squales, des dents de crocodiles, des pates d'un homard, des huitres, des cérithes de plusieurs espèces, des turritelles, des balanes, des patelles, etc.

85. Description accompaning a collection of Specimens made on a journey from Delhi to Bombay; by J. B. Fraser. Sur une collection d'Échantillons faite pendant un voyage de Delhi à Bombay.

La distance de Bombay a Delhi, en ligne droite, est de 
200 lenes de poste frameaises. Jees iedrantillons sont dis roches primitives recucilliendims la parie contrale des Indse, entre 1)elhi et Neymuch; des roches seconditires prises anttour de lhodepoer, a loecident des montazues primilises; des roches traprennes, fort analnones ans rachen hasaltiques

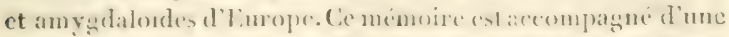
carle de cerle partie des Indes, situde culre le golfe du Bengale et les montagnes de l'llimalaya, montrant en un senl conp dieil combien il reste at explorer siologiquement dans ce vaste pays.

Cet extrait nons a été communiqué par Mr. Undervoon, les Transactions géologirues ne nous étunt pas encore parvenues.

86. Mŕmone sur les terrains de sédiment supérienrs calcaréo-mappréens du Vicentin, et sur fuclepues terrains d'ltalie, de Frauce, dAllemagne. cetc., qui peuvent se rapporter à la mime épuegue, et efui présentent quelyues particularités; par M. BinoGNART. I vol. in-f; avee 6 pl. Clez Levaule

Nous rendrons compte incessamment de cet ouvrase qui vient de paraitre.

\section{$700 \mathrm{~J}, 0 \mathrm{GH}$}

87. Histome matromere nes mamifines, arec dies figures originales enluminces, dessinées daprés des avimaux vivans: publiée sous l'autorité de l’administration du Muséum d'histoire naturelle, pas

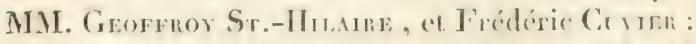
M. de Iasteyrio edicur, rue du Par. 11. 59. 36 a $38^{\circ}$. livaisons, infol. Imp. de Rignonx. Pris, $15 \mathrm{fr}$. la livraison.

36e. Lemarson. Fille renferme les descriptions ef les fignres de six animanx, savoir: i'. 1'Ouandexen de Buffin, Simia Silenus, Linn.; 2' Ie Press, Clatolutes formegineat Tred. 
Cuvier; petit animal de Sumatra, fort remarefuable en ec qu'il présente à la fois un systeme dentaire analogne a celui des musaraignes, et des formes extericures trís - voisines de celles des écureuils : il avait déja été indiqué par M. Díard, sous le nom de Sorex-glis, et décrit par MM. Raffles et Ilorsficld, sousle nom de Tuper) a ferruginea; 3'. lo Singe à queue de cochon, d'Edwards ou Maimon de Buffon et d'Audebert, Simia nemestrina Gmel.; q". le Blaireau commun, Taxus 'ulsaris, Cuv.; 5. la Corinne, Antilope Corinna, Gmel., qui n'est qu'une simple variété de la gazelle proprement dite; Antilope Dorcas, Linn.; 6. le bouc dn Nepaul, dont la femelle est décrite dans une livraison précédente; race domestique rematrquable par l'élévation de ses jambes, la grande courbure de son clanfrein et la longueur de ses oreilles, qui sont étroites, plates et pendantes.

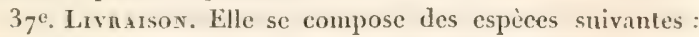
I". Ie Caracal du Bengale, Felis Caracal; 2". la Beletted'Europe, Mustela wu'gutris, Linn.; 3\%. le Nonax grris, Arctomys Empetra, 4․ le Iérot du Sénégal, Myoxus Corpeii, Fréd. Cuv., espéce nouvelle, plus petite que celle du lérot d'lírope, se rapprochant un peu du loir, par sa coulcur qui est d'un gris clair, légèrement jaunatre en-dessus et sur la queuc; blanchâtre aux parties inféricures du corps, et d'un blanc pur sous la mâthoire inférieure ainsi que sur les joues; 5". le Chevrolain Napu de Sumatra, Mosihus Nupu, Fred. Cuv., espéce nouvelle de la taille du lievre, d’un brun mé. langé irrégulierement de reflets gris-noirâtres ou fatures, ayant la mâchoire inférienre blanche, le poitrail d'un beun foncé et marqué de cinq raies blanches convergentes, denx de charque côté et une inférieure; son corps est lourd, épais et ses jambes sont tres-fines; MI. Raffles l'avait déja indiqué sous le nom de Mischus juranicus, mais cette désignation doit être résersée pour le chevrotain de Jita, décrit par Buffon, et qui appartient a une espece differente; 6 . le kirt du Bengale (la figure seulement se trouve dans celle livraison).

38". Jivnasox. Les quadrupédes compris dans cette livaí son sont : I". Ie Tehineon de Jiva et de Sumatra, Semu(y):the'usmaurus, lirél. Cuv, sinģe deja nommé guenon maure 
par M. Groffroy Saint-Hitaire, et Simic cristulu, far Raffles vol. 13 Trans. soc. Linn): il appartient à un greure nouvellement proposé par M. Frédéric Cuvier, pour placer quelques especes de gruenons, et dans lequel il fait rentrer aussi le Nasique ougenre Nasralis, Geoff.; et le Doue (genre Lasio-

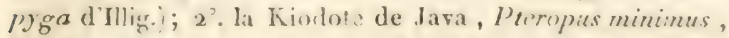
Cocoffr., ou l'teropus rostratus, Horsfield, dont M. Frédéric Cuvier forme un nowven genre sons le nom de Macroglosse, caracterise par la longueur extrème de la tète, et par plusieurs differencen dans le systeme dentaire comparé a celui des roussettes; 3 . la Tortule de totre pays; Iespertilio Notula, Gmel.: 4. Ie Schermaus, Lemnus terrestrix, Frid. Cur, qui parait étre le Blus amplibus terrestris de Linn. (Foun. suec. 3r, et Syst. nat. édit. 12, page 82 ), et qqui, tris-semblable par son aspect général au rat s!'ean, en differe réanmoins par les proportions générales, les formes de quelques paries de la téte, la longueur et le nombre des vertibres de la quene; 5 ". le. Muscardin, Myoxus asellanarius, finel.; $6^{\circ}$. l'Fncoubert, cspere de tatom, harquelle apparticut viritablenent re nom, et gui cot differente d'une au-

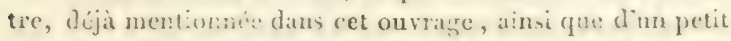
taton velu, qui y ent figraré sans disignation spécifique; $c e$ dernier ne defferint par de celui que H. Desmarest ( Mammalogrie, $\left.n^{\circ} .595\right)$ a dérit comme étant le tatom pichiy de d'A cara, tuut en lui a pportant par erreur la tigure duprenier encoubert de M. Frédéric Cuvier. Drsm.

88. Essar nour servir a lhistoire des animanx du midi de la Trance; par Marcel de Sranes, anciun inapecteur des arts et manulartures, etr. In-f. de ra feui!. et demie. Imp) de Ricard, à Marseille, prix, a fr. A Paris, chez Gubon; is Nontjullies, chez $A$. Crabon et compagric.

Ce travail, du a un meturaliste bien connu, a cle entrepris comme le dit l'auteur, pour forner un des chapitres de la statistique du dipartement de l'terault, et pour donner une idee des animaux qui caractérisent le midi de la france. Il se comprose de deux parties listinetes dans !e fiti, quoi- 
qu'elles soient réunies sous un titre commun, qui est le suivant : Des diverses espéces d'animaux que l'on obscrve dans le département de l'Hérault, et particulièrement des espéces qui peuvent servir à caractíriser la bande isotherme de 15 it $20^{\circ}$, dans laquclle se trouve compris ce département. Ia première partie offre des observations générales sur le's lois de la distribution des animaux sur le globe. La seconde présente une énumération systématique de tous les animaux observés dans le département de l'Hérault. Nous observerons d'abord qu'il est fácheux de voir un semblable travail englubé dans une statistique départementale; il n'y est point à sa place, tant dans son intérêt que dans celui de cette statistique, et c'est se tromper étrangement sur cette science que de croire qu'elle puisse admettre des catalogues nominatifs de toutes les productions d'un pays. La statistique n'envisage que les productions utiles ou nuisibles à l'espèce humaine; elle les considère sous les points de vue de l'ćconomie publique; à plus forte raison une dissertation. sur les lois générales de la distribution des animaux sur le globe est-elle déplacée dans une statistique partielle, où l'on ne doit présenter que la situation particulière du territoire en question. Quoi qu'il en soit, ce nouvel ouvräge de M. Marcel de Serres est d'un véritable i térêt, et mérite d'ètre lu et médité. On trouvera dans la première partic un apercu de la plus grande partie des faits connus sur lesquels se basentles lois indiquées par MM. de Humboldt, Latreille, besmoulins, etc., relatives à la distribution des animanx sur le globe, ainsi que quelques vues nouvelles et quelques rapprochemens intéressans dus à M. Marcel de Scrres. Dans la scconde, a laquelle la premiere sert d'introduction, on trouve, comme nous l'avous dit, un cataloğne systématiciue d'apris le regne animal de $M$. Cuvier, pour tous les animanx reconnus jusqu'a présent, selon l'auteur, pour être indigenes dins le déprattement de l'Hérault. Chaque espèce est accompanné d'une citation synonymique, et quelquefois de notes intéressantes. Les espéces des côtes de la Mlédiler rance offriront surtout de l'intérèt aux naturalistes, pon lesquels ces sortes de catalogues sont très-importans, surtout lorsqu'ils sont dressés par des hommes qui sont faits, comme 
11. de Serres, pour inspires une juste conliance par la réputation méritée dont ils jouissent.

F.

By. 1)Es nrives nes mumines considérées comme caractiores zoologipues, par M. Frédéric Cerner. In-s. aver roo plamblies lithographices, ouvrage formant g on ro livraisons. Prix, 3 fr. la livaison. Chez li-ti. Levrault, éliteur is Strashourg; et rue des Fossés-1I.-Je-Prince, n. 33 , à Paris.

L'anteur de cet ouvrage purement didactique a cu pour objet spécial de donner les moyens de comparer entrelles les formes des dents des mammiferes qui, ainsi qu'on l'a reconnu depuis long-temps, fournissent les meilleurs caracteres qu'on paike employer pour lit ciassification naturelle de ces animatux, parce quelles sont toujours en rapport avec leurs mocurs et arec le reste de leur organisation.

Il a divisé son travail en un nombre assez consitírable de petits chapitres, renfermant dhacun la description d'un systeme de dentition et l'indication des espèces qui présentent ce système.

Dans chacun de ees chapitres, les dents sont dérritesavec détail pour un seul côté dés deux mathoires, et les ligures qui laecomparnent représentent leurs sirics supéricure el infericure vues de profil el de face : un dessin particulier donne une idle de leur situation respective, lorsque l's mâchoires sont fermées.

Toules les dents sont renfermées diuns la meime dimension en longuene sur les differentes planelies, quelle que soit la taille des animams ansquels elles appartiennent; mats pour faire comnatre kne juste dimension, lantenr a en woin d'indiquer te rappost du dessin avee la granderer naluretle des objets qu'il représente.

Le discours froiminaire de cet omvage ne paratra qqu'ited

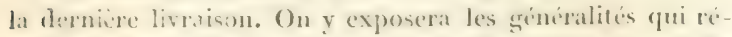
sulteront des observations consigners dans soll étendue, et la therie de la formation et du diveloppement des dents. Celle derniere livaison contiendra aussi une syounguie de. genres ct des espèces qui auront été cités.

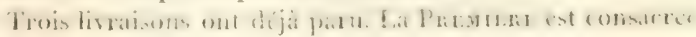




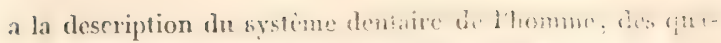

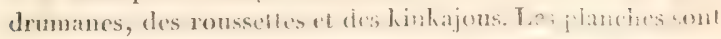
relatives, sclon lenrs numéros: $x^{\prime \prime}$ a l'homme, 2"à l'thurng outang, 30. au Pongo, 30. ( lis) aux Gibbons, fo anx Sem-

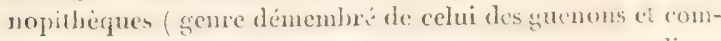
prenant six especes), 5", anx Guenons proprement dites, 60. aux Macaques et anx Cynociphales, $7^{\circ}$. anx Alouates, Atèles, Sajous et Samiris; 5 ". anx Sahis , 9 ". anx Ouistitis, $10^{\circ}$. aux Mahis et Indris, I $1^{\circ}$. aux Galagos et Loris, $11^{\prime \prime}$ (bis, au Tarsier, 12, au Potto ou Kinkajou.

La Scconne a pour oljet les mammiferes insectivores; savoir : 133 . les Roussettes, dont le système dentaire ne difficro pas essenticlement de celni des Ceplualotes, des Cynoptises,

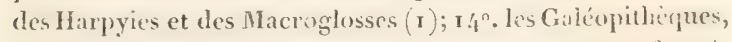
150. les Chauves-souris, comprenant tous les genres formés anciennement par is. Geoffroy, el de plus celui que l'aiteur nomme Dy sopes (2) (Dys. Moops de l'Inde), et le genre MOrmoops de Leach (Trans. Linn. t. 13 ); $166^{\circ}$. les Hérissons, I $7^{\circ}$. les Cladobates de M. Frél. Cuvier, ou Tupaju de Riufles et Inorsfield ; $18 \%$. les Chrysoclores, r 9". les Tamreci, 20". les Musaraignes, 21․ les Desmans, 22". les Scalopes, $22^{\circ}$. (his)

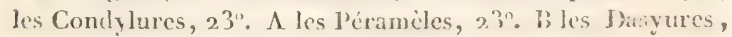
et 23. C les Sarigues ou Didelphes.

La Trorsime livraison se compese d'une partic des carnassicrs, savoir: $24^{\circ}$. Ies Chats, $25^{\circ}$. les tiyenes, 26 ol $27^{\circ}$. Ies Putois, Zorilles et Martres; 28'. Jes Moulfetles et Mlydaus,

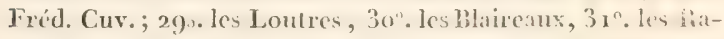
tels, $32^{\circ}$. Ies Grisons, Tairas et Gloutons; 33 . les Clicinis et Thenards, 3/\%. les Civeltes, Mamgoutes, Genelles of l'aradexures, $34^{\prime \prime}$. (bis' l'Jetide, nonvean genre chabli parr MI. Talencicnnes; $35^{\circ}$. Le Suricate, 36". les Ratons ot Coatis, $37^{\prime \prime}$. les Ours.

Desy.

(1) Les genres Cynopteric et Macroglosse sont nonveaux et proposés par M. Frédéric Cuvier. Le premier renferme le P'ternpusmarsinalus de M. Geothroy, ct le sccond le Plerepus minumas an hio dote du même naturaliste.

(2) Il ne faut pas confondre ce genre Dy-sopes avec celui ufu'li liger nomme ainsi : ce dernier se rapporte an genre Mlolossus de A. Gcoflinay. 


\section{Galeme des Oiseacx, etc.; par M. L. P.} Vieirlot et Paul Ordant. $\mathbf{8}$ e. livraison, in-4. d'une fenille et 4 planches coloriées. A Paris, chez Aillaud. Prix pour les souscripteurs, $5 \mathrm{fr}$. : pour les autres, $6 \mathrm{f}$.

Le but que l'auteur parait s'étre proposé dans cet ourrage est de classer méthodiquement les oiseaux connus des cinq parties du monde; ne croyant pas que pour y parvenir, il fiut nécessaire de donner les figures de plus de quatre mille individus, il s'est borné à n'en publier qu'une seule par division générique, ou deux à quatre, quand un groupe cst composé de plusieurs sections.

Le bec et les pieds, parties les plus essentielles à la détermination des genres, soat figurés isolément sur trente-deux planchəs, distribuées gratuitement, ainsi que le texte, aux souscripteurs. Chacune de ces planches contient au moins neuf objets : ainsi, on présente à l'oul l'explication du discours, qui quelquefois n'est pas assez intelligible, nu qu'on est forcé de se servir de termes techniques, qui ne sont pas connus de tout le monde. Par ce moyen, celui qui veut s'atonner à l'étude de l'ornithologie parviendra facilement à la connaissance des caractères, et pourra, avec ce seul aide, classer les oiseaux.

Le texte contient non-seulement une description en languc française, mais encore, pour cenx qui ne savent pas cette langue, une phrase linnéenne et latine du vieux, de l'adulte, du jeune et de la femelle. En outre, cliaque division est accompagnée d'une généralité qui peut s'applicquer à toutes les espèces qu'elle contient, et qui donne une idéc suffisante du genre de vie de la totalité.

Les dessins, exécutés par un des éleves les plus distinguis de Vanspansdonck, lithographiés, coloriés sous sa direction et sous ses yeux, ne laissent rien à désirer, et s'approchent de la nature autant qu'on le peut.

Il a déji paru vinght-cinq livraisons de cette ouvragge. DEsn. 91. Moni:zes ne Céphllopones microscopiques vivans et fossiles, reprisentant un individu seulement de chacune des principales divisions d'une nouvelle 


\section{Zoologie.}

méthode basée sur le mode d'accroissement des coquilles; par M. Alcide Dessalines d'Orbigny, fils.

La souscription se compose de quatre livraisons, qui comprennent chacune vingt-cint modèles, et en outre trois à quatre coquilles, pour les premiers souscripteurs : l'extrême rareté des originaux ne permettant pas maintenant d'en promettre dasantage. (Ces originaux sont renfermés dans de petites boîtes de verre qu'on ne doit ouvrir qu'avec la plus grande précaution.)

Les quatre livraisons seront expédiées dans le cours des six premiers mois de 1823 . Le prix de chacune d'elles est de 20 fí, payés, soit à La Rochelle, chez l'auteur, jardin des Capucins; soit à Paris, au bureau du Bulletin, rue de l'Abbaye, n. 3, ois l'on peut voir la première lirraison déja publicé, ainsi çu’au Musíum du Jardin du Roi.

Les souscriptenrs recevront avec la quatrième livraison le tableau méthodique de la distribution de ces Céphalopodes, qui indiquera par numéros correspondans à ceux des modèles, le nom des individus envoyés et l'ordre de leur classification.

Nota. Le diamètre des modèles a été porté de 40 jusqu'a 200 fois celui des coquilles originales, afin de rendre plus sensibles tous leurs caractères. Les Céphalopodes fossiles sont colorís d'une teinte rousse uniforme, less vivans sont blancs. Le lieu et la forme des syphons ou des orifices de la dernière cloison y sont indiqués par des traces ou des points noirs.

C'est une heureuse idée que celle de rendre sensibles à la vue simple, en les grandissant, ces petits Céphalopodes microscopiques, qui forment le sable de certaines plages, et qui remplissent certaines couches des terrains tertiaires. Ces modèles, infiniment supérieurs à la meilleure figure, et qui suppléent à toutes les descriptions, sont d'une parfaite exécution. En continuant à produire ainsi une suite de livraisons, on pourrait avoir la collection complète de ces petits êtres aussi merveilleux par leur prodigieuse fécondité, que par leur admirable construction et l'inconcevable diversité de forme qu'ils offrent. 


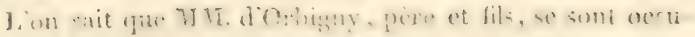
pés avec un zèle ex une patience inouis à observer ecs in-

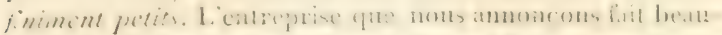

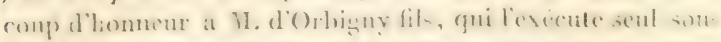

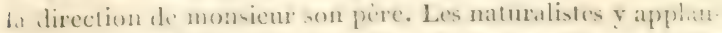

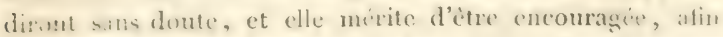

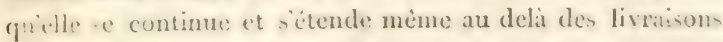
promises.

L.

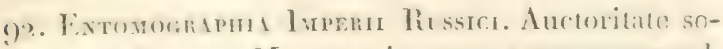

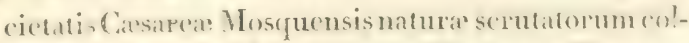

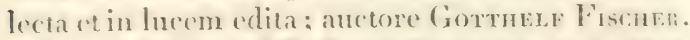
Iolumen I , cum X. VI tithulis aneis, in- $\{$. Mosqux, I $820,1822$.

Crit onvagye, écrit en francais et en latin, et qui se composera diun nombre indetermine de volume., pent etre consdlexé comme une des entreprines les plu's gemargualhlin de nutre siccle; elle honore en meme temps le souveain cint la favorise et le sarant distiugut qui se livre tont entior at wh exiention. Ie premier volume, dont lantem a fiti lomm-

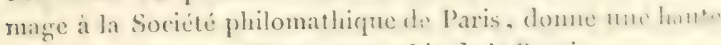
idée de ce que sera lFintomograplete de la liumbe.

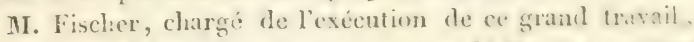
par la Sociéte Impériale des naturalistes de Mloston, expos. dans sa prelace le plan de l'ouvrage : il sera divine en de:1: sections; l’une, l'Entomographie de la Fussie proplenmentelite.

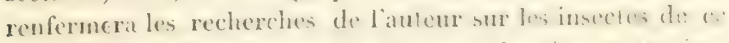

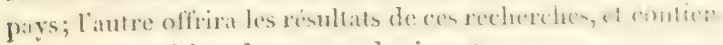
dra une exposition des genres des insectes.

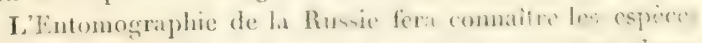

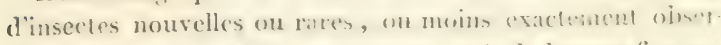

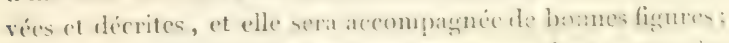

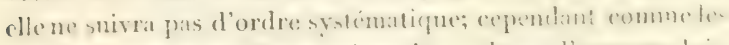

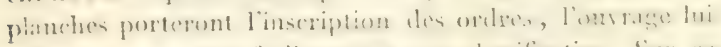

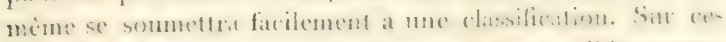

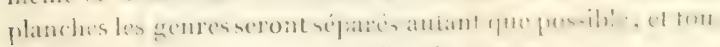
les insectes seront représentés d'apeès wature.

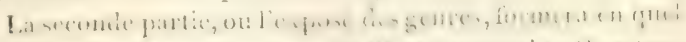

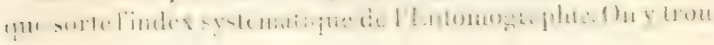




\section{Zoologie.}

vera toutes les especes observées jusqquà ce jour ; ctles scront rangées dans un ordre systématique el stons le genre auquel elles appartiennent. Chacune d'elles sera acconpagnée d'une très-courte notice.

Ilans le premier volume, que nous annonçons, l'autiun suit exactement le plan que nons venons de tracer. Lintemographic russe commence ce volumc. Il y décrit et fignut avec le plus grand soin plusieurs espèces de divers ordres trouvées dans l'empire russe, et qui appartiennent anx genres Cicindele, Carabe, Nebrice, Cyminde, Criquet, Myrmelion. Frigane, etc., etc. Il établit mème plusieurs genres nouveaux, et en donne avec détails les caractères. - Les figures colorićes qui accompagnent la description de chaque espece ne laissent en général rien à désirer. Sonvent les parties esscnticllcment caractéristiques sont représentées par de simples traits à côté de l'insecte entier.

La seconde partic ou l'exposé des genres des insectes porte ce titre : Genera Insectorum systematice' cxposita et analysi iconogra phici instructa; cuctore Gotthelf Fischer. MIosqual, I 8 I. Cette seconde partie, placée à la suite de la premiitre, en est entièrement distincte; sa paģination est mème différente. L'auteur lit fait précéder d'une préface dans laquelle il amnonce que claque genre sera accompagné d'une analyse dew parties de la bouche. Entrant ensuite en matière, Mr. Fischer jette un coup d'oil sur les insectes en général, établit leut division en ordres, donne les caracteres de chacun d'enx, et s'arrète à celui des Coléoptères; il présente, dans un tableau, le nom de tous les genres rangés en familles, en adoptant à peu de chose près la méthode que M. Latreille suit dian le troisième volume du Rè g̀ne animal de M. Cuvier, et après avoir offert dans une liste alphrabétique lia synonymie de tous les genres de l'ordre des Coléopteres, il passe à l'objet principal de cette seconde partic, l'esposé rles caractires die claque genre Il fait connâtre ceux qui portent les noms

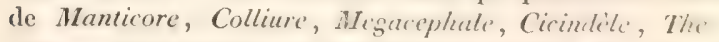
rate. - Une planche, représentant une espece de chayme genre, offre à côté les caracteres de lit bouche. Mr. Firchres plice entre les Colliures et les Hligacéphales un genre nol:ar ruil nomme Caride. 
I.e plan de l'ourrage étant tracé, nous ferons connaitrele contenu des volumes suivans à mesure qu'ils arriveront à notre connaissance.

V. Aonouis.

93. Descriprtox d'un nouveau genre d'oiseau, nommé Brachiurus, par M. C.-P. The xneng. (Mém. de

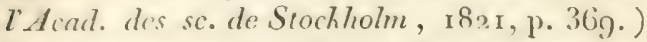

Deux oiseatux apportés du Brésil, et figurés de grandeur naturelle, dans la planche iv du second volume, ont donné lieu a l'auleur de créer ec genre, dans lequel il plave égitlement l'oiseau qu'il avait apporté de Coylan, et donné au Muscém Carlsonianum : il est decrit et firuré dans la description de ce cabinet, sous le nom de Turdus triostemus. Le caractere distinctif de ce nouvau genre est d'avoir la quene arrondie et pas plus longue que les ailes. Ceux qui jugeraient ce caractire trop peu tranché pour motiver l'établissement de ce genre, voudront bien observer, dit M. Thunbers, que le genre turdus était déja surchargé d'espéces, le șistema nuturce, de l'édition de Gmelin, en indiquant jusqu'a I 26 ; an moins pourront-ils l'admettre comme sous-division de ce genre.

C. MI.

9\}. Notvenz genres et nouvelles espèces d'insretes, décrits, par M. D.-IV. Dilan (en latin), avec figures. (Mém. de l'Acad. desse. de Stochholm, I S2 I, pag. 3 I2.) $^{2}$

Les deux nouveaux genres sont de l'ordre des coléoptères, savoir:

I․ Famille ou section des Staphylins.

Zinophonts. 3 espéces. Le fronticornis, dont la patrie est inconnue, le penicillatus, de la Guadeloupe, et le spinosus, de l'Amérique méridionale.

J'ai donné ce nom, dit l'auteur, à ce genre ol clytra bre-

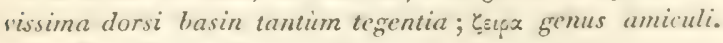

2. Famille des Pentamères.

Porxtonus. C'est le Rhipicera de M. Latreille.

MI. Dalman décrit en outre deux nouvelies espèces de genres déjà connus, savoir :

Le Phaleria furcifere de Rio-Janeiro, et l'Aplerogyna slobularis du cap de Bonne-Espérance. C. M. 


\section{Zoologie.}

95. Sur tre nouvelle espice d'insicte nomméc

Pimpla ovivora, dont la larve habite parmi les reufs des araignées, et se nourrit de ces exufs, par M. Ch. H. Bonemix, arec une figure. (Mcim. de l'Acad. des sc. de Stochiholm, I 82 I, p. 335.)

96. Reminks on the identity, etc., Ou Remarques sur l'identité de certaines lois générales, qui ont été demièrement observées, pour régler la distribution naturelle des insectes et des champignons; par W. Sinnpe Mac-Lfay. Lu à la saciété linnéenne de Londres. (Annals of pluilosopliy, N. XXV, janv. 1823.)

Ce mémoire, qui a occupé plusieurs séances de la Société limnéenne, parait avoir pour but de comparer les bases de classification des animaux, et particulièrement des insectes, adoptées par MI. Mac-Leay, dans ses Horee entomolocicice, avec celles données jar Fries pour les champignons.

Les Annales de philosophie n'en donnent qu'un extrait trop court pour qu'on puisse en bien-apprécier l'oljet.Ao.P.

97. Onservations str le gente Pelnniare; par J.-R. Jouxson, D. M., membre de la société royale de Loudres. (Philos. Trans. of the lioyal sucieity of London. 1822. Part. II. $V$.)

MI. Johnson fait connaitre dans ce mémoire les habitudes de plusieurs espèces de ce genre curicux, Leur anatomis difficile a laissé voir une cavité commune avec des cellules divergentes, comme dans la sangsue médicinale. Lanteur :1 observé deux ouvertures circulaires, ventrales et inférienres, dont l'une, postérieure, conduit à l'ovaire, et dont l'autr', antirieure, livre passage à un tube long et flexible, lequel sirr a saisir les animaux dont ils se nourrissent. M. Johnson leur ayant tranché la tète, a reconnu que c'est au moyen de cet organe tubulaire qu'ils introduisent dans leur corps les sulotances nutritives. L'auteur s'est aussi assuré que plusieurs de res planaires sont ovipares; mais qu'ils ont encore un autre mode de reproduction, par nue division nalerelle de livm Томе. 1 . 
corps en deux parties, la tète reproduisant alors une queu", et celle-ci une tête.

V. Aun.

98. Ye célebre cheralier Poux dans un avis imprimé ra français, date de Naples du 12 octobre r $\$ 22$, et qu'il a bion voulu adresser à M. de Férussac, par l'entremise de M. Sitvirny, fait cesser les craintes des naturalistes, en lenr annoncant la prochaine publication du troisieme et dernier volume de son magnifique ouvrage intitule Testar " utriustu!? "Wiliue, corumque historia et anatome tabulis ancis illustrata, dont les deux premiers ont été publiés on 17-92. Lon sait que cet ouvrage gr. in-fol., avec des planclies d'une superbe exicution, colorices, nu en noir, accompannes de planclies au simple trait en regard, a puissamment conIribué à l'avancement de l'histoire des Mollusques, dont it offre les plus belles anatomies.

M. Poli, obligé de suivre le roi de Naples en Sicile, pris chargé d'importantes fonctions, n'a pu jusqu'a présent publier le dernier volume de cet ourrage, impatiemment atiendu... Ce dernier volume comprendra les Lnivalves de: Linné; le prix sera le mème a peu près que celui des denx premiers volumes.

11. Savigny a vu chez M. Poli une grande partie des planches déjà gravées.

I.

99. Consmératrons générales sur les insectes, où l'un traite du rang que les insectes paraissent devoir occuper dans léchelle des ètres, de leur conformation, de leurs fonctions principales, de quelquesumes de leus particularités intéressantes, de leur clitssification et de leur distribution en genres, avec leurs caractères essentiels, et enfin des auteurs qui ont écrit sur l'entomologie. I vol. in-8, arec plus de 6 o $\mathrm{pl}$.

I.e libraire Levrault annone comme devant paraitre pi:chanement ce nouvel ouvrage de II. A. Constint Dusias, que tous les entomologistes verront arec lintixit que com:1mandent les productions de l'auteur de la Zoologice trialytique, etc. 
BOT A N IOUE.

ioo. Mrcologia Eeropea, seu completa omnium fungorum in variis Europe regionibus detectorum cnumeratio, methodo naturali disposita; descriptione succinctà, synonymià selectà, et observationibus criticis additis; elaborata à C. H. Persoon: sectio prima cun tabulis XII coloratis. Erlangx, ap. J.-J. Palmium, 1822 , in- 8,556 pag.

Cette premiere partic renferme une portion de la première classe de la méthode adoptéc par Persoon dans cet ouvrage, qui comprend les champignons à sporules ou à capsules (thece) placées extéricurement et non contenues dans un peridium. Le premier ordre de cette classe, auquel il donm: le nom de Trichomyci, renferme tous les champignons filatmenteux, ou bissoïdes des auteurs.

Il a créé dans ce premier ordre plusicurs genres nouveaux, particulièrement aux dépens de l'ancien genre Byssus; mais il a surtout rendu un véritible service à la science, en réunissant plusieurs des genres établis récemment par les mycologistes allemands, et fondés la plupart sur des caractères trés-légers. Nous citerons pour exemple le gyenre Botrytis, ancuel il a réuni les genres Claclobotrym, Iirgaria, Stachylidium, Verticillium.

Son second ordre, qu'il nomme Sarcomyci, renferme les champignons proprement dits; il n'en a décrit dans le premier volume qu'une partie, savoir: 10 . Les Tremelloride: renfermant les genres Tremella, duricularia, etc. 2". Le's Thelephorei. 3o. Les Helselloülei, qui comprennent lis genres Clasairia, les Liolia, les Helvella, les Peziza, cte. et plusieurs genres nouvenux.

Les figures qui accompagnent ce volume sont giniralement bien exécutées.

In. $B$.

i I. Botanieve médcale, ou histoire naturelle et midicale des médicamens, des poisons, et des alimens tirés du règne végétal; par Achille Ricuano, D. NI. 
Premiere partice i rol. in-s de fís pars. A Paris. chez Bechet jeune, 1823 . Prix, 6 fr. $50 \%$

Sous ce titre, l'autcur a donné une description exacte el délaillée des plantes le pius érnéralement employies en mi decine et de celles qui servent dialiment, particulierement en Europe; il a passé sous silence ou n'a fitit qu'indiquer quelques plantes qui, quoique insérérs dans plusicurs anciranes matieres medicales, sunt tout-à-fait hors diusaye on ne sont jlus employées dans la médecine europienne. Les plantes décrites dans cet ouvrage, qui sera d'une grande utilití a toute personue qui voudra étudier la botanique specialement sous le point de vue de la médecine, sont distribues suivant les familles naturelles qui présentent le grand avantigne que les propriétés des plantes d'une mène fimille sont presque toujours analogrues; ce qui permet de donner sur chacune de ces familles des généralités intéressantes, et facilite le medecin dans le choix des remeiles qu'il peut en tirer. Le caractere des familles et des genres ent exposé avec un soin particulier; les especes y sont dérites avec beaucoup de précision, et à la suite de chacune l'auteur a joint quelques observations sur leur analyse chimique, leurs propriétés et leurs usages. Enfin, à la fin de chaque famille il at récapitulé les propriétés générales des plantes qu'elle renferme et leur mode d'action. Cette premiere partie comprend les acotylédones, les monocotylédones, les dicotylé. dones apétales et monopétales.

L'histoire des differentes especes de quinquina, q̨ui ter mine presque ce volume, peut être indique comme lun des articles les plus intéressans. A. B. 1 .

mon. Srmorsis puatancu quas in itinere ad plagam equinoxialem orbis novi collegrerunt, Al. DE l:t vmolnt et Am. Bosilano. Auctore C. S. Kintu. vol. in-8. Tomus primus, 49r pag. Parisiis, Levrault, 1822 . Prix, 9 fr.

Cet ouvrage, comme son titre l'indigue, est une sorte d'abrégé du enrand ouvage publié par M. Kunth, sous le titre de store gene'se it species plantarum, que son prix 
methait au-dessus des moyens de beaucoup de savans. Mrais ce riest pas cependant un simple alorégé, car l'auteur y a tait plusicurs additions importantes; telles sont principalement la deseription des plantes cryptogames rapportées par MM. de ILumbolàt et Bonpland, 'fui, à l'exception des Fougères, Lycopodes, Marsiléaccées, Equisetacées et Charawes, avaient été omises dans les Norra generaet species plantarum. La description des algues est due aux soins de 11. A gardh, botaniste suédois, qui a fait une étude spéciale de cette funille. MI. Hooker, professeur à Glascow, a rédigé 1.t partie relative aux Champignons, aux lichens, aux IIépatiques et aux Mousses.

Parmi les algues, on remarque le nouveau genre Dic:tonemu, voisin de l'Hydrodiction. Tous les autres genres de c.e cryptogames étaient déja connus, mais plusieurs espices sont nouvelles.

Parmi les additions ou les changemens apportés à la partie deja insérée dans les Torra genera et species plantarum, on remarque que le Dracontium pertusum est rapporté au genre C'alla. Le Virstus cinusque forme un souveau genre, sous le nom de Cluusquea ; et is cette occasion, l'auteur indique une nouvelle division du grand gene liambusa, en 5 geneses;

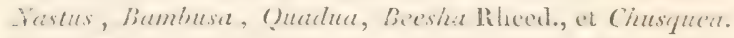
On y trouve un nouveatu gुenre, sous le nom de Radia, idus l. fimille des bromeliaces; un antre dans celle des orchidées, sous le nom d'Epistephium.

Outre les genres cque nous venons d'indiquer, ce volume renferme plusieurs espéces qui avaient été onises dans le srand ouvrage; il comprend jusqu'aux chénopodies inclusivement.

Charpe expèe est accompagnée d'une pirase caracteristique de lat synonymie et de lindication des lienx où elle croit ; on n'a done setranché que les descriptions détailléer, It les observations ginerales sur la géographic botanique, follacées a la suite de chacple famille naturelle. AD. B.

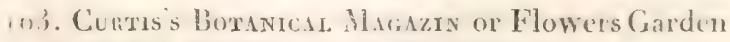
- lisplayed, dans lequel le's plus belles plintes crotiyues cultivées en plein air. dans les serres compu- 
rées et les serres chaudes, sont représentées aver exactitude et arec les couleurs naturelles; ouvrage dédié aux amateurs des deux sexes; public pirr John Sius. M. D. numéro 432. (Prix 3 sch. 6 d.) Londres, chez Sherwood, Neely et Jones.

Ce calier se compose, ainsi que les précédens, de huit planches in-8'. colorices, et d'une feuille de texte intercalaire.

Les plantes qu'on y trouve figurées sont les suivantes :

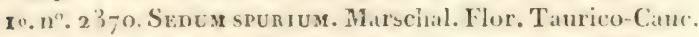
I, [1. 352 et 3, p. 312. Originitire du Caucise, celte plant. a été désignéc par Haworth sous le nom d'Anacampseres ciliaris, dans son Traité des plantes grasses On la cultive en pleine terre dans les jardins.

$2^{\prime \prime} \cdot \mathrm{n}^{\circ} \cdot 23-\mathrm{r}$. Cistus barretieri on Helianthemum Barrelieri. Tenore prodr. dellis Flor. Napholitana, p. 3x. Cette espice peu remarquable croit a l'ile de Capri, dans la baie de Naples : elle craint le froid.

3. No. 23-2. Lo is. мiтins. Iamk. Enc. 3, p. 581. M. de Jussieu avait déja figguré cette plante ( $\Lambda$ un. du mus. 5, t. 2 , f. 2 ), et ce genre, d'abord placé parmi les Onatgritires, est devenu le type d'un ordre naturel nouveau que ce célebre lotaniste nomme Lodśxes. Le loasa nitidla est une plante amnuelle originaire de Lima. Fille se reproduit de graines.

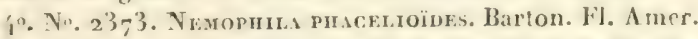
6r. Cette jolie plinte fait partie du groupe de la fimille des Borraginées a fruit capsulate, dont on a formé une section a part sous le nom d'hydrophyllées.

5”. XN. 237 - Achaxia mollis. Willd. sp. 3 , p. 839 . On cultive cette julie espece, yui est originaire de l'Inde et de l'Amérique-1Iéridionale, dans la serre chaude.

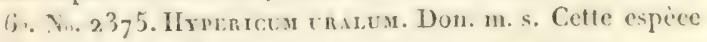
est originaire du Nepaul.

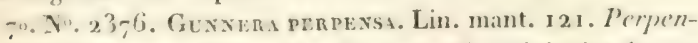
sum blitispersum. Burm. prodr. cap. 26. Originaire du cap de Bonne-Espérance.

8". N". 2377. Geravium wathichiagu. Don. in swect's fencraniacea, t. go. Cette espèce croit dans l'Inde. A. B. 


\section{Botrinigrie.}

111. Oesenvarions on the natural group of plantic called Pomacea by M. J. Lindery. Observations suy le groupe naturel de pliantes appelées Pomaccís, par M. J. Lindley. 20 pag. in-8., avec 4 planchus.

sectateur de la doctrine professée par l'école francaise, Mi. I indley a cru devoir couphtiter sa Honographie des roses, "'! publiant des observations sur un groupe voisin, celui des frumarées. M. de Jussien considérait ces plantes comme une :mple section des rosacies; mais M. Richard proposa depuis d'en faire une famille particuliere. Le botaniste anglais passe (n revue, dans une savante dissertation, les caractères des pornacies; $\mathrm{el}$ les retrouvant tous dans les rosacées proprement dites, il se décide pour l'opinion de l'auteur du genera plantarum. Après avoir terminé cette discussion, M. Lindley trace en latin le diagnostic des pomacées, et il les divise en douze genres. Ce sont, le chanomeles, caractérisé principalement par son fruit à cinq valves; l'amelanchier, le cyclonia, lont le fruit est polysperme, et le tégument propre, mucilagineux; le pyrus, dont les pétales sont presqueronds, l'endocarpe cartilagnineux, et les loges dispermes; l'osteomeles, qui a des pétales oblongs, des ovules solitaires, le fruit clos, et l'endocarpe ossenx; le mespilus, qui a le fruit découvert au sommet, e. . endocarpe osscux; le cotoneaster, ou l'on tronve des ovaires distincts; l'eriobotrya, dont les cotylédons ecourrent la radicule; le photinia à ovaire semi-supéricur et 2-toculaire; le charamele's, ou le jeune fruit est r-loc. et '>-ү.; ; le raphiolepis, dont le calice a le limbe infundibuliforme, el lovaire 2-loc.; enfin, le crato'gues, qui a le fruit obong et fermé, el des novaux osseux. - Les caractères de resdouze genres sont tracés par M. Lindley avec une clarté "t une précision qui ne laissent rien à désirer ; et latuleur se? montre digne de mareler sur lé traces de son illustre compatriote M. Robert Brown.

Aug. de S.-IIllaire.

10.5. Sphomen rotanicum exhibens synopsin graminum indigenarum Bagii partis septentrionalis, olim YII. Provinciarium, unà cum appendice, quâ nonmulla indigene nos a indicantur. ()uod favente Der, 
preside vir. Clar. Jano Kops. ad publicam disceptationem proponit auctor IIermannus Christ. Yat Hall, Amstelodamensis, Med. Cand. die xis dec mocccxis. Trajecti ad Rhemum. Ex oft. O. 1. vant Padelenburs Academiac typographi. 1\$:2, in-S, I 68 pag.

Dans la prenice partic, l'auteur donne la description des sraminées qui croissent dans la Hollande. Leur nombre est de 97. La seule esprece qui, jusqu’a présent, n’avait pas éti observec dans l'ouest de l'Europe, est le Milium sernule. Mar:ch. fler. tuur. caur. MI. Van Ifall l'a figurée dans une planche sravée qui accompagne cet onvrage; mallicureusement il n'a pas donné les détails de la fructification. Toutes les autres sont des plantes de France ou d'Allemagne. L'autcur a adopté giniralement, pour la clissification, les genres de Palissot de Beauvois.

Dans la seconde partic, on trouve la description de quelques plantes qui n'avaient pas été indiquées dans la Flore des Pays-Bas de Gorter.

AD. B.

yo6. Chavis agrostographiz antiquioris. Uebersicht des Zustandes der agrostographie bis auf Limne; und versuch einer reduction der alten synonime der Graser auf die heutigen trivial nalmen; ou Coup d'ueil sur l'état de lagrosingraphie, jusquà Linné, et essais diune rédurtion des anciens synonymes des graminées a lrurs noms spéciliqurs actuels, par D. Cart. Brmunten Truns, comseillex impérial russe, de l'ordre de Saint-WVladimir, ete., in-s de 4 ra pag. arec une planche lithographice. Coburg, chez Bichermann, 1822. Prix, 5 fr. $50 \mathrm{c}$.

Cet ouvage renferme deux parties distinctes : la premiere historique, la seconde synonymique.

Dans la premiere, l'auteur passe d'abord en revue tous less ateurs qui, depuis les Grees et les latins jusqua Linné, ont frarlé des graninées dans leurs ouvrages; il écharcil quelques points de synonymie sur les noms downe's par les anciens au- 
ieur's grecs. C'est ainsi qu'il prósume que le farehurm des Grees n'est pas la canne à sucre, mais ane esjece de lhanbun; et que c'est du tabaxir qu'ils ont voulu parler, cn iadiruant une matière sucrie dins lintérienr de leurs tiges. Il fimmère ensuite tous les divers nons sous lesquels ces differens anteurs ont désigne les organes des graminées. Enfin, il présente un lableau des classifications agrostologiques des anciens auteurs, depuis Tabernemontana, en 1 5888 , jusqu’a Micheli, en x 729 , le dernier auteur, antérienr à Linné, qui ait traité les graminées en général.

La seconde parlie de l'ourrage est entiérement synonymique. L’auteur y énumire, pitr ordre alphabétique, tous les noms donnés aux divers graminées par les auteurs antérieurs à Linné, en rapportant le nom générique et spécifique, actuellement adopté, de la plitnte a laquelle ce nom parait se rapporter. - Cette partic est terminée par un catalogue alplabétique des noms de griminces actuellement adoptés, renvoyant aux divers noms qui leur ont été donnés par les auteurs anciens.

La planche qui accompagine ce volume représente trois graminés, copiés probablement d'apres quel gue ancien ouvrage; du moins on doit le présumer a la maniere dont ils sont dessinés, mais sans ancun nom ni renroi au texte; de surte qu'on ne peut savoir ce qu'elles représentent. An. B.

in7. The Botinical ragister, etc. Chaque mumúro renferme huit figures coloriées de plantes exotirgues, aceompagnées de leur histoire et de leur node de culture. Les dessins faits d'aprés les plantes vivantes; parSroenna Eowarns, etautres. Londres, imprimé chez J. Ridgway. Prix de chaque numéro, 4 shell. No. XCV. Janvier i 823 .

Ce numćro renferme sous le $\mathrm{N}^{\circ} 6-6$, le 7 anda terctifolia. (lipidendrum teretifolium, Iink., enum. Hort. Ber.), deji figuré par Lindley, Collect. tab. 6.

677. Passiflora albida, espéce nouvelle.

6-8. Cassinia spectabilis, R. Br., Trans. linn. NII. 128; Calea spectabilis, Labill., etc. 
679. Crinum amnlile, var. B., Ausustum, Cunismat. $\$ 605$; crinum superlsum, Roxb. 11. ind. ined.

68o. Acaciu longissima, Wendl.acac., 45, tab. 1 ; ; link. enum. hort. Ber.

681. Athrixia capensis. Ce genre nouveau appartient a la famille des compusées ou synanthérées; l'auteur le caracetérise ainsi :

A тиrıxı: flores adiati. Calyx oblongus, polyphyllus, ubequalis, aristatus, recurvo-squarrosus; foliolis angustis; ratdiis plurimis; flosculis bilabiatis, labio interiore minimo integro. lappus plumosus. Recept. alveolitum. Stigmata disci a pice subtruncata subpenicellati. Frute $x$, folia syorise, inferm: adnata, integra.

682. Dichorisandra thyrsifora, Mikan. Delect. flor, et Faun, brasil. fasc. I.

C.e genre très-voisin du campelia appartient comme lui a la famille des commelinées.

AD. B.

108. Tire Botanical cininex, consisting of coloured delineations of plants, ete., consistant en figures coloriées des plantes de tous les pays, avec l'indiration de leur culture; par Conrad Lonnice et Soxs ; les figures, par George Coоке. Chaque partie comprend dixplanches. Londres, chez J. et Arthur $\Lambda$ reh, etc. Part. LXIX. Janvicr I 823 .

Cet ouvrage parait destiné plutôt aux cultivateurs qu'aux botanistes, car il ne renferme pas de description réelle des jolantes qui y sont figurées, mais simplement quelques notes -ur leur culture. Le's planches sont mal gravées et coloriées à motiti seulement. Yarmi les figures de ce numéro, la sente qui pourrat offrir guelyue interit anx botanistes est eello du Murice semi-aperta, assez bien exécuté, mais sans aucun détail analytique.

Av. B.

109. The Butusn вotanist, or a familiar introduction of the srience of botany, retc. Le Botaniste anglais, ou introluction familiene a la botanigur, expliguant la physiolosie végétale et les principes des systemes artificids et naturels de $L_{1}$ yé et de Jissret. 
suquel on a ajouté un synopsis de genres des plantes d'Angleterre, dans lecpucl on a domé l'étymologicet la signification du nom de chaque genre; our rage spécialement destiné aux jeunes persomes. In-1?, avec 15 planches. Prix, 7 shell. 6 den.; coloric ro shell. 6 den. A Londres, chez F. C. et J. Rivington, 1822 .

$\Lambda$ D. B.

Iro. Hontus ANglicus, or the modern english garden, ete. Hortus anglicus, ou le Jardin anglais moderne, ccntenant une description agréable de toutes les plantes qui sont cultivées dans le climat de l'Ángleterre, soit pour leur usage, soit pour l'ornement; et un choix des plantes les plus remarquables cultivées dans les serres chaudes et tempérées, disposées suivant le système de Linné, renfermant leur caractère générique et spécifique, et des remareques sur les propriétés des espèces les plus remarquables. Par l'auteur du British botanist. 2 vol. in-r?; chez F. C. et J. Rivington. Londres, 1822 . Prix, 16 shell.

AD. B.

IIr. Eiements of the philosophy of plants, containing the principles of scientific botany, nomenclature, theory of classification. Phytography, anatomy, chemistry, physiology, geography, and discases of plants; with a history of the science and practical illustrations. By A.P. Degandolte and K. Sprengel. Traduit de l'allemaind.

C'est une traduction de l'édition allemande, de la Théorie élémentaire de la botanique de MI. Decandolle, publié par M. K. Sprengel. AD. B.

12. Botanische grammatik, etc. Grammaire botanique, pour servir à la classification, tant naturelle qu'artificielle, des plantes, avec un exposé du système de Jussieu; ourrage traduit de l'anglais, de 
J. E. Smonr. In-s', ave 21 pl. Prix, r rise, $18 \mathrm{gr}$. Weimar, 1822 .

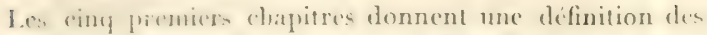
parties de lowgentsation der plantes. Je sixitme traite des principe a da tamification; le septieme est un tablean du systime serned de Linne, avee quelefues modilications. I.e linitione contient un expesć du systeme naturel de Jussieu ; il remplit les deux tiers du live. Le neuviène renferme une companaison entre ces denx systemes. Une table des genres at des fanilles mentionnees dans louvarge le termine. lees plimelie- reprisentent $2-6$ flenrs et plantes. (Jourmal simiral de la litcérature, etc. Octobre $\mathbf{1 8 2 2 , p . 2 9 1 . ) ~} \mathbf{F}$.

113. Citalone Des runtres ranes, culivérs et multipliées dans le jardin de Fronose, pris Paris. In-r? de 3 feuilles. Imp. de Fain, il laris.

Ce catulunue n'eat quine simple émmeration des plantes cullis a dans ce jurdin, sans description et queldguelois soun ales noms de jardin peu connus des lotinistes. On y remaryue particulierement une nombreuse collection de masnolie, d'asulen, de rhodedendrum, ete. Cie jardin ost situe at six lieue de Paris, sur la route de Fontainebleau. Ad. li.

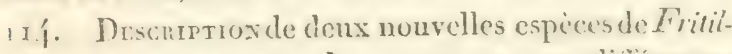

laria, aceompagnée de remarques sur difierentes "spieces de plantes, appartenant au meme sente, par

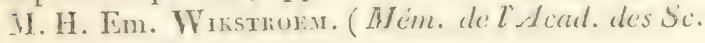
de Stockitiolm, I82 r, 350.)

ces esperes unt éte nommés par lanteur lititllaria lusifanica et Fritillaria ruthenica.

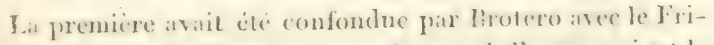

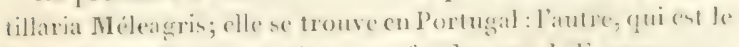

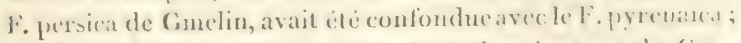

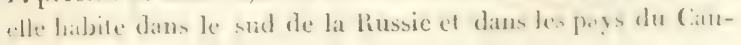
case.

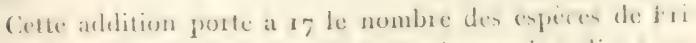

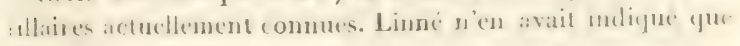
quatre.

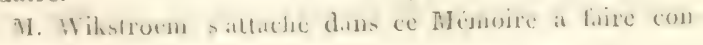




\section{Botanique.}

zailbe non-seulement les caracteres qui distinguent les dins espèces nouvelles des autres espèces précidemment dicuile, mais encore les caracteres distinctifs des especes les jlun woi sines; de sorte que son travail peut servir pour une nomographie du genre Fritillaria.

Toute la partic descriptive est en latin.

C. II.

I 5. Sur un nouveau grente de plante qui a élé nommé Tritistroemia; par M. C. Sprengel. (Ce mémoire est en latin. Mém. de l'Acal. des Sc. de Stocholm, $\left.\mathrm{r} 8_{2 \mathrm{I}}, \mathrm{p} . \mathrm{x} 6 \mathrm{7}.\right)$

Ce nouveau genre, qqui a cité nomané par l'auteur du Mhémoire en l'honneur de M. le docteur Wiskstrocm, botaniste suédois, se borne jusqu'ici à une seule espèce, trouvéc dans l'Amérique du sud, province de Cunana, près la nouvclle Barcelone. Il appartient a la famille des composées, ordre des eupatoires.

C. MI.

i i6. Description de deux espèces d'Équisclum, piai

M. Wristroem. (Mém. de l'Acad. des sc. de Stockholm, 182 r, p. 36o.)

Ces deux espèces sont l'Equisetum giganteum de Linné, qui est une plante des Antilles, et une autre que Thunbers, dans sa Flore du Cap, a nomméc aussi gigunteum, mais qui, différant spécifiquement de celle de I.inné, a reçu die: M. Wikstroen le non d'Equisetum Thunberri. C. M.

I 7. Description de nouvelles especes de Lichens, par M. Élias Fries. ( La partie descriptive est en latin.) (Mém. de l'Acad. dessc. de Slochholm, rison, p. 320.)

Il s'agit $x^{0}$. d'un genre que l'auteur nomme Coniangrum, qui a le plus grand rapport avec le Conioloma; l'espèce uni. que e'st le Conirungium sulgare, qui croit sur le bois et licoree de différens arbres; 2 ". d'un senre P'yrenoter, que l'auteur place entre les genres Varioluria et Calicium.

Il a indiqué trois especes de P'yrenotea, savoir: l'inconstans, le leucocephala et le stictica.

A cette occasion, H. Fries propose une nourclle division 
des lichens en quatre groupes ; division analez̧ue à celle quii avait proposie frécédemment jour les clampignons. If nomme aes groupes Comiothulumi, Muzblinti, Gasterothi lami, ct Hymenothalami.

C. $\mathrm{M}$.

Ix. Remare es sur la constitution physique de lilı. d'OFland, et sur les végétaux qui s'y trouvent; pax M.Alor.Anecrst; suiviesd observations sur le mèn:e sujet, par M. Goelda Wahlenberg. (Mém. die l'Acad. des sc. de Stockholm, 1821, p. 37-.)

Ce Mímoire, fait connaitre la manière dont sont distribuies dansectle île quelques plantes que le nord de l'Euroje. offere rarement, et qui ont fixé l'attention de Linné, ais:'que celle de tous les botanistes qui l'ont visitéc depuis lt:1 noun nous bornerons a indiguer les plantes suivantes:

Melien ciliuta, bromus asper, glubularia wulgaris, ci. tus alandicus, ranunculus illyricus, coronillat emerus, artemisia laciniata, panicum viriele, gralium austriacum, verbascum condensatum, olontites tenuissima, grysopleylia fastigiata, lychnis alpina, potentilla fruticosa, malva atcea, chrysocoma myosyris, etc.

M. Wahlenbers, qui a vu l'ile d'OEland en minéralogiste et én géologue, joint ses observations a celles de M. Ahlquist pous "xplieguer ce qu'offe de particulier la flore de cette ile, qui, placée a une haute latitule $\left(56 \mathrm{a} 55^{\circ}\right)$, et ayant des hivers tres-froids, doit a la nature de quelques terrains et it la forme de quelques hauteurs, de poséder des plantes propres à de's climats beaucoup plus temperies. C. M.

119. Tue Wonnis of the vegetalle lingdom displayed in a series of letiess; hy lhe author of Selece female biograpliy. Les Merveilles du règne végétal; lettres par l'auteur de "Select f'male biography. " In r2. 252 pas. Whittakers. 1822. Prix, 7 sheil.

Cet ouvrage parait destiné particulierement aux amateus: de la botanique, et surtout a présenter dans un style agría. ble les plénomenes les plus remarquable du rénne végéta!. ( Monthly review, decembre 1822 , rol. $99, n^{\circ} .4_{4}$.) An. B. 


\section{Botanique.}

120. Greville's scottisit cryptogamic Flnta, n. 5 ct n. 6 .

Ces numéros renferment la description et la firure des espèces suivantes :

No. 5. Tab. 21. Erineum betulo, DC. - 22. Erineum $\mathrm{Py}-$ rinum, Pers. - 23. Agaricus tuberosus, Bull. - 24. Hysterium rubi, Pers. - 25. Echinella paradoxa, Lynghbe.

No. 6. Tab. 26. Hysterium juniperi, Greville. - 27. Cylindrospermum concentricum, Greville, nouveau gence d'Uredinées (Fusiloidece, Greville. - Epiphyte, Link), caractérisé ansi : Plante minutissima in foliis vivis parasitica, non ruptì epidernide. Sporidia cylindrica truncata non septata, nuda, libera, coacervata. - 28. Agaricus odorus, Bull.29. Puccinia fabe, Greville. - 30. Gloionema apiculatum, Greville. (Tilloch's philosophical magazine and Journal, n. 296 , déc. 1822, p. 458 .)

AD: B.

I2 I. Sur LA floraison automnale des Narcisses, par M. le Cayma.

L'auteur pense que la floraison de certains Narcisses, en automne, qui s'observe assez souvent, n'est pas due aux causes annoncées par le docteur Forster (1'hilos. magazin, n . $29^{5}$ ), c'est-à-dire à l'absence de la foraison printanière des mêmes bulbes. (Tilloch's philosophical magazin, $\mathbf{n} \cdot 29^{6}$. déc. $1822, \mathrm{p} .426$.

Av. B.

1 22. - KNowesLex (Angleterre ), I 7 septembere 1822. On voit ici un phénomène de végétation très-cxtraordinaire sous notre climat: c'est un poirier de jargonelle qui a fleuri quatre fois cette année. Une poire provenant de lat première floraison avait deux pouces trois quarts de haut, et six pouces et demi de tour; une autre de la troisieme floraison avait à peu près la même grosseur, quoique plus allongée. Jusqu'a la fin de la belle saison, cet arbre a été chargé en même temps de fleurs et de fruits.

(Extrait du haleidoscope, ou miroir scientifique et littéraire. Revue encyclop. déc. 822, p. 621.)

F. 


\section{PALONTOGRAPHIE.}

1.)3. Ox momerates to the study of fossil organic remains, rk, ou Introduction it l'étude des corps organisés fossiles particulierement de cem yuom trowe en Andererre, drstiné à aider les étudians dans lems rerherehes sur la nature des fossiles, ot sur leur rappost aree les formations de la terre. Par James Parkinson.

Cet onrage est imprimé de mème format que lespuisse de la ciolugie de lingleterre et du pays de Gialles, par MIM. Conybeare et W. Phillips.

AD. B.

1: $\{$. Rircum de planches des coquilles fossiles des environs de Paris; par M. de Limstick, avec leurs explications. On y a joint deux planches des Limmces fossiles, et autres roquilles qui les accompagnent, auxem irons de Paris; par E. Br.tru; ('nsembletrente planches gravées en taille douce, in-1. A Paris et a Anstredam, chez G. Dufour et P. $\Lambda$. d'Ocagne.

Depuis long-temps les naturalistes qui s'ocenpent des coquilles fossiles, recrettaient que la description et les fisures de celles des environs de Paris, que l'on doit an célebre et rénerable chevalier de I.amarek, fussent dissemincen el perduen on quelque sorte dins les 20 whlumies des Ánmales du Mussum, recueil important, mais à la portie de pen de personnes. Lat difículte de comparer les descriptions aur figures placress souwent dans des volumes doignés, ren-

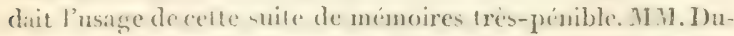
four et docagne, powesseurs des mives a du reste de l'idition des Annales, ont rendu un wat service anx sas ans, en faisint lirer at juat les plinches qui rejuesentent les fos-

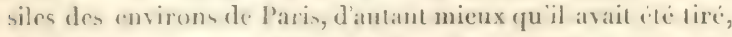
dam le temps, un extein nombre dexemplates du texte descriptif, et que: rese qui le postident pourront formes dorenatrint un recucil complet. Mallecureusement beancoup 
d'espèces décrites par M. de Lamark n'ont point été gravées. Il cite alors les vélins du Muséum; mais an moins avecle recueil des planches actuelles on pourra mieux déterminer celles dont on n'avait que la description. Chaque planche est accomparnée de son explication, telle que M. de Lamarck l'a donnée. MH. Dufour et d'Ocagne y ont joint les deux

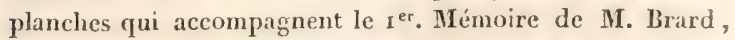
sur les terrains d'eau douce des environs de Paris. F.

125. M. W. D. Conyenare vient de donner des additions à son mémoire sur les genres fossiles Ichthyosaure et Plésiosaure, accompagnées de 8 planches. (Voyez Transactions géologiques de Londres, deuxième série, volume $\mathrm{I}^{\mathrm{e}}$, planche $\mathrm{r}^{\mathrm{re}}$.)

126. M. le professeur Sискоw, à Manheim, va publier un travail important sur Jes ossemens fossiles des bassins du Rhin et du Necker, sur ceux des environs de Darmstadt, de Pappenhein, d'Altdorf et d'Eichstaedt, dont le Muséum d'histoire naturelle de Manheim est fort riche. Les figrures de chaque objet seront lithographiées gr. in-fol. Après avoir fini cet ouvrage, M. Suckow publiera peut-être les Coquilles fossiles, et surtout les Bélemnites des mèmes contrées, dont le Musée de Manheim possède aussi une belle suite. (Ext. d'une lettre de M. le docteur H. Bronn d'Heidelbers, is M. de Férussac, ${ }^{\mathrm{er}}$ novembre, 1822.)

$\mathrm{F}$.

\section{PHYSIOLOGIE.}

i 7. Tératoscopie du fluide vital et de la mensambulance, ou démonstration physiologirgue et plychologique de la possibilité d'une infinité de prodiges réputés fabuleux, ou attribués, par lignorance des philosophes, et par la superstition des ignorams, a des causes fausses ou imaginaires; par C.-R. H. , in-8 de 25 feuilles. Imp. de madame veuve IluctPardoux, à Orléans. A. Paris, chez l'aureme, cloître St.-Méry, n。 16.

Ia mensambulance de l'autenr est une sorte de somnambulisme qui, délachant l'homme de hi-mène, sćparerat, s Томе I. 
vlle castait, la matiere corporelle du primeipe qui lanime. C. live contient une multitude de fits tous plus elommans

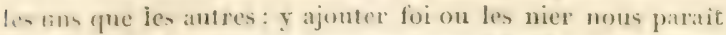

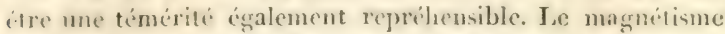
animal diservé par des hommes diun rare mérite a tronve des partisans ef des incrédules. I.e liver que nous annoneons veut explinguer par les lois de la plyysiologie et de la physique, des faits que tontes les religions ont tonjonrs atduis comme base le leurs dogmes. Si le style, qui ent lat vie d'un ouvrage, rifondait atas choses extraordinaires qui s'y rencontrent, l'otlvage $\mathbf{l}_{1}$. M. II aurait un grand nombre de lecteurs; mats, tel quiil se prérente, nous craignons rquil ne demeure dans l'onbli : an isste, fout l'ouvage repose sur ce principe, quil y a dar: l'lonme nue cause supérieure à la maticre qui a le droit de lui rommander et de s'en faire obéir. Bentrevis.

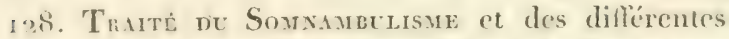
modifirations quil présente; par A. Bratraxo. In-80 de an feuilles trois quarts. Prix : $6 \mathrm{fr} . \Lambda$ Paris, chez J.-G. Dentu.

12). Minorie phrsiologique sur le colimarom, Cuchle pomalia, ete.; par B. Gispino, D. M.

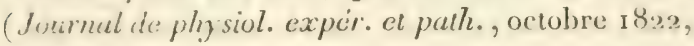
1). (3). )

Ce minnoive fort interessant montre pour la premiere fois une suite de recherches physiologiques sur le limacon, fuites avre la procision, l'habileté et les comnaissances comparatives at varies que l'état actuel des sciences a rendu necessaires pour tont ce qui lient à ce genre dinvestigntion; car, malgré les importans travaux de Swammerdam, Lister, Redi, Spallanzani, Errhart, Harder, Hérissant, Muller, Duverney, Rivamur, (te. efe; il n'est presque aucune question fonchant la pliysiologive des mollnsques en grénéral, et celle des limarons en particulie's, qui udit besoin d'ètre examince de nouveau d'apros les principes anjourd'hui adoptes. M. Gispard a rendu un virilableservice a lhistoire naturelle de ces animaur, en publiant l'ouvrage que nous annoneons, et on doit visement divirer ya il continue at sommettre at son examen 
ane foule de questions obseures de lem hivoire; "füil dtiermine les fonctions encore peu connues de divers organen 'qu'on trouve chez certains mollusques; et surtont quitil ob-

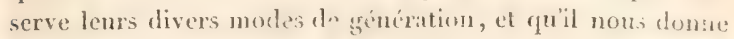
enfin au vrailit ther:a de la reproduction spontance de leus. parties amputée, atin de déteminer les limites et les circonstances influentes de ces reproductions.

Les recherches de M. Gaspard ont eté sprécialement dixiyées vers l'état de sommeil ou d'hivernation du colimaçon, et par occasion il présente de's observations sur les orgimes et les fonctions de ce mollusque.

Il décrit d'abord l'état du limacon à lapproche de l'hiver, les precautions gu'il prend pour s'enterrer, la formation de's cpiphragnes. Il montre par diverses expériences le degré de: empérature qui peut déteminer le limaçon à sortir de terre: un à y rentrer. Il parle de l'opereule, de sa composition el le sa formation; puis il recherche les causes occasioncllen qui peuvent porter le limacon à sengourdir et a so cacher, et rapporte à ce sujet une foule d'expériences curicuses. It examine l'état des diverses fonctions vitales pendant l'engont. dissement. Il détermine le maximum de froid qui les fait perir pendant leur hiveruation; et enfin soumet à l'examen l'engrourdissement des limacons pendant la sécheresse, ob servé par Pline, etc. M. Gaspard parle ensuite de l'accouplement des limacons, de leur ponte et de la crobsince des petits. Il examine le sang de ces animaux, les organes de leur sensations, et dit un mot sur la rigénération de lexorstarties amputés. Ce mémoire, plein de fats positís et emienx ye nous ne ponvons rapporter ici, offe baneoup d'observations nouvelles et d'expériences dílicates qui peuvent diriger les recherches de méme genre que vombrient entreprendre d'antres naturalistes.

F.

130. Le temps nous manquant pour olsenir lievati the importans mémoires du no. d'octolure du journal de 31 . Mi: gendie, nous allons au moins en donner les tires.

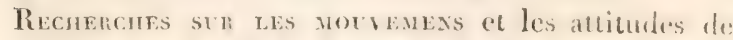

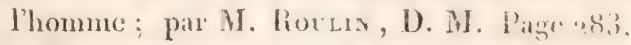

Ce Hemoire interessant parat remplir parfirement son but; il est écrit avec clarté et méthode. 


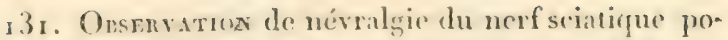

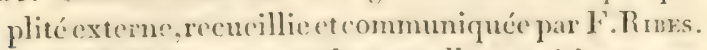

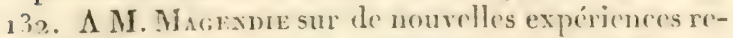
latives anx propriés medicamenteuses de liure, et sur le genre de nort que produit la noix romique; par M. Segalas.

Nous donnerons dans le numero prochain un extrait de ce mémoire qui a été imprimé à part.

133. Nocter.es rechercues sur le systeme nerveux; par Ch. bezl.

13. . Exprexices sur les fonctions des racines des nerfs qui naissent de la moolle épiniere; par M. F. Magende.

I35. Rı pront fait à l'académie des sciences, sur des expériences relatives aux fouctions du systime nervens; par M. le baron Cuvien. (Ućtermination des proprictés du systime neveux, ou recherches pli) sigues sur limintilite et la sensibilité; par M. J'lourens.)

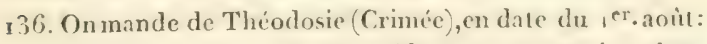
Depuis plusieurs années nous possédons un centenairc dans notre ville; c'est un Arménien nommé Soas-Ogflou; il est né en I zo2, à Erzerum, ou il était porte-fais. Son corps, courbe par ses anciens travaux, laisse voir qu'il éfait d'une taille moyemne. Il a les épaules largges, les jambes sèches et la vue bonne; il a conservé la mémoire, il monte facilement les ciraliers, et peut encore porter quelques firdeanx. Il soulevait, dans son temps, trente pounds ? environ 1200 lires; : il a tonjours dé solore; it ame beaucoup a fumer. Merie deux fois, il a eu luit enfans, dont il ne reste qu'une fille de trente-cing ans.

Ce vieillard offe dcux singuliers phénomines, qui ne sont pourtant pas sans exemples. Sa barbe, jadis grise, a noirri; el, a l'itye de cent ans, il at eu deux dents machelieres; actueblement il lui en pousse une troisieme. M. Le conseiller d'etat Boncles, halitant cetle ville, a fait son portrait, et l'a présenté, accompanné d'une notice, à H. Ic comte de Iangron. Son age est cufisimment constate parle 


\section{Anatomie.}

0.

récit qu'il fait lui-mème. « J'avais dix-lruit ans, raconte-t-il, lorsque Thamas-Koulikan commença ses cuncuettes et vainquit trois pachas dans le Korasan, où il commit d'horribles cruautés. Je me rappelle lui avoir vu porter un sabre pesant quarante-huit livres, poids de liussie; j'ai encore devant les yeux le jour oì il fut assassiné par le tartare Jagale. " Kin eff et, l'on sait que Thimas-Koulikan commença ses incursions en 1720 ; et, comme notre centenaire en avait alors dix-huit, il est donc actuellement dans la cent vingtieme. année de sa longue carrière. (MIoniteur du 4 janvier, 1823 .)

F.

\section{$-\infty$}

137. Rechenches D'tndtome pathologique sur l'endurcissement du système nerveux; par M. Prixel fils, médecin. Mémoire lu à l'académie des sciences, le 27 mai 1822 , et suivi du rapport de MII. Portal et Magendie. In-8 de 2 feuilles. Imp. de Cosson, it Paris. A Paris, chez Béchet jeune.

M. Pinel fils, dans ce Mémoire, s'est proposé de faire connaitre une altération du système nerveux sur laquelle l'attention des obscrvateurs parait s'être peu fixée. Il rapporte quatre observations dans lesquelles la pulpe nerveuse présentait une induration très-remarquable, soit dans le cerveau, soit dans le cervelet, soit mème dans le prolongement rachidien. Voici les caractères principaux de cette lésion : "le tissu nerveux ressemble à une masse compacte, " inorganique; on ne peut mieux comparer sa densité qu'à - celle du blane d'ouf, durci par l'ébullition; la substince " cérébrale est affaissée et déprimée; elle prarait entièrement "dépourvue de vaisseaux sanguins; l'œil n'y aperçoit ancune - trace de vaisscaux capillaires; sounise à l'action du feu clle " se racornit, produit une odeur forte et tenace; laisse un " résidu noirâtre, vernissé et solide; une portion de cerveau " sain, exposée à la même action, donne des résultats oppo"sís; elle se dilate, l'odeur est presque nuile, le résidu n lormatre et léger; la pulpe nerveuse se déchire par fai"sceaux et par fibres dont la direction varie dins te cel- 


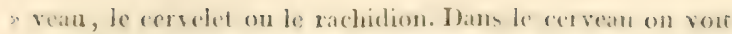

- de loule la prepiplicrie de cel organe lés fibses rayomer - el se rendere en convegearit vers le corps strie; l'endur, cissement rend tris-apparente ecte disposition des fibres; elles senfoneent dans le corpes strie, traversent les conches - optiques, se mélent dins les pidoncules à un peru de substance grriso, sortent au-devant du pont de varole, et, arri* ves an bosd inferieur de la protubérance amulaire, se contiment avec la moelle épiniere, dont elles sont evidemment le prolougement,

Linsi, relle dieposition des fibres nerveuses lonstemips mace, mais mise hors de doute par les travanx de Winston, de Yic-d'Azir, de Mill. Cuvier, Gall, Claussier ot bediad, se irouve encore confirmée par lanatomic pathologique.

Si l'endurcissement peut faciliter la commaissance de la structure de l'onéphale, il doit igalement jeter quelipue jone sur l'histoire de ses fonctions; lappliation de la physieloaic pallologrique aux fonctions corribales ne peut que fournir des notions positives; cle fera combitice, stirant M. P'inel fils, que toutes les facultés morales et intellectuelles, mene les plus élevies, la raison, le sentiment du uno, sont Ie's produits et les résultats de l'organisation saine, funisque Ialtiration profonde de cette organisation, l'endurcissement, frat exemple, du cerven, cause si sorvent lidiotisne el entraine lix perte de toutes ces ficultés.

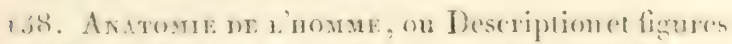
linhegraphices de toutes les parties du corps humain:

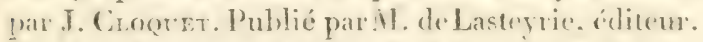
bixicme livaseon. In-folio de 3 fenilles et f planHes. Imprimerie do Rignoux, à Paris. A l'aris, chez M. de Lasteyric.

les nenf prenieren livraisons de est ourage ont ile ron-

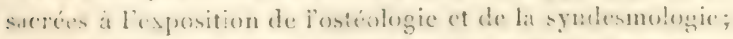

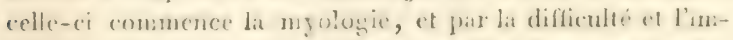
pertaner du sujet, comme pror la manice heureuse dont il

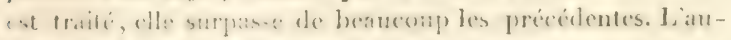

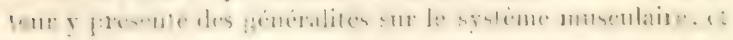




\section{Anatomie.}

il passe i la description particulière des muscles du crine, dic la face et de la langrue, tous représentés de grandeur natuvelle d'après des dissections faites avec un art clui permet de saisir parfaitement les rapports mutuels de toutes ses parties Les planches sont fort belles tant sous le rapport du dessin que sous celui de l'exécution lithographique. Ce grand onvrage ne peut manquer de devenir classique et d'indispensable utilité aux médecins, chirurgiens, peintres, sculpteurs, et enfin à toutes les personnes qui s'ocenpent de l'étude des sciences physiques ou s'adonnent aux beaux-arts. Le style de l'ouvrage est concis et d'une extrème clarté. L'Anatomie de l'homme ne peut que faire beaucoup d'honneur à l'auteur, MI. Jules Cloquet, déjà connu par les utiles ouvrages qu'il at publiés sur la chirurgie, et à l'éditeur, M. le comte de Lasteyrie.

139. Discours n'intronuction à l'ouvrage des Monstruosités humaines, formantle deuxième tome de la Philosophic anatomique; par M. Georfroy ne $S_{\mathrm{T}}$ Hilatre. In-8 d'une feuille et demie. Imp. de Rignoux, à Paris.

L'anatomie emploie les animaux, chez les Grees, dans lit vue de faire connaitre l'organisation quant à son ensemble; - Clle est déja par consiquent à son début une science propre, se faisant remarquer par son caractère philossphlique.

Restreinte a la renaissance des lettres en liurope, cest a devenir plyysiologrique dans des cas déterminés qu'elle s'ipplique; auxiliaire de la médecine, sa forme est toute midicale.

Plus tard, dans le 18 e. siècle, elle examine successivenent la structure intérieure d'un grand nombre d'animaux : s'en. richissant de faits particuliers, et, tant par la multiplicité qque par la nature de ses travaux, toujours subordonnée, die disvient anatomie zoologique.

Mais enfin, dans le ige. viecle, n'opérant plus sur ha stre des espices, et ne potasant s'arreter à tous les faits particts Jiers et varie's a linfini qu'elle embrasse au contraire dam

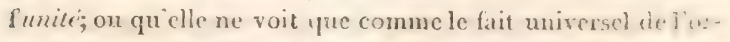

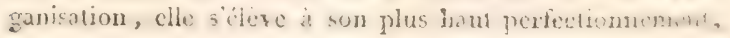


seience pour elle-méme et philosophirpue, comme a son point de départ, dle prendra le nom d'anatomie transcendunte.

Son principal perfectionnement tient à la découverte d'un nouvel instrument ou d'une nouvelle méthode de ditermination, larpuelle se compose de l'intime association des quatre règles ou principes, dont la définition est concentrée sous les formes appellatives suivantes:

La therie des analngues, le principe des connexions, les affiniti's ciluctives des matciriaux organiques, et le balancement des organes.

r fo. Plaxches anatomeu f's du corps humain, exécutées d'après les dimensions naturelle's, accompagnées

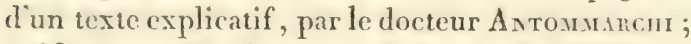
publićes par M. le comte de Lasteyrie, éditeur. In-4 d'une demi-feuille. Imp. de Rignoux. A Paris, chez le comte de Lasteyrie, rue du bac, n. 58. (Prospectus.)

Les figures de l'ourrage que nous annoncons forment une carte topographique complete et fidile de tout le corps liumain, à l'exception des tigumens, tout ce qui les concerne "yant été démontri dans les planches du prodrome de la grande anatonie de Mascagni, publić par le doctenr Antommarchi, Florence, 1819 .

La grandeur et les proportions des figures sont celles diun homme de taille ordinaire.

L'ouvrage de MI. le docteur Antommarchi sera composé de 45 planches ombries, et de 35 environ representant dis esquisses au simple trait. Les 2', premieres qui paraitrom, étant réunies trois prar trois, forment un corps entier, et donnent ainsi la représentation de huit figures humaines entieres, sur lespuelles on pourra itudier la couche superficielle, et sucessivement les suivantes, juscju'au squelette; le rébeau sons-cutane, forme par les nerfs superficiels, les artires et les veines superficielles; enfin, les differens plans musculasires avec leurs nerfs, leurs vaisseaux sanguins et leurs vaisconux lymphatiques. Six de ces figures repréentent les trois conrles de muscles aver lenrs vitisseanx, leurs nerfs; et les deux dernieres fignures, le squelette naturel, recourert de san 


\section{Anatomie.}

périoste, avec ses cartilages et ses ligamens. Itans fuus ce's desseins, le sujet représenté est vu sur ses faces antérieure et postérieure.

2 I autres planches comprennent un certain nombre de figures particulieres, savoir: $x^{\circ}$. les parties que la disposition des grands dessins ne permettait pas de faire voir en détail; 2 . les viscères de trois grandes cavitis du corps, leurs nerfs, leurs vaisseaux sanguins, et leurs vaisseaux lymphattiques.

Toutes les parties représentées dans les figures de cet ouvrage sont caractérisées par le genre de dessin qui leur est propre, ainsi qu'il sera expliqué dans l’introductiun. Lat désignation précise des muscles, des tendons, des os, des vaisseaux, des nerfs, donne à ces figures, quoique en noir, presque toute la clarté ct l'exactitude des planches coloriées.

Pour faciliter la description des dessins, on a joint une esquisse an trait à chacun de ceux que la multiplicité des parties pouvait rendre confus. Outre les indications nombreuses faites, soit à l'aide de lettres ou de chiffres, sur chaque esquisse, on a encore eu soin d'y joindre les dénominations latines les plus usitées.

I.e docteur Antommarchi fera paraitre chacune des parties de ce grand ouvrage dans liordre suivant lequel elles viennent d'ètre énuméríes. Ainsi, les planches représentant les différentes couches musculaires du corps hmmain jusfu'aux os, paraitront les premiceres; viendront ensuite les fiznres de détail; et, en dernier licu, celles des visceres des trois ynates cavités du corps, dans l'ordre suivant: $I^{\circ}$. ceux de la téle; $2^{\circ}$. ccux du thorax; $3^{\circ}$. ceux de l'abdomen.

L'ouvrage sera divisé on 5 livraisons, dont la prennite paraitra dans le mois de janvier 1823 .

Chatue livaison se composera de 5 ou 6 planches avec l'explication, et paraitra régulierement de trois mois en truis mois.

Le prix de chaque livraison, sur grand-aigle, en noir, $25 \mathrm{fr}$; sur vélin colorié, zo fr.

La souscription est ouverte cliez MI. le comte de Tanferrie, éliteur, rue du Bac, n⿳. 58, passage Sainte-Maric, et flez les principaux libraires franeais ef etrangers. 


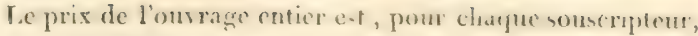
en noir, $3-5 \mathrm{fr}$.; colorié, $1050 \mathrm{fr}$.

La souscription restera ouserte pendant un in; apres rette epoque, dhaque livaison serat anmentée de 5 ou 10 francs.

sera dipose, a Paris, chez l'Editeur, et à l'étranerer, chez les principanx Iibraires, une éprenve colorice, (gui fera connatre an public la manere dont sera tratiélouvase.

Les lettres ct demanies devront itre adressées, frane de port, à M. le comte de Lasteyric.

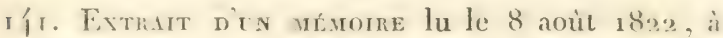

linstitut par M. A. Desmotelns, pour servir de complement i ses recherehes sur le systime nerveus des poissons. (Journal de phlys. experr. et palh., octobre $18 \% 3$, pag. 3is.j Voxez lextrait de ses premieres recherches. (Joumal de pluss., arril, I822. )

F.

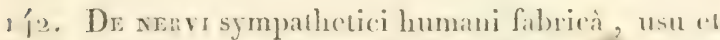
morbis. Commentatio anatomico-plovisolonicon-pattholugrica; auctore, J. Fr. Jobsters, modicinar, prof. Argentinensis; adelit. tabulis lithographicis et pictis. In-f, pap. jésus fin. Prix, $15 \mathrm{fr}$.

Se libraire I.evault annonce cet onvage comme desaut paraitre sous peu.

\section{MEDECINE.}

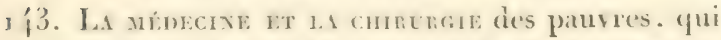
contiennent des remedes choisis, faciles à préparer, et sins dépense, pour la plupart des maladies inter' nesctevernesqui attarpentlecorpshumain: par *** . Sourdle cilition. In-1', de an fenilles at demie. Imp. de Bumsy a Lyon. A Lyon, chez Sats.

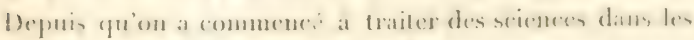

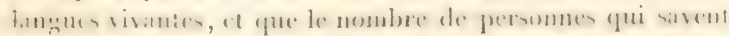

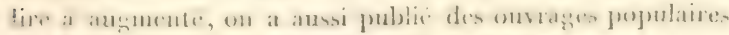

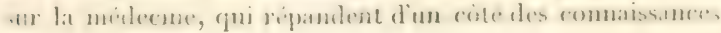




\section{Múdecine.}

ntiles, ef qui perpétuent de liutre l'ancion prejugés. Lees onvages pour les pauvres ont. orighatrencut dé composépour des dames de la charité attachées aux hospices et aus hôpitaux; on cu at composi aprés pour toutes les classes de la société, ct M. Ie doctenr Capuron a domne il y a queldues. ammées une nouvelle ídition d'un des onvrages les plus ripandus dans ce genre, ouvrage surtont utile anx gardes-malades. Le livre que nous annoncons n'est qu'une réimpres sion d'un ouvrage moins au niveau des connaissances actuelles.

For.

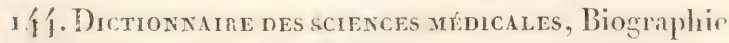
médicale, tome $\{$ ( Ebel-fiwin), in-\$. de 35 feuil. Prix, 6 fr. Inple de Panchoule, à Paris. A Paris, chez Panckouke.

Tout le monde a reconna l'utilité du Dietionnaire de M. Éloi, publié en ${ }_{77} 78$. La Biographie des múdecins, souvent si nécessaire pour apprécier la solidité de leurs travau, a cependant été tellement négligéc, que la Biographie médiale que nous annoncons est devenue un ouvrage indispensalhle. Personne ne possede plus de connaissances littéraires que le principal rédacteur de ce Recueil. On doit cependan! segretler que de jeunes médecins, d'ailleurs estimables, se: permettrut de juger quelquefois les hemmes supériours, avec none présomption qui dínonce, d'un còté, le port de comnaissance de leurs travaux; ct de l'autre, peu d'iclées de ce grual faut particulierement fairc ressortir dans la vie dinn lomme. Cist l'article Jean-Picre l'anck qui nous porte a faire celle olservation. Cel homme, justement celebre, a cxeré pendant un grand nombre d'annces la plus grande influence sur la ligislation et la police médieale de l'stlemagne; il a éte appeli pour organisere colle de la haute Italie el de latiossic. II a, ch outre, marqui comme anteur, comme profisicur de cliniefue et romme praticien, a foltungue el a lienne, it

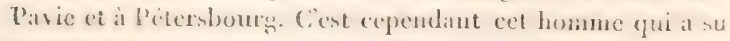
se placer à une hatuteur parcille, e'est cel homme qu'on semble vouloir deprecier, en doutant de létembe de se

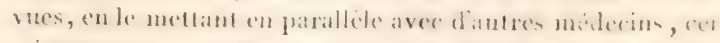

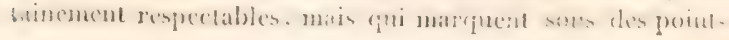


te vae bien différens, et peu analogues, par les circonstances dans lesquelles il se sont trouvés placés. For.

1 $\{5$. Nocveau nictionarae de médecine, chirurgie, pharmacie, phy sique, chimic, histoire naturelle etc., où l'on trouve l'étymologic de tous les termes usités dans ces sciences, et l'histoire concise de chacune des matiores qui yontrapport; par $\Lambda$. Becland, Chomi, H. Clocelet, J. Cilocelet, M. Onfila. Tome II. (II. Z.) In-8 de 4 r fruilles 3 quarts. Imprimerie de. Cellnt, ̀े Paris. A Paris, chez Méquignon Marvis, chez Gabon, chez Crochard. Prix $9 \mathrm{fr}$.

L.'s auteurs de ce Dictionnaire, dont le but a été de mettre les jeunes médecins à méme de vaincre toutes les difficultis qu'ils peuvent rencontrer dins lat lecture des livres de médecine et des sciences accessoires, y ont riuni la plus grande quantite de muts possible; et sous ce rapport leur ouvage est le plus complet de tous cenx publitis jusqu'à ce jour dans le mème but. Les termes les plus inusités et leu plus bizarres de l'alchimie, de la plarmacie, tons ceux de médecine abandonne's maintenant avec les anciennes doctrines médicales oì ils ćtaient employés, s'y trourent consignés, le plus souvent avec une itymologie arabe, sreegue ou latine, en mime tomps tous les noms les phos nouveaux du langage chimicue et de la momenclature des sciences naturelles y trouvent auss leur place, avec medefinition succinte, mais toujours suffisamment pricise. J) aillenrs tons lesanticles importans ont ete traté diune manieve plus etendue : nous nen citerons anem parfieulierement, mais it nous suffira de dire que, rolatirement a la partie de la medecine, on y tramve, dans les difinitions anatumiques, de tableau le plus fidele quoirgue abrége du corps humain, dans celles de physiolonite, de pathologic et de therapentique, lexposition complite de son fonctions, thistoire des maladies qui peuvent en deranger ilatmonie, et les moyens d'en prévenir les causes ou d'en combattre les effets.

En petit suppliment ajome a lat fin du second volume qui 


\section{Médecine.}

vient de paraitre, achève de mettre cet utile ouvrage an niveau des découvertes nouvelles, des changemens les plus récens apportés depuis le commencement de sa publication au langage des sciences qu'il embrasse.

I 60. Dictionnare a biégé des sciences médicales, de Min. Anelon, Alment, Bunemer, Biyle, ete., par une partie des collaborateurs. Tome 7. (FpinFlore). In-8 de 37 feuilles. Imp. de Panckouche, à Paris. $\Lambda$ Paris, chez Panckoucke.

III. Panckoucke est certaine ment le libraire qui donne le plus d'occupation aux médecins, et qui a le plus contribué dans les temps modernes à répandre lés connaisiances médicales. Le Dictionnaire abrégé dont nous annonçons déja le septieme volume, ne devait ètre que l'extrait du grand Dictionnaire en 59 volumes qui l'a précédé; mais le concours des médecins de l'école de M. Broussais, en a fait presque un ouvrage original, qui présente les faits connus d'après le systime nouveau, et qui cherche à suppléer aux lacunes par des observations nouvelles.

For.

147. OEuvres complètes et inédites de Cibanis, membre du sénat, de l'institut, de l'école et société de: médecine, de la société philosophique de Philadelphie, ete., accompagnées d'une notice sur sa vie et ses ourrages, par M. Tиunot, professeur de plilosophic au college roval de France. In-8 de I quatt de feuille. A Paris, chez F. Didot père et fils; et chez Bossange frères. (Prospectus.)

r 4 8. Meniziniscme Toposrapiry des gouvernemens und der stadt Kasan, ete. - Topographie médicale du gouvernement et de la ville de Casan; avec des notices historiques, géographiques et statisliques; par J.-F. Enmunn, professeur de clinique à l'université de Dorpat, membre de diverses socion tés savantes. Riga et Dorpat, 1822 , in-s, avec un pliun Jithographié de Casan et des environs. l $^{\prime}$.... 


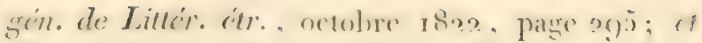

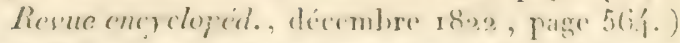

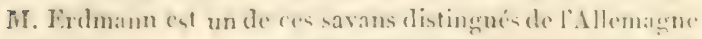

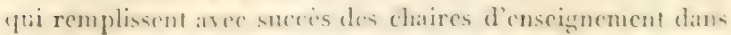
les mivernites meses. Arant de passer a celle de Dorpat, il avait, pendant rept ans, proferse dans celle de Casan; et, dans linterralle des cours, il aviat pareomu les diverses confrees dent se compone ce vaste gouvernement. Lourages qüil vient de publier est le fruit de ses observations, faiter aver sigate Aftè la description plysique du sol ct colle du climat, riennent les observations sur la population, lin. dustric, le régime dieletiqque et moral, les maladies, les tables de naissances et de mortalite, on un mot, fout ce dont se compose une toprograplie médicale soigneusement cxécntee.-A la suite de l'ouvrage, viennent des chapitres ditaillés sur le pars, les antiquités bulgares, les peuplades nomales et celles qui sont apricoles, leur religion, mourset usignes. Ce livre, qui atteste les talens de M. Erdmamn, l'étendne de ses comnitsanes, at lis perspicacite de son esprit obsersateur, e fait lire avec un vif intérèt, et mériterait dètre traduit. F.

I ́́g. Vorace mineal mitala, précédé d'une excursion, ete.; par le doctenr Louis Vilentrx. Extrait de la bibliothéque médicale. In-8. de 3 quarts de: feuille. Imp. de Cellot, à Paris .

Ia premiere partie de ce royage contient l'historique d'une excursion au volcan du mont-lésuve, et am ruines d'llerculit num at de Pompétia en 1820 , pour voir les effets de l'éruption: qui durait depuis le mois de decembere 1819 . Tat seconde? jartie traite de l'état des hippitaux de Xiples et du reste de I'Italie. On n'a pas adoptí dans le royaume de Napules la milhode exclusive des dibiliuns et des contrastimulans. Les: Brunoniens sont revenus a la midecine hippocraticue.

f.abus dies saignies est toujours régnant a liome; les fievres intermittentes font consommer une quantité énome de quinguina, flus de 10,200 lives par an. On rommence at diblir dans ces pars des cabinets danatomic et d'histoire niturelle, dans tous les ctablissemens d’instruction pour lat medecine. - La Tuscane est depuis long-tempre riche en ca- 


\section{Médecine.}

binets de ce genre, et surtout en modeles en cire. M. Valentin, ayant fait des recherches sur la fieve jaune qui a régni: a Livourne, a été confirmé dans l'idée qu'clle n'est pas contagieue. - Bologne est le centre d'une doctrine nouvelle qui a été inventée par M. Rasori (le mème qui autrefois a répandu le systene de Brown). Cette doctrine dite des rontrastimulans, a surtont recu sa plus grande impulsion par MI. Giacomo Tounmassini, qui prétend avoir enseigné avant M. Broussais les idées sur la non-existence des fièvres essentielles. Le reste de l'ourage parle de l'université de Padoue, de Milan et des autres villes de la haute Italie. Il est en général plein te notes intéressantes pour les médecíns qui veulenf comnatre l'état do ce pays dans le temps ou il a été visité par l'auteur.

For.

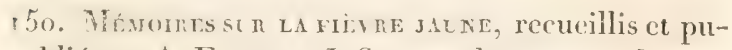

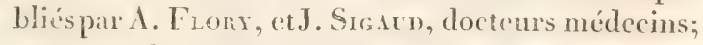
premicre livaison. In-S de 6 feuilles un quart. Imp. de Guion, à Marscille. 1 Paris, chez Gabon. Il y aura 3 livraisons de 6 at 8 fenilles. Prix, payable en recevant la premicre livraison, so fr.

Nons ne savons si les denx autres livraisons ont paru. Il a été publié à Marseille un autre ouvragre sur la Fievre jaune qui a régné au lazartet de celte vilie, par hi. Rošrt, médecin de ce lazaret.

151. Maladies de la croissance; parM. Dichimp, docteur-médecin. In-s de 3 fenille's. Imp. de Fain, it Paris. I823. A Paris, chez l'auteur, place Sorboune, n. 4.

152. Mémorre sur l'usage des bains dans le tétanos, par' M. Méglin, docteur en médecine à Colmar, in-8. de 2 feuilles et demie. Imp. de Levrault, à Stras bourg. A Paris et à Strasloourg, chez Levrault.

153. De r.'urnorisene portrine, et des palpitations du coeur, promptement dissipées par la digitale pourprée; par J.-13. Conne, docteur en módecine, cte.; deuxieme édition, considérablement augmentéc. 
In-8, de a fenille's eq demic. Tmpe. de Belin, it Paris. A Paris, chez Croullebois, el che\% l'autens, rue du Colombier, n. I 5 .

15 f. Mlisu ridu pére de famille, ou Méthodes nouvelles de lablaitement artificiel, et de faire prendre aus confuns et mime aus adules, les licpuides dans certains cats: par Z. Leselire, ancien chirurgion major des armers; ormé de gravures. In-s, de 3 fenilles trois yuats; plus les planches. Imprimerie de \on-

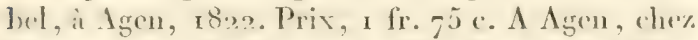
lauteur, rue des Arénes n. 68; a Paris, chez Méquignon-Marvis.

155. Instrection sur les premiers soins ì domner aux persommes asplyyxiées par les vapeurs du vin ou de la hiere en fermentation, parcelles du charbon ou de la braise allumée, gaz carboni(que; par U. Hсxris, in- 2 d'un sixieme de feuille. Imp. de Lamort, à Metz. $\triangle$ Paris, chez Audot.

Cette Instruction parait moins étendue et par consiquenl moins complete que celle que vient de publicr an mois de dícembre MI. le préfet de police de Paris, et qui a éle rédigée par les membres du comité de salubrité de la capitale.

156. Dissertation sure li peste, par M. Chomzis (Stanislas), d'Migues-IIortes, ancien chirurerien, etc. Jn-s' de a fenilles un quart. Prix, 1 fr. 50 e. Imp. de Rouchon, à Marseille.

157. Rivine ne goitre et de lat gontr. In-8 d'um demi-feuille; par De trente, ex-capilane d'ardillerie cet ancien éléve de l'école polytechnique. Imprimerie de Monnoyer, au Mans.

153. Riғzтtrox de quelques prejugés qui se somt répandus contrela vacrine, et moyens de pratiquer

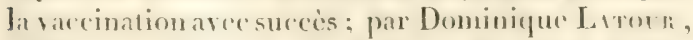

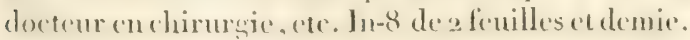


Imp. de veuve Tislet, ì Toulouse. A Tonlouse, chezlantenr, me St.-Renisi, no 7 ; chez F. Vieusseux; chez Senac.

i59. Notice st fies observations mídicales du derteur L.-F. Brg Eox, médecindes épidémies, etc. In-í, d'une demi-fenille. Imprim. de Boacher, à Paris. Prix, ro c. A Paris, chez Lance.

x6o. Letre minicate sur Paris. In-8, d'une feuille, signée de Sr.-Gerun. Imp. de Boucher, à Paris. Prix, 75 e. A Paris, chez les principaux libraires de l'école de múdecine.

1(i. Cinn.ucteristik der Franzosischen Medizin. La médecine française comparée à la médecine anglaise; par J.-L. Cispel. Leipsick, 1822, che\% Brockhaus. (Juurn. gén. de la lit. étr., oct. 1822.)

J.es onze chapitres qui composent cet ouvrage sont intitulés: Esprit de l'école de Paris. - Enseignement public.La médecine pratique. - Les hospices et höpitaux. - Rcmarrues sur la médecine pratique. - Ophathahmologie. Ho-pices des aliénés. - Hospices pour l'acronchement et les enfins trouvés. - Médecine légalle. - Sur le Dictionnaire des sciences médicales.

162. La menecina cunativa o la purga dirigida conted la causa de las enfermedades, probada y malysada en esta obra por Lenoy, decima edixion, revista, corregida y aumentada con muc has casos praticos. In-12, de 15 feuilles deux tiers. A Bordeaur, chez Gayet.

163. Laziret de ha Méditenranese, à fonder daus la rate de Marscille, par souscriptions. In-s, de fenilles, imprimerie de Romson, a Marseille. $\Lambda$ Marseille, ches Camcoin ; à Paris chez les priucipanx libraires du Palais-Royal.

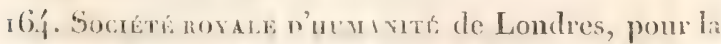
promaration des moyens propers a mppeler a lat so Томк 1 . 


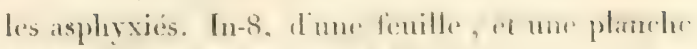
gravéc. Jupp de Cordier, at Parris.

CIIRURGIE.

165. Touveaux prancipes becmetratr, rédigésuivan! leplan de louvrage de C. Delafaye, contenant, rete par F. MI. V. Legoris, docteur en médecine de J: faculté de Paris, ete. Quatrieme édition, revue, corrigée et angmentée. In-8, de fo fenille's un quart. Imp. de Cellot, à Paris. A Paris, chez Miquigüon-Marvis. Prix, $8 \mathrm{fr} .50 \mathrm{c}$.

166. Iiapponts fa notes sur les bandages et appareils inventés par M. Vennire, chirurgien-herniaire de lit marine royale, ete. In- 6 , d'une feuille et demis. A Paris, rue Montesquieu, n. f́. Imp. de madame: veuve Scherff, à Paris.

\section{ART VETERINAIRE.}

167. Traité de h'irt víténzame, ou description raisonnée desmaladies du cheval et de leur traitement; suivie de l'anatomie et de la physiologie du pied, de's principes et de la pratique de la ferrure, aree des observations sur le régine d'écurie, sur la nourriture et l'exercice du cheval, et sur les moyeus a enployer pour le préparer au travail et à la course; par J. Wurte, ex-nédecin-vétérinaire des dragons rowaux d'Angleterre. Dédié à S. A. R. les duc: d'York. Onzième édition. Traduit de l'anglais, par Henri German; revu et annoté par Dretritette, vétérinaire des gardes-du-corps du roi. Iu-f́ d'une page. Imprimerie de Delagnette i Paris. A Piris, chez Mongie ainé. Cet ouvage formera I volume in-s, qui sera mis en vente au commen- 


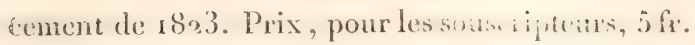
50 c.; pour les autres, 6 fr. 5o.c. (P'ospectus.)

J'ai l'ourage orignal sons les yeux; il en rompon de 'puatre volumes grand in- I 2, qui fous ont pour tite' "ommun : A treatise of veterinary medicine.

Le premier, qui porte sur le titre onzieme édition et lo inillésime London, 1815 , contient un compendium de l'att vétérinaire, ou une description soignéc des malulie'; du cheval et de leur tratement, plus l'anatmie et lit physiologie du pied et les principes lle l'art de ferrer; il ent orné de dix-sept planches, lont sept coloriées, sur l'anatomic du pied du clieval et sur la ferrure.

Le second volume trate de la matière midicale et de la pharmacologie; on lit sur le titre, nouvelle édition et le millésine London, 1814 .

Le troisieme renferme des observations pratiques sur quel. ques maladies dangerenses du cheval, telles que la morve, le farcin, la péripneumonie, les entérites, les boiteries, plus les précautions a prendre en achetant un cheval; il porte sur le titre seconde édition et le millésine London, 1814; il contient quatre planches sur la ferrure.

Le quatrieme, enfin, renferme des observations sur les maladies des bètes à cornes, des betes a laine, des cochons et des chiens; sur quelques maladies de l'oeil dans le cheval, et sur la rage; sur la maniere de pratiqner quelques epérations importantes de lit maréchallerie, telles que l'action d'abattre un cheval, la castration, la coupe de la queue, la cautérisation, etc.; on lit sur le titre le millésimeLondon, 1815 , sans indication d'édition; c'est la première. On y trouve cinq planches; la cinquième représente différens états de la pupille dans le cas de maladie de l'ceil.

Ces quatre volumes forment autunt d'ouvrages séparés qui ont paru à différentes époques, qui ont eu plusienrs éditions, le quatrième excepté, et qui ont été réunis sous le même titre.

J'ai sous les yeux la première édition du compendium senl, datée de Canterbury, 1802 , in-12, avec I/4 planclies plus petites, mais mienx soignées que celles de la onzièn: érlition; trois sont coloriées. 


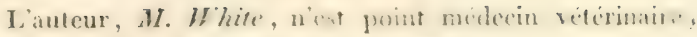
mais bien chirurgien vétérinaire (surgeon).

Il y a dans les quatie volume de quoi romplir avantagensement le prospectus que je vions de fare comatre.

\section{Huzarn.}

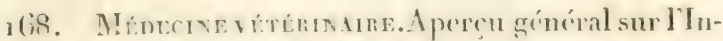
flammation, par F. L. Morre, vétérimaire, a Chaumont-0ise, ref. In-s, de 2.3 pares. 18.3 .1 Paris, rhez Demonsille, imprincur, rue Christine, 11. 2; et chez Compère, libraire, rue de lícole de Hédecine, n. I 7 .

Cet ousrage traite sulement de linflanmation en cénéral, comme son serond litre l'iadigue; ot il pourrait aussi-bien sappliguer à la méderine humaine qu'a ba medecine viderimaire. It parait tait pour prouver, I". Fue tous les atsens qui

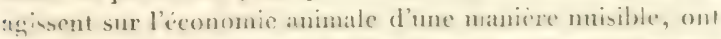
pour resultat, quelle yue soit leur maniere darerir, de pro-

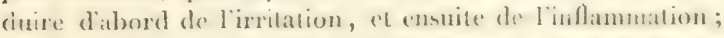
2". que les cures obtenues par len movens ot les cubstance foniques, irritantes, stimulanies, ete, ne promeni par- l'in. verse, et viennent, au contraire, en analysant bion leur matnière d'agir, donner de la forer à la premiare propusition.

"Je répete donc que l'inflammation, on du moins lirrita"tion, qui en est le premier degré, existe chapte fuis qu un 2 derangement plus ou moins fort, plus on moviss virible, se - discleppe dans l'organisme, n’uporte stou quello apple"rence; et que toutes les canses, sans distinction, profluivenl ce -phinomine, et rien de plus. v (Päre 10, ligne 21 el sulis.) - Quand une phlegnmasie es dévelojpée, tous les moyens "anti-phlogistiques doivent èlre mis en usage pour la com"battre. " (Page 15, ligne 18 et suiv.)

"Cependant lobservation demontre le contraire, et tons s les jours, malgré l'uspge abusif des tonicques, des irrilans,

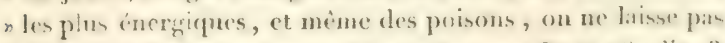
. dioblenir des cures souvent inesprirées. a (l'age 16 , lig. 3 et suiviustes.)

"Quoi qu'il en soit, il est pourtant vrai de dire que lis surs rison de beaucoup de phlegmasies apprartient a ces moyens. 


\section{Art vélérinciive.}

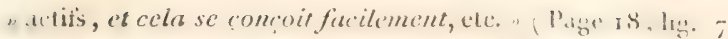
et suivantes.)

(Huzaru fils.)

y(j). Le nouvelu p.trFit bouver, ou traité compla! sur le gouvernement des bexufs, des vache's, des clate vaux, des moutons, dess chivres, etc. Ouvrage ou lion apprend à connaitre tous les animaux utiles au villitge, leur âge, leur aptitude au travail, les symutomes de leurs miladies, les moyens de les guevir; par quels soins les Espagnols rendent leurs troupraux si productifs et si sains; ce que doit être la ferrure lorsque le pied se trouve en santé, ou quil est vicieux ou malarle; et enfin comment les bergुers et autres labitans des campagnes doivent se soigner cux-mèmes, daus le cas de certains accidens résultant de la garde des troupeaux, ou même do toute autre cause. Par M. II. L. 1822. In-I2, de 406 pages, avec quatre planches. $\Lambda$ Paris, ehez Jiclin-Leprieur, libraire, quai des Augustins, 11. 55. Prix , $3 \mathrm{fl}^{\circ}$.

Cet ouvrage est une réimpression pure et simple, frase

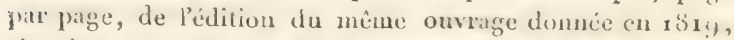
ehez le même libraire. Il est composé de sept sections, at deux articles supplénentaires. Lat premiere section tratw. des bêtes à cornes; la seconde dis bêtes à laine; la trobicme de's chevres, hones et chevaux; la quatrieme des malitlien dies cochons; la cinquieme des animaux de la basse-cour ; la siseme des aheilles; la septiime des chevaux; le premier artirle supplémentaire, des maladies on accidens communs arl labitans de: la campagne; le second arlicle est un traite su lés mérinos.

Un des principaux onjets est de donncr des pomedes contur

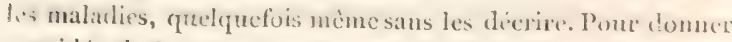
:ane idé de la rélaction de cette partic de l'ouvrage, nou 3. pporterous le jassage suivant, page 59 :

\section{farcin et gale.}

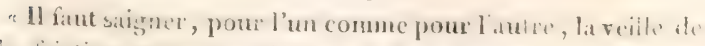

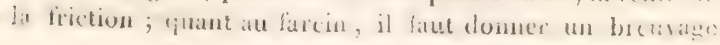


, thaque jour, emprose d'une chopine d'ean dans baquello *auront honilli, nu demi-quart d'heure, denx onces de raa cines de dosne ou de patience, et ee, pendant six jours: "pour la gale il n'en est point nécessitire. "suit une description d'une graisse pour le farcin et la gale. Hzzann fils.

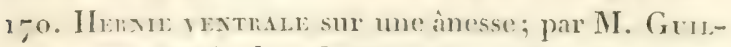
I.A1 11: (evtrait des observations vétérinaires, envorées par lui ì la sociéti centrale d'agriculture). (Ann.d'agric., octobre 1822, page 1 Iо.)

z un cheval; par le même.

172. Sith lis cotrises de cinevatx; par M. Hezhin fils. (Id. page 2 Io. )

i 7 . Note sur l'opération de la clavelée pratique en

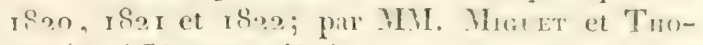
irrenes. ( $I d$. page 248. )

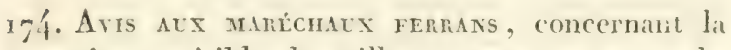
pratique muisible de tailler, rogner et couper lit corne de la fourchette. (Ann, ill page 325.) li.

175. Compte rexde des travaux de léeole rojale diconomie rurale et vétérinaire de Lyon, pendant lamméc scolaire; et de la correspondance pratipue des vétérinaires; par Racisarin. (Imn. de Tregricult. francaise, décembre i822.) Bosc.

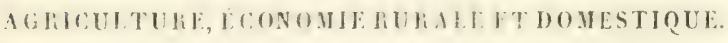

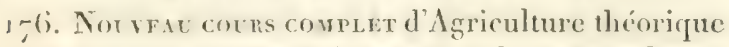
et pratique, contenant la grande et la petite culture, lécononice rurale et domestique, la médecine vétérinate, ete., ou Dirliommane misomé of miversel d'Aerieulume. Ourrage rédiése sur le plan de celui dre fen l'ablé Rozier. duquel on a conserve les ar-

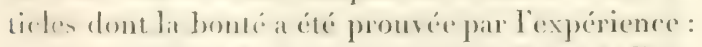

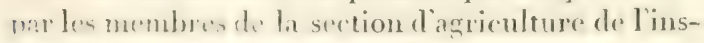


timt de lrance, ete., avec des fig. en taille-douce. Nouvelle édition, revue, corrigée et augmentée. Tomes 13, 1.́, 15 et 16 (lial, Zuc), quatre vol. in-8, ensemble de r fo ferilles et 6 planches. Prix, 30 fr. Imp. de madane Huzard, ì Paris. A Paris, chez Deterville.

La nouvelle édition de cet important onvage a été publiée avec une rapidité qui n'a pas empéché que des développemens utiles et étendus n'aieut ajouté un nouveau prix à un tres-grand nombre des articles de ce Dictionnaire. Il est devenu un livre de premiere nécessité pour les agronomes; il donne, dans une juste proportion, de trés-bons documens sur toutes les parties de l'économie rurale et domestique, et if s'itend spécialement sur la pratique convenable pour toute espece de culture, d'éducation des animaux domestiques, ef de préparation des produits ruraux. La premiere édition a itépromptement ipuiséc; de nombreux souscripteurs se sont prisentés pour obtenir cette seconde édition perfectionnée, et les rédacteurs ont convenablenent répondu à leur attente.

SiLv.

17.- Essar sur les moyens damciliorer lagriculture en

France, particulierement dans les provinees les moins riches, et uotamment en Sologne; par M. le baron de Mokon fs, membre de plusieurs sociétés savantes, natlonales ef étrangères. 2 vol. in-8, de 4 à 500 pag. chacun. A Paris, chez Touneux, et Huzard-Courcier; Huet-Perdaux.

LE sol de la Sologne est árilemment un sol d'atherrissement; ce terrain col preu productif, tout y est obstacle aux progrés de l'arriculture. Fialternation d'un sol argileux et quarterux, les meurs des latirans, leur indolence, leur répugnance a ablopter des usareses qui different de la routine, la superstition, les prejuges, le difart de population, le peu de capilaus a comployer, sont autant de callses epui sopperem a l'asnélioration de lat culdure. I.cs conseils de M. le baton de 
Iorogues pour obtenir ec résullat lui ant serride division pour son ourrage, qu'il partage en r plives, dans lerguts if irailedes

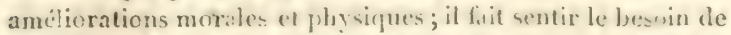
changemens dans les methudes de culture; il en voudsat introduire de nouvelles. Si . Ti. Le baron de Mrrognes est icoutio, on verua des plantes inconumes sacelinater dans les landes

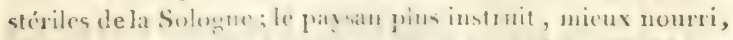

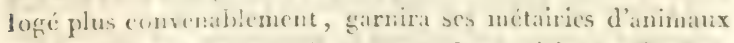

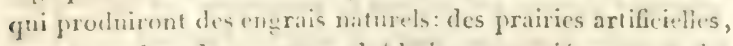
srand rombe de moutents, ded bois apjropriés, scront des sources de produits successifs : les romtes onvertes reniront Jes communications du commeree plus faciles; les jaclicres disparaitront : l'industric naira; chaque annéc signalera une erperience nouvelle: dejja le pin a triomplé des prejjugés, et il seleve de tous cotés: la pomme-de-terre a púnétré dans des villages affanés, et les genetiires se multiplient. Tout tend vers un mieux sensible, et le 'Traité que nous a donné cet agriculteur, aussi zélé que savant praticien, ne jent qu'cn accélérer l'action. Ce livre est un des meilleurs que nous ayons vu paraitre depuis long-tenuns sur ces matieres.

\section{Bartuevis.}

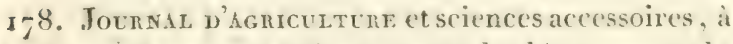
l'usage des proprićlaires yuraux du département du Tarn; public par M. Lmuszx-L, worme, phannacien à Alli, mombre du jury mídical du dip. du

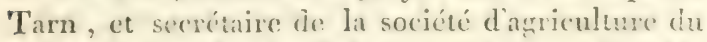

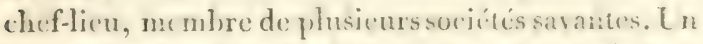

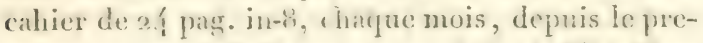
mier jan jer 18̈23. Prix, par an, 5 fr. A Albi, chez

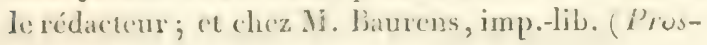
pectus. )

'Fel est le titre d'un nousean journal domt on vient de

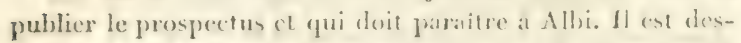
liné à répandre dins les communes ruxales din departinent du Tarn les sanes doctrines de l'agriculeme, et a imbiquer aux habitans des campangnes les moyens qui penrent conconrir a conserver leur sante. (eete utile culreprise at engitgé M. le préfet diu dipartement, a alloner lo montant de la- 
bonnement sur le budget des diverses communes. Cet exemple mérite d'être signalé, et il serait à désirer qu'un journal semblable, s'établit dans plusieurs départemens. Fin Allemirne comme on le sait, chaque état, chaque province a sa fenille de correspondance pour tout ce qui intéresse l'économie rurale et l'agriculture.

F.

779. Coxsmérations génćrales et particulicres sur la jachere et sur les meilleurs moyens d'arriver graduellement à sa suppression, avec de grands avantages; par J. A. Victor Y vant, membre de l'Institut, professeur d'agriculture, etc. I vol. in-8, de 150 pages, avec figures. $\Lambda$ Paris, de l'imp. de madame Iluzard, rue de l'Éperon, n. 7: 1822 .

On appelle jachere le repos qu'on laisse à un champ après Iui avoir fiit produire quelqus chose pendant un, deux, trois ans.

Généralement on sème la première année du froment, la seconde année de l'orre ou de l'avoine, et la troisieme vient la jachère, pendant laquelle on laboure la terre deux, trois, quatre fois et plus.

Ce mode de culture nous est venu des Romains. Il prédomine malbeureusement encore en France, malgré les bons écrits et les bons exemples offerts aux cultivateurs depuis un demi-siècle.

La jachiere n'est point dans la nature puisçue jamais on n'a vu un terrain se dépouiller des végétaux qui y croissaient pour se reposer; donc son adoption est fondéc sur de faux principes.

Cependant l'expérience des siècles prouve aussi qu'un excellent terrain qui a porté deux ou trois années de suite des céréales, ne produit plus que des récoltes clétives; et qu'iprès une jachere d'un an il reprend un peu de sa fertilité premiere. Les mavais terrains exigent une jachere consécutive de deux, trois, quatre, six ans et plus.

Les avantages de la jachere sont de pouvoir labourer lat terre plusieurs fois dans le cours de lanne, ce qui 1 ". fait perrer les mavaises herbes produites par la germination des

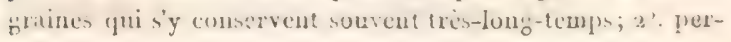


mul : $14 \times$ saz atmosplieriques de rentre soluble la portion d'huens qui ne l'ititit plase, et de la rendre par-la propere a servir a lat mutrition des récoltes futures; 30. favorise lintroduclion des eatux pluviales dans les conches inférieurs, etc.

Mais ces avantages sont de beaucoup compensés par le: manque de produits du terrain pendant lannée de jachire, ainsi que par la dépense des labours quil recroit; et il a été découvert, il y a 50 à 60 ans, un mode de culture qui les offre égilement, et qui ne laisse pas lit terre sitns produits: c'est celui des assnlemens, qui consiste à ne jamais semer deux annies de suite la mème plante, ou des plantes de méme fiunille, dans le méme lieu, et entreméler des récoltes sarclies, de's rícoltes étouffantes, des prairies artificielles, à la culture du froment et autres céréales.

Ce nouveau systeme de culture est fonde sur ce que les plantes qui ont crù plus ou moins de temps dans un terrain l'épuisent de l'aliment qui leur est propre, disparaissent $\mathrm{et}$ sont remplacées par d'autres de genres différens, qui seront remplacées à leur tour. Ce phénonomine se remarque partuut, mats surtout dans les maurais terrains. J.e chine, ultraséculaire, l'offre comme les céréales, comme l'humble violette.

L'ouvrage annoncé plus haut a pour but de faire connaitre. combien la culture par assolement est préferable, sons les rapports du produit, à lit culture avec jachere. Ce ne sont pas des théories vagues qu'on $\mathrm{y}$ tronve, mais des raisonncmens foncles sur des faits et sur des expérienees indubitables. 11 y est prouvé que si la jachere n'est point encore abolie parfout en France, celat tient an defaut d'instruction et d'aisance des cultivateurs, an morcellement des proprités, a l. brieseté des baux, rte; que la prospérité de quelques cantons des dipartemens du mord, de quelques comtis de l'Augleterre, est due à sa supprescion.

"Xometerminerons, dit l'anteur, nos considerations šreneraleset particulices sur cel important objel, con observant que

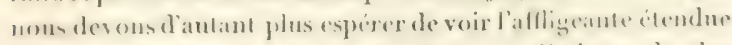

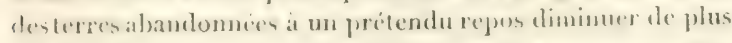

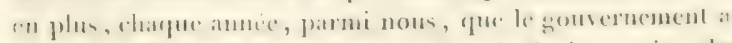

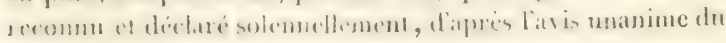


conseil général d'agriculture, dés l'origine de sa formation, que l'abolition des, jacheres est un grand principe d'umilioration, et qu'il a sişnalé à l'estime particulière de sa majesté Jes correspondans du conseil, parce que la plupart d'entre eux les ont bannies de leur exploitation."

Je crois que l'onvrage de M. Y Yvart mérite d'être l'objet des méditations de tous les proprictaires de terres jaloux d'améliorer leur aisance et d'augmenter les prołnits généraux de la richesse publique.

Bosc.

180. Quelquesobservations pratiques sur la théorie des assolemens, par M. Monel de Vindé, pair de France, etc. In-8, de 3 fenilles, plus ume planche. Imp. de madame Huzard, à Paris. $\Lambda$ Paris, chez madame Huzard. Prix, i f. 25 c.

L'auteur présente dans les quatre premiers paragraphes l'état dans lequel il suppose l'exploitation avant de donner' sa méthode d'assolement; il combat surtout la méthode du pare, prétendant que par-là l'engrais n’arrive qu'à la superficie. Quelques principes généraux sur la fumure des terres le conduisent à ce théorème d'agriculture; "pour atteindre la "quantité suffisante de fumier, il faut obtenir des animaux " que l'on éléve une quantité de tombercaux de fumier égale "à six fois le nombre des hectares que l'on cultive.n La rotation des cultures essayées par l'auteur est quadriennale, elle se compose de céréales fournissant pailles, des prairies artificiclles, de cultures successives, de plintes sarclées; enfin de trefles en rapport.

Un paragraphe est consacré au moyen de tirer parti de ecs produits. Si on suivit les procédes de culture de M. de Morel de Tindé, on pourrait diminuer les craintes inspires aujourd'bui, d'oblenir des produits sans pouvoir les écouler; il en fixe l'ouploi. $\Lambda$ u reste, l'auteur a expérimenté par luiméme, et tout ce qu'il dit ne sert qu'a confirmer cet axio. me "que par la variété des cultures, l'assolement et. "l'nesras, la terre peut indefinment produire." Cette bro chure ne peut 'pu'ijouter is la réputation de l'auteur, et se meommande à l'altention de tous les agronomes. Le tablean 
d'assolement qualriennal dicompone, offrint ente qualue vingls combinaisens, cst nouveau et intéressant.

BERTIRVIN.

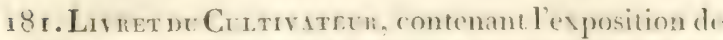
la maniere de passer dusystime de culture en tonis saisons, à celui de quatre soles; ct d'obtenir ainsi les plus grandes arantiges des teres labouraliles, e't surtout de celles situées dans les plaines privées de friches, bois, prairies naturelles et pallurages quelconques; arec un tableat figuré de derus rotattons quadrienuales, complites et suecessives, fresntile et tres-commode pour fare valoir; par un agronome qui a cultivé. In-12, de a feuilles ce demie, et le tableau imprimé. Prix, I fr. 25 r. A Eveux, chez J. A. Despierres, dit Lalonde, prire. 183. Mímone sur létat de l'Agrieulure daus le Jusa, les amćliorations qu'clle a recues, et exl!es don:? clle parait susceptible; par M. (ienmen, doyen du consilil de préfecture de ce département. Th-8 de 10 feuilles, trois huitiemes. Imp). de Gitultier, il Lomsle-Samlnier.

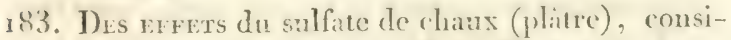
déré conme engrais; par J. A. F. A A.1. licutenant-géneral des armés duroi, we. In-ii de i feuilles et demie. Inp. de: Roch, à Jevers.

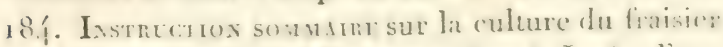

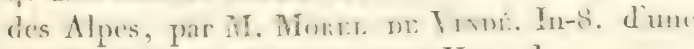
fenille. A Paris, chez madame IIuzard.

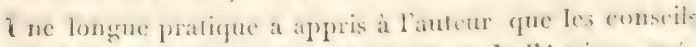

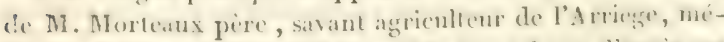

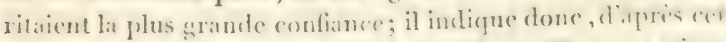

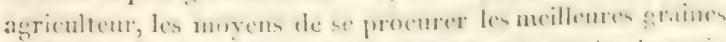

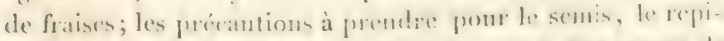

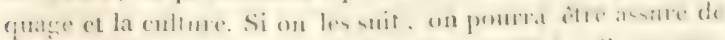

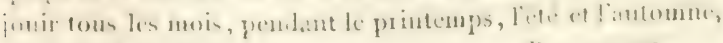
de ce fruit délicicux.

di:nturino 
835. LA Not verie rucue a mel du mois de nuai 822 , d'une seule pière et du procédé le plus simple ct le? plus naturel qui ait encore paru; se dirigrant à dicouvert, et à vue des oljets, sans rien donner au hasard; dégigée de tous grands systomes scientifiques, problématiques, minutieux, et de tout ce qui le plus souvent séduit limagination aux dépens de la raison; petit ouvrage trés-abrégé, avec figures; par M. Delavibue de Mrrist, ancicu capitaine de cavalerie, etc. In-8, de 2 feuilles 3 quarts, plus les plinches. A Paris, chez Louis Colas.

asci. Extrait du cinquieme conrs théorique pratique

et gratuit sur liducation et la Conservation des Aheilles, fait en i 822 ; par M. Lonвalit, dans lecpuel (nn trouve la solution d'une question importante, qui n'a élé traitéc par aucun des nombrewx auleur's qui ont écrit sur les abeilles. (Ann. de l'agricult. fiuncaise, novembre $\$_{22}$, page $\left.\mathrm{I} 8_{9 .}\right)$ Wosc. i87. Mémoire sur l'époque la plus convenable de semer les graines céréalesen France, adresséà la socićté royale et centrale d'agriculture; par I3. Gasinn. (Alun. de lagric. française, octolore is 23 , page 5.)

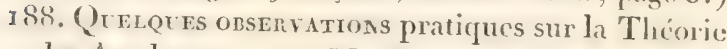
des Assolemens; par M. Monel de Vixdé. ( $/ \mathrm{m} / \mathrm{m}$. de l'agric francaise, novembre 1822 , page 1 . 5.$)$ C'est de cet ouvrage, dont il a été tiré quelques exemplaires à part, qu'il a été rendu compte plus haut.

iṣ. De l'état de l'Agriculture dans le département. de l'Areyron; par M. A. R. (Ann. de l'agricult. francaise, décembre 1822. )

190. Sir l'empor we scere pour conserver le poisson; par J. Mac Cil zoch, D. M. et membre de la sociétć royale de Londres. (Repertory of arts, janvier, 1823 , p. 109 .)

Une cuillerée à bouche de sucre brut suffit pour conserver 


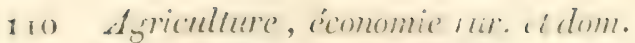

un saumon du poids de 5 a fi livere. Il sufrit dionvir le poisson et d'en soupoudrer de sure la partic munculaire, en le laissant en suite a plat pendant 2 ou ; jours, pour que le sucre pénetre bien; aprés quoi on peut le secher, et il u'y a plus qu'a l'essuyer et l'erposer à l'air, de temps en temps, pour emprécher qu'il ne se moisisse. L'auteur a conservé de la sorte et pentant trés-long-temps, non-seulement du saumon, mais aussi des merlans et du cabilliaud. Il dit gue le goìt du poisson ainsi conservé, est beancoup meilleur (que s'il était salé. Au surplus, on peut ajouter si l'on veut un peu de sel.

C. II.

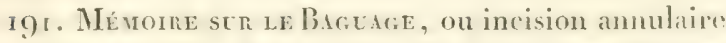

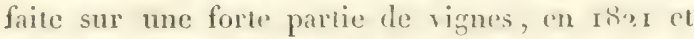
1832, par II. de Besss; imprimé par ordé de son excellence le ministre de lintérieur. ( Amn. dr? lagricult. francaise, novembre 1823 , page 230.$)$

192. Nocvelde expénence sur le Cuvage du vin; par M. H. Drdisaxt. (Ann. de l'agricult. fiancaise, novembre 1822 , page 258.$)$

Bosc.

193. Des Cinpagnols ou souris des champs. ( $1 \mathrm{nn}$. de l'agric. française, norembre 1822 , page i 38.)

19'. Lettre ne M. Th. Creslin, adressée à son cacellence le ministre de lintérieur, sur un nouvrau procídé contre les ravages des souris. ( A l nn. de l'agricult., novembre 1822.$)$

195. De ra récrion b́coxonact, convoquéc le ro oc:tobre 1822, à Saint-Onen, par M. Termax, ayint pour objet la conservation du blé dans des silos, la polenta de la pomme de terre, la vente des cherres

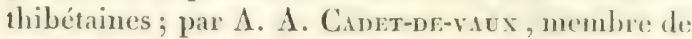
diverses académies, etc. In-8, de deux fenilles. Imp. de Fain, a Paris. A Paris, chez Louis Colas. 
GÉOGR A PHI E.

196. Cours abrícé de Géographie physique, civile " politique, où il est traité de tontes les partic's du monde, des mours et du caractiere des peuples et des productions de chaque pays. Ce Coursest précédi d'un traité de la sphiere, et tout l'ouvrage est termine: par une table des elimats, et une table des distances des degrés de longitude entre eux, selon les diverses latitudes, avee la maniere: de s'en servir; par B. J. Cnéper, in-r 2 , de 4 funilles et demie. Prix, i fr. A Lille, chez le Fort et cliez Martin Muivon.

197. Ëlémens métuongues de Géographie, disposés d'apres un ordre nouveau; par J. Ch. But.tert, ancien dépuié. Seconde édition. (Prospectus on annonce. ) I vol. in-12, de i 7 feuilles et demie. avec une Mappemonde. Prix, 3 fr. 50 c. $\Lambda$ Paris. chez Ant. Bailleul.

198. Annégé de la Géographie de Crozıt, par demandes et par réponses, avec la nouvelle division de la France par départemens, ou préfectures, souspréfectures, évéchés, d'après les dernières bulles de circonscription, ete., ete., précédé d'un Traité de la Sphère, adopté par plusicurs collèges. Vingldeuxième édition, revue, corrigée et augnentés d'une table alphabétique des villes, avec cartes géographiques, i l'usage des jeunes gens. In-12, ro feuilles et demie, plus 5 cartes. A Paris, che\% Théodore Leclerc, jeune.

199. Nouvel Abrégé de la (iéographic de Crozit, par demandes et réponses, aughmenté de la nouvelle division de la France, par départemens ou préfectures, sous-préfectures, archevéchés, et évéchés; des changemens les plus récens, survenus en Europe, dia- 
prés les derniers males de 1 imme et de Paris: précédé d'une idríc grénérale do la géngraphie mise ren vers, et d'un traite de lit spliere, suivi d'me tabib? alphabélique des villes of des rendroits principanx dont il est fait mention dans ext ourage, ì liusary de la jemesse. Yourelle édition, revue etaugmentér. avec des cartes nouvelles. In-Is, de I/f feuilles et demie. A Ljon, chez Rusan.

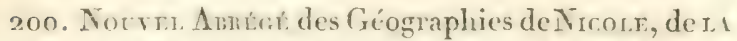

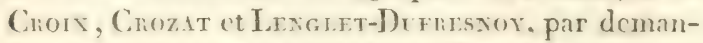
des et par réponses; précédé d'un Traité de la Sphirr. d'apres le systeme de Copernic; orné de neuf cartes nouvellement gravées: Mappemonde, Furope, France, Italie, Allemagne, Asie, Afrique, Amérique méridionale, Amérique septentrionale: jar un professcur de géographlie. Nouvelle édition, revue et corrigée diapris les avtes du congrès de Viemue, les traités de Porris, en 181 fet rsis, ete. et augnenté d’un abrégé de la Géographie ancienne, extrait des meilleurs anteurs, par J. (r. Missein, à lusage des commercans. In-I2, de ro feuilles I sixième. $\mathbf{A}$ Paris, chez Delalain.

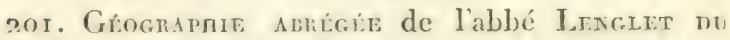
Frisxor, nouvelle édition, contenant les changemens relatifs a létat présent r. liturope: précedes d'un nouvel abrégé élémentaire de la Spliere; suivie d'un abrégé de géosraplio ancienne, et d'un précis historique sur le's principales contrées du monde; par Bomorer-Lixg ious.In-1 2, de ríf feuilles. Prix, I fr. 50 c. A Angers, chez Pavie.

202. Pricis ne Grocindentes ancienne et moderne comparées. Ouvrage composé pour l'nuscignenent de la géographie, dans les classes de sivieme des colliges rovaux, redestinc it faciliter löntelligence des anecurs dassicpes. Premicre partie. Géographe an- 
cienne. In- i a de i f feuilles. $\Lambda$ Paris, chez madame Camille Defrène.

303. Geografia moderna universile, ete. Géographie moderne universelle, ou description physique, statistique, topographique de tous les pays du monde; par G. R. PAGnozzi, tome premier, contenant l'introduction et l'Asie occidentale. In-8, I 822 , à Florence, chez Batelli.

Cet ouvrage sera composé de dix volumes avec un atlas de sept cartes.

204. Dictionnare géograpmique universel, contenant la description de tous les lieux du globe intéressans sous le rapport de la géographie physique et politique, de l'histoire, de la statistique, du commerce, de l'industric, etc., par une société de géographes, en 8 forts vol. in-8. chacun d'environ 800 pages. $\Lambda$ Paris, chez les éditeurs : $\Lambda$.-J. Kilian. lib.; et Ch. Piequet, géographe. (Prospectus.)

En lisant les ouvrages nouveaux consacrés, soit aux scienres, soit au récit des événemens qui se sont succédés depuis une trentaine d'années, il n'est personne qui n'ait senti le besoin d'un dictionnaire géographiqque où les descriptions fussent moins surannées que celles reproduites par les abréviateurs de Moréri et de la Martinière.

Des états, des provinces, des villes ont entièrement changé de face et de rang, et cependant les éditions les plus nouvelles des compilations à consulter, non-seulement conservent encore des empreintes dont les trait, caractéristiçues sont totalement oblitérés, mais présentent aissi des omissions inexcusables dins des ouvrages que l'on devrait considírer comme classiques. En vain l'on y cherche des noms devenus à jamais célebres par les hauts faits qui les ont illuł trés: Valmy, Rivoli, Auerstaedt, echappent à lit phumo d'inteurs dont la tâche parait s'itre bernée at un alsrew. servile. Heurcusement pour les seiences, ces lacumes somt : in veille d'etre remplies!

Томе I. 
Nous sarons qu'en er moment est soms presse lat pre. miere partic du premier volume du grand Dictionnate grengraplaique de MII. Kilian et l'icquet, dija annoncé depuis quelque temps.

Des articles imprimes, parvenus a notre connaissance, promettent le plus heureux succés a cette utile entreprise. Nous pouvons assurer du moins, d'apres la richesse et l'exactitude des détails nouveaux qu'ils contiennent, que les ćlliteurs de cet ouvrage n'épargnent ni soins ni dépenses pour recueillir, dans tous les pays, les résultats les plus récens de l'astronomie, de la géologie, de la navigation et de: la géographie.

DEN.

205. Grograme mistonique, physique et statistique du royaume des Pays-Bas et de ses colonies; par J.-J. de Ci.oft. 2 parties, in-8, i 822 ; Bruxell's, cliez Vanderstracten. (Revuc encyclop., décembre 1022, pag. 582.)

Les écoles réclamaient la rédaction de ce livre qui manquait à l'enseignement. Les élèves y apprendront à bien connaitre et à respecter leur patrie. M. de Cloet était déjà connu avantageusement par sa traduction de l'ouvrage de Schiller sur la révolution de la Belerique, au XVI ${ }^{e}$. siecle.

I.

\section{STA TISTIQUE.}

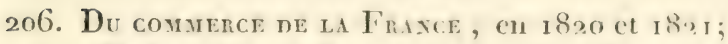
par M. le comte de Vaunaxe, ministre d'atat, député du Calvados. In-8, de a 3 feuilles et demice. Imp. de Trouvé, a Paris. A Paris, chez Trousé; chez Goujon.

La balance du commerce d'un état résulte de lit différence de la valeur des objets importés ou exportes, valeur exprimée, ou cn quantitis ou en muméraire... I des tableanx dressés par l'alministralion des douanes offrent, prour 1\$20, 450 millions d'exportations, et seulement 360 millions d'importation; la balance est done de go ruillions; et comme lexcidens est en exportations, la balance est, suivant la douane, en 
motre faveur, ele toutecette somme. Le hurean de la balance du ministere de l'intéricur porte les exportations a f r a millions, et les importations à 499 ; différence, r3 millions de balince favorable. MI. de Vaubline atopte ce dernier chiffe, et même ensuite l'affectant d'une considération particuliere, relative, le fait descendre a 3 millions. Il défend les consiquenees de son choix, et représente notre situation commerciale comme étant dans un état de langueur et de dépérissement. Son ouvrage est plein de vie, sit polémique est forte, animée; il défend, avec la chaleur de la persuasion, les intérêts du commerce en général, et puis avec un intérêt de propriété, ceux des colonies. Ici, ses connaissances, l'habitude d'eavisager cette question lui prêtent encore de nouveaux moyens.

Mais, en général, a-t-il bien connu, apprécié les faits? Il est permis d'en douter ; son livre est l'ourrage d'un homme de bien, ses vues sont pures; mais, adopties, auraient-elles le résultat qu'il s'en promet? on peut douter encore. Doiton, comme il le propose, suivre en tout point, le systime de prohibition qui, selon lui, a si long-temps fait la prospérité de l'Angleterre? Ice doute peut encore s'étendre: faut-il regardant le commerce de l'Inde comme onérux, cesser de le protéser et l'interdire à notre marine? Ici lo doute cesse et nous nous prononcons négativement.

Quoi qu'il en soit, la difference qui existe entre les calculs de deux administrations, qui opérant sur la même quantit: ont été conduites à des résultats si dissemblables, est une anomalie qu'il n'est pas sans intérèt pour l'arithmétique politique d'expliquer. Il est aisé d'entrevoir que cette dissimilitude tient au mode d'évaluation des deux administrations. La douane, et c'est notre opinion personnelle, a pensé qu'il fallat s'attacher a donner la valeur du prix d'extraction pour les marchindises d'importation, et du prix sur notre sol pour celle d'exportation : le bureau de lit balance a, au conIraire, opéré en prenant dans les deux ly potheses, pour hase, le prix du marché de France, ce qui ajoute au prix intrinces que de la marchandise celui quelle recoit du prix du transport et des frais accessoires. I a cause de la difference apercue, ce fait vient à l'aplui du systeme commercial, qui 
cherche at procurer a ia marine du pays lit plus grande masse de fret, puisque cette canse senfe alferte la valcur des objels de prés d'un cinquieme. lae fret peut done étre consideré comme un gain dû à la main-d'ouvre.

Nous n'avons pu, dans le pen d'espace qui nous est acrordé, que faire connaitre libut de la question. I.e live de M. Te comte de Vaublanc doit che lu par fout homme qui s'occupe d'economie politique; nous roudrions pousoir dure êre médite, mais il y a tant de choses hasarites dans cet ouvrage, qu'en conscillant sa lecture nous recommandons de le lire avec réserve.

Bertievin.

207. Eximex gexicind et détaillé des récoltes et des consommations de blé en France, ave indication des moyens propres à remédicr à la surabondance et aux disettes; par le chevalier Lexomle, ancien ordommateur des guerres, ete. In-8, de ro feuille's. Imp. d'A. Clò, à Paris. Prix, 3 fr. A Paris, chez les principaus libraires.

Cet ouvrage, qui a principalement pour objet d'établir le rapport des récoltes de grains aree la consommation gincrale, et d'indiquer quels scraient les meilleurs moyens de prévenir les diseltes et les renchérissemens exagérés des subsistances, est divisé en chapitres, dans lesquels l'auteur expose d'abord les principes divers qui ont successivement réglé l'importation et l'exportation des blís on France, depuis 1677 jusquen 1816 . Il consacre un chapitre a faire connaitre et à discuter les calculs publies par divers icrivains sur le produit des récoltes et sur la quotite des consommations; il examine ensuite, dipartement par département, quel est l'érat au vrai de ces récoltes et de ces consommations; il expose les résultats on plus et en moins de clacun d'eux, et indique le poids moyen de lhectolitre de froment, et son prix moyen pendant huit amées; l'auteur énumere ensuite les causes des disettes rielles on factices; il pose en principe que de grandes mesures de prévoyance peurent senles remidier an refour frequent de ces ealamites publiques; et il ennsacre des derniers chapitres de son ouvrane a examiner quelles sent les mesures de preveyance qui, jusqua ia 


\section{Strtistique.}

ce moment, ont été employées ou proposées, et à justifier la préférence qu'il donne aux magasins de réserve sur lout autre moyen. Il expose les bases de ces établissemens ainsi qu'il les conçoit, et l'utilité dont ils peuvent être pour aider les agriculteurs, diminuer les variations dans le prix du blé, ct remédier aux disettes réelles ou simulées.

Sicv.

20\%. Du Commerce des Grains, dans le système général d'économie industrielle; rapport présenté, au nom de la commission spéciale, à la société d'agriculture de l'arrondissement d'Étampes, sur l'ouvrage de M. Labovinuene, sous-préfet de cet arrondissement; par Louis Rousseau. In-8". de 4 feuilles un quart. Imprimerie de Guiraudet, à Paris.

309. Méditations sur l'écononie politique, du comte Verri, auteur des Nuits romaines; traduites de l'italien par Frédéric Néale. In-8, de 14 feuilles. Imp. de Nouzou, à Paris, 1823 .

l'ouvrage du comte de Verry étaít déjà connu en France par la traduction qui en fut donnée en l'an VIII (1800). C'est un des premiers Italiens qui aient traité de l'économic politique. Son livre a eu un succès surprenant en Italic; mais en France il fut peu goûté. $\Lambda$ l'époque où il parut, l'attention était trop absorbée; et comme il ne faisait que retracer les idées émises pour ou contre l'économisme; cet écrit fit une bien faible sensation. Aujourd'hui, que les matières, plus souvent discutées, ont été éclaircies avec plus de scru. pule, on ne lira que difficilement des chapitres où la confusion perpétuelle de valcur et prix appliqués aux marchandise's, laisse le lecteur dans une obscurité toujours croissante. Des erreurs palpables, des ides bizarres, des aperçus quelquefois inaltendus sor.t les caractères de ce traité en general. Donnons des exemples.

L'anteur émet cet axiome : Le prix d'une marchandise est en raison direte du nombledes acquereurs et en ration interse du nombere des sondeurs. Il y a bien des chemens, des circonstances an moins aussi influantes qui viennent 
augmenter le prix des objets échangeables. Errour trop pialpable pour soutenir l'examen. M. le comte de Verry regrarde lat dette publique d'un grand état comme un élément de prospérité; et cette proposition, il la soutient en cherchant a prouver qualors la nécessité de limpoot est un besoin de travail, et ce besoin une cause immédiate de production. Comment enncilier ce principe avec celui, qu'il avait avancé avec plus de raison, que l'impôt mis sur une marchandise, sur une industrie, arrête lit reproduction, et que c'est souvent une bonne politique de le faire cesser. Y a-t-il rien de plus bizarre que la comparaison suivante, et lat conclusion quion cherche à en obtenir? Tne feuille, un brin d'herbe laissés à eux-mémes, ou pourissent, ou se dessèchent; rassemblés, ils deviennent des alimens; entassés, ils s'enflamment et éclairent l'horizon. Toute cette perte d'esprit aloutit a conclure qu'il faut avoir des villes médiocres de peur que les hommes ne soient trop isolés; pas de grandes villes, parce qu'elles finissent par ruiner les états.

Mes éloges, si l'espace me le permettait, tomberaient sur un grand nombres de passages, ou lauteur a, il y a environ 36 ans, deviné ce que nos études suivies ont confirmé. La nouvelle traduction uous parait plus conforme au texte que l'ancienne. Les Mréditations du comte de Verry doivent entrer dans la bibliothéque d'un homine d'état.

\section{Bertuevin.}

310. Essar politione sur le rovaume de la Nouvelle Espagne, par Alcxandre de Hr ubolot ; traduit 'n espagnol, par D. Viernte Gonzalez Ariso, aree deux cartes géngaphiques. \& vol. in-1's, ensemble de 1 r a feuil. ct demie, plus les planches. Prix, 36 fr. $\Lambda$ Paris, chez Rosa.

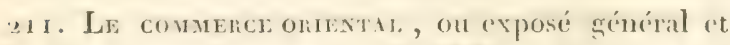
auhentique du conmere des diflérentesnations enropéennes, particulierement de la compasnice des Indes dingletereavee l'Inde, la Chine ed le Japon, et toutes les contrées à l'est du Cap de Bomme- Espérance, depuis la derouverte de ce cap) jusegu it nos 
jours. Traduit de lianglais de Vill. MlLbt rn , employé supérieur de la compagnie des Indes d'Angleterre, par M. AI. de Rosily. In-fo. d'une demifeuille. A Paris, chez Leblanc. (Prospectus.)

Dès que cet ouvrage paraitra, il en sera rendu compte.

2r2. Lor de Moïse, ou Système religieur et politique des Hébreux, par M. J. Sulvador. I vol. in-8, de 620 pag. Prix, 7 fr. Chez Ridan, libraire, rue de l'Université, n. 5, et clez les principaux libraires.

Quoique ce livre rentre plus spécialement dans le domaine de la politique et de la morale, nous l'annonçons ici parce qu'il tient aux sciences, surtout à l'économie politique par l'esprit de sa composition, et par plusieurs de ses chapitre's. M. Salvador a voulu développer tout ce qu'il y a de positif dans la législation et dans les livres de Moïse, et détruire ainsi une foule d'erreurs de fait et de préjugé, d'autant plus dangereux qu'ils ont été accrédités par des hoinmes plus célèbres. Du sein des contradictions et de la confusion qu'offrent, au premier coup d'oeil, les écrits de ce législateur, il fait sortir un système complet, système qui a recu plus tard des modifications nombreuses, mais dont l'influence n’a janais été détruite entièrement : c'est à elle que la nation hébraique doit la force de vie qui l'a si longtemps conservée.

L'auteur divise sa première partie, intitulée PoLirigue, en donze sections aui embrassent tout ce qui tient à l'ordre social. Les plus remarquables sous le rapport scientifique sont la troisième, qui traite des riche'sse's; et la neuvième, sur la sante publique: dans celle-ci surtout on s'apercoit que M. Salvador a fiait de la science médicale l'objet d'une étude particulière.

Ia seconde partie de son ouvrage comprend, sous le titre de Punosopmif, cinc sections, dont l'une a pour but de donner une explication simple des premiers chapitres de la Genise, et de faire voir comment plusieurs grands principes scientifiques reconnus de nos jours $\mathbf{y}$ sont indiqués.

Cet ouvage imporlant par le sujet qui en est l'oljet ren- 
ferme des recherches curieuses, ou l'auteur montre beaucoup d'érudition. L'esprit de modération qui rigne dans cet ouvrage ne peut que faire honneur a M. Salvador, et son livre sera sûrement lû et médité.

$\mathrm{R}$.

2 3 . Prix morfo de lihectolitre de froment, dans les differens départemens de France, d'iprès les merruriales adresseres au ministre secrétaire d'état de l'intérieur, par MIM. les préfets, septembre $1 \$ 22$. ( Inn. de l'agriculture française, décembre I822, page..... )

Bosc.

214 . Statistieve du départenent des Bouches-du-Rhóne. Cette statistique si importante par la situation, l'étendue, le commerce et les souvenirs de ce départenent, qui renferme deux des ports principaux de France, Marseille et. Toulon, vient de paraitre. Le premier volume, n'a pas moins de 1000 pages in-4. On sait par l'introduction qu'en a publice il y a plus d'un an, M. le comte de Villeneuve-Bargemont, prefet de ce département, auquel on doit son exicution, toute la part que M. Toulouzan de Saint-Martin prend a ce travail, pour lequel M. de Villeneuve n'a pas borné son zéle à des soins et des encouragemens.

Nous donnerons sous peu l'analyse de cet ouvrage. Il s'imprine à Harseille; le deuxième volume suivra de près le premier.

F.

215. LA consomation de nos deux colonies, la Martinique et la Guadeloupe, est estimée à 7,000 quintaux métriques par mois.

Il a été embarqué dans les ports de France pour ces deux colonies :

En janvier 1822 1,644 quintaux métriques de farine, et 5 ig quintaux d'autres farineux.

\begin{tabular}{|c|c|}
\hline En février & - $\quad 9,89^{6} \mathrm{q} \cdot \mathrm{m}$. de farine \\
\hline & et $79^{5}$ q. d'autres farineux. \\
\hline En mars & $\begin{array}{l}\text { - } 1,455 \text { q. m. de farine, } \\
\text { et } 26 \mathrm{x} \text { q. d'autres farineux. }\end{array}$ \\
\hline En & $\begin{array}{l}\quad 4,060 \text { q. 1n. de farine, } \\
\text { et } 1,373 . \text { \%. d'autres farineux. }\end{array}$ \\
\hline
\end{tabular}


En juin - $6,0 / 42$ T. m. de farine,
et 322 q. d'autres farineux.

En Angleterre, il suffirait de signaler au commerce un scmblable déficit cutre la consommation et les approvisionnemens, par la métropole, pour que dorénavant il n'cût plus lieu.

F.

2 16 . Une lettre écrite de Saint-Louis du Sénégal, doune les détails suivans sur la situation, au $x^{\text {er }}$ septembre dernicr, des établissemens de culture libre sur la rive gauche du fleuve.

"Onze grandes plantations sont en activité, et présentent đéjà crviron 800,000 pieds de cotonniers, nombre qui ne lardera pas à être porté au double. L'une des plus consirléritbles appartient à M. Bíruchet, négociant à Saint-Louis, ancien élève de l'École polyteclınique.

"Six concessions nouvelles viennent d'ètre faites, et vont recevoir aussi des plantations de cotonniers.

"La culture de l'indigo et de plusieurs autres plantes équinoxiales a été également tentée avec succès sur divers points.

* On ne manque pas de bras pour les cultures; les travailleurs affluent spontanément des pays voisins.

"Des encouragemens de toute espèce sont accordés jar le gouvernement; ils résultent de deux arrêtés de l'administration locale que je-joins ici, et qui me paraissent propres à faire diriger sur cette contrée une portion des capitaux et de l'industrie dont la France surabonde."

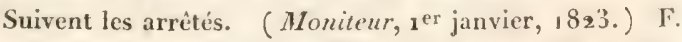

217. Le gouvernement anglais a établi une ligne télégraphique entre Calcutta et Chunar, forteresse bâtic sur le Gange, à 150 milles au sud de Bénarès. La distance est de 336 milles anglais ( 139 de nos lieues de poste de 2,00o toises). Le service se fait à raison de 12 minutes pour 100 milles, ou $4 \mathrm{I}$ lieues. Les Européens et les Naturels qui se livrent au commerce, ont demandé à profiter de ce moyen expeditif de communication; ct, pour jouir de cet avintage, ils ont offert des conditions très-favorables.

(Moniteur, $\mathrm{x}^{\mathrm{er}}$, janvier $18 \% 3$.)

I. 


\section{PLANS', CARTFS TOPOGRAPHIQUES ET GEOGRAPHIQUE.}

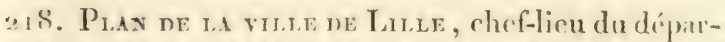
tement du Nord, dédié à la ville, et agrévi par leconseil municipal dans sa séance du 1 zoùt Isoo; indiquant le's principaux élifices, le détail de toutes le's propriélés, les rues, places, marehés, cte.. ainsi que les nouvaux projets de percemens et redressemens qui y sont relatifs: levé par F.-F. Rorssi: lr, génmetre du cadastre; réduit et dessiné par V.-J. Biston; gravé par J.-M. Darmet, ex-dessinatrur géographe au dépòt général de la guerre, employé au ministère des aflaires étrangères; écrit par Ifacr. Paris, is22, une feuille graud aigle, à l'échelle de -5 ou de 2 millimetres pour 5 metres. Prix, r. fr. A Paris, chez Pirquet, grographe, quai de Conti, n. 17; et à Lille, chez tous les libraires.

Ce plan, remarquable par son exnctiturle et la quantité prodiçieuse de détails qu'il prísente, est très-soigneusement sravé; c'est une véritable topographie de Lille. Les halbitans di. cette ville et les personnes qui ont des relations avec eux ne peuvent mieux faire que de ser pourvoir; ils y verront jusquatu n". des maisons dans chaque rue, dont une ligende sert à trouver facilement les positions.

B.

2 in. Axt.1s Anuisistritif de la France, par M. MIure, géographe. Prix, $25 \mathrm{fr}$. Chayue carte se und siparément a raison de deux fr. A Paris, chez l'auteur, rue de l'Université, n. I3.

Cris ouvrage présente dans un cadre de $/ 2$ centimetres en largeur sur 33 en hauteur, une carte de la France ripétec 17 fois sous antant de divisions diliérentes en conlenr :

Ia preniere donne la France divisie par départenens et arrondissemens;

La densieme par séries pour le renouvellenent des departemens; 


\section{et géographiques.}

La troisième par arrondissemens électoraux ;

La quatrième par' divisions militaires;

La cinquieme par divisions des places de guerre;

La sixième par régimens de cavalerie;

La septième par légions de gendarmerie;

La huitième par archevêchés et évêchés;

La neuvieme par arrondissemens de cours royales;

La dixième par arrondissemens d'académies;

La onzieme par inspections des ponts et chaussées;

La douzieme par inspections des mines;

La treizieme par terrains de mines de houille;

La quatorzieme par arrondissemens des foréts ;

La quinzieme par arrondissemens forestiers de la marine;

La seizièmé par inspections de douanes;

La dix-septieme par inspections des tabacs et poudres.

DEN.

230. Atras pontatif et complet du royaume de France, contenant les quatre-vingt six cartes des départemens, précédé d'une carte générale, avec un texte en regard de chacune d'elles, et comprenant, io. la description des principales villes, de leurs antiquilés, des curiosités naturelles et industrielles qui s'y trouvent; $2^{\circ}$. des détails précieux sur la nature du solet de ses produits, soit végétaux, soit minéraux; l'agriculure, les manufactures, les rivières, les canaux et les prineipaux sites; 3o. l'indication des uibunaux, du nombre d'habitans, des distances des dillérentes villes cntrelles, etc., etc.; un index alphabétique des noms des villes, bourgs et contmunes dont il est parlé dans le texte, avec la désignation du département; ouvrage entièrement neuf, utile e indispensable pour le voyageur étranger ou national, curieux ou négociant; par X. Ginten, ex-gégraphe des postes, et Royen ainé, autrurs proprichaires. Prix de lexmplaire cartomé, sif. A Paris, chez Dondey Dupré, pire et fils. ( P'rospectus.) 
2:1. Notvel Atras de la France, cartes des 86 départemens, précédées des cartes de l'ancienne France, et de la France actuelle; dressées par MII. Arpick et Perrot; gravées par MII. Malo frères ; avee se tableaux historiques ou statistiques, imprimés par F. Didot, pire et fils; publié par MII. L. Duprat Duverger et J. A. S. Collin de Plancy. A Paris, chez Duprat Duverger, rue des Fossés Si.-Germaindes-Prés, 11. 13, et rue Montmartre, n. i21. (Prospectus.)

L'onvrage paraitra de mois en mois, par livraisons séparées, chacune de trois cartes coloriées et de trois tableaux statistiques; tout petil in-f'. carré.

Chacque livraison sera du prix de 6 fr. pour les souscripteur's; on a tiré dix épreuves qui seront numérotées, sur papier de Chine, avec texte sur papier vélin. Le prix de la livraison est de $25 \mathrm{fr}$.

A la quatrieme livraison, terme de rigueur, le prix de lit livraison sera de $7 \mathrm{fr}$. 50 c., au lieu de 6 , pour les personnes qui n'auront pas souscrit.

Les cartes de chaque département se vendent séparément, avec la statistique disposée sur les deux côtés de la carte, $2 \mathrm{fr} .50 \mathrm{c}$.

222. Carte de l'Eunope par Defoln, Paris, $182 \%$. Chez Basset.

Cette carte est détachée de l'atlas du même auteur. F.

223. Carte ne l'Océane, ou de la cinquieme partic du monde, par Bucé. 1822. A Paris, chez Goujon, rue du Bac, n. 6.

224. Cante ues Routes de poste el itinćraires d'Fispagne et de Portugal, en a feuille; par L.ipe, is e. $\Lambda$ Paris, chez Ch. Picyuet, quai Conti, 11. 1 .

Cette carte, dont la premicre edition a paru con 1810 , indique toutes les distanus itincraires, of itre utile anx 
voyageurs : on a tracé, conjointement avec les limites des anciennes divisions, celles des nouvelles provinces, arréties par les cortés en janvier 1822 , et qui sont au nombre de 5 I.

B.

225. Carte d'Espagne et de Portugal, en une feuille: colombier, dressée par le chev. Lapie, géographe. A Paris, chez Basset, rue Saint-Jacques, 11.64. Prix, 5 f.

Cette carte, dédiéc et présentée au roi, est la première sur laquelle on trouve figurées avec exactitude les différentes chaines de montagnes qui couvrent ces belles contrées; l'auteur a fait usage d'un grand nombre d'itinéraires qui l'ont mis à mème de rectifier de graves erreurs, et nous pensons que dans les circonstances préseutes cette carte ne peut manquer d'être favorablement accueillie du public.

B.

226. Cintede l'Italie, en i feuillecolombier, dresséc

par le chev. Lapie, géographe. Prix, 5 fr. A Paris, chez Basset, rue Saint-Jacques, no. 64.

Avant les belles vbservations astronomiques de M. le capitaine de vaisseau Gauttier, la partie méridionale de l'Italie changeait souvent de place : tantôt on la portait à l'ouest et tantôt à l'est; aujourd'hui son emplaçement est invariablement déterminé, puisque des observations plusieurs fois répétées n'ont offert que de légères différences. M. Lapie, qui a fait usage de ces documens, n'a rien négligé pour rendre celte carte aussi parfaite que possible, et nous ne croyons pas nous compromettre en assurant que c'est encore la plus parfaite qui ait paru sur ces contrées, que tant de souvenirs rendent si intéressantes.

B.

227. Archipel toscan (Carte topographique de l'), ou de l'ile d'Elbe et des iles adjacentes, à l'é-

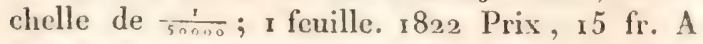
Paris, chez Picquet.

Cette carte a été gravée au dépôt général de la gnerre. F

228. Cante giverare de la chaine des $\Lambda$ lipes, contenant la haute-Italic, la Suisse et l'dllemagne meriliomale. dressée pour lintellience de l'histuire des nueres 
de la révolution; par le: sénéral Jomus. Quatre fenilles, papier dit gramb-aigle. Pris, 24 f. Clery Anselin et Puchated, sucresseurs de Masimel, libraires pour lart militaire, rue Dauphine, n. ()

Cotle belle carte topugraphique a bi construite aver beaucoup de soins sur d'excellens materiaus: tous les mouremens et accilens du terrain y sont exprimes arec la plus scrupuleuse exactitude, ot le trait est de lia plus grande puretí. Elle a été gravée par Orgiazzi, attaché au dépòt général de la gucrre.

II. S.

220). Cifite givénale ne í Allemigne, comprenanta confédération germanique; grand colombier. Carte de l'Amérique méridionale; demi-colombier. - Carte de l'Amérique septentrionale; demicolombier. - Lidsie, lifrique, IUcéanic on l'Australasie et la Polynésic.

Toutes ces cartes sont des réimpressions des cartes de MI. Ilérisson; Paris, 1822 , chez Basset. F.

230. Cinte ne li Scindinive, ou rogamede Suido. de Norwége et de Danemarek; dressée parlechevalier Lirie, géographe. I feuille colombier. Prix, $5 \mathrm{fr}$. A Paris, chez Basset, rue St.-Jacques, n. (if.

It manquait a la géocrajphie francraise une carte de ces contrées septentrionales; M. Lapie vient de remplir cette litcune. Il a fait usage, pour la rédaction de celte carte, de: celles d'Hermelin et de l'ontoppidan pour la Sude et la Xorwege; et de celles publites par l'Acadimic de copentagune pour le Inanemarck; plusieurs cartes marines of des relations de voyageurs sont venues ausmenter la masse des documens dont il a fait usage, et nous pouvons affirmer que, sous le: double rapport de l'exactitude et de l'exicution, cette carte ne laisse rien à désirer.

B.

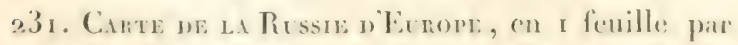

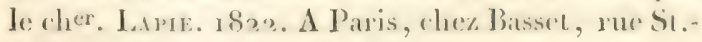
Jacques. Prix, $5 \mathrm{fr}$.

Cetle carte est une reduction carcle de celle en 6 ferulles 
dresie par le míme autenr : les nonveaux accroissemens de l.t Russie y sont indiqués, ainsi que le royaume de l'ologne.

13.

232. Le fouvel $\boldsymbol{A}$ tras de l'empire de Russic, du myaume de Pologne et du grand-duché de Finlande, est acheví. Cet ouvrage, dont l'auteur est M. le colonel Pranischer, cht magnifiquement gravé, et composé de soixante-dix carles grand in-folio. (Revue cncyclop. déc. 1822, 1).625.) F.

233. Carte gínérale de a Tunqued'Europe, en i 5 f. dressće sur les matériaux rassemblés par M. le: lieutenant-général comte Guillemixot, directeur général du Dépót de la guerre, et M. le maréchal de camp, baron de Tromelin, inspectewr-général dinfanteric, par M. le chevalier LApre, oflicier supéricur au corps royal des ingénieurs géographes militaires. Cette carte paraitra en 5 lirraisons de 3 feuilles; le prix de la livraison est de $5 \mathrm{fr}$. A Paris, chez Ch. Picquet, quai Conti, n. i g, ct chez tous les marchands de cartes.

Depuis d'Anville, qui devina plutôt qqu’il ne connut la construction physique de la Turquie d'Europe, il n'a ricn paru de satifaisant sur cette importante contrée; et lorsque le savant géograplie M. Barbier du Bocage voulut de son cabinet nous aider a suivre le jeune Anacharsis dans ses voyages en Grèce, il fut le premier à recomnatre l'insuffisance des matériaux que l'on possédait.

Les éditeurs de la carte qu'on offre anjourd'hui au public ont l'immense avanfage d'avoir eux-mêmes parcouru la phis grande partic des pays qu'ils décrivent, et de s'ete trouves en position de rassembler une immense quantité de matériaux et d'itinéraires, parmi lesquels nous citerons cenx de MIM. Ies généraux Haxo, Foy, Andréossi, Sorbier; et les colonels Trezel, Fabvier, Riollay et Boutin.

Cette carte, appuyée sur les excellentes observations astronomiques de M. le capitaine de vaisseau Gautier, est la premia're sur laquelle on trouvera tracíes, d'une maniere satisfaisante, les nombreuses chaincs de montagnes qui cou- 
vrent ces vaster contrées, ou fant et de si grands intéréts politiques se trouvent en présence.

Faudra-t-il suive la marehe des armées belligirantes: lat carte que nous annoneons indiquera les passages accessibles. a travers ces chitines qui divisent l'empire du Croissant, et divoilera aux yeux de l'lomme d'état le the'àtre de la gruerre.

Le voyageur voudra-t-il, pour des temps plus heureus, se préparer a ces généreuses excursions qu'inspire lamour des monumens de l'antiquité: ces mêmes chaines de monlagnes, tracís telles qu'elles existent réellement, et les caux, qui en découlent, offriront une direction à ses savantes recherclies; ct soi a ail avide reconnatra désormais plus facilement, dans les ruines qui couvrent ces belles contrées, les villes anciennes drnt l'emplacement n'avait pu être, jusqu'à ce jour, que vaguement déterminé.

Les deux livraisons qui viennent de paraitre donnent la plus haute idée de ce que scra cette carte, qui, dans les circonstances présentes, ne peut manquer d'ètre de la plus grande utilité.

B.

23. Carte de la Turoqued'Europe, destinće al usage des colléges, 1822. - Carte des Pays-lias, id. Carte des Iles Britauniques, id.-Cárte de litalie. id. A Paris, chez Selves fils, rue des Lions SaintPaul, n. I 4 .

235. Reinef de la Suisse.-Les royagenrs qृui parconrent la Suisse et qui passent a Geneve, visitent avec intérêt le beau modele en relief de la Suisse et des pays environnans, expesi cette annie par M. Gaudin, associe honoraire de la sucirti pour l'asancement des arts de Genére. On peut étudier avec facilite, sur ce modile, le pays qu'on va pareourir, ou se faire une juste idée de celui qu'on a parcouru, en eximinant avec attention les hautes chaines de montagnes, les glaciors aree foutes leurs sinuosités, les vallées et leurs coleures di verses, les rivieres qui les arrosent, les laes qui les orment. enfin les routes qui serpentent dans tout le pays. It s'etend de

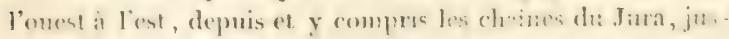

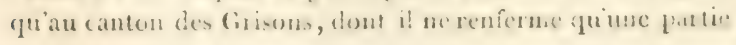


th nord an sud, répuis Zurich jusqu'atu hont-Bhanc, alu Saint-Bernard et au Mont-Rose, exclusivement. Ce plan, 'qui at vingt-quatre pieds de longueur sur dix-neuf' de largeur est dì à un travail assidu de plusieurs années : l'auteur, déjà avantageusement connu par l'exécution de relicfs sur des échelles moindres, a voulu montrer, dans celui-ci, ce que pouvait faire un seul individu, avec de la persévérance. Ce relief est renfermé dans un bàtiment isolé, construit tout exprès, et fort hien éclairé, situé aux Pâques, à un petit (quart de lieue de Genève : il est disposé de telle manière que les curieux peuvent aisément en faire le tour, et en observer les parties centrales.

Le premier relief de cette espèce que lon connaisse, celui construit par le général Pfyffer, et qu'on montre encore i Jucerne, comprend cent quatre-vingts pieds carrés et n'a que vingt-deux pieds et demi de long et douze de large. (Revue Encycl. Décembre 1822, p. 63i.)

F.

\section{ART: NAUTIQUE.}

236. Le priote du golfe du Mexique et du canal de Bahama, ou description desiles, bancs, hauts-fonds, ports, rades, rivieres et baies qui bordent toutes ces cótes, et celles de l'ile de Cuba; augrmentée de détails sur la navigation du fleuve Mississipi, et d'observations sur les vues de terre, la direction des courans, et la manièe d'entrer sans pilote, dans les ports, rades et autres mouillages. Par M. Dusserra, Capitaine de frégate en retraite, etc. In -8 de 7 feuilles. Imp. de Rottier, à SaintMalo. Prix, 2 fr. A St.-Malo, chez Rottier.

Cet ouvrage inconnu à Paris, et dont nous n'avons pu, par conséquent, rendre compte, serait-il un extrait du suivant, dont le Dépòt général de la marine va faire paraitre une traduction, annonce qui ne peut manquer d'intéresser

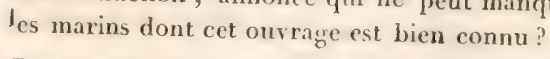

Denoteno de las islas Autillas, de las costas de TierraFirme, y de las del seno Mexicano, formado en la
Tome $I$. 


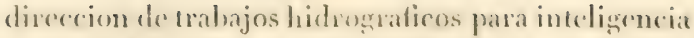
y uso de las cartas que ha publicado. ae. edicion, corregiclay aumentada con noticias nuy recientes, y con un appendice sobre las corrientes del Oceamo allantico: de: orden superior. Imprenta nacional, Madrid, 1820 .

\section{$\triangle R T$ MILITAIRE.}

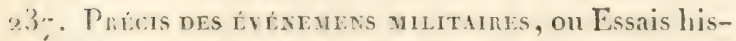
tnriquessur les compagues de 1799 à 181 亿, avec cartes etplans; par N. Ir comte Mathieu Dius, lieutenant général des armées du roi. Tomes 1 1, 12, 13 et 14. Campagnes de 1805. I, 2, 3, 4. volumes in-s. , ensen:ble de 16 feuilles; plus un atlas in-folio, renfermant 2 i planches, dont 15 doubles. Imprimerie de Crapelet, a Paris. A Paris, chez Treuttel et Wurtz; à Strasbourg et à Londres, cliez Treutel et Wurtz; à Hambourg, chez Perthes et Besser. Prix, 48 fr.

238. Histome ne Lexpldition de Russie, par $\mathrm{M}^{* * *}$; avec un atlas, un plan de la bataille de la Moshwa, et une vue du passage du Niémen. 2 tomes in-S. de 56 fenilles et drmic, \& tableaux, et 1 atlas in-8. de f́ cartes et un plan. Imp. de Pillet ainć. Paris, I $8: 3$, , wez Pillet ainé, Anselin et Pochard, libraires. Prix, $18 \mathrm{fr}$. et $22 \mathrm{f}$. par la poste.

Composé par un fémoin oculaire, sur les documens franrais les flum anthentiques, érite avec clégance, angmentéc de notes instactives sur l'art militaire, l'Histoire de l'expédition de liusie, que nous annoncons peut étre mise an rombre des ouvatices remarquables qui ont ilé publits sur les campannes des armées francaises. Lille est divisie en quatre livres, pui son pricédés par une preface tres-courte, et une note préliminaire sur la Pologne.

Le premier live se termine au combat de Smolensk: le second rontieut le récit de linvasion en Moskovic; le troi- 
sieme, le récit de lat relraite jusqu’i Orsza sur le I)nieper; le guatrieme, le reste de la retraite. Chaque livre est suivi de notes dians lesquelles l'auteur traite les questions d'art militaire qui naissent du sujet; en sorte que l'ouvrage est tout a la fois historique et didactiqne. $A$ la fin des notes de chaque livre, se trouvent des lettres de Napoléon, ou du major général de l'armée, qui étaient inconnues, et qui servent de pièces justificatives; phusicurs d'entre elles font connatitre les motifs des résolutions de Napoléon, et son opinion sur quelques parties de l'art de la guerre.

La marche que suit $\mathrm{MI}^{* * *}$ dans Ia partie historique est simple et claire; après avoir mis tous les corps d'armée en mourement et les avoir fait pénétrer sur le territoire russe, il s'attache à la partie de l'armée que Napoléon commandait immédiatement; lorsqu'elle s'arrète, il reprend le récit des opérations des autres corps d'armée. Il donne son opinion sur les diverses opérations; il y ajoute des rélexions sur la nouvelle méthode de guerre suivie par les Français, sur la difficulté de l'employer en Russic, et sur les maux infnis dont clle accablait les habitans et les combattans cux-mêmes.

Cet ouvrage n'est point une description aride d'événemensmilitires, mais une historie compléte de l'expédition de Russie; la prartie de la politique européenne qui se rattache à ce grand ¿'vétement y est surtout très-bien traitée. Les réfexions de l'anicur sur le caracière de Napoléon, les anecdotes qu'il rapporte concernant cet homme célebre, le portrait qu'il en atraré sous le rapport physirque, dans le second live, le font parfititement connatre, et le metient en quelque sorte sous les yeux des lecteurs.

Ia vue du passage du Jiémen qui se trouve au commencement du quemier live a cité réduite d'après celle de Bagetti, ingénicur-réograjh" qui l'avait dessinće sur les lienx mêmes. Ie's cartes soiat exéutes avee le plus grand soin; celle pui est intitules? Cirte greminale du thécitre de la guerre est coloriec et reprúsente linterieur de l'Europe, telle qu'elle était en 1312 ; les trois antres qui représentent : la première, le: noril de la Lithuanie et la Vieille-Prusse; hadenxieme, le midi de la Lithunie, ce une partic du grand-duché de Taroovie; la troisieme, les genvernemens de Noshow et de: Smolensli, contiennent fidblement tous les noms qui sont dim, 
l'ouvage, mais ceux-lit seulement. La pratigue de cofte righe merite d'être recommandée dans l'exécution des cartes destinées à faciliter lintelligener d'opriations militaires.

A.

23.9. Extrats nes services ne cimpigne ct de la place, pour la thénie de MM. les officiers et sous-olliciers dinfantrie; par demandes et réponses, avec des noteset un plan d'un camp diufanterie et d'artillerie: par un officier supérieur. In-18 de 5 feuilles; plus, la planche gravée. Imı. de Verounais, à Metz. $\Lambda$ Metz, chez Veronnais.

Le service des places et de campacrne forme une des branches si essentielles de l'instruction militaire, qu'on doit toujours applaudir aux efforts des officiers qui veulent bien se charger du soin d'en rendre l'étude plun furile, en clénageant les ordonnances d'une infinité d'articles alorogis, on d'une application extrénement rare. C'est ce qua fait aver discernement l'atuteur de l'extrait ci-dessus.

K.

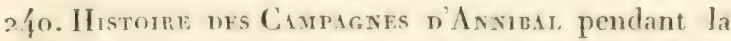
denviène guerre punique, suivie d'un abrégé de la lactique des Romains et des Grees, etenrichie de plans et de cartes topographiques; par M. le génés

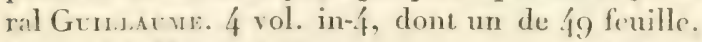
Prix, -j f. Milan, de limprimerje royale : et à laris, chez Barrois l'aîné.

Quoique Polybe et Tite-live nous aient linise IHistoirc de la deuxieme gnerse punique, l'on n'avait en France qu'une idec imparfaite, inexacte, des campagnes d'A nnibal en Italie. Travesti plutôt que traduit par un bénédictin; tourmenté par les commentaires systématiques de folard, l'historien gree, défiguré à claqque page, qui écrivait prour l'instruction de Scipion, n'offrait plus, aux yeux des militaires, que des fragmens historiqques sans liaison, où l'on cherchait envain des lrecons de tactique et de strategic. I'un autre còté, l'historien latin par cela mème gu'il contenait une multitude de faits que le premier avait negligris d dxsein, fut taxé d'ignorance et d'imposture, comme si te eadre d'une histoire cireonstanciéce devait 
être le méme que celui d'ua ouvrage purtuent milidile.

C'était à l'un des compagnons de ent autre capitaine qui sut conquérir deux fois l'Italie, qu'il appartenait de ramener l'opinion des militaires francais, de mettre Tite-Live d'accord arec Polybe, de redresser les nombreuses errems dans lesquelles le traducteur de ce dernier était tombé, de jrísenter enfin une histoire complète des campagnes du héros carthaginois en Italie. Le général Guillaume, que ses fonetions ont mis à même deparcourir l'Italie dans tous les sens, de visiter les champs de bitaille, et de recucillir une foule de renseignemens précieux sur la géographie ancienne de la pé. ninsule, a heureusement exécuté cette entreprise, au milieu des I ravaux les plus pénibles, à l’aide des matériaux que Guischard et Maizeroy lui avaient laissés.

Son ouvrage est recommandable sous les rapports de l'é rudition, de la critique, de l'ordre et de la clarté. Toutes les opérations d'Annibal, depuis son départ d'Espagne jusqu'à la bataille de Cannes, y sont parfaitement développées, et bien que depuis ce moment, le secours de Polybe manqua à l'auteur, on s'en apercoit a peine, tant il a su étudicr Tite-Live.

Cette histoire est très-précieuse, parce qu'elle fixe d'une maniere irrévocable le degré de perfection auquel les anriens étaient parvenus dans l'art de la guerre. $\mathrm{K}$.

z.j. Consintrations genterales sur linfanterie francaise, par un général en retraite. Strasbourg, I 822. Chez Jean Henry Heitz, imp.-libr. rue de l'Outre. n. 3 ; ct à Paris, chez Anselin et Pochard, rue Dauphine, n. 9. Prix, I fr. 50 c.

Dans cettc brochure de I I 7 pages d'impression, l'auteur parcourt successivement les écoles du soldat, de peloton, de bataillon et les évolutions de ligne, et démontre les améliorations dont quelques-uns de leurs articles sont susceptibles, dans l'esprit de l'ordonnance méme, quelquefois pour répatrer ses lacunes ou pour redresser ses erreurs. Passant ensuile au régime intérieur, il propose divers exercices gymnastiques, autant pour rendre les sollats adroits et vigoureux, que: pour fortifier leur santé et les tirer de l'étal de torpeur ou les plongre souvent la vic de grarnison. Venaut cufin a l'ha- 
billement, a l'équipement et a l'armement, il indique ce que ces parties ont de défectueux, et propose souvent des moyens aussi simples qu'ingénicux d'y remédier. $\Lambda$ ux nombrenses et utiles observations que renferme cette petite brochure, il est facile de juerer que son auteur a manié long-temps de l"infunterie, et je ne serais pas étonne quelle ne fü le réve d'un ancien inspecteur qui lui consacre encore les loisirs de sa retraite. K.

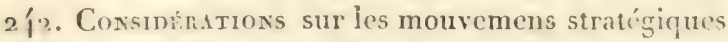
des armérs francaises, dans quelques-umes de's campagnes de Napoléon Bonaparte, et particulierement dans les quatre dernières, etc., avec trois

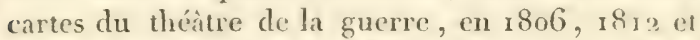
r8 3 , ou sont tracées les difiérentes marches; par L.-C. Dichitele, lientenant colonel d'élat major, chevalier de l'ordre roval militaire de Saint-Louis. Paris, 1822 . Chez Anselin et Pochard, suceesscurs de Maginel, libraire pour l'art militive. In-S de I 2 pag. dimp., aver tableaux. Prix, $3 \mathrm{fr}$.

Cet opuscule consiste en devix mémoires. Dans le premiex l'auteur propose une manière d'apprécier la valeur des monvemens strategiques. D'après sa méthode toute griométrigune, la plupart des plans de campagne et des plus brillantes opurations de Napoléon semblent pécher contre les principes fondamentanx de l'art militaire. Dans le second cet offecier réfuse le memoire de M. C. sur la drifense de la Franee jer Les places fortes, concurremement ave l'artion des armer.; et pense qu'il faut comfier la defense de litut at une bone armee, sanf a saisir au monetent du danger les points stratigicques par des camps retranches. Cefte opinion, partagie pare des militaires distingrue, arquiert un nouveau poils par les raisons dont i! lappuic. Ja lechre de cé deux mimotren, d'un mérite bien difterent, fera natre des ictes a tous cem qui s'occupent des hantes combinaisons de greme et de . - litique.

K.

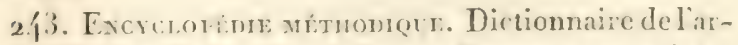
villerie ; par le colonel Cotrr, directeur genéral des manufactures royales d'anmes de gueree cheralier

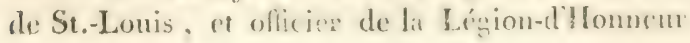


$\Lambda$ Paris, in- $\{$. Chez madame veuve $\Lambda$ gasse, imprimeur-libr., rue des Poitevins, n. 6. I832.

Les comaissances de l'auteur, les fonetions importanter dont il a été chargé, et la place quil occupe maintenant an ministère de la guerre, sont de sûrs garans de la bonté de ce: Dictionnaire. M. Ie coloncl Cotty s'est borné à ne donner, autant que le comporte la nature de son ouvrage, que des choses de principes ou fondées sur l'expérience. Il a écarté, avec beaucoup de sagacité, le faur du vrai et le douteux du certain; il a dégagé des mathématiques transcendantes et mème de l'analyse simple tous les articles qui auraient pu en renfermer; il n'en a présenté que les résultats; il a mis ainsi son ouvrage à la portée d'un plus grand nombre de lecteurs, et l'a rendu utile aux officiers de tous les corps. Dans une notice sur une bibliothéque d'artillerie, it indique aux officiers de cette arme qui veulent approfondir leur métier les ouvrages qülils doivent consulter pour atteindre leur but.

L'artillerie a des relations avec presque toutes les sciences, tous les arts et tous les métiers; on trouve donc dans ce Dictionnaire des termes communs à ces diverses parties des connaissances humaines; mais ils n'y sont présentés que sous le ratport des travaux de l'artillerie : par exemple, en parlant des métaux employés pour la fabrication des armes, des projectiles, etc., l'auteur a laissé a la minéralogie à expliquer les moyens de les extraire die la terre, de les combiner, de les travailler et d'en rectilier les usages généraux. Il a fait connaitre dans les articles importans le peint de départ de to science de l'artilleur, le chemin qu'elle a parcourn, et l'état ou elle se trouve maintenant en france; il y a consigné, aulint qu'il a ćé possible, lés découvertes qui se sont succédées si rapidement dans ces derniers temps ot qui sont relatives it l'artillerie, telles que la fabrication de la poudre ronde, la manipulation des poulres fulminantes et l'usage des armes it perension. Finfin cet ouvage est rédigé avec la précision at la clirté qui distinģuent tous les écrits de l'auteur. B. P.

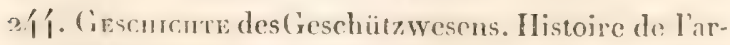
tillerie, depuis son origine jusqu à nos jours, princi-

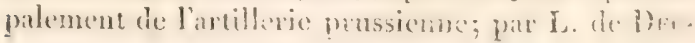


Mr. Soronte cidition, entierement refondue. In-s. Prix, 18 gros. 1822. Berlin, chez ilister.

\section{VOY $\triangle G E S$}

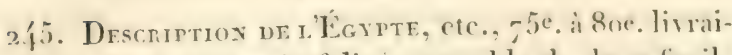
son; deux cahiersin-folio, ensemble de deux féuil. avee les couredures, et 22 planches, dent \& doubles. Imp. de Panchouke, a Paris. A Paris, che'z Panckouche. Prix de chaque liv raison, $10 \mathrm{fr}$.

Ces cinq livraisons renferment, entre antres planches intércssanic's, $1^{\curvearrowleft}$. les collections de meubles et de vases sculptis dans les ruines de Thebes, et enrichis de précieux ornemens qui constatent l'état des arts et la prospérité de l'ligypte a une époque très-reculée, avec les indications de chiffes, dont l'objet est l'énumération de toutes ces riclresses; 2 ". des papyrus trouvés sur les monies et converts de l'écriture ézyptienne, dont les caractires mèmes étaient inconnus autrefois; $3^{\circ}$. un plan topographique tris-étendu de lancienne et de la moderne Alexandrie; $4^{*}$. les roches de pierre dure que les Égyptiens ont exploitées pour l'exécution de leurs statues colossales, de leurs obéliques et de leurs monolithes; 5". les monumens souterrains connus sous le nom de tombeaux des rois, célèbres par leur magnificence ei l'éclat de leurs peintures.

2.f6. Deschiption de L'ÉGrte, ou recueil des observations et des recherches qui ont été faites en Frpte, pendant l'expédition de l'armée francaise; deux. édit., tome 7 . In-8 de $36 \mathrm{f}$. trois quarts ; plus Io tableaux imprimés. A Paris, chez Panchoule,

Ia réimprescion de la Description de l'Égyte avance ratpidement : So livraisons, de cime planches chacune, sont publices. On a vu peu d'exemples et d'un debit anssi prompt et d'une publication anssi rapide, surtout pour un

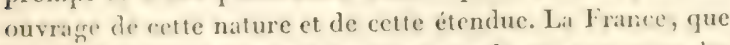
le sort des armes at prive de plusieurs de ses avantages les plus brillans, semble ressaicir anjourdhui arece empressement un ature gene de gloire: ses conduètes littraires et semtitirnes sont des titres que nul érenement ne ponra 


$$
\text { Toyages. }
$$

lui ravir. Sil est une science qui soit relevable is l'expédition l'Érypte, c'est sans doute la Géographie. Indépendamment de la description topographique de tout le pays et des contrées environnantes, on trouvera dans cet ouvrage une multitude de recherches approfondies et de renseignemens préris sur la géngraphie physique, sur les productions naturelles, sur la statistigue et sur le sol du pays; on y compare aussi la géograpbie moderne avec la géographie ancienne, et plusieurs Mémoires sont consacrés à ces rapprochemens intéressans. Le tome VII que nous annonçons a pour objet l'une des questions qui intéressent le plus l’ancienne géographie, savoir : le système des mesures qui ont été en usage dans l'Égypte; et qui fut la source ou puisèrent les Hébreux, les Grecs, les fiomains et les Arabes. Dans cette exposition du systime métrique des anciens, l'auteur établit Io. que les mesures usuelles dérivent du degré terrestre égyptien; $\mathbf{2}^{\circ}$. que leur progression était sexagésimale depuis le schœne jusqu'au doigt, qui fait la $24^{\mathrm{e}}$ partie de la coudéc; 3 . que les grands monumens renferment exactement dans leurs dimensions $\therefore$ :'s parties aliquotes du degré; $4^{\circ}$. que la grande pyramide en par ticulier présente ces mesures avec une étonnante précision; $5^{\circ}$. que le stale olympique n'est autre chose que le stade égyptien que les Grecs ont cmprunté; $6^{\circ}$. que les principaux théorèmes de géométrie et les grands phénomènes astronomiques ont été connus des anciens habitans de l'Égypte; $7^{\circ}$. que res peuples ont possédé des connaissances géographiques très-étendues pour une époque aussi reculée que celle à litquelle remonte la prospérité de l'empire égyptien. Ce trivail, qui remplit à lui seul le tome VII, est accompangú d'un grand nombre de tableaux; il sert de base aux recherches de géographie ancienne que l'on doit au mème auteur. 2彷. Promfine autour du moxde, pendant lesannées

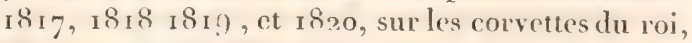
l'Uranie et la Physicienne, commandées par M. de Fregcinet; par J. Arugo, dessinateur de: l'expédition. 2 vol in-8, ensemble 358 pages; mu atlas in-fol. de: 25 pl. et une carte litographicis. Prix, 35 fr. $\Lambda$ Paris, chez Ed. Garnot.

Le nom d'Arago, l'importance et la celebrite de lexperli- 
tion commandes par NI. le citpitaine Freprinel, sont de' moyens de suecés qui ne fromperont certanement pas l'an teur du Vovase gne nous ammoneons, frere de M. Araeno, membre de l'Acarimic des sciences. Beancoup d'esprit et d'imagination, des vues piquantes, des rapprochemens inatlendus et des tourmures originales; un style facile et chaud, la variete et la singularite des peuples, des habitudes ef des aspects que M. Arago a pu observer dans sa promenado en passant par le Brésil, le cap de Bonne- Espérance, da Nouvelle-Hollande, les divers archipels de lamer du Surl, Ju cap llorn, etc., feront lire cet ouvrage avee aviditi par un grand nombrede lectcurs. MI. Aragoayant senti queles risultuts scientifiques d'une expédition faite par ordre du roi appartemaient a gonvernement de S. M., qui a chargé M. Le capitaine Freycinet d'en diriger la puljlication, et qu'il ne pouvat entrex dans aucun détail de cette nature; les sciences proprerment dites y trumeront peu à gagner, mais cet ourage pispularisera dans le beau nonde des idees instructives, of il atra sans doute plus de lectenrs que s'il s'adressiat aux senis savans. Nous n'aurons done, d'après le but de notre jumrnal a citer qu'un tableau, platé en trite din premier vol., oii l'un imlique la plus grande hanteur du barometre, du thermometre et de l'hegentetre dans nue vinglaine de slations im portanies de celle longue traverses: tablean qui contraste arec le reste de liomiane et guon trouvera d'ailleurs at a place dans la rebialion de lexperdition de M. le eapitane Irey -

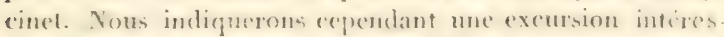

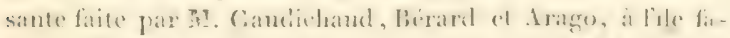

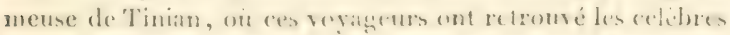

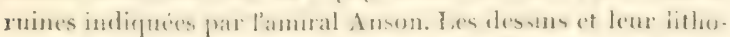

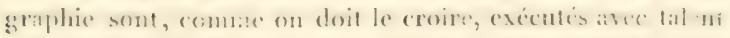

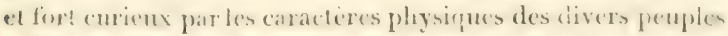
guils sont destince a relracer. On rematequera cependant mote

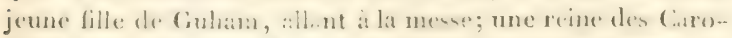

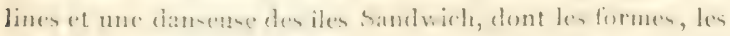

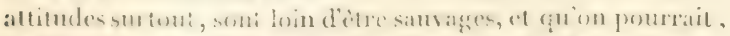

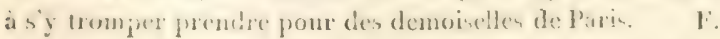

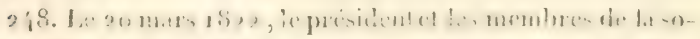

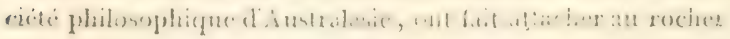


grès duquel débaryuerent le capitaine Cook et sir Joseph Banks, au cap sud de Botany Bay, l'inseription saivante:

" L'an du Seigneur $x 77^{\circ}$, sous les auspices de la science? "britamique, ees rivages furent découverts par lacques "Cook et sir Joseph Banks; l'un le Christophe Colomb. et "l'antre le Mécene de leur temps. Ce lieu les vit autrefois x pleins d'ardeur dans la poursuite des connaissances; an" jourd'hui cette inscription est consacréc à leur mémoire:

"La première année de la Société philosophique d'Austra" lasie; sir Thomas Brisbane, membre de lat Societé royale. w de Londres et de celle drimboug, correspondant de "l'académie des sciences de Paris, $1822 . "$ (Moniteur, I $^{\text {er }}$ janvier, 1823. )

F,

\section{DEUXILME SEGTION.}

REVUE.

\section{PROGRES DES SCIENCES.}

249. Limportance et l'intérét général de l'Extrait suivant nous ont engagés à le dunner en entier, mal re qu"il sorte par son étenthe de's bornes de notre Joumal : mats les travaux des savans snédois sont si pen connus dins une grande partie de liburope, que nous pourons espérer qu'un tableatu qui fail comnaitre l'etat ascer récent des sciences dans la patric de Linne sera lu avee satisfation patr les savins. L'ouvrage d'où il est tiré, envoyé a l'fustitut de linuce, par MI. Berzélius, n'a été recu qu'an mois de novenbre dernier.

Aorsberottelser om vetenshapernas framsteog, aifgifuc af Kongl. Vetenshaps-academiens embetsman, cest-

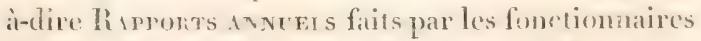
de l'Arallenic Royale des Scienes, lo 31 mars. s's, surles progres des sciences. Stochlooln, riss, in-\$. de 364 pag.

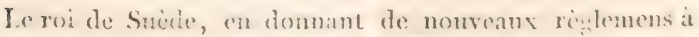

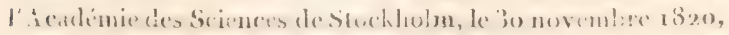
asail preserit, par l'art. I I $\$ 6$, de ces rightenens, qu'il

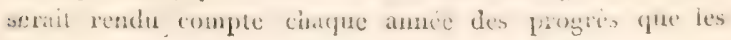


sciences andaient fills, soit en Suede, sovit dans les autres pays, pendant lannée précidente. En consequenee, le rapport relatif aux sciences physigues a eté confie at Mi. lieve: lius, secrétaire de l'Académie;

Celui des sciences mathemotiques à M. Cronstrand, astronome ;

Celui de la zoologie à M. Dalman;

Celui de la botanique ì MI. Wikstroem.

Ces differens Rapports ont été lus dans la séance solennell. du 31 mars I 821 , et réunis dans le volume que nous avous sons les yeux. Ils sont précédés d'un Discours, dans lequel le bitron de lirsen, alors président de l'Académic, rappelle? en peu de mots les serrices que cette Compagnie a rendus aur sciences pendant l'espace de 82 ans, depuis l'année $1-39$, époque ou sir savans de ce pays, du nombre desquels était l'illustre Linneus, en formerent le noyau. L'orateur paic rapidement un tribut de lonanges a ces premiers fondateurs de l'A cardémie, et à ceux qui, marchant sur leurs traces, ont ajouté chaque année à sa célébrité. T'Académie de Stochholm comple, depuis sa création jusqu'en 1821 , '38; inembres suédois et $2 \mathrm{I} 6$ associés étrangers. Ians ce nombre 'n remarque deux dimes, live de la Gardie, éponse du comte Ekcblarl, et la princesse Dasclikow, née Woronzow, qui présidait l'Acarlínie des Sciences de Pétersbourg.

Un phénomène qui mérite d'ètre cité, c'est que l'A cadémic voit encore assister à ses séances, à l'àge de 89 ans, M. Watvid Schultz de Schultzenheim, qui fut recu dans celle Compagnie il y a soirante el un ans, et dont un des premiers tratvaux fut un Mímoire sur les moyens d'alteindre un cige avarué.

Passons aux comptes rendus, qui sont l'objet essentiel du présent volume.

M. Berzéliu, divise son travail en 5 chapitres, qu'il intitule: $x^{\prime \prime}$. Physique et chimie inorganique; $2^{\prime \prime}$. Chimie minerale, el mineralogie; 3っ. Chimie végétale; $4^{\circ}$. Climie animale; 5\%. Géologic.

Yous nous abstiendrons de citer les derouvertes des savans francais, anglais, dont M. Berzélius fait mention, et qui sont bien connus parmi nous. Nous nous attacherons particulièrement a indiquer les travanx des savans suédois, el quelquesins de ceux des savans de l'Allemagne. 
Nous trouvons indiqués dans le premicr chapitre le Mrimoire de M. Berzélius sur l'emploi du chaluméru duns a a chimic et la minciralogie, que M. Fresnel a traduit cn francais; et les Élémens de physique, du professeur J'roberg, dont le $\mathrm{I}^{\text {er. }}$. volume a paru en 1820 à Westeraos.

Dans l'article minéralogié, après avoir cité les attaques dirigées, par M. Mitscherlich de Berlin, contre quelques points du système minéralogique de M Haüy, ct. dont il a été rendu compte dansle tome xiv des Amnales de chimie et de physique, de MII. Gay-Lussac et Arago, M. Berzélius parle de l'extension que M. Mitscherlich a domnée à ses découvertes, en groupant ensemble les bases d'où résultent des corps isomorphes. Le premicr groupe, par exemple, se compose des huit bases suivante's, savoir: la chaux, la magnésic, le fer oxidulí, le manganèse oxidulé, et les oxides de zinc, de nickel, de cobalt et de cuivre. Au second groupe apparticnnent la baryte, la strontiane et l'oxide de plomb. Le troisieme se compose de l'oxide de fer, de l'oxide de manganise et de l'alumine; et ainsi des autres groupes. Pour faire micux sentir l'importance que ces découvertes ont pour la minéralogie, il cite les travaux de divers chimistes suédois sur les pyroxènes et les ampliboles, noms sous lesquels MI. Hauy, se fondant sur les caractères distinctifs qu'il avait adoptés, réunissait un grand nombre de minéraux différens par la couleur, la dureté, la pesanteur spécifique, et la composition chimique, mais semblables quant à la forme cristalline. Liun de ces chimistes, M. Rose, en comparant ensemble plusirurs sortes de pyroxènes, a fait voir que tous sont desbisilicates de quelquesunes des quatre bases isomorphes, conformément au système de Mitscherlich, savoir: la chaux, la magnésic, le fer oxidulé, et le manganèse oxidulé : ici cest un bisilicate de manganése oxidulé et de chaux; là un bisilicate de chaux et de fer oxidulé: dans la salite de Sala et la malacolithe de Tioetten, Jes bisilicates ont pour bases la chaux et la magnésic. Ainsi disparainsent, grâce aux découvertes de M. Mitscherlich, les contradictions qui existaient entre le résultat des analyses chimiques et le caractère fondé sur la forme des cristaux. M. de Bonsdorf, professeur-adjoint de chimie, à Abo, a soumis à une semblable analyse comparative les minérazix que M. Hauy comprenait sous le nom d'amphibole. Il a trouvé que ce sont des sili- 
cates dombles de chame of de masnesie, dont la eornposition est telle, guim atome de trivilicafe de chaux est uni a trois atomes ele bisilicate de magnesie, ot qüils comliemont eonstamment du fuafe de chanx en quantiti plus ou moins considérable. Dam la sorte la moins pure, la masmesie cat remplace en partic par le fer oxiluke, et dans une autre la chaux est reprinentie par la magnésie, gui dans ce cas l'emporte, comme base, sur le fer oxiduli. Quant aux ampliboles noires, arsilliferes, M. de Bunsilorf s'est cru fondé à soupeonner que latumine n'y cxiste pas comme base; mais qu'en qualité de partie constituante électro-ncgative, cile représente la silice, attendu que la quantite de celle-ci diminue à proportion que celle de l'alumine angmente; et il regarde comme certain que trois atomes d'alumine en représentent deux de silice.

Crs analyses ont élé faites dans le laboratoire de NI. Berzelius, qui rend un témoignazge très-favorable de lexactitude avec laquelle ces jeunes chimistes ont opéré.

Le même M. Rose, en opérant par distillation à sec sur différentes sortes de mica, a retiré de toutes une forte proportion d"acide spathirpue, mais moins des micas qui sont dans le calcaire primitif que de ceux qui sont dans le granile.

Lit lépidolithe de Rosena et de L toe contient, de plus que le mic: , une certaine quantité de lithion : à cela pris, ce minéral a beaucoup d'analogie avec le mica.

M. de Grotthuss a décrif, daus un journal allemand, l'aprés des échantillons conservés pendant I3 3 ans, une substance semblable a nu papuer noir tres-frippé, gui tombat

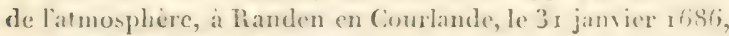
pendint qu'il neigeait. On ne donne pas l'analyse de celle espèce particulière d'aérolite.

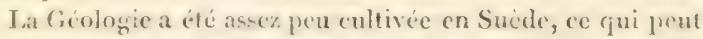
provenir, comme l'obsesve M. Berzilius, de ce que ex pily noffre presque qu'une seule clatse de terrain, savoir la formation primitive, fort importante, sars doule, sous le point de rue ćconomique, a raison des mines gu'elle renferme, mais bien moins intéressinte pour le griolognge que les ferrains varies dureste de l'Europe, ou lon trouve la sucession des differentes formalions, depruis les plus anciemnen junqu'aux plus récentes. 
Cependant deus savans suédois ont écrit d'une manière fort distinguée sur la gúblogie de leur pays. L'un est le docteur Wahlenberg, dans un Mémoire sur Ce que la Sucde renferme de terrains secondaire's contenant des fossiles; l'autre est M. Hisinger, dont on a une Géographie minéralogique de la Strède, et un Disconrs prononcé dans une séance publique de l'Acadéne de Stocklıolm, en 18 I 1 , sur la constitution géologique de ce royaume; ouvrages auxquels il a joint, en 1819 et 1820 , un recueil d'observations sur la physique ef la géologie faites en parcourant une partie de la Norvége et celles des provinces du nord de la Suède qu'il n'avait pas visitées précédemment.

M. Nordenshiold a publié, en 1820 , un ouvrage du mème genre sur la Finlande.

Zoologie. - M. Tréviranus a donné, à Bremen, in-4\%, des Recherches, en allemand, sur la structure et les fonctions du cerveau dans les animaux de différentes classes.

M. Blumenbach a comparé la tète d'un ancien habitant de la Grande-Grèce, trouvée par le prince royal de biavière, dans un tombeau près de Naples, avec celle d'un individu de la nation, encore anthropophage, des Botocudos du Brésil ; comparaison qui fait voir, d'une part une analogie parfaite avec les profils des statues grecques que quelques auleurs ont regardé comme un genre de beauté purement idéal et de convention, sans modèle effectif dans la nature, et de lantre au contraire une forme de têtc encorc plus semblable à celle de l'ourang-outang que ne l'est celle des races nègres les moins civilisées. A cette occasion M. Dalman recommande à ses compatriotes les Suédois de comparer soigneusement les têtes des squelettes qui se trouvent dans les plas anciennes sépultures de la Suede, et qui doivent appartenir à des hommes de la race Mongole, avec les tètes des Scandinaves modernes, issus d'une nation venue de l'Asic occidentale. Il rapporte, d'après la Funne scandinave de Nilsson, que dans la province la plus míridionale de la Suède, en Scanic, on a trouvé, dans un marais a tourbe, a cote de bois ou cornes de renmes, une tite qui partult ètre celle d'un Lapon; ce qui semble pronver qu'a une ipocine reculie les Lapons savancaient arec 


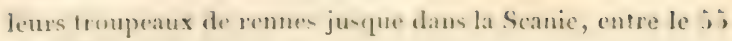
et le $56 \mathrm{e}$ degré de latitude.

M. I.inngren, de Lund, a donné en latin une these sur les extrémites anterieures de la taupe, comparies au boras de l'homme.

D)ans lat Mestologrie (c'estainsi qu'est désigntere icilhintoire naturelle des mammifiese), on cite un travail de M. I.chtenstein, inséré dans les Mémoires de l'teudemie de berlin, sur quatre especes du genre Lonehere, de l'ordre des rongeurs, tous de l'Amérique Méridionale.

I. professent Thunberg a décrit, dans les Mimoires de Stor hholm, une espece de Ifyène, qu'il nomme brumnea, qui pourrait être la mème que la littoralis et l'unirolor. Le mème a donné et figuré, dans les Mémoires de Pétersbourg, l'Lrius Urasiliensix; et le prince Max. de Neuwied, dans l'Isis de $\mathbf{8} 80$, Ie Cavia rupestris, aussi du Brésil.

On doit a M. Hellw irg une nouvelle édition de l'exeellent onvrage de Hilliger, intitulé : Prodromus systematis animalium et avium.

M. Nilsson a donné à Lund, en 1820 , un ourrage sur les mammiféres de la Scandinavie, ou il en décrit - f especes, et qu'il a enrichi d'observations sur les principales diffirences anatomiques propres à chaque espece. Il n'a pas négligé de mentionner les ossemens qui se rencontrent dans les marais a tourbe de la Scanie, et dont les uns appartiennent à des animaux de contrées plus méridionales, tels que le sanglier, le bison, l'urus, l'ours, etc:; tandis que les autres, au contraire, tels que les élans, les renne;, les castors n'habitent plus que dans le nord de la Suède.

MI. Pander et d'Alton ont publié, en allemand, un trèsbel ouvrage accompagné de 7 grandes planches sur le Megatherium de M. Cuvier (Bradyms giganteus), dont le squelette se trouve au cabinet de Madrid.

Orniehologie.- Q Quant aux viseaux de Suede, M. Nilsson, qui s'itid déja exercé sar celte matière, dans les Mimoires de I'Acadimie de. Stocklolm pour les annés 1816 et 1817 , particulierement en ce qui concerne la synonymie, a fait paraitre en $x 820$ et 1821 , a Copenhaicnue, les deux parties de son ornithologire suerica, on il dícrit afjo especes d'oiseaux seronnus juscu'ici pour habiter la Suede. Cet onvage se distinguge 


\section{des sciences.}

de la plupart de cenx qui l'ont précédi, par des ditails sur la manière de vivre des oiseaux, la composition de leur nid, le nombre et l'aspect de leurs oufs, leur ramage, leurs migrations, etc.

Iththyologie. - M. Quensel a donné, dans le tome 27 des Mémoires de l'Académie, un Essai sur la détermination des espèces de Pleuronectes des mers de Suède; et M.Holberg, dans le tome III des Mémoires de la Société des sciences de Gothenbourg, un Traité sur les poissons du Bohusleen, où il joint a leur description intérieure des détails anatomiques, et ce qu'on a pu savoir de leur manière de vivre.

M. Retzius a fait imprimer, a Lund, une thèse de sa composition, sur l'Anatomie des squales et des raies.

M. Oken a confirmé, d'après l'inspection de la pièce originale, l'opinion que M. Cuvier avait conçe, d'après la seule fifrure de Sonnini, touchant l'animal fossile des schistes marneux de Sehlenhof, savoir que cet animal aurait été un amphibie volant ; il le reconnait pour une espèce de lézard vossine du Crocodile et du Caméléon. Par ses soins et ceux de MI. Soemmering, on en connait déjà deux espèces fossiles, désignées par les noms de Pterodactylus longirostris et bresirostris, outre des fragmens d'une espèce beaucoup plus grande, déposés au Muséum de Carlsruhe.

En creusant le canal de Gotha, on Ostrogothic, on a trouvé, 15 pieds au-dessous de la surface du sol, quelcques carapaces de tortue.

Entomologie. - Cette branche de la Zoologie est bien cultivée en Suède, mais seulement quant à la partie ilescriptive et systématique; peu de personnes ont suivi les traces du célèbre de Geer, en étudiant les métamorphoses et les mœurs des insectes.

MIM. Quensel et Thunberg ont déterminć, soit dans les Mémoires de Pacadémie de Stockholm, soit dins des dissertations académiçues, les insertes rapportés de la Laponie.

On doit a M. Schionherr un ouvrage important ćcrit en allemand, mais publié en Suede, sur lat synonymie des insectes, formant 3 vol, in- $8^{\text {n }}$.

On cite aussi, comme publices on 1820 , des Observations académiques du professeur Fallen, et quelques Mémoires parıni ceux de l'académie de Stockholn.

Tom $\mathrm{I}$. 
NI. Schweigger, ayant esamine tris-attentivement les insectes renfermés dans les morceaux d'anbre jaune ou snecin fes còtes de Prusse, et qu'on jugerait, au premier asponet, atre les mémes que les insectes acturls de ce pays, a recomnu que ces insectes appartiement souvent en effet aux memes genres, mais non pas aux especes actuellement vivantes. Parmi In petit nowbre dinsectes cites et figurés dans l'ouvrage de cet auteur, on remarque particulièrement une espece inconme de scorpion, et une arainnéc qui differe de toutes les expeces actuellement vivantes en ce qu'elle n’a point la téte d'une senle piece avec le corselet. II. Germar, professeur a Iralle, a donné un travail semblable dans un journal d'Entomolncie, oir il a cherché à déterminer quelques espéces d'insectes du succin, dont on ne connait pas les analogues vivans.

Iat dernière édition de l'Introduction à l'Entomoloşite de MT. Kirby et Spence, fournit un fait que nous croyons diroir ciler, a canse de sa singulariti, guoique nous nons abstenions cu géneral de parler des ouvrages anglais, presque tou bien conus de nos savans. Il s'agit d'un insecte "Amérique, nommé par Fabricius Redurius scralus, 'qui, suirant une observation du géneral Davies, serait doué de la proprité de donner une forte commotion électrique.

On inouve, dans l'ouvrage périodique allemand, intitulé Isis, des recherches de Mr. Nees de Fssenbeck sur les parties de la bouche des insectes, et d'autres sur les plantes desderniers rangrs, telles que les Conferves, qui se clangent, dit-on, en animalcuies, at réciproquement. Ce dernier sujet a fourni aussi ln matiere d'une these de Vetamoryhosi algarum, soutenue à Lund, en Sneste, sous lit présidener de M. $\Lambda$ gardl.

l.e singulier petit coléptére nommé Clasiger, qui, suirant tes obsvation de M. Muller, est nourri avec grand soin par les fourmis, lesquelles profitent d'une liqueur sucrce qui sort d'une pelite touffe de poil plarie sur son dos, a été o.)servé en Suede comme en Allemagne, et dans les mèmes rirconstances.

Suivant M. Goling, la plus grande durée de la vie de la mere abeille n'e veèle pas cinq années. ( 1 sis, $1820,(6$, p. 5 go.)

I. IInller a $\mathrm{eu}$ ocrasion d'étudier avec soin un gruepier qui sit:ait forme dans une ruche vide. La mere guêpe étais desoue tre-familiere, of rlfe avait transmis cotte qualite a 


\section{des sciences.}

sa progriniture. (Magasin d'entomologie de Germar. T. III.)

iI. Wiedemann a décrit, dans son Magasin soologique, plusienrs nouveaux genres et quelques espèces exotiques de diptères.

Nous ne parlerons pas ici des ouvrages latins, tels que $A$ ug. Ahrensii Fauna insectorum Europa, espèce de continuation de la Fauna germanica de Panzer, et Dipterorumnovagenera.

L'Isis, ouvrage périodique allemand, de MII. Oken et Germar, contient un catalogue nombreux, quoique encore incomplet, d'ouvrages entomologiques.

Helminthologie. - L'Helminthologie ne parait pas avoir flé cultivée en Suède avec autant de zèle que les autres branches de l'histoire naturelle. Cependant M. Nillson a donné a Lund, en 1817 , un dissertation académique, intitulée: Collectanea Zoologice Scandinavica, où il décrit quelques espèces d'oursins et d'étoiles de mer; il a fait connaitre aussi dans les Mrémoires de l'académie de Stockholm, pour 3818, trois espèces de Pedicellaria, petit animal analogue aux polypes, qui habite comme parasite entre les pointes des oursins.

On trouve aussi dans la Zoolngie suédoise la description et la figure d'une nouvelle et belle espèce de Méduse (Medusa Palmstruchi) des parages du Bohus-læn.

Nous citerons encore ur travail de Mr. Lichtenstein sur les Séches, où il propose de faire un genre particulier, sous le nom de Onychotheutis, de celles dont les tentacnles sont armées d'espèces de crochets durs.

M. Otto a décrit, dans les Acta Acad. nat. curios. X. r 120 , une belle et nouvelle espèce de Diphyllidica, de la Méditerranée, qu'il désigne par l'épithète de lineata : on ne connaissiit précédemment que deux espèces de ce genre, toutes deur de l'Océan indien. Il a également disséqué, décrit et figuré deux nouvelles espèces de vers aquatiques des parages de Naples, savoir, le Sternaspis thalassemoides et le Syphonostoma diplochaitum.

M. $\boldsymbol{\Lambda}$ gardh a publié des Observations sur l'enimalcule infusoire nommé Vorticella convallaria, analogue à celles que Roesel avait consignées, long-temps auparavant, dans ses $A m r^{-}$ nités entomologiques (Insekten Belustigungen, III, 9). MI. A gardh soupronne que cet animal attire a lui des animal- 
cules encore plus petits, dont il fait sas proie, au moren dïnc espece de fiscination analugne a celle quon a attribuie a qual. ques serpens.

M. Jule Leo, de Berlin, a confirmi, par de nouvelles ohservations, ce que Swammerdam avait deja dit an sujel des vers de terre, savoir 'ju'ils se multiplient par des cenfs qu'on trouve au printemps, et qui laissent apercevoir non-seulement le petit ver qui doit en sortir, mais meme la circulation de son sang. Cés observations ont été confirmép, Isis, 1820, 4 , page 386 j par M. Rudolphi, suivant lequel ce que quelques naturaliste's modernes ont trouvé dans le corps des virs de terre, et qu'ils ont pris pour les petits vivans de ces vers, n'est antre chose qu'un ver intestinal dit improprenent dicaris lumbrici, qu'il rapporte au genre fibrio, et quil a trouvé non-seulement dans les vers de terre mèmes, mais aussi dans leurs œufs.

Enfin l'on doit à MI. de Schreiber, de Vienne, l'enumération des richesses que sept naturalistes revenus du Brésil ont rapportées au cabinet de l'empereur d'Autriche. Quclques collections et même les Musées de Suède seront aussi enrichies par les soins d'un autre royageur allemand, M. Freyreis.

Nous bornons ici cet extrait, nous réservant de rendre compte de ce qui se rapporte a la Botanique lorsque nous aurons reçu le compte rendu qui doit paraitre cette annés, et dans lequel les auteurs promettent de compléter ce quils ont dit relativement a cette science dans l'ouvrage que nous avons sous les yeux, et de traiter en méne temps de lit géographie des plantes, de leur anatomie et de leur physiologie, ainsi que des régétaux fossiles. 
J OUR NAUX.

:50. Juurnaux fiancais quiont par udans les 25 prentiers jours de Janvier.

Les uuméros ci-dessous cutre parenthéses répondent à ceux de la liste des journaux de France

Journal de physique, août I 822 . (I)

Gazette de santé, $\pi^{05}$ I, 2, 3, de janvier 1823 . (2)

Journal universel des sciences médicales, etc., décembre 1822. (3)

Journal de pharmacie, etc., décembre 1822 . (4)

Annales ouropéennes, etc, octobre 1822 . (5)

Annales de l'agriculture française, etc, décembre 1822. (6,

Cours d'agriculture pratique, décembre 1822 . (7)

Annales maritimes, etc., décembre $\mathbf{1}_{222}$. (8)

Journal des voyages, etc., décembre $1822 .(9)$

Journal des savans, etc., décembre $1822 .(29)$

Revue encyclopédique, décembre 1822 . (30)

The Paris monthly Review, décembre 1822 . (33)

Galignani’s liter. gaz., décembre 1822 . (34)

Galignani’s monthly Review, décembre 1822. (35)

Annales de l'industrie nationale et étrangère, décembre $182 ? \cdot(38)$

Bibliographie de la France, $\mathbf{n}^{0:}$ I à 4 , de janvier 1823 . (40)

Journal général de la littérature étrangère, novembre 1822. (41)

Bulletin des séances de la Société philomathique, novem. lire 1822 . (14)

Bulletin de la Société d'encouragement, no. 219. (15)

Journal asiatique, janvier 1823 . ( 15 bis)

Annales de mathematiques, octobre et novembre 1822. (16)

Bulletin de la Société d'agriculture de l'Herault, norembie $1822 .(17)$

Bulletin de la Société d'agriculture de l'Eure, janvier 1823. (18)

Bulletiu des sciences médicales del'Lure, janvier $18 \% 3 .(9)$

L'Observatur des sciences médicales (Marseille), octohre et novembre 1822 . (20)

Le Jycée armoricain (Nantes), janvies 1823.91 . 


\section{TROISIEME SECTION.}

\section{TRAVAUX DES SOCIÉTÉS SAVANTES.}

\section{SËA KCES.}

251.Pan1s, Société philomathique.-Séance du 7 decembre I822. - M. Clément communique a la société des résultats d'expériences qu'il a faites avec M. Parkins sur la compressibilité des liquides, en soumettant l'eau à une pression de I 20 atmosphères, et danslesquelles il a obtenu une diminution de volume de 6 pour 100.

Sćance du xí décembre. - M. Laugier lit un mémeire sur l'analyse de deux variétés de cobalt arséniaté, provenant d'Allemont et du duché de Wurtemberg.

Séance du 11 janvier 1823. - M. de Blainville communique verbalement quelques détitils sur le crocodile et le's serpens que l'on montre en ce moment à Paris. Le crocoilile est de l'espéce du Nil et a six pieds et demi de longueur. Les serpens sont des Pithons et non des Boas, comme on l'at annoncé; ils appartiennent à deux espèces différentes, très-remarquables par la beauté de leurs couleurs. Les deux plus grands ont treize et seize pieds de long.

252. PARrs.-Société d' II istoire naturelle. Siance die 8 novembre $\mathbf{8 2 . 2 . - \Lambda}$ M. Richard présente un travail sur le genre Ophiorhiza de Linné; duquel il résulte que les deux espaces de ce genre connues sous les, noms d'Ophiorhiza Munghos, et d'Ophiorhiza Vutreola, constituent non - seulement deux genres nouveaux, mais que ces deux genres doivent ètre plikcés dans des familles distinctes: le premier qui conserve le nom d'Ophiorhyse, et qui comprend la première espece, doit étre rangé dans la famille des rubiacées; et le second, nommé Notreola, et qui renferme la dernicre espece, appartient à la famille des gentianées. - On lit un mémoire de M. Le Sauvase, correspondant de la Société à Caen, sur un noureats 


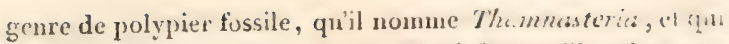
a été trouvé aux environs de Caen et à berneville, département du Calvados.

Sćance du 22 novembre. - M. Desmoulins communique verbalement une observation qu'il a eu l'occasion de faire sur la conformation de lit rétine et du nerf optique du vautour, qui sont plissés sur cux-mêmes, ainsi que cela existe dans quelques poissons disséqués également parr M. Desmoulins. - Le même membre fait part de quelques remarques qui lui sont propres, sur les hémisphères cérébraux dans len jeunes animaux, comparésaux mêmes parties dans les adultes. - M. Deshayes lit un mémoire géologique sur les cornilles fossiles de Valmondois (département de l'Oise), et principalement sur les coquilles perforantes découvertes au mème lieu, dans le grès marin inférieur.

Séance du 6 décembre. - M. Desmarest lit un mémoire sur un nouveau mammifère de l'ordre des roncreurs, internédiaire aux rats et aux marmottes. - Cet animal, appelé Ltia, dans l'ile de Cuba, d'où il est originaire, recoit de M. Desmarest la dénomination générique de Capromys. On lit, pour M. Bourdest, correspondant de la Société, une notice sur un gisement de baryte sulfatée dans les montagnes de Weissenstein, près Soleure, en Suisse. - M. Deshayes lit une note sur le genre de mollusques, appelé Gastrochène, et qu'il pense devoir être réuni à celui des fistulanes.

Siance du zo décembre. - M. Choisy, correspondant de la Société, lit un mémoire sur un genre de gruttiféres, appelés Micranthera, et sur l'arrangement des genres de cette famille, qu'il partage en quatre sections sous les noms de Clusiées, de Garciniées, de Calophyllées et de Moronobées. Son genre Micranthera appartient à la section des garcinićes. M. Bertrand-Geslin lit une note sur une mine de plomb sulfuré argentifére de Cogolin, département du Var. M. Brongniart fils lit une note sur une variété de structure dans le maïs. - M. Breschet communique verbalement une observation de M. Andrul fils, sur des vers acéphalogristes trouvés en abondance dans les veines pulmonaires et dans le tissu propre du poumon et du foic.

Séance du 3 janvier 1823 . - II. Underwood, correspon- 
dint, communique une leflre de M. liuekland, sur la dicouverte fate dans les monts Hymalaia, a 16,000 pieds de hauteur (c'est-a-dire dans la rigion des neiges), d'ossemens fossiles de chevaux renfermes dans un sable calcaire.

\section{Tiravuludes Sociètés savantesdes départemens, pendant l'année 1822 .}

Ce n'est joint un compte rendu des traviux de chaque séance de ces Socićtés que nous allons présenter, mais l'exposé de ce qu'elles ont publié en outre de leurs mémonres pendant 1822 ; nous insererons avec soin, dans notre bulletin, l'extrait des procès-verbaux de leurs séances, lursque ce's Sociétés nous les feront parvenir, ayaut élé invitées à les adresser, franc de port, au bureau du Bulletin. (Voyez, pour les meimoires de ce's Sociezis, la liste des journatix des départemens:)

Arx. Séaxce pumieve de la Société des amis des sciencés, des lettres, de l'agriculture et des arts, tenue a Aix le 15 juin 1822. in- $8^{\prime \prime}$. Prix : 3 fr. A Aix, chez Ponthier.

Axrmiac. Drscoers lu en séance publique de la Société d'agriculture, arts et commerce d'A urillac, le 8 juillet 1822 , sur le développement successif de ces trois branches de l'industrie humaine dans le département du Cantal, depuis les temps les plus reculés jusqu’a la fin du xiI ${ }^{\circ}$ siecle de notre ére. Par C. F. Ravlnac, membre de cette Société. In-S". de 4 feuilles. Imprimerie de Picot, à Aurillac.

Borneuzx. Academe royari des sciences, belles-lettres et arts de Bordeaux (sciance publique du 27 aout $1 \delta_{21}$ ). In-8. de dix fenilles et demie; plus, un tableau. Imprimerie de Brossier, à Bordeaux.

Notice des travaux de la Société royale de médecine de Bordeaux, depuis sa dernière séance publįue jusqu'au 28 aoùt $1 \$ 22$; par M. Dupeca-Laponte, scerétaire général. In-8. de 3 feuilles. A Bordeaux, chez Iawalle.

Bornc. Exposi hictorique et statistique des travaux de la Sociétí départementale d'émulation et d'agriculture de l'Ain.

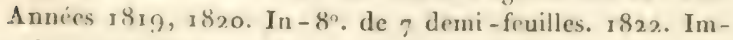
primerie de Bottier, à Bourg.

Cir.y. Phogr ame des prix proposís par la Socicti royale 
d'agriculture et de commerce de Caten. An 1822. In-8". de 2 feuilles. Imprimerie de Poisson, à Caen.

Dison. Académie des Sciencis, arts et belles-lettres de Dijon. Séance publique du 2'ł août I 82 I. In-S". de 22 feuil. Juillet 1822 .

Étampes. Stancer publeue de la Socićté dingriculture de l'arrondissement d'Etampes, tenue le 6 octobre $1822 . I_{1 \mathrm{i}}-8 \mathrm{*}$. de 6 feuilles. A Paris, ehez Mme. Huzard.

Limoges. Société royalr n'afiricultune, des sciences ot des arts du département de la Haute-Vienne. Séance publiq̧ue du 26 août 1822 . In $-8^{\circ}$. de 5 feuilles. Imprimerie de Dalesmes, a Limoges.

Lxon. Compte rendu des travaux de la Société royale d'atgriculture, histoire naturelle et arts utiles de Lyon, depuis le premier mars 182 I jusqu'au premier avril 1822 ; par M. L. F. Grocmier, professcur à l'école d'économie rurale et vétérinaire de Lyon, etc., secrétaire de la Société. In-8 de 21 feuilles. Imprimerie de Barret, à Lyon.

Macon. Conpte rendu le 6 décembre $8_{21}$ des travaux de la société des sciences, arts et belles-lettres de Màcon, pendant l'année i82i; par M. Cifarles de Latoughe, secrétaire perpétuel.

Marseille. Rèclement de la Société royale de médecine de Marseille. In- $8^{\circ}$. de I feuille (juillet 1822 ). De l'imprimerie d'Achard, à Marseille.

Séance publique et Exposé des travaux de la Sociélé royale de médecine de Marseille pendant l'année 1822 . In-80 de 4 feuilles 3/4. Imprimerie d'Achard, à Marseille.

Provins. Séance publique de lia Société libre d'agriculture, sciences et arts de Provins, tenue le 18 décembre 1822. In- $\mathrm{n}_{\mathrm{n}}$. de 4 feuilles. Imprimerie de Lebeau, à Provins.

Rouen. Rìclement de la Sociélé de médecine de Ronen. In-1 2 de $3 / 4$ de feuille. Imprimerie d'Émile Périaux, à Rouen.

Préeis analytique des travaux de l'Académie royale des sciences, belles-lettres et arts de Rouen, pendant l'année. $182 x$. In-8 8 de 12 fenilles. $x$ 822. Imprimerie de Périaux pere, ì Rouen.

Séance publique de la Société centrale d'agriculture du département de la Seine-Infericure, tenue le is mai $182 \%$ In-8. de / feuilles. Imprimerie de Périaux père. 
Toulouse. Séarce pubroce de la Société royale de medecine, chirurgic et pharmacie de Toulouse, tenue le $;$ mai 1822. In-8\% de 4 feuilles et demie. Imprimerie de Ibouladoure, à Toulouse.

954. Suciéte caxtoxyal ne Largaxie. Deux Mlimoires très-interessans ont été lus dernièrement à cette société; le premier, qui est dù à M. Zinck, est relatif à la gruerison de lu tumeur blanche par l'iorle; et le deuxième, dont l'auteur est M. Chavannes, a pour objet l'anatomie du Cormoran, oisean dans lequel on trouve un os particulier, dont M. Chavannes explique l'usage. (Reruc encyclop. déc. 1822. p. 632.) I.

\section{PRIX PROPOSÉS.}

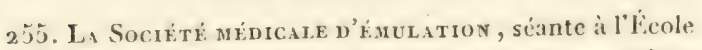
de medecine de Paris, propose plusieurs prix pour l'année 1823 ; savoir:

Deux, un premier et un second, qui seront décernés aux auteurs des deux meilleurs mémoires sur l'anatomie, lat physiologie et l'anatomie pathologique.

Deux autres prix, ésalement un prenier et un second, seront aussi décernés aux auteurs des deux meilleurs mémoires sur la pathologie médicale ou chirurgiciale, soit particulière, soit générale.

Les sujets sont au choix des auteurs.

Les deux premiers prix seront chacun une médaille en or de la valeur de 200 francs, et les seconds une médaille en or de la valeur de 100 francs.

En outre, un prix de la valeur de 200 francs sera domic a l'auteur qui aura le mieux traité la question suivante:

* Déterminer le caractère propre de l'inflammation, et exposer la thérapeutique de cette affection considéréc dans les différens tissus, dans les différens modes dont elle est susceptible, et dans toutes les circonstances caprables d'influer sur le traitement."

Les mémoires, écrits très-lisiblement en français ou en latin, devront arriver chez M. L. R. Villermé, docteur en médecine, secrétaire général, francs de port, avant le 31 décembre 1823 . Ils seront distingués (Ies concurrens étant tenus de ne point se faire connaitre) par une epigraphe qui sera 


\section{Prix proposés.}

répétée daus un billet cacheté contenant les noms et l'adrense de l'auleur. Les membres correspondans de la société peuvent concourir.

256. PAR1s. - La socicité d'histoire neaturelle ayant recu d'un anonyme la somme de huit cents francs, destince a fonder deux prix égaux dontil a indiqué les sujets, et qui devront étre décernés, s'il y a lieu, en 1824 ; après avoir entendu le rapport de la commission qu'elle a chargée d'examiner cette proposition ainsi que le programme proposé par le donateur, a accepté cette donation aux conditions mises par le doinateur, et a admis le programme des prix tel qu'il l'a proposé.

Premier prix. "Il sera donné, en avril i 82 \%, une médaille "d'or de la valcur de quatre cents francs à l'auteur du meil"leur mémoire de gćologie organique sur une partic quel» conque de la France. $"$

On voit qu'il est nécessaire que ce soit un espace plus ou moins étendu, dont le terrain renferme des débris organiques, animaux ou végétaux; et qu'on devra faire connaitre non-seulement les roches et minéraux qui le composent, leur ordre de superposition, etc., mais encore donner la ditermination précise et comparée des débris organiques renfermés dans ses couches.

Second prix. "Une médaille d'or de la valeur de quatre n cents franes sera remise, en avril i 824 , à l'auteur du meil- leur mémoire sur le sujet suivant: Déterminer, par l'exame't - des corps organisés fossiles, et par tous les moyens chimi" ques, les diffirences des houilles ct des lignites, et celle's "des terrains houillers et des terrains de lignites, en faisant " connuitre avec précision les lieux d'oit parviendront les * substances analysées ou décrites."

La Société pense que les personnes qui voudraient diriger leurs recherches sur ce sujet pourraient le considérer de lis manière suivante :

Sous le point de vue chimique. - Prendre, dans des terrains bien géneralement et bien évidemment reconnus pour terrains de houille ancienne ( $\mathrm{I}$ ) (c'est-à-dire présentant la réunion des circonstances d'être de la houille inférieure an

(1) Tels qque les houilles de St. -Etienne, d'Anzin, de Neweastle, elc. 
gris bigaré, de la louille acompanne de feulles de fougeres sans coquilles, de lat houille grasse ou matigre, mais non al l'itat d'anthracite, etc. ), des échantillons nombreux, prownant de la masse mème des couches exploitées; et les ixaminer chimiquement pour en fare ressoria les caractieres esseutiels.

I'rentre, dans des terrains bien ginerilement reconnus pour ère des lignites (par l'existence d'une ou de plusieurs circonstances, telles que celles-ci : la position au-dessus du calcaire du Jura on de la craie; la présence de parties a structure lignease, accompasnant la masso; l'absence des fenilles de fomarese f la présence des feuilles d'arbres ou celle de quelyues coquilles, etc.), des échantillons nombreux, choisis surtud parmi ceux qui, par leur aspect exterieur, resscmblent le plus a la houlle. Les examiner chimiquement pour en faire ressortir la composition et les caracteres, f:ar opposition avec cenx de la houille.

On pourrat examiner, dans le mème but, des échantillons de louille ou de lignite muins hien caractérisés; mais, si lon ce livre a ce travail (ce qui n'est point de rigueur), il faudra wigneusement distinguer ces analyses des jrécédentes.

Sous le point de vue des corps organisés fossiles.-Choisir, atutant qu'il sera possible, les mènes mines, soit de houille, suit de lignite, qui auront fourni lés échıntillons examinés chimiquement, pour donner une énumération raisonnée, avec des rapprochemens anx corps organisés actuellement vivans :

I". Des genres de vingitaux et de leurs principales especes, observés dans l'ensemble de ces mines choisies dius ehaeun de ces terrains, pour en conclure quels seme les genres el les appices particulieres a chacun d'eux, et ceux qui leur sont communs.

2". I) (s coquilles et autres débris animaux, considerén sous les mêmes points de vue.

On pourra se contenter de nommer, avec citation critique? de lir description et de la figure, les especes végetales el animale's deja observées par les naturatistes; mais on destat Jitre connaitre, par des descriptions el des dessins, celles guion jugera caracteristiques, et qui naturunt fats encote eti figurées. 
On voit que cette question est double, et quil est possible que la méme personne ne puisse pas en résomble lis deux parties. Dans le cas ou il n'y aurait qu'une personne en nom, le prix total serait adjugé a cette personne. Dans le cas où un mémoire, renfermant les deux solutions, serait en nom collectif, le prix total serait adjugé anx auteurs du mémoire, si la Société trouvait que les doux questions aient été également bien résolues. S'il n'y en avait. qu'une qui fìt satisfaisante, on n'adjugerait que la moitic du prix aux auteurs.

Si l'une des deux questions seulement éfait traitée, mais qu'elle le füt convenablement et complétement, la Sociétéadjugerait la moitié du prix à l'auteur de ce mémoire, et l'autre moitié appartiendrait à l'auteur qui aurat également bien résolu la stconde question.

En présentant cette question complexe, le fondateur du prix et la Société ve se dissimulent pas qu'ils offrent une apparence de travail considérable; cependant on doit romarquer que la partie chimique n'a pas l'étendue de délails qu'elle semble présenter, et qu'il n'est pas nécessaire de domner une analyse complete d'un grand nombre d'échantillons de houille et de lignite; mais qu'on doit trouver, s'il est possible, les caractires chimiques distinctifs de ces deux combustibles fossiles, ou prouver qu'il n'y en a aucun qui soit général.

La deuxième partie exige beaucoup plus de travaux de détails : aussi la Société pense-t-elle que, si l'on entreprend seulement de la résoudre, il sera convenable d'étendre, autant qu'on le pourra, ce qui est relatif à la géologie, en faisant connaitre si les débris organiques, renfermés dans les terrains de houille et de lignite, indiquent pour chacun d'eux des époques de formation différentes.

Conditions grencirales. - Les membres honoraires de la Société d'histoire naturelle de Paris, parmi lesquels seront choisis les juges du concours, sont seuls exclus d'y prendre part.

Les mémoires, portant une épigraple ou devise qui sira rípétée avec les noms, prénoms, qualités et demenres de l'autcur où des auteurs, dans un billet cacheté joint au matnuscrit écrit lisiblement en français on en latin, seront adres- 
ses au secrétaire de la Societi, rue d' Injou-D.uphine, avant le y er janvier 182 f.

257. Arras (Pas-ale-Calais). - Lab Societi rovale pour tencouragement des sciences, des lettres ce des arts propose pour prix a diecrner en $x 8 \% 3$ les sujets suirans:

$\mathbf{x}^{\circ}$. Agrionlure. Déterminer l'état actuel de lagriculture dans le departement du Pas-de-Calais, et indiquer les amcliorations dont elle serait susceptible. Las société, indépendamment des autres détails inhérens à la question, désirerait connaitre quelle est la proportion des cultures, et si chacune est relative aux besoins? (Quels sont les divers modes d'assolemens? Quel est l'état des prairies naturelles et artificielles, et si elles suffisent aux besoins? (Quelle est la pratique des engrais, et quel motif a pu faire chercher chez l'etranger dés engrais pour le sol du département, tandis que les engrais du département sont exportés pour les mémes pays d'où l'on tire cenx qu'on utilise? Quel est l'état des troupeaux, et quel est l'état des races? Quels sont les procídés aclucls de culture, et quel est l'état des cultures particulieres, telles que celles du houblon, de la garance, etc. ? Si la vaine páture est nuisible à l'économie rurale dans l'etat actuel de nos cultures?' Chacune des subdivisions de la question devra être accompagnée du tableau indicatif des améliorations qu'elles comportent.

2". Commerce. Donner l'historique des anciennes manufactures d'Arras, et rechercher, d'après l'état actuel des conmaissances physiques et industrielles, quelles seraient celles qui pourraient y être établies avee le plus de probabilités de succès.

3". Économie rurale. La société délirrera, dans la séance annuclle du mois d'aout 1824 , une prime de la somme de 300 franes à la personne qui, dici au 30 septembre 1823 , aura construit, dans litendue de l'arrondissement d'Arras. un toit ou rine portion de toit d'une etendue de, au moins, soixante mères carrés, suivant la méthode dite ignifuge de MI. Lagavrian, laquelle est décrite dans la troisième livraison du tome IIle. de ses Mémoires.

Les ouvrages envoyés au concours, pour 1823 , devront itre adressies au secrétaire perpétuel de la societe royale d'Arras, et etre parvenus avant le 15 juillet 1823 . (Rerue encyclop. Dicembre $\times 822$, pagc 64 .) 


\section{Nominations et Éloges.}

258. Macon. - La société des sciences, arts et belleslettres vient de publier le programme du prix qu'elle a proposé dans sa séance solennelle : " $x^{\circ}$. Quels seraient les résul* tats de l'indépendance de l'Amérique, relativement à la „richesse commerciale et monétaire de l'Europe?"

Les ouvrages devront ètre parvenus, francs de port, au secrétaire perpéiuel, avant la fin de juillet 1823 . Le prix sera une médaille d'or de la valeur de $300 \mathrm{fr}$.

$2^{\circ}$. La même société décernera une médaille d'or de la valeur de 600 fr. aux meilleurs ouvrages sur l'Histoire naturelle et physique du département de Saóne-et-Loire.

Le concours sera fermé le $\boldsymbol{I}^{\boldsymbol{e}_{\mathrm{r}}}$ janvier I 823 . Les menbres résidens sont exclus de ce concours.

Nota. Les exemplaires des ouvrages imprimés on manuscrits, cnvoyés à la société, lui appartiennent de droit.

NOMINATIONS ET ELOGES.

$25 \mathrm{~g}$. M. le docteur Lassis, auteur d'un ouvrage estimé sur les Causes des Épidémies, traduit à Barcelone et à Madrid, que l'on sait s'être rendu en Espagne pour faire des recherches sur la maladie qui a ravagé ce pays l'année dernière, vient de recevoir le diplôme d'associé intine de l'Académie de médecine de Barcelone.

(Journal des Débats du I I décembre I822.) F. 260. Éloge de Xavier Bichat, suivide notes historiques et critiques. Discours qui a remporté le prix proposé par la Société d'émulation d'agriculture, belles-lettres et arts du département de l'Ain, pour. l'année ${ }_{1} 822$. In-8. de 4 feuilles. Prix, 2 fr.

Éloge de Panmentier, discours qui a remporté lo. prix proposé par l'académie d'Amiens, pour l'année 18r9. In-8. de 6 feuilles. Prix, 2 fr.

Par Ant. Mrever, doct. en méd., membre de plusieurs sociétés savantes. A Paris, au bureau de la Gazette de Santé, rue Bergère, n. 19.

Ces denx éloges réunis se vendent ensemble $3 \mathrm{f}$.

On se tromperait si l'on considérait ces deux éloges comme n'ayant un intérèt direct qu'avec les deux savans illustres 
dont ils cribhent la vie et les travanx. Conduit, dit M. Miqquel en parlant de Bichat par la suite naturelle de ce discours, au milien de la grénération prósente, forcé de rappelericiles noms des contemporains dui ont continue les travaux de bichat, je vais errouper antour de lui tout ee qui appartient à son feole, et sisnaler la tendance générale qu'il a impriméc aux diverses branches de l'art de suérir. Fn effet, l'éloge de bichat sich, a proprement parler, que le tibleau fudele de la medecine frameatise depuis le commencement de ce siecle jus$\mathrm{r}_{\mathrm{i}}$ ü it nos jours. Des notes pleines d'interit, de concisinn et de clarte, jettent un grand jour sur me quantité d'opinions controversées en médecine et dans la science plı̣siologique. M. Niquel peint Bichat à grands traits; il analyse rapidement les travaux anatomiques et physiologiques de ee jeune professcur, el montre l'influence queses travaux curent et ont encore sur les progres de la science médieale. Le style de 31. Miquel est digne de la renommée de lichat et du brillant. colat qu'il a fait rejaillir sur les sciences anatomiques et pliysiologiques. Il est plein d'éloquence, de force et de mouvemens oratoires.

L'eloge de íarmentier, dont le nom appartient à la fois a l'agriculture, à la médecine, à la pharnacie, est traité avec le méme talent. La part de gloire de ces deux hommes célebres est faite avec discernement et équuité. Ils paraissent sous lit flume du panégryiste également grands. Il raconte aver art les travaux d'un homme occupé continuellement de faire le bien des hommes on de le préparer. Il le montre dans les diverses fonctions ou il fut élevé, usant de son influenee pour faire adojter les vues utiles. M. Miquel rénssit parfaifement non-seulement à montrer lous les litres que Parmenlier possede a la reconnaissance de nos contemporains et de la postérité, mais encore à populariser davantage sa mimoire par le labieau de tout le bien qua fait ou preparé ce licenfaiteur de Thumanite; et cel doure, comme celui de Bichat, est aussi un tablean plein de vie des sciences que cultivait Parmentier.

F.

${ }_{2}$ frr. L'Áloge historique de Broussonnet, premier fondateur de la societé Iiméenne, a éti prononcé dans la séance publique de celle societe, le 28 dicembre $1 \$ 22$, par M. Thiebaut de Berneaud, secretaire perpétuel de celte socití. 


\title{
QUATRILME SECTION.
}

\author{
ANNONCES DIVERSES.
}

\section{ENTREPIISES SCIENTIFIQUES.}

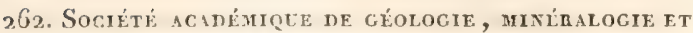
BOTANQQE D'AUVERGNe. - Quelques savans et amateurs viennent de former sous ce nom, dans la ville de Clermont (département du Puy-dé-Dòne), un établissement qui peut devenir d'un liaut intérèt pour les géologues de tous les pays. Cette Sociétí se compose de vingrt membres résirłans en $\mathbf{A} u-$ ver rne, dix membres honoritres, d'un nombre indéterminé d'associés étrangers, et de correspondans. Son but est, ${ }^{\circ}$. de former un Iusce public renfermant des collections aussi complètes que possible de la géologie, de la minéralogic et de la Vlore d'Auvergne, et subsidiairement aussi de toutes les autres branches de l'histoire naturelle de ce prays;

20. De réunir toutes les notions qu'elle pourra se procurer sur la géologie et la stratification des montagnes d'Auvergne, spécialement par rapport aux vo'cans anciens et modernes qui remdent ce pays une terre classique pour leur ćtude.

3n. Elle se propose de fíne mieux connaître cette intéressante partie de la France, d'encoarager et d'étentre les conmaissances d'histoire naturelle parmi les habitans, et de fournir aux savans etrangers les moyens de lit parcourir avec plus d'utilité pour les sciences.

La Société a éln pour président MI. le colonel comte de Iaizer, militaire listingné, et ardent amateur des sciences, anx soins duquel cet établissement est principalement dù. Deja Ia ville de Clernont a mis a sa disposition un trés-benu local dans le bitiment de la bibliothéque de la ville, et elle s'est. chargée des frais nécessures a la confection des armoires, vitrages, etc. Dója le savant ablé Iacoste, directeur du musée de la Société, a tanspurti: dans ce local la totalité de: si collection, à lacpuclle messieurs les mombres s'empressent de joindre leur contingent.

Тонк I. 


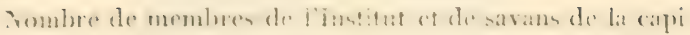
lale se sont empressés de s'associer anx utiles travaux de lit Société naissante.

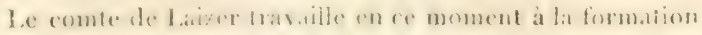

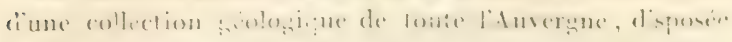

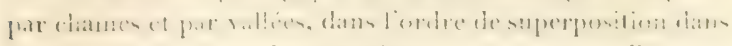
lequel charpue formation se présente; et, comme il est en mème temps président de la Commission pour lat recherclie des antiquites et la conservation des mommens bistoriques du departement, il s'occupe à former dans un

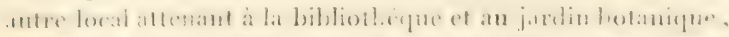

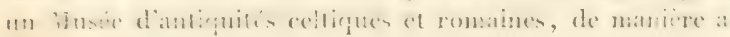
réunir en un seul point tout ce que cette importante pro-

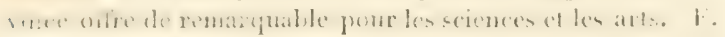

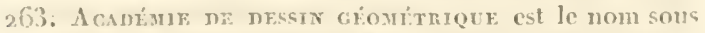

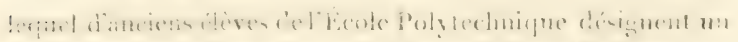

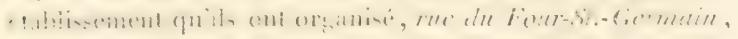
$n^{n} \cdot 37$, pres celle de Bussi, destiné, sons la direction de

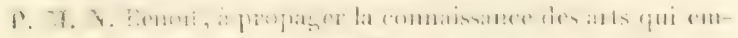

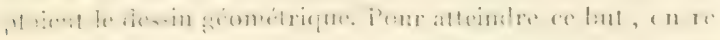
mil tous les jours, de neuf à quatre heures, dans un bean

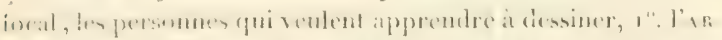

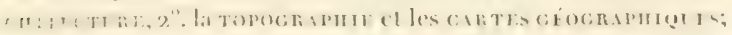

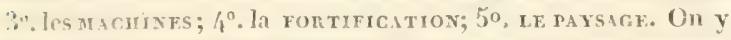

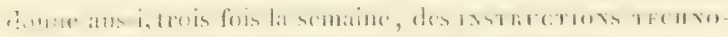

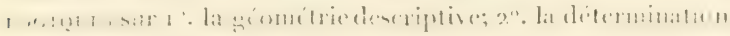
des comberes et la perspective; $3^{\circ}$. La coupe des pierres et lat clan-

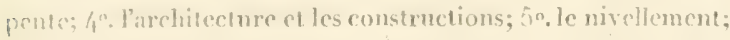
î. Ies levés topographicpues, larpentage et les opérations catiasirales; $7^{\circ}$. la géodósic et la projection des cartes géogratphiques; $8^{\circ}$. la composition et l'analyse des maclines; $9^{n}$. la rasirametalion of la fortification passigère; ro". la fortificalion permaneate, l'ittacque et la défense des planess. Fufin, fir antorisation dn conseil royal de l'Université de France, in y a onvert des covns de Matminteuse, qui ont iscaleuent lieu trois fois la semaine. Un choix considérable d'ouvrages technologicues anciens et modernes, me collection

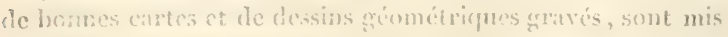
at la disposition des habieités de l'Aendemie, pour les gnider dans lenes recherches. Le prix de labonmement pour les 


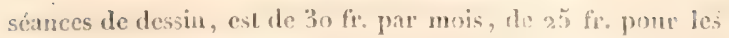

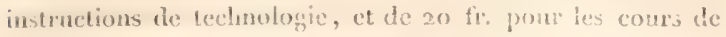
mathématiques.

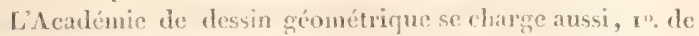
copier, graver et lithogaphier los dessins gúomćtriques de toute espèce; $2 "$. de lever les plans el cartes topographiciues,

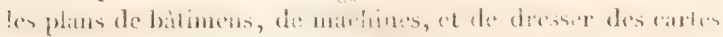

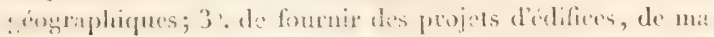
chincs, d'usines, de canaux, etc.; $4^{\circ}$. de rédiger tontes sorten de devis; 5\% de donner des reiseigneinens sir l'exécution, en petit el en grand, de tons le's objets et travaux d'art qui

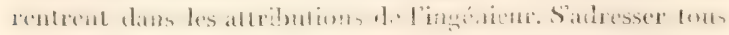

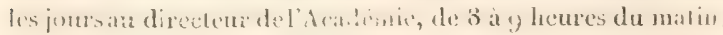
et le dimanche avant 2 heures.

L.

26\%. M. Maclere, si comu par ses voyages ef ses travaux

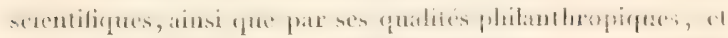

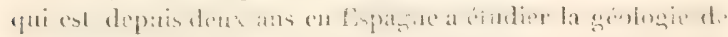
ce royaume, où it s'est rendu en quiltant l'ltalie, vient d'atcheter une étendue de cinc mille anglais de terrain, swe le

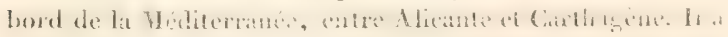
le projet d'y établir une école d’agriculture et d'y fomlex

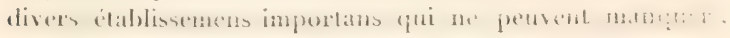
sous sa direction, d'avoir une utile influenec sur la prosperité intérieure future de l'Espagne.

li.

265. Foyages de 31. F. Cailliand it Ateroë, an fleuse Blanc et dans les Oasis. - On s'occupe avee activite de lat

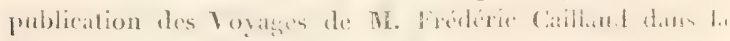

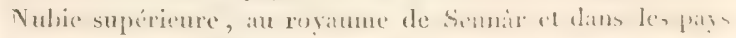

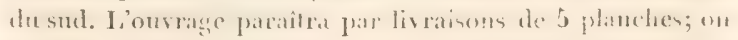
espère ponvoir en donner une ou deux par moss : la première paraitra le $I^{\mathrm{er}}$. unars prochain. $\mathrm{I}_{\text {ail }}$ snuecription est ou-

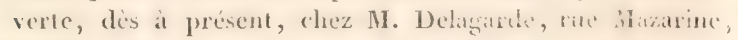
no. 3.

\section{INDICATIONS SEIENTIFIOUES.}

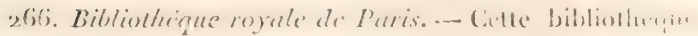
ne possédait, en 179 r, gue 150,000 volumes; elle est fir

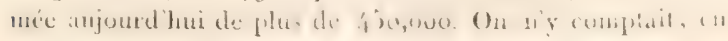




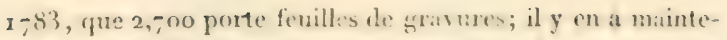

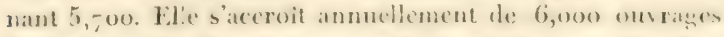
francais et de 3,000 itrangers; ce qui pernet desperes qu'en 50 ans ce magnilique établissoment aura dubble see richesse's litteraires et scichtiaiques. ( Rerue encyctop). Dicembre 1822 , page $6 / 8$.)

\section{VENTE D'ODJETS SCIENTIFIQUES.}

26\%. Vexte de la Collection de Mnéluax ine feu M. I'Aвье́ Ilıx, compuse denviron douze mille échantillons de choix. Les fersonne's qui voudratint en fitie l'arquisition sont prices de s'adresser it M. et a M Mac. VuteLexот-HaUs, au Jardin,du Roi.

Cette magnificgue coliection, destince pour l'étude de lit minérologie, et disposce de la manière la plus favorable jour servir a son enseignement, est en mine temps l'une des plus completes que l'on connaisse. Fille se recommande par le choix cl l'intiret des morecanx qui la composent; par le nombre prodigieux de cristans qu'clle contient, et dont lat plupart joignent au mérite d'une rareté extrème, celui d'une jurfaite régularité; enfin, par le précienx avantage de dewir sa formation et son arrangement aux soins atsians ef iclairés de M. Hauv. Les connitisenes aftacheront surfout nngrand prix à une cuistribution métliodique fate par .1. Hany

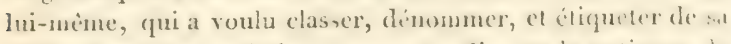
main jusqu'anx moindres morecaux. 11 a en lat patience de

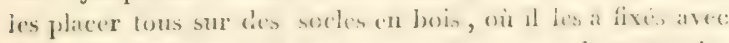
de la cire, et par-la il sest procuré liasantige de modre les formes cristallines en relation de position les une aree les antres, ce qui donne beauconje de farciliti pour létude decen formes. Chatque socle purie en outre une dituetle oi sont

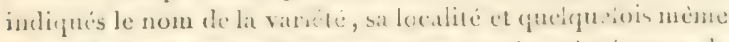
ses principatux caractires; an sorle que le releve dexact de toutes ces itipuettes, on le catalogue ratomne de la collection offrimat a lui seul un traté abrésé de la hience. lin tête des séries relatives anz différentes espices, setrouvent les morceanx qui presentent la forme primitise de lasubstance, ou du moins les indiees des a division mécanique, les cffets 
de la réfraction, ctc. Ce sont ceux que M. IIany a employís a la détcrmination des caractères qu'il nomme spécifiques. T iennent ensuite les variétés dans l'ordre de leur plus grand. perfection, et les morceaux qui indiquent les relations gronlogiques des espèces.

Un tel cabinet mérite d'étre conservé comme un monn:ment de la science, ct il serait digne d'un gouvernement d'en fire l'acquisition, pour le deposer daus un établissement d'instruction publique.

$\Lambda$ la collection principale se rattachent d'autres collections accessoires, également précicuses en elles-mêmes et par l'esprit dans lequel elles ont été formées; telles qqu'une suite nombreuse de roches classées et dénommées minéralogiquement; la série des pierres précieuses et des pierres fines, toutes montées en or; la collection entière des modeles en bois, pour l'étude des cristaux; et enfin l'assortiment complet des instrumens nécessaires au minéralogiste.

M. Hauy, qui s'était adonné avec succès dans sa jeunesse à l'étude de la Botanique, avait composé un lerbier d'environ dix-huit cents plantes des environs de Paris, dont il était parvenu à conserver les couleurs à l'aide d'un procédé de son invention; en sorte cu'elles sont encore aussi fraithes apres un intervalle de plus de quarante ans, que si elles venaient d'itre appliquées sur le papier. Cet herbier, unique en son genre, se trouve également à vendre.

268. Citaloge des lives rares et précieux, des manuserits, etc., de la bibliothéque rassemblée par fett M. Pajgुnou Dijonval, et continuée par M. le vicomt de Horel-Tindé, pair de France; dont la vente se ferat le lundi 17 mars 1823 et jours suivans, ì sis hemres trés-précises de relevéc, en l'une des sallesde: Thitel Bullion. In-s. de ass parges. Prix, broché $\therefore$ fr. 50 r., et frane de port 5 lir. A Paris, ches Debure frères.

l'ne des dernicres gramdes billiotheques commes de liatfis, we de celles qui formée longr-tenups atsant lat révolution,

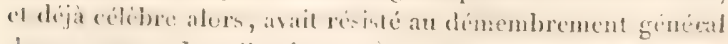
de ces sortes de colleetions, va se vemle dans le mois aie

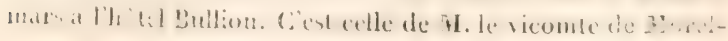


Vinde, qui posscilat an nène tempes un des plus riches cabinets destumpes qu'un particulier eht j:mais rasscmbli, et des collertions de dessins et de tableaux d'un sran:l prix.

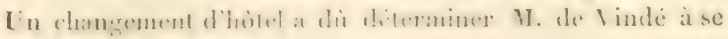
défaire de toutes ens collections.

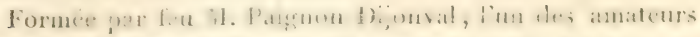

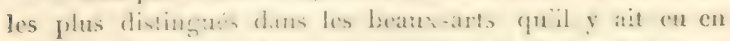

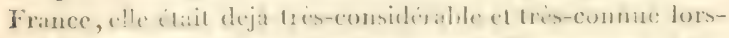

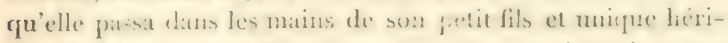

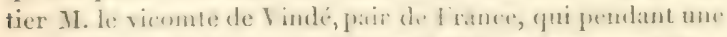
partie de sat vie s'est occupe a l'ombellir en y ajontant dans tontes les classes des livres pricienx, et principalement les

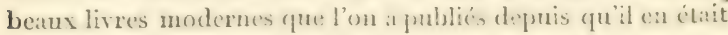
devenu possesseur.

I.e catalogue que nous amonems ne: comprent pas une foule de petits ouvrages et de hrochures qui dépendent de cette bibliotheque, il contient cependant 3yzo articke. Vous ne pouvons citer ié la guantité de livas raras et precicas sur les sciences qui ornent eetle riche biblientheque, surlout les ouvrages a figures sur lit botanique, les oisans, les arts, etc., ct les atlas de: toutes les ipocines. Il fatit an prondre connaissance dans ce catalogue.

\section{CINPUTHE SECTION.}

NECAOLOGIE.

269. Éloge de M. Nils Dalberg, inélecin.

Ce mederin était attaché an prince royal d. Sucde, qui rénna depuis sous le nom de Gustave III ; il accompragna ce prince dans son voyige a Paris, en r $70_{0}$ et 1771 , et ne nowligea pas, est-il dit dians son élogre, de vinter les principaux etablisemens que ronferme cefle capitale, tamt pour

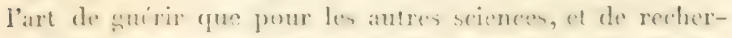

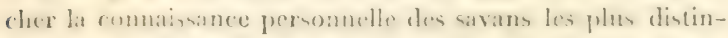

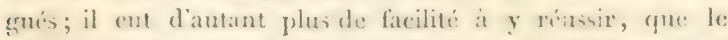

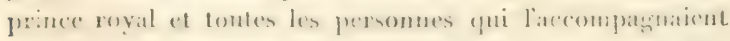
excitaient alors mu vif inférêt. Il se lia bientôt avec les

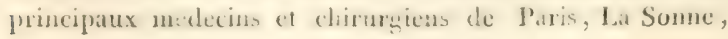




\section{Nécrologie.}

Louis Fabre, Petit, ainsi quavee Jussien, le Iimne de lat Jrance. (P.85.) Il obtint même accés anprès de lioussean, re qui n'bait fas une chose facile, et il en recul des lettres. Il fit encore en lrance la comaissance de d'Alembert, de I a Condamine, de Cassini; ct en Allemane, celle de Gledituth, de spalding, du célebre midecin et chimiste licireis, et do Mechel, habile anatomiste de Berlin.

On conserve dans la bibliothécue de Linkopinzs, on Suede, le Jommal de Toyage de Dalierg, ierit de sa main.

Le reste de la vie de ce savant a moins d'intérêt pour les Francais. Disgracié à la cour en $I 78 I$, il n'y reparut un

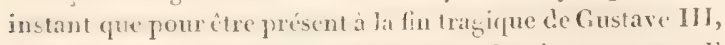
aupres dnquel on l'avitil appele dans les derniers momens. Il est mort à Stochlolm, à l'ige de pres de 85 ans, le 3 junvier 1820 .

On cite de lui quilunes mémoires, parmi crux de Meridémie des sciences de Gtoch.holm, not?munest, dans le volume

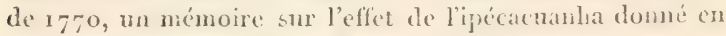

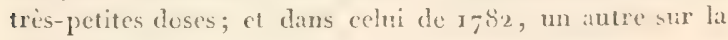
coloquinte (Cucumis colocynthis.)

Deux fois président de cette même acadinie, il prit pour sujets du discours que le président somtant prenonce stivint l'usage: la promiere fois, "les avantage's et les inconrénicns du climat de la suerle, pour lat sante; "la seconde fois, "les propriétés de l'air ćans les villes grandes et populcust"s."

C"est en l'honneur de ce savant midecin, et da colonel Dalbergyson frère, également naturaliste, que linté le fils á domné le nom de Dalbergia à un genre de plantes de lit famille des légumineuses.

C. Ir.

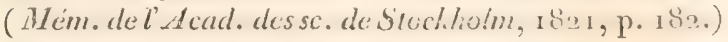

270. Éloge du baron Samuel Gustave Hermelin.

La mémoire du savant dont il sagit dans cet éloge doit être également chere aux grérraphes et aux personnes qui cultivent l'art des mines.

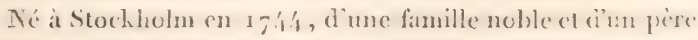
qui parvint à la dignité inmente de śnateur du res.d1:ne, il se destina de bunne lieure al la carriere administrative la

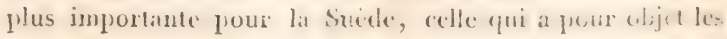


mines ef les usines, qui sont la principale richesas ale ce pay Il commenes par visiter les prineipatus établissemens de e.. genre que la Sucile renferme, et s'arrita particulierement a la celdere mine de cuiv re de Fahlun on il contribua a for:der une fabrique de vitriol, de soufre et de colcotar. Il s'occupa anssi activement de la mine d'or d'Estelfors.

Apres avoir acepuis une connaissance approfontie de tout ce que ha Scandinase lui ofirait de plus remarquable en cesenre, il obtint la permission de visiter ansi le: pays ctrang rs. Il traversa l'Allemanne, les Pays-Bab, l.e France, et se remlit en Am rirgue chargé en même temps, de: lat part de la Suede, d'une mission politique aupresetes litut, Z nis. 11 en parcourut presme toutes les parties, et revint cn Suède par l'Angleterre vers la fin de 17.84 .

Mais son désir le plus vif fut bicntot de répandre un nonveau jour sur la géographis, la géologie et la satistique de la Sucde, qui lui semblaient tres-imparfaitement connues.

En conséquence il lit parcourir à ses frais les provinces du nord de ce royarme, sur lesfuelles on avait encore moins de renseignemens exacts que sur les autres. Il résulta de ces voyagres une carte de lit Westro-pothnie et de la Laponie, et ce fut la le commenement de la grande entreprise géorraphliciue à laquelle le baron llermelin consacra pendant quinze années ses soins les plus assidus et une gramle partie de sa fortum. Malleureusement la publication des vingt-six premicres cartes ayant épuisé ses ressources pécuniaires, il fut oblizgé en 18 o de céder lat suite de ce tratvail a une compagnie; mais il jout un roble principal dans celte associalion, et il eut fe bonhenr de voir, avant de: mourir, ce srand atlas de sueite enticrement arheve, montument, dit l'auteur de l'lloges de llermelin, devé au sciences et à la prutre par un seul individu au prix des plus grands sacrifices.

Ses efforls patriotiques ne s'itaient pas bornés a cette seuk. entreprise, queldque stanele qu'elle fut : touclá de l'e trène pauvete a laduelle sont rélaits les habitans de's provinces, les plus septentrionales de la sutede, et comnaissant l'abondance et lat richesse des mincrais de fer gue ces prosines

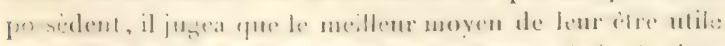
statit d'y etablix des fon 
fit Bolhnie trois de ces usines; il ouvrat de nouvelles routes, et perfectionna les moyens de transport par ean; il attira des colons, et il introduisit une culture jusqu'ilors incommur. Mais ces entreprises, qui ne furent point secondés, et yue des circonstances accidentelles confrarièent de piusionrs mat nières, épuiserent a latongue les ressources de M. Alemalin; il fut obligé d'abandoniner toutes ses propriétés a ses creanciers, et it éprouvat comme bien d'autres, que dims les mutreprises les plus ntile's et le's mieux calculces, les profits sont rarement pour les premiers qui les ont tentées.

An moins n'eut-il pis a se plaindre de l'isgratitude de tous ses concitoyens: Ic collinge des nobles a la diete de 1 Soo fit frapper en sun honnear ane medaille, avec cette légeade en suétois: "Offert a Hemelu par ses concitoyens et "amis, pour avoir fait mienv comaitre notre patrie, et " pour avoir enrichi et peuplé des déserts."

L'académie des sinces de Stockholm lui ouvrit son sein dès l'année 1771 ; souvent il seconda par ses libéralisés les recherches scientifiques de celte compagnie, et les voyiges qu'elle faisait entreprendre.

Lorsqu'il quatta, en 1355, l'administration des mines, apres cinquante-quatre ans de service, non-seulement ses appointemens lui furent conservis, maisles étals duroyaume y ajoutirent ane pension de mille rixdales; secours bien merié, mais modique, ajoute lhistorien de l'académie, si l'on considere les satrifices de tout genre que cet excellent eitoyen avait faits au bien public.

Supportant ses revers avec un courage philosophique, il jonissait dans la retruite du souvenir de tout le bien qu'il avait fait, lorscrue la mort mit fin a son utile et glorieuse carrière le 4 mai 1820 .

L'ál yge du baron IIermelin est terminć par l'énumération des ouvrages qu'il a composs, au nombre de treize, y compris ses mémoires acalimirues, et par celle des ouvrages 'qui n'nuraicnt pas été publiés sans ses secours généreux.

Tous nous bornerons a citer ici les ouvrages suivans de Hermelin lui-même qui ont été imprimés à part.

Sur la fonte des mincrans de conive. Stockholm, r-66.

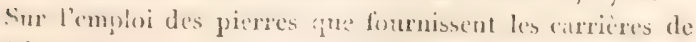
Si:ite. 17:1. 


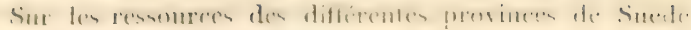
1773.

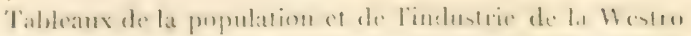
Bothnie. Stockholm, I So3.

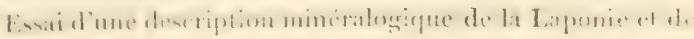
la Westro-Bolhuic. Stockholm, $180 x$.

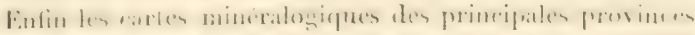
du sud de la Sucde. (Truluit du suidois et extrait des mém. de l'stcad. des sc. de Stochtrolm, 1821 , pas. qoy.

$$
\text { C. MI. }
$$

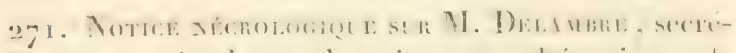

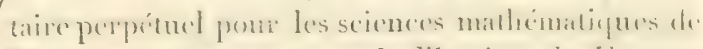

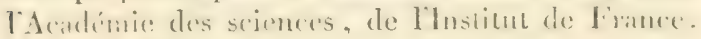

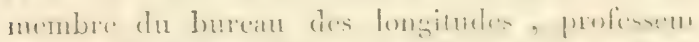

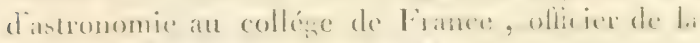

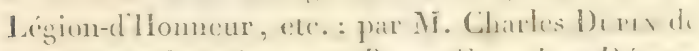

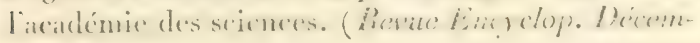
Zre 1823,1$) .417$ is $466^{\circ}$ )

Cette notice est cmpreinfe du sentiment le plus vai, el

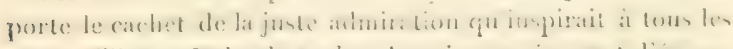

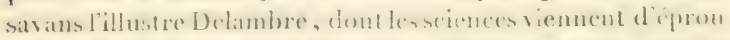

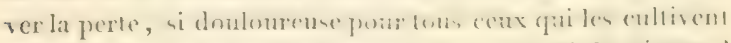

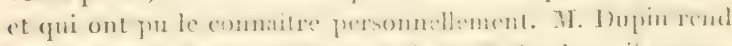

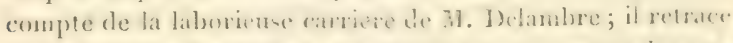

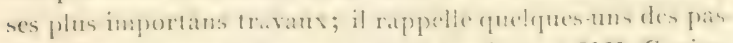

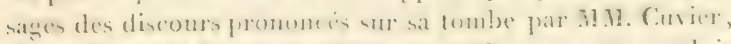

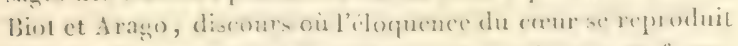

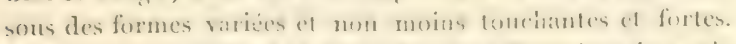

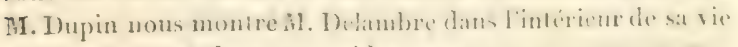

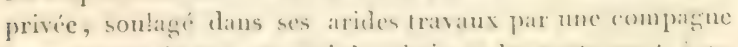
inconsolable de sa perte, quicherchat, coltes partagenat, a en alléger le poids.

it. J) lambre a ete cnleré anx scieneres, à ses amis, i sol patrie, le 2 ) aout 1822 ; il vicut d'elre remplace commo se-

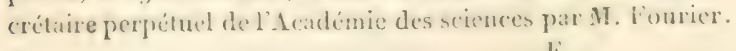
l:.

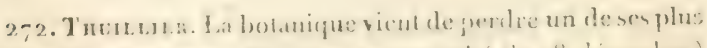

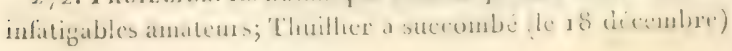


a une malatic donlournse. 1)(onc par la sature d'une

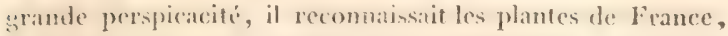
daus tous leurs ditats el dans toutes les variations ou la naine se plate a les offrir. Ne dans une rlasse inferverure, il "IVait recu aucune instruction; son intelligence l'ileva bionlùt au-dessus de l'omploi de jardinier qu'il exereat. On Ini acordat un grand fonds d'honneur, et la connatssance parfaite des plantes indignenes; ces qualités étaient ternies par de lineomblute, suite de sa prenicre education, et un caractere qui le prisa de ses amis et de la protection des hommes dont il altendail des movens d'eristence. 11 est anreur de denx édilions dune Jlore eles environs de Parit, is largurle: on croit que le chlibre botaniste Richard avait conseati a travailler. Thullier composait des herbiers, et faisit profession de le, veudre, ansi-bien que denseigner à irombre è recommitre les plantes sur lis lieux ou elles croissent. Une blessure a la jambe lavait force d'interrompre ses escursions depuis quelques amues; il est mort de ecte affertion, dans un itat voisin de lindigence. (Rerue encyelop). Déc. I $S_{22}$. p. $66_{2}$.)

2-3. T'université de Moscon a perdn, l'annie derniere,

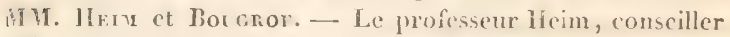

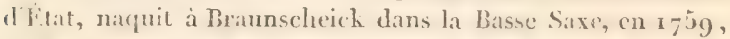
il fit ses itudes dans les aniversités de Hclmolited of de

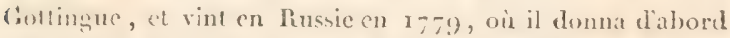
des lions ditis des maisons particulieres. En $x=g$ fi, il fut 'ommin professeur de langue allemande et d'antiquités ct en 1804 , d'histoire, de statistique et de. la science du

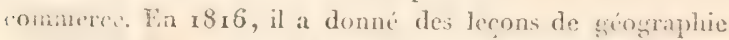

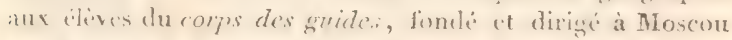

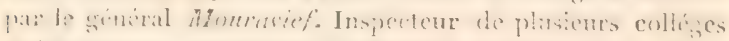

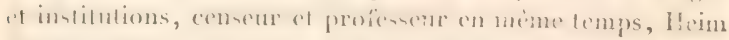

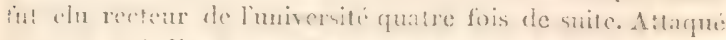

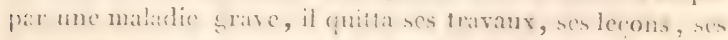

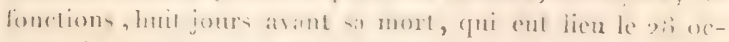
iobre ( 16 octobre, ז. st.) 1 821 , léğuant à la postérité les vertus d'un chrétien et les travaux d'un érudit. Il commais-

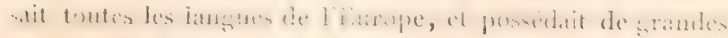

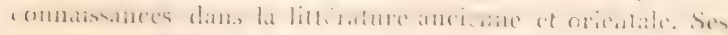

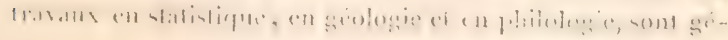




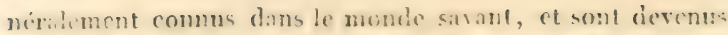
dassirues en Russie : voici coux de ces ouvagges cyul se rapportent aux sciences.

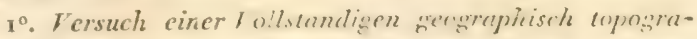
phischen Encyolopedie der liussiche'n reichs nach alphabetischer ordmus... Insai d'unc Encychopedie complete sur la gengraphie et la topographice de l'eupire de Russie par ordre alphabétique.) in-8 . Gottingue, $179^{6}$.

2." Lstruisse de Gécomplice universclle. in-\$". Moscon, I8Ix.

3". Principes fondementuux de la gergrephic moderne, in- $8^{\circ}$. Moscou, $x 8 \times 3$.

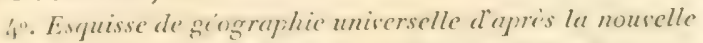

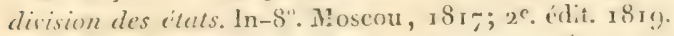

5.. Lissai de statistique de's principaux Ftats. In-8"., Tom. $x^{e r}$. Anscou, r 821 . Ce tome contient la statistique de la linssie, de l'Autriche, de la France, de l'Angleterre et de la Prusse.

Heym rédigea la Gazetle de Inscou (Mostendis. he zcitunir), depuis janvier 1811 , jusqu'a septembre I 8 I 2.

liougrof, mathimaticien, is qui de grandes connaissances dans l'astronomie préparaicnt une juste célébrité, et qui était destiné par le gouvernement à voyager dans les pays etrangers, pour y recueillir des notions sur cette science, ayant été atteint d'hypocondrie, s'est brìlé la cervelle be 25 aoùt (13 aout, v.st.) 821 . Il était fort jemue, et conmu par une Dissertution sur le mousememe elliplique des astres, publice a MIoscon en 1822 . (Rerue Inc) (Rlop. Dicembre 1822. p. 626 et suiv.) 


\section{BULLETIN}

GÉNÉRAL ET UNIVERSEL

\section{DES ANNONCES ET DES NOUVELLES SCIENTIFIQUES.}

\section{PREMIERE SECTION.}

ANNONCES DES OUVI $\Lambda$ GES, EXTRAITS DES JOURNAUX E'T NOUVELLES SCIENTIFIQUES.

OUVRAGES ENCYCLOPÉDIQUES.

274. The Panorama of Science and Ait, etc., ou Panorama de la science et de l'art, contenant l'aréostatique, l'agriculture, le jardinage, l'architecture, l'astronomic, la chimic, l'électricité, le magnétisme, la brasserie, l'horlogerie, etc.; par J. Surtr, nouv, édit. 2 vol. in-8, avec 49 grav., prix I 1. $5 \mathrm{Sh}$. cart. Londres. H. Fisher.

375. Dictronnarre chronologique et raisonné des découvertes, inventions, innovations, perfectionnemens, observations nouvelles et importations en France, dans les sciences, la littérature, les arts, l'agriculture, le commerce et l'industric, de $17{ }_{9}$ à la fin de 1820 ; comprenant aussi, etc. Ourrage rédigé d'après les notices des savans, des littérateurs, des artistes, des agronomes et des commercans les plus distingués ; par une socicite de gens de lettes. Tome $6(\mathrm{FLE}-\mathrm{FER})$ in-8 de $35 \mathrm{f}$. A Paris, chez L. Colas.

'T'OME I. 
$\because 76$. A complete axn exrensus. english dichonary, etc., ou Dictionnaire anglais complet et universel, contenant l'explication des mots difficiles et des termes techniques pour toutes les professions; pour l'algèbre, l'anatomie, l'arithmétique, l'astronomie, etc. Ouviage du Rev. J. Barclay, revu et augmenté par W. Snontox. I vol. in-f. arec un beau frontispice et un titre gravé. Prix a l. Gish. Londres. H. Fisher.

27-. Les Anglais possèdent déjá plusienrs grandes Encyclopédies, Iencyelopadist Briennica, en 20 vol.; celle de Rees the New Encyclopedin or universal Dictionary, par A вr. Rees); et celle d'Édimbourg (the Edinburgh Encyclopedir, conduced by $D$. Brewster), qui toutes sont dans le genre de nos $1^{\text {res. }}$. Encyclopédies, mais ou les diverses sciences sont cependant traitées à certains mots d'une manière générale, et quelquefois très-remarquable, par des savans connus. M. $J$. Mitchell, profess. à l'univer. d'A berdeen, s'est décidé à entreprendre une Encyclopédie par ordre de matieres, c'està-dire une série de dictionnaires séparés qui seront au niveau de l'état actuel des sciences. Il pense que 12 vol. portatifs suffiront; le $1^{{ }^{e} \mathrm{r}}$ vol. (l'Histoire) vient de paraitre, les autres se publieront de mois en mois jusqu'a la fin de l'année. Il y aura un grand nombre de belles gravures. Si ces promesses sont remplies, l'on verra une Encyclopétlie se publier en un an eten 2 vol.! Il semble qu'il fitudrait bien plus de temps pour réduire ainsi les matières à ce qui serait réellement fondamental; du reste nous sonmes bien persuadés que toutes nos connaissances positixes générales, tous les prinripes fondamentaux, peuvent se renfermer en douze vol., mais alors cette Encyclopédie ne ressemblera guere a lit nôtre.

\section{MATIEEMATIQUES ELEMENTAIRES.}

278. Stgcio sulla Storia delle Matrmatiche, ete., ou Fssai sur lHistoire des Mathématiques; par J'. Francmin. In-8. i 82 . Lucques.

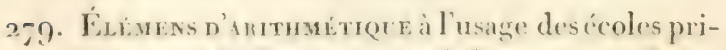
maires; par M. ( ) venet, chef d'iustitution à Sint- 


\section{élémentaires.}

Malo. In-12 de \& f. $\frac{2}{3}$. Prix, 90 c. 1822. A SaintMalo, chez L. Hovius.

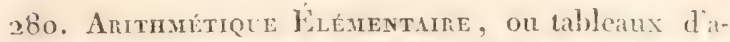
rithmétique composésselon les principes, et assujétis aux procédés de l'enseignement mutuel, Par M. Jomand, secrétaire de la Société pour l'enseignement élémentaire. Ouvrage adopté par la Société, et parle conseil d'instruction primaire établi par M. le Préfet de la Seine pour l'usage des écoles élémentaires; ML. nUel eXPliciter pour servir a l'intelligenee des tableanx, et pour en diriger l'emploi. I vol. in-s. avec 60 tabl. r822. A Paris, chez L. Colas.

Depuis l'Arithméliquede Condorcet, aucun traité ne nons a yaru mériter mieux que celui-ci le titre d'élémentaire. Décomposer des idées regardées comme simples, urraduer les nouvemens des oṕciations, les réduire à une action presque mécanique, voili ce qu’exigeait lia spécialité de l'ouvrage, et M. Jomard n'a rien laissé à désirer dans l'exítution. Jous avons surtout remarçué avec une vive satisfaction le míratzuisme ingénieux qu'il a employé pour transmettre lés notions lies plus étendues sur le système métrique, et son application usuelle. Yous ne ponvons que conseiller la lecture de col ouvrage í tous les professenrs charécés de donner des lecurs d'arithmétique, méme à ceux qui, dans leurs cours, vont au delit des élémens; ils y tronveront une clarté, une precision que nous aimoais a faire apprécier à nus lecteurs, et quon peut proposer comme modèle.

Bertievix.

shi. Recuerl donervations ex De bruemes sur l'Egypte ancienne et moderne, on Desipiption, ete.: par M. Jomard, membere de linstiun royal de France, cte. In-s. Paris, sis.?. Tome 3, Sisteme métrique des anciens Égyptiens.

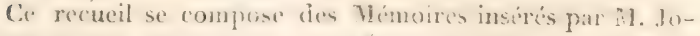

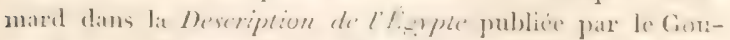

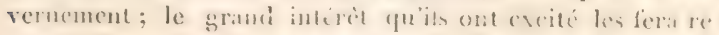

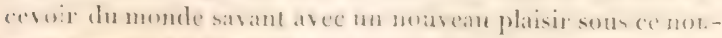

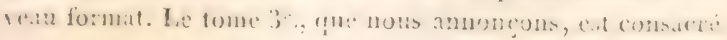


tont entier a lexposition dusstime metrique des anciens forptions, ot a den recherdes sur lenes commatissances géomitriques, giongraphiques at astronomicyes. Ce sujel appartient aux premienes pages de Ihistoire des connaisancen humaines; ce n'ent done pas trop de létemdue que le sastant acalemicion a cru devoir donner it son travail, surtout en

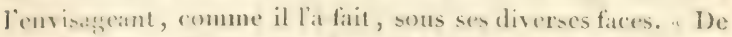
bons ceprits, dit-il, cherchent a etablir les tieres de l'antiguite dans les seiences posilives, et à fire voir re gue thatgre peuple et chague àge ont apporté à lédifice commun

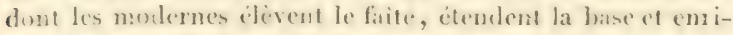

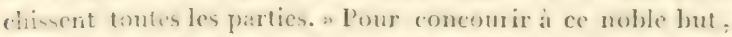
31. Jumari comsulte et compare à la fois les anteurs et les monumcus de l'antiquité, cherche lat vaie experession des premitrs, et donne les mesures des plus cediblores d'entre les seconds; il en deduit le type des diverses mesures usifees dans l'ancienne ligypte, et lesconfirme par des mesures sembiables encore en usacre dans l'figyle moderne. Recherehant ensuite les valeurs du pied gree et du pied romain, it on fait voir l'accold avec les mesures isyptiennes, ct de cellesci avec les mesures usitées rhez plusienrs peuples anciens. Tous ces résultits sont appropriés à nene savante discu-siun sur une des plus importantes "prations de la géonctrie, la mesure de lis terre, discussion dans laquelle MI. Jomard examine et cheache a expliquer ce qui a ete rapporti sur toutes les mesures de la terre attribares aux ancions ot an: Arabes, et les rénllats de cet immense tavail, acompragné d'un grand nombre de tabreaux, oblige at siaproser dans les autcur; du systeme metrigue des conmaissmers positives de: geometre et de groggraphe mathematigue. I.antenr a aissi

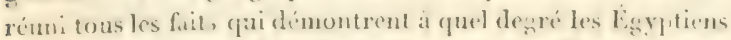
avient porté ces mémes combaisances, et a l'apjonide ses opinims il ajoute plusicurs notes et cilatreisemens, et quel-

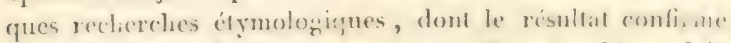

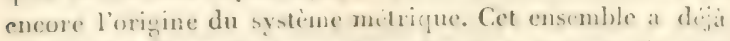

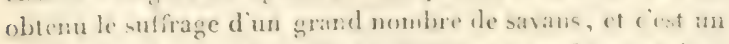

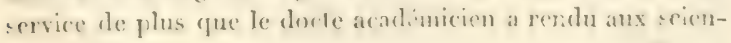
$\operatorname{ces} \mathrm{ct}$ à l'untiquité. 
383. Fvaliation de leereur qui peul afecter les quotiens et racines approximatifs; par un anonyme. (Ann. des math., : Je. vol., nov. I822, p. гоj.)

La plupart des nombres décimaux qu'on emploie dans les calculs sont des nombres approchés; alors on ne peut compier sur l'exartitude du résultat que jusqu’à un certain ordre de décimales. Il est trés-important de connaître à l'avance quel est cet ordre de décimales. Tel est le but de l'auteur de ce travail. Voici une des regles qu'il donne: la plus grande erreur à craindre sur le quoticnt de la division de deux nombres enticrs, approchés à moins d'une demi-nnité, cst le qu zvient de la division de la deni-somme de ces nombres par le carré du điviseur.

B. $x$.

383. Sut les rursancret sur les racines des nombres;

par M. Queriet, chef d'institution à Saint-Malo. (Ann.des mathémat., nov. 1822, p. 162.)

L'auteur fait l'application de sa méthode a la formation de I: cinquième puissance, d'un nombre, età l'extraction de la diicine cinquième d'un autre nombre.

B. $\mathbf{x}$.

28\}. Covrs pritroe et théorique d'arifhmétique, d'apres les principes de Pestalozzi, avec des modifications: par II. L. D. Rrval, disciple de Pestalozzi. (Prospectus.)

Cet onvrage sera du prix de $6 \mathrm{fr}$., pour les souscripteurs qui s'inscriront jusqu'an premier juin, rue de la Harpe, $n^{7}$. I r 7 ; les non-souscripteurs le paieront $7 \mathrm{fr}$.

285. Finnents of geometry and trigonometry with notes, ou lilémens de Géométrie cte Trignnométric, traduits de l'ourrage francais de $\Lambda$. M. Lrgender, membre de l'Institut, des Sociétés royales de Londres et d'Edimbourg, et public's par D. Bnewstri, docteur en droit, avee des notes, des additions et un chapitre d'introduction sur les proportions. in-s, avee des figures graves sur luis. Pris. ro sh. Edimbourg. Olivier et Boyd.

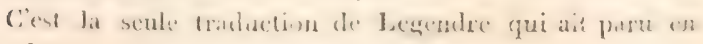
Angleterre. 


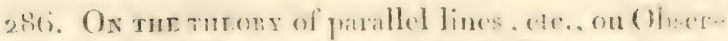

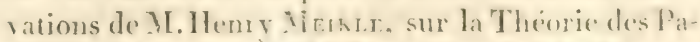
ralleles, domnces par . Hi. Trory dans le n". de mets I $8_{22}$ du mines Journal. (The philos, magraz. and journ., n'. 296, déc. $1822, p$. 423.)

Ces observations portent sur la troinieme proposition de N1. Ivory, gui avait pour but de dimontrer pue la somme dos trois angles d'un triangle est égale a deux droits, sans sippuyer sur les proprietés des parallèles. Ibrr.

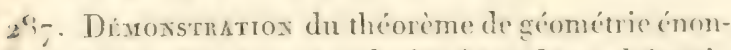
cé a la p. 3:s d du vire.vol. des Ann. de matlómati-

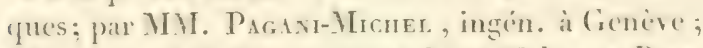

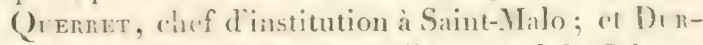
raxne, profes. de phys. au collige royal de Cihlors.

Voici l'enoné de re thiorime : la circonference qui plishe for les centres de trois quelconques des quatre cerches qui touchent à la fois les trois còtés d'un triangle quelcongue c'st donble de celle qui passe par les trois sommets de ce triangle.

13. $\mathrm{Y}$.

288. Tést vé et application des principes ćlémentaires de la Perspective; par C. Fancr. I ${ }^{\mathrm{er}}$. cahier. In-

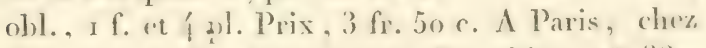
Climent, ot clacz l'auteur, rue I)auphine, n". 3S.

Liatenr a en pour but de presenter les principales méthodes de perspertive praticue appliquere an dexsin du paysase et de larchitecture, et, pour en faciliter l'application, il comne des exemples ombres a leffet, demines el lithograplicis avec soin.

II. S.

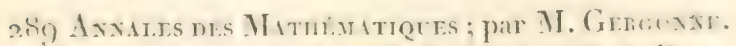
Bolume 33 , dece r8se. A Paris chez Bachelier.

\section{Perspective linciaire; par MT. Gengoxis.}

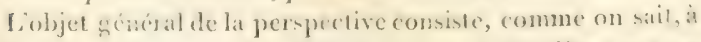
colorer wne simple surface de telle sorte qua elde offre, pour un spectatener convenablement plare, le meme aspet que lui offriraient des objets en relief distribues daus l'espace dium. manière déterminée. 
MI. Gergonne assigne clairement la différence qui existe entre la perspective aériemne et la perspective linéaire. Il établit une juste distinction entre les objets originaux et la perspective. Il définit successivement le plan vertical et le plian horizontal, passant l'un et l'autre par l'œil; la ligne verticale et la ligne horizontale du tableau; le point de vuc; le rayom principal; la ligne de terre; le plan géométral; le point de vue accidentel.

11 montre ensuite comment on représente en perspective un point, une ligne droite, un polygone rectiligne plan ou gauche, wne courbe plane ou it double courbure; un corps quelconque; il applique la perspective aux cartes géographiqques, à la théorie des ombres, à la gnomonique.

II. Sur la construcition d'un cercle tangent is trois cereles tonnés.

III. Solution des deux problemes suivans; par M. Gr: -

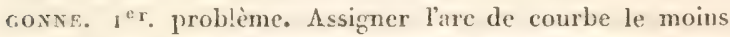
long entre tous ceux qui se terminent aux deuxextrémités de la base d'un triangle isocèle donné, et qui, étant en ces points tangents aux deux autres côtés du triangle, partagent son aire en raison donnée. $2^{e}$. Problème. Assigner la portion de surface courbe la moins étendue, entre toutes celles qui, se tcrminant à lit circonférence de la base d'un cône droit donné et touchant sa surface convexe suivant cette circonférence, partagent son volume en raison donnée.

IV. Question proposée. Théorème : Deux liyperboles équilatères quelconques tellement disposées l'une par rapport ì l':autre que les diamètres principaux de chacune sont lesasymplotes de l'autre, se coupent toujours à angle droit.

Nons nous bornons à l'énoncé des articles II, III ct $1 \mathrm{~V}$, parce qu'ils semblent se refuser an genae d'analyse eompritible avec la nature de notre recueil.

B. $Y$.

9go. Tables of Leganthus, ou Tables de Lozizrithmes de tous les nombres depuis a juscquit 10,000 , et des sinus et tangentes de chaque second dı quadrant, par M. TAYLon. Avee des rimgles pour les expliquer, et la manière d'en faire nsage: par N. Maskelyas, astronome royal. In-4́. Prix, 3 1. Londres. 


\section{MATHEMATIOUES ThANSCENDANTFS.}

291. Applations df gioméxne el de mécinique à la marine, aux ponts et chaussées, etc., pour faire suite aux Développenens de Géométrie; par M. DLrix, membre de l'Institut (Acad. les sc.). I vol. in-4. Paris, 1822. Chez Bachelier.

Ce volume contient cinf mémoires. I. Théorie nouvelle de la stibilité des corps flottans, fondée sur les principes de la courbure des surfaces. Ourrage important pour les arts relattifs a la navigation sur les mers, sur les fleuves, el mème sur les canaux.

II. Construction des rontes isolées. Les ingenieurs de lit marine sont souvent chargés de tracer et d'ouvrir des routes pour effectuer l'exploitation et le trinsport des bois propres à la charpente ainsi qu ì la mâture des vaisseaux. ()n indirque ausi d'apres quels prineipes ils deivent exécuter les voies militaires, a travers les forèts et les montagnes, sils sont appelés aux armées de terre avec lours ouvriers. L'anteur désire enfin que cet essai puisse être consulté avec quelque fruit par les ingénicurs des ponts et chaussées.

III. Routes quil faut suive pour opérer les transports connus sous le nom de deblais et de remblais, transports également opérés daus le génie maritime, le génie miitaire, l'artillerie, les mines, etc.

IV. Routes suivies par la lumiere et par les rayons sonores, dans les phenomenes de la riflexion et de lit réfaction; elles sont soumises à des lois qui reproduisont avec une entière fidélité les règles géométriques des transperts les plus avantagenx, sur des routes mathématiques, et pour des prix donnés.

V. Dans le cincuieme mémoire, on revient aux applications qui concernent la marine. On montre comment on peut combiner les connaissances données par lia pratique sur la structure des bittimens de guerre, avec les lois scientifignes qui rérgissent la forme, la stabilité, la force et la durce de ces grands corps floltans. (On sail que déjat notre marine ch a tiré avantage. Ciela scul fait l'clegéfe diun pareil travail. 
En finissant, ajoutons qu'il est digne du professeur de mécanique industrielle, au conservatoire des arts et méticrs, et de l'auteur du grand ouvrage sur les Forces de terre et de mer de l'Angleterre, devenu classique chez les Angiais euxmêmes.

B. $\mathbf{Y}$.

292. A System of Mecinanic, etc. Systime de Mécanique; par le Rev. J. A. Roninson. In-8. Prix, I8 schel. Londres.

203. Ménome sur lis intégrales définifs, où l'on fixc le nombre et la nature des constantes arbitraices, ('t des fonctions arlitraires que peuvent comporter les valeurs de ces mèmes intégrales quand elles devicunent indéterminées; par M. A. Carcuy. (Bull. des Sc. de la Soc. philomathique, nov. 1823.)

L'auteur, après aroir rappelé, $\mathbf{I}^{n}$. son premier mémoire sur les intégrales définies, présenté à l'Institut le 22 aout I 8 I 4; $2^{\circ}$. ses lecons à l'École polylechnique, et celles qu'il a données au collége de France en $1807 ; 30$. son Mémoire sur les Solutions particulieres, présenté a l'Acarlémie royale des sciences, le 13 mai 1816 , ajoute qu'il at pensé qu'il serait utile de montrer toutc l'extension dont sont susceptibles les méthodes qu'il avait suivies. Tel est l'objet du Irlémoire qu'il a présenté le 28 octobre dernice à l'Académie des seiences, et dont celui que nous annonçons contient l'analyse. $\mathbf{B}$. x.

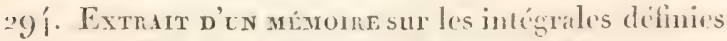
et sur la sommation des séries; par M. Porsson. (Bul. des Sc. de la Soc. ptrilunathiciue, sept. I\$22, p. I34.)

C'est la suite des mémoires que l'auteur a donnés sur le méne sujet dans les trois derniers rahiers du Journal de l'école polytechnique.

Le premier article est relatif à la sommation des séries de quantités périodiques. Ce sont principalement les sériès qui procedent suivant les simus ou les cosinus des multiples d'un angle variable. I'oljet du sccond article est de transformex une fonclion donnée, en une série de sinus ou de cosinus. I). bernouilli, Lagrange, et l'autcur lui-múme avitem! dijat tratte 
celte matiere; il y revient pour complefer ses premiers travatux.

En soccupant de ces nouveiles recherches relatives aux intiograles définies, M. Poisson a été conduit ì d'autres formules qui pourront itre ntiles en astronomie, et que, pour cette raison, on a insérées dans la Comnaissance des Temps de 1825 , déjà publiée.

Le Mémoir que nous annoncons fait partic du r 9 . calnier da Journal de l'école polytechnique, qui paraitra incessamment.

B. $\mathbf{~ . ~}$

9y. Démostnatron de la proprićté de minimum. dont jouissent la circonférence du cerele, entre les périmetres des figures planes de mème surfare, et la surface de la sphére entre les surfaces des romps de mìme volume; par un abonné. ( $A$ nnn. de mathématiques, oct. $1822, p .132$.

Le but principal de l'auteur est d'introduire dans les élimens de géométrie la propriété dont jouit la sphère, l'ètre le corps de moindre surfice entre lous cenx de mime volume, ou le corps de plus gratnd volume entre tous ceux de mème surface.

B. $\mathbf{x}$.

2y6. Solcton nouvele d'un probleme (dit probleme? du chien), énoncé dans la Correspondance de l'F́cole polytechnique; par M. T. de Sт.-Lar nist, lieut. , aide-major au $7^{\circ}$. régim. d'artill. à pied. ( $/ \mathrm{m}$. de mathémat., nov. 1822, p. 145.)

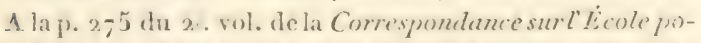
frlechnique, publice prar Ml. Irachethe, on tronve ce gui suit : "Ln ancien élexe (de léecole polylechnique), 11. Inubeis- Aymé, se promenait sur le bord de la mer; il apereut une - personne de sa comnaisance et se mit a courir pour hat "tinter son chien, qui s'etait éarte, courut vers lui, en " décrisant une courbe dont leupreinte resta sur le salle. " M. Dubois, revenan sur ses pas, fut frappri de la réşularité de colte courbe, et il en chercha l'équation, ensuppo, sant: $\mathbf{~}^{\prime \prime}$. que le chien se dirigeait constamment vers l'en- droit oi il voyait son maitre; 2 ". que le maitre parcourait une ligsie drevite; 3". que le's vitesses du matte et du
" clien étaient uniformes. "
B. $x$. 
297. Essir sti les fonces qui déterminent les dirers ćlats des corps; par M. II.-G. Scmuntex. ( $A$. de mathématiques, oct. 1822, p. I2I.)

Nous sommes forcés de nous borner à indiciuer seulement quelques principes rgui ont servi de hase à l'analyse de l'autour." On pent, dit-il, regarder la mattiere comme un assemblage de points d'oin émanent des forces répulsives et des forces attractives. Celles-ci sont constantes dans le meire corps, mais celles-là sont variables. »

Il arrive at un résultat qui lui semble devoir être l'explie:ttion des changemens bruscques qui s'opirent dans plusieurs corps, par un cerlain changement de température.

I u firit général pour tous les solides, dit-il ailleurs, c'est qu'arrivés à un certain degré de iempérature, cette tompé. rature n'est plus augmentée par une accroissement des forces répulsives, jusqu'i ce qu'un changुement d'état l'ait rendue susceptible de recevoir un nouvel accroissement.

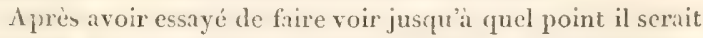
possible de se rendre compte des principaux phénomines rui accompagnent les différens états des corpes, MI. Sehmidion ne dissimule peint la nécessité où l'on est de se borner a des considérations trop générales.

B. $x$.

\section{MACHINES ET CONSTRUCTIONS.}

99h. Recterl des michuves, instrumens et apparcils yui servent à l'économic rurale, tels que charrues, semoirs, herses, moulins, tarares, machines a cilever l'eau, presses in vis, presses hydrauliques, hachepailles, coupe-racines, machines à broyer, ete. ete., 't dont les avantages sont consacrés par l'expérience; publice, aree les détnils nécessaires à la construction, par Lrebisc, dessinateur-graseur du Conservatoire royal des Arts et Métiers. Prix de lat livr., contenant $(6 \mathrm{pl}$. in-fol. ol,., papier vélin soli.. papier ordinaire of fi. A Paris, chez lantem, rue de Crussol , $n^{\circ}$. 5 .

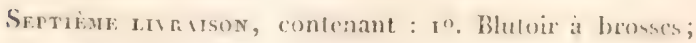
?." presse a cylinders el continue; 3". charrue amérieaine. 
I.es six premieres livaisons, précédemment publiées, renferment, savoir : la r re. Moulin i ecraser les pommes de terre; macline a methoyer les graines de trifle; idem, a ceratser les graines olaugineuses; hache-pailles hollandais; coupe ceps et coupe clicots. Ia 2e. Charrue de Brie perfectionnéc; Charruc a bulter; charrue du Brabant; presue levdraulique de P. E. Mongolfier. La 3e. Extirpateur a chesal; machine i faner; presere a vis. La $4^{e}$. Machine a battre le ble, dite Sućdoise; mance pour les machines à battre le blé; moulin a bras, par M. Ovide; coupe-racines. Lat 5e. Semoir a bras, perfectionné par M. Scipion Mourgues; pompe portalive pour larrosement des jardins; rou'eaux brise-molles subdois; houe a eleval renversée; charrue hrandilloire écossaise. Ia (je. Moniins a blé établis chez M. Couggonilhe, près SaintQuentin, par A. Maudsliy, de Londres, en 1818 ; monlins a blé de différentes constructions; extirpateur rotatif de MI. Morton d'Édimbourg.

Ln des plus grands obstacles qu'éprouve la propagntion des machines, instrumens et appareils qui servent a l'industrie, est sans contredit le manque de dessins assez exactement tracés el ofrant les détails nécessatres pour en faire conmaitre toutes les parties integrantes avec assez de clarté, ct sur des échelles assez grandes pour qu'un constructeur puisse les exícuter. N1. Ieblane, pénétré de cette vérité, à cu l'heureuse idé de réunir les meilleures machines, dont les avantages sout constates par l'expérience, tant en Fance que chez l'etranger, et il a parfattment atteint le but qu'il s'est proposc. Ln texte deseriplif accompagne chapue planche et indrupe le jeu, l'effet et le produit de charque madhine, en un mot tout ee que le dissin ne peut exprimer : il serat a désirer, pour ripandre les arts, que les planches des ouvrages de technologie fussent dessinées avec autant d'intelligence, et gravér, arce autant de soin que celle's de l'ouvrage de MI. L.chlane. Cit artiste recommandable par ses tatens a rech du gouvermment, à titre d'encouragement, une midialle a l'exposition de $18 \times 9$.

II. S.

39). Collecton de machises, dinstrumens, ustemsiles, constructions, apparcils, cte., cmploy dans léconomic rurale, domestique et industriclte. 
Japrés les dessins faits dans diverses parties de l'Europe; par le Ce. de Lasternes. se cilit., tom. I er , $2^{e}$ livr. In-í, d'une f. et ro pl. A Paris, chez Arthus Bertrand.

Louvrage aura 20 livraisons, qui formeront 2 volumes. Chaque livraison coûte $4 \mathrm{fr}$.

Les personnes qui roudraient payer d'avance les 20 livraisons, ne donneront que jo fr.

30o. De l'Art de Fonthiner sondect, et des Puits Antésmens, ou Mémoire sur les différentes espices de terrains dans lespuels on doit rechercher des eaus soutcraines, et sur les moyens qu'il faut cmployer pour ramener une partie de ees eaux ì la surface du sol, à l'aide de la sonde du mineur et du fontainier; par F. Girxren, ingén. atu Corps royal des Wines, ancien éléve de lícole polytechnique. (Ce Mémoire, imprimé par ordre du gourerncment, a été corronné par la Soc. d'encourigement de Paris, dans la séance générale du 3 oct. I 82 I.) Prix, 13 fr. 50 \&. A Paris, chez Madame Iluzard.

Ce inémoire forme un vol. in-4". de $133 \mathrm{pag}$. de texte et de $19 \mathrm{gr}$. pl. J'autcur a divisé son ouvrage en $\{$ chapitres. Dans le I , il fili connaitre tout ce que l'expérience a appris sur la composition des terrains où l'on a pratiqué des fontaines jaillissantes de fond, tant dans l'ancienne province d'Artois que dans d'autres contrées. Il établit, sux la cause de ces fontaines, une théorie très-simple et très-satisfaisante qui fait conmaitre dans quelle nature de terrain il faut les chercher. Dans le $2^{p}$. chap., l'auteur donne quelques remarques particulières sur les circonstances qui se présentent dans la recherche des eaus souterraines jaillissantes. Il rend parfaitement raisun de tout re que l'expérience a fait observer dans les différens terrains oin l'on a sonde. On trouve dans le 3c. chap. la description très-exacle et tre-s-claire de tous les instrumens qui composent la sonde du fontainier, pour percer toute es-

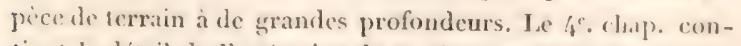
lirut le ditail de lopération dix somdange, exposée de maniere 
qu'on puisse la diriger dans tontes les circonstances : les procédés pour enfoncer les coffres y sont parfaitement decrits. Enfin, l'auteur termine par exposer avec le plus grand soin comment on doit s'y prendre pour établir les buses dan les trons de sondage, pour amener l'eau jaillissante a la surfitce du sol. On tronve a lit fin de ce chapitre quelques clemens pour évaluer ce que peul couter l'établissement de ces fontaines a differentes profondeurs, eu égard a la nature du terrain. On a inséré au commencement du volume le rapport fait a la Societé d'encouragement sur le concours qu'dle avait ouvert sur le sujet traité par l'auteur. On trouve a la fin derux tubles, l'une par ordre de matieres, et l'autre par ordre alphabétique, et une légende explicative en 16 pages, des ro planches ou sont dessinées les machines et les outils, aver toute l'exactitude qu'on peut désirer.

Après avoir lu cet ouvrage avec attention on sera en étal de faire établir des fontaines dites puits artésiens, ot mims de faire construire les maclines et les outils nécessaires an fontainier sondeur.

G. C.

3or. The Mecnaxe, or Compenelium of practical Inrentions. La Mécanique, ou abrécé de's inventions pratipues, en 2y 5 articles, divisés sous les rubricpues : Manufactures et commerece - Apjurial philosophiegue et beaux-arts. - Economic rurale et domestique. - Mélanges. Aree ron srave cl un grand index anily lique; par J. Smitu. ? rol. in-s. Prix, I 1. I 5 sh., cart. Londres. H. Fisher.

Cet unrage parait avoir une grande xeputation parmi len mécaniciens anglais.

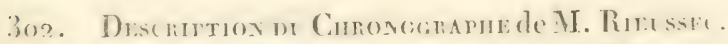

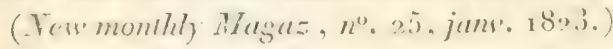

Ce chronograple a la forme et les dimensions dun stamel

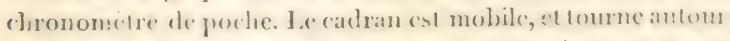
d'un ase gui traserse son rentre perpendiculatement a son

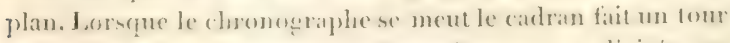
far minute; et comme al circonference est divise en sosante parties, le mourement angulate d'une divinios: 
correspond à une seconde de temps : les minutes sont marquées à part. L'observateur qui désire connaitre l'instant d'un phénomène presse un bouton de cuivre, itlors une plume ou pointe métallique qui traverse le sommet d'un cône ouvert, rempli de noir de fumée et placé vis-à-vis le point zéro, d'où le cadran commence à se mouvoir, marque sur sa circonférence un point qui sert à faire voir à quelle seconde et fraction de seconde correspond le commence ment et la fin du temps qu'il s'agit de mesurer. Le jeu du mécanisme qui lance la plume n'arrête ni ne retarde le mouvement du cadran autour de son axe: le bouton de cuivre peut en conséquence être pressé plusieurs fois pendant la durée du mouvement, et fournir autant de points noirs dont chacun indiquera, par sa position, l'instant précis où il a été marqué. La petitesse de ces points esi telle, que l'on peut aisément estimer un quart de l'intervalle qui sépare deux divisions consécutives. Cette estimation sera d'autint plus exacte et facile, que le cadran sera plus grand. Elle a bien réussi dans la mesure de la vitesse dans les courses de chevaux, les machines en mouvement, l'eau courante, etc. Ro.

3o3. Handeiding tot de kemnis van den scheep sbouw (en hollandais), ou Instruction sur l'art de Ia construction des vaisseaux, à l'usage des ofliciers de la marine des Pays-Bas; par J.-C. Rrek. In-千 avec p]. Prix, 9 fl. Roterdam, I822. Arbon.

3of. Notice sur une nouvelle machine souftlante cmployée au Itartz; par M. Chapeyron, aspirant au corps royal des mines. $7 \mathrm{p}$. et $1 \mathrm{gr}$. ( $\mathbf{f n n}$. des mines, $\boldsymbol{t} .7$, p. 3.)

Cette nachine est un chapelet qui, au lien d'ètre cmployé a dever de l'eau suivant l'usage ordinaire, recoit de l'eau sur ses palettes at me certaine hauteur, entraine dans sa rotation une portion de lair compris entre les palettes, of comprime cet air dans un réservoir d'où il s'échappe par in tuyau.

D. B.

305. Descruptoon ats rste dine espice de mantean nommé Ducilimetre. pour estimor ce comparer lis 
ductilité de différens métaux; par M. Regnera.

2. p. 1t 1 gr. (Amn. des mines, 1. 7, p. 13.)

I'instrument dont il s'isgit vient d'ètre établi à Paris pour l'usage des douanes de France, afin de connaitre et de choisir les plombs les plus doux, propres au plombage des colis.

D. B.

3o6. Srecmicition of the patent, etc., ou Détails sur la patente accordíe à F.-MI. VAN IExtuersex, pour nue nouvelle méthode de faire mouvoir les bateaux et les voitures légires. (Rípert. of arts, manuf. and agric., janvier $1823, p .65$.

Linventeur a trouvé le moyen de remplacer les rames ordinaires dont un homme fait mouvoir une couple par le système de deux roues à rames, fixées aux extrénitís d'un axe, qu'un seul homme fair touner avec les pieds, à l'aide d'un mécanisme convenable. Ce systeme de deux roues à rames est assez léner pour qu'on puisse l'enlever et le replacer avec beaucoup de faciité. On en met deux sur un batean: l'homme qui fait mouvoir les roues de derriere, peut cn même tempss manouvrer le gouvernail. Avec les rames ordinaires, un liomme ne frappe l'eau que trente-quatre fois par minute, arec ces roues, il la frappecent vingt fois par minute, et l'on évite une perte de force considérable.

Le même mécanisme que l'inventeur adapte a l'axe de ces roues pour qu'un homme fisse tourner cet axe avee les pieds, il l'applique aux essieux des voitures légères qu'un honme ferait marcher.

G. C.

30z. Patente accordée à sir W. Coscriere, pour un nouveau procédé de consuruction des machines à vapeur. (London, jour. of arts and sc.,n. 25, p. 16.)

Ce procrudé consiste principalement dans l'emploi d'une roue a angets, tournant dans un fluide metallique, érlauffé a une tempéxature suffisamment élevere. Le mouvement est communiqué a la roue par la vapeur aqueuse, introduite au bas de cetic roue, qui s'eleve dans ses augets, el qui s'échappe quand elle est parvoute an point le plus bevé. 
Cette invention a beaucoup d'analogie avec une invention plus ancienne, lue à M. Cagniard Latour, et d'après laquelle l'a:r ćchauffé faisait tourner une roue plongée dans une cau également échauffée. Plusieurs raisons doivent faire présumer que linvention de M. Congreve n'est pas susceptible d'être utilement appliquée.

R.

3os. Onservatross on the evils, etc., ou Observations sur les mauvais résultats du systéme de construction. actuellement usité pour les roues et leurs essieux, moutrant que, par les effets ruineux de ce système, toute tentative pour le perfectionnement des routes devient en partic inutile. (The monthly Magazine, janv. 1823, p. 499.)

L'auteur, M. Hutchinson, annonce qu'il fabrique des voitures depuis un demi-siècle, et qu'il emploie depuis quarante ans une plus grande variété de voitures qu'aucune personne ne l'a jamais fait.

Cet article contient diverses observations sur la forme des roues et la construction des routes. L'auteur trouve surtout un très-grand inconvénient dans la disposition légale d'après laquelle toutes les voitures (à un petit nombre d'exceptions près ), doivent avoir leurs roues également espacées. Il pense, avec raison, que celte disposition facilite beaucoup la formation des ornières, et propose un nouveau réglement, d'après lequel l'espacement intérieur des roues varierait depuis 5 pieds 9 pouces, pour les jantes de 16 pouces de largeur, jusqu'à 4 pieds 9 pouces pour les chaises et gigs ayant des jantes de 2 pouces. Il propose aussi de placer dans les stages conches (diligences) les roues de devant et de derrière à une plus grande distance les unes des autres, et de donner plus de hauteur aux premières. Les voitures tonrneraient plus facilement, et le centre de gravité de la charge pouvant ètre placé plus bas, elles seraient moins exposées à verser. Ro.

309. M. Prorixs a dernierement obtenu une patente pour un perfectionnement dans la construction des machines à vapeur, ayant, comme il le pense, découvert certaines propriétés physiques dins la vapeur, et dans les lois deson action mécanique, jusqu’a préseut inconnues. Ces perfectionTone I. 
semens apportent me enomomie considerable dans le con-

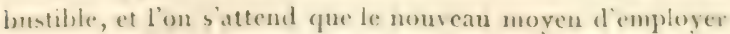

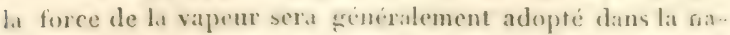
vigation. On eroit que le poists de la nouvelle machine, comparc a colui diune machine ordinaire de sueme foffel, sesa reluit an moins aux $\frac{3}{4}$; le mexanisme en est dailleurs extrimement simple, et sa construction met il Labride toule espéce de danger.

\ous avons su une machine en activite, construite d'apris les principes de M. Perhins; son piston moteur n'a pas plus de denx ponces de diametre et de douze ponees de course: sa force ćprouvée est celle de dix clievaux, et son volume a l'exception dn volant n'oecupe qu'une étendue sujerficielle de six a huit pieds. La chandiere est d'une construction et d'une forme chtierement nouvelles; et, quoiqu elle ne contienne pas julus de trente gallons, cette quantité d'ean suffit pour un long voyage.

Si M. Perkins ne s'ent pas trompé lui-méme par de fausae? domnés, ce qui souvent a dirangé les projets de soiłznenx praticiens, son invention, qui a recu l'approbation de plu sieurs ingénieurs pleins de talent, scra la plus importante des découvertes faites depuis la premicre invention des machines a vapeur. (The London jour. of arts and sc., n". 25, p. ifi.) b.

310. M. Herryarz, imprimour à Fifure a inventé une nouvelle presse próférable à colle de limig. Celle machine, quelion pent construire de toute grandens, et qui imprime jusqu'a luit fenilles en formes a la fois, fournit en 12 heures, de chaque feuille, 7, oon ipreuves, par consiquent des lmil feuilles, 56, (100 exemplaires imprimés des deux cotés. I a machine est facilemenl mice en mouvement par un cheval; trois hommes suffirent pour mettre le papier sur le chissis et pour l'en òter, saus arréter la machine, les formes imprimées se deplaceut d'elles-mines, et les antros se replacent. La machine est simple et solicle, par eomsiquent

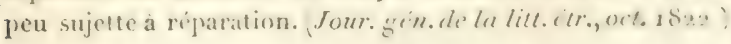

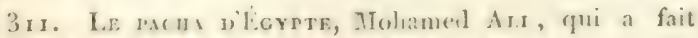

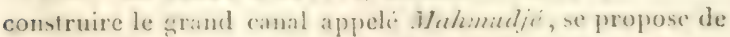
refalshir atussi colui fail par Darius et P'olomie jour la jonc-

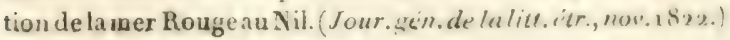


TOPOGRAPHIE, GÉODESIE.

3i2. Cocrs complet de Topographe et di créodésie, à l'usage de l'École d'application du Corps royal d'état-major; par P. M. N. Bevort, professeur adjoint de topographie et de géodésie a l'École d'application du Corps royal d'état-major, etc. Première livraison, Traité des Levés à la planchette, précédé de généralités sur les descriptions graphiques des corps eq du globe terestre en particulier. In-8. de r 3 f. et 6 pl. Prix, ffr. 50 c. A Paris, chez Barrois l'ainé.

Ce cours entier sera composé de so livraisons, et les matières distribaées de la manière suivante. Deuxieme livraison. - Traité des Levés al la boussole et des Levés au goniometre ( sous presse). - 3e. Traité d'Arpentage, comprenant les Leve's ì la chaíne et les Levés à l'équerre d'arpenteur, avec leur application a l'évaluation des surfaces et à la construction des plans et des édifices, des usines, etc. $-4^{\text {e. Traité }}$ complet de Vivellement. - 5e. Traité de l'Expression du relief du terrain sur les cartes topographiques, suivi de divers procédés pour construire les pluns et les cartes-relief. 6e. Traité du Dessin et du Lavis des plans et des cartes, contenant des problemes relatifs à leur réduction et a leur amplification. $-7^{\circ}$. Traité des Levés militaires, suivi d'une classification et nomenclature des formes du terrain, et de détails sur la rédaction des mémoires relatifs aux levés topographiques. - 8c. Traité des Oprirations trignnométriques, ou moyen d'obtenir le canevas trigonométrigue des grandes cartes topographiques. - y". Trati de Céchesse, ou moyen d'ohtenir le canevas des carter chorographiques et géographiçues, suivi d'un esai de metrolugite - 10e. Traité de la Construction des cartes géographiques.

Lianteur expose dans un pronier chapitre les divers procédés que l'on emploie pour reprisenter par le dessin les formes des corps, et particulierenent la suriace du terrain. II distingue spérialement les profits dir sections par des plans 
verticaux parallides, les sections horizontales on lignes de niverue, et les lignes de folus strunde inclinaison. 11 indique ba maniere dont ces procides sappliçnent a la description graphique des corps de divers genres.

Le deuxième chapitre contient des généralités sur les curtes destinces a representer la surface de la terre ou de ses parties.

On trouve dans le troisieme chapitre tout ce qui regarde les irhelles employérs dans le dessin des cartes.

Les six chapitres suivans sont destinés a la description de la planchette, a la rectification de l'alidade, et a l'application ditaillé de l'usage de ces instrumens, soit pour la levée des joluns, soit pour la solution de divers problemes de géndésie.

Cet ouvratre est rédigé avec méthode et clartí, et il offre dans une juste mesure les connaissances utiles aux personnes auxquelles il est destiné.

R.

313. Notice ser M. Messat, chef descadron au Corps royal des Ingénieurs géographes militaires, suivie de Notices sur la carte des ex-quatre départrmens réunis de la rive gauche du Rhin, et sur M. Tranchot, colonel au Corps royal des Ingénieurs géographes militaires; par MI. Argortr, capit. au Corps royal du Génir. In-s. de a f. $\frac{?}{4}$. Prix, r fr. Paris 1822. Chez Anselin et Pochard.

Ces notices offrent de lintérêt par les renscignemens qu'elles contiennent sur les progrès de la topographic et sur la carte des quatre ex-départemens seunis de la rive ganche du Rhin. La triangulation de cette carte fut exécutée avec toute l'exactiturle qu'on doit apporter dans les travaux de ce grme. Le figuré du terrain et les détails topographiques furent egalement trè-soisnés. Le terrain fut figuré suivant le systeme des lignes de plus grancle pente; les details furent levis a la bonssole, par des procédís nouveaux, plus expéditifs, et surtuut plus exacts, que ceux qui élaient connus antrefois.

La Notice sur M. Messiat fail commaitre la part que cet ingénieur-eriographe (ul daus ces travanx et dans le perfec- 
romnement des míthodes et des instrumens. Les Notices sur la carte des départemens font connaitre sommairement les risultats de la triangulation, et les règles que l'on suivait dans l'exécution des cartes-minutes. La Notice sur M. 'T'ranchot rappelle les principaux services par lesquels cet ingénieur-géographe avait acquis la réputation dont il jouissait dans son corps.

T.

3r f. Méxorne où l'on rend compte des expériences faites pour déterminer les degrés d'accélération du pendule, à diliérentes latitudes; par le Capit. Ed. SAuine, du corps royal d'artillerie (lu à la Soc. royale de Londres, le 8 mars $18_{21}$ ). (Addit. à la Connais. des T'emps pour I 825, p. 265.)

Les pendules dont s'est servi M.Ed. Sabine sont de Shelton et étaient sur le vaisscau du capitaine Cook, pendant le voyage de ce marin autour du monde. Les expériences ont été faites a Londres, aux îles de Brassa, d'Hare et Melville, dont les latitudes respectives sont: $51^{\circ} 31^{\prime} 8^{\prime \prime}, 4-60^{\circ} 9^{\prime} 42^{\prime \prime}-70^{\circ} 6^{\prime}$ $17^{\prime}$ et $74^{\circ} 47^{\prime} 12^{\prime \prime} 4$. La longueur du pendule battant les secondes a été trouvée de $39,13929-39$, 16929-39, 1984, et 39,207 pouces anglais; ce qui donne pour la diminution de lit pesanteur, o055066 - 0055082 - 0055r39 et oo55258, et pour l'ellipticité du globe terrestre un divisé par 31,4,33 I $1,2-3$ I 3,6 et 3 × 2,6 .

B.

315. M. Bexor, qui s'occupe arec beaucoup de zèle et de soins des observations barométriques et thermométriques, a obtenu sur quelques points aux environs de Màcon, sa patrie, les résultats suivans. Dans une partie de ses opérations il a été sccondé par M. Mathieu, son compatriote, membre de l'Académie des sciences.

Moyenne météorologique de Berzé-la-Ville, résultat de quatre années d'observations.

Hauteur moyenne barométrique, $733 \mathrm{~mm} .50=$ environ 27 pouces I ligne.

\section{Hauteurs moyennes thermométriques.}

Thermomitre (centigrade) intérieur, joint au barométre 14 $\mathrm{d} .62 .=$ de Réaumur. $11 \mathrm{~d} .6 \mathrm{~g}$. 
Thermomelre fentigrate extericur, 13 d. 3i $=$ de licinmur. 10 d. 6 \%

Fivellement par le caleul de's moy ennes barometriques.

Hauteur de Berzé au-dessus de

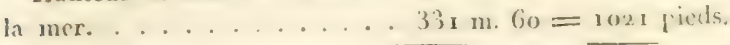

Hanteur de Berzé an-dessus de lObservatoire de Paris. ...271 $=834$

Hauteur de l'Observatoire audessus de la mer....... $\frac{65}{336}=\frac{200}{1034}$ différence.....4 $4 \mathrm{~m} .40=13$ pieds.

Deux procides differens, donnant des résultats si rappleochés, semblent garanlir l'exactitude de cetle mesure.

MII. Mathieu et Benon ont fixé ensemble les différences de niveau de plusieurs autres localités.

Élévationde Berzéau-dessus du baromère de M. Mallieu, placé au premier étagn de la maison $n^{\circ} .3$ de la rue SinintBrice, à Mácon, $155 \mathrm{~m}$. = environ 477 pieds 5 pouces.

Élévation du baromètre de M. MIathieu, au-tlessus du no ro de l'ancienne échelle du pont, 2 I m. $\mathrm{r}_{2}=65$ pirds.

Élévation de Berzé au-dessus du no so de l'échelle, i 75 m. $y=5,42$ pieds 5 pouces, ce qui donne à la Sasine 1 jug m. $82=499^{2}$ pieds d'élévation au-dessus du niveau de la mer. La hauteur de Berzé au-dessus de la maison de MI. Mathieu résulte de 26 olsservations, et c'est par un nivellement que MM. Mathieu et Favier ont obtenu l'ilévation du prenier éligge de cette maison au-dessus du no ro de l'échelle du pont.

Voici trois antres nivellemens qui ne résultent que d'une seule observation.

Au-dessus de la mer. mitres pieds "poutes

Hauteur du chàteau de Monceaux. $2.29,5=7235$

Id. du passage du bois Clairfarrond.

de Charolles). ................

1d. du télégraphe de Soligny. . $556=17$ I

An-dessus de la Saône.

Hauteur du château de Monceaux. 7 I, $7=220$

Id. du passage du bois Clair. . . 221, $5=680$

Id. du télégraphe de Sologny. . 3960 
Lilévation moyeme du harometre est, a Micon, de $\% / 87 \mathrm{~mm} .=27$ pouces $8 \mathrm{lig}$.

Id. ì Monceaux, $7 / 3 \mathrm{~mm} .=27$ p. 5 lig. $\frac{1}{3}$.

En résumé, l'auteur conclut que les plus hautes montagnes du Maconnais n'ont grucre plus de 2,000 pieds antichsus de la mer, el 1500 preds au-dessus de la Siúne.

(Ext. du compte renin le 6 déc. 1821 des travaux de la Sor. eles srienes, arts "t belles-lettres de Maron, pendiut s.s. par M. Chase de la Touche, serét. perpetuel; p. 5ti.:

3i6. Relatuon des opérations pour la mesure dim are d'un parallele de latilude, exécutées en 1892 e 1822 .

()n sait qu'apres avoir terminé l'opération impotate de la mesure d'un are du midien de Douhcregue a formentarat, Ic hureau des longhodes dirigea ses vues vers la mesure dun are d'un parallele de latitude le plus etendu possible.

Le aninistre de la gucre, à l.ı sollicitation du célébre Laplace, chargea en $18 \mathrm{x}$ I divers officiers du génie, de former une suite de triangles qui, commencant sur les rivages de l'Océan près de Bordeaux, viendraient couper le méridien de Jiris, et sétendre jusqu'aux rives orientales de l'A lritityu". On devait en méme tempes unir dans cette série et déterniner

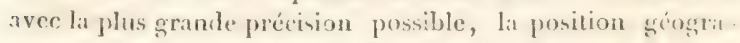

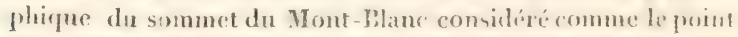
le plus remarquable en Enepe, et le plus propre at fixer la position diun premier méridien. Louvrage entrepris fut exécuté avec beancoup d'activité, de sorte guen risiá, la triangulation s'étendait, d'une part, de Finme à liroli, el de l'antre, de la Tour de Cordouan, prén Bordeanx, aux montafrines qui séparent la France de la Savoic.

lin 1820 M. de Laplace s'aclressi an gouscruement du l'iemont pour l'engager a urdonner une triangulation en Sa-

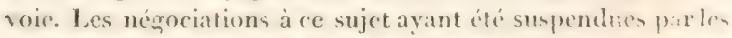
wenemens politigues, be gowvernement antrichien, borrpue labanquillité fut rétablie, s'offrit pour contribuer atus d.-

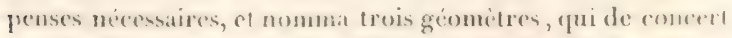

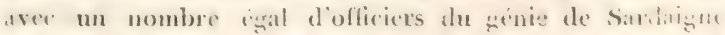

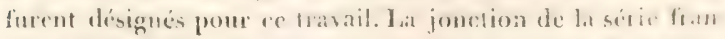

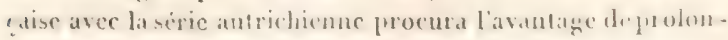


ger l'arc du parallele à neuf degrés de longitude an deli de Fiume en se servant d'une triangulation précédente, qui s'étendait depuis l'Adriatique jusqu iz Orsora en traversant la Croatie et l'Esclavonie; ainsi nous arons un are de 2.4 degrés de longitude, dunt 5 sont en France, 3 en Piémont, et I5 dans les états de l'empereur d'Autriche.

Cet arc est coupé a des intervalles presque égaux par les méridiens de Paris, de Milan et de Vienne.

Ro.

\section{ASTRONOHIE, COSHOGRAPHIE.}

3i 7 . Astronome des gens du monte, ou exposé du systeme planétaire, aree l'explication des phénomènes célestes, mis à la portéc des persomes qui ne sont pas rersées dans lés mathématiques, et suivi de la description et de l'usage des globes; par A. O. Panilut́. In-r 2 de 5 f. :; ; plus, 8 pl., 1822. Prix, I fr. So r. Paris, Delarue; Lille, Castiaux.

318. Trinté d'Astrovome théorique; par FrédéricThéodore Sirunert. 3 vol. in- $\{$, aree des planches. $2^{e}$ édition, 1 $\$ 22$. Saint-Pétersbourg, imprimeric de l'Académie des sciences.

La I $^{\text {re }}$ édition le cet ouvrage parut en allemand, en $r 79^{8}$. Parmi les motifs qui ont porté l'auteur à donner la seconde édition dans une langue qui n'est pas la sienne, il alliegue qu'en Russie, sa patric adoptive, dins les classes ou il pouvait compter sur des lecteurs, la langue francaise est bentuconp plus répandue que la langue allemande. Il ajoute que ce qui l'a complefement décidé, c'est l'aceueil flatteur fait en France a

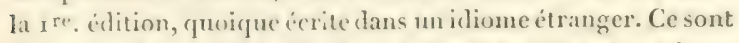
les savaus de ce pays, dit-il, qui mont cucouragét par leurs suffrauses a devenir le traducteur de mon propre ouvrage.

Le plan de cette dition est le méme que celui de la précédente; mais on a fait tous les changemens et toutes les additions qu'exigeaient les progrès de la science et les renseignemens communiqués à l'autcur.

I.e dessein de ce traite est diexposer les vérités astronomi- 
ques dans le mème ordre qu'elles ont été découvertes. Ce qui donne lieu aux trois divisions suivantes : L'astronomie sphérique, l'astronomie théorique el l'astronomie physigue.

Le premier vol. contient l'astronomie sphérique; elle est divisée en cinq livres dont voici un aperçu.

Livre $I^{e r}$. Sur le mouvement diurne. Moyens pour mesurer des angles; premières observations du ciel; la sphère avec ses cercles; détermination de la ligne méridienne; détermination de la hauteur du pôle; situation des astres relativement à l'équateur.

Liv. 2e. Sur le Soleil. Orbite a pparente du soleil; obliquité de l'écliptique; position des points équinoxiaux; position du soleil relativement is l'équateur; les saisons; les quatre époques principales du jour; longueur de l'année; longitude moyenne du soleil; longitule vraie du soleil; anomalie du même astre.

Liv. 3e. Sur la mesure du temps. Temps sidéral; temps solaire moyen; équation du temps; détermination du temps vrai par olservation; longitude géographiqqu; lever et coucher des astres.

Liv. $4^{\text {e}}$. Sur les parallaxes. Fignure de la terre; calcul des parallaxes dans la sphitre; calcul des parallaxes dans le spléroide elliptique; calcul des parallaxes suivant l'hypothèse de Bouguer; déterminatiou de la parallase par observation.

Livre 5e. Sur les réfractions. Déconverte des réfractions; méthode pour les observer; théorie physique pour les expliquer.

Le second vol. comprend l'astronomie théorique ou rationnelle; on y trouve une introduction et luit livres.

Introduction. On assigne les limites où finit l'astronomie sphériciue, et où commence l'astronomie rationnelle. Celle-ci, dit l'autcur, est entierement fondce sur cette proposition très-philosophique: c'est que les lois de la nature sont extrêmement simples, et qu'en consérquence si les mouvemens célestes nous semblent irréguliers, c'est que nous ne sommes pas placés au point de vue convenable.

Liv. I er. Sur les monvemens de lat terre. Son mouvement diurne; son mouvement annuel.

Liv. $2^{e}$. Sur les ćtoiles fixes. Distance, grandeur et posi- 
tion des ctoiles; heur mourement atplarent; leur mouvenent propre; antres proprieter remaryuables que meinente le ciel étoilé.

lir. 3". Sur les planeles. Phénomenes generand de ces corpes célestes: systimes planctaires; systeme de Copernic; cencle encentrique appliqui an soleil; le meme applirgue :ux

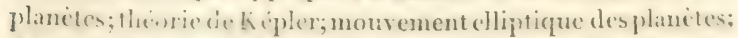
délemitation des orbites par observation; grandeur des Planètes; leur rotation; nouvelles planètes.

I.iv. qe. Sur la lune. Phénomenes grincraux de cet atslre; mois divers; apsides et excentricité de la lune; sa lonsiInte; nowuls et inclinaison de son orbite; son mourcment horaire et sit parallaxe; sa grosseur, sa rotation.

Liv. 5r. Jecultations et eclipses. Classification die ces phinomenes; éclipses de lune; éclipses de soleil en gencral: riclipse de la terre en ginéral ; éclipuse des différens lieux de la terre; edipse de soleil dans un lieu defernine de la tere: nsagers des éclipses de solril; iclipses ou occultations der civiles et des planètes.

Liv. 6*. Parssiges des planetes sur te soleil. Periodus de res passages; calcul des passages pour les divers lieux de la terre; calcul de's mèmes pour un licu déterminé de lis terre; parallaxes de vénus.

Liv. 70 Sur les satcllites. Découverte des satellites; leurs orbites, leurs éclipses, leurs autres élénens; satellites de saturne et d'uranus.

Liv. Se. Sur les cometes. Propriétés génesales de a. astres; calcul parabolique de leurs orbites; calcul ellyptirpue; reffexions gienérates sur le systeme solaire, oit lin tronve celte remarque de lauteur de la meanigue céceste (MI. de Laplace) : Les élumens du systeme solaire sunt oidhit. nés de maniere qu il doit jouir de la plus grante stabilitu.

le troisiene volume est destiné a lastronomie plysigure Nous ne l'avons pas sous les yeux; cependant, pour en don ner une idce suflisante a nos lecteurs, nous croyons qu it snd fit d'extraire yuelques passages de la priface de colle secomi: édition.

Liauteur y declare que les plus grand rhangemens qüil a litils a son ouvrage se trouvent dars la twoinieme partice. It rend phus de justice a hepler, con montrant combien ce phit 
losophe était près de découvrir la loi de lattraction. Il a suivi Laghra (l'autcur) au lieu d'Euler, dans ce qui concerne la précession et la rotation de la terre, ainsi que la libration de la Iune. Ses guides, pour la figure de la terre, ont été Macliurin 't Clairant, il a donné aussi un abrégé de la méthode de M. de Laplace sur le même sujet. C'est encore d'aprés ce dernicr géomètre qu'il a refondu entièrement la théorie des perturbations; il se flatte avec raison qu'une graude partic: de ses lecteurs lui sauront gré d'alvoir cherché à éclaircir plusieurs passages de la Mécanique céleste, ajoutant qu'ıl seri content si l'on veut bien regarder celte partie de son ouvrage comme un commentaire sur un texte ancquel personne ne porte une plus profonde vénération que lui. Pouvons-nous aussi nous flatter qu'on nous pardonnera d'avoir pent-être frunchi nos limites ordinaires, en fitisant cette annonce du traité d'astronomie de M. Shubert?

B. $\mathrm{x}$.

319. Astronomie de Fenguson, traduite de l'anglais en Bengali (langue du bengale), proposée par souscription au nom de trois Indous. I vol. ind'environ 200 p., sur le plus bean papier de Patna, avec des $\mathrm{pl}$. gravées en cuivre à Calcutta. Prix \& roupies pour les souscripieurs, et 6 roupies pour les non souscripteurs. (La roupie vaut à peu près $2 \mathrm{fr}$. fo c., ann. du bureau des longitudes.)

L'astronomie, disent les traducteurs, outre les services qu'elle rend ordinairement à la société, ne peut mancpuer de contribuer efficacement a détruire, dans l'esprit de nos jeunes compatriotes, les préjugés et les superstitions qu'une longue suite de siecles a dû y enraciner. (Voir, pour plus de détails, le I5e. vol. , p. 53, de l'asiatic Journal.)

32o. Dre entrerinen der Sonne von der Erde, efe. La distance du soleil de la terre, démontrée pax l.

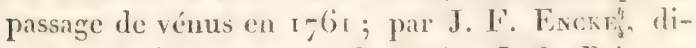
recteur de lobservatoire de Cotha. In-8. Pris: i rixd. $12 \mathrm{gr}$. Gotha 1822 . Becker.

I'auteur promet une snite a cet onvayge, qui contivithe les observations sur le passige de vinus en 1 f 69 . 
32. Dis m.ent und die Wrelgegenden, ate. La Jizmiere et les pays du shlobe, avee un traité sur les conjonctions des planètes, et l'étoile des trois mages; par J. G. Praff. In-8. Prix: I flor. $45 \mathrm{hr}$. Bamberg I $8_{2}$. Kunz.

L'auteur cherche a refuter la thérie de lit lumiere et des couleurs de Newton et à en démontrer l'erreur. Le second Traité est astrologique.

322. Uximstchungen über die Bahn des grossen liometen rom Jahr i $S_{2}$ I. Recherches sur la route de la grande cométe qui a paru en $182 \mathbf{2}$; par Fr.

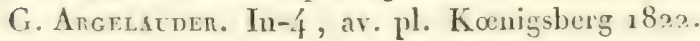
Borntrager.

Cette comete a ćté découverte, avant son passage près du soleil, par M. de Flauguergues; elle a ensuite éti observéo par M. le bar. de Zach ; et finalement, dans la derniere période, par II Wisniewshi, à la nourelle Tschulask, en Russie. 323. Astroxochroxoneter, or planisphere, ete. Astronorhronometre, ou planisphire des plus importantes constellations boréales dont on peut facilement apprendre les noms et la position en les comparant avec le ciel, pendant une seule nuit étoiléc; contenant les régles à suivre pour ohtenir la mesure du temps au moren de ces astres, des instructions sur la maniere de déterminer les rumbs de vent par l'étoile polaire, et lindication de différens objets utiles anxquels on peut, dans la vie ordinaire', appliquer lastronochronomètre et la connaissance des étoiles principales. Prix so $\frac{3}{2}$ flor. Londres, chez....

324. Métuone de M. Litrrow pour obtenir la latiunde diun lieu par des olserrations de hauteur de létoil. polaire, en un point quelconque de son cercle diune; par MI. Fraxcoech. (Bull. des Sc. de la Soc. Philomatique, oct. 1822, p. 1.17.)

"Cette méthode, dit M. Francaur, est non-seulement 
"dine facile application, mais elle a encore toute la préci" sion désirable. M. Littrow l'a exposée dans le $3^{\mathrm{e}}=$ vol. du "Journ. de $\boldsymbol{M}$. Lindenau, p. 208, et dans la Corresp. "astron.de M. de Zach, (n. x, I822, p. 70 ; etc.)"

Nous croyons devoir saisir cette occasion pour ajouter que M. Littrow, attaché à l'observatoire de Vienne, a publié une Astronomie théorique et pratique, en allemand, et quil en prépare une nouvelle édition.

B. $\mathbf{Y}$.

325. Remirques ser la latitude géocentrique des Américains, comme applicable aux occultations d'étoiles par la lune. (The quarterly Journ. of science, p. $4 \mathrm{I} 2$, etc.janv. 1823 .)

Des ingénieurs américains ont pensé qu’il serait plus correct de compter la latitude d'un point, par l'angle que fitit avec l'équateur le rayon mené du centre de la terre à ce point, que par l'angle de la verticale avec l'équateur ou la hauteur du pôle, comme on le fait toujours. Les États-Unis gagneraient, à l'adoption de cette manière de compter, un terrain de dix ou douze nilles en largenr, et de plusieurs centaines de milles en longueur, sur la ligne qui les sépare des colonies auglaises.

La latitude géocentrique est toujours moindre que la latitude vraic. On l'obtient en retranchant de la latitude ordinaire I' I / "multipliées par le sinus du double de la latitude; formule qui est réduite en table.

La considération de ce genre de latitudes, que l'on ne peut guère songer à substituer aux latitudes ordinaires, peut étre utilc dans le calcul des occultations. On voit, dans une opération de ce genre, une différence de $3^{\prime} 4^{6 \prime}$, entre 12 durée d'unéoccultation calculée avec ces latitudes d'une part, et par la néthode ordinaire de l'autre; l'effet de l'aplatissement de la terre étant de raccourcir la durée de l'occultation.

BA.

326. Situation grogriphique de Calcuta, Madras et Bombay, dans les Indes-Orientales; par M. J. Comprama ur, Fisf; dela Soc. Roy.-Différences des 
Inngitudes trouveres par les chronomitres, et les celipses des satcllites de jupiter, ete. par le mème.

Ces observations, jueres fort eractes, donnent :

Iongitude de lobserratoire de Madras (a l'est de Greenwich )............ 80 $80^{\circ} 17^{\prime} 21 "$

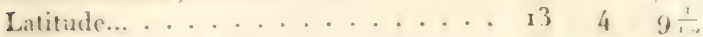
Longitude $d u$ fort William.. . . . . . . $88 \quad 23 \quad 39$ Latitude... . . . . . . . . . $22 \quad 33$

1.ongitude de Bombay (au fanal).... . $72 \quad 53 \quad 36$ Latitude................ I8 $54 \quad 25$

Iongitude de la pointe de Gallé(au pavillon). 80 r7 2

Latitude.............. 6 o 50

Longitude de Friar's hood.. . . . . . 8 8 ז $36 \quad 3 \frac{1}{4}$

Latitude... . . . . . . . . 72935

Iongitude de Canton............ I1 3 17 39

Toutes les latitudes sont boréales. (Journal of sciences lit. and arts, n. $28, p .386$.) BA.

327. Trois nonvearx oesenvatorres ont été établis dans des pays les plus éloignés les uns des autres : a Niholajen, aux bords de la mer Foire; au Cap de Bonne-Espérance, et à la Nouvelle-Hollande. (Jour. gén. de la lit. eitr., oct. I 822.)

328 . M. Insury de Chainpier, à la Trinité, vient d'inventer un instrument ingénieux pour déterminer la longitude. Il a été soumis à six officiers de la marine qui saccordent daus b'opinion (iu'il répond complétement au but qu'on se projose sur ierre ou sur mer par un temps calme. Mais ils sont décidement d'avis que son usaege est impraticable en mer par nn temps d'orage, a cause du mouvement violent du vaisseau. Cette objection cependant, si on ne peut y répondre, dest s'appliquer at tous tes antres instrumens de la mème esfece. M. Harley a porté le sien à Londres pour l'y faire examiune. Lit récompense proposéc pour un instrument partait pone la mesure des longitudes est, a ce que nous croyous,

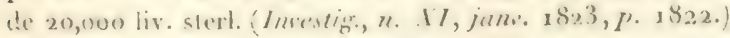




\section{P H Y I Q UE.}

3थ. Kлtтrsche Untersuchungen der allgemeinen polaritats gesetze. Examen critique des lois générales de polarité; par E. A. Nacmanv. In-8. Prix: I rxd. 4 gr. Lcipsic 1822 . Wienbrack.

330. Unterschenengen über den magnetismus der Frde. ete. Recherches sur le magnétisme de la terre, traduit du damois du professeur Hansteen, par Treschow Hanson. Tome I. In- 4 avecallas de 7 rart. r3 rixd.8. gr. Copenhague 1822. Gyldendal.

Ce premier volume, divisé en huit chapitres, contient tes phénomènes mécaniques de l'aimant.

33r. L'Elettrowotone perpetLo. L'Élcctromotcur perpétuel; par Gius. Zambon. Tome II. In-8 avec fig. Vérone x 822 . Merlo.

332. Onservations thermouétrieves faites dans la mine de Fahlun en Suede, par M. Cr.afs IValt.miv, anxquelles M. de Tolisertes a joint des remarques. (Me'm. de l'Ácad. des Sc. de Stochholm, I $82 \mathrm{x}, \mathrm{pp} . \mathrm{I} 6 \mathrm{~g}$ et $\left.\mathrm{I}_{7} 3.\right)$

Ces obscrvations, quoique bornées à un trop court espace de temps pour fournir des résultats positifs, nontrent cependant, suivant M. de Forselles, que les lumières des lan?pes nécessaires à l'exploitation des mines, éléve de plusieurs degrés la température de celles-ci, et que l'influence de celte cause augmente avec la profondeur des mines, parce qu' elle esi moins contrebalancée par l'action de l'air extérieur. Il s'ensuit, ajoute-t-il, rque l'on peut rapporter principalement à des circonstances accidentelles l'angmentation de chaleur observie dauns beaucoup de mines d'linrope et d'Amérique, à mesure que l'on y descend plus avant, augmentation qui a fait penser à quelques savans que la température propre de la terre allait en croissant dans la profondenr. Si l'on veut soumetter

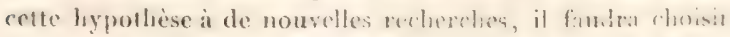

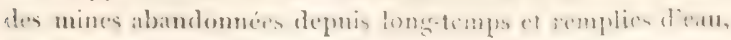


de sorte quelles soient complitement refroidies et a l'abri de font conrant d'air. Il faudra amen répeter les expériences as de conrts intervalles of a plunieurs reprises pendint l'annee entice, afin de juger à quel point les résultats sont subordonnés aux chanomens dans la température de l'air exterienr; et meme, comme il est probable que ces changemens agrissent, pent-ètre jusqu’a une profondeur assez considéralile, dins l'ean tranquille de ces mines, il conviendra de s'assurer, comme point de comparaison, de la température constante du sol, en observant celle des caves de lit manière ordinaire.

C. $\mathrm{MI}$.

333. Tísentat nes fxpíriences fiites par ordre du bureau des Iongitudes, pour la détermination de la vitesse du son dans latmosphere; par M. Aricio. (Add. it la connais. destemps pour $1 \$ 23, p .36 \mathrm{r}$.

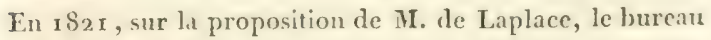
des longitudes nomma une commission prise dans son sein et composie de MII. de Prony, Bouvard, Arago et Mathieu, auxquels furent invités de s'adjoindre MM. de IIumboldt et Gay-Lussac, pour faire une série d'expériences réciproques, sur la vitesse du son dans l'atmosphère.

Ces expériences ont eu licu les 21 et 22 juin 1822 vers les $x$ heures du soir par un temps assez calme, entre Yillejuif et Montlhéry. Le son parcourait la distance du canon de Villejuif à la tour de Montlhéry, trouvée par Mr. Arago de 95.9 toises, 6 , dans la durée de $5 \%$ ", 6; ce qui donne pour la vitesse du son I 74 toises, $9^{\circ}$ par seconde sexagésimale, sous une pression de 756 millimetres, 4 , lat température étant de r $\tilde{y}^{\prime \prime}$ g centigrades, et l'hygrometre marquant $72^{\circ}$. Ia diminution de celte vitesse, pour charne degré d'abaissement du thermomitre, est de o toise, 32 I. A $10^{\circ}$ de tempréralure, lit vitesse du son, trouve en 1738 par les académiciens francais, ne surpasse que de o toises, $83^{\circ}$, celle résultant des expériences précedentes ramenés a cette tompérature, vitesse qui est de $1-3$ toises, 10 ou de 337 métres, 2 , dans une seconde sexagésimale.

M. Rieussec ansistait aux expériences du 22 juin, pour faire l'essai de son chronograple décrit au n." 302 du Bulletin.

B. 
334. Sur lascrision nes nuaces dans l'atmosphire; par M. Fresnel. (Bull. des Sc. de la Soc. Plilom., oct. 1822, p. 159.)

L'air et tous les antres gaz incolores ne s'échauflent que par le contact des corps solides ou liqquides dont la température a été dlevéc par les rayonı solaires ou le calorique rayonnant. Cela posé, Soit un nuage formé de très-petits ghtolunles d'eau ou de cristaux de neige excessivement déliés. L'air compris dans l'intérieur du nuage ou très-voisin de sa surface pourrat devenir plus chaud et plus dilaté que l'air environnant; il devra donc être plus légrer. Lorsque le poids total de l'ean et de l'air du nuage sera moindre que le poids d'un volume égal de l'air environnant, le nuage s'élèvera jusqu’à ce qqu'il parvienne à une région de l'atmosphère où il y ait égalité entre ces deux poids. Voir, pour plus de déveIoppemens, le texte de M. Fresnel, également dans les $A n-$ nales de chimie et de physique, nov. 1822 . B. $\quad$ B.

335. Extrart d'un Mémone sur la double réfraction particulière que présente le cristal de roche dans la direction de son axe; par M. A. Fresnel. (Bull. des $S_{c}$. de la Soc. P'hilom., déc. $1822, p$. 19г.)

Cet exlrait forme près de 8 pages in- 4 ". Le mémoire auquel il se rapporte à été lu par l'auteur ( MT. Fresnel) à l'académic des sciences, le 9 décembre.

Lat polarisation de la lumiere est une dicouverte toute française, due au célètre Mlalus. Lille avait reçu de grands développemens par les expériences de MIM. Arago, Biot et Brewster; clle en doit de nouve:auxà M. Fresnel, qui, danases dernieres recherches, est parvenu à plusicurs thiorimes fort curicux et remarquables.

B. $\mathrm{x}$.

336. Note st k LA Dozble néfraction du verre comprimé; par M. Fresnel. (Bull. des Sc. de la $S o c$. Philom., sept. 1822, p. 139.)

M. Brewster a le premier reconmu qu'on pouvait donmer an verre, en le comprimant, la propriété de coloes lit lumiere polariséc. 1)e trés-habiles physiciens n'avaient pas considtéré les expériences de M. Brewster comme une grenve Tоме I. 
suffisante de la bifureation de la lumiere; mais f'apries ses propres expériences, M. Fresnel avone qu'il ne lui reste plus aucun doute sur lexistence de la double refraction dans le verre comprimé et la séparation angulaire de la lumiere en deux faisceaux distincts, lorsqu'clle le pénetre sous une incidence oblique.

B. $x$.

33-. Sir l'ítit ne l'eau et des substances aíriformes qui se trourent dans les carités de certains Cristaux; par sir IIumphry Divs. ( Tr. Ph. pour 1823, et Ann. of Philosophy.Janv. 1823, p. 13.)

Plusieurs échantillons de cristal de roche contiennent daus leur intérieur des cavités tout-a-fait isolées et remplies en tout oụ en partie d'un liquide dont lépoque du dépôt scmble. remonter juscu'au temps de la formation du cristal. M. 1)avy a analysé ce liquide qui s'est trouvé de l'cau presque pure, avec une très-petite quantité de sulfates alcalins. Le gaz était de l'azote pur, autant que M. Dayy put s'en assurer avec d'aussi petites quantités. Ce gaz était toujours fort raréfić et son volume se réduisait généralement à $\frac{1}{5}$ ou $\frac{1}{i n}$ du volume primitif. Cette expansion des gaz est importante par les aperçus qu'elle pourrait fournir sur la température a laquelle ils ont été renfermés dans ces cavités.

MI. Davy chercha done à répéter les mêmes expériences sur des cristaux dont l'origine est ordinairement rapportic au feu. Le liquide fat toujours trouvé être de l'eau presque pure, le gaz de l'azote; mais il ćtait beaucoup plus raréfie que dans les cristanx précédens. Il était entre 60 et $z^{0}$ fois plus rare que l'air atmospliérique et les cavités contenaient proportionnellement une plus grande quantité d'eau.

Pour savoix si le gaz renfermé n'avait point été orighinairement de l'air ordinaire dont l'oxigèene aurait été absorbé par l'eau, une calcédoine percéc fut mise sous de l'eau puraxír d'air dans un récipient; elle fournit du gaz qui, examiné par l'aride nitreux, scmblait contenir autant d'oxigene que l'air ordinaire, l'eau de la picrre ayant probablement abandonné dans le vide, l'oxigène qu'elle avait absorbé.

M. Davy pense qu'il est possible qu'une combinaison d'ean ot de silice existe a l'blat liquicle à une haute pression et a 
nne haute température, tenant de l'air en dissolution; l'air et l'eau s'en sexaient séparés par le refroidissement.

Dans un appendix, M. Davy amonce asoir trouvé un cristal dont la cavité était tout-à-fait vide de gaz, et un autre qui contenait du gaz comprime dix a douze fois plus que l'air atmosphérique: le liquide de celui-ci était de l'eau, mais dans le précédent se trouvait un liquide visqueux de nature différente.

BA.

338. Notre sur une disposition particuliere de la glace; par M. Creric, ingénieur des mines. $6 \mathrm{p}$. et x grav. (Ann, des Hines, tome: VII, p. 15.)

Espece d'efflorescence ou de végútation de glace en petits faisccaux ou cylindres cannelés, disposés en conches séparées par des conches d'argile, et s'élevant jusqu'a 8 décimètres au-dessus de la surface du sol; phénomène analonne à celui que M. Desmaret asait observé et décrit dans le Journal de Physique, de mars 1783 . B. D.

339. Extratt d'en mémone de M. Ampère, sur les phénoménes de l'électro-magnétisme. (Bull. des Sc. de la Soc. Philom., oct. I822, p. х 45.)

$\mathrm{I}^{\mathrm{re}}$ partie : Résultats de trois expériences que $\mathbf{M}$. Ampire a faites a Genive avec M. Aug. de La Rive. II' partie : conséquences déduites des lois trouvées par lui en 1820 , prar rapport à l'action mutnelle de deux conducteurs voltaiques.

Enoncé des trois nouveaux faits contenus dans la Ire. partie: $I^{\circ}$ Les différentes portions d'un mème courant électrique rectiligne se repoussent mutuellement comme dans le cas oir ce courant parcourt successivement les deux cótés d'un angle quelconque, en passant de l'un à l'autre par le sommet de cet angle. $2^{\circ}$. Un conducteur fixe plié en are de ccrcle dans un plan horizontal ne pent exercer ancune action sur un conducteur mobile d'une forme quelconque, si ce conducteur ne peut se mouvoir qu'antour d'un axe vertical passant par le centre de l'arc. $3^{\circ}$. Il s'établit dans un conducteur mobile formant une circonférence completement fermée, un courant électrique par l'influence de celui quion produit dans un conducteur fixe circulaire redouble, place 
tru-prex du conductenr mobile, mais sans communication avec lui.

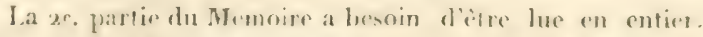
Yous sommes forcen dy renvoser nos lectents. B. S.

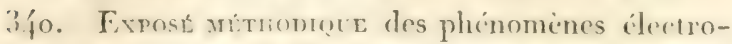
dynamiques, ct dis lois de: ees plónomines. (Bull. des Si. dela Sor. Philome, no: $1822, p .157$.

On doit a M. Oryted la découverte de l'action dircetrice des comlucteurs voltaiques sur les aimans; a M. A ragen, celle de la fropricte quont ces mémes conducteurs, de rendre mannelirue, le fer et l'acior; a MI. Ampere, tout ce qui est relatif a leur artion mutselle et a la force qui est exercie sur enx par le silobe terrestre, ainsi que la rolation d'ua aimant ou d'un fil conducteur autour de son axe; a M. Faraday, la notion de l'action révolutive, toujours daus le mème seus, qui a lieu entre un conducteur et un aimant.

L'ordre que nous venons de suive, est celui des découvertes; M. Ampèse, dans son nuscau travail, a préfiri lordre naturel ou celui qui convicnt a l'exposition methodique des faits, et nous pensons qu'il a eu raison.

Ce mémoire, de 11. Ampere, a été imprimé isolément in- 8 d'une feuille, chez M. Plassan.

B. $\mathrm{x}$.

34 . Expénences ex oesenvations sur le développement du magnétisme dans lacier et dans le fer par la percussion; par W. Sconesux, jun. esi. (Trams. I)ill. 1822 , part. 11 .)

Des barreaux d'acier doux, tenus verticalement et frappés avee un martean, s'aimantirent par l'action de la terre. lietlet edait d'abord progressif, mais apres un certain nomlie de coups, en continuant de frapper, on n'ajoutait phus xich it la force misnuctique. Avec quatre barreaux aimantés jusquat un certain point par cette mithode et denx contacts de fer doux on peut aiuanter ces barranx à saturation. Ba.

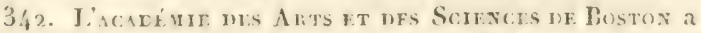

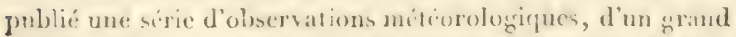
intert. Flles sont le remeltat de trente-trois années d'exper-

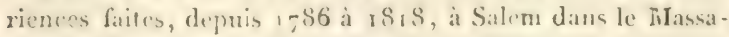
chuset. (Jour. gin. de la lit. eitr., oct. 182.2.) 
CHI MIE.

343. Grendnuss des systems der Chemic, etc. Plan d'un système de chimie, ou classification des corps simples et composés, d'après Lavoisier et Bexzélius ; par A. Limpidies. In-8. Prix, I rxd. $18 \mathrm{gr}$. Freyberg 1822. Craz.

34任. Description du laboratoire de chimie de l'icole d'artillerie de la garde royale, construit sur les plans de M. d'Arcet; par le capitaine Brianchon. (Extr. des Ann. de l'industrie nat. et étrangère. Sept. 1822. ) In-8 I f. - et 2 pl.

345. Extrate d'en mévorne sur la carbonisation du bois; par M. le chev. de la Chabedessiene. $20 \mathrm{p}$. et I pl. (Ann.des Mines, t.VII,p. 246.)

Ce mémoire indique cinq méthodes différentes employée. pour la carbonisation du bois, et particulicrement une méthode due a MI. Foucauld, perfectionnée par M. de la Clatbearnssière, et qu'il regarde comme préférable à toutrs les antres. L'anteur décrit les fourneaux et appareils de cette opérat. tion, dont les plin et coupes lithographiés sont joints à lix livraison.

B. $\boldsymbol{~}$.

346. Nouvelles expénences sur l'huile volatile d'amandes amères; par MI. Robiquet. (Extrait du Bull. des Sc. par la Soc. Pluilom。, oct., p. 150.)

347. Str lrs Sulferas qui proviennent de la réduction de quelques sulfates, par le mojen du charbon ; par M. Bertuier. 24 pag. (Aniz. des Mines, I 822,3 livi. , p.)

L'auteur a fait un grand nombre d'expériences en chauffant les sulfates dans des creusets brasqués de charbon, mais sans les mélanger avec le combustible. Il obtient par ce moyen des sulfures à un grand état de pureté et que l'on peut recueillir sins perte; de sorte qu'il peut conclure, par différence, le poids des matiires volatiles dégagées pendant 
Poperation. Il trouve que pour les sulfiles de baryte, de strontiane, de claux, de sonde et de potasse, la perte est precistement igale au poids qui représente la somme des quantités d'oxignène contemues dins l'acide et dans la base. Il conchut de ce fait, el d'autres expériences exartes qui concordent arec lui, que, dans les sulfures, les alcalis et les terres alcalines sont dépunillés d'oxierene, ou à l'itat métallique. M. Berthier a également reconnu la déromposition de jhusicurs sulfures par le charbon, et la formation d'un sulfure de carbone. Enfin ses expériences l'ont conduit a reconnaitre l'existence et la proprićté de plusienrs sulfures a double base, qui n’avaient pas encore été observés: $\quad$ D. B.

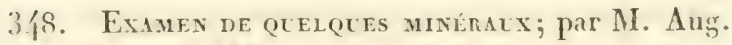
Arfwenson. (IÉm.de l'Acad. de Stuchholm, 1821. p. 147.$)$

En soumettant a une nouvelle analyse la cyanite cristallisée prismatique du Saint-Gothard, l'auteur a trouvé que ce minéral était composé de

oxygene.

Silice. ... : $34.33-17.26$

Numine. . . 64. $89-30.31$

Ce qui, dit-il, s'accorde bien avec la composition du silicate bi-aluminique (silicias bi-aluminicus) qui est

Silice.... 3 $3 x \cdot 7 \mathrm{r}$

Alumine. . . 68, 29

la cyanite de Reraos en Torwege contient les mèmes terres dans une proportion à peu près semblable.

L'analyse de la néphéline par M. Vaupuclin et celle de la cyanite par Kklaproth avaient présenté des résultats tellement conecordans qu'on aurait cru pouvoir rapporter ces deux minérans à une méme espece. Cependant leur aspert extérienr suffit pour indiquer une grande différence cntre enx et le chalumea semblait entre les mains de M. Berzedius avoir confirmé cette indication.

Fn effet, il. Arfuedion a trouve dans la néphidine en prisme à six pans du Vésuve 
oxygene.

Silice.... 44. 11 - 22. 29

Alumine . . 33. $73-15.75$

Soude.....20. $46-5.23$

Le méme chimiste ayant analysé aussi la sodalite du Véstve a reconnu qu'elle était composée de la manière suivante.

oxygène.

Silice. . . 33. $7^{5}-16.97$

Alumine... 35. $50-16.58$

Soude....26.23-6.07

Acide muriatique ... 5. 30-3.09

C. M.

349. Sur la nature des Scories des forges catalanes et des foyers daflinerie; par M. Bentmer, ingénicur des mines. (Ann. des Mines, $1822,3^{\mathrm{e}}$ livr. )

L'auteur, qui a annoncé, il y a déjà long-temps, que les scories de forge étaient composées de silice et de protoxide de fer, et qu'on pourrait, en les fondant au haut-fourneau, en retirer autant de fer que des meilleurs minerais, fait connaitre aujourd'hui les résultats de l'analyse de 3 r scories de forge différentes, provenant: $\mathbf{I}^{\circ}$. đu travail des anciens; $2^{\circ}$. des forges calalanes actuclles; $3^{\circ}$ des grosses forges d'affinerie, dans lesquelles on convertit la fonte en fer en une seule opération; 4". des petites forges d'affinerie, dans lesquelles l'affinage se compose de deux opérations distinctes. Toutes ces scories lui ont donné de 36 a So pour cent de protoxide de fer, et tontes, excepté peut-îtrecelles des forges catalanes, pourraient être tratées avec un grand avantage dans les hauts-fourneaur. L'autcur calcule l'économie qui en résulterait pour les maitres d'usines. et indique les fondans qui conviendraient a chaque espèce de scories.

D. B.

350. Sili l'ixploitation des makis dela Corse, pour en fabriguer de la potasse; par M. Gizхмиво, ingén. des miness. (Lun.des Mines, $\left.t . V^{\top} I I, p .26 ;.\right)$

Celle notice extraite d'un rapport général sur une mission semplie en Corse par l'auteur, indique, comme une tes phlas 
belles andeliontions dont lit Corse soit susceptulse, lat fabrication en grand de la potasse, au moyen de l'exploitation des makis (bois de 7 a 8 pieds de hauteur) qui convent une grande partie du sol, qui se renouvellent en 5 a 6 ans, et dont les cendres donnent $0,1,5$ de salin. J'auteur donne les ditails des procides ficiles quon peut suive pour cette fatbrication.

D. B.

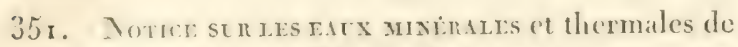
St.- Iectaile; par M. P. Bertumen, ingén. des mines. (Ann. des Wines, tom. VII,p. 208.)

MI. Berthier donne un apercu fort interessant de la contrie oi est situé St. - Nectaire dont less caux minirales étaicut connues des Romains et dont bn trouve cucore d'anciemnes construrtions appropriées à l'usage des bains. Il cite les lieux remarquables et les monumens intéressans qui méritent l'at tention du voyagenr. Il indique la température des diverses sources de Saint-Vectiire et donne lanalyse detaillie des eanx et de leurs dépôts.

I.

35\%. AxAlyse Des eAtx mixtrales et thermales du Mont-dOr; par M. P. Berturer, ingén. des mines. (Ann. des Mines, tome VII, p. 20I.)

L'auteur fait précéder cette analyse par un apercudes embellissemens et des constructions nouvelles que reçoit le village du Mont-d'Or. Il cite le nouvel édifice thermal qui ne le céclera pas aux plus belles constructions romaines.

Lat source du puits de César, fournil 56 méres culuen d'eau par 24 heures. Elle marque $45^{\circ}$ therm. cent. J'anteur decrit avec soin l'etat de la source et donne cnsmite les résultats de l'analyse de l'eau qu'elle fomrnit; puis cemx de lanalyse d'une matice siliceuse et ocreuse quise dépose dans des conduits sonlermins ou dans le pruils; et par occasion l'analyse d'un dépot analogue quion ramasse dans a grande source salée de Mouticrs en Tarentaise. IV. 35.). Note sur la présence de l'iode dans l'eau minérale do Silles, en Piémont. (Bull. des Suc. pur la Sc. Philom., déc. , p. rgo.)

On vient de conslater la présence de l'iode dans les eanx mincrales de Sules, province de Vostera, en l'iémont; et 
Mi. Duponchel, membre de la société médicale d'émulation de Paris, nous a fait connaître les résultats des recherches fitites à ce sujet par plusieurs hommes de mérite, et consignćes par le docteur Berrini, dans un ouvrage estimé sur les eaux minérales de Sardaigne.

354. Or trouve dans les Annales desmines, tom. 7, p. 22 ; et suiv. les analyses chimiques des substances mincírales dont voici l'énumération.

$\mathbf{1}^{\circ}$. La Caluate ou Turquoise de Perse; $2^{\circ}$. les Amphituoles; 3०. les Proxines; $4^{\circ}$. Ia Pyrallolithe de Pargas; $5^{\circ} .1^{\prime}$ Épidote; $6^{\prime \prime}$. les Grenats; $7^{\circ}$. les Idocrases; $8^{\circ}$. les Micas; $9^{\circ}$. l'Eesensinter de Freyberg. (Eisenpecherz de Klaproth.)

ıо". Sur la P'imélithe de Kosemütz; $11^{\circ}$. sur l'argent sulphuré aigre. (sprod-glauzerz.)

$12^{\circ}$. Sur la Sordewalite par M. Nondenskrou.

Nola. Ces divers articles sont extraits de louvrage de II. Berzélius, intitulé de l'Emploi du chalumcou dous les analyse's chimiques, etc. i vol. in- $8^{\circ}$. à Paris, chez Nićquignon-Marvis.

355. Analyse chmieue du uatt des chevres de Cachemire, importées par MII. Ternaux l'ainé et A. Jaubert; par M. Burrees, chef des traviux chimiques à la faculté de médecine de Paris.

Il résulte des expériences faites par M. Barruel, que les proportions butireuses du lait de chèvre sont:

9 prarties sur rooo pour les chèves de Cachemire nies en France.

8 sur 35 pour celles de l'mportation Ternaux el Jauberi.

7 sur $9^{5}$ pour les métis, provenizut du croisement avec les indigènes.

5 sur 50 pour les chèvres indigènes.

M. Barruel en conclut :

1\%. Que le lint des clìvres de Cachemire est beancoup plus riche en matiere sucrie que celui des chères indigènes.

$2^{\circ}$. Que lis matière casiuse y est beaucoup plus délicate ct par conséquent plus digestive.

30. Que la matiere butireuse y est aussi trés-abondante, moins âcre et bcaucoup plus agréable.

4". Enfin il est probable que les médecins retircront un 
grand avantage de l'usage du lait de ces chevres, dans les cas de maladie, pour lespuels ils ordonnaient le last de cheve ordinaire. (Moniteur du 26 janvier 1823 .) I.

\section{GEOLOGIE.}

356. Essiy ox ture Theory of the Earth by M. Cuvier, ete. ete. Essai sur la Théorie de la Terre; par M. Cuvien, secr. perp. de I'Institut, ete. tracl. en anglais, avec des notes minćralogiques, et un précis des découvertes géologiques de M. Cuvier, par le prof. Janeson. I vol. In-8 avec $6 \mathrm{pl}$. 行. édit. Prix a 2 sh. Edimbourg. Blackwood.

35-. Die Unwelt und dis alterthum, erlahtert durch die Naturkunde, ou le Monde primitif et l'Antiquité expliqués par l'histoire naturelle; par H. F. Lisk, professeur de médecine à Berlin, directeur du jardin botanique. Tom. I, 89 I. In-8 de $350 \mathrm{p}$; ; tom. II , $\mathrm{r}_{22}$, de $303 \mathrm{p}$. Berlin.

Cet ouvrage, très-connu en Allemagne l'est très-peu en France et dans le reste de l'Europe, il mérite copendint de l'ère, et il est précieux par la quantité d'indications qu'il fournit, et les rapprochemens qu'il présente. La r re. sect. du I er. volume renferme des recherches sur le monde primitif, basées sur les corps pétrifiés qui composent la croute du globe. Les $2^{e}$. et $3 e$ e sect. s'occupent de la dispersion de lhomme ef des corps organiques sur la surlice laprestre. La fi". sect., des langues consillérées comme signnes de la dispersion. La 5e., de la patrie des animanx domestiques et des pliantes usuelles. La 6re sect., de l'exploitation des

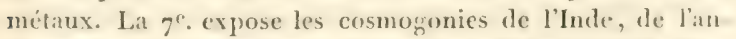
cicnne P'erse, la création de Mloise, les mythes phénicienues les cosmogonies des Babyloniens, des Ryptiens, les mythes greçues, et se termine par des considérations génirales sur ces diverses cosmogonies.

Daus le 2e. volume, qui a parn, i ce qu'on dit, col 1822, les $3 \mathrm{I}^{\mathrm{ret}}$, sect. renferment des atditions at celles du $1^{\mathrm{er}}$. wol.; dans les 3 sect. suivintes, M. Link soccupe des changemens suhits 
par le globe dans les temps héroüques; il examine les traditions sur tous les déluges, les irruptions de la mer, l'atlantide. L'auteur s'occupe ensuite des exhaussemens des terrains bas et de l'abaissement des terrains élevés. Il discute toutes les opinions à l'égard des animaux fabuleux; l'état des connaissances chez les anciens, ce qu'il savait sur les produits du Nord.

M. Link se décide, dit-on, dans ce 2e. vol., pour l'unité de l'espèce humaine, et regarde l'Afrique comme en étant le berceau. Il croit que le déluge de la Genése n'a été que partiel, etc. Il faut lire dans l'ouvrage l'ensemble des questions qui y sont traitées.

$\Lambda$ cette occasion nous annoncerons ici une critique de la $r^{\prime}$. partie de cet ouvrage intitulé seconde lettre adressée is la Socitite Asiatique de Paris par M. L. de L'on, ancien officier de cavalerie, $8^{\circ}$ de 45 p. Paris. Chez Dondey Dupré. (La $I^{10}$ lettre était relative à l’ouvrage de M. Adelung, neveu du célébre auteur du Mithridate, intitulé Aperçu de toutes les Langues connues et de leurs dialectes.) Dans cette seconde lettre le savant M. de L'or, dont la science trahit le secret de son véritable nom, combat avec des armes victorieuses les opinions de M. Link sur le nombre des races humaines et l'origine des diverses langues; mais il $\mathrm{P}^{\mathrm{pa}-}$ rait que M. de L'or n'a pas connu le $2^{c}$. vol. de l'ouvrage du savant Link, de Berlin, qui n'est point encore parvenu à l'aris. L'extrait de ce 2.e vol. que nous venons de donner, est tiré de la Revue Encyclopédinque qui, sans doute a reçu ce volume de Berlin, en communication, avant sa public:tion.

F.

35. Fssai Géognostigue sur le gisement des roches dans les deux hémisphères; par Alex. de Huıвozdo. Un vol. in-8. de 379 p. Prix, 7 fr. Paris, 1823 , chez F. G. Levrault.

I'n article composéprar. M. de Inumboldt pour le Dictionnaire des sciences naturelles, et inséré au mot Indipendance des formations mérituit sans doute d'ètre donné au public comme un ourrase sépare; en effet, cet articte

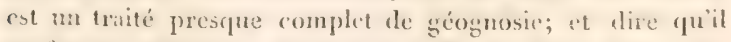
senferme l'ensemble des fitts que M. de Humbolatt at re- 
cucillis dans ses nombreux voyigges, ainsi que les résultats: fénérax que ec savant celebre a obtenus de ses observa-

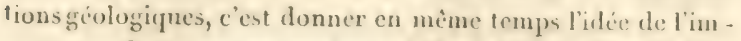
portance de l'ourrage et de la difficulté d'en firire l'objet d'une courte analyse.

Liun des buts que l'antenr s'est proposés a été de faire connaitre la structure géognostique de l'Ancirique, on lit comparant a celle de l'ancien continent; il a voulu anssi faire ressortir de tontes ses observations la véritable idée qu'il faut prendre de la composition générale de l'ícorce terrestre, et la difficulté que l'on rencontre dans la niture a ítablir des lignes de démarcation tranchies entre les froupes ou séries de couches auxquels on est convenu de donner le nom de formation.

II. de Humboldt a adopté la division générale de terrains primitifs, termains de transition, terrains secondaires, turains tertiaires; et an lieu de placer à la suite de ces diverses clisses et comme hors lisne celle des terrains voleaniques, il la met immédiatement à lat suite des terrains de transition, mais parallelement avec celles des terrains secondaires of tertiaires; et cette disposition dicolomique est la cousequence des rapports nombreux qui lient, par leur nature, d'une maniere intime, beancoup de roches, des terrains de: transition et mème primitifs avee les produits des volean, bien que par leur époque de formation ces produits soicnt contemporains des terrains secondaires ct tertiaires.

L'ouvagge est terminé par l'exposition d'une méthode pasigraplique, employée depuis lons-temps par M. de Humboldt, et qui donnerait un moyen facile d'exprimer jar des signes les rapports de superposition des roches et ceux des formations entre elles.

C. $P$.

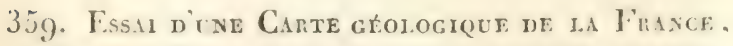
des Pays-Pas, et de quelques contrées voisines; dressée par J. J. n'Omales n'IILloy, d'aprén des matériaux recueillis de concert aver M. le barom

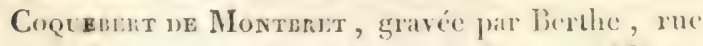
Saint-Jacques, 12. 66, à Paris. (Amll. des Mlines, 
$1823,3 e$ livr., p.) Celte (nite est accompasné d'Observations, par M. d'Omalius d'Halloy.

L'iutcur ne donne cette carte que comme un premier ésai, et pour inviter les géologues a en perfectionner les differentes parties. 11 expose les motifs quil'ont porté à sacrifier à l'uniformité le désir de présenter les détails ruil possédait sur plusieurs contrées, détails dont il a consigné une partic sur une autre carte d'une plus grande échelle, qui se trouve chez II. Delamarche, rue du Jardinet, $\mathrm{n}^{\circ} . \mathrm{I}^{3}$, a Paris. M. d'Halloy fait connaitre ensuite les principes qui l'ont guidé pour réunir les terrains en groupes généraux qui pussent être distingués sur la carte par des teintes de coulours différentes. Ces groupes sont au nombre de six seulcment : le premier comprend, sous le nom général de terrains primordiuux, la rémion des terrains primitifs et des terraius de transition parmi lesquels l'auteur range les terrains houilliers du nord de la France et des Pays-bas. Le second groupe, désioné sous le nom de terrains péméens, comprend le todtcliegende ou grès rouge ancien; le macigno des Toscans, ct plusieurs terrains houilliers, notamment ceux du centre de la France. Lituteur nomme terrains ammonniens son 3c. groupe, sous lecguel il range le zechstein ou ancien calcaire des $\mathrm{Al}$ pes, le griès bigarré, le muschelhalk, le queuder santstein et le calctire jurassique. La formation de la craic, en y comprenant les tuffeaux, les sables et les marnes, qui sont au-dessus de la craie proprement dite, constitue à elle seule un groupe, sous le nom de terrain crétacé.

L'auteur réunit dans le 5e. groupe, sous le nom de terrains mastozootique's, tous les terrains postéricurs à la craie, dont l'origine aqueuse n'est pas contestée.

Finfin le $6^{\circ}$. groupe, ou celui des terrains pyroüdes, comprend les produits des voleans actuels, ainsi que les terrains, basaltiques et trachytiques.

v. $B$.

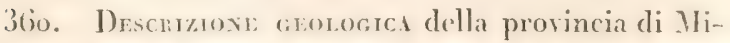
Jano, ete. Discription géologieque de la province dr Milan, publiće par ordre du gouvernement de Lom--

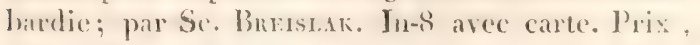
6 lire. Milan 1822. 


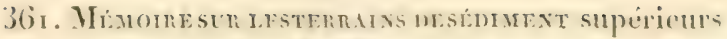
calcarén-trappéens du Vicentin, et sur quelques terrains d'Talie, de France, dillemagne, etc. qui pertvent se rapporter a la mème éporque, par Ales.

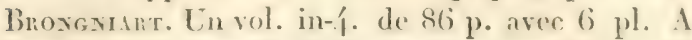
Paris, I 823 , chez F. G. Levrault.

Dans la description minéralogique des environs de Paris, TI. Brongniart a compris sous le nom de terrains de séliment supérieurs toutes les conches de la terre qui ont éti formies depuis le dépôt de la craic. Dans son nouvean mimoire, l'auteur at pour objet d'établir à quels ordres de cette classe les terrains de plusieurs localités célebres, quil a en l'occasion de visiter dans ses voyages, doivent ètre rapportés.

Le mémoire est divisé en trois parties:

Premiere partie : Sur les terrains de sédiment supéricurs calcare-trappéens du Vicentin; c'est ainsi que 11. Brongniart désigne les terrains qui se voient principalement an pied méridional des Alpes dans lat Lombardic, et qui sont formés de couches calcaires de sédiment, síparées par dés dépòts dits trappéens, plus ou moins abondans. Les principales localités décrites sont celles du F al-iera, du fallionca, de Honterdhio-Hagraiore, de Honte-tiale, de Wonto-Eolca. L'auteur regarde ces terrains comme analogues cntre cux et aux couches inférieures des terrains parisiens, c'est-a-dire a celles qui sont comprises entre la craie et le: sypse.

Dexieme partic: Sur quelgnes terrieins qui offent certaines particularités, comparćes avec les terraitus de sidiment supérieurs des environs de Paris, tels que ceux de la colline de Supergue, pres Turin; des environs de Mayence; du Banyul-des-Aspres, an pied des Pyrénies-Orientales; den sommites de la montagne des Diablerets, ćleries de 3200 metres au-dessus de la mer; de colles dies montagnes do Glaris, etc.

Troivime purtie: Description de plusicurs des corps organisés fossiles renfermés dans les terrains de sédiment supérieurs, décrits ou mentionnés dans les deux premiẹres parties. 
Six planches lithographiés avec le plus grand soin, qui représentent six coupes de terrains et près de r jo figures de coquilles, accompagnent ce mémoire important. C. P.

363. Mémorre géologique sur l'Allemagne, par $\Lambda$.

Boué (Jour. de Phys, mai, juin, juillet, cloitt).

C'est après avoir parcouru et visité non-seulement presque toutes les parties de l'Allemagne, mais encore l'Angleterre, l'Écosse, et la France, que l'auteur essaie de tracer it grands traits le tableau de la composition géognostique du vaste pays qqu'entourent le Rhin, l'Océan, la Baltique, les plaines de la Pologne, la Hongrie et les Alpes; tout ce cu'il a vu et observé a pu le mettre à mème de saisir les véritables caractères communs ou différenticls que présentent entr'elles les diverses formations étudiées isolément dans leur pays par les géologues allemands, anglais et francais, et de chercher à concilier leurs opinions divergentes sur plusicurs points. Tel a été un des buts que M. Boué s'est proposé d'atteindre dans un travail trop riche de faits et de détitils, pour que nous puissions espérer de donner une idée de son importance par une courte analyse. Nous nous bornerons done à annoncer que le mémoire sur l'Allemagne... inséré par partic, et successivement, dans les suméros des mois de mai, juin, juillet et aout, du Journal de Physique, n'est pas terminé, et qu'il continucra à être publié dans les numéros suivans qui restent à paraitre pour 1822 .

Ce qui a paru comprend l'histoire des terrains de l'Allemagne, depuis le sol primitif jusqu'au calcaire du Jura inclusivement.

C. P.

363. Noticesur les Brieches osseuses de l'île de Corse, par M. Bocroet, (de la Niève) (Joum. de Ply)s., aout 1822, pag. I/43 à 1/5.)

MI. Bourdet, qui a visité en 1816 les brèches asseuses des environs de Bastia, décrites précédemment par M. Rampasse, a trouvé dans ces breches des ossemens de mammiferes que 19. Cuvier n'avait point rencontrés dans les échantillons qu iil avait recus de M. Rampasse. Ces ossemens ont paru ètre a M. Bourdet, I $^{\circ}$ l'extrómité inféricure du fémus d'un ruminant voisin du daim, à peu pres semblable à colni figure 
par M. Cuvier, comme vennut des breches ossenses de: GiBrallar; 2. une portion de machoire analogrue à celle du lapin ordistire, ef ne differant pas de celles observes dans les roches de Celle. Ces nouveaux faits confirment l'identile reconnue entre les briches osseuses de l'ile de Corse, de Gibraltar, de Nice, d'Antibes, etc.

C. $\mathrm{P}$.

36\}. Sit le gistucat ufs ossemens fosines des envirms didrgenton (Indie), par M. de Basterot. (biull. des se. par la Soc. Philom, déc., p. I88.)

I'anteur décrit la localité ou ont été trouvés les ossemens de lophiodon, de crocodile et de tortue mentionnés pax M. Cuvier, dans l'Histoire eles ossemens fossiles: lit marniche dess Prunes, oủ ces ossemens ont été trouvés, remplissait une espice de ravin ou d'enfoncement creusé dans le terrain Oolitique; M. de Basterot ripporte ce terrain marneux à la formation d'argile plastique at de liginite; il pense que la présence des ossemens de lophiodon peut étre regaricé comme un des caractères de cette formation.

F.

365. Noticfs su le Intixz; par M. de Bowxinn, ingrén. en chef des mines. 28 p. (Ann. des Mines, 1om. VII, p. 41.)

Ces notices sont au nombre de 4 : la $x^{r^{e}}$. est un apereu physique ou topographique du Hartz. Ia $2^{c}$. fait connailre la nature des terrains du Harlz et examine leur ancienneté relative; l'auteur s'attache surtont à discuter lat [rimordialité attribuce gréneralement aux granits et aux autres roches cristallines du Hart\%; il les regarde comme étant probablement. de formation intermediaire et entemporaines de celles de Granwache. Ja $3^{\prime}$. notice traite des mine: de plomb et d'argent du Hartz considerées sous le point de vue géounostique. La '́ notice a prour oljjet les gites de minerai de fer. 366. Pricis n'rexe cotre dans le pays du llartz; par MII. I.IIne ct C.lapernon, asp. au corps royal des minc's. 2o p. (Inn. des Mines, lom. III, p. 2. . $)$

Ce pricis renferme des observations sur la griologie, lixploitation des mines of la métallargie du Hartz, relutives seulement anx diffirems points que les auteurs ont visitís dam leur course.

n. B. 
367. La Mine De sel gemae de Vic (Meurthe), surlaquelle on est arrivé le 10 décembre, est toujours exploitće à l'aide de la poudre, moyen sans lequel on aurait eu beaucoup de peine à arracher quelques morceaux de sel, qui est aussi dur et aussi brillant que le cristal. Cependant, la croûte de la couche est chargée de nuages gris; mais ils deviennent rares à quelques ponces plus bas, et il est présumable qu'on ne sera pas à deux pieds dans le sel sans le trouver parfaitement blanc. MM. les ingénieurs conviennent que la beauté de la mine surpasse toutes leurs espérances. On s'est arrêté à dix pouces de profondeur dans la couche, pour s'occuper de travaux urgens (Journal de la Meurthe). Nous ajouterons qu'une personne qui arrive de Vic nous a fait voir des morceaux de sel du poids de plusieurs livres, d'une beauté et d'une blancheur étomantes, dans lesq̨ucls on ne découvre pas la moindre molécule de matiere hétérogène. Son degré de salure est, dit-on, le double de celui du sel fabriqué par l'évaporation. (Rerue Encyc., déc. 1822, p. 640 .)

\section{HISTOIRE NATURELLE GÉNẺRALE.}

368. Dictionnare des sciences naturelles, dans lequel on traite méthodiquement des différens êtres de la nature, considérés soit en eux mèmes, d'après l'état actuel de nos commaissances, soit relativement à l'utilité qu'en peuvent retirer la médecine, l'agriculture, le commerce et les arts; suivie d'une biographic des plus célébres naturalistes. Ouvrage, ete.; par plusieurs professeurs du Jardin du Roi, et des principales écoles de Paris. Tom. XXV. (Iact-Leo.) In-S de 3 o f. $\frac{1}{2}$. Id. 22 et 23 cahiers ens. de $\div$ f. et $40 \mathrm{pl}$. Paris, F. G. Levrault.

La publication de cet ouvrage se continue avec beaucoup d'activité. Le 25" vol. que nous annonçons renferme un assez grand nombre d'articles importans, tels que ceux-ci :

Lagroni et Laves par M. Brongniart. Dans le premier, ce sivant naturaliste dévelople son opinion sur les lagonis de Toscane, sorte d'amas d'eau bourbeuse et noiråtre agitée 'ооме I. 
par une ébullition apparente, ot doui serhalent avec impe tuosite des vapenrs tris-risibles et tresodorantes. Il consilere les pliénomines qui constituent ces laggonis comme représentant en petit reux qu'on observe dans les voleans, el il atribue notamment le degagement de gaz et de vapeurs à des actions chimiques qui ont lieu dans lintericur de la lerre au-dessous des terrains anciens. Enfin il explique la formation des dessins ruiniforwes de lat pierre de Florence par unc altération que ces vapeurs ont fait subir à des ruches calcaires remplies de fissures naturelles, qu'elles ont traversées pour s'échapper.

I.nurier, par M. Poiret._- I. inumineuses, par MI. de Jussien.

Laidice et Laurose, par M. Loiseleur-Deslon champs -

Laminavia, par M. Léman.

Labiatiflores, Lachnospume, Lactuciés, Lä̈nneide' Lasuscree, Lagenophore, Lnilion, Laitur, Lampourde, lampsune, Lam!r, Lappu, Lusiosperme, Leibnitsie, Leontony: etc, par MI. Cassini. Ces articles sont la suite du Irarail de cebutanisie sur les synanthérés, et renferment non-seulement la description desplantes dont ils portent les noms, mais encore des additions à plusieurs articles imprimés dans les premiers volumes de ce dictionnaire.

I.rniogere, nouveau genre de mollusques, par M. de Blainville.- Labre, par M.II. Cloquet.

Lamie, Lampyre, Lnoue, Lave, par M. Duméril.

Lubbe, par Mi. Dumont. - Einticulite, par M. Defrance.

Lama et Lamantin, par MI. Frédéric Cuvier.

Lait, par 1I. Chewreul. 1)ans cet article l'iuteur délaille successivement les proprictés chinaques du latit de vache, de brebis, de chirre, de femme, danesse et de jument; et fait connaitre le résultat de l'action de la chalcur, des acides, des alcalis, des sels neutres, de la gomme, du sucre, de l'alcolıol et de l'éther hydratirque sur ce liquide animal.

I.e $22^{r}$. cahior de l'atlas 'jui accompanne ce vol, contient 8 pl. de botanique : \% pl. représentent des insectes apteres; 2 , des coquilles univalves; 5 , des poissons de divers ordres; et 3 , des oiseaux échassiers.

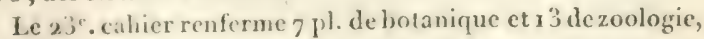
savoir: Treprésentant despuissons d'ordres varicis; 4 , des rejtiles sauriens; et h, desoiseaux échassiers et palmipedes. Da:sa. 


\section{Zoologie.}

ZOOLOGIE.

369. Zoologicar. Researches insava, and the neighbouring islands, ou Recherches zoologiques sur Java et les îles voisines; par T. Ilonsfield. Londres I $821,-1822$.

Les Moluques, situces sous l'équateur, et couvertes d'une riche végétation, sont peuplées d'un grand nombre d'animaux remrrquables par lit grandeur de letr taille, par la variété de leurs formes, et par l'éclat de leurs couleurs. Depuis Rumphius, il n'avait paru sur ces iles intéressantes aucun ouvrage dins lecquel on décrivit leurs animaux. Les sc"-les notious qui nous en étaient parvenues étaient fournies par des navigateurs qui y relächaient; mais ils n'y faisaient pas un séjour assez long pour en explorer la grande étendue.

Dans ces dernières années, deux jeunes naturalistes franrais, MMI. Diard et Duvaucel, ont fait à Java et à Sumatra un séjour assez long. Ils y ont formé de nombreuses collections, qui, en augmentant les nỏtres d'une grande quautité d'espèces nouvelles, nous ont fait connaitre la richesse du règne animal de cette contrée du globe. Pendant ce temps, les Angrlais rassemblaient les mêmes productions pour les déposer dans le Muséum de la compagnie des Indes; et les Hollandais, par les soins du professeur Reinwardt, faisaient les mêmes recherches avec la plus grande activité. Plus heureux que Kuhl, dont les amis des sciences déplorent la perte prématurée, M. Reinwardt est de retour dans sa patrie avec les trésors quili a amassés sous ce beau ciel.

Quelques-uns des plus curieux des animaux que ces recherchies nous ont procurés avaient été déjà publiés par MI. Cuvier ou par M. Temminck ; mais il manquait un ouvage particulièrement destiné a laire connaitre la zoologie des Moluques.

Sir T. Ilorsfield, qui a séjourné dans l'ile de Java et qui y a fait des collections, a entrepris ce travail. Il sera composé de $\mathbf{S}$ cahiers in- $\mathfrak{4}$, dont il prarait un numéro tous les trois mois ; chaque cahier contient la figure exacte et la description détaillée, avec la synonymie et les noms malais de \$ animaux , 4 mammiferes, et 4 oiseaux. Une 9 . planche est 
employéc à représenter avec dítails la forme des dents ef des pieds des nouveanx genres de mammiferes, et le bee et les pates des nouveaux grenres d'oiseaux que l'auteur a jug̨é convenable d'établir. Quatre numéros ont deja paru; nous allons fitice connaitre les oljets qu'ils contiennent, en commencant par les mammifëres.

Dans le cather $n^{\prime \prime} .1$, on tronve: $\mathbf{I}^{\circ}$. le Felis junanensis; non encore figure, mais decrit par MI. G. Cuvier, dans ses recherches sur les ossemens fossiles, art. de's Carnassiers.

2. I.e Ficlis gracilis; d'aprés l'examen de ses dents, et le facies de l'animal, nous pensons qu'il appartient au genre Viverra.

3". Le Tiverra musanga, paradoxurus typus, F. Cuvier. Ayant vu cet animal vivant, nous remarquerons que c'est a tort que l'on a représenté sa queue droite et allongée. Il la fient toujours cnroulée sur elle-méme, comme M. F. Cuvier l'a fait peindre.

$4^{\circ}$. Le Tapjrus malayamus, dont M. G. Cuvier a donné la description et la fignre du squelette dans la deuxieme ílit. de ses Ossemens fossiles. M. I. Cuvier l'it fait également dessiner dans son histoire de la ménagerie du jardin du roi, sur un dessin envoyé de Calcutta par M. A. Duvaucel. Ce nouveau 'Tapir, l'oiseau décrit, cahier $n^{\circ}$. IV, sous le nom de Culyptomena viridis, et plusieurs autresespèces non décriles sont de la plus haute importance pour l'étude de la distribution géographirge des animaux sur le globe. Ces formes avaient été jusqualors resrardées comme propres a l'Amerique. Lorsque ce continent sera mieux connn, je ne doute pas que l'on ne trouve dans ses foréts encore vierges beaucoup de formes que nous n'avons jusqu'a présent rencontrées que dans l'Inde. Ciest ainsi que nous avons atu cabinet du Roi, une espece inédite de Roussette (Pteropus), qui nous vient du Brésil; et que M, de Saint-Lilaire a rapporté des provines méridionales de ce royaume une espèce nouvelle d'oiseau du genre Rhynchie que je propose de nommer du nom de ce savant voyigeur, Rhynchara llilairea.

Les mammiferes du eahier $\mathrm{n}^{\prime \prime}$. I I sont : 1\%. le Wylaus meliceps dont les dents et les pieds sont figurés sur la planclic des détails, ainsi que les grandes anales qui sécrètent une liqueur d'une odenr infecte comme les Mouffettes d'Améri- 


\section{Zoologie.}

que, dont cette espèce et ses congénères se rapprochent. 2. Le Gulo orientalis; 30. Le Tarsius Bancanus; 4". Le Felis sumatrana. Aucun de ces animaux n'avait encore été publié.

Dans le calier $n^{\prime}$. 3 , on trouve, $\mathbf{I}^{\circ}$. Deux espéces d'un nouveau genre nommé Tupaia; leur crâne, leurs dents et $\mathrm{l}_{\text {eurs pieds, sont aussi figurés dans ce calier. Ces animaux ont }}$ le port d'un écureuil, et se rapprochent des Sorex, par les caractères de leurs dents, d'où M. Desmarets a proposé pour eux (Mammalogie) le nom générique de Glisore. M. F. Cuvier les a nommés Cladobates. $2^{\circ}$. Une nouvelle espèce de Gibbon dont le nom Simia syndactia indique la singulière conformation des doigts du pied. 3". Enfin, une espèce de Roussette, Pteropus rostratus.

L'auteur a figuré dins le cahier $n^{\circ}$. IV , les caractères des dents d'un nouveãu genre de singes près celui des guenons. Il a été nommé par M. F. Cuvier, Semnopithèque, et l'espèce que M. Horsfield a représentée est la Maure de M. Geoffroy. L'Ursus malayanus, le Pteromis genibarlis, et le Pternpus javanensis (Pteropus Edulis, Geoff.), sont les derniers mammifères que l'auteur ait décrits et figurés.

Les oiseaux dont on trouve la description dans le cahier $n^{\circ}$. I sont : Io. L'Trena puella (Coracias puclla, Latham) (Drongo azuré, Temminck).

$2^{2}$. Sous le nom de Phenotrix Temia, le Temia de Le Vaillant. M. Horsfield, en établissant ce nouveau genre, n'a pas examiné le Glaucopis cinerea (Forster) dont j'ai vu plusieurs individus dans le cabinet de Surgeon's Hall. Je pense que le Temia doit être classé dans le genre Glaucopis. 3. NOotacilla speriosa, Enicurus Coronatus, Temminck.

Dans le cahier $n^{\circ}$ II on trouve $1^{\circ}$. L'Eurylaimus jasanicus (Eur. Horsfieldii, Tem.) Nouveau genre de la famille des Gobe-Mouches. $2^{\circ}$. Podargu javanensis. $3^{\circ}$. Turdus s'arius. $44^{\circ}$. Dacelo pulchella. Ces deux dernières espices encore inédites.

Dans le cahier $\mathrm{n}^{\circ}$. III, l'auteur a figuré, $\mathrm{I}^{\circ}$. le Falio ichthycetus, nouvelle espèce d'aigle pecheur. $2^{\circ}$. Le mäle et la femelle du Faleo cerulescens Lath. (Hoberean moineau, Temminch.) 3". Deux especes d'un nouveau genre, Timalia pileata, et Timalia sutaris. 4". Une nouvelle espece de Coucou, Cuculus .Yantho ilynchos. 
Dans le cahier nn. IV, MI. Itorsfield a figuré sur la 5e.pl. un des plus beaux oiscaux que l'on connaisse. Outre la vivacitć de sa conleur vert d'émeraude, il se rapproche par l'ensemble de ses caracteres des cons de roclies fripru rupicola , Gm.); et sous ce rapport il est, comme nous l'avons dit, très-intéressant pour les naturalistes. L'auteur en fait un nouveiu grenre ru’il nomme Calyptomena; mais il me scmble difficile de séparer cette espere des Rupicola de l'Amérique. I.es $6^{e} ., 7^{`}$, et 8 e., représentent Ie Strix budiut, l'Alcelo biru, et le Turdus gyancus, trois espèces qui n'ivaient pas cncore été publiées.

On voit, d'aprés cet exposé, que l'ouvrage de M. Ilorsfield fait connaitre des espices encore inconnues aux zoologistes qui n'ont pas à leur disposition les grandes collections de Paris ou de Leyden. Ce travail, fait arec beaucoup de soin, sera done d'une grande utilité pour l'avancement des sciences naturelles.

A. VAle NCIE NNES.

370 . Anmilia nova qux in itinere, jussu et auspicis

Max. Jos. Bar. Regis, a. i 8 I $7^{- \text {I } 820, ~ p e x ~ B r a s i l i a m ~}$

suscepto, olsservavi et depingi curavit Jo. de Sprx.

Cet ouvrage sera composé de 30 pl. coloriées, in-fol., ou 6 livr., contenant les mammifures, les singes et les chauvesouris. Le prix de chaque livr., de 6 à 7 pl. colorićes, sera de $2,4 \mathrm{fr}$. Les autres espèces d'animaux seront publiées format gr. in- $4^{\circ}$; il en paraîtra 4 à 5 livr. par an, chacune de 6 pl. color., du prix de 14 fr. 50 cent. On souscrit chez Lindauer, lihr. a Munich; et chez Treuttel et Würtz, à Paris. Londres et Strasbourg.

37r. Mammlogre nu description des espéces de mammiféres, par A. G. Deswarest, profes. de zoologie, à lifenle Roy. vétérinaire d'Alfort; membre titulaire de l'Acad. Roy. de Médecine, des sociétis philomathique et dhistoire naturelle deParis, etc. r vol.g. iu-f́, de $556 \mathrm{p}$., dépendant de l'Encyclopédic par ordre de matieres. P'rix, $27 \mathrm{fr}$. A Paris, chez Mad. Ve. Agasse.

Cet ouvrage avait pour but principal, la description des planches de I'Lncyclopedie, exécutées depuis long-temps 


$$
\text { Zoologie. }
$$

sous les yeux de feu l'abbi Inonnaterre; mais l'anteur a cru sevoir le transformer en un véribble specises des manmifires connus jusqu’a ce jour, en donnant à son travail toute lifendue convenable pour bien faire distinguer ces itres.

Le nombre des mammifères qu'il admet séleve à sís, en y comprenant environ 80 espexces fossiles, la plupart dicouvertes par $M$. Cuvier, et les cétacés dont il ne traite que sommairement, afin de ne point recommencer le travitil de Bontaterre sur ceux de ces animaux qui itaient anciennement connus.

Gmelin en réunissint dans la $13 \mathrm{e}$ e édit. du systema nalurce, plas d'especes de mammiferes (4 40$)$ qu'on n'en trouve dans aucun des ouvrages systématiques qui ont précédé le sien; Gunelin avait admis sans crilique, toutes les inlications des voyageurs relatives à l'existence de beaucoup d'animaux, ce qui avait donné licu à des dombles emplois et à la création de plusieurs espéces factices. M. Desmarest s'est attaché à écarter ces données incertaines, el áles remplacer par les notions positives que l'ont mis à méme de recueillir les nombreux voyages scientifiques entrepris dans ces demiers temps, les nombreuses collections dont notre Muséum s'est enrichi, ainsi que la publication de plısicurs ouvrages importans, parmi lesquels nous nous bornerons à citer la Menagerie du Muséum, par MII. Geoffroy Saint-Hilaire, et Frédéric Cuvier.

Néanmoins parmi les espèces dont il traite dans son ouvrage, x 45 , quoique proposies par des naturalistes esacts, ne lui paraissant pas irrivocablement constatees, sont marquées d'un signe particulier (un astérisque) qui a pour objet d'inspirer la défiance à leur égard, et de portor de nomveaux observateurs à approfondix la question de la réalité de leur distinction.

Pour compléter son tableau des mammiferes, l'auteur n'a pas nérgligré de rassembler les notes, méme les plus fugritives, qui abondient dans le; récits des voyageurs, ou que se bornent à publier quel(pues naturalistes superficiels; mais il n'a lonné à ces indications que la place qu'elles méritent, en les ajoulant, comme par ippendices, anx articles avec lesquels elles paraissent avoix le plus de rapport.

Conraincu que rick u'st plus nuisible aux postes de liz 
science, que decréer un nouvcau systime de classification, uniquement pour se donner la satisfaction d'en avoir un à soi, ainsi que cela se pratique assez généralement de notre temps, M. Desmarest s'est contenté de choisir parmi ceux qui existent, celui qui lui a paru le plus en rapport avec la nature des animaux, et c'est celui de $\mathbf{M}$. Cuvier qu'il a cru devoir adopter.

Les 850 espèces vivantes ou fossiles qu'il décrit, sont ainsi réparties dans les dirers ordres: Bimanes $\mathbf{I}$, quadrumanes, 14x. - Carnassiers, 321 (subdivisés en cheiroptires, $9^{8}$; insectivores, 29 ; carnivores, 147; marsupiaux, 47); - rongeurs, 149 ; - édentés, $24,-$ patchydermes, 55 (la plupart fossiles); - ruminans, 97; cétacés, 62 .

Plusicurs de ces espèces sont nouvelles; d'autres, imparfaitement counues, sont l'objet de descriptions détaillées.

II. Desmarest ayant l'intention de publier unc carte zoographique plus complete que celle de Zimmermann, a cu l'attention de donner à charpue espèce un numero différent, afin qu'il puisse lui servir d'indication sur cette carte.

Dès a présent il reconnait que les espèces dont la patric est connue, sont ainsi répandues sur le globe : 181 , dans l' $\Lambda$ mérique mérid., - 54 dans l'Amérique septent., - Io communes aux continents de l'Amérique et de l'Asie, - ix propres à l'Asie septent, - 88 à l'Europe, - ro7 à l'ifrique, -29 à MIudagascar et à Mascarcigne; - -8 ì l'Asie mérid. et à Ceylan, $-5 \mathbf{1}$ aux iles de l'Archipel indien, - 33 à la Nouv.-Hollande et a la terre de VanDiimen; 3o cétacés ou phoques, habitent les mers du nord, $x_{4}$ celles du Sud, et a peu pres 28 se trouvent dans les latitudes moyennes.

M. Desmarest s'est particulierement altaché à décrire les races domestiques, ce que les nomenclateurs négligent de faire ordinairement ; et suus ce rapport, dans son ouvrage, l'espiece du cheval est celle qui présente les résultats les plus neufs.

Eufin le Traité de mammalogie est terminé par une table alphabétique de tontes les enpeces qui y sont decrites, table a larpuclle l'auteur a cu lidie d'attacher un certin degrie d'utilité, en désignant par une lettre (II) toutes celles 


\section{Zoologie.}

de ces espèces dont il existe des dépouilles, plus ou moins complètes, dans les collections du Muséum d'histoire naturelle. Il en a fait, pour ainsi dire, un catalogue trèscommode pour l'étude.

372. Beitrage zur Vogelkunde, etc. Mémoires pour servir à l'ornithologie, ou description de plusieurs espèces d'oiseaux d'Allemagne, rares ou nouvellement découvertes; par Chr. L. Brens. In-8, avec fig. To. II. Prix, 3 rixd. Neustadt i822. Wagner.

Les oiseaux rares en Allemagne, décrits dans ce volume, sont : Cinglus melanogaster; - Regulus procephalus; Sy-ria It'olfii; -Sylvia striata; - Certhia brachydactyla. $\Lambda$ la fin se trouvent des observations sur les mojurs et l'instinct des oiscaux, leurs amours, etc. L'auteur promet un 3e.v., qui doit paraitre sous peu. (Journ. grin. de lat Lit. étr.) 373. Ornithologische Bertnage, etc. Mémoires pour servir à l'Ornithologie de l'Allemagne; par F. Bore. in-8. I $^{\text {ere }}$ et $2^{\mathrm{e}}$ livraisons. I 822 . Kiel.

37 亿. Zusitze und berichtigungen zu Meyers und Wolfs taschenbuch derdeutschen Wònelhunde, etc. Additions et corrections au Manuel dOrnithologic des prof. Meyer et Wolf; par Bern. Mrexen. In-8. Prix , 2 flor. 3o kr. Francfort tog2. Bronner.

375 . IIstome natertele des Lépidortimes, ou Papillons de France; par M. J. B. Gondr't, ancien proviseur, et l'un des rédactenrs de l'article $P a-$ pillon de l'Encyclopédie méthodique. Ouvrage Jasé sur la méthode de M. Latreille; avec les figures de chargue espèce, dessinées et coloriées d'après nature, par P. Duménil. Prix, 3 fr. la livr.et 3 fr. 50 c. fr. de p. Imp. de F. Didot. Paris, Crevot.

I'on sait que feu Génouville D. M., et M. Vauthicr, peintre distingué, ne sétaient proposé en entreprenant cet ouvrage que de faire comnaitre en six livraisons les papillons de jour des environs de Paris. Cette entreprise étant passíe dinls les mains d'un des naturalistes de l'Europe quia le plus appro- 
fondi l'histoire des lépidopteres, MI. Godart, ancien proviseur an lycie de Bonn, un nouveau texte basé sur la méthode de AI. Tatreille a remplacé celui livré an public, et les dessins ont été confrés à M. Duménil. Une nouvelle souscriptiona été ouverte pour leslépidoptères crépusculaires et nocturne's, c'est-i-dire pour les sphinx et les phalenes; ct, sur le désir manifesté par plusieurs naturalistes, les papillons diurnes des départemens méridionaux ont été livrés au publie; ce supplément touche à sa fin. La totalité de l'ouvrage aura 6 vol. qui se vendront séparément.

Les descriptions sont d'une fidélité scrupuleuse, leur synonymic est rectifiée et faite avec le plus grand soin. M. Godart mentionne les habitudes de ces especes et celles de leurs chenilles dont il a élevé un grand nombre. MI. Duménil a souvent surpassé l'onvage d'Hübner, le plus estimé en ce senre, et celni-ci lui est bien supérieur sous le rapport de la gravure et du mírite littíraire. Le format est plus commode et les planches plus remplies.

Le premier vol, contient en totalité g' espèces de diurnes propres aux environs de Paris, avec des instructions sur la chasse, la conservation et la préparation des papillons; la maniere de chercher et d'úlever les chenilles, etc.

Ie deuxieme vol. sera de I 4 livraisons, dont ix déja publies. Il renfermera les diurnes de nos montagnes alpines et du midi de lit France, au nombre de 85 espéces.

Te troisieme vol., achevé, depuis plus d'un an, est conacriaux cripuseulaires ou splinx, dont le nombre se monte pour toute la France à environ 60 espèces.

Les tomes 4, 5, 6, sont destinés aux nocturnes, ou papillons de nuit. Il a paru jusqu'a ce jour 7 livraisons du quatrième.

Chaque vol. a une table alpluabétique et synonymique des esprices qu'il renferme, afin qu'on puisse sur-le-champ les trouver far les differens noms qui leur ont été imposés.

Le tome deux sera terminé par un tiblean contenant la plirase caractíristique ou le signalement en latin et en francais de tous les diurnes, avee l'indication des lieux el de l'epoque ou ils se trouvent. Ce tablcau atura le double avantage de présenter dans une serie continue les especes decrites et figurées dans les deur premicrs volumes, et d'ctre jour les 
amateurs un rade-mecum détaché et extrêmement commode.

Il y aura un pareil tableau à la fin de chacun des trois derniers volumes.

Parmi les espèces publićes jusqu'ici, il en esi d'inédites, dont une, propre aux montagnes de la Corse et appartenant à mon genre satrae, communiquée par M. le Fébure de Cérisy, ingénieur des constructions maritimes à Toulon ; lautre, nommée Cossus lírbasci par Fabricius, mais devant élre placée avec les espèces provenant de chenilles à queue fourchue dans la division de mes bom вxx sans crin. MI. de Luxer, procureur du roi à Nancy, a aussi envoyé une nouvelle t́cAILLF ou ARCTIE qui avait échappé jusqu'a présent aux recherches des entomologistes.

Enfin, l'auteur et l'éditeur sont tellenent animés dudésir de présenter un ouvrage utile et classique, qu'ils sont convenus en sus de mettre à la fin de chaque genre la description des espèces europiennes qu'on ne trouve pas sur notre territoire, en sorte que, sous le titre de Papillons de Frunce, ils publient réellement les Papillons d'Europe, et qu'il ne leur restera à donner pour compléter ces derniers qu'un faible supplément de planches.

LATreitLe.

3-6. Mémoire sur deux oiseaux du comté de Nice, observés en nov. et déc. I\$I9; par le chev. Albert de la Manmora. ( Miem. della reale Accad. delle Sc. di Torino, 10. 25, p. 253.)

La première espèce figurée et décrite par l'autcur, appartient au genre Sylvia; il la nomme S. Cetti, elle est indubitablement la même que l'Usigmuolo di Fiume du père Cetti (Ĺccelli di Sardegna p. 216.) Temminck la rapporte di la Fauvette, figurée dans la pl. enluminéc de Buffon, no. 655, fig. 2, sous le nom de Bouscarle et la nomme becfin Bouscarle (Sylvia Cetti de la Marmora). Bonelli et de la Marmora ne partagent pas celte opinion. (Quoiqu'il en soit l'auteur dicrit, et représente le mâle, la femelle et le nid. Cet oiscau est sédentaire et habite les broussailles qui bordent les caux courantes.

La sccomle espice est le Motteux noir, improprement nommé Mexle à queue blanche, Turdus Leucurus, dont on nc 
connaissait que le mile par lat description assez imparfaite qu'en a donné Latham; il se tient sur les rochers et se rapproche beancoup par cette habitude constante du Turdus Saxatilis. L'anteur décrit et représente le màle ainsi que la femelle. Temminck n'a pas connu celle-ci, et il donne au mâle le nom de Traquet rieur. Saxicola cachinnans. V. Acn.

377. Drscimpton nes Porssons du Bohuslaen, par M. Hen.meng, $2^{c}$ enhier. Extr. des Nouv. Mim. de lasoc. Roy. des Sc. et des Lettres de Gottenbourgr, $4^{e}$ partic. I 82 r (en suédois.)

Nous n'arons sous les yeux que le deuxieme cahier de ce travail. Lauteur y décrit le's espéces suivantes de poissons et en donne des figures colorices avec soin. $1^{\prime \prime}$. La Chimere arctique de Lacépède (Chimern monstrosa, I.), elle habite le fond de l'eau et s'y nourrit de mollusques, de petits crabes, et queiqquefois mème de harengss. On retire de son foie une huile qui est employée, en Angleterre, pour les rhumatismes chroniques, et qui est aussi considérée comme la meillenre lnile pour préserver les instrumens de la rouille. L'autcur donne plnsicurs détails sur son anatomie et représente les os de la tête.

2. Raja dessbatus punctatus. L'auteur pense que celte Raie nouvelledont il donne la figure et qu’il décrit, appartient à une espéce de Dasylatus mentionnće par M. de Blainville dans son prodrôme d'une nouvelle distribution du régne animal.

3. Gade rouge, petite merluche, sadus ruber. (Lacépicde, $2^{\circ}$. suppl., t. v, p. 673.) Il est rare.

4. I'leurnnectes Flesus, L. La description anatomiquede ce poisson est assez complete. Il a 35 vertibres, ce qui le dintinguc essenticllement du Plenr. passer. Cette différence est toujours liće a la position des yeux sur le cóté droit.

5. Pleuronectes passer. L'auteur regarde cette espice comme entierement distincte de la précédente, lit position des yeux au cófé gauche de l'animal est un caractere invatriable auguel vicnt s'ajouter le nombre des vertebres qui ici riest plus quede?. M. Cuvier (Régn. anim., 1. 2, p. 220) réunit, comme virieté accillentelle, celte espece an Pleur flesus.

6. Gallus mustela, L. Céte espice cot ritre dans les mers 
du Nord, la figure en est bonne et la description assez complète.

$7^{\circ}$. Pleuronectes (uensclii; Cette espice fort rare parait nouvelle, l'auteur la représente et la dícrit avec beaucoup de soin; elle se distingue de toutes celles connues jusqu'à ce jour par une belle couleur rouge acajou, sur laquelle sont dispersées des taches étroites et transversales. Voici d'ailleurs sa phrase spécifique: oculis dextris, corpore ovato-oblongo, lavi, rubro nigroque sariegato, maculis carule is sparsis, pinni cauda rotundath. Le nom qu'elle porte lui a étédonné en l'honneur du docteur Quensel, qui le premier a rangé dans un ordre naturel les Pleuronectes de Suède; dans les Mém. de l'acad. de Stockolm, année I So6. V. Avv. 378. Des causes qui déterminent les abeilles à construire leurs giteaux parallelement, sur des plans verticaux en ligne droite, et selon des dimensions déterminées; par le comte df Loche ( Mem. dellı reale Accad. delle Sc. di Torino, to. $25, p$. I 7 . .)

Huber n'avait fait aucune recherche pour découvrir la cause du parallélisme des rayous dans les ruches de quelque forme qu'elles soient. L'auteur de ce mémoire s'étant aperçu qu'un essaim d'abeilles, supposé très-petit et placé dans une ruche très-vaste l'occupait toute entière, cxamina comment cettedilatation considérable de volume pouvait avoir eu lieu et il vit qu'elle était uniquement produite par une suite de rideaux parallèles, formés par les abeilles, accrochées les unes aux autres et disposées de manière à occuper précisément l'emplacement de claque rayon futur, dont il figurait à la fois le tracé et le relief. M. de Loche assigne pour cause de ce singulier arrangement, le besoin de respirer, ce qui n'aurat lieu que trés-difficilement si les abcilles étaient agsglomerées en une seule masse. La direction des rayons en ligne àroile parait être une conséquence du mème principe. L'air, parcourant librement ces allées longitudinales toujours ouvertes an bas de la ruche of vers la porte, est sans cesse: renouvelé dans tonte l'habitation, et cet air n'est pas moins indispensable aux insectes parfaits, qu'aux larves contenues dans les alvéoles.

V. AUD. 
3-9. Novs somares asseres de procurer une vire satisfaction anx savans et it tous les amis des sciences en leur annongant que M. Savigry, apric une maladie longue et prínible sur les yeux, fruit de ses immenses travaux microscopiques pour le grand ouvragre d'Egypte, vient de se remettre à la téte de la partie qui le concerne dans ce célebre ouvrage. Un voyage de 7 à 8 lutit mois en Italie a raffermi sa vue, et l'on peut enfin esprirer de voir l'histoire naturelle de ce beau momument achevie dans quelque temps. Nous donnerons dims un prochitin numéro une notice détaillée des travaux de M. Savigny, Iravaux bien peu connus, parce que l'ouvrage d'Ésypte est dans un petit nombre de mains, et que le texte des planclies n'a pas paru. Si cet ouvrage cùt pu être étudié, labsence du fexle n'aurait point empéché que la science ne profitit de toutes les découvertes qui s'y trouvent; car ces planclies sont d'une telle perfection, et les figures de toutes les parties essenticlles de chaque animal tellement en rapport, 'qu'on peut y lire aussi bien que dans la meilleure description.

\section{BOTANIQUE.}

380. Theophrist's naturgfachichte der gewàche, übersetzt und criatert ron K. Sprengel; c'est-ìdire llistoire naturelle des plantes de Théophraste, traduite et commentée par M. Kunt-Spnengel. $\mathbf{r}^{\text {re }}$. partic, contenant la traduction. In- $8^{\circ}$. de 358 pag. Altona, I822, chez Hammerich.

Cette traduction allemande d'un des principaux ouvrages de Théopliraste, etait attendue avec impatience d'apres l'échantillon qui en avat été inséré dans l'Annuaire agricole de 'Tubingue, prour l'annce 1 So 3 . On savait que M. Sprengel y travaillait depuis fort long-temps. Lui-mème nous apprend dans son avant-propos ru'il regardiat celte entreprise comme si difficile qu'il a cru devoir s'y préparer par de longs travaux, notamment en composant son histoire de la Botanique, dont lat $1^{\text {re }}$. idit. fut donnée en 1806 et dont la $2^{\mathrm{e}}$. a paru en 1817 .

Il ajoute arec une mod:stic bien remarquable qu'il n'au- 


\section{Botanique.}

rail pas même cru pouvoir se décider à publier cette traduclion de l'Histoire des plantes du diseiple d'Aristote, s'il n': vait eu pour devancier et pour guide l'illustre Se-hneider, auquel on doit une excellente édition du Texte de Théophraste, fort altéré dans les éditions antérieures, et qqui a été trèsheurensement rétabli dans celle-là par la collation du manuscrit dit d'Urbino. On ne peut qu'attendre avec impatience le commentaire promis par M. Sprengel.

C. $\mathrm{M}$.

38 r. Exumeratro Plantarum quas in insulis archipelagi ant littoribus Ponti-Euxini, annis 1819 et 1820 , collegit atque detexit J. De mont n'Urville. ( $1 / \mathrm{cm}$. de la Soc. Linnéenne de Paris, t. I. I822.)

Cefte énumération des plantes récoltées par M. D'Urville, dans les iles de la Grèce et sur les côtes de la mer Noire, peut intéresser les botanistes sous deux points de vue: $\therefore$. comme catalogue géographique d'un pays encore imparfaiteınent connu. M. D'Urville a donné l'énumération de $9^{5}$ o espèces, dont plusieurs sont ou nouvelles, ou n'avaient pas été indiquées dans les pays où il les a découvertes, ou enfin n'étaient connues que d'après les herbiers ou les manuscrits de Tournefort. Sous ce rapport on peut regretter que ce catalogue ne soit pas distribué par familles naturelles, la scule classification qui puisse donner une idée exacte du genre de végétation d'un pays.

$2^{\circ}$. Comme botanique descriptive, on doit remarquer dans le travail de Mr. D'Urville, la description de plusieurs espèces nouvelles, et une synonymie faite avec soin d'après l'herbier de Tournefort et les dessins d'Aubrict; les espèces nouvelles sont au nombre d'environ 6o. Le plan de ce journal ne nous permet pas de les citer, nous dirons sculement que la plupart appartiennent aux familles des Graminées, des liubiacées, des Caryophyllées, des Ombellifëres, des Labiées, des Légumincuses, des Composées, des Orchidées et des $\Lambda$ lgues; ces dernières, dont 6 espéces et plusicurs variétés sont nouvelles, ont été déterminées et décrites par M. Lamouroux. A $\mathrm{D}$. B.

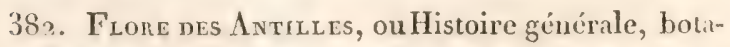
nique, rurale, et économique, des végétaux indigines de ces iles et des cxotiques qu'on est parvenu i y 
naturaliser, décritsd’apris nature, ot classés suivant le systime sexuel de Limú et la méthode naturelle de Jussiéu ; enrichie de planches dessinées, gravées et coloribes aree le plus grand soin par les premiers artistes de la capitale; par M. le cliev. F. R. de Tussac. T. II, fr. livraison in-f. de 4 a $5 \mathrm{pl}$. A Paris, chez l'auteur, rue des Touruelles, n. 30.

383. Fione ménicile des axtilles, ou Traité des plintes usuelles des colonies francaise's, anglaises, espagnoles et portugaises. Par M. E. Descocrtizz D. M., peinte par J. T. H. Descourtilz. AParis, chez Pichard. Tom. I. I82r. Tom. II. Liv. I8 à 21 . 1822. Les souscripteurs paient chaque livr. , $3 \mathrm{fr}$.

La Flore médicale des Antilles doit renfermer la description et la figure de 600 plantes cmployées en médecine; il en a déja paru 2 l livaisons, dont cliacune comprend quatre planches, etles descriptions qui les accompannent, renfermant le nom vulgaire des colonies, le nom botanique de Linné et de quelques-uns des principaux auteurs qui ont écrit sux l'histoire naturelle des Antilles; l'indication de sa place ditns le systeme de Tournefort, de Linné et de Jussieu; le caractère du genre, une description assez détaillée de la plante, l'indication de ses proprićtés, et l'usage médical qu'on en fait dans ces colonies, ainsi que le mode d'administration. Ces plantes sont classies d'aprés leur mode d'action, et par consépuent suivant l'usage qu'on en fait en medecine, en 25 classes. L'auteur a adopté en général la classification de la therapentique d'Alibert; les 2 I premieres livraisons renferment les plantes dites stomarhiques, divisées en stomachiquess aromatiques, amires fubrifuges, anti-scorbutiques, vermifuges, et astringentes, ou styptiques.

I.e plan de cet uuvrage, est comme on le voit, plus médical que botanique; on regrette, sous cedernier point de vue, que les descriptions ne soient pas plus détaillées surtout pour lat fructification, et que les planches qui sont agréablement colorices et qui donnent bien l'idéc de la plante, ne présentent pas plus de détails analytiques.

Cet ouvrage cependant ne peut manquer d'être utile aux 
colons auxquels l'auteur l'a particulièrement destiné ; il leur donnera le moyen de reconnaitre facilement les plantes qui croissent autour d'cux, et leur indiquera l'utilité qu'ils peurent en tirer. Il sera surtout utile aux médecins qui u'auraient pas les connaissances botaniques nécessaires pour déterminer les plantes médicales des colonies. $\quad$. v. M.

38. A flona of nontu Auenica, illustrated by original coloured figures drawn from nature. Flore de: l'Amérique du nord, avec des figures colorićes, dessinées d'après nature,par W. P. C. Bırton, D. M. Profess. de botanique à l'Lnjversité de Pensylvanie. 2 ' cahiers in- $\left\{\right.$. dont le $\mathbf{1}^{\text {er }}$. porte la date du

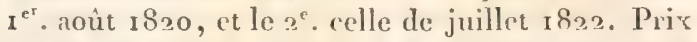
de chaque cahier, un dollar. A Philadelphie, chez H. C. Carey et J. Lea.

Tous les mois il parait un cahicr de 3 pl. et d'une, 2 ou $3 \mathrm{f}$. d'impression. Les 24 cahiers annoncés forment 2 vol. dont le $x^{\mathrm{er}}$ a a $36 \mathrm{pl}$. et $338 \mathrm{pag}$; le $2^{\mathrm{e}}, 34 \mathrm{pl}$. et ro pag. d'impression. Le texte et les figures sur beau papier vélin. L'auteur a l'intention de faire pour lia Flore des États-Unis d'Amérique, ce que Jacquin a fait pour la Flore d'Autriche, Oeder, Vahl, Müller et Hornemann, pour la Ilore Ianoise; Palmstruch et Vénus, pour la Flore Suédoise; Smith, pour la Flore Britannique ; c'est-i-dire de publier successivement les figures de toutes les plantes qui croissent spontamément aux Ėtats-Unis, sans s'astreindre à aucun ordre, autre que celui dans lequel les objets qu'il est appelé à décrire se frésentcront naturellement à lui. Plusieurs plantes, ou entièrement nouvelles, ou mentionnées pour la première fois, en I $\$$ I , pax Nuttal, sont figurées dans les cahiers que nous annoncons, et l'ouvrage offe sous ce rapport un grand intérêt; mais it laisse beaucoup à desirer relitivement au texte, au choix des échantillons, au coloris des figures. On regrette surtont que l'anteur se soit cru dispensé d'ijouter à ses planches ces détails anatomiques grossis, qui constituent la partie lit plus essentielle de l'iconographie botanique.

J. GAX.

385. Recherches sur Licchorsement et la reproduclion des végétaux, par. XI. II. Dusnocner, conesp. de 'Іоми I. 
linstitut de France. ifs'm. du (.Mus. dhist. nalurelle, vol. VIII, page 12 et 2 fr; pl. r. 2.)

I'auteur, apres avoir, dans la premiere partie de son me moire (sect. I et II. Mém. du Muscum, vol. VII, p. 379), examiné la maniere dont s'opére l'accroissement en diametre des végétaux dicotylédons et monocotylédons, recherche dans la troisieme section du méme mémoire (vol. I III, J. 12) comment se fait l'accroissement en longueur des tiges et des racines des végétanx; il remarque que dans les racines qui naissent de tiges submergées et rampantes, telles que celles des Nymphea, des Sparganium, etc., ou de racines principales, le systeme cortical de la tige ou de la racine principale ne. se continue pas sur les radicelles, mais que ces dernicres wrtent de dessous, et sont entourćes a leur base d'une sorte de gaine, formée par l'icorce qu'elles ont percée; il donne a celte gaine le nom de Colcorrlize, qui n’avait été appliqué jusçu'a présent qun'à la gaine qui envoloppe la radicule dés vigrtaur monocotyledons, et a larpelle on devrait, a ee qu'il nous semble, réserver ce nom.

Il examine ensulte comment s'opère l'allongrement de la tive, et particulierement celui des bourgeons. Il distingue dans cet accroissement l'èlongation gemmatire de l'élongation caulinaire : la première ayant licu par le développement des parties contenues dans le bourgeon, et lit seconde par l'allongement des parties déja développées; la différence entre ces deux modes d'accroissement nous parait à peine sensible, l. dernier ne pouvant ètre regardé que conme une con-. tinuité du premier.

La quatrieme section ( vol VIII, p. s? I ) a rapport au développement des ovules et de l'embryon dans l'ovaire, elle renferme des observations trés-intiressantes sur le développement des diverses parties de la graine, et surtout sur l'origrine du périsperme, et des tégumens de lovule; enfin, sur l'origrine des différentes parties de l'embryon. A 11 . B.

386. Onsenvations sur nes fietns monstrucuses de Cirsium pyrenairum: par MI. H. Cassizi. ( Bull. de la Soc. Philont., oct. 1822.)

Cette monutruosite consiste dans l'avortenent phus on moins complet de lovaire changé en une sorte de tige, dans le chan- 
gement de laigrette en écailles vertes et verticillées, dans la iransformation de la corolle en cinct petites feuillev verfer a nervures marginaler; les étamines étaient vides, presçue libre; le style très-allongé s'élargiscait à son sommet en une sorte de calathide présentant à sa base deux écailles vertes opposcer.

II. Cassini en déduit, $x^{\circ}$.que l'ovaire et le style sont des or ganes analogues à la tige, tandis que les squamellules de l':igrette, les 5 pièces de la corolle et les deux stigmatophores (Cass.) sont analogues aux feuilles; $2^{\circ}$. que l'aigrette n'est point un calice adhérent né de la base de l'ovaire et colléa sa surface, mais un vari calice épigyne; 3 ". que, dans les Synanthérées an moins, l'ovaire ne pouvait pas ètre regrardé comme la réunion de plusieurs feuilles, mais plutot comme un tronçon de tige creuse renfermant la graine.

A. $\mathrm{n} . \mathrm{B}$.

38- . Note sur une nouvel.le plante de la famille des liosacées, employée avec le plus grand succès en Abyssinie contre le teenia, et apportée de Constantinople par.I. Brazer. (Bull. de la Soc. Philom., ocl. i 82\%.)

La plante qui fait le sujet de cette notice vient de l'Abyssinie au Caire par les caravanes; il parait que son efficacite contre le tenia ou ver solitaire y est bien connue. Le docteur Brayer a été témoin d'une guérison très-prompte opérée par ce remede à Constantinople; il a suffi de donner une seule fois une infusion de 4 i 5 gros de poudre de cette plante pour faire rendre le ver. - M. Brayer ayant rapporté at Paris des débris de cette plante, M. Kunth y a reconnu a' nouveau genre de Rosacées très-voisin de l'Agrimonia et auquel il a donnéle nonide Brayera; le Brayera anthelmintica est un petit arbuste, a rameaux velus, i ferilles alternes, à fleurs quaternées, et entourées d'un involucre. A n. E.

35. Descruption d'une nouvellespère d'Eupatorium. par M. H. Cassın. ( Bull. de la'soc. Philom., sept. 1822.)

NI. Cassini donne a cette erpice le nom d'Eupatorium microstemon; clle est cultive an jardin des Plantes de P'arrs. On ignore d'ou elle vient.

A is. B.

389 . Descriptos d'une nouvelle espiece de Buphtul- 
mum, par M. H. Cissixr. (Lull. de la Soc. Philom., sept. 1822.)

Cette espèce nommec Buphitalmun longipes a été recueillie à Madagascar, par Commerson.

A D. B.

3)о. Mevorfe sur une nouvelle famille de plantes, les Balunophorés; par M. L. C. Ricnsis. (Mem. du.Mus. d'hist. naturelle, vol. VIII, pag. fof.)

Des quatre genres actuellement connus qui composent la famille des Balanophorées, deux seulement avaient été decrits d'puis assez long-temps, mais d'unemaniere si incomplete. qu'on n'avait pu jusqu'a prisent les rapporter à aucune famille. Ces deux genres sont le Cynomoriumet le Balumophora; le dernier méme n'est encore connu que par la descitiption très-imparfaite que Forsteren a donnée. MI. Richard a ijouté deux genres à celte famille: l'un, le Langssdorffar, découvert jar M. Martius au Brésil, avait été décrit par lui dans Es chrege, journal von Brasilien. I, quatrieme, entierement nouveau, a été nomme Hilosis; il renferme deux plantes rapportées au genre Cynomorium, par Swartz ( $1 \%$ ind. occidl. t. I p. I I-I 3 ), sous les noms de $C$. jamaicense et $C$. carennense. La famille des Balanophorées est ainsi composie des quatre genres: Helosis, Langselorffa, Balanophora et (ynomorium. Ces quatre genres se font remarquer par leur consistance charnue, fongueuse, labsence de feuilles qui, dans quelques especes, sont remplacées par des écailles imbriquées sur la tige; ces plintes croissent parasites sur les racines des autres plantes, les fleurs sont monoiques, réunies en têtes très-serrées et compusces d'un grand nombre de fleurs; loplus souvent des deux sexes, guelquefois d'un seul sexe langreforfia) . Les fleurs miles prisentent en géneral un calice a trois divisions profondes, et trois atamines dont les filets et les antheres sont rémies en une seule colonne centrale; dans le Crmonorium le calice est à une scule disision tronquée et charnue, et il n'y a qu'une seuls: étamine.

Dan, les fleurs femelles, on observe un ovaire infere mifoculaire, monosperme, a erraine pendante, couromé par bu c. lice a 2,3 ou í divisions, et sumonté de denx styles dans l'Helosis, el d'un seul dins les trois autres gemes. 
La graine renferme un trés-petit embryon monocotylédon, entouré d'un périsperme charnu, très-épais.

Ce Mémoire, extrumement intéressant par la singularité des plantes qui y sont décrites, est le dernier travail de 1. C. Richard, et n'a été publić qu'après sa mort par son fils; il est une nouvelle preuve du talent et de l'exactitude généralement reconnue de son auteur, et atteste la perte que la botanique vient de faire par la mort d'un si habile observateur.

A. D. B.

301. Fitalissement d'une nouvelle famille de plantes sous le nom de Cyclanthea, les cyclanthées; par M. A. Portenu. (Mém. du Mus, d'hist.naturelle, Tom. IX. page 34, tab. 2 et 3 .)

La fimille que Mr. Poitean propose d'établir sous ec nom ne renferme encore que le seul genre Cyclanthus, genre nouveau d'une organisation très-singulière et décrit pour la première fo is dans ce mémoire quoiqu'une des espèces qu'il renferme en̂t déjáté observéepar Plumier et qu'elle soit décrite et figurée dans ses manuscrits; celte famille est très-voisine des Aroïdes et surtout du genre Carludovica dont le Cyclanthus à tout-àfait le port.

M. Poiteau caractérise ainsi le genre Cyclanthus : Spitha polyphllla. Spadix glindraceus, florilus diclinis spiraliter aut circulatim dispositis tectus.

IIAs. Calix obscurus, calici foris feminei adherens, wix apice liberus, in cirrulum aut spiram longam suprit spadirem expansus. Stamina numcrosissima fundo calicis inserta.

Fin. Calix major, calici masculo inferne junctus, in lamimes ciurs divergentes apice desinens, uti masculus suprit spridicem expeansus; stigmata numerosissima, laminata fauce calic is exserta Ovarium alherens, uniloculare, polyspermum. Semina orata minina parieti affixa.

J.e fruit n'est pas encore conm. D’après la figure méme donnée par M. Poiteau il parat difficile de regarder lit partie quil indique comme le calice des fleurs miles comme appartenant a ces fleurs, il pasaitrat plutût dépendre des flcurs feunelle's, tandis que l'orane décrit dans ces flenrs comme un calice adhiremt ne seratit que les parois externes de l'ovaire. 
I.es etamines seraient alors nues, ee qui est plus en rapport avec leur insertion irrégulière.

Des denx espreces dicritesdans ce mcmoire l'une, Cylanthus Bipurtitus, acte oburber frar M. Poiteau a la Cinyane, lautre; Cyclanthus Plumieri, a eti. Irouve par Plumier a la Martinique. Toutes deur ont les feuilles bifides comme plusieurs Carludorica.

A. 1) $B$.

39?. Atalsse ne freit de biobay ( Adansonia Bao-

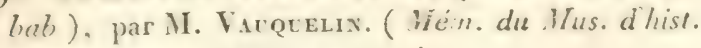
naturelle, tom. VIII, page r.)

Lat partie de ee fruit citue 3 . Vauquelin a soumise a l'analyse cist la matiere parenchymateuse et amylacie qui entoure les graines et remplit presque toute la cavité du fruit : elle est comnorie; $\mathbf{r}^{\circ}$. d'amidon; $2^{n}$. d'une gomme parfaitement sem-blable à la gromme arabique; $3^{\circ}$. d'un acide resscmblant beancoup a lacide malique qui n a pas pu cristalliser, $q^{n}$. de surre incristallisable comme le sucre de raisin. 5". entin dun parenchyme formé de ligneux et d'amidon.

Ia fienle sucrée du fruit du baobabaclonne lieu facilement a la fermentation alcoholique; mais elle est passée presque immediatement a la fermentation acide, probablement a cause de la petite quantité de sucre et de la srande masse de matière mucilagineuse et de lacide qui existait déja dans cette fécule.

ni. Vanquelin rapporte quelques faits citis par Adanon

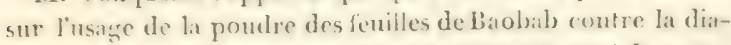

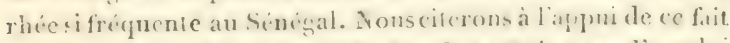
les observations faiter par le J). Frank au (aire sur l'emploi

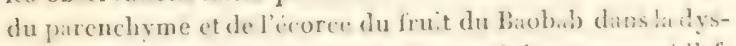
senterie et dont des experiences directes lui ont prouse l'effic:uté. Toyez Bull. des Se. par la Soe. Philomathique, juillet $x 322, p=105$.

A. D. B.

ig3. Onenvationssur le genre firrostemon, descriplion dimi nouvelle espéce qui lui appartient: par M. Desvoxtaxis. ( Mem du Mus. dlist. naturelle', vol. VIII, page II 4.)

Fo divivint le genre Gyrosicmon dans les Memoires du

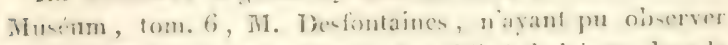
les fruits mors de cette plante, fint oblige de lifeser dans le 


\section{Botanique.}

donte la place qu'il devait oceuper parmi le's familles naturelles; il présuma seulement qüil pouvait appartenic a la fa mille des Euphorbiacées. M. Gaudichanh, botaniste du voyage de M. Freycinet, ayant rapporté une nouvelle espece de ee genreavec des fruits murs, MI. Desfonlatines sist assuré que l'embryon était recourbé à radicule infere, ce qui éloigne ce grenre des Euphorbiacées et indique son analogie avec les Malvacées, parmi lesquelles MI. Desfontaines le range. L'espece nouvelle sur laquelle l'auteur a fait ces observalions a Sté nouméc Grostemon cotinifolium. Elle croit a la Nouvelle-Hollandesur les côtes de la baie des Chiens-Marins; une excellente figure de celte espece, présentant une analyse dilaillée du fruit, accompagne ce Ménuire.

A. 1). B.

3 í. Descruption d'un noureau genre, par M. Desfoxtanis. (Mém. du Mus, dhist. nuturelle, tom. VIII, page Ir9. )

Ce senre auquel M. Desfontaines a donné le noru Condylocarpon appartient a la famille des Apocyues: on u'en connait encore que le fruit, mis il prischte un carictere assez remarquable pour le distinguer de tous les autres genres de la même famille. Les deux follicules qui composent ce fruit sont articulés transveralement et disidis ansi en trois on quatre segmens monospermes indehiscens; les graines sont linciares, allongeies, sillonnces sur une de leur face, sans aigrettes; elles renferment un cmbryon invere, a deuxcotylédons linéaires, plats, enveloppé d’un pírisperme clarnu.

I.a seule espéce connue de ce genre porte le nom de condylocurpon öujumense. Elle a été ripportée de la Guyane par J. Martin. Elle est figurée, pl. I r. A. D. B.

ig5. Notice sur re cannerien de l'ile de Ceylan, sur

sat culture et sur ses produits, par BI. Lescie-

NAUt, DE IA T'OUR, naturalistedu roi. (Mem. diu

diass. d'hist. malurclle. tom. YIIT, iage 436.$)$

liobjet de ec mómoire est particulierement de dierire la

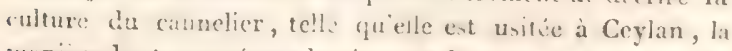
maniere dont on pripare les aurces de camnelles, el dont on

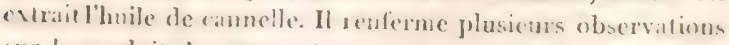
sur le produit de celle culume, sur l'exportation de exlec 
substance à Ceylan, et sur la quantité d'huile qu'on peut en extraire.

A. D. B.

?.g6. Fxpositiox plus exacte des caractères du genre deplante Luíoria (Carludovica Fl. P'er.) tenant à la famille des Aroidées. (Mém. du Mus. d'hist. naturelle, tom. IX, p. 25 , tab. $\mathbf{I}$.

M. Poiteau décrit dans ce mémoire deux especes nonvelles de ce genre, sous les noms de Ludovia funifera et de Ludovia terrestris. Toutes deux croissent à la Guyane, et prísentent comme les especes déjà connues des feuilles profondément bifides. C'est sur ces deux espèces qu'il a étudié et nodifie le caractère du genre qual itablit ainsi :

spatha polyphylla; spadix cylindraceus, floribus declinis tectus.

Iasculi flores quaterni, formineis interjecti. Calix obvers: ronicus, apice duplici serie multifidus. Stamina indefinitn, parieticalicis inserta.

Fieminei flores. Calix $4-p$ hyllus. Filamenta, 4 sterilia, Inngissima, lyposvna, foliolis calicis opposita. Ovarium litherum, tetragronum, superne incrassatum, retusum. Stigmaia, 4 obtusa, olscura, ex angulis orarii prodeuntia. Fruetus, iaccatus, obverse', pramidatus, unilocularis, polyspermus. S.emina primium numerosa, dein abortu pauciora, osata, $p^{\prime \prime-}$ rietibus quadrifariàm inserta.

La principale difference entre ce caracterc et celui donné far Ruiz et Pavon, consiste, comme on voit, dansla deseription du pistil; les anteurs de la Flore du Pérou ayant pris les 4 filamens stériles qui entourent l'ovaire pour des styles.

Willkenow, qui a décrit ce nème genre sous le nom de Salmia, differe aussi beancoup dians le caractere qu'il lui assigne; et il ent probable que la plante d'apres laquelte il l'a trací, doit former un genre particulier. A. D. B.

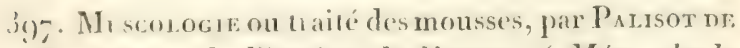

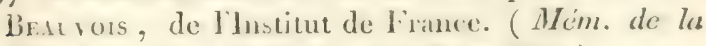
Soc. liuncenne de P'aris, vol. I, p. 388.)

Ce mimoire n'est presque qu'une seconde édition du $I^{\prime}$ ro-

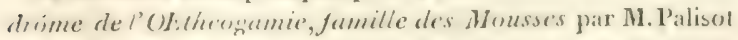

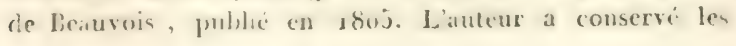


mèmes divisions, les mêmes principes de classification et souvent les expressions elles-mêmes; un seul genre est ajouté à ceux qui étaient dans le Prodrôme, il porte le nom de Codriophorus; l'auteur l'indique comme intermédiaire aux Dicranum et aux Trichostomum il a le péristome du premier et la coiffe du second. Aucune des espèces qui appartiennent à ce genre n'a été indiquée par l'auteur, il est probable pourtant qu'il y rangeait les Dicranum oratum, pulvinatum, etc. Quoique ces espèces soient placées dans cet ouvrage parmi les Dicranum oules Trichostomum; il est à remarquer en effet (qu'ancun changement, aucune addition, n'ont été apportés à ces inumérations d'espèces placées à la suite des genres. Elles sont telles dans cet ouvrage que dans le Prodrôme et cependant combien depuis $\mathbf{x}$ so5 les ourrages de Schevœgrichen et de llooker n'ont-ils pas augmenté le nombre des genres et des espèces connues.

Ne sachant pas à quelle époque M. Palisot de Beauvois a rédigé ce travail, ne l'ayant peut-être pas destiné à être publié tel qla'il est, on ne saurait lui reprocher d'avoir passé sous silence beaucoup de changemens apportés à cette science depuis son premier ouvrage.

La senle addition importante faite à cette seconde ćdition est celle de ir planches in $-4^{\circ}$. représentant les caractères des divers genres adoptés par M. de Beauvois. A. v. B.

3y8. Le prenier cahier d'un ouvrage italien intitulé : $F a-$ miglie celchri Italiane, Milano, 1822 , in-fol., renferme dés détilils intéressans sur la vie et les ouvrages de Frédéric, prince et due de Cesi, qui, âgé à peine de 18 ans, fonda, le 17 août $15 y^{3}$, l'académie des Lincei de Rome, dont il fut chu président perpétuel. Celte société savante, qui compta parmi ses premiers membres, Galılée, Culumna, Porta, Stelluti, Terenzio, Velser, Jean Fabri, et qui promettait de faire faire aux sciences naturelles de grands progrès, éprouva, dés sa naisaince, toutes sortes de contrariétés et mème de persécutions. Ie fondateur étant mort de chagrin, le 2 août 163 , les membres de son acidémie se dispersèrent, et leurs utiles travaux demeurèrent suspendus. Cependant les papiers des tincei avitent été recueillis par l'un d'eux, et ils se trouvaient dims la bibliotheque Albani, d'ou ils ont disparu lors des 
rouble's qui celaterene a Rome, a la findu siecle dernier. Ors a presdu aussi un grand ouvrage entrepris, et a ce quion eroit. fermine par Friblérie Cen, sous le titre de Theatrum totius nizlure; il nest iehappéa la destruction que ses Tables phitosoptiques, imprimies à Rome, en $16 \%$, vers la fin du cruxiene volume de la Flore du Mrxique de Ilernandez. Cos fubles, pour leaquelles l'auteur avait obtem une approbalion dés l'annie 1628 , ont éte pen cilés parce qu'elles se trouvent jointes a un ouvrage tris-rare, et quelles manquent d'ordre; mais elles meritent l'attention des botanintes par les idees quelles renferment sur la classification et la nomenrlature desplantes, sur la figure et l'usage de leurs differenies parties, sur leur ermination, leur reproduction, etc. i.auteur n'a pas mome nighligé d'indiquer la difterence des oxes dans les viocilaux, en prenant pour exemple le chanve ct la mercuriale. (Voy. table 18 vers la fin.)

Apres san sierde et demi d'oubli l'arademie des Rincer a

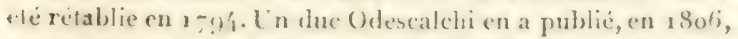
une hisfore, et l:- eoncitoyens de Frideric, lui rendant une fardive jastice, o ti place ston buste dans le Capitole.

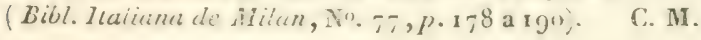

\section{IINRRAQGIF.}

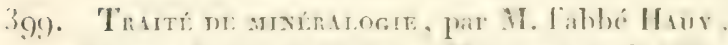

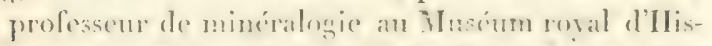

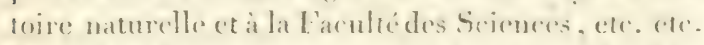

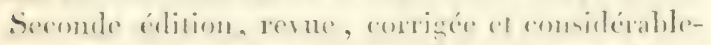

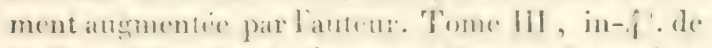

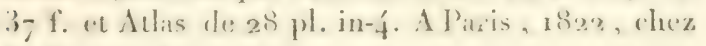
Bachelier, Prix, i $5 \mathrm{fr}$.

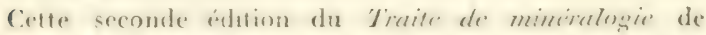
M. labhe Hany peut elre combidere comme un uonvol

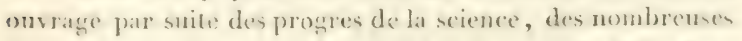

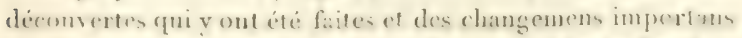

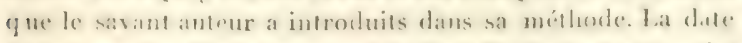

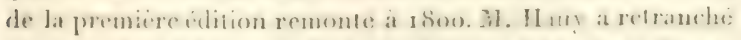

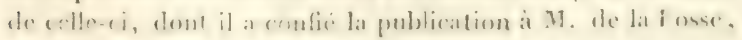
son éleve, la parsie relative a la théorie grénérale de la 


\section{Minéralogie.}

crislallisation qui forme maintenant un ouvrage a part (Tresite de cristallographire, 2 tol. in-\$", avec atlas in-4", de $\$ 4$ planches, $1 \$_{22}$ ) et qui est destimé a servir en quelque sorte d'introduction à l'onvrage que nous annomions.

Le premise volume de ce dernier renferme un discours préliminaire asse\% ctendu qui présente l'histoire abrexice de la minéralngie depuis vingt-deux ans qu'a paru la $I^{\mathrm{re}}$. édition, et indigue les motifs qui ont dirigé l'auteur dans la redaction de celle-ci. Il consacre ensuite pres de trois cents pages a l'exposition des notions préliminaires sur les minéraux; a celle de la cristallisation el de sa propre méthode, au sujet de laquelle it examine les systemes de flerner et de Berzeliun; à la nomenclature, ot au développenent des caractires, tant pliysiques, que chimigues ou geometriques, cmplovés pour recommatre ou caractégiser les minéranx. Coux qui ont rapport aux variélés de formes indéterminables, al lélectricité, au magnétisme et aux couleurs, sont traités avec beaucoup plus de détails que dans la premicre édition. Cetle introduction est termince par une table fort itenduc des pesanteurs specifiques des mineraux; un tableau du regne minéral consikléré sous le rapport de l'électricité produite par le frotlement et des amnotations xclatives anx différentes classes; et enfun par le tableau des formes cristallines des espéces minérales, suivi de l'exposé du plan de l'auteur dans la description des especes. La seconte moitie du premier volume contient lat dastiption de la premiere classe renfermant les circiles liures, laciole sulfurigue et l'acide boracique, el celle des neuf premieres especes de la seconde classe, gue 11 . Haïy nommail antrefois substances acidiferes, et actuellement substances meital-

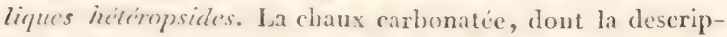
tion est enrichie d'un grand nombre de variétés nouvelles, et spos melanges, est la fremierre esjece de cette classe; viennent ensuile les dencriptions de laragnonite et eelle des autres expecs du sone chanx gui terminent le volume. Iess annolations relatives a la siluation gónlogique des esperes ef l'indication des jays ou relles se trourent out recu aussi beancoup de diveloppronens. I.e premier volume est com-

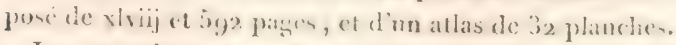

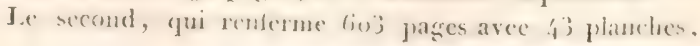


contient la suite des espices de la seconde classe, au nombre de vingt-sept; elles appartiennent aux genres baryte, stronliane, magnésie, alumine, potasse, soude et ammoniagque. Flle est terminée par un appendice dans lequel sont rangées les espèces connues ordinairement sous le nom de pierres et qui formaient dans la première édition la classe des substances terreuses. Ces dernières sont aujourd'hui au nombre de cinquante-trois, dont 38 sont décrites dans ce volume. Plusieurs des substances de la seconde classe et de lappendice sont nouvelles; d'autresn'avaient élé qu'imparfaitement ciérites. Le corindon, classé jusqu'à présent parmi les pierres, forme actuellement la première espèce du genre alumine. En général cest dans la distribution des espèces minérales de cet appendice que la méthode de M. Haüy a subi le plus de changemens. Celles que l'on nomme communiment gremmes sont décrites avec un très-grand soin, quoique avec moins de développenens techniques cependant que dans le Traité des pierres pricieuses ( 1 vol. in- $8^{\circ}, 1818$ ) de notre célèbre professeur.

Le troisieme volume enfin, qui rient de paraitre, est composé de 5 g 8 pages, et de 28 planches. Il offre lá fin de la deseription des espèces qui forment l'appendice à la scconde disse, et celle de la plus grande partie de la troisieme qui est consacréc aux substances metalliques àutopsides, on proprement dites. Ces dernières sont an nombre de quarante-trois : elles appartiennent aux genres platine, iridium, or, arghent, mercure, plomb, nichel et cuive. La description des deux premieres especes du genre fer termine le 3\%. volume, qui sera suivi d'ici à deux mos d'un quatrieme, lecpuel completera l'ouvrage. L'on remarquesa particulieremont dans le volume qui nous occupe les considerations siviriales qui précédent les articles de l'or, du fer, des pierres météoriques, et celui de la théoric du magnétisme.

M. II:iir a fait entrer dans cette nouvelle édition le's nombreuses observations qu'il a publiées a part depuis 1800 , soit dans son tableau comparatif des résultats de la crist:llographic relativement au classement des minéraux ( 1 vol. in- $8^{\prime \prime}$, avee 4 planches, 1819 ), soit dans les Amuales du Muséum dhintrire naturelle, ou le Journal des mines.

1,a partic typographique de cette seconde édition est 


\section{Minéralogie.}

soignée, ainsi que la gravure des planches qui l'accompagnent. Un perfectionnement qu'offre celles de cette édition c'est d’avoir placé le nom de chaque variété à côté de la figure qui la représente.

La distribution méthodique des espèces minérales scra publiće avec la dernière livraison de l'atlas.

Lucas.

40o. Tentamen mineralogicum, seu mineralium nova distributio in classes, ordines, genera, species; cum varietatibus et synonymis auctorum : cui additur lexicon mineralogicum, etc. Auct. Z. Kick in-8. Prix, 2 flor. Bruxellis, 182 r. Delemer.

4́r. Lexicon Mineralogiccir enneaglottum; par

Michel Konatz. I vol. in-8. Pest, i82 I.

402. Traitéde cristallographie, suivi d'une appli

cation des principes de cette science à la détermination des espèces minćrales, et d'une nouvelle méthode pour mettre les formes rristallines en projection; par MI. l'abbé Hacr. 2 vol. in- 8 de plus de $600 \mathrm{p}$. , avec atlas in- $\{$ de 8 亿 pl. Prix $30 \mathrm{fr}$. Paris, 1822 . Bachelier.

I'auteur s'est proposé de développer dans cet ouvrage la théorie de la structure des cristaux, qui dans son Traité de mineralogie est l'une des bases de la détermination des espèces. Ce travail est divisé en quatre sections, dont deux relatives à l'exposé des principes de la cristillographic, et les deux autres aux applications de cette science à la méthode minéralogique. L'exposé des principes comprend naturellcment deux partics, l'une synthétique, et l'autre analytique. Dans la partie synthéticue, l'auteur présente les résultats généraux de sa théorie à l'aide du simple raisonnement, et sans faire aucun usage du calcul, empruntant seulement à lat géométrie le secours des figures pour rendre sensible anx yeux ce que le raisonnement montre at l'esprit. Dans la partie analytique, il traduit ces résultats généraux en formules algribriçues, toutes préparées pour la solution immédiate der probiemes, dont le principal a ratport au calcul des anciles; il donne une explication détialice de certains acridens de: lat cristallisation, tels que les hémitropies et les transpositions, 
les arrondissemens des crivtaux et les simenularifés de lenu stoupement. lat trobieme pattie renferme des considerations

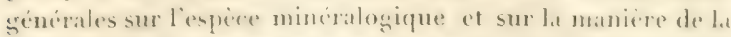
determiner; sur les ss stemes gimeran de cristallisation el les differencen cutre les ststemes particulien's relatifo a des fornes primitise du meme semre. L'anteur trate ensuite des formes communes a diverses especes; des mincians mélangies de matieres heterogines, et de cenx dont les formes sont indeterminables. 11 termine cette partic par une histoire abs a gree des progres de la cristallographie, depuis environ trente ans. Fnfin, dans la dernite section de l’ouvage, il indigue le procedé quil a suivi pour tracer les projections des cristaux, si nécessaires al l'itude de leurs formes et de lours principales propriétés. On wit, par cette comme analyse du Traté de cristallographie, qu'on peut le considerer à lat fois comme une sorte dintroduction et de complément an sirand

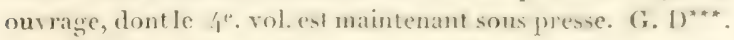

foj. Ser ia Nipuér.rxe trourée dans une Dolérite au Katzenbuhlel, prés Heidelberg; par NL. de Léoxhard. - Sir ra Néphéline de Kayserstuhl; pax M. Lemax. ( Bull des Sie par la Suc. Philom, nov. I $822, p .174$ et 176 .)

qó. Notice ser la marenisite du bassin de Paris, et sur le gisement de celle roche dans divers lieux;

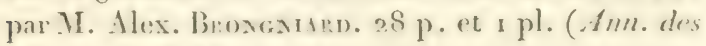
Mines, to. VII, p. 270.)

L'auteur réunit en une sente eqpere la masnivite plastique compones de mannésie, de silice ol d'eau, of la magnesile effersesernte, composie essentiellemont de mannesie of da ride carbonique. Il dierit lat magnesite parisienue et son ghise. ment, quilil a observe pres de Coulommiers, en lits minces, interposis dams des couches diun ealcaire marneus, lequel doit itre rapporte à la formation d'ean done movenne des terrains textiaires, cest-a-dire à celle du calcaire siliceur; il retrouve la macnesse dans le méme forrain, a Crecy ot a Saint-Onen pren Paric. I, 'autur complare enonite arec ce gisement, colui des magnésiom de Silinelle (Gard), de Vallecas, pres Madrid, de Castellamonte et de Baldisioro, an 
Piemont, et y recomait les indices de lit méme formation. xe $\$ 4^{\mathrm{e}}$ de ce ménoire renferme des observations sur la composition de la magnésite et de la masnésie carbonation, et la" analyses de neuf varictés de cees substances par nil herthier

B. $\mathbf{D}$.

405. Notice sur les mines ancicmement exploitées près de Pont-Gibaut (Puy-de-Dòmo); par

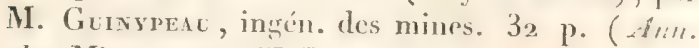

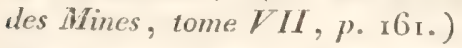

Cette notice renferme: I". un apercu de la constitulion physirue de la vallée de Pont-Cribaut; $2^{\circ}$. des renseignemens wur les mines difes de Combres, de Burbecot et de Roure, ret sur les fonderit's de Pont-Gibitut, d'après d'anciens manuserits, des communications verbales faites a l'atuteur et ses propres observations. L'auteur conclut que la reprise de ce, anciennes mines seraient un oljet dinge d'interet et quiun ponrait en esperer annuellement un produit de Soo à aone quintaux de plomb avec 1200 mares d'aresent. DE Bus.

fu6. Notes ser le minerit de roond argentifèe d." Chéronic (Chasente), et sur le minerai de plomb de Chenclone (Pliome); par M. Brnturer, ingén. des mines. 6 p. (Anm. des Ifines, $t$. VII, p. 1.4o.)

Ces deux noles ont pour objet la description et l'analyse des minerais de res denx gisemens dont l'mu (celui de (charonie ) est remarquable par la richesse en argent du plomb carbonaté qui est uni a la galène, et l'autre par la gratitie proportion dn plomb phosphaté qu'il contient et qui forme la partie la plus importante du ininerai.

B. 11 .

foz. Secono Ripportit analylique sur quelques ('.peces de micas; par M. J. Pescher (de Geneve) (Journ. de Plysique, aotit $1822, p .137$ a 143 ).

L'auteur avait díja fail connaitre, dans le tome 43 du Journal de pliysique, pagne 2 a par l'analyse de trois va. rietes de micit, que l'on rencontrait lacide fluorique of l. titane parni les principes constituans de cette substanco minérale. Iyant andiysé depuis sept autres varictés, il at porti par ses recherches a conclure qu'il faut admetive 
comme principes constituans des micas la silice, l'alumine, le fer, le titane, la potasse ou la soude. Le mica vert du Vésave et le mica noir du Saint-Gothard ne lui ont offert ni titane, ni soude, ni potasse; et M. Peschier fait observer que cette dernière variété de mica présente tant de différences dans les proportions deses principes, que peut-être les minéralogistes seront conduits à la séparer des autres. C. P.

(4o8). Lfs Expéntexces faites par M. Regxifr, ancien conservaleur du musée d’artillerie, avee le ductilimitre de son invention. ont montríes : $1^{\circ}$. que le plomb refondu dix fuis de snite, loin de s'aigrir, gagne en qualité ; $2^{\circ}$. que le plomb mélangé avec un dixième de zinc se durcit sensiblement; 3.. que l'etain de Cornouailles serait quatre fois plus dur que le plomb. (Ann. des Min. tom. 7 p. I/.) F.

\section{PALONTOGRAPHIE.}

409. Sur ta classification et la distribution des végétaux fossiles en général et sur ceux du terrain de sédiment supérieur en particulier; par M. Ad. Bronganint. (Mém. du Mus. d'hist. naturelle), vol. VIII, page 203 et $297^{\circ}$ )

L'auteur a eu pour but dans ce mémoire d'établir parmi les vigétaux fossiles de quelque terrain qu'ils fussent, des coupes génériques bien caractérisées qui permissent anx géologrues de déterminer et d'indiçuer les especes qui se rencontrent dans les diverses couches du globe. Ces genres sont nécessairement artificiels puisqu'ils ne peuvent ètre fondés que sur les caracteres qu'offre la partie du véggétal çui a passé à l'ctat fossile. Ces caracteres paraissent pourtant fournir quelquefois des groupes assez naturels. L'auteur a distingué I9) genres de plantes fossiles qu'il a distribuées dans quatre classes.

I". Tiges done l'oranisation interne est reconnatissuble. Ce sont les bois fossiles divisés en denx genres suivant qu'ils sont dicotylédons ou monocotylédons.

2.". Tiges ciont lorgranisation interne riest plus distincte, mais qui sont saracteriseses par leur forme enterieure. Presque tous ces ventiax appartiennent aux terrains houiller ot 


\section{Palonthographic.}

paraissent provenir de végétaux monocotylédons, Fougères , Equisetum, Lycopodes, etc.

30. Tiges et feuilles ríunies et feuilles isolies. Cette division très-artificielle renferme huit genres appartenant à différens terrains.

$4^{\circ}$. Organes de la fructification divisés en deux genres, les Antholithes et les Carpolithes.

L'auteur examine successivement ces différens genres et cherche à déterminer les plantes actuellement existantes dont elles se rapprochent le plus.

Dans la seconde partie de ce Mémoire, il indique les véGétaux propres aux terrains de sédiment supérieur ou terrains tertiaires, et il décrit plusieurs fruits ou autres portions de végétaux qui leur appartiennent; tels sont plusieurs pins fossiles, une espèce de noix des environs de Turin, les diverses gyrogonites ou fruits de Chara des environs de Paris, une tige des terrains d'eau douce de Paris que l'auteur a reconnue pour une tige de Nymphea, etc.

Ce Mémoire est terminé par quelques considérations générales sur la distribution des végétaux fossiles dans les divers terrains et sur les principales différences que ces fossiles paraissent indiquer entre la végétation de ces diverses époques et celle qui couvre actuellement la surface de la terre.

Ce mémoire qui offre les principaux résultats d'un travail fort étendu et dans lequel on ne sait qu'admirer le plus de la sagacité ou de la persévérance de son auteur, est accompagné de planches parfaitement lithographiées représentant un exemple des différens genres et de leurs principales divisions, quelques portions de végétaux vivans pour faciliter leur comparaison, et les fossiles des terrainstertiaires. V.AUD. fio. Sur des os humarvs ( réputés fossiles déconverts en Allemagne avec des débris de différens animaux), par M. Th. Weaver membre de l'Académie d'Irlande, etc. (Ann. of Philos. Janv. 1823)

Les ossemens dont il s'agit dans cet article ont été trourés dans les environs de Kocstritz, village de la principauté de Reuss, sur la rive gauche de l'Elster, au-dessous de Géra. Ils ont été décrits par M. de Schlothcion, dans son ouvrage sur les pétrifications (Petrefactenkunde), Gotha 1820 , et dins Tоме I. 
Ie supplément qui a paru en $18 \times 2$. C"est ce travail dont MT. Weaver donne ici une traduction on anglatis, avec un petit nombre de remarques, oin il insiste particulierenent sur Les faits suivans : $x^{\circ}$. le ossemens dont il s'agit, se trouvent dans des fissureset des carsites qui, soit dans la pierre caleaire. soit dans le sypse, sont remplies par le méme terrain d'al. luvion qui recouvre la surfice de toute cette terre, a plusieurs milles a la ronde; et ce ferrain ne se compose pas seu. lement de terre franche (loam) mais aussi de gravier fulus or moiurs gros, non-seulement calcaire, mais aussi de rockes de differente's natures et mèmede granite, quoique cotle derriere roche ne se trouve qu'à plusieurs milles de là.

2 . Les os humairs ne se trouvent que dans les fissures et tes car ites du gypse de Koestritz; ils y sont accompagnes d'us de renard, de chien on de marte, de belette, de musaraigne, de mulot, de rat, de hamster, d'écureuil, de lievse, de tanpe, de corf, de hibou, el de grenunille, d'espuces dont lese analogues existent, a un pelit nombre d'exceptism pres, qui semblent exiger un nouvel examen.

3o. Parmi les ossemens communs aux cavites du calcaire el a celles du gypse, il y en a qui apparticnuent a des espéces qui ont disparu, savoir un Rhinocéros, quelques expicess du govere C'errus, une Iyène ct un animal approrhant du Jasuar; d'autres, savoir ceux de Bouf, et probatblement aussi ceux de quelque espèce de Cerf, appartiennent aux especes actuelles.

40. Enfin, on ne trouve dans le calcaire qui entoure et domine le grpse, que des ossemens de chevaux, les uns d'une espèce éteinte, les autres de l'espèce existante.

M. Weaver recommande anx naturalistes d'examiner avec la plus frande attention, toutes les circonstances dans lesquelles peurent se tromer ces ossemens, soit dans le grype rt le calcaire, soit anssi dans le terrain supérieur d'alluvion (supposé que celui-ci en renferme). Il insiste aussi à ne pas néglicer de noter les différens niveaux auxquels on les rencontre.

Il regrette que nous ne sachions pas encore quels sont les grands animanx dont on trouve les restes fossiles dans les contrées situées entre les tropiques.

C. II. 
PIITSIOLOGIE.

fir. De la stéritrté de linomine et de la femme, et des moyens d'y rénérlier: par V. Moxn.1T, ete. 2". édit. entièrement refondue, in-8. de $13 \mathrm{f}$. $\frac{1}{3}$, et a pl. Prix, \& fr. 50 c. A Paris, chez l'auteur, rue Culture Ste.-Catherine, n. 19 .

412. Imflexee de cenvelú sur la forme du crâne, diffecultés et moyens de déterminer les qualicés et les facultés fondamentales et de déconvir le siége de leurs organes. Exposition des qualités et des facultés fondamentiles et de leur siége, on organologie; par F.Z. Gact, Tom.1II, in-8. de 32 feuil. Prix 7 fr. A Paris, chez l'anteur, rue de Grenelle-Saint-Germain.

4r3. Rippont de la nature à l'homme, et de I'homme à la nature, ou Essai sur l'instinct, l'intelligence et la vie; par M. le baron Massias, ancien chargé d'affaires de Trance, près la cour de Bade, ete. Tom. III, in-8. de 28 f. Prix, 6 f. Imp. de F. Didot, à Parìs.

亿年. Recmenches ser ifes smpatmes et sur d'autres phénomènes qui sont ordinairement attribués, comme exclusifs, au systime nerveux; par Mic. Fonfia D. M., etc. Paris, I823, chez J.-B. Baillire.

II. le d'octeur Fodéra se propose de prouver dans ce mémoire :

" $x^{\circ}$. (Due la présence du systime nerveux n'est point une * condition absolue pour la production des phénomenes " sympithicques.

"25. (Que de, physiologristes ont atribué trop exclusive"ment l'habilude et le retomr pérodique des fonctions an 2) systeme nerveux, avant de se rembe comple des phino"mines que nous prénentent fous les etres vivans; il mous " semble cofin que ciss un pur jeu de mols que de faire 2 dipendre les phénomines des viogétaux de l'irritabilik, et 
" cenx des animanx de la sensibilite, lursogülos sont donés des - memes proprietés, quits ant sensibles, ont des habiludes, "des sympathies et offrent des phénomenes périodiepues."

Pour arriveraces deux conséquences, l'anteur, aprés avoie distingue dessensations aree cons sience ou pereption, et des sensations sams amandiate; telles seraient pour ces dentieres l'impression recue ster la man quand on dort on qu'on est préoccupé, le plénomene qui a lien daus les animanx decapités, et mene dans tonte partie détichée du corps, etablit d'aprés le sens tries - général qu'il donne aux mots sensation et stmpathie, que la différence entre ces deux phínoménes consiste: "cn ce que, dans la premiere (sensation) l'im"pression s'arréte sur le lien affecté, oil clle arrive tout au "plus au cerveau; dans la deuvieme ( sympathie) l'irritation " s'étend au delà de ces limites, et le reste de lorganisation "souffre de son influenee. "Cela posé, U. le doctear Fodéra jette un coup d'xil rapide sur diverse's classes d'animaux, et dapres ses propres exprérences et celles de M. Lamouroux sur les zoophytes, et entre autres sur la Lucernaire campanulée, il conelut que des sensations et des sympathies existent avec cette même différence dans toutes les classes d'animaux. Il va beaucoup plus loin, car, prenant la Sensitive et les plantes congénires pour objet de ses recherches, il trouve dans le régne végétal des exemples de sensation et de sympathie avec la différence qu'il a établie précédemment. Dans la première expérience, il touche légerement une foliole sur une sensitive, el celte seule foliole se contracte; dans la deuxième expérience, il conpe, pique ou irrite par les rayons solaires une foliole, il y a dabord contraction de la foliole irrite, puis suceessivement des autres et du pétiole qui les supporte. Le premier fait offe a M. Fodera ce qu'il appelle une sensation simple, et la deuxieme une sympathie qu'il distingue des effets de l'absorption en comparant les résultats qu'il obtient at celui de l'expérience de M. le professeur Desfonlitines qui, ayant arroné la tige d'une sonsilive avec un acide mineral, vit quelque temps apres, à mesure que l'al sorption avait lieu, les pélio'es se contracter, puis les folioles, qui tous meurent avec la tige.

M. Fodera pense donc qu'il y a similitude entre les phénomenes que presente la sensitive, les plantes conger- 
xreres, et ceux analognes, qui ont lieu chez les animaux des classes supérieures jusqu'à l'homme inclusivement. D'aprèsces faits, que les suivans tendent à fortifier, l'auteur tire sa première conséquence, et tente, à l'aide du raisonnement, de repeusser la proposition de M. Bronssais qui dit : point de sensation ni de mouvement transmis sans sympathic, point de sympathie sans nerfs.

L'auteur cite des expériences faites avec M. Magendie qui prouvent que la sensitive se contracte sous l'influence galvanique; il rassemble les observations faites par M. Desfontaires, les ingénieuses expériences de M. Decandole sur la sensitive, celles faites par d'autres observateurs sur l'hedysarum girans et quelques autres plantes, qui toutes prouvent que des individus du règne végétal ont des habitudes, que leur sensibilité s'émonsse par une action répétéc, ct qu'elles peuvent contracter de nouvelles habitudes, mème contraires à cclles qu'elles ont naturellement. Notre auteur ajoute que des expériences faites avant lui prouvent que plusieurs plantes perdent de leurs propriétés par l'action de l'opium, et de l'alcohol et en retrouvent par celle dia camphre.

Tels sont les faits qui viennent étayer la première conséquence de M. Fodéra et lui fournir la seconde.

En un mot, voir une série progressivement graduće, formée par l'homme, les animaux et les végétaux, tenter d'établir une similitude générale entre les sensations et les sympatthies de ces différentes classes d'ètres, prouver que ces phénomènes peuvent être indépendans du système nerveux; tel est, je crois, le but que l'auteur veut atteindre. Mig.

415. Aveettexg zur phrsischen und moralischion Erzichung des weiblichen Geschlechtes. Instruction pour l'éducation physique et morale du sexe féminin, d'aprés F. Darwin, avec des not's : par C. (x. Hefeland. In-8. Leipsic ris22. Brocklatus.

Extrait de l'ouvrage de Darvin: Plan the conduct of at female edrication.

fí. Latetre a M. MAcexune sur de nourelles apririences relitives any proprictés médicamenteuses de l'Urée sur leseme de mort que produit la noix 


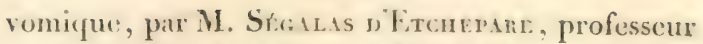
particulier de physiologie et de pathologie.

La lettre de M. Ségelas a M. Magendie contient deux notes communiquere a lacadimie de médecine sur lurée, et quelques faits pour checher à apprécier le genre de mort produit jar la strychnine. Dans la premiere note, M. Ségalas confirme les experiences de MM. I'révest et Dumas qui prouvent que l'uréc a dans l'économie animale une existence indépendante de la séerétion rénale, et il rétracte le premier résultat des recherches qu'il avait faites avec M. le professeur Vaupuelin, en annoncant que ce célebre chimiste a rencontré de l'urée dans le saner d'un chien privé dés deux reins.

Dans la seconde note, M. Sigalas rapporte denx experiences qui tendent à prouver l'action diurétique de l'urée, que cette substance introduite dans l'ceonomie animale 'n est promptement éliminée, et qu'elle ne parait pas y avoir d'action nuisible. Les essais tentes sur l'homme par M. Fouquier semblent confirmer l'action médicanentense? de cette substance et prouver qu'elle n'a pas d'action nuisible.

$\Lambda$ ces deux notes, M. Sigalas ajoute des expériences sur l'effet de la noix vomique comparée a l'asplyyxie, et il vent prouver que la strychnine administrée à haute dose produit la mort par une action directe sur le systime nerveux, à pru prés comme pourrail le faire une forte commotion électrique; ces expériences n’infirment en rien l'idée émise par M. Magendie qu’il y a asphysie dans ce genre de mort, car il n’a point nié l'effet produit sur le systeme nerveux, puisqu'il a décrit le tétanos qui a lieu alors; mais il a dit qu'il y avait aspliyxie, et cest ce dont il est facile de se convainere, en donnant une petite dose de strychnine; la mort est plus lente a arriver, et les degrés de l'a ipliyxie plus marqués.

On trouve un extrait des expériences de M. Sígalas sur la noix vomique, dans la Resue míl., déc. 1822 , et son mémoire en entier dans le journ. de Physiol. Exprir., I $\$ 22$. M. 4і. Observitiox sur l'existence simultanée de l'alloumine et du sublimé corrosif dans l'estomac d'un cheval. l'ar J. I. LAssaig.ne. (Revue. mét. jam. x833.)

Tous les anteurs, el principalement M. Orfitit, avaient donne lablhumine comme lambidose du sublime corrosif, ou 


\section{Anatomie. - Médecine.}

deuto-chlorure de mercure. Mais dans cetze observation on a trouvé que l’albumine ingérée aprés le poison ne l'a point neutralisé, et l'animal est mort. Ne se pourtait-ii perist que les phinomènes chimigues fussent suspendus daus l'estomac sous l'influence de la force vitale?

\section{ANATOMIE.}

4IR. Rechencmes sur la structure du foic, par M. MI ppe.

(Lond.med. reposil., ॥. I o9, janv. I 8:23, p. 3.)

11 résulte de ces recherches que le fuie est composé de deux substances distincies; J'une granulie, dins: d'une couleur jaune et formant des circonvolutions, tantôt semblables à celles des intestins, tantòt prenant d'autres formes; l'autre cellulo-vasculaire d'une couleur brune, qui remplit les interstices de la premiere. W'apris la disposition des vaisseaux qui se rendent a ces deux substances, M. Mappe pense que li bile est probablement séparée du sangr par les radicules de la veine hépatique.

P. V.

419. Notrce sur de nouvelles pièces anatomiques artificielles, exécutécs con rarton, par M. Amelixe, associé national, à Cacn. (Jostr. de méd. et de chir.oct. 1832, p. 28.$)$

La piece présentée par M. Aneline a pour but de faire connatue l'anatonic des hermien, at be mécanisme de leur formation. Flle offec une representation exacte da eanal inguina!, da eanal fimoral, des saiseaux ensiromnans, etr.

P. $\mathrm{V}$.

MED DCINE.

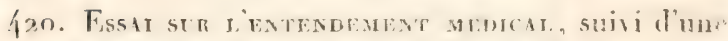
nouvelle méthode pour appremorre la méderine, par J. P. FAм 5 feuilles. Prix g fro rono : a Harsoille imp dA-

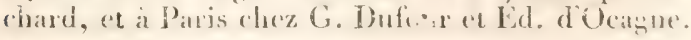

L'anieur a sommi, son ouvrage, avant de lo rende public, a l'andinie rovale de midecine de l'aris, comme ataml lat

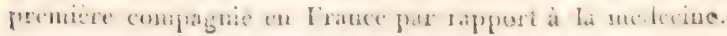


Il a cu la satisfaction de recevoir une lettre de remerciment dans laquelle on lui dit que l'académie a été frappée de la grandeur de l'entreprise et du bonheur de l'exćcution. D'après cet exposé, nous n'avons plus le droit de rien dire sur le mérite de cet ouvrage, dont l'auteur a été traité avec tant de bienveillance. On voit dans l'introduction, composée de 48 pag., que tonte la médecine est renfermée en 5 divisions: en siméiotique, pronostique, dianostique, indication, et pronotion, et que toutes ces parties doivent étre réunies pour former le préjugement général. Chaque division est composée de symptòmes ou signes que l'auteur a réduits en tableaux. Il ne donne dans ce volume que les tableaux des indications et des pronotions. L'éléve commence à prendre note des signes qui constituent l'observation. Il cherche dans les tablètux ce qu'on a employé pour chaque symptôme ou rénnion de symptômes; si on a saigné ou fait vomir, ou purur', ou mis un vésicatoire, ou donné du quinquina, ou enfin si on a cndormi le malade avec l'opium. C'est de la réunion des synutòmes avec leurs remédes que résulte le caleul de probabilité sur ce qu'on a à fiare dans claque cas particulier. Le plan du Bulletin ne nous permet pas de nous étendre davantage sur l'exécution de cette production extraordinaire. Nous devons d'ailleurs attendre les autres tableaux avant que de former un jugement complet.

For.

42i. Couns ellementaire d'uygiene par Mr. Rostan, médecin à la Salpétrière, deux vol. in-8. à Paris, 1822 , chez Bécnet.

L'idée dominante dans cet ouvrage est que tous les pliénomènes de la vie défendent de l'organisation. P'artant de ce principe, l'auteur examine les différences que présente cette organisation selon que prédomine tel ou tel appareil; de lit diverses especes de constitution. Les modifications apjor. tées par l'age, par le sexe, par les idiosynerasies, par le's hiabitudes, forment également la plus grande partic des prole-

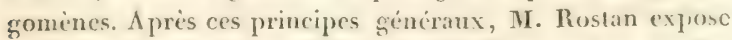
les effets des divers modificateurs de l'économie animale, suivant qu'ils agissent sur les appareils digestifs-respiratoi-res, exhalans, secriteurs, ete; ou bien sur ceux de lit vie de relation, le cerveau et ses dipendances: ici sont compris les 


\section{Médecine.}

excitans des sens, cenx qui exercent la partie du cerveau qui préside à l'intelligence, et ceux qui mettent en action la portion à laquelle ( suivant l'auteur) sont confiés les mouvemens. Cet examen est terminé par l'exposition des phénomènes qui concernent les fonctions de la génération.

Jusqu'ici M. Rostan n'a considéré les agens de l'hygiène que dans l'lypothèse qu'ils concernaient un individu adulte et d'une moyeune constitution. Dans la troisième et dernière partie, il examine leurs effets lorsque prédomine tel ou tel système d'organes, suivant l'àge, le sexe, le climat, ete. C'est l'hygiène spéciale.

On voit par cette analyse rapide que cet ouvrage est entièrement basé sur l'anatomie et sur la physiologie; nous ajouterons que, sous ce rapport commesous celui de son exécution, il diffère heureusement de tout ce qui a été écrit sur cet important sujet.

P. f.

422. Ser tine nocvelle organisation de l'enscignement de la médecine; par Philibert Duvors, ancien secrétaire-général de l'académie de médecine de Paris, ete. In-8 de 7 f. $\frac{2}{6}$. Prix $x$ fr. 25 c. A Paris, chez Delaunay.

433. Toxological cuart exhibiting, ete. Tableau Toxologique offrant d'un scul coup d'ocil les symptôme's, le traitement et la manièe de découvrir les diflérens poisons minéraux, végétaux et animaux; par \$тоwе, deux f. grand in-fol. qe. éd., Londres, $18_{22}$. 4:4. Remanques sur le traitement des fièreres muqueuses à caractères ataxiqques; par N. Cartifn, dorteur en médecine, ete. In-8 de 3 f. $\frac{1}{2}$. Paris, I822, chez J. M. Barret.

4:5. Letetre a M. Lejcueav de Kenginanic, doc-

teur en médecine de la faculté de Paris, etc., en réponse à son Nímoire sur l'auscultation appliquée à l'étude de la grossesse; signé Fonestien. In-8 d'une f. Imp. de Chaigneau jeune, a Paris. 426. Examex de la doctrine medicale de M. Brous-

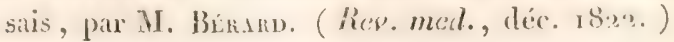


L'irritation fibrile est le sujet ou le texte de cert artucte. dins leciucl l'auteur cherche a faire vorr, diapren les idies mime du réformateur, que l'irritation fibluile est neressiturement une irritation generale et sympathique, et que la localisation des firrers dans un seul or ane est une crreur physiologique de la doctrine physiologique.

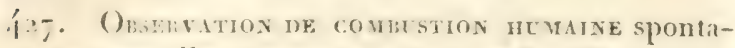
née particle, eommuniqué par L. Manchast, médecin à Bordeaux. (Revue méd., dée. 182.)

Ce fait est tres-important a cause des cireonstances particulieres quil a présentées. Il offre la combustion de lat cuisse "t de la main isolées, et sans aure aceident ultérieur : l'individu ctat forgeron, et la chaleur du jour etait tres-forte. Le malade est partititement guéri de cette combustion.

far. Srr re muturasise, on maladie pédiculaire;

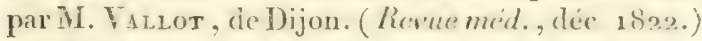

Cemedecin a observe deux fois cette maladie, qui, tenant d'ahord a des canses liygiéniques, derient en queldque sorte constitutiontelle. L'emploi du quinquina à l'intirieur produit, dansce dernier cas, de trim-bons effets, quand on place l'individu dans un lieu sain et propre.

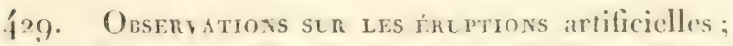
par le docteur Itexier. (Resue méd., dée. 1823.)

Apres avoir regeneré l'criece bumane par la vaccine, le docterar Ienner poursnit encore ses decomertes. Il es parvenu, en provoquant des éruptions par la pommade émétision, dïja conmue, at gutrir extains maniaques el un grand nom bre $d$ 'autres maladies internes.

f́ro. Note ser 3.5 porsox apprele Arpua tofana; par M. Ozanam. (Revue múd., déc. 1822.)

l'usieurs anteurs ont doute de lexistence de ces poisons glui unt acquis une si efirayante renomméc mais M. ()/a-. nam jense, aproce heaucoup de recheretes, quion en con-

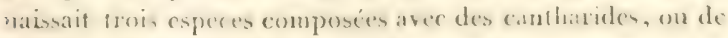
Parsenic, ou de l'accitate de plomb.

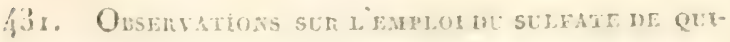


Nine dans la névralgie faciale; par I'. Rubes. (Revue méd., déc. І 82.2.)

Cette maladie est si sonvent rebelle a tous les moyens employés, qu'on doit se féliciter des succés oblenus par la quinine. L'internittence des acces en avait bien fonrni l'indication, mais le quinquina brut n'offrait pas des rertus assez energigues. Deux malade's, trattés de celte maniere, sont. parfaitement guéris, et n'ont pas encore eu de rechule.

43?. Note sur le dauger de donner de l'huile dans

l'empoisonnement par les Cantharides; par le docteur Pailas. (Revue méd., déc. I822.)

Celle observation est d'autant plus importante, que l'huile est la panacic de tous les empoisonnemens; mais, dans ce cas, l'huile dissout le principe actif des cantharides, et le rend beancoup plus dangerenx. Le doctenr Pallas et M. Orfila ont fait des expériences qui toutes confirment ces résultats.

133. Recheriches sun L'emplot de linute de térébenthine rectifié ; par W. Moner. (Revue méd., déc, r 822.$)$

Ce médecin administre cette substance à très-haute dose pour guérir le tonia, l'épilepsie, et quelques autres affections du cerveau ou de l'estomac : mais ce moyen est perturbateur a cette dose, et peut occasioner des accidens. Son effet le plus spécial est d'exciter les voies gastriques et de porter sur les voies urinaires.

亿3. Oeservation sur les effets de l'acétate de plomb, administré à lintérieur; recueillic par F. Gr Borssetu, D. M. P. \{ pages. (Joum. univ. des Sc. méd. tom. 28, p. 229.)

Quelquefois pour faire cesser les sueurs füchenses qui accompagnent la phlhisie pulmonaire, on fait usage de l'acétale de plomb qui remplit effectivement l'indication propesere, mais exaspère souvent les autres symptòmes. C"est ee ' qui est

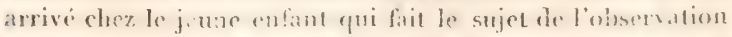

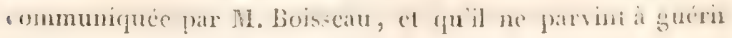


qu'en supprimant l'emplloi de ce remède et lui substituant des évacuations sanguines.

435. Onservation surl'usage de lextrait de la Tanhia, employé avec succés pour prévenir l'avortement chez une femme qui avait fait trois fausses couches; par le docteur Tocnes, chirurgien aide-major des hussards de la garde royale. 4 pages. (Journ. univ. des Sc.méd., tom. 28, 225.)

Lne femme âgée de 30 ans environ devient enceintc pour lat premiere fois et accouche heureusement; bientot une nouvelle grossesse se manifeste, et vers le septicme mois une perte, accompagnée de douleurs dans les lombes, détermine l'avortement; deux nouvelles grossesses sont encore suivies de fausses couclies qui eurent lieu vers le second mois de la gestation. Dans le courant de janvicr 1822 , celle dame, enceinte pour la cinquième fois, et menacéc des mèmes inconvéniens, réclama les soins de M. Tournel, qui lui administra l'extrait de Tanhia, parvint à se rendre maitre des accidens, et a conduire sa malade jusqu'au terme ordinaire de la grossesse ordinaire, ou elle accoucha heureusement.

\section{CIIRURGIE.}

436. Traité des malades chrorgicales et des opérations qui leur conviennent; par M. le baron lBorfa. Tome VIII. In-s de 36 feuilles. A Paris. chez lauteur, rue de Grenclle-St.-Germain, n. 9) Prix $6 \mathrm{fr}$.

437. Die Kr:nst, die in die Luft-und Speisrohre gefillenen Kixper zu entfernen, ete. L'art d'éloigner les corps tombés dans la trachée-artère et dans loesophage, ainsi que de traiter les maladies de la vessie et du rectum; par une société de méderins et de chirurgiens. In-S avee pl. To. I. Prix : 2 rixd. 12 gl. Gotha 1822 , Henning.

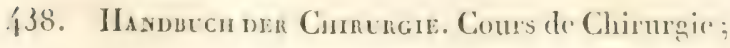


Par M. J. Cnezrus. Tome I. In-8. Ifeibelberg, I822. Groos.

Ce volume, divisé en sept partics, traitc des inflammations el des opérations chirurgicales.

439. Nosologie und tuenapie der chirurgischen hrankhieten. Nosologie et thérapeutique des maladies chirurgicales; par Z. M. LAngeneecn. In-s. ave pl. Cottingue, 1822 . Dietrich. Tom. I prix : 3. rixd.

Ce volume traite des inflammations.

仵. Hinderch den Geburts nülfe für IIebammen.

Manuel à l'usage des sages-femmes. Par Cl. ScirWanzer. In-S. Prix 2 fl. $45 \mathrm{hr}$. Vicme i 822. Heubner.

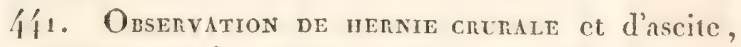
guéries par la même opération; par M. le baron Larrey. (Revuc Méd., déc. r822.)

Ce fait a présenté une circonstance qui doit le faire distinguer, puisque M. Larrey, en opérant une hernie crurale, a aussi guéri une ascite, dont toute l'eau s'est évacuée sants accident. Cette observation ne pourrait-elle pas indiquer un moyen nouveau et plus sùr pour guérir l'hydropisie abdominale?

fí Opservation sur les mernies du pétinée, par M. Bompard. (Rev. méd. Dée. I822.)

Eu rendant compte du Mémoire de M. Scarpa sur ce sujet, NI. Bompard cite une observation nouvelle qu'il a faite et qui confirme encore l'existence de cette espèce de hernie dont quelques auteurs et Scarpa lui-mème avaient douté jusqu'ici.

413. Case of sancocitice (Scirrhous degeneration of the testicle). Observation de sarcocèle ou dégéniriat tion squirreuse du testicule, traité par l'opération. Par Drapes. (Edemb. méd. and sur. Journ. p. 5!).) Cetle observation ne renferme aucun fit remaryuable. H. F. 
許. Case of a Carcunus in the Liethat, efe., (O) servation diun calcul formé antour diun anmean de cuivre, dans le ranal de lliche, par Iastos. (Fid. med. and sur. Jour. janv. p. 57.)

A l'àge de ? ans le malate étant affecté d'incontinenr: d'urine, introdnisit sa verge dians un anneau de ridean pour remédier a cet inconvénient pendant la nuit. Le lendemain le gronflement l'empècha d'oter ce corps qui díterminat bientot l'ulcération de la pean, et a mesure qå il s'enfonca dans la pean le gonflement diminua, et enfin les tónumens se rémirent de manière à l'envelopper. Les fonctions de cette partie se rétablirent parfaitement. (Quarantesept ans après, il sadressa a M. Tiston, étant tonrmentée d'incontinence diurines. On sentait près de la symphise du pubis, un corps résistant; on en fit l'extraction, et on tronva qu'il était forme par un calcul minaire, renfermant les denx tiers de l'anneau de cuivre. II.-E.

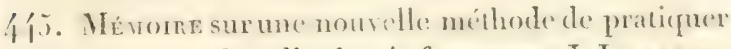
l'opération de lataille chez la frmme par J. Lısfatsc. (Rev. méd. janv. 1823).

Cette découverte fit honneur an génic de ce chirurgen, et il n'est pas douteux que bientòt cette opération ne soit préférée a celles qu'on avait l'usage de pratiquer. Ta facilité d'extraire les calculs les plus considerables et le peu de danger ancuel ce procédé expose ajoutent le complément a celte nonvelle métlode qu'on pourrait appeler Souspubienne. Jine planche inséréc dans la Resue Medicule avec en mémoire donne tous les détails anatomiques nécessaires. (Rer. Med. Jans. $x 8_{23}$.)

4.f6. Ciss of urtuotous, with singulaty shaped calculus rete. Opération de la taille eteveraction diun calcul dour forme singuliere; par \. Moxts, dhirurgien de Theipital des enfaus malades. (fhe Tomdon méd.et phys. journ. n. 288 p. I I6.)

Le malade etat age de 11 ans. On me derouvrit frats dia-

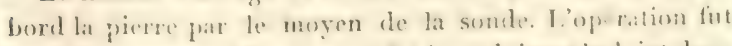

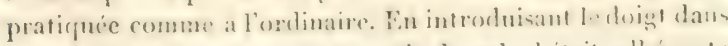
lit plate, on reconnut qu'use partic du raleul ctat athirrote 
et logre dans wn hyte de la vessie. ()n le rempit, en essayant de le degager: cependant on parvint à extraire les deux fragmens : ce ealcul était allongé, arromdi par ses pxirémités qui étaient rémies par une sorte de colérruil a pen près comme une courge.

P. .V.

447. Extraction ne cilcels chinitres qui avaient pour noyaux plusicurs pièces du squelette d'unfortus. Observation communiquée par M. le doeteur Lecraux. (Bull. de la Soc. méd. d'émulation dér. 1822. pag. 477 .

Cette observation n'offre d'intéressant que le passage des débris du fotus de l'utérus dans la vessie, passage qui parait avoir été déterminé par une chute violente pendant la grossesse.

P. $f$.

4.48. Sul taglo retto-vescrcale per l'estrazione della pietra. C'est-i-dire : sur la taille recto-vésicale praticuéc pour l'extraction de la pierre; par le professeur Scaria. I pages. (Journ. univ. des Sc. méd., tom. 28, p. 268. )

M. L.J. Samson rapporte dans cette notice une lettre du professeur Scarpa, adressée à M. Mannoire, sur la taille rectovésicale, et où l'on trouve quelques considérations sur les chances variées que peut offrir cette opération dans les circonstances diverses où il convient d'y avoir recours. Il termine en réfutant les assertions de M. Tortis D. M. P. qui parait opposé à cette nouvelle manièred'opiérer. G. V.

49.9. Perforation de la vessie par les sondes fixes. par L. Lamlemand. (Bull. de la Soc. méd. d'émulation, déc. I 822. pag. 230.)

450. Historne d'une texituon de la vide du fémur sur la branche ascendante de lischion, vers son union avec la portion desecudante du pubis, el description d'une mithode nouvelle pour procider à la réduction de re seme de déplacoment; suivie d'un léger aperecu sur l'état de la chirurgie chez les ancions, et juscuu à nos jours ; par J. n’ $\mathrm{A}$ u. 
ntann, chirurgien et accouchenr, etc. Broch. in-S, avergrav. (Journ. gein. de Mid., oct. s \$s2, p. 133.)

M. Gaultier de Claubry pense que le procédé n'est pas nouveau, qu'il est extremement incommode, et que si l'opérateur n'est pas d'une force athlétique, il est completenent inutile.

P. V.

绾. Notice ser cne mét, rison des renversemens de l'intestin rectum; par M. le profess. Dr pertifis, (Jour. sén. de Mćd., ort. x\$32, p. 85, et Bibl. Mécilic., p. 128.)

MI. Dupuytren excise, a l'aide de pinces a ligature et de ciseaux courbes, un plus ou moins grand nombre de plis saillans qui se porient de la circonférence au centre de la marge de l'anus; il les saisit au dehors et les excise le plus haut possible dans l'intestin. L'hímorrhagie, quand elle a lieu, doit étre réprimée par la cautérisation.

Dix ou douze malades ont été ainsi complétement guéris par cette opération.

P. V.

452. Ser la nítro-stition des dexts incisives et angulaires, dites canines, de la màchoire supérieure, dillormité vulgairement appelée menton de galoche; par M. Derois-Foccou, membre résidant, dentiste du roi. (Jour. gén. de Méd., nov. 1822, p. I 75.$)$

L'auleur décrit un instrument dont il se sert avec succis pour replacer les dents rétro-stitionnćes : c'est un levier du premier genre, qui consiste en denx branches d'acier croisées et retenues par une vis ; une des branches porte un erochet, et l'autre une vis, at laquelle est fixée le point d'appui, qui peut ainsi se rapprocher ou s'éloigner du crochet à volonté : cet instrument est aussi trés-commode pour arracher les dents antérieures.

P. V.

453. Dissentation sur la possibilité et les moyens de faire eder le col, posur corriger les diflormités provenant de fractures vicieusement consolidées; par E. J. Jiceresus, I). M. P. (Journ. gén. de Méd., de Chir., elc. p. 267.) 
Tel est le titre d'une these dans laquelle on essaie de risoudre par l'affirmative la question suivante: Quand une fracture, quoique solidement réunie, ne date cependant pas d'une époque éloignée, et qu'il en résulte une lifformité désagréable, de la géne daus l'exercice des mouvenuens, de fréqrens retonrs de douleur, faut-il changer la mauvaise disposition des fragmens, ou, comme on dit, redresser la fracture?

M. Villermé, qui a fait l'analyse de re travail, le regarde comme digne de toute l'attention des chirurgiens, par le choix du sujet et la manière dont il est traté. P. V.

¡5i. Botatron saw, newly invented, etc., Scie de rotation, nourellement inventic, par le professeur Thal, à Copenhague. (Edimb. med, surg. Jour. janv., p. 55.)

Cet iustrument permet de faire sur des os plats et larges, des incisions droites, d'une profondeur et d'une itendue quelconques : chose très-difficile ou même impossible avec les autres espèces de scies. H.-E.

455. Pratioue mes acconchanens, ou mémoires et observations choisies sur les points les plus importans de l'art; par Mad. Lachappelze, sage-femme en chef de la maison d'accouchemens de Paris: publiés par A. Dugies son neveu. (Journ. gén. de Méd., nov. I $822, \mathrm{p} .272$.

Le premier mémoire comprend l'énumération, le diagnostic, etc., des positions du fotus, et l'cxposé des procédés opératoires.

I.c second, les positions les plus remarquables du vertex, les circonstances qui nécessitent l'emploi des instrumens, etc.

Le troisieme, les positions de la face; il est plein de préceptes utiles.

M. Devilliers termine cette analyse en recommandant la lecture de cet excellent ouvrage. P. V.

156. Nouvelles mimonstrations D'accolcurmisas, Tоме 1 . 
aree des planclies en laille-doner, acrompagnées d'un texte raisonnci propre à en faciliter l'explication; par D. G. Mascrier, doet. en médecine, de.

La premiere lisratison de cet ouvrage, contient lat description et la représentation du bassin et des os yoi le composent. Les explications sont courtes et cependant tres claires, t les figures de la plus exacte vérité. P. V.

位. Two cases of casarean operaton, ete. Deur observations dopération Césarienne, dont liune a cté suivic de succes; par J.V.I an Buren. Communiquices par le Dr. Carson, de Lireppool. (The Lomd. meit. and phys. journ. n. 288 , fév. 1823, pag. 9 r.)

Ia première opération fut pratiquée sur une esclive âge de 35 ans. Le travail durait depuis Go heures environ. Ine exustose place sur la face interne de la symphyse du pubis et se portant vers le sacrum ne laissait que deux poures d'ouverture au bassin et rendait l'accouchement impossible. L'enfant était vivant et se présentait bien. On fit une incision depuis l'ombilic jusqu'au pubis sur la ligne blanche; on divisa cette opomorose et on ouvrit la matrice vers son fond avec un bistouri concave, et on retira anssi vite que possible l'enfant et le placenta : les lèvres de la plaie extérieure furent réunies au moyen de la suture. La malade n’éprouva d'ibord pas d'accident; mais, vers le cinquieme jour, il se manifesta des symptomes de péritonite qui furent combattus avec succes, et ro jours après l'opération elle état guérie.

Ia d. uxieme ojération fut fate sur une néurresse. L'accouchement était rendu impossible par une excroissance dure tris - crrosse qui unissait les grandes lères : on n'osa pas enlever cette tumeur, dans la crainte de l'hémorrhagrie, et on préféra l'operation césarionne. I.e travail durait depuis 2 f heures. Loperation fut faite comme la précédente. I.a matlade mourut de péritonile; l'enfant vint trés-bien. T. V.

fis. Histoine n'in roetes renfermé dans la trompe saurlie de Fallope; par F. Jis.r. (Rev. méd. jamv. 1823. ) 


\section{Pharmacie.}

Ce fait est assez. remarquable en ce que le fretus se développa dans cette partie, et ce ne fut que plus de deux ans après que les os furent successivement évacués par l'intestin rectum, sans amener d'accident fàcheux.

\section{PIIRMACIE.}

459. Fonyulaire magistril et mémorial pharmacentique, recueilli par Ch. L. Cadet de Gassicount, $5^{e}$. édit. rev. et augm. d'un grand nombre d'articles, par M. B.LLy, D. M. membre titulaire de l'Acad. roy.de médecine, I vol. in-ı 8, prix : 4 fr. A Paris, chez L. Colas.

Cadet de Gassicourt, que les sciences et la pharnacie ont perdu depuis peu, avait conçu l'heureuse idée de recueillir les formules magistrales habituellement prescrites par les médecins les plus célèbres des temps modernes; il y avait joint quelques prescriptions de médicamens officinaux extraits des pharmacopées étrangères, et que l'usage avait fait adopter parmi nous. L'utilité du recueil de M. Cadet fut généralement reconnue. Quatre éditions de cet ouvrage sont épuisées. Cette cinquième, revue, corrigée et augmentée par M. le docteur Bally, n’aura sans doute pas moins de succès que les précédentes. Nous engagageons les médecins et les pharmaciens à se procurer cet ouvrage, qui leur évitera de longues et pénibles recherches.

Peil.

f(io. Formulane pour la préparation et l'emploi de plusicurs nouveaux médicamens, tels que la noix vomique, la morphine, la strychnine, la vératrine, les alcalis du quinquina, l'émétine, l'iode etc.; par F. Magende, membre de l'institut, etc., 3 . édit. A Paris, chez Méquignon-Marvis.

?.es progrès que l'analyse vérgétale a faits dans ces derniers temps ont permis de retirer des végétaux les plus actifs les matciriaux immédiats anxquels ils doivent leur action sur l'écunomie animale; mais l'énergie mème de cette action aurait été long-temps un obstaclé à l'emploi médical de ces nouvelles sub- 
stances sans laide d'un guide sir et expérimenté. Liourrage de M. le docteur Magendie remplit ici l'anvre du temps; on peut: dés a présent, en prenant cet ouvrage pour guide, employer es agens puissans, et qui ne sont víritablement dangereux que par l'abus ou l'usage inconsidéré qu'on en peut faire. On frouvera dans le méme ouvrage l'indication des doses et des formes sous lesquelles on pent administrer l'acide prussique. emplové aver tant davantages dans les cas ou l'irritibilití pulmonaire est vicieusement augmente; l'iode et ses préparations, administrés avec tant de succés dans le traitement du goitre, etc., etc.

Pell.

f(ir. Pharmacopée ustelde, théorique et pratique. par J.-B. Van-moss, 2 vol. in-8. Prix : i 2 fr. Louvain , 1822 .

Ces deux volumes, ensemble de 1280 pages, renferment une collection nombreuse de matirianx pris dans des lives ćcrits dans toutes les langues.

463. Codex medicamentaru s Furopaus, seclio Get 7. Pharmacopoeam Ilispanicam et Lusitanicam et littcraturam pharmacopoeorum continens, in-8. Prix : 3 rixd. 20 gr. Lipsix, 1822, Fluscher.

463. Phamicopoea austrica. Edilio tertia emendata. in-8. Vindob, I822, Gerold.

464. Thomson's conspectes. A percu des pharmaropées des colléges de médecine de Londres, d'Édimbourg et de Dublin, par Tuomson; in-12. Prix 5 shell. Londres, Underwood.

465. A Syñopss of thechimical decomposition that take place. Abrégé des décompositions chimicfues qui ont lieu dans la préparation de la pharmacopéc de Londres. Prix, 9 den. Londres, liurgess et Hill.

466. Grav s eiements of phanmacr. Élemens de pharmacie et dhistoire chimique de la matiere médicale, par Grax, avec fig. Londres, Underwood. 
; 6 \% Vegemabe matera medica of the United States, Matière médicale végétale des États-Unis ou Botanique médicale; avec des gravures coloriées par P. C. Barton. 2 vol in- 4 . Prix, 6 1. 5 shel. Londres, Hurn Robinson.

368. A treatise on the materia menica. Traité sur la matière médicale regardée comme faisant suite à la Pharmatcopée des Etats-Unis, par J. Bigelon, M. D. in-8. Boston, 1822 .

4(ig. Extract from $D^{r}$. Ainslie's materia medica. Extrait de la matière médicale publiéce en 18 I 3 à Madras, par le D. Ainslie.

íjo. Haxdecth der pharmaceutischen Waarenhunde. Manuel du droguiste. Par J.-B. Thomsdonf, $3^{\text {e. }}$ édit., augmentée. in-8. Gotha 1822 . Ilenning.

47r. Catalogue of pregs. Catalogue de drogues, spécifiant leurs proprićtés, leurs doses pour les enfans et les adultes, leurs véhicules convenables, les maladies pour lesquelles elles s'emploient et les prix, ete. par Heece, neuv. édit. augm. Prix, 2 sh. 6 d. Londres, Sherwood.

472. Prinuncologra, comprehending the art of prescribing upon fixed and scientific principles, etc. Pharmacologie, contenant l'art de formuler d'après des principes fixes et scientifiques et une description des substances médicinales; par J. A. Paris. M. D. F. N. S. ete. (The Lond. medic. and physic. Journ. n. 287 , janv. 1823, pag. 78 .)

C'est la 5c. édition d’un livre très-estimé en Angleterre: elle est augmentéc d'un volume par les nombreuses additions que l'auteur y a faites.

G. V. 4-3. Découvente de la composition du sirop antisyphilitique de Lathecteur, (Bull. de la Soc. méd. d'émulation, déc. I832, p. 214.) 
Voici la recette que le docteur Savaresy rigarde comne identique à celle de Laffecteur.

F Salsepareille.............. Ib. 9. p.

Bois de gaïac.

Racine de squine.

Sassafras.

Quinquina jaune........... th. 3

Fleurs de bourrache. . . . . . . . It. 1. $\frac{1}{2}$.

Semence d'anis.......... $\tilde{\tilde{z}} \cdot 4$.

MIélasse clarifiée............ tb. 3o. P.

\{́f. Rappont sur les sucres acidules de M. Levrat, par MM. Bundix ainé, Plaxche et de Lexs, rapporteurs.

Les rapporteurs se sont assurés gue ces sucres ne sont réellement formés que de fruit et de sucre. Ils proposent a la Société de médecine de Paris d'accorder son approbation à l'entreprise utile de M. Levrat.

La Société approuve le rapport et les conclusions. (Jour. gén. de méd., de chir., etc., oct. 1822, p. 23.) P. V.

4-5. Composition des gouttes noures de Lancastre.

(Revue. méd., déc. I822.)

Ce médicament, qui est fort employé en Angleterre, a loner temps été un remède caché et secret; mais on sait maintenimt qu'il se compose d'acide acétique et d'opium. Calle préparation peut être utile quelquefois, mais non en la fırodiguant d'une manière empirique à tous les cas.

\section{ART VÉTÉRINAIRE.}

4-6. Tnatrt concernant la connaissance du cheval. la manière de le nourrir, tant en voyage qua l'écurie, et de le préserver des accidens et maladies occasionés par le mauvais traitement; suivi des principes d'équitation moderne; par le vicomle E. Detonet, in-12. Prix : 2 fr. Bruxelles, $182 .$. Jemat. 
Agriculure, économie rurale et domestique. 275 177. Résumé axalytreue des différens rapports des vétérinaires commissionnés par l'administration dans le département du Nord, sur les maladies qui ont régné sur les chevaux, pendant les années $18_{20}$ et 1821 ; ainsi qu'une notice sur la maladie épizootique qui a régné sur les bètes à cornes, dans l'arrondissement de Douai, pendant les amées I8I 4 et I 8 I 5 , redigé suivant les ordres de M. le comie de Murat, préfet du Nord, par Tressigniez, vétérinaire de l'arrondissement de Douai, ete. In-8, d'une feuille. Imp. de Donel, à Lille.

Cet ouvrage étant inconnu à Paris, on n'a pu sc le procurer pour en donner un extrait.

478. A Treatise on the monbid respiration, of domestic animals, ou traité de la respiration morbirle des animaux domestiques, avec le traitement reconnu comme le meilleur, par E. Causer, chirurgien, in-8. Glascow, 1822 .

\section{AGRICULTURE.}

1:9. Encyklop edre des gemein nützigen weiblichen wissens, etc. Encyclopédie des sciences économiques, ou manuel pratique d'économie domestiq̨ue, à l'usage des femmes de ménage.Par $\mathrm{A}$. Bondonff, 4 vol. in-8. Prix : 4 rixd. Quedlinbourg, 1822 . Basse.

Cet ouvrage contient une instruction pour toutes les connaisances utiles aux femmes, à l'exception de l'art de la cuisine.

480. Mémoire sur l'agniculutue et le commerce,

par M. le comte de Turefrner Beavvois, in-8. de 9 f. $3 / 4$. Imp. de Boucher, à Paris.

L'on a dit, et l'ouvrage de M. le comte de Thieffrier Beauvois en est une prenve, que l'ordre rend courts les ouvrages les plus longs, tandis que ceur qui sont écrits sans méthode, quoique courts, se refuicut a l'analy:e. Dans ce 
Mlémoire, d'environ xoo pages, il y a au moins cinq a six cents propositions diverses incohérentes entre elles; toutes les matières s'y confondent, rien n'y est classé; cependant comment se fait-il qu'on le lit avee une sorte de suite, et je puis dire avec utilité? la raison en est sensible : il y at des faits; il y régne de la bonhomic et de la persuasion: quelques vues silges rachitent la monotonie de redites perpétuelles; quelques alinéa présentent des vues saines et partent de la plume d'un homme zélé ami du bien public, mais mallheureusement peu éclairé.

Le Mímoire présente quatre sections. Dans cellc intitulée Dessichemens et difrichemens, l'auteur énonce avec prédilection l'idée de recréer des monastères propriétaires des terrains vagues, et qui les fíconderaient en soignant leur culture; il voudrait inféoder les terres non défrichées: ce node de possession lui paraît celui qui revivifierait l'agriculture et la replacerait au point de perfectionnement où il la suppose avoir été en $x_{7} 89$, car i! croit que l'aggriculture est sur son déclin.

Dès l'année rzzo, l'auteur, simple sous-lieutenant, conseilla et pratiqua un desséchement de marais qui produisit i 2,000 francs, plus en revenus que ne valait le fonds. Fn 1787 , il publia une constitution militaire, qu'on trouva un excellent ouvrage; il voulait créer une sozte d'armée composée de soldats qui, pendant la paix, seraient, suivant les besoins, cultivateurs. Cette constitution remarquable fit plus de sensation que n'en fera l'ouvrage dont nous nous occupons, et il y eut plusieurs géneraux qui en adoptèrent quelques bases.

Le titre suivant entretient le lecteur de l'aménagement des forèts : il joint à des conseils pratiques une vue que l'anteur dit devoir a son expérience; mais nous avons sur les bois, considérés par rapport a la marine, des Mémoires ou la natière est plus approfondie, et traite avec une bien gramde supérioriti; néanmoins nous livrons anx méditations de: écrivains anronones l'opinion de l'anteur, qui prefére les futaies sur taillis aux futaies en masse.

L'article des harats et des bestiaux n'offre que des lieux communs rebattus.

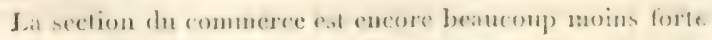

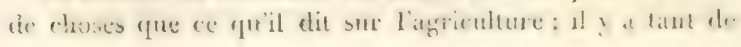


choses entassées dans vingt pages, que nous désespérons de pouvoir en suivre la série; et d'ailleurs il nous faudrait raisonner dans la supposition fausse que le commerce n'a pas de numéraire, que les manufactures en général sont perdues, et les arts négligés. Il est fícheux que ce Mímoire, ou il y it de bonnes choses, les offre confondues avec tant d'inutiles.

Bentuevin.

48r. Mémolre sur l'agriculture, l'industrie et le commerce de l'Égypte, par M. P. S. Girand, ingénieur en chef des Ponts et Chaussées, de l'institut, ctc. I vol. in-fol. de 224 pages. Imprim. royale, à Paris.

483. Comparaison des trois charrues; par Ch. Pictex. In-s. de $8 \mathrm{f}$. et une pl. prix i fr. So c. A Paris et à Genève, chez Paschoud.

483. Faits et observatioxs sur la fabrication du sucre de betterave; par C. J. A. Mathien de DonBASLE, $2^{e}$. édit., corr: et aug. d'un article sur la distillation des mélasses, par le mème. In-8. de $2 \mathbf{q}^{\mathrm{p}} \mathrm{p}$. aree I pl. Prix 4 fr. et $4 \mathrm{fr} .75$ cent. fr. de port. Paris, 1823. Chez mad. Huzard.

Cet ouvrage se compose d'un avertissement sur la seconde édition, d'une introduction qui fitit voir les avantagnes que la fabrication du sucre de betterave peut procurer, et qui donne l'historique de la fabrique établic par l'auteur. Le corps de l'ouvrage se compose de trois chapitres. Dans le premier, il est tratić en dix articles de la culture de la betterave destinée à la fitbrication du sucre; dans le second, l’auteur décrit spécialement lit fabrication da sucre, aussi en dix articles, dont le septieme traite non-seulement de la concentration du sirop, mais encore de la cortstruction des chaudières ot de leurs foyers, du combustiole et du noir animal. Un onziemes article additionnel a ce chapitre a pour titre : De la distillation de's milasses. C'est cet anticle, qui manquait a lis pre-

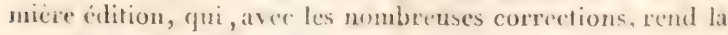

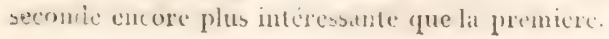


Le troisième chapitre, composé de six articles, roule sur des objets généraux, tels que le prix du sucre de betterave, les produits accessoires d'une sucrørie, l'influence qu'exercerait l'introduction de la culture de la betterave sur l'agriculture française, les intérèts coloniaux, etc. La planche représente les instrumens principaux de fabrication.

Cette seconde édition vient bien, dans un moment ou la guerre en enchérissant le sucre donnera probablement un nouveau degré́ d'importance à cette industrie, et relèvera des établissemens que le bas prix du sucre des colonies avitit fait tomber.

H. F.

48 f. Fabrication du fromage de Parmesin, par MI. Huzind fils, corresp. de la Soc. roy. et centr. d'agriculture. Broch. in-8. de 44 pag. avec 1 pl.; 1823. A Paris chez M. Huzard. Cette brochure est extraite des Ann. de l'agric. française, $2^{2}$. série, t. 2 r. $\mathbf{n}^{\circ}$. de janv. 1823, p. 5 .

Ce petit ouvrage contient d'abord une espece d'introluction sur les avantages qu'il y aurait à fibriquer en France des variétés de fromages de longue conservation; ensuite, deux articles. Dans le premier, l'auteur détaille exactement et minuticusement en onze paragraphes les procédís de fabrication : il donne les raisons de ces procédés. Dans l'article second, il donne des détails sur lit race des vaches qui foursissent le litit, sur les soins qu'on leur donne, et enfin sur la manière de cultiver les prairies qu'on appelle marchita, et qui fournissent la nourriture aux vaches. La planche ajoutie a la fin de la brochure donne une figure suffisante de lat chauclicre et du fonrneau, qui sont les deux instrumens principanx de fabrication.

Son but est de prourer qu'on peut fabriquer du fromage de Parmesan, sans avoir les vaches et les paturages du pays; qu'il suffit seulement d'avoir de bon lait, en quantité sulfisante et à un prix modéré.

II. F.

185. Nouvelu systime de cultune sans emploi de chatux ou fumiers, et sans jachere, ainsi qu'on le pratique à la ferme de Knowle dans le comté de 
Sussex; par le major général Al. Beatson, ancien gouverneur de l'ìle de Sainte-Hélène, etc. avec 3 grav. prix 63 $\frac{1}{2}$ centièmes de dollar. (Extrait de la délibération de la Soc. de Philadelphie pour le perfec. de l'agricul. )

Le président ayant précédemment mis sous les yeux de la Société, avec invitation à ses membres de lire et de consiciérer avecattention, le contenu de l'ouvrage ci-dessusannoncé, la résolution suivante fut adoptée, et une copie en fut délivrée à MII. Mathew Carey et fils, éditeurs.

La Société de Philadelphic, pour le perfectionnement de l'agriculture, ayant vu, et plusieurs de ses membres ayant pris lecture, et d'autres ayant fait l'essai avec approbation, de quelques-uns des instrumens décrits dans un nouveau systène de culture, sans chaux, fumiers ou jachères, ainsi qu'on le pratique sur la ferme de Knnowle, dans le comté de Sussex, par le major général Al. Beatson. Considérant que cette publication contient dans l'opinion de la Société des changemens importans dans le systène du labourage, plusieurs ćtant considérés comme des améliorations en agriculture, et dignes de l'attention de tout cultivateur praticien qui désire un grand bénéfice de l'emploi des moyens annoncés, et que s'il ne trouve pas que tous puissent être également adoptés, on peut faire choix de différentes parties qui seront reconnues très-avantageuses méme pour l'ancien système de culture. Sachant que MIM. Mathew Carey et fils sont convenus de réimprimer cet ouvrage et de le vendre à un prix modique, la Société, afin de les y encourager, s'engage à prendre 60 exemplaires de cette réimpresion, satisfaite des avantages importans que le public en retirera.

Signé, Rozerts Vaux, secrétaire.

Wardo: 496. Avis des plus intéressans pour messieurs les propriétaires des forêts et des parcs tant français qu'étrangers, qui désireraient passer leur temps agréablement à leurs campagnes, relativement à un piége de mon invention, des plus ingénieux et des moins dispendieux, pour la destruction des loups et de tous les animaux nuisibles. In-8. I f. Signé le ch. Lissenin, tue Geolitoy-Lasmier, 13. 22. 
48 - Notice str le papien glace de M. Quénedey; par M. Gillet de Lilmont, 5 p. ( A n d des mines, tom. 7, p. 8.)

Ce papier a été présenté en 18 ro à la Société d'encouragement, et a été décrit ensuite dans la Biblioth. physicoeconom., 18 I6, t. 8 , p. 3955 . Il est fort recherché pour calquer et mème pour graver au trait ou au lavis, etc.

B. D.

GÉOGRAPHIE.

488. Geognafa moderna universale, etc. Géographie moderne universelle, ou description physique, statistique, topographique de tous les pays du monde; par G. R. Pignozzr. In-8. Florence I82\%. Batilli. Vol. I, contenant l'introduction et I'Asic occidentale.

Cet ouvrage sera composé de ro volumes, avec un atlas de 7 cartes.

439. Cochs ŕlémentaire de géographie ancienne et moderne, rédigé sur un nouveau plan; par M. LETroxif, inspecteur-général des études, etc., adopté par le Conseil royal de l'instruction publique pour les établissemens de l'université, et par S. Exc. le ministre de la guerre pour les écoles royales militaires. 6.e édit., avec a $p^{l}$. In-ra de ro f. $\div$. A Paris, chez Aumont.

49o. Dictonmane griographece et descriptif de Titalie, servant d'itinéraire et de guide aux étrangers qui voyagent dans ce parss, divisé en deux parties; par J. Banzus ancien professeur à Ferrare, etc. In-12 de 23 f. A Paris, chez lauteur, rue Montmartre, n. 144.

4.91. Gema da Mrano a Ginevia pex Sempione. Cruide de. Milan à Genève par le Simplon, aree 30 vues et une carte géngraphique; in-f et in-s. Milan 1820. Astritit. 
492. Hrstothe ta nescription DFs hlis ionientas, depuis les temps fabuleux jusqu'à ce jour, avee un nouvel atlas contenant, cartes, plans, vues, rostumes et médailles, par un ancien officier supérieur en mission dans ces îles; ouvrage revu et précédé d'un discours préliminaire, par M. le rolonel Bony ne St. Vincent, corresp. de l'Acad. des sciences, etc. I vol. in-8. de 5 r f. $\frac{3}{4}$, plus l'atlas petit in-folio, de 5 f. et i 8 pl.grav. Prix, 25 fr. A Paris, chez Dondey-Dupré père ct fils.

Nous ne saurions mieux faire, pour donner une idée juste et exacte de cet ouvrage que d'indiquer le plan qu'a suivi lauteur, M. $S^{* * *}$, officier supéricur en mission dans ces iles. Il se compose de six livres: les cinq premiers sont relatifs à l'histoire des iles Ioniennes, depuis les temps les plus reculés jusqu'à nos jours; et le sixième donne une esquisse de la statistique de chacune d'elles, ontre celle de l'île de Naxos dont l'auteur rapporte aussi l'histoire, quoique cette île ne fasse point partie de la république septinsulaire. Dans son discours préliminaire, M. Bory de Saint-Vincent passe en revue l'histoire des différentes îles, et donne ainsi une sorte de résumé de l'ouvrage.

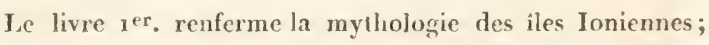
cette mythologic est traitée pour chaque ile dans des chapitres particuliers. Le livre un comprend l'espace de temps qui s'écoula depuis les siècles communément appelés fałuleux jusqu'à l'arrivée des Romains dans la Grèce (environ depuis l'an 600 jusqu'à l'an 230 avant J.-C. ) - Le livre IIr sétend depuis la première expédition des liomains dans la Grèce, jusqu'à l'occupation de l'empire d'Orient par les Latins $\left(\mathbf{r O}_{2} 3\right.$ ans depuis J.-C. ) - Le livre xv offre l'histoire des sept iles sous le gouvernement de leurs ducs, et s'étend jusqu'à la mort de Soliman II. ( 1556 ).- - Ie livre v va de 1556 à nos jours; et enfin le livre vr traite de la statistique de chacune des îles en particulier, statistique complétée par d'intéressans tableanx analytiques de la population, des jroductions, du commerce. Ces tableaux très-détaillés présentent des résultats précieux.

L'auteur y parle anssi avec une judicieuse sagacité et en 
lıonme de l'art des difficultés du terrain, des divers points militaires et des moyens de défense propres à mettre ces ile's à l'abri de toute attaque extérieure.

Cet ouvrage est enrichi d'un atlas à la tête duquel sont les tableaux statistiques. On y remarque aussi une carte de la Grèce ancienne, pour faciliter l'étude de l'histoire et notamment celle des alliances et des lignes de la Gréce. Outre cette carte, il s'en trouve plusieurs autres qui représentent les iles: de plus l'atlas offre des plans et des vues de villes, de forteresses dans leur état actuel, et une planche de costumes. L'auteur y a joint une suite chronologique de médailies frappées à Corfou, depuis les temps fabuleux jusqu'a Constantin, et quelques médailles grecques frappées dans les autres íles, et dont le catalogue raisonné est à la fin du volume. Toutes ces planches lithographiées sont une preuve des grands progrès qu'a faits la lithographie, et laissent entrevoir le degré de perfection auquel elle est susceptible de parvenir. Nous ne croyons pas qu'il ait été rien exécuté en ce genre d'aussi parfait. Yous remarquerons cependant que les cartes n'ont pas tontes été dressées surles matériaux les plus récens. $\Lambda$ Lex. B. du B.

493. Découverte de l'ile Élisabeth. Fxtrait d'un voyage fait à l'ile Piteairn par le capit". H. Ring en 18 I9. (Ann. marit., déc. I822, p. 562 ¿ 56.ł.)

Cette île est situce par les $24^{\circ} 26^{\prime}$ de latit. S. et par les $127^{\circ} 30^{\prime}$ de long. occid., méridien de Greenwich. Elle pent avoir 6 lieues de circonférence. Le capit. H. Ring y

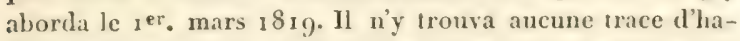
bitans, ni mème de quadrupedes. Il en prit possession an nou de S. M. B., et lui donna le nom d'Élisabeth. Joziv.

49í. ExpĹditron tennestre dans les régions polaires, commandéc par le capit. Frankur. (Nouv. Aun. des Toy.,t. 6 , oct. I 822.)

L'exploration de la côte septentrionale de l'A nérique a été pousséc par le capitaine Franklin jusqu'i la distance de plus de 500 milles a l'est du Copper-mine-river; il s'est assuré que l'embouchure de ce fleuve se trouve a $67^{\circ} 48^{\prime}$ de latitude, ce qui donne 4 degris de moins que la position indi- 


\section{Statistique.}

quíe par Hearıe, et qu'aucune portion de la côte vers l'est ne dépasse $68^{\circ}$; elle descend même dans un scul endroit jusqu'à $66^{\circ} 20^{\circ}$.

L.

495. Extrait o'un Itinéraire de Hhaleb (Alep) à Moussel (Mosul) par la voie du Djezire ( la Mésopotamic); par M. R.... In-8 de 3 f. $\frac{1}{4}$. (Jour. des Voyages, n. 5o.)

496. Extrait d'une lettre adressée à M. Barbié du Bocage, membre de l'Institut, etc., parM. N. VIDAL, interprete du consulat gén. de France à Bagdad, 5 sept. 1821. ( Bull. de la Soc. de géogr., n. 3 , p. IOI.)

Ces extraits offrent des renseignemens intéressans sur les ruines de Babylone; mais pour la géographie moderne on n'y tronve qu'un calcul de la direction des diverses sinuosirés du Tigre, dans son cours, depuis Moussol jusqu’à Bagdad.

F.

\section{ST $\Lambda$ TISTIQUE.}

497. Historre rolitioee et statistique de l'Aquitaine ou des pays compris entre la Loire et les Pyrénées, l'Océan et les Cérennes; par M. de Verneilit Puiraseau; tome $I^{e r}$. in-8 de 20 fcuilles $\frac{x}{4}$. A Paris, chez Guyot et chez Ponthicu.

498. Essai statistreue sur le royaume de Portugal et d'Algarve, comparé aux autres états de l'Europe, et suivi d'un coup d'oil sur l'état actuel des sciences, des lettres et des beaux-arts parmi les Portugais des deux hémisphères. Dédié à S. M. T. F. , par $\Lambda$ dr. Busbr, ancien profes. de géographie, etc. 2 vol. in- $S$, cusemble de 72 f. $\frac{1}{4}$ et 6 tableaux. Prix 16 fr. A Paris, chez Rey et Gravier.

L'auteur a voulu faire connaitre un pays sur lequel nous r'avions que des données inexactes ou incomplètes. Il donne d'aborl un tablean de la géographie du Portugal depuis les. temps les plus ancicns jusqu'à nos jours; cnsuite il expose 
les principaux faits qui composent son listoire, y comprenant la revolution qui vient de s'y opirer. Il passe ensuite a la géographie pliysigue, qui a un développement convenable, el ctablit pour divers pounts des tableanx de comparaison avec d'antres pays, notamment arec les litatsUnis de l'A mérique septentrionale.

I a grégraplie politique et administrative comprend les tableaux de population a diverses époquess, et celui de ses monvemens de 1815 a 1819 . Cette population est estimce, an $\mathbf{I}^{\mathrm{er}}$.janvier $\mathbf{1} 822$, à $3, \mathbf{I} 73$, 000 ûmes.

$\Lambda$ res tableaux sont joints ceux des mouvemens de la population des différentes parties de l'Europe an XJ ". siécle, pris on examine les diverses canses qui ont arrete en Portugal les progrès de la population.

liennent cusuite les details relatifs an gouvernement avant 1820 , puis ceux qui concernent la forme actuclle. Fn parlant dit droit porlugais, on voit qu il résulte de graves inconvéniens de la cumulation des lois rendues a diffirentes époques, et que le besoin diun code nouveau et uniforme se fait généralement sentir.

L'exposé du commerce du Portugal, à différentes ipoques, contient beaucoup de faits nouveaux; ils sont authentiques, puisque lautem les a puisés dans les archives du rovamme. On voit successivement les rapports commeriaux de ce pays avec clatpue contrée de liurope et des autres parties du monde, ainsi qu'avec ses colonies.

I.e chapitre de la géographie ecclésiastique donne sur cet objet important des renseignemens précicux; il en est de méme de celui oi il est question de la géngraplie litéraire, qui comprend tout ce qui tonche au caractere des habitans, a la langue, aux établissemens d'instruction publicgue, aux academies et aux bibliotlicques. Le Porturgal est mone compare, sous ces divers oljets, avec tous les pays de l'Europe.

Ia topogmalie du royame, un apercu des pars gui forment la monarchie portusaise, terminent louvrage.

In appendix offre le tableau de tons les ouvraren publiés en Portugal de 1800 à 1820 .

Errits.

499. Colzecton of costranes des liversos prorinces de lEspagne, lithographiés par Prost, diaries des 


\section{Statisplique.}

dessins originanx de Wute, publiće à Paris par Clément frères, MI . d'estampes, quai Volt. n". I, et boulev. des Ital. n". 9. Cent cost. col. Prix 50 ir.

Dans un moment ou tous les regards ore fournent yers l'Espagne, cette publication, qui dans tous les temuls sit été recherchée des amateurs, acrjuicrt un nonvel interèt. La vérité des costumes y est gardée jusçu’an scrupule, ot la pureté du dessin de M. Pigal permet de suivre le plus petit détail. D'ailleurs, la modicité du prix anquel on a fixé cet nuvrage, nous fait croire qu'il aura un grand succès.

La vue de tous ces costumes nous a fait nattre une réflexion pénible; c'est qu'én général le peu de rapport qu’il y a dans les halillemens des habitans d'une province d'Fispiugne à une autre, annonce quelque chose de disparate dans leurs mours, et l'on sait qu'un peupnle çui n'offre pas une sorte d'homogénité de mæurs, présente moins de chances pour le bonheur individuel et pour la félicité publiq̨ue. Brntus.

50o. Apfrgev du Conmence de $\mathbf{S}_{\mathrm{T}}$-Pétenseoure, durant la navigation de 1822 . In-4 d'une demic fenille. Imp. de Bailleul, à Paris. Ne se veud pas. 501 . Adstract of the answers and returns made pursuant to an act for taking an account of the population of Great-Britain sccexx, ordered by the house of commons to be printed a july 1823 ; ou Fxtrait des renseignemens relatifs à la populaiion de la Grande-Bretagne, en $182 x$, recueillis en exécution d'un acte du parlement. Un vol. in-fol.

Cet ouvrage, composé sur des matériaux officiels fournis par les chefs eivils et ecclésiastiques des différentes communautés d'habitans et paroisses de la Grande- Bretagne, a été rídigé par M. Jolun Rickman, dont le nom se trouve a la fin de l'avant-prop̧os. L'impipession en a été ordonnée par dílibiration de la cliambre des eommunes, 1: 2 juillet $182 \%$, mais seuiement pour l'usage des mombres du parlemenn; if n'a point été mis dans le commerce. Nous en devon: la pronsession aux soins obligeans de Mi. le ducteur Youner serertaire de la Société royale de Londres.

T'ONE I. 
A la téte de ce volume le rédacteur a placé des observations: préliminaires qui comprennent trente-cinq pages. (ha y voil quelles ont été les questions adressées anx personnes chargéede fournir les élémens de ce travail. Ces personnes átaicnt, pour le recensement des mainons et des familles, et pour celui deshabitans par sexes, àges et profensions, en Anerleterre, les inspecteurs (orerseers); en beosse, les matres d'ecole? (schoolmantese); et pour les mouvemens de la population, c'est-a-dire pour les mariagres, les naissanees et les enterremens, les ministres des différens cultes, mais principalement ceux de l'éğlise anglicane.

M. Rickman a joint a cet avant-propos des tableanx sommaires qui font voir, d'un coup-d'œil, la population par sexe. et par ige de chaque comté de l'Angleterre propre, du pias de Calles, de l'Écosse; celle desiles de Gnernesey, Jersey, Min; et cn outre, un état spécial de la population des villes de Tondres et de Westminster avec leurs faubourgs of leur banlicue, formant ensemble ce que les $A$ uglais comprenuent sous le nom général de métropole ou capitate (mitropolis) ćtat qui se monte à $1,225,69 \%$ ûmes.

Il a offert aussi, dans des tablenx eomparatifs, les résul tats des trois recensemens exéculés de dix ans en dix ans, depuis le commencement du siecle présent (cn 1801, 1811 , 1821), ct on doit lui savoir un gré particulier d'avoir fait reinarquer les diverses sources d'erreurs qui ont pu influer sur l'exactitude des renseignemens.

Cette introduction est suivie des détails du recensement proprement dit, en anglais enumeration. Ces ditails sont exposés en forme de table:ux, comprenant dix colonnes, dont quatre se rapportent au nombre des maisons et des fatmilles ou feux, trois a celui des principales occupations des familles, et les trois autres au nombres des personnes de l'un et de l'autre sexe.

Dans restableaux, dhaque communanté d'habituns (Parish, 'Lownlip, (Chapelry, Hamlet, ete.), noecupe qu'une liguc. Il y a une récapiblation pour charpe arromdisoment (Hundred, Waprentibe, ele.); une antre pour chacpue pro-

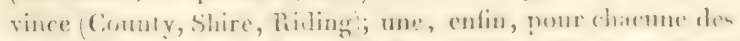

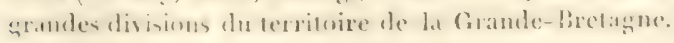

Cette partie de l'ouvrage occupe 55 r pages. 
La seconde partie en contient cent cinquante, avec une pagination séparic; elle renferme les tableitux du mouvement de la population, pendant dix ans, de Is I I à I 820 , non par paroisses, mais par arrondissemens, avec des récapitulations par comtés.

Ces renseignemens ont été fournis par les ministres de l'église andicane, et par quelques-uns de ceux des communautés dissidentes, d'après les registres de leurs églises respectives.

Le volume que nous avons sous les yeux ne contient rien de relatif au mouvement de la population en Écosse. On n'y frouve également rien qui se rapporte à l'Irlande, sous quelques points de vue que ce soit. C'est que les recensemons tentés dans ce dernier pays nont pu réussir. Il faut donc se contenter à cet égard d'une approximation dont nous ferons usage dans le relevé suivant :

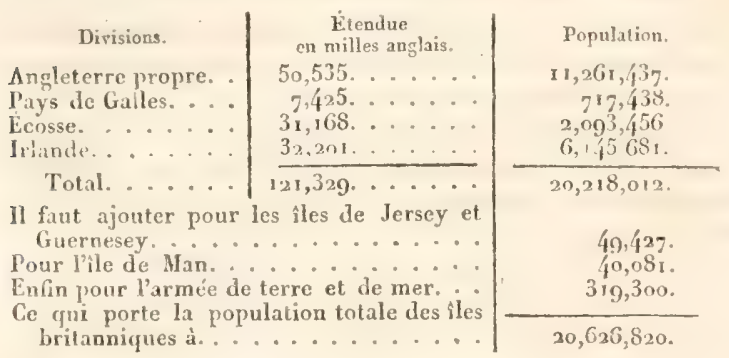

La population parait s'étre accrue très-rapidement depuis le commencement de ce siècle, dans la partie méridionale de la Grande-Bretagne. comprenant l'Angleterre propre et le pays de Galles. En effet, le recensement de $\mathbf{r}$ So I n'avait donné qฺ 9, I68,000 habitans; celui de 18 I r en donnait déjà x $0,502,500$. Ce qui supposait une augmentation de $14 \div$ pour mille par an. Le recensement de $\mathrm{I} \mathrm{S}_{2} \mathrm{I}$ suppose une aurmentution encore plus forte et quiva pour chapue ammer a 16: pour mille, puisqu'il porte la population de la méme et tendue de pays à $12,218,500$ habitans, y compris les militairs.

Fin ce qui concerne le monvement de la population, le fravil que nous avons sous les yeux ne samait être p.urlite. ment exact, par la ratison que les ministres de la relisien 
remplissent exclusivement les fonetions d'officiers d'ulat civil, et que beaucoup de naissances, de décés el mitme di. mariages leur restent inconnus.

Nous noterons an moins un fait constant, c'est yue I. nombre des garcons à été à celui des filles comme le nombri. 10,433 est a ro.ouc): an contraire le nombre des décés a ili. égal, à très-peu près, dans les deux sexes; d'où l'on pent conclure que l'excédant de la population mâle est moissonné par la mort hors du pays.

Les baptèmes enregistrés n'ont été (année commume) a la population totale, dans l'Angleterre et le pays de Galles, que comme I est à 35 .

La proportion des mariages connus y a été de I sur $13 \%$ individus; celle des décès enregristrés de r sur 58. C. M.

5oz. Remaris on the comparatrve health, and populalion of England, etc. London. I\$22. 3y pages.

Le journal qui se publie a Londres, sous le titre de Medi"al repertory, en rendant compie daus son calier du moic

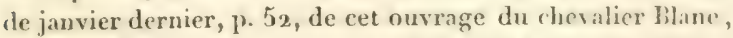
médecin du roi d'Angleterre, sur l'état de la santé publigne dans la Grande-Bretagne à différentes époques, donne le 1ableau comparatif suivant, calculé, pour cet auteur, par M. Finlaison.

Durée moyenne de la wie en Angleterre.

$$
\text { Vers } 169 \text {. }
$$

Vers 3789 .

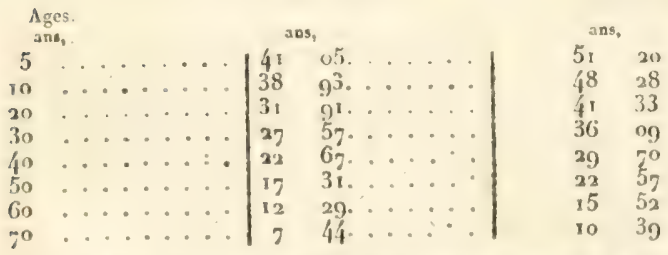

I.e uneme journal donne, pour chaque comté de l'Angleterre, lit propurtion des naissances, des mariages et des décès, a la population totale. Nous avons fait voir, dans l'article pricédent, ce qui emprehe qu'on ne puisse accorder une entiere confiance aux données sur lesquelles ces calculs sont fondés. Les anomalies qu'on observe dans le tableau, ct dout 
lanteur convient qu'il ne peut découvrir les causes, sont un antre motif de dontes légitimes, lorsqu'on voit, par exemple, daus le comté de Kent un décès sur 50 individus, et dans le conté de Sussex, qui touche au premier, un décés sur 72.

Prut-être peut-on en dire autant du tableau qu'il donne de la longévité par comté; car pour étab!ir, comme il le fait, le rapport du nombre des individus décédés dans l’âge tries avancé de go ans et an-dessus, à la totalité de la population, il faudrait, avant tout, qu'on put compter sur l'exactitude les resistres mortuaires; quoi qu'il en soit, voici le résultuı „rénéral, tel que l'offre le journaliste anglais; il l'a rapportéau nombre 20,000; anquel nous préférerons le nombre 100,000

Sur une population de cent mille âmes, il menrt, suivant se's calculs, le nombre suivant d'indivicius àgés de quatre vingt-dix ans et plus, savoir :

En Angleterre. . . . 5 5120 , dont 170 centénaires.

Dans le pays de Galles. 92.31, - 250

En Écosse. ....... 75 80, - 5 ×5

C'atuteur sur suris lui-mêne que daris le pays de Galle, la mortalité soit si faible et la longévité si commune. On peu s'étonner également que la mortalité de la vilk de Lon. dres, qui étail, suivant lui, en 1750 , de I individu sur 21 , se trouve ne plus eire, en 1821 , que de $x$ sur 40 ; it cherche à expliquer cela on disant qua la prenière de ces époques, le peuple abusait beaucoup plus des liqueurs fortes quil ne peint le faire anjourd'hui, les droits ćtant beauconp plus considérables; il dit encore que le peuple est moin. eltanger aux soins de la propreté qu'il ne l'étitil y a 70 ans, que la ville de Landres est mieux fournie d'eau; que l'on s'y nourrit plus abondanment et avec de meilleurs alimens, enfin, que la médecine s'est perfectionnée; que beancoup dabus rui avaient lieu dans la première éducation des enfan. ont eté réformés. Il est juste d'ajouter à toutes cescausew ríelles ou présumées, le bienfait incontestable de lit vacel nation.

Mais, nous le répetons, avant de fonder aucun raisonue ment sur les donmies que fournit le recensement anglais, relativement au mouvement de la population, il faudritit que: ces renseignemens eussent atteint le degré d'exactitude dont cette matière est susceptible. 
Il nous semble, en géniral, que la statistique de la France est loin d'avoir rien a envier a celle de l'Angleterre par rapport aux lumières que les deux pays empruntent de l'uluninistration. Nos resistres de l'elat civil en particulier somt dignes de servir de modele aux autres nations. C. M.

5o 3. La Gafta uf Colmora, qui parait à Bogota, estime le nombre des hahitans des sept provinces (Orinoco, Tenezuela, Sulia, Bayacca, Cundinamara, Cauca, Mardalena) a $2,644,600$. Ces provinces, qui étaient autrefois connues sous le nom de Quito, envoient au conrrès 28 sénateurs et 9'3 représentans. (Journ.gineir. de la Litt. eitr., now. I 822.)

5o. . St lr le climat de l'Afriece australe; par M. Colebrooke. (Juurn. de l'Instit. royale de Londres, janv. I823.)

Le caractere particulier du climat du cap de Bonne-Espérance résulte de la succession róguliere qui a lieu entre lit mousson du sud-est, qui règne dans cette contréc du nilieu de septembre au milieu d'arril, et la mousson de l'ouest, qui occupe les cinq autres mois.

La mousson du sud-est n'est autre chose que la prédominance du vent alisé auquel est soumis fout le centre de l'Afrique; celle de l'ouest est la prédominance du.vent d'ouest, lequel règne habituellement dans les mers australes. Ainsi, pendant l'été de l'hémisphère autral, lextrúmité de l'Asie vers le sud fait jartie de la région des vents alisís, ct, au contraire, pendant l'hiver de cet he'misplère, elle se trouve appartenir a la region des vents d'ouest, la ligne qui sipare ces deux rigions s'éloignant de l'épuatenr pentant que le soleil est dins l'hemispliere austral, et s'en rapproclant le reste de l'année.

Les montagnes qui oecupent le centre de la colonic élablissent des différences importantes entre la partie de l'est et celle de lourst. Ia premiere est plus seche et plus froide; l'autre a seuk le bénéfice des pluies que procurent les vents d'ourst, ansi est-elle la plus fertile et la miens peruplec. I 'air qui arrive de l'A tlantique en hiver, chaseg de vapeurs, depere son thumidite sur les flancs des premieres monlagnes

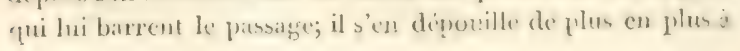




\section{Statistique.}

mesure qu'il rencontre des montagnes de plus en plus élevies, et par conséquent de plus en plus froides. Il ne reste pas de quoi alimenter la moindre source dans les plaines qui sont au delà. Aussi, dans celles de Karrou, bien connues par leur aridité, les pàtres guettent les symptômes d'orage qui se manifestent à l'horizon, et ils dirigent leurs troupeaux du côté où ils ont aperçu des éclairs, dans l'espérance de profiter d'un peu de végétation qu'une pluie d’orage aura pu ranimer.

On manque jusqu'ici de bonnes observations sur la météorologic du cap. Celles que M. Colebrooke a pu recueillir sont d'une trop fiible durée, et le thermometre, au lieu d'étre exposé a l'air, était suspendu dans une grande chambre. Elles ont donné pour résultat de près de trois ans une température moyenne de $67^{\circ} 17^{3}$ Fahr., un maximum de $96^{\circ}$, un minimum de 45"; pour la moyenne des trois mois d'été $77^{\circ}$, pour celle des trois mois d'hiver $58^{\circ}$.

C'est en janvier et février que le baromètre est le plus devé; en juiltet qu'il l'est le moins. La différence est de près d'un pouce anglais. Celle qui a licu en 24 heures excède rat. rement un dixieme de ponce, n'arrive presque jamais à deux dixièmes. On cite, comme un fait remarquable, qu'au mois de janvier 1821 , à l'approche d'un ouragan, le mercure descendit de quatre dixièmes de pouce, et remonta de six dixièmes lorsque l'orage eût cessé. Le baromètre monte ordinarement quand le vent passe à l'est et au sec, et rice rersa. Les variations les plus grandes ont lieu quand la sècheresse est accompagnée de froid, et lorsque l'humidité l'est de chaleur.

505. Il est utile de rassembler les données diverses d'ilpres lesquelles on peut dédure des termes moyens pour la slatislique. C'est dans ce but que nous offrons les renseisnemens suivans sur les revenus et la dette publique des États de lit confélération germanique, extraits du Moniteur du vendredi $x 7$ janvier $x 823$, dont nous avons rapproché les évaluations fournies par Lichstenstern. Les ren-eignemens du Monileur sont datés d'Ausboures, 8 janvier, "t sont donnés comme tirés de sources autlienliyacs.

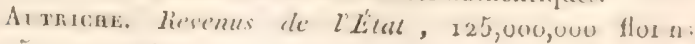
$(325,000,000$ francs $)$. 


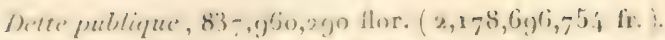

1.e montant des revora des blats allemands antricluens, comprois dan, la somme ci-lenesus, est de $68 \div$ millionn de florian. Vers la fin du i II". sieve lat totalité des revonus de

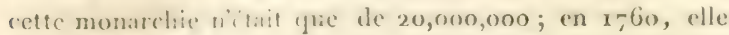

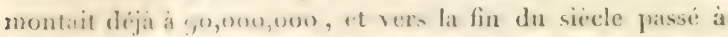

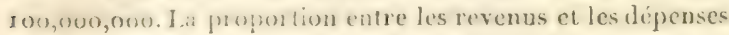
actuelles de l'État n'est pas connue.

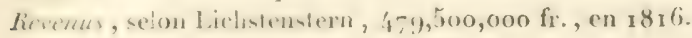

Detle, selon Hassel, 4,000,000,000 fr.

Pux ss., Ri's. 50,000,000 rixd. ( $185,000,000 \mathrm{fr}$.).

Dép., 50,000,000 rixd. ( $185,000,000$ fr. $)$.

Dette, I8o,og1,720 rixd. ( $656,339,364 \mathrm{fr}$.).

I.e montant des reventis provenans des États allematuds prussiens, compris ditns lir somme ci-dessus, est dt $29,399,744$ rixd. ( $108,779,053 \mathrm{fr}$.).

Rer., Dans Liclistenstern, $\mathrm{x} / \mathrm{I}, 700,000 \mathrm{fr}$.

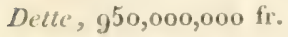

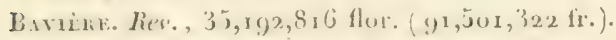

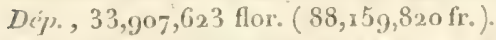

Ditte diares le butjet de r\$19), $105,740,925$ florins. $(274,925$, I $05 \mathrm{fi}:)$.

D'après Lichstenstexn. hev, , 3,600,000 fr.

Dette, 2 I 8,ooo,oon fr.

IInxoxre. On mangque de données exactes. ()n sait seulenent que le fermage anmuel des domaines ast de $4,000,000$ de rixd. ; que les cuntributions directes et indirectes excé.

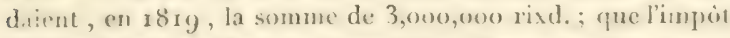
foncicr a ćle, on 1821 , angunente de 150,000 rixd., et que, par une ordonnance du 25 juin 1822 , il a éte chalili uns: nouvelle taxe sur l'industric et les revenus.

Selon Lichstenstern. Res', 20,60r,000 fr.

Detle, \{3,600,000 fir.

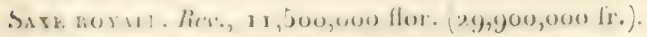

Detle, $21,553,50 /$ rixd. $(82,324,385 \mathrm{fr}$.$) .$

Letat financiar de ce pays est reste conore un mystiere, mémo jom les membres de ses etats-géneranx. Ie montant ci-clesslos indigue des revenus parat ère beaucoup trop

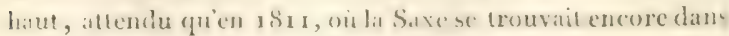




\section{Statistique.}

son integrité, ses revenus n'excédaient pas la somme de $11,606,000$ rixd.

D'après Lichstenstern. Rev. 29, 430,00o fr.

Dette, $96,000,000$ fr.

Wuntemberg. Rev. 10,028 , 458 flor. ( $26,073,990$ fr. $)$.

Dép., 10,542,52 I flor. (27,410,555 fr. $)$.

Dette (d'après l'élat de $1819^{-1} 820$, présenté aux chatul bres), $21,985,62.0$ flor. $(57,162,612$ fr. $)$.

Selon Lichstenstern. Rev., 34,88o,ooo fr.

Dette, 65,400,000 fr.

BaDE. Rer., 9, 185, 288 flor. (23,33o,632 fro).

Dép., 9, 185,288 flor. ( $23,330,632$ fr. ).

Dcte, , 6,036, ooo flor. $(40,731,440$ fr. $)$.

Selon Lichstenstern. Rev., I 1,990,000 fr.

Dette, $47,96 \mathrm{~s}, 000$ fr.

Hiesse-Cassel. Reve, 4,000,000 flor. ( $11,520,000$ fr.).

Dette, $1,297,130$ rixd. $(3,735,734$ fr. $)$.

Dans le montant de la dette on ne trouve pas comprise celle des pays de IIanau et de Fuide, évaluée r, ooo, ooc de flor

Selon Lichstenstern. Rev., 8,720,00o fr.

Dette, $4,79^{6,000} \mathrm{fr}$.

Hesse-Darmstant. Rev, 5,996,5 io flor.

Dép. $, 5,99^{5,735}$ flor.

Dette (d'aprés les états pour $\mathbf{1}_{21-1} \$_{23} 3$, prísentés itus chambres), I 2,574, 282 flor.

Rev., d'après Lichstenstern, $7,630,000$ flor.

Dette considérable.

Saxe-TWeimar. Rew. 640,987 rixd. $(2,448,570 \mathrm{fr}$. $)$.

Dép., 58o,869 rixd. (2,018,920 fr.).

Selon Lichstenstern. Rev., 3,2 \% o, ooo fr.

Dette peu considérable.

Mick Lembotc-Scminerin. Rev, 2,200,000 flor.

Dette , 3,000,00o flor.

Celte quotité a été garantie par les états-généraux. F. outre, il existe une autre dette de la cliambre, dont le mon tant n'est pas connu.

Selon Lichstenstern. Rev., 3,924,00o fr.

Delle, $\mathrm{x} 9,600,000 \mathrm{fr}$.

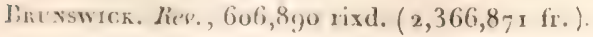

Dip., 7i5,800 rixd. $(2,791,620$ fro $)$. 
Detle, 3,606,303 rixil. $(14,054,581 \mathrm{fr}$. $)$.

Selon Lichstenstern. Rev., 3,92/,000 fr.

Dette, 14,300,000 fr.

Nassau. Res., $\mathbf{5}, 557,78$ r flor.

Dép., 1,533, 亿 1 0 flor.

Le montant ci-dessus des revenus ne comprend que les contributions. Il fant y ajouler cnrore ceux des domaineivaluées g on Soo mille florins, dont les étuls n'ont point ete jusqu'ici communiqués aux chambres.

F.

jof. Il y a maintenant à Liverpool un jeune lomme natil de lat tere de Van-Diemen. On lui a cnseigni a lire et a dire ses prieres, nais fuoiqu'il soit derenu ob́rissant et traitable, et qu'il soit corrigé de ses labutudes vagabonder, on apereoit encore tres-peu de proserie dans son intelligence. 11 parait qu'il coneoit assez bien la loi du mien et du tir" et qu il ne volerat rien excepte jour manger. (Tous. Inn des Voyages, oct. 1822.)

\section{PLANS, CARTES TOPOGRAPIIQUES ET GEOGPAPIIIQUES.}

joj. Cirtes ex Plaxs publiés par le dépôt de la mavine, en 1822 . Toutes ces cartes sont sur papier grand aigle, feuilles ou demi-feuilles. Flles seveudent chez Mr, Dezauches, rue des Noyers, n". 40, wat dan. les ports, chez ses correspondaus.

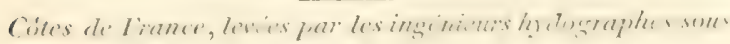
les ortres de M. BEauTEars-I3Eaupré.

Carte de la partie septentrionale du chenal du fiour, et de environs de l'ile d'Ouessant. Prix 4 fr.

Carte de lentrice de la rade de Brest, et de la partic méri dionale du chenal du Four. Prix \& fr.

Plans des emirons d'Argenton et de Tabérildut. Prix 2. fr Plan de la cliaussée des Pierres Noires. Prix $4 \mathrm{fr}$.

Plan des pouts de Camaret et du Conquet. l'rix 2 fr.

Plan du goulct de Brest. Prix 2 fr.

Plans des enviroun du cap de la Chense, et dea passigeses de:

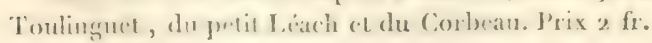

Plan des environs de Douaruenez. Prix. 2 fr. 


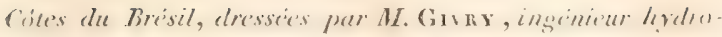
graphe sous les ordres de M. le Baron Roussin.

Carte de la còte du Brésil, comprise entre lile Santa Cat. tharina et le cap Frio. Prix $5 \mathrm{fr}$.

Plan du mouillage situé an Ñ. O. de l'île Santil Cathatrina. Prix $4 \mathrm{fr}$.

Carte des îlots et du canal des $\Lambda$ brolhos.

Carte du mouillarge de Cayenne; par M. Grinserer, ingi:nicur hydrographe sous les ordres de M. Roussin. Prix 3 fr.

Carte réduite de l'archipel des Bissagots, à la côte occidentale d'A frirue; par M. Grver, sous les ordres de $\mathbf{M l}$. le Baron Roussin. Prix 3 fr.

Cirlede la mer Noire, par M. Guutter, capitine de vaissean, secondé par MI. Benoist, ingénieur hydrograplle. Prix 4 fr:

Plan de lit baie Saint-Georges, còte de Terre-Teuse; par M. Le Sivlar de Vauneloo, enseigne de vaisseau. Prix e fr.

Plan de la baie de la Conception, à la còte du Chili ; par II. Chaucieprat, enseigne de vaisscau.

Plan de l'entrée du Para (d'apres les Portugais'. Prix 2 fr.

Carte de la còte méxidionale d'Anghetere, depuis bouvres jusqu'à Winchelsea (d'aprés la carte anghlitise publiée par l'amirauté).

Cartes des còtes d'Islande (d'apres les cartes redigues [r.r. M. le contre-aniral Low naxon, directeur du dépôt royal des cartes marines du Danemarck).

I'. De Reikianos à Snefields-Jokul.

2 ". De Sucficlds-Jokul au cap TYord.

3․ Du cap Nord jusqu'à l'île Malmey.

Carte de lise de lis Mitratrita et du goolfe de Carineo: (d'après les nouvelles cartes espagnoles).

I.

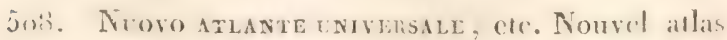

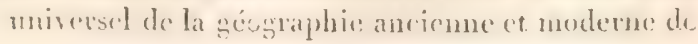


11MI. Arrowsmith, Poirsmn, Sotanamu, d’amblh. bemme, cte. ; traduction nouvelle, augmented des nouvelles découvertes, aver une introduction at lit grographie générale ancienne et moderne. hn-1 arec cartes. Milan 1822. Vallardi. Livaisons in et 18 .

Ces deux livratisons contiennent les cartes du P'iémont, de la sitvoie, de l'Italie et de l'Empire romain. Prix de changu carte, in-folio, 2 lire.

509. licu M. Riez, ci-devant consul britannique a Jiagrdad, a dessiné un plan de l'ancienne Ninive, cl découvert les ruime. dupalitis et des jardins de Sardanapale. (Journ. gin. de la Lir. citr., nov. 1822.)

jo. CEOgRAFIsK, mLitarisk statistisk karta Sverige weh forrige, ete., ou Carte géographique, mili. taire et statistique de la Suede er de la Por wege, publiée avec l'autorisation de S. MI. le lioi. par U.J. IlAcenstu, licutenant-colonel an rorpe du genie maritime, thev. de lordre de llipex, "t member de l'araténie des sciences militaires de Stuchholm. Stochholn, 1830 , aree des adclitious faites en $183 \mathrm{I}$ ( $\mathrm{f}$. gr. monde, en subluis); ste trouve à Paris, chez Goujon. Prix, $18 \mathrm{fr}$.

Celte carte est sans contredit une des plus remaryualbl. qui aicnt chi publiés depuis tris-long-temps, et peut-ître linnique de son genre par l'immense varicte de notions qu elle présente.

La partie purement géographigue est fondée sur une xiduction soigne des cartes des provinces de Suede, publicen par fen le baron d'ilermelin; des cartes hydrographiques des. róten de Vorwegr, dues a la direction du dépit des carten de la marine de Copenhague; des itineraires des voyageurs, entre antres de V. I. de Bucle; ainsi que sue les abervation presonaclles die l'auteur: l'échelle est at peu pris an

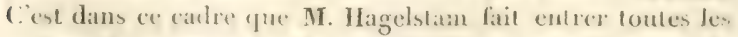
molions de grographe plysique, militiare, maritime ct de thlistique que l'esprare lui a permis of a accumbles. 


\section{Art nautique.}

A l'inconvénient pres de cotte accumulation, qqui crpenGant n'empêche pas de tou distinguer, on rend un entiey hommage a l'excellent travail de M. Hagelstam. I.es tahlean de population militaire sont authentiques, el l'on a porté la publicite jusqu'à indiquer les jours d'étape ou de marche forcée de chaque garnison à tel point de la frontière.

Ia dircetion des chaines de montagnes, leur élévation, leurs pentes, leurs versans, les plateaux, les valiées, les défilés sont marqués sur cette carte avec une exactitude serupuleuse qui manquait jusqu’à présent aux cartes générales. La hauteur perpendiculaire an-dessus du niveau de la mer, de plus de 200 montagnes ou plateaux, et de 270 lacs ou conrs d'ean, s'y tronve indiquée en pieds suédois. Six profils, pris dans des directions intéressantes, donnent une idée de la puninsule scandinave. Une foule de renseignemens précieux redatifs à la température et à la végétation s'y tronvent aussi réunis, en sorte que cette carte offre un ensemble de faits nouveaux et du plus haut intérêt pour l'Europe septentrionale. (Voyez le rapport fait à la Soc. de Creographic, sur cotte carte, par M.M. de Humboldt, Eyriès et Malte - Brun, Bulletin de cette Soc., no. $3, p \cdot 9^{\mathrm{r}}$ )

\section{ART NAUTIQUE.}

5ix. Extrait de deux Rapports faits à S. Exc. le? ministre de la marine; par M. le Capit. de vaisseau Philibert. (Ann. marit., i $822, n^{\circ}$. г o $p .28_{1}$ is 38 r.)

Les gabares de S. M. le Rhône et la Durance partirent de l'ile d'Mix le rer. janv. 1819, sous les ordres du Cinpit. de vaissean Philibert, pour se rendre a Manille en prassant par la Guiane française.

Elles arrivèent à Cayenne le $\mathrm{I}^{\mathrm{er}}$. fúvrier, en repartirent. le 27 , relàchìrent de nouvean à la Praya (île du Cap) Vert), reconnurent la Trinite, le banc des Aisuilles, false Bay an Cap de bonne Espérance, l'ile Maturice, et moullirent a Bombon le 26 juin.

Ayant appareillé de celte ile le 27 juillet, la division alla reconnaitre la còte N. O, de lia nouvelle Hollande, passa par 
Io detroit d Allar, et apres divers monillages sur plusicur points de la cote de bully, ainsi qu a la pointe de Penha, elle entra le ro sept. à Sourabaya daus l'ile de Java.

Elle remit à la voile le $1 j$ oet., s'eleva daus l'est, traversa Jes ditroits de Micassar et de Rasselan, et entrat le 22 nov. dans l'etablissement espagnol de Sambouangan.

Le 3 dre. alle quitta ce poste, prit an N. des iles de Santa-Cruz, cotuya la jartic S. O. de Mindanao, passal par le détroit de Mari Cahan, et arriva le 22 déc. a Cavilte, port de Manille dans l'ile de Tuéon.

La division, aprés aroir séjourné à Manille jusqu’au i 7 mars 1820 , fit son refour en passant par le detroit de lat Sunde, et rint mouiller à Bourbon le 5 mai. La les denx batinens se séparèrent. Le Rhóne fut diriggé sur Madisgasear, "t de lia sur Cayenne, d'ou il revint en France. La Durance's y rendit directement, et jeta l'ancre à l'ile d'Aix le 17 seppt.

Dans le cours de ce royage, M. le capitaine de vaissean Philibert a été a portée de faire plusieurs observations qu il a consignées dans ses rapports, et qui intéressent lart nantique et la géographie. Il a rectifiéla position de divers points mal indiqués par les navigateurs et les géographes, et il a recucilli des notions utiles tant sur l'état physique que surp la situation politique de quelques-unes des contrees quiil a visitées, notamment de l'ile de Java et de celle de Lucon. Jos.

5ja. Relation de la reconnissance hympoilaphige de la cóle occidentale du golfe de la Syrte.

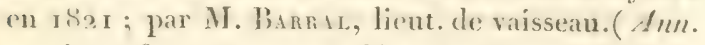
marit., $1822, n^{\circ}$. то , p. $33_{2}$ i 346 .)

Citte relation en forme de journal contient l'expose technicue de loperation, commencée le i a avril a 82 I, et terminée le 5 mai.

L'anteur y a ajouté des détails intéressans sue le port de Bengizi, to les regences de Barbarie, ainsi que sur le lintoral et la navigation du golfe. Joi.

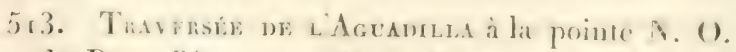

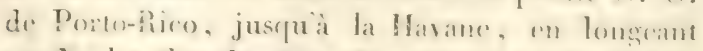
au 1. Les iles de Sain-l)omingue el de Cabal: et retour de la Havane en Furope, amée isss, 
i bord de la frégate du Roi la Junon; par M. L.

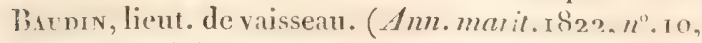
p. 346 i 36я.)

514. Extrait d'une litwe de Fernambouc, du 7 mai 1822. Je saisis cette occasion pour vous faire part de quelyues notions générales sur la navigation des côtes de cette province, ainsi que sur les différentes monssons qui existent à Fernam bouc, et qui exigent que les capitaines prenuent leurs précautions pour ne pas mançuer leur attérage. Les saisons sont ici divisées en été et en hiver; le premier détermine la mousson du nord-est, le second celle du sud-est. I'une commence en septembre; avec clleviennent les grandes chaleurs, el. rarement quclques pluies temperent-elles lit sécheresse. Mars amene l'autre, et avec elle les pluies, qui sont d'ordinaire presque aussi continues qu la sécheresse l'avait été. La duré de chacune est ainside six mois. La derniere est le temps le moins propre à la navigation. Les brises du sud-est souffent souvent alors avec violence, sans pourtant se tourner jamais cn ouragans, et les courans règnent avec beaucoup plus de force; ils suivent généralement la direction du vent: ccux de la mousson du nord-est sont à peine comparables à ceux de la seconde : de toute manière ils sont ordinairement assez. forts pour qu'un bàtiment qui se seriat laissé tomber sous le vent de son port de destination, ne puisse se relever qu'avec bien de la peine; souvent mème, s'il n'a pas de qualités, il n'y pourra réussir. Un navire qui part de France pour Fernambouc, en septembre, doit toujours attérir au moins vingt lieues plus sud; antrement s'il attendait qu'il fût E. et $O$ avec le port pour compléter les degrés qu'il lui resterait it parcourir en longitude, entrainé vers le nord d'environ une liene par heure, il lui fiudrait pent-être ruinze on vingt jours pour regagner; on a mème des exemples de bitimens qui ont été obligés de rétropiquer.

Les navires partant de France, en mars, doivent au contraire attérir au momd; dans la mousson du sud-est, on vient. reconnatre le cap Saint-Augustin ; dans celle du nord-a.t, lo eap d'Olimba, et un fanal a trois feux, dont un somere et clenx blanes, qui est chabli a l'entré du port, front l.r muit indigner ie monillage de lat rade an eapilatene qui comnat 
dejit le port; et à relui qui n’y est jamais venu, sil doit serrer le vent on se laisser arriver. Ce fanal est allumé depuic le couché du soleil jusqu't sou lever ; un bitiment pent fran chement attaquer lia terre, ear il n'y a de danger que le bane dOlinda, au nord de Fernambouc, qui s'étend a une liene au large, et au sud il n'y en a aucun depuis la riviere de Saint-Francisque. Les navires doivent mettre le pavillon de la nation au mat de misaine, pour demander un pilote.

Telles sont les différentes précautions qu'un capitaine doit prendre pour ne pas manquer le port de Fernambouc, comme plusieurs l'ont déjà fait.

F。

5ı. On a construit dans le district de Kala, sur les cótes de la mer Blanche, deux tours pour servir de fanal aux vaissaux marchands. L'une d'elles se trouve au cap Orloff, a 66 toises du rivage. L'autre se trouve au cap Pulongne, a 125 toises des bords de la mer; sa base est de $\{2$ pieds andessus du niveau de la mer. Ces deux tours sont peintes en blanc. (Journ. gén. de la lit. étr., nov. 182..)

\section{ART MILITAIRE.}

5i6. Carus Juzres Cesan ad Codices parisinos recensitus cum varietate lectionum, Julii Celsi commentariis ; tabulis geographicis et selectissimis eruditorum notis, quilous suas adjecerunt N. L. Acuruntre et N. F. Lenarae. Vol. quart. (etult.) in-s de 3r f. Imp. de F. Didlot, 1822. A Paris, chez N. E. Lemaire, édil, boulev. Italien, n". 22.

517. Mémorill pour la dífase des places, faisant suite au mémorial pour lattaque; ourage postume de Conmontaigre, marichal de camp, directurur des fortifications des places de la Mhosclle." celit. In-8. de 25 f. et :5 pl. Prix, y) li. Paris, 1822. Anselin et Pochard.

Le merite de cel onvrage ent comm des hommes de larl.

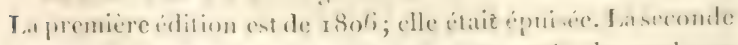

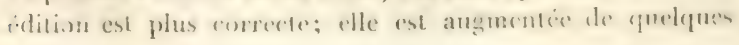


notes, d'un extrait des décrets sur les devoirs des gouverneurs des places, et de trois passages qui ensemble ont quarante lignes (pages 198,267 et $27 \mathrm{I}$ ); à ces différences près, elle est parfaitement conforme à la premiere. H.

518. Essai d'Une nouvelle méthode de fortification régulière pour la défense des grandes places de guerre, d'un diametre de 900 toises et au-dessus; par le prince E. D'Arenberg. In- $\{$ de 9 feuilles; plus 5 pl. Imp. de J. Didot l'aîné. Paris, 1822.

Nous regrettons de n'avoir pu nous procurer un exemplaire de cet ouvrage, afin d'en donner l'analyse.

5 19. Lennurucr der militargeographievon Europa, etc. ou Élémens de la géographie militaire de l'Europe, pour servir à l'instruction des écoles militaires de l'Allemagne, par A. - G. HAunzog, aumônier et prof. à l'école militaire de Magdebourg. $I^{\mathrm{er}}$. vol. 1820 : prix, \&f. 50 c; 2". vol. 1823 : prix, 8 f. Magdebourg, chez Rubach.

Cette géographie, bien qu'elle ne remplisse pas son but, puisque toutes les grandes considérations de l'examen des divers états, sous les rapports de l'attaque et dc la défense, n’y sont pas abordées, n'est cependant pas sans intérét.

C'est un traité élémentaire asscz commode où l'on trouve les renseignemens les plus généraux sur la géographie physique et politique des divers états de l'Furope; mais qui est très-loin d'offrir les renseignemens détaillés nécessaires pour la guerre. On n'y trouve ni la description des lignes frontières, ni des notions suffisantes sur les places de guerre, qui y sont à peine indiquées.

Ce livre est du reste le scul ouvrage général qui existe en Europe sur l'application de la géographie à l'art militairc. F.

520. Naмnкunnifa Svenska Faltherrars Lefverne (en suédois.) Vies des célèbres eapitaines suédois. In-s', avec portraits. Stockholm, x 82 I. Bernder. Tome I.

Ce volume contient les hauts faits et les aventures du felumaréchal comte de Stenbock, connu par l'histoire de Clatrles XII; avee son portrait.

Томғ. I. 
52x. On a trouvé depuis peu, au fond du puits de l'ancien chàteau de Coucy ( Aisne), le fruşuent d'une couler rine: ou est trace le millesime de 1258 ; e e pui semble promver que l'usage du camon est d'un siecle an moins flusancien qu'on ne l'a cru jusqu’à présent.

\section{VOYAGES}

522. Antict nes Vordges moneranes, depuis igho jusqua à nos jours, contenant, cte. ; par M. Fyrtis. lum des principaux rédacteurs des Anmales des Ioyages, rete. To. III at IV. 2 vol. ens. 5y f. '. Prix, 12 fr. Pap. vél., 2 ffr. Paris, E. Ledoux.

Les deux premiers volumes de cet ouvrage contiennent les voyages de la Pérouse autour du monte; dintrecasteanx a la recherche de la Pérouse; de Portlock of Dixon autour du monde, et a la côte norkt-ouest de l'Amérique; de Meares dans les mines parages; de Marcliand autour du monde, de Vaucouvert autour dumonde, et surtout dans le srand orian septentrional; de Broughton dans la partie septentrionale du frand Océan; le naufrage du paquebot l'. Amitope, sur les iles Peliou, et le voyage de Mac-Clucr à ce mème archipel.

On trouve dans le troisieme volume les découvertes de plusieurs terres dans le grand Océan, faites par plusieur. navigateurs anglais en 1789 . fe voyge de bligh dans le stand

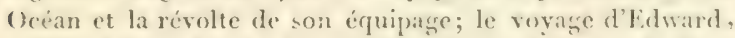

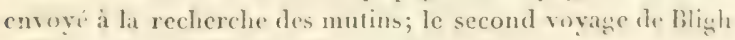
à 'Taiti ; celui de 'lumbull autour du monde.

Le quatrieme volume offe le voyage de Prampton el $1 \mathrm{ll}$, de l'ile Yorfolk à Bataviat, en 179 3; la relationde la dicomserle dudétroitentre la Nouvelle-IIollande, et la terre Vin-Dienen par Bass, en 1795 ; le voyage a la terre Australe, par Flinders; ceux de Grant et de Tuckey a la partic méridionale de la Nouvelle-Hollande, et le tableau de la colonie anglaise de la Nouvelle-Galles méridionale de 1788 a 189.9.

Plusieurs des relations navaient pas encore dit tradutes en franeais, les traductions ancienues ont ede revues sur lee originaux. 
523. Voxages dats l'intérieur du Brésrl, fait par ordre de S. M. le roi de Bavière, dans les amnées I 8 r 7 à 1820 ; par MIN. J. de Sprx et Cu. de MArruss. 2 vol. in- $\{$, avec atlas in-folio. Munich. (Ouvrage proposé par souscription.)

Le premier volume de cet ouvrage conticndra les voyages par les capitaineries de Rio-Janeiro, Saint-Paul, Minas-rieraës, Goyaz et Bahia. - Le second renfermera la description du voyage dans les provinces de Fernambucco, Piauchi, Maranhâo, Para et Rio-Negro, jusqu'aux frontières du Pérou. $\Lambda$ chaque volume sera joint un atlas in-folio, contenant sur ${ }_{15}$ planches lithographicées des portraits d'Indiens, des vues pittoresques, des carles géographirques, de géologie et de botanique. Les cartes géographiques sont dressées sur les propres observations des voyageurs, et sur les matériaux les plus nouveaux qu'ils se sont procurés dans les archives brésiliennes, portugaises et espagnoles. Le prem. vol. paraitra en 1823 , à la prochaine foire de Leipsic; le sec. en 1824. Prix de chaq. vol, pap. vél. impér. avec l'atlas complet sera de 4 charles d'or; sur papier ordinaire sans atlas $9 \mathrm{fr}$. Ia carte générale géographique séparée coûtera 34 f. 50 c.

$\mathrm{La}$ description des animaux et des plantes les plus remarquables du musée brésilien, sera en latin, les planches seront lithographiées, et elles formeront deux parties séparées ( voyez $\mathrm{N}^{\circ} .370$ ). Le tout sera publić par livraisons.

On souscrit chez Lindauer, libr. à Munich, et chez. Treuttel et Würtz, a Paris, Londres et Strasbourg. F. 5á. Voyages de M. le doct. J. Divy, de la Soc. roy. de Londres, dans I'ile de Ceylan. (Nou'. Ann. des Voy. To. XYI, oct. i 822.)

(ies contrées n'avaient pas encore élé décrites par des voyageurs qui les eussent parcourues; nous allons par ces motifs indiquer les divers voyages du docteur J.-Davy.

loyage au pie d' Actum. - Le célébre voyageur rapporte qu'un observateur habile a trouvé, par une mesure trironomét. faite it lat hâte, que l'éévation du pic d'ldam est de 7,000 pieds. Lui-mème, at raide dun baronètre, a trouvé une hauteur approximative de 6,152 pieds. Par une antre 
estimation aussi approximative, il porte cette hatteur a 6,68 o picds.

\section{Observations mettorologiques (awil 1817 ).}

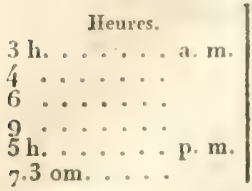

\begin{tabular}{cc}
\multicolumn{2}{c}{ Températ. } \\
54 & $(7,77)$ \\
52 & $(8,88)$ \\
51 & $(8,44)$ \\
51 & $(8,44)$ \\
53 & $(9,32)$ \\
59 & $(11,99)$
\end{tabular}

Vent N.-E.... modéxé. N.-N.-E. . dilo. dito.... doux. dito. ... dito. dito. ... frais. dilo. ... doux.

On trouve dans le même voyage une description intéressante de la montagne dont il s'agit, et surtout de son sommet où l'on voit l'objet de la vénération des Chingrulais, le Sriri-Pada, on l'empreinte sacrée de pied de Bouddah.

Foyage a Candy. - La description de Candy ou MahaNioura (la grande cité) offre des particularités assez curieuses. C'est la capitale de l'intérieur de l'ile. Elle est située à $x, 400$ pieds au-dessus du niveau de la mer dans une position fort agréable. Sa population est au plus de 30 o âmes.

Le voyageur fait d'abord la description du pays qu'il a parcouru depuis Colombo jusquả Candy qui est éloigné de 85 milles.

Voyage dans le Doumbera.-Le voyageur fait une petite excursion aux environs de Candy dans le Doumbera, province que l'on n'avait point encore visitée. $\Lambda$ près quelques détails intéressans sur le pays et les mours de ses habitans, il fait la description d'une grolte à nitre, et celle des procédés qui sont employés pour l'extraction du salpêtre.

Voyage is Trinkemale. - Ce voyage s'est fait de Candy, par Nalandé, Mainery, et Candellé, sur une étendue d'environ 130 milles. Cette partie septent. de l'ile est beaucoup moins habitée à cause de son insalubrité. Elle est la retraite favorite des bétes sanvages. On trouve dans ce voyage des détails intéressans sur la nature du pays.

Nota. Les quatre voyages dont on vient de lire une analyse très-succincte sont accompagnés d'une carte de l'ile de Ceylan qui parait dressie avec beaucoup de soin, et qui fait comnatre la direction des chaines de montagnes de cette ile, particularité que l'on chereherait vainement ailleurs. I.

525. Letroes sur quelques contrées de l'Espagne; 


\section{Voyages,}

\section{par M. Léon Dufoun, D. M. (Nouv. Ann.des Voy., oct. 1822.)}

L'auteur, dans ses quatre premières lettres, fail voyager son lecteur de Tarragone à Lérida et à Sarragosse, et lui fait franchir les Pyrénées à Jaca. Il se propose, dans les suivantes, de rentrer en Espagne par Irun, de continuer sa route à travers la Navarre et l'Arragon, de parcourir plus en détail le beau royaune de Valence, de mettre un pied dans celui de Murcie, et de sortir de la péninsule par Perpignan, après avoir visité plusicurs cantons de la Catalogne.

C'est en r 8 I q que l'auteur exécuta lui-mẻme ce voyage. Cette époque le met à mème de raconter quelques événemens militaires auxquels il a pris part. On trouve dans ces lettres des observations intéressantes pour l'histoire naturelle.

L.

526. Onservations ser les Grotues d'Adelsberg, en Carniole; par M. Broccur (Bib. Italiana, n. 74. 1822. p. 275.)

$\Lambda$ delsberg, que les Slaves de la Carniole appellent $P_{o s-}$ toina, est un petit bourg de la partie méridionale de la Carniole, sur la route de Goritz à Lubiana. Les montagnes voisines, qui sont de calcaire du Jura, renferment deux grottes à stalactites, dites l'une de Postoina, et l'antre de la Mlatleleine. Celle-ci est célébre parmi les naturalistes, parce que c'est dans un petit lac, situé au fond de cette grotte, qu'a été découvert le singulier reptile nommé proteus anguinus, qui est privé d'yeux, du moins apparens, et qui respire par des branchies, à la manière des poissons et des larves des batraeiens, ainsi que cela a été dénontré par M. Rusconi, anteur d'un ouvrage publié à Milan en 1821 , sous le titre français d'Amoursdes Salamandres aquatiques, et d'un autre ouvrage italien intitulé: Description anatomique des organes de la circulation dans les larves des salamandres arquatiques, qui a Jaru en 1817 . Ne peut-on pas supposer, dit M. Brocchi, que le protce n'est autre chose qu'une larve de quelque animal du gemre des salanandres, qui, condanné à passer sa vie dans une obscurité profonde, ne peut subir de métanorphose comme les autres batraciens, faute de recevoir l'influence de la lumiere? M. Rusconi a trouve on effet dims le protice denx 
petites vessies qu'il regarde comme des poumons flasques et trop peu développés pour servir à la respiration; les rudimens d'yeux qui demeurent cachés sous lat peau du même animal, sont de méme impropres à la vision. Ne conviendrat-il pas d'intergoger l'expérience et de rechercher ce qui arriverait à des Salanandres privées absolument dés leur naissance de l'accés de la lunière? L’autre grotte remarquable par son étendue et par la beauté de ses stalactites, l'est aussi par la découverte que M. Lovengreiff y fit, il y a 2 ou 3 ans, des ossemens fossiles d'un animal qu'il nomma d'abord Palaotherium, mais qui n'est pas celui auquel M. Cuvier a donné ce nom. M. Brocchi a reconnu que c'était l'ours des grottes (ursus spelatus) : à cette occasion ce naturaliste demande si la destruction successive des espèces n'entrerait pas dans le plan général de la nature aussi bien que celle des individus.

C. M.

527. Exprart de lit 4". et de: la 5e. lettre de M. I'. Cambracu, à M. Jomard. Sennir, nov. Is'za. (Bull. de la Soc. de géogr. n. 3. p. 1 r. )

M. Cailliand annongait d'abord son départ pour la province de fazoële, puis son départ de cette province, pour retourner à Sennatr et en Egypte. Les réflexions de M. Jomard, qui terminent ces extraits montrent toute l'importance de's renseignemens fournis par M. Cailliand, qui est parvenu jusqu'au dixiéme degrtóde latilude, rest-a-dire plus au sud cqu'aucun autre vorygeur, ef a plus de joo lieues de la derniere cattaracte du Vil. M. Citilliaud fait comnatre le fleuve lilane et cest le premicr Luropren qui en donne nue relation de visu. Il faut lire daus celle courte notice qui ne peut s'extraire, les réflexions judicieuses de M. Jomard, sur les cours du Vil et du Niger, et sur l'etut et les rapports probables des arrands cours d'ean daus cette partie de l'A frique. F.

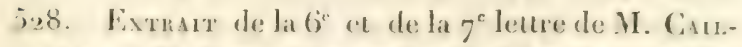
them i M. Jomard, membre de linstilut, sur les antiquilés de la Nubie, eq Observations sur les lettres précédentes; par M. Jovano. ( Amm. des Voyages, oct. 1822, ) 
Le voyageur croit avoir découvert !es ruines de l'antique cité de Naka ct les restes du collége de Héroè.

M. Jomard se livre à une discussion scientifique sur la position de la résidence des prêtres de Méroé, et de leur observatoire. Il fait veir qu'un résultat très-intéressant du voyage de M. Cailliand est que plusieurs des antiquités de la Nubie sont postérieures aux monumens de hẻbs. Le T.

529. Śtour ne trois mors dans les montagnes prís de Rome, pendant l'année I8I9; par Marie: Colima , trad. de l'anglais, \ vol. in-8. Paris, 18̆22. Béchet. (Amn. des voyages, oct. 1822).

Madame Graham sattache surtout à peindre les moeurs de la population des habitans des campagnes, les habitudes de rette race à deni civilisée, et les ressources de cette contrée si peu connue. Elle donne des détails qui foumissent des lumières précieuses pour expliquer beaucoup de particularités sur les mours antiques dont la trace s'est nieux conservée au milieu des canpagnes que dans les villes.

L.

530. L'Arrieue ou histoire, moeurs, usages et contumes des Africains (Dahome'), Guinee); pas M. John MAc-Leon, D. M ; traduit de l'anglais, par M. Ed. Gauttier. 1 vol. in-16, avec fig. Piris, 1821 . Nepveu. (Ann. desvoy ases, oct. 1822).

Les détails que M. Mac-Leod donne sur les mours des negres qu'il a vus, montrent que cenx du Dahomey, sont. ansi féroces que leurs voisins. Ils ne mangent pas de la chair hmmaine, comme on les en avait accusés; mais, excepté cet acte de cruauté révoltante, ils se traitent les uns les autres d'une maniere qui fait horreur. Ce petit ousrage est bon à lire par les particularité curieuses qüil renferme. L.

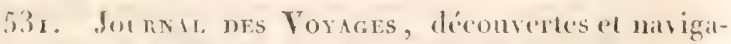
rioms modernes, ou Archives géographiques du XIX" siecle; public par MT. Velinear, membre dr la commission contrale: de la Société do: Géngrat-

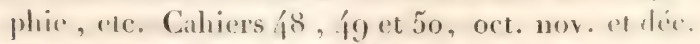
rise, formant le: 10m. I I de la collection.

Les articles prineipaux contenus dans ce volume sont les 
suivans : Deseription historique, gúograplique et statistiqne de la ville d'Alep et de ses environs. - Souvenirs du pays Basrue et des Pyrénées en I8I9, par M. Boucher. - Notice sur les montagnards de Rajemahaul dans l'Inde. - Journal d'un voyage fait à l'ile Pitcairn par le capitaine $H$. King, en 18 rg. - Découverte de líle Elisabeth. - Fragmens d'un voyage inédit en Italic. - Notice sur l'expédition des Francais dans le Texas. - Mémoire sur la sphère et le zodiaque.Itinéraire d'Alep à Mossul par la Mésopotamie, par M. Rousscau, consul-général a Bagdad. - Voyage dans la Valachie, la Transylvanie et la Hongrie, par l'abbé Sestini._Esquisses du haut Canada, par Howison. - Voyages en Syric et à lat Terre-Sainte, par Burckhardt. - Atlas miversel de géographie moderne, par M. Brué. - Explorition de la côte orientale du Groënland, par le capitaine Scoresby. - Exploration de la cote septentrionale d'Afrique, par le capitaine Sinyth. - Statistique de Paris. - Population de I'Angletcrre. - Statistique de la Bucharie. - Statistique du royaume de Suède. - Commune origrine des insulaires de la mer Pacifique. - Tableau de la nouvelle circonscription des diocéses de France. - Notice sur l'expédition pédestre arctique du capitaine Francklin. - Voyage de M. Rüppel cn Egypte et en Arabie.-Voyage en Vubic par M. Cailliaud.Exploration de la nourelle Shetland méridionale. - Expédition russe à la côte N.-O. d'Amérique. - Reconnaissance des côtes de la Floride. - Missions au Groënland et au Thibet. - Ile de glace dans la mer du Sud. - Plusieurs récits de naufrages et autres articles détachés, etc.

532. Note sur les voyages de II. A. de Str-Huane, dans l'Amérique méridionale.

M. de Sant-Intainr partit pour l'Amérique le $\mathrm{x}^{\mathrm{er}}$ avril 1816 ; et après de courtes relaches a Lisbonne, Madere et Ténériffe, il arriva le ${ }^{{ }^{e r}}$. juin à Rio de Janeiro. Il consacra quelques mois à explorer les environs de cette ville, et préluda à ses voyagres par une excursion sur les bords du Parahyba. Ce fut alors que, ne se bornant plus a des recherclies botanipues, il commenca à former des collections d'animaux, collections qu'il at continuées jusqu'a son diprart pour l'Europe. Il prartit en décembre pour la eapitancrie des 


\section{Toyages.}

Mines, qui égale presque la France en étendue, et il étudia avec soin le caractére de diverses sortes de végétation qu'on y observe; celle des bois vierges, celle des campos, où l'on ne trouve que des herbes et des sous-arbrisseaux; la végétation des carascos, espèces de hois nains, composés d'arbustes de quatre à cinc pieds; des cattingas, autres bois qui ressemblent à nos taillis de 18 ans et perdent leur's feuilles cha que année; enfin celle des tabuleiros cobertos, pâturages parsemés d'arbres tortueux et rabougris. M. de St.-Hilaire, après avoir passé quelque temps a Villa Rica et Villa do Principe, et observé le travail des chercheurs d'or, s'enfonca dans les bois vierges situés à l'est de la capitainerie des Mines, et visita les restes de plusieurs peuplades Indiennes, les Malulis, les Monoxos, les Maconis, etc. Il passa ensuite dans le district de Minas-Novas, si abondant en améthistes, en aigues-marines, en chrysolithes, etc.; et il séjourna quelqque temps au milieu des Botocudos, Indiens belliquenx, qui ont l'usage de se faire un large trou au nez et aux oreilles, et qui virent dans les forêts entièrement nus et sans habitation. De Minas-Novas, il se rendit sur les bords pestilentiels du Rio de San-Francisco, parcourut les déserts qui aroisinent ce fleuve majestueux, et, revenant sur ses pas, il entra dans le District des diamans. Après avoir vu tous les lieux où l'on extrait cette précieuse pierre, il retourna à Villa-Rica en faisant un long détour, et revint a Rio de Janeiro en mars $18 \times 7$. Partant pour la seconde fois, il suivit jusqu'à l'embouchure du Rio-Doce la côte qui s'étend au nord de la capitale du Brésil. Il vit le Cap-Rio, la ville de Campos et son territoire fertile, la capitainerie du Saint-Esprit et ses différens ports. S'étant embarqué à Villa da Victoria, il revint à Rio de Janciro, et, vers la fin de janvier 18 ig, il commença son troisième voyage. Il traversa la partic orientale de la capitainerie des Mines quil ne connaissait pas encore; il vit la magnifique cascade à laquelle le Rio de S.-Francisco doit son origine, les caux sulfureuses d'Araxa, la ville de Paracatu située comme un oasis au milieu des déserts; et, suivant ce long plateau qui lonne naissance au Rio dos Tucantins et au Rio de Sinfrancisco, il arriva dans ia capitainerie de Goyaz. Il penctra jusqu'à lat frontièe de Matogrosso, visita les Indien: 
Coyapos, et prit le chenin de Saint-Paul. Arrive dans cette ville, il y laissa les collections quil avait formées jusqu'alors, et se dirigrea vers le midi, en passant derrière la grande Cordeliere parallèle a l'Océan. $\Lambda_{\text {près avoir vu les }}$ villes d'Hytu, Porto-felix, Sorocaba, etc., il entra dans les Campos-Geries, pays charmant, celui qui de tout le: Brésil conviendrait peut-être le mieux à des Européens. Il examina, a Curitiba, la fameuse lierbe du Paraguay, qu'il reconmut pour un Ilex; il descendit l'effrayante Serra de Parannagroa, se retrouva sur la côte, visita les îles de SaintFrameois et de Sainte-Catherine, et se rendit dans la capitainerie de Rio-Grande, l'une de celles que la nature a fivorisées le plus. Il y reconnut les limites de la canne a sucre, du cotonier, etc., et suivit avec attention les dégradations successives de la végetation du tropique. Après avoir passé un mois a) Porto-Allegre, ville sitnée à l'origine d'un lac immense, devant l'embouchure de quatre rivières navigables, il vit hio-Crande de S. Pedro do Sul, autre ville bàtie au milieu des sables, à l'extrémité du lac dos Pathos. Au dela du fort de Sainte-Thérése, il entra dans les possessions espagnoles, il visita Rocha, Saint-Carlos, Mitdonado, Montevideo, la colonie du Saint-Sacrement; il suivit les bords du Rio de la Tlata dont la Flore a tint d'analogie avec celle de l'Europe, et ensuite il voyagea sur les rives de l'U ruguay. Au nord de Belem, il passit treize jours dius un désert uniquement labité par des jaguars, des autruches et des cerfs, et il fitllit y périr pour avoir mangé quelques cuillerées d'un miel fourni par la gruépe fecheguana. Il prarourut les sept hoursates qui seules subsistent encore des trente qui formaient Ies anciennes missions du Paraguay, et il eut a gremir de ne: plus trouver que des mines daus une contre qui fut jadis si florissante. Revenu a Porto-Allegre, il s'embargua sur le lar dos Pathos pour se rendre d'abord à Rio-(irande et de la a Rio de Jinciro. Cn dernier voyage qu'il fit de eette ville a Saint-Paul, en passint par les Mines, Jui procura loecasion de visiter quelques lautes montagnes qu'il ne ronnassait pas cneore. It s'embarqua pour l'Europe en juin 18\%, e. il est arrive a Paris ave 2000 ofiseanx formant

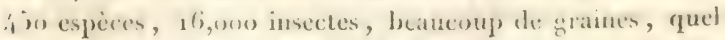
gues quadrupides, des reptilis, des proinosis, quelques. 
minéraux parmi lesquels se trouvent la gangue des diamans, ct environ trente mille échantillons de plantes formant sept mille espèces. M. $\Lambda$. de Saint-Hilaire a fait l'analyse botanique de tous les véngétanx qu'il a recueillis, et s'est principalement attaché aux plantes usuelles, telles que les quinas, les ipecacuanhas ou poayas, etc. Il a fait en outre le journal détaillé de son voyarge et a pris tous les renseignemens qu'il a pu sur la statistique des pays qu'il a visités, les mours des habitans, leur agriculture, leur commerce et leurs usages.

\section{DEUXIEME SEGTION.}

\section{TRAVAUY DES SOCIETES SAVANTES.}

SE A N CES.

533. Panis. - Institut royal de France--Academie des Sciences séance du 6 jansier $\mathbf{1}_{22} 3$. M. Pailhis, inspecteur de la navigation, adresse à l'Académie le journal des crues et diminutions de la Seine olsservées au pont de la Tournelle pendant 1822 .

M. Vaurguelin rend compte de l'examen qu'il a fait d'un arćclithe tombé aux environs d'Épinal le i 3 septembre 1822 , a l'entrée de la forêt de Tannière, à un quart de lieue de la Baffe, département des Vosges.

On donne lecture d'une partic d'un rapport sur les procédés chimiques et mécaniques employés par M. Puymaurin fils pour la fabrication des médailles de bronze moulées et frappées.

M. Coquebert de Montbret fait un rapport verbal sur un ouvrage suédois intitulé: Expériences hydrauliques faites anx mines de Fahlun en Suéde par M. Laycrhyelm.

Le mème dorne une indication sommaire des mémoires et des éloges contenus dins le Recueil de l'Académie royale des sciences de Stockholm pour l'année 1821 .

On donnc lecture d'un mémoire de $\mathrm{H}$. Alix Tissot, intitulé : rues nouvelles sur les maludies des riscére's awee l'indication d'un moyen pour s'en préserver. 
Séance du $x 3$ janvier. - M.II. Bose et Molard font ur ripport sur le piége de l'invention de M. Licent pour pren dre les loups, les renards, etc.

II. Aragro lit, pour M. Fresnel, le résumé d'un mímoire sur les lois de la modification que la reflexion produite par les corps transparens imprime à la lumiere polarisée.

On lit un mémoire de N. Casteron, médecin, sur les hydatides acéphalocystes.

Séance dus 20 janvier. - MI. Delire envoie un ouvrage manuscrit intitulé Histoire des Lichens, genre strictu. MM. Desfontaine et Bosc sont nommés commissaires pour l'examen de ce mémoire.

M. Percy lit, au nom d'une commission, un rapport sur un mémoirc qui avait été présenté par M. Bancal, et qui a pour titre: Mémoire sur un nouveau kistitome cache pour l'opération de la cataracte par extraction.

Il est composé d'une gaîne étroite, longue ct plate, ayant un petit couloir à son extrémité supérieure et d'où on fait sortir, en pressant un bouton placé latéralement, une petite lame aiguë et tranchante qu'une languette attenant au couloir rend inoffensive, et qui, mise en mouvement, açit avec autant de facilité que de certitude.

Nous ne répéterons pas ici les détails descriptifs que l'auteur a consignés dans son mémoire, relativement a cet instrument que MII. les commissaires ne regardent an surplus que comme une modification de celui de Lafaye auquel peu de personnes hésiteront désormais à le prefírer.

M. Dupin lit la premiere partie d'un rapport fait an nom de la commission chargée d'examiner le travail de M. Mareslier concernant les batcaux à vapeur. Ia seconde partie sera Juedansla séance prochaine.

Sinnce du 27 janvier.-I'A cadémic recoit m mémoire manuscrit sur l'Agriculure de la Flumdre, par M. Cordier, ingénieur des ponts et chaussées; 2 vol. in-folio avec un atlas. (Renvoyé à l'exiunen de MM. Bose et Yvart.)

M. Cauchy présente un mémoire intitulé: Recherchess sur 1. mousement de derux fluides superpose's, l'un compressible, l'autre non-compressible.

MM. de Juscien, Desfontaine, Latreille, Geoffroy $\mathrm{S}$ Hilare et Brongraiast font un rapport sur les Collections of 


\section{Séances.}

les Observations recueillies par M. Aug. S. Hilaire dans sou voyage au Brésil.

M. Dupin termine la lecture du rapport commencé à la dernière séance sur l'ouvrage de M. Marestier.

Séance du 3 février $\mathrm{r} 823$. - L'académie recoit un mémoire sur le rétablissement d'une chaire d'Hippocrate, par M. le chevalier' de Mercy.

MI. Girard lit, au nom d'une commission, un rapport sur un mémoire manuscrit ayant pour titre, De l'Arrondissement des Cames ou des dents des roues et de leur frottement dans les engrénages; par M. le comte de Bucquoy.

La commission pense que le travail de M. de Buquoi mérite l'approbation de l'académie, qui approuve ce rapport et en adopte les conclusions.

Sćance du 10 jésrier. - MI. Dupetit-Thouars lit un mémoire intitulé, Observation sur un bourrelet produit par la décortication compléte pratiquée sur une branche de pommier, et envoyée par M. Ducrochet dans la séance du 9 novembre 1822 .

M. Moreau de Jonnès lit un mémoire intitulé, Recherche sur l'Origine géographique des plantes des Antilles transportées dans ces íles par les agens naturels.

534. Société de géographie. Séance du 3 janvier. La commission centrale vote une lettre de remercimens à $M$. le comte Orloff, pour l'offre d'un prix de 500 fr. Le secrétaire général donne communication d'une lettre de M. de Krusenstem, contenant des remercimens pour la commission centrale, de l'ouverture qui lui avait été faite de correspondre avec elle. Cette commission renouvelle ses bureaux, et admet comme membres de la société, MM. de Prigny, capitaine de vaisseau; Lecesne, capitaine au corps royal des ingénieurs géographes; Dumont Pascal; d'Entend et M. le baron de Derfetden de Hinderstein.

Síance du x 7 jansier. - M. Bessayrie, membre de plusicurs sociétés savantes, annonce à la société qu'il prépare un volume sur la Guyane française. Il adresse à la société deux discours lus à la société du Var.

M. Guitlemin, consul de S. M. à lat Nouvelle-Orléans, offre à la société de correspondre avec elle, et de lui cn- 
vover les journaux, publiés dansle pavs et contenant des relations géographiques. Ia section de correspondance est chargec de lui adresser me serie de questions.

M. Langles propuse à la commission centrale de publier, pour premier résultat de ses travaux, un volume contenant la traduction ou la publication de quelques ouvrages importans sur lit géngraphie. Il indique a la société les ouvrages suivans qui se trouvent à la Bibliothéque du Roi, et entre lesquels elle pourra faire un choix.

I. Tne Mappe-Monde sur vélin, manuscrite, en catalan, de $13: 6$.

2. The relation complete des Voyages de Marco-Paulo, in- $-^{\circ}$. de 46 pages. Imprimécen catilan en $129^{8}$.

3. La relation manuscrite en slavon d'une ambassade du srind-duc de Moscowie, à Pékin, écrite en 1685.

4. Relation du Congo, a traduire du portugais, en 3 volumes in-fol., dont on pourrait inprimer un extrat.

5. Ia traduction francaise du Géographe ture. Petil in-fol.

M. Langlés s'arrête plus particulièrement sur Vareo-P'inlo, dont il existe huit manuserits à la Bibliothéque, que l'on pourrait consulter pour les variantes.

La commission centrale arrète qu'il sera publié à ses frais un volume, et charge la section de publication de lui faire un rapport pour déterminer sm choix entre les ouvrages proposés par M. Langlès.

On admet comme membres $\mathbf{M 1}$. le prince de Labanoff, aide-de-cimp de S. M. l'Eimpereur de Russie; Frnest de Beaufort, officier de la marine royale; ct Guillemin, ronunl de France à la Nouvelle-Orléans.

535. - Socivte: Linnienne. Siunce du 28 dicember, jour anniversaire de la morl de Tornasont. - Diseours douverture, par M. le comte de Lacépede, pair de France, président. - Compte rendu des travaux de la Sociéti Linnéenne, pendant l'année 1822 , par M. Thiébant de Lerneand, secrétaire perpétuel. - Notice sur des aras bleus, nés en France et acclimatés dans le département du Calvados, par M. Lamouroux, correspondant, a Caen. - Ihiscours sur IC:s moyens d'arriver, dans les scienees naturelles, a l'unite dopinion, par M. J.efebure, menbre résident.... Observations 
sur le naturel du chat, par M. Charles Lemesle, mombre auditeur. - Lecture du programme des denx prix proposés pour 1824 , l'un de zoologie, l'autre de botanique, par M. Desnarest, l'un des vice-présidens. - Eloge historique de Broussonnet, premier fondateur de la Société Linnéenne, par le secrétaire perpétuel.

Séance du 9 janciér. - La Société reçoit de MI. Plassiart, de Coulanges, une notice sur des plantes alpines trouvées dans le département de l'Yonne; et de MI. Deshayes, correspondant, une grande carte botanico-médicale. Des commissaires sont nommés pour rendre compte de ces deux ourrages.

M. Desnazières, de Lille, adresse une notice sur les lycoperdons de Linné, et sur une nouvelle espèce de carpobolus, genre nouveau à ajouter à la flore française. - Ce mémoire est réservé pour le troisième volume des Mémoires de la Société.

M. Duvau lit une notice sur trente-un manuscrits de Plumier, existans à la Bibliothéçue royale et à celle du husénm d'histoire naturelle de Paris.

M. Deslayes, correspondant, lit une pièce de vers sur le système floral.

M. Lachevardière en lit une autre à la mémoire de Joseph de Jussien. On en ordonne l'inpression dans le deuxiéme volume des Miémoires qui cst sous presse.

Srance du 23 janvier 1823. - On reçoit plusicurs cmpreintes végétales fossiles des environs de Chalonne, département de Maine-et-Loire, et des détails sur la végétation extraordinaire des mois de novembre et décembre 1822 , dans le département de la Meuse.

M. Noyer lit un mémoire sur le manioc, sa culture et ses produits.

On lit, pour M. de Cliesnel, correspondant à Montpellier, des réflexions sur le sommeil des plantes, que ce naturaliste voudrait qu' on appelàt simplement état nocturne.

II. le docteur Vallot, correspondant, envoie de Dijon trois notes: l'une est relitisc a une substince composce, que l'on trourve dans les fourneaur des charbonniers, et que l'on nomme fleur du fourneau; c'est le produit de la fumé blene qui :ort du four quand il brùle convenablement; la seconde sur les huit insectes qui vivent aux dépens du fraisier, fra- 
garia vesca, I. et la troisieme sur une nouvelle espece de stapele a branches quadranģulaires quil nomme stcipelio nostrata.

Sur le rapport de II. Vicillot, la Société donne son approbation à la partie ornithologique de la faune du département de la Moselle, qui lui a été adressée par M. Horavork, son correspondant, et l'engage à terminer cette intéressante entreprise.

536. - Socrété Ro yale ex centrale d'AgriculturF.Sécunce du 5 férrier 1823 - - Io. Méricart de Thury a fitit un rapport sur des Mémoires envoyés au concours, relativement à l'introduction d'amendemens ruraux dans des cantons où ces amendemens sont encore inconnus, et sur des essais comparatifs de l'urate calcaire employé en grand pour différens genres de culture. Il résulte de ce rapport : $x^{\circ}$ qu'un limon argileux laissé par la mer dans les hautes marées a été employé avec succès aux environs de Saint- $\mathrm{I}_{n-}$ leri, par M. Caron, et que ce propriétaire a tiré un parti avantageux de l'addition de la suie et d'une poudrette préparée à l'aide de cendres de tourbe; $2^{\circ}$. qu'aux environs de Pamiers, dans le département de l'Arriège, M. Lajoux a composé un urate économique en mêlant du plâtre à l'urine des écuries; $3^{\circ}$. que dans le département de Vaucluse, H. Waton s'est servi avec beaucoup de succès d'une poudrette composée de plâtre et de matières des vidanges. Les auteurs de ces applications nouvelles recevront à la prochaine sćance publique des témoignages de la satisfaction de la Sociétc.

2". La Société des Sciences de Rochefort a adressé quelques premiers essais sur la culture comparée des blés dits d'Odessa et de Tangarock, ou mieux Taganrock, avec les blés du pays; on a remarqué dans la Sociétí, que des expériences de ce genre acquéreraient beaucoup d'intérêt, si l'on pouvait fixer la véritable variété on l'origine positive des grains en expérience; la désignation du port de mer qui a fourni des blés, ne donne aucune certitude que la qualité de ces blés sera toujours identique; ceux tirés d'Odessa notamment proviennent de contrées diverses d’une immense itendue et situies a de telles distances les unes des autres, quil n'y a aucun ripport entre la nature de leur sol, la tempéra- 
inire qu'elles éprouvent, et la qualité des semences qu'elles emploient: une désignation scmblable d'origine ne parait donc point suffisante pour établir des expériences comparatives.

$3^{\circ}$. M. Coquebert de Montbret a fait un rapport verbal étendu sur plusieurs cahiers imprimés des feuilles agricoles d'Hoffwill, qui avaient été adressés à la Société par M. de Fellemberg, et qui contiennent notamment des détails curieux sur les instrumens ruraux perfectionnés, sur les procédés de culture suivis et successivement développés par l'auteur, sur les produits obtenus de ses assolemens, et sur son enseignement-pratique de l'agriculture, tant pour les élèves qui font partic de son institut, que pour l'école des pauvres qu'il a établie à Hoffwill.

$4^{\circ}$. M. Lasteyrie a rendu compte de plusieurs ouvrages allemands qui ont été adressés à la Société par M. de Hazzy, et qui ont rapport à liirrigation des prairies, à la salubrité des villes, à la construction des moulins à huile, à la conservation des moutons à laine superfine; et aux travaux divers de la Société d'agriculture de Munich, dont l'existence parait avoir eu une influence remarquable sur les perfectionnemens de l'éconiomie rurale de la Bavière.

5\%. MI. Brun-Neergaard a communiqué la traduction manuscrite de denx ouvrages danois : l'un rédigé par M. Viborg, et relatif à l'éducation des bêtes à cornes dans la Moldavie ; lautre rédigé par un anonyme, et qui a pour objet des détails. sur la race des chevaux du royaume de Maroc. Silv.

\section{PRIX PROPOSÉS.}

537. Paris. - La Société Linnéenne décernera, en sa séance publique du 28 décembre 1823 , une médaille d'or de la valeur de trois cents francs, à l'auteur du meilleur mémoire dans lequel :

$x^{\wedge}$. On exposera les conséquences qui résultent naturellement des observations et des expériences faites jusqu’à ce jour, sur les mouvemens et l'état de la séve dans toutes les phases de la vie végétale et dans les diverses saisons de l'année;

$2^{\circ}$. On confirmera ces résultats, et on y ajoutera, par des faits récens, par des expériences réitérées, des considérations nouvelles;

Tомк 1. 
3". On offrira, enfin, en évitant toute explieation pure.. ment hypothétique, une thérie de la marche des fluide, végétaux, aussi probable, aussi complite que le permet l'état actuel de la science.

Prix propose's puur 1834. - Prix de Znologie. - I Ius observations, dont quelques-unes reposent sur des faits attestés par des naturalistes instruits, semblent pronver que parfois on découvre dans des masses de pierres plus ou moins dures, dans des troncs darbres, et mème dans dies couches de houille, des êtres vivans, tels que serpens, crapauds, lézards, insectes, ete., sans qu'on puisse se rendre compte comment ils y ont pénetré, comment ils ont cun. servé la vie.

La Société Linnéenne de Paris désirerait quion rass: blit tous les faits analogues qui ont éte rapportes par les écrivains; qu'on établit leur degré réciproque de probabilité ou de certitude, en rapportant textuellement les preuves sur lesquelles ils reposent, et en s'attachant à réunir toutes les circonstances critiques qui peuvent écliarer sur l'existence et la cause probable de ces faits, et que le touf fùt traité de manière à établir d'abord les pièces d'où l'on peut et l'on doit partir pour expliquer, s'il y a lieu, le plénomène en question.

Quelques observateurs ayant pensé, à l'égard des animaux trouvés dans des troncs d'arbres, que l'individu qui y avaif pénétré, jeune encore, par un accident quelconçue, s'y était développé et $\mathbf{y}$ avait. acquis l'accroissement ordinaire qu'il prend a l'air libre, la société Linnémne désire que l'on examine cette singuliere opinion, et que l'on montre si les. lois de la physiologie permettent ou non de l'admettre.

Enfin, par rapport aux animaux trouvés dans des bloes de pierre, il importe de voir si la meme thérie peut leur ètre appliquée, ou s'ils ont été enveloppés dans l'étit oú on les trouve par la matiere liquide, laquelle, en se durcissant, a produit la masse pierreuse qui les renferme, et dans ce cas explirguer comment la vie a pu ne pas cesser; constater, autint qu'il sera possible, par la nature des masses pierreuses, leur grisement relatif, leur homoşinéité, l'époque géologique a lapuelle on pent rapporter l'emprisonnement de ces animanx, en atyant igard anx canses acriden- 
lelles qui peuvent diminuer l'intérèt et l'importance de tel ou tel fait.

Une médaille d'or de trois cents francs, on sa valeur, scra remise, en séance publique, le 28 dicembre 1824 , a celui qui répondra le plus complétement possible aux différentes questions proposées. La meilleure monngraphie, qui satisfera entiérement aux vues de la première partie du présent programme, obtiendra, en cas de non solution satisfaisante sur la seconde partie, à titre d'encouragement, une somme de deux cents francs.

Prix de Botanique. - Dans la fleur, il existe un organe qui sícrète une liqueur mucoso-sucrée, premier rudiment du miel que l'abeille nous fournit. Cet organe a reçu le nom de Nectaire(I). Il manque dans les trois quarts des végétaux connus; et, dans ceux où on le trouve, il n'est pas également le mème aux yeux de tous les botanistes: on pent dire que c'est le point dogrnatique des élémens de la science, le plus obscur dans tous les ouvrages publiés jusqu'ici.

Selon Linné, on doit entendre par nectaire les corps glanduleux, les pores, les appendices, les formes anomales, et généralement toutes les parties de la fleur étrangères aux organes sexuels et à leurs enveloppes.

Quelques botanistes justement estimés nient l'existence du nectaire, ou, s'ils la reconnaissent, ils placent cet organe tantôt à la naissance des pétales, autour des ovaires, on dans la gorge de la corolle; tantôt sur le réceptacle, à la base des anthères, entre les étamines ou sur le pistil. Chez les uns, le nectaire est un cornet, une écaille, une glande et mème une espèce de poils; ou bien une fossette, un sillon, une excroissance. Chez les autres, c'est l'épcron court que l'on voit près du style; c'est toute portion quelconque de la fleur

(1) L'Académie des sciences et belles-letires de fruxelles arait mis au concours, en 1820, une question ainsi conçue : "La défi" nition du nectaire, donnéc par Linné, convient-elle à tous les "organes désignés jusqu’à ce temps sous ce nom? En cas de ré„ ponse négative, on demande une classification physiologique de " ce mime organe. " - Cette question a été remise au concours pour $1 \$_{23}$, et, comme elle est demeuréc saus réponse, elle a et: retirce. L'indiférence apportée à cel appel, est due, sans aucun clonte, au peu de développement de la question. 
qui se présente éminemment prolongéc ou difforme; ce sont les taches plus on moins remarquables que l'on observe a lie base des pétales ou des corolles d'un certain nombre de fleurs. En un mot, on n'est point d'accord sur ce que l'on doit exclusivement appeler nectaire, et l'extrème diversité d'opinions à ce sujet tend à prouver la nécessité de s'entendre.

Dans la vue de faire cesser toute incertitude et de fixer invariablement re qu'il convient de nommer nectaire, la Société Linnéenne de Paris fait un appel aux botanistes et leur propose de résoudre les questions suivantes:

Quel est l'organe dans la fleur auquel on doit exclusivement donner le nom de nectaire? A quel caractère peut-on le reconnaitre? et de quelle importance est-il pour les végretaux qui en sont pourvus?

Une médaille d'or de trois cents franes, ou sa valeur, sera remise, dans la séance publique du 28 décembre $182 /$, à l'auteur qui aura pleinement satisfait à toutes les conditions du présent concours.

Conditions générales. - Le's mémoires portant une épigraphe ou devise qui sera répétée avec les noms, prénoms, qualités et demeure de l'auteur, dans un billet cacheté joint au manuscrit écrit lisiblement, seront adressés, francs de port, à M. Thiébaut de Berneaud, sccrétaire perpétuel de la Société Linnéenne de Paris, savoir : poar le prix de pbysiologie végétale, avant le $\mathrm{I}^{\mathrm{er}}$. avril $\mathrm{I} 8_{2} 3$; et pour les deux autres prix, celui de zoologie et celui de botanique, avant le $\mathrm{I}^{\mathrm{er}}$. juillet 1824 . Le terme fixé est de rigueur.

Les membres résidans, les menbres honoraires, domiciliés à Paris, ainsi que les auditcurs, sont seuls exclus du concours.

La Société Linnéenne prévient qu'clle ne rendra aucun des écrits qui auront été envoyés au concours; mais les auteur ou leurs fondés de pouvoirs auront la liberté d'en faire. prendre des copies s'ils en ont besoin.

338. Prognamae des pux proposés par la Socrété d'Encoeragement pour lindustrie nationale, dans sa séance générale du 3o octobre 1822 , pour itre décernés en $1823,1824,18.5$ et 1830 . In-8. de 5 . 
feuilles, et 2 lableaux imprimés. Imp. de Nadame Huzard, à Paris.

10. Prix pour un moyen de procurer aux areugles indigens le travail le plus utile pour eux et le mieux approprié il leur situation. La Société demande qu'on indique le travail le plus productif pour les aveugles dans leur condition donnée; elle exige essentiellement que le produit de ce genre de travail soit d'un débouché facile, général, et que le travail aille chercher l'aveugle, l'aveugle ne pouvant guère aller le chercher lui-même. Il faut que l'apprentissage puisse avoir lieu à tout âşe, ne soit ni long ni difficile, n'exige ni le déplacement de l'aveugle, ni le secours d'un maitre. Le prix est de rooo fr.

$2^{\circ}$. Prix pour la conservation des substances alimentaires, per le procédé de $\boldsymbol{M}$. Appert, exécuté plas en grand, ou par tout autre moyen analogue. La Socicté propose un prix de $2000 \mathrm{fr}$. à celui qui aura formé en France un établissement dans lequel, en euployant un procédé quelconque on pourra conserver, au delà d'une année, les substances animales et végétales a l'état frais ou de préparation récente, sous un poids réel d'au moins 8 ou ro kilog. et dans un mème vase, pendant le voyage a une de nos colonies. Une condition de rigueur est que la vente annuelle des objets conservés s'éleve à $20,000 \mathrm{fr}$ au moins, et que les frais de manipulation et de conservation ne montent pas annuellement à un prix tellement élevé, qu'ils excluent l'emploi le ce procódé comme moyen économique et d'un usage général.

30. Prix pour l'importation en France et la culture de plantes utiles it l'agriculture, aux arts et aux manufarlures. La Société propose un $\mathrm{I}^{\mathrm{er}}$. prix de $2000 \mathrm{fx}$, et un second áe rooo fr. pour l'introduction d'une ou de plusieurs plantes, pouvant étre cultivées en pleine terre, soit dans le midi, soit dans le nord de lit France, et dont les produits irouveraient un cmploi important dans l'agriculture ou dans un art quelconque. La Société distribuera des médailles aux uersonnes qui se livreront d'une maniere plus spéciale a lit culture ou a la fabrication des produits de ces plantes.

$\therefore$. Pour la construction d'une machine propre a trabail- 
Ler les verres d'optique. La Suciété propose un prix de $2500 \mathrm{fr}$. pour une machine a l'aide de laquelle on puisse fabriquer, avec la plus grande précision, des verres de lunette d'une courbure déterminée. On pense qu'il doit y avoir dans la machine uncentre fixe de rotation dont le verre se tienne toujours à la mène distance, et qui, par cette raison, doit finir par donner à la surface du verre, à l'aide d'un frottement la forme d'une portion de surface sphérique dont le rayon serait égal a cette distance, quelles que soient d'ailleurs les imperfections du bassin contre lequel se frolte le verre, et lors mème qu'il serait plan. Les concurrens sont tenus de présenter le $\mathrm{I}^{\mathrm{er}}$. mai i $\delta_{2} 3$ les machines mèmes qu'ils auront inventées, et de les faire fonctiouner en présence des commissaires de la Société, qui se réserve la ficulté de publier dans son Bulletin la description de la machine qui aura été jugée digne du prix.

50. Prime pour la construction d'un moulin is moudre et it concasser les grains, qui puisse être culapte it toutes les cxploitations rurales. La socité offre une prime de $4000 \mathrm{fr}$. a celui qui aura appliqué pendant deux ans avec succès à une ou plusieurs exploitations rurales, un monlin d'une facile et solide construction, d'un prix modique, et qui soit habituellement mù par le vent, au moyen d'ailes placées sur le toit du bâtiment. Le moulin devra ètre ajusté de manière que le grain tombant sans cesse dans la trémie, l’opération de la mouture se continue pendant, tout le temps qu'un vent quelconque règne sur l'horizon, et que la farine puisse itre recueillie et divisée dans un blutoir annexé an moulin sans que le proprietaire ait besoin de donner aucune attention à l'opération, jusqu'a ce que la totalité du grain qu'il a mis dans le réservoir supérieur soit complétement épuiséc: il serait à désirer qu'on phut, au besoin, substituer à l'action du vent la force des hommes et des animaux. Les démens du moulin demandé se trouvent en modeles, au conservatoire des $\Lambda$ rts et Métiers à Pars ; il ne s'agrit done que de les réunir avee: intelligence, et d'en former un ensemble qui soit at la portée du fermicr peu fortuné.

6o. Prix pour la fulrication des aiguille's it coudre. On propose un prix de 3000 fr. a celui qui ama formé en France. une fabrique d'aiguilles a coudre, comparables par la varieté 


\section{Prix proposés.}

de leur forme on grandeur, la perfection et le prix, à celles que le commerce préfère. Il est nécessaire de faire parvenir à la société d'encouragement, avant le ${ }^{-r}$. mai $x^{8} 33$, des échantillons avec l'indication des prix, et des certificats des autorités locales qui constatent que la fabrique est montée et organisée de manière à ne laisser aucun doute sur la permanence et le succès de ses travaux, et qu'elle a versé dans le commerce des produits pour une valeur annuelle de $1000 \mathrm{fr}$. Indépendamment des aiguilles fabriqquées avec du fil de fer cémenté, les concurrens devront adresser des aiguilles en acier fondu.

$7^{\circ}$. Prix pour l'application de la machine is vapeur aux presses d'imprimerie. La société propose un prix de $2000 \mathrm{fr}$. a celui qui mettra en action, par le moyen de la machine a vapeur, une ou plusieurs presses typographiques construites soit d'après le système ancien, soit d'après un autre système. Celte presse devra produire dans un temps donnéun plus grand nombre d'épreuves que les presses ordinaires; et le bénéfice net qui en résultera devra être plus considérable que celui ?qu'on obtient actuellement. Les concurrens adresseront, avint le xer. mai 1823 , des mémoires descriptifs accompagnés de dessins sur échelle, des presses qu'ils auront employées et justifieront par des certificats des autorités locales qu'elles ont été enactivité pendant 3 mois consécutifs, et qu'elles offrent les avantages que la société désire.

Nous donnerons incessamment la suite du détail de ce programme.

539. Rourn. - Académie royale des sciences, belleslettres et arts. - L'Academie propose, pour sujet d'un prix a décerner dans la séance publique de 1823 , la question suivante :

"Est-il prouvé par des observations exactes, qu’il existe "des fuivres qui se communiquent par infection, sans cepen- darit être contagieuses? en admettant l'existence de ces * fièvres, quelles sont les principales causes qui donnent " lieu à leur développement et à leur propagation? quels sont "les moyens propres à les prévenir, ou à en arrèter les "progrès?"

J.e prix sera une médaille d'or de la valcur de 300 francs. 
Chacun des auteurs mettra en téte de son ouvrage une devise qui sera répétée sur un billet cacheté, ou il fera connaitre son nom et sa demeure. Le billet ne sera ouvert que dans le cas où le mémoire aurait remporté le prix.

Les académiciens résidans sont seuls exclus du concours.

Les mémoires devront ètre adressés, francs de port, au secrétaire perpétul de l'académie pour la classe des sciences, avant le $x^{\mathrm{er}}$. juin 1823 . Ce terme est de rigueur.

54́. Tourouse. - La société de médecine propose, pour sujet du prix à décerner en 1823 , la question suivante : "Déterminer le mode d'action de l'iode sur l'homme dans "l'état de santé ou de maladie, et assigner les propriétés "médicales de ses diverses préparations, tant à l'intérieur "qu'à l'extérieur."

Elle propose en outre, pour sujet du prix à décerner en $\mathbf{1 8 2 4}$, cette autre question : "Déterminer, d'après une "bonne théorie, et surtout d'après les résultats précis de "l'expérience, les effets salutaires d'un ou de plusicurs agens "thérapeutiques, pris dans la classe des poisons végétiux " et minéraux. "

Chaque prix sera de la valeur de 300 francs; les mémoires devront ètre adressís à M. Ducasse fils, secrétiairegénéral.

541. Amsterdan. - Institut royal des sciences, lettres e't beaux-arts. - La $2^{e}$. classe a mis au concours, pour cettc année, la question suivante : Qu'est-ce qu'on peut indi" quer, surtout d'après des données historiques, relative" ment aux changemens que la surface du royaume actucl "des Pays-Bas a subis, sous le rapport des bois, tourbicres, dunẹs, rivières, lacs, et en général pour ce qui rcgarde lat " partic terrestre et aquatique? „

5.,2. U'тrecht (Pays-Bas). La Socicté des arts et de's sciences a publié l'extrait suivant du programme des prix proposés pour les années $1_{12} 3$ et $x_{2} 8_{4}$.

Questions de plyssique. On demande : $x^{\circ}$. Un tableau historique et critique des améliorations qqu'on a essayé de faire aux barometres de mer, peudant les quarante dernieres années, surtout pour les rendre propres à observer l'ćlévation et les moindres changemens du fluide dans le tube, nonob. 


\section{Prix proposés.}

stant le mouvement du vaisseau; $2^{\circ}$. Quelle est l'influence que les canons de fer, qui se trouvent a bord, et le fer qui se trouve dans la construction d'un vaisseau, exercent ou peuvent exercer sur la déclinaison et sur les sondes observées et constatées au moyen du compas maritime? Comment peuton anéantir cette influence, ou bien la déterminer, de manière à ce qu'on puisse, avec une exactitude suffisante, en dégager les observations qui ont été faites. On désire, en mène temps une critiqne des indications, que Flivders (appendix $n^{\circ}$. $\mathbf{x}$, de son Voyage aux terres australes) SABINe (Philosoph. Transact., part. x, 1820), Banlow (an Essay on masnetic Attractions, by Peter Barlow, 1820), Lecourt (a Description of the changeable magnetic Properties possessed by all iron bodies, by $\boldsymbol{P}$. Lecount, 1820 ), et autres ont données à cet effet, et qu'ils ont déduites de leurs observations 3. Quelle est linfluence que les canons de fer qui se trouvent a bord, et le fer qui se trouve dans la construction d'un vaisseau, exercent ou peuvent exercer sur la marche d'une montre de mer qui se trouveà bord? Quels sont les meilleurs moyens pour anéantir cette influence, ou bien, pour la déterminer de manière à ce qu'on puisse la considérer comme une quantité connue dans la computation de la marche d'une montre de mer? -- L'auteur de la réponse à cette question augumenterait beaucoup le mérite de son ouvrage, s'il pouvait l'enxichir des résultats d'expériences et d'observations satisfaisantes, faites par lui-même. $4^{\circ}$. Comme il est connu que la chaleur ôte la force aux aimans artificiels, et que d'un autre côté, il semble résulter des expériences de Scoresby et Erman (Voyez Edimb. pliil. journal, vol. Iv, et abhandlungen der kongli. aliademie der Wissenschaften im Berlin, 1814,1815 , 134), que non-seulement la chaleur augmente l'action de l'aimant de terre surle fer, mais qu'on peut même, par la chaleur, donner au fer une force magnétique permanente, tandis qu'il est reconnu que l'intensité de la force magnétique de lat terre auggmente vers les pôles, ou il règne le plus grand froid. On demande comment toutes ces contradictions apparentes peuvent se concilier, et.siles phénomènes galvano-magnétiques récemment découverts peuvent jeter quelqque lumière sur ces difficultés.

Questions de medecine. I'. L'huile de foie de morue 
olenm jecoris aselli) ayant ite applique dans plusirurs endroits des Pays-las avec succès contre le ruhitis, au commencement de cette maladie, et de mème lorspu'il existait déjà un amollissement considérable dans les os des enfins; et le témoignage de médecins espérimentés et dignes de foi ne laissant aucun doute que, dans bien des cas, ce remede n'ait été plus efficace que tous les remedes qui ont été appliqués jusqu icei, et n’itit procuré un rétablissement plus prompt. On demande: 1 . Ln développenent court et raisonné des qualités sencibles de l'huile de foie de morue non falsifiée, ct une analyse chimique et exacte de ses parties constituantes; 2? une démonstration thérapeutique pour prouver jusqu'à quel degré ct de quelle maniere ce remède contribue probablement à corroborer toute la constitution des enfans, et surtout à améliorer le système des os; 3 . une notice fidèle des observations et des expériences faites par l'autcur, ou qui lui auraient été communiquées par des gens de l'art dignés de foi, et qui serviraient à prouver clairement l'efficacité et les avantages de ce remède appliqué empiriquement à ladite ma ladie; $4^{\circ} . s^{\prime i l}$ y a des circonstances, et surtout si l'on a observé des cas où ce remède doive ètre considéré comme nuisible à l'économie animale; s'il a eu de mauvais effets, principalement en gènant ou en ralentissant l'opération des premiers organes de la digestion? 5'. quelle est la meilleure maniere, et quelles sont les doses convenables pour administrer ce reméde, qui dégoûte les malades?-- - II'. quelles mesures a-t-on prises dans les différens pays de l'Europe, pour surmonter l'opposition que rencontre la vaccine? (Quel a été le résultat de ces mesures, et quelles sont celles applicables aux PatysBas? Les mimoires seront envovés a $\mathbf{~ I I . ~ J . ~ F . ~ L . ~ S c h r o - ~}$ der, secrétiire de ha société, et profess. à l'université d'Itrecht, avant le premier octobre 1823 . Ils peuvent itre écrits en hollandais, francais, allemand, anglais ou latin. Les auteurs n'écriront pas leur mémoire de leur propre main; ils n'y mettront pas leurs noms, mais seulement une devise, qu'ils répéteront sur un billet cacheté, qui contiendra leur nom et leur adresse. Les némoires qui anront obtem un prix deviendront la propriéte de la société, clles les fera imprimer parmi ses ouvrages, et personne ne pourna les pu- 
Éloge. - Entreprises scientifiques.

blier en tout ou en partie d'une autre manière, sans l'aveu des directeurs de la société.

\section{ELOGE.}

543. Éloge de M. Hallé, prononcé le $\mathbf{1} 8$ novembre 1822 , devant la faculté de médecine de Paris, par M. le baron Desgenetres. In-8. d'une fenille et denie. Imp. de Didot le jeune, à Paris.

\section{TROISIÈME SECTION.}

\section{ANNONCES DIVERSES.}

\section{ENTREPRISES SCIENTIFIQUES.}

544. Leıpsick.-Société des sciences nuturelles et médicales.-Le 15 septembre 1822 plusieurs naturalistes et médecins se sont réunis, sur l'invitation de M. le docteur Oken. On a remarqué parmi eux M. le professeur Blumenbach, de Gattingue; M. Frosriep, de Weimar; MM. Carus et Reichenbah, de Dresde. Ces savans ont formé une société de naturalistes et de médecins allemands, dont les règlemens ont été arrêtés sur-le-champ, et dont le but principal est de créer, entre les savans de l'Allemagne, des relations personnelles. Tout auteur d'un livre sur l'histoire naturelle ou sur la médecine en devient membre de plein droit. Tous les ans, au i5 septembre, il y anra une assemblée générale des sociétaires, qui resteront réunis pendant plusieurs jours. Un administrateur et un secrétaire résidans sont charéés de la correspondance et des affaires de la société, d'une session à l'autre. Il est défendu de proposer aucun clangement aux règlemens avant la sixième assemblée générale. (Rev. Encycl., janv. 1823, ए. 176.$)$

5/15. Irvenuoou. - Sociel) of Travellers. - Sociéti de Voyageurs. - Il s'est établi i Liverpool une société composée de 
nocrsonnes qui ont visité les pays éloignés dans la vue de sinstruire, tant dans les sciences en général, que dans l'histoire naturelle, et l'on espère que cette société donnera au monde des renseignemens précieux, qui, autrement, eussent éte perdus faute de moyens de publication. (London Mugr., fév. $x 823$, p. 224.)

5.,6. Enmuotra.-Society of urts in Scotland.-Sociétedes arts en $\dot{k}$ cosse. - Ceux qui sont zélés pour l'encouragement des inventions mécaniques apprendront avec plaisir qu'une société des arts vient de s'établir en Écosse, sur le même pied que culle d'Angleterre. Le principal objet de cette société est de procurer des secours à ceux qui, faute d'occasions convenibles, ne peuvent compléter leurs découvertes. (London Mag., fév. 1823, p. 224.)

Cette société tenant maintenant ses séances, les auteurs des nouvelles inventions ou procédés sont priés de les transmettre, franes de port, aux secrétaires, John Kobinson, it Coats - crescent; T. G. Wright, Charlotte-Square, a Edinburgh. (Phil. Jour, no. 15, 1823, p. 207.)

\section{VENTE D'OBJETS SCIENTIFIQUES.}

5. 7 - Notrce des livres rares, des manuscrits précieus et des recueils de peintures faits dans linde, la Perse, la Chine, le Japon, la Turquie, l'Espagne, IItalie, etc., de suites de vignettes et gravures, de lettres autographes, provenant du cabinet de M. $N^{* * *}$, dont la vente se fera le 3 aviol, 18.3 , et jours suiv. rue des Bons-Enfans, n. 30. In-8. d. 3 feuilles $\frac{x}{2}$ A Paris, chez Silvestre.

\section{NECROLOGIE.}

5:4. Un coup d'apoplexie foudroyante a fappo dans la soirée du 17 décembre dernier, à Florence, le célébre che. valier Jean Jabbroni, el y a répandu un deuil universel. 


\section{Nécrologic.}

La patric a perdu en lui un de ses citoyens les plus mérisans, l'Italie entière un de ses enfans les plus illustres, les sciences et les lettres un des génies les plus marquans. Doué d'une rare sagacité, plein d'amour pour la gloire et pour les nobles travaux, né avec une aptitude merveilleuse a toutes les connaissances humaines, et avec une supériorité décidée pour les affaires publiques, Jean Fabbroni sut déeouvrir quelques trésors et recueillir quelques fleurs dans chaque partie du vaste champ du savoir.

Il traita toujours avec succès les choses les plus importantes, et remplit les postes les plus éminens. Sa vue pénétra les secrets de la nature, et sa plume féconde enrichit le monde littéraire d'excellentes productions. Il s'occupa spécialement d'agriculture, de découvertes physiques et d'économie politique. Tous ses efforts tendaient au bien général; it trouvait du plaisir à se rendre utile. Sa réputation fut celle d'un des meilleurs esprits d'Italie, son nom retentit outre mer et au delà des monts.

Il fut secrétaire de l'açadémie des géorgopliles, directeur du cabinet de physique et d'histoire naturelle de Florence, professeur honoraire des universités de Pise et de Wilna, correspondant de l'institut de France, l'un des quarante de la société italienne des sciences; député toscan du nouvean système des poids et mesures, l'un des membres composant la députation des finances, sous le gouvernement de la régence d'Étrurie, député au corps législatif en France, directeur des ponts-et-chaussées de l'empire français, pour les départemens au delà des Alpes, directeur de la monnaie de Florence, commissaire royal des forges et des mines, ct l'un des commissionnés pour le cadastre universel dans la Toseane, chevalier de la légion d'honneur, et décoré par S. A. I. le grand-duc, de l'ordre du mérite, sous le titre de Saint-Joseph. Dans toutes ses fonctions diverses, il se fit remarquer par son activité, son zèle, son intelligence et son intégrité.

(Ext. du Moniteur, du 20 janvier.) _ F.

549. Le docteur Си. Нuтton, mathématicien distingué, est mort a Londres le 27 janvier de cette année, à l'âre de 85 ans. Il était né à Newcastle, sur la Tyne, en ${ }^{7} 737$. 11 a rendu de grands services aux sciences pendant une longue. carrière de plus de fio ans; il fut pendant 10 ans professeur 
a l'ecole militaire de Warwick. Outre ses premiers ouvrages sur l'Arithmétique, la Te'mue des lisres et le Toisce, il publia en r $77^{2}$ un ouvrage sur les principes de la Construction des ponts; en 1777 , un Traite dimentuire dess sections coniques: en 17 8. des Tablesdes produits et des puissances des nombres; en $x 785$, des Tables de logarithmes qui ont en 5 cilitions jusqu'à $x 8_{11}$; en 1786 , des Tables d'intercits, et des Traités mathimatiques et philosophiques; un Abréné d'arpentuge; en I $79^{6}$, un Distionnaire matheinatique et phitosophique; plusicurs autres traités sur les Mathématique's, les Projectiles et la Physique, et un Abrégé des transactions philosophiques, avee les docteurs Shaw et Pearson. La tactique militaire, l'artilleric et le génie surtout, lui doivent de nombreux perfectionnemens et inventions qui ont été même adoptés par plusieurs des premicrs professeurs du continent.

550. Le docteur Alex. Marcet est mort à Londres, le is octobre dernier, à lâge de 52 ans; aucun médecin n'at été aussi universellement regretté; il a été pendant lorg-temps médecin' de l'hopital de Guy; il fut un de ceux qui contribuèrent le plus à former et à soutenir cette grande et utile association médicale, l'une des plus importantes tant de son pays que des pays étrangers. Ses nombreux ouvrages attestent ses travaux et ses talens. (Médical intelligencer, no. 38, janv. ז 823, p. 28 . )

55 r. Le doct. Jesner, célèbre propagateur de la vaccine, est mort le 26 janvier a Berkley dans la $74^{\circ}$. année de son âge. (Phil. mag. Janv. 1823. p. 70.)

Liste des Sournaux Francais qui ont paru depuis le 25 Janvier jusqu'au ro ${ }^{\text {er }}$ Mars.

Annales de chimic et de pliysique, I $\delta_{22}$. (7)

Annales des mines, tom. 7 , 3. livr. de 1822 . (8)

Journal de physique, septembre 1822. (9)

Gazette de santé, n. 4 , 5, 6, févr. 1822. (10)

Jour. comp., etc. (11)

Nouveau journal de méd., nov., déc. 1822. (12)

$\Lambda$ rclives éénérales de médecine, $n^{n}$. 1 , janv. 1823 . (13)

Nouvelle bibliothéque médic., $\mathrm{n}^{\circ}$. 1 , janv. 1823 . (15)

Revue médicale, janv. 1823 . (16) 
Annales de la médecine physiologique, déc. 1822. (1 7) Journal de physiologie expérimentale, janv. I 823.(18) Journal de pharmacie, etc., $n^{\circ}$. I , janvier I 823 . (I!)

Annales Européennes de phys. végét., nov., dée., $\left(9^{\mathrm{e}}\right.$ et roe. livraison.) (2I)

Bibliothéque physico-économique, janv. et fév. I 823.(22)

Annales de l'agriculture française, janv., fév. 1823 . (23)

Journal des voyages, janvier 1823 (26)

Nouvelles Ann. des voyages, octob., nov., déc., 1822 , et janv. $1823 .(27)$

Journal des savans, janvier 1 823. (29)

Revue encyclopédique, janvier, février i 823. (3o).

Tablettes universelles, décembre i 822 . (3i)

Galignani's littér. gaz, n'. 245 à $247 \cdot(34)$

Annales de l'industrie nationale et étrang., janv. 1 823 (36)

Annales françaises des arts, etc., novembre $1822 .(37)$

Bibliographie de la France, no. 5 à 8 , février. (39)

Journal général de la littér. de France, octob. 1822 . (40)

Journal génér. de la littér. étrangère, nov., déc. 1822. (4 I)

Mémoires du Muséum, 5e. année, 2e cahier. (43)

Bulletin des sciences de la société philom., déc.x 822. (44)

Journal général de médecine, janvier. (47)

Annales du cercle médical, décembre 1822 . (48)

Bulletin de la Soc. Nílic. d'Em., déc. 1822, janv. 1823. (49)

Bulletin de la Soc. d'Encourag., déc. 1822. (53)

Journal asiatique, février. (54)

Journal d'agric. du dép. du Tarn (Alby), no. I, janv. 1823 .

Le Musée d'Aquitaine (Bordeaux), $\mathbf{n}^{\circ} .3$.

Journal d'agric. de l'Ain (Bourg), déc. 1822 ,

Bulletin de la Soc. d'agricult. de l'Eure (Evreux), février.

Ii. des sciences médic. de l'Eure, février.

Journal d'agric. de l'Arriége, juil., oct,' 1822.

Gazette hebdom. de santé (Lyon), no. 1, 2, janv. 1823 .

Recueil agronom. (Montauban), tom. 4, no. $x$. (12e. rec.)

L'asclépiade (Montpellier), $\mathbf{n}^{\circ}$. r, janv. 1823.

Annales de mathém. (Nimes), déc. 1822 , janv. 1823 .

Extrait des trav. de la Soc. d'agric. de la Seine-Infericure (Rouen), 8e. cahier.

Bulletin d'industrie agricole (St. Étienne), (1 ${ }^{\mathrm{re}}, 2^{\mathrm{e}}$. livr: oct., déc. 1822 .

Journal des propriét. ruraux (Toulouse), junv, fiv. I 8 s. 3. 


\section{TABLE DES MATIERES.}

\section{PREMIERE SECTION.}

Annonce des Ousrages, Extraits des Journaux et Touselles Scientifiques.

Ourrages encyclopédiques. ..........................

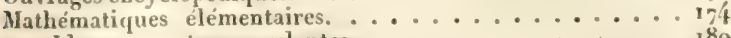

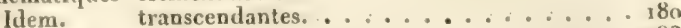

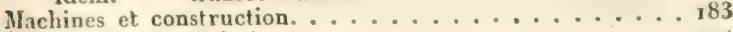

Topographie, géodésie..........................

Astronomie, cosmographie.................. 196

Physique $. \ldots \ldots \ldots . \ldots . \ldots . . \ldots 203$

Chimie .................... 209

Géologie. .............................. 314

llistoire naturelle générale. ......................

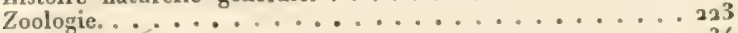

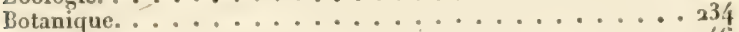

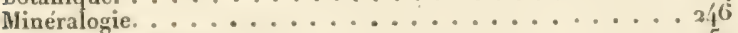

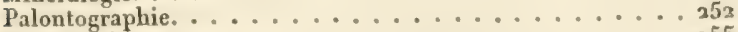

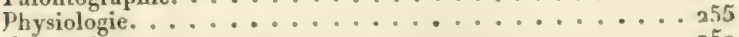

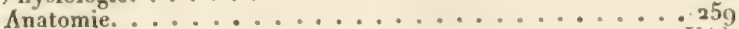

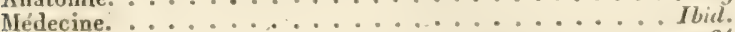

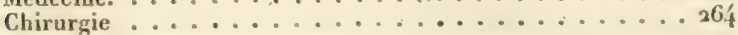

Pharmacie. .............................. 7 I

Art vétérinaire ......................... 174 $\Lambda$ griculture; économie publique, rurale et domestique. . . 275

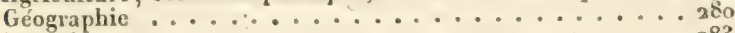

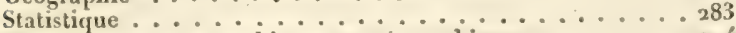

Plans, cartes topographiques et géographiques ..... 29 i

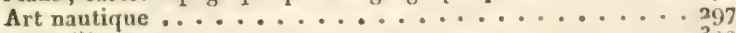

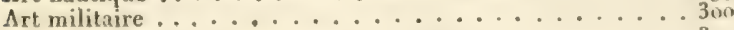

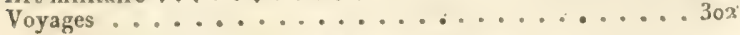

DEUXIEME SECTION.

Travaux des Socićtés savantes:

Séances, ..............................

Prix proposés. ....................... 3r

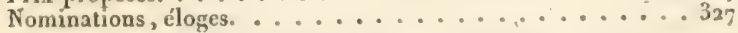

TROISIEME SECTION.

Annonces diverses.

Entreprises scientifiques............... 32m

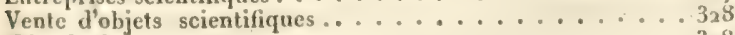

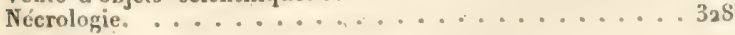

IMPRIMEHIE DE: IAIN, PLACE IHF L'ODEOX. 


\section{BULLETIN}

GÉNÉRAL ET UNIVERSEL

DES ANNONGES ET DES NOUVEILIES SCIENTIFIQUES.

\section{PREMIERE SECTION.}

ANNONCES DES OUVTAGES, EXTRAITS DES JOURNAUX ET NOUVELLES SCIENTIFIQUES.

OUVRAGES ENCYCLOPÉDIQUES.

553. Imperial encyclopada, etc. Encyclopédie Impériale, ou Nouveau Dictionnaire universel des sciences et des arts, embrassant dans un système raisonné un tableau exact des arts et des sciences, dans l'état actuel de leurs progrès et perfectionnemens, ainsi que tous les objets qui sont du ressort de l'esprit humain, par W. Moone Jounson, et T. ExLey, prof. de math., à Bristol, aidés de plusieurs hommes de lettres distingaés. Enrichi de 2 I 5 grav. explicatives; en 18 part., du prix de 8 sh. chacune, formant 4 vol. in- 4 . Londres; Robins et Underwod.

553. Encyclopdeda britannica, etc. Encyclopédie Britannique, ou Dictionnaire des arts, des sciences et des mélanges de littérature; 6" édit. revue, corrigée et augmentée, en $20 \mathrm{vol}$. in- 4 ., avec près de Goograv. Londres; Hurst, Robinson.

$55 \%$. Supplement to the Encyclopedia Britannica.

Supplément a l'Fucyclopédie Britannique, par 'J'OME I. 
Macney Nipieit, en 10 part.; prix, i 1.5 sh. chaque : Ie premier vol. upui a paru est accompagné de saviuntes dissertations, qui forment sa préface. Londres; Hurst, Robinson.

555. An reimghal techyologtcal nictionany, elc, Dictionnaire techologique miversel, ou explication des termes employés dans tous les arts et toutes les sciences; contenant les définitions données par les meilleurs auteurs : enrichi de $60 \mathrm{pl}$. et de gr. sur hois, par G. Crane, 2 vol. in-4. 1823. Prix, 51. 8 s. sterl. Londres; Baldwin.

556. Lecteres on select subjects in mechanicks, ete. Leçons sur des sujets choisis de mécanique, d'hydrostatique, dhydrauliçue, pneumatique, optique, géographic, astronomie, et gnomonirue; par J. Fragrsox; $3^{\text {e }}$ ćdit. avee des notes, et un volume de plus, contenant les découvertes les plus modernes dans les sciences et dans les arts, par D. Brewster. 2 vol. in-8. avec 27. pl. Prix, 25 sh. Londres; Whittaker.

Dans liappendice qui forme le $2^{e}$. vol., on trouve les descriptions de la plupart des machines et instrumens précieux nouvellement inventés, avec 2 chap. sur la double réfraction et la polarisation de la lunière.

55-. A nectoxany of universal history, etc. Dictionnaire dHistoire et de hiographie historique nuiverselle, eurichie de cartes explicatives et de portraits des principaux personnages; publié sous la direction de J. Mitchell : in-8. Prix. so sh. 6 d. cart. et $x_{2} \mathrm{sh}$. rel. en veau.

Ce vol. est le 1". d'une suite de 12 Dictionnaires destini's à former une Encyclopédie méthodique; les volumes suivans iraiteront :

20. Chimie, minéralogie;

3". Nathematigues et philosophie naturelle;

4. Géographie; 
Ђo. Les arts utiles et modernes;

6. Biograplie;

7․ Droit;

8. Médecine, chirurgie et pharmacie;

$9^{\circ}$. Théologie et morale;

xoo. Histoire naturelle et botanique;

I ${ }^{\circ}$. Métaphysique, logique et belles-lettres;

12 . Archéologie.

C'est cet ouvrage dont nous avons annoncé la publicalion, $\mathbf{n}^{\circ} .277$ du Bulletin de février.

F。

558. Tinsskrift for natur videnskaberne, c'est-à-dire Journal des sciences naturelles, pub. par MM. Orrsted, Hornemann et Reinhardt, professeurs à l'université de Copenhague. in-8. Copenhague , 1822. (Tous les deux mois un calicer de 8 à ro f. depuis le mois de fév, 1822 . Prix de la souscription, pap. ord. six Rbdlr.)

Nous avons sous les yeux les $\&_{4} \mathrm{I}^{\text {ers }}$. cahiers de ce journal. Voici le titre des articles qu'ils renferment :

$I^{\circ}$. Sur les progrès de la chimie depuis le commencement de ce siècle, par M. Oersted.

$2^{\circ}$. Sur un laboratoire public de chimie, établi par le gouvernement danois, pour l'instruction pratique des élèves, admis à y manipuler eux-mêmes.

$3^{\circ}$. Sur le jardin de botanique de l'université de Copenhague, par M. Hon nemann.

$4^{\circ}$. Traduction du mémoire de M. de Humboldt, sur la limite des neiges dans les montagnes, etc.

$5^{\circ}$. Quelques observations communiquées par M. HoFmar, sur l'apparition subite de certains végétaux.

$6^{\circ}$. Sur une substance semblable à la houille, trouvée en Seclande dans la tourbe; par MIII. Brensnorfr et ZeIse.

$7^{\circ}$. $15^{\circ}$. et $17^{\circ}$. Sur les progrès de la botanique, depuis le commencement de ce siècle, par MM. Hornemann et Scrouw.

8. Sur les ossemens fossiles de mammifères, trouvés en Angleterre, en Allemagne, ete; par M. Reinirardt.

9". Sur quelques anciens volcans de la France, par M. Brinsnorr. 
ro. Sur la purification de l'en-de-vic de frrain, an moyer du muriate de chaux, par M. Zersf.

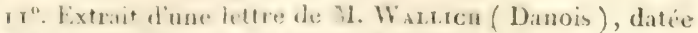
de Katmandu, dans le Nepit, le 1 s juillet 1821 .

I'20. Instructions sur la meilieure maniere de faire des observalions métiorologirpues, par MI. Scuovw.

130. Détermination du degré de chaleur, auquel l'cau-devic entre en ćbullition a proportion de sa force, et sur l'emploi du thermometre comme alcoolomète, d'après un écrit de M. Groeninc, fabricant.

1 f̊. Touvelle expérience galvano-magnétique, par $\mathbf{M}$. OtRSTLD.

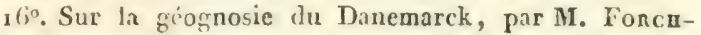
IIABMFR.

1\$. Sur les progries que la géologie a fails depuis l'annéc I 800 , par M. BREDSDoRf.

19. Extrat d'un mémoire publié dans la Collection de la sociélé royale des sciences de Copenhagnue, sur l'acide xanllogene (hydro-carbosulfurique), par M. ZEISE.

$20^{\circ}$. Analyses de quelques minéramx des iles féroë, par M. pu Mŕsıl, demeurant à Wunsdorf.

$21^{\circ}$. Note sur unc masse de fer météorique, tombée en Islande au moyen âge.

C. DI.

550. Axines Flumenses de sciencias, autes elitteraunia, publicadas por huma sociedade philotcehnica. Anmales des sciences, des arts et de la lituérature, pour Rio de Janciro, par une socićté philotechnique, a vol. Prix, \&fr. Rio de Janciso. Isz2.

I.es autcurs se sont proposé pour but de signaler a leurs compatriotes les progre's que les sciences et les arts font chatque jour en Europe, et dindiguer aux Europiens les découvertes des sarans du Brésil. Le premier volume, que nous annoncons ici, peut etre en quelque sorte consideri comme lavant-scine de l'ouvrage. Dans un discours préliminaice, les aulearajeflent un comp d'ocil géneral sur leseonnaicances humaines, et cherchent a prouver aux Braciliens, combien il leur sexut ulile de s'appliguer a l'étude des sciences qu'ils ont eté trop long-temps forcés de nigliger. ()n trouve, dans un serend article, des détails sur la banque de hio de laneiro, 
les causes de sadécadence, et les moyens de rendre à cet établissement sa splendeur primitive. Lacfe de réunion de Montevideo et de toute la province Cisplatine, a lempire Brasilien, forme le $3 e$ article. Le $4^{e}$. et dernier se compusellun mémoire de M. Ie conseiller A. R. Veloso de Oliveira sur la division du Brésil en évêchés, et sur la statistigue de cette vaste contrée. Des tableaux joints a ce mémoire font connaitre la population des différentes provinces brasiliennes, et la portent à 2,488,7/3 hommes libres, $1,107,389$ esclaves, et 800 , 00o Indiens encore sauvages. Le nombre de ces derniers est probatblement exagéré; mais nous pensons que M. Veloso ne fiit point monter assez haut celuides blancs et des hommes de couleur. Quoi qu'il en soit, on ne peut que faire des voux pour la continuation de l'ouvrage que nous amnoncons. Il deviendrait extrèmement utile si les Brasiliens qui cultivent !a science unissaient leurs efforts à ceux des collaboratcurs; si M. J. B. de Andrada e Silva fournissait des articles sur la minéralogie et la botanique, M. le colonel F. Marlins sur la minéralogip et la géologie, M. Ferreira dit Camara Bithancurt e Sá, sur la minéralogie et l'art du mineui, Mi. ic colonel J. Gomez sur les mathématiques, le P. Leandro du Sacramento sur la botanique et l'entomologie, MM. Melo Franco, père et fils, sur la médecine, H. J. 'Texcira sur l'agriculturc, etc. $\Lambda u g$. de S.-IIL.

MATHEMATIQUES ELEMENTAIIES

560. AN ELEMENTATy tneAtise on the mathematical principles of arithmetic. Traité élémentaire d'arithmétique, trad. du fr. deS. P. Lncronx. in-S. Prix, 5 sh. cart. Londres; Whittaker.

56i. Tine arithuetician's goide. Le guide de l'arithméticien, ou livre d'exercice complet, à l'usage des écoles publiques et des précepteurs, par W. TAx Lon, in-1 2. prix, 3 sh. rel. Loudres; Longman. 560. A new sxstem of arithmetic. Nouveau syslime d'arithmétique, sur un plan tout-à-fait original $\mathrm{ct}$ tendant à abréger le travail des instituteurs, ainsi quà faciliter les progrès des éleves, par J. Walker. Prix, $2 \frac{1}{2}$ sh. Loudon; J. Souter. 
563. Buтterwontr's useful arithmetical tables. Tables d'arithmétique, par Butrenwortu, if. in-4. Edimburgh; Oliver et Boyd.

56. A complete course of Anitumetic theoretical and practical, etc., ou Cours complet d'arithmétique théorique et pratique, en 3 part., àl'usage du public et des écoles particulières, par W. H. IVmite, inst. cn chef de l'école commerciale et mathématique, fondée à Bedfort, par sir IV. Iarpur. In-12 ; prix, 4 sh. Londres; Longman.

565. Arithmetical Questions on a new plan. Questions d'arithmétique sur un nouveau plan, par W. Buttlen. $3^{e}$. édit. in-1 2 ; prix, 6 s. Londres; Harris.

566. Arithmetrcal tables. Tables d'arithmétique, par W. Buttlen. ıoe édit. in-ı 8 ; prix, $8 \mathrm{~s}$. Londres; Harris.

567. La clef des réductions des prix de toutes espèces de marchandises de l'univers; ouvrage, etc., accompagné des règles nécessaires qui ont servi pour créer cet ouvrage, des rapports des poids, des mesures, des monnaies, des changes étranger's réduits au pair, et de quantités de notes utiles pour faciliter les opérations; par B. Tscingento in-8. de $5 \mathrm{f} . \frac{1}{2} \Lambda$ Paris, chez l'auteur, rue Bourgl'Abbé, n. 36.

568. Stenirotse's reckonen and tables of monies, etc. Calculateur et tables des monnaies, poids, mesusures et cours de change de toates les nations; ou Le prompt calculatcur Britannique et C'ambiste universel à l'usage des banquiers, négocians, fermiers, commerçans et hommes diaffaires en gínéral; rédiqué d'après les sources les plus authentiques, par W. STEnouse, comptable a Edimbourg, auteur do la table d'Intérèts; nouv. édit., consi- 
dérablement augmentée et améliorće. In-32. Prix, 3 sh. rel. Edimburgh; Oliver et Boyd.

569. The univensal cambist and commercial instructor, etc.Le Cambiste universel et instituteur commercial ; ou traité complet et exact des changes, monnaies, poids et mesures de toutes les nations commerçantes et de leurs colonies, parP. KeLLy, Doc. en droit. 2e. édit., considérablement augmentée. In- $\{$. Prix, 4 1. 4 sh., Londres; Lawington et comp.

C'est l'ouvrage original dont nous avons annoncé la tra duction sous le $n^{\circ}$. xi du Bulletin de janvier.

5 jo. $\Lambda$ concise system of mensuratron, etc. Système alorégé des mesures, adapté à l'usage des écoles; contenant l'algèbre avec les fluxions, la géométric, pratique, la trigonométrie, la mesure des surfaces et des corps solides, les levées des plans, le jaugeage, et avec un appendice étendu offrant les démonstrations des rìgles contenues dans l'ouvrage, et des tables y relatives : par A. Ingrum, mathématicien à Leith, auteur des ćlémens d'Euclide, ete. Édit. aug. de l'arithmétique de Melrose. In-12. Prix 4: sh. rel. Edimburgh; Oliver et Boyd.

j7:. An introdection to algebra, etc. Introduction a l'algèbre dans laquelle les règles fondamentales sont clairement démontrées, et le tout rendu facile: et familier, ete, avec une appendice, contenam la solution de cent questions d'algebre; par li. Smsnr, maitre de mathématiques. in- 12 , prix, $33 !$. sh. rel. Edimburgh; Oliver et Boyd.

572. Dr. Woov's eleurats of algebri. Elémens dalgebre du docteur Woon. Prix, 7 sh. (11 a anssi du même auteur, les traités suivans. Principes de mécanique, 5 sh. Elémens d'oplique, 6 sh. Londres; Mawman.

573. Algebriatcal problems. Problemes dialgibre, 
qui se résolvent par les équations du $\mathbf{r}^{\mathrm{cr}}$. et du $\mathrm{z}^{\mathrm{s}}$. degré, pour servir d'introduction à l'analyse supérieure, par M. Bland, 3e édit. in-8. Prix, rosh. 6 d. cart. Londres; Whittaker.

57 4. Élémens de géométrie, avec des notes: par A.

M. Legenne, membre de l'institut et de la légiond'honneur, etc., $\mathbf{r}^{\text {e }}$. édit. in-8. de 27 f. $\frac{3}{8}$. plus les planches. Prix, $6 \mathrm{fr}$. A Paris, chez F. Didot.

5 75. A treatise on geonetrix. Traité de géométrie contenant les 6 prem. liv. des élémens d'Euclide, avec les élémens de la géométrie des solides, par D. Creswell. Un gr. vol. in-8. Prix, $x$ \&́sh. cart. Londres; Whittaker.

576. A Supplement to the elements of Euclid. Supplément aux élémens d'Euclide, avec plus de 200 grav. sur bois, par D. Cnesweld, in-8. prix, to sh. 6 d. cart. Londres; Whittaker.

577. Grometrical pnoblems. Problemes de géométrie, déduits des VI xers. liv. d'Euclide avec leurs solutions, et un appendice contenant les élémens de la Trigonométrie plane par Mrefs Bland, in-8. Prix, I f sh. cart. Londres; Whittaker.

578. A Treatise on plane and spherical trigonometry. Traité de trigonométric plane et splıérique par R. Woophorse, 3e. édit. corr. et aug. In-8. Prix : 9 sh. 6 d. cart. Londres; Whittaker.

579. Algebraic geouetny. Géométrie Algébrique, par

Dinnistes Lardoner, a vol.in-8; le I ${ }^{\text {er }}$. vol. comprend la géométrie des courbes planes.Londres; Whittaker.

580. A grometrical system of Conic sections, etc., Système géométrique des sections coniques à l'usage des étudians en mathématiques de l'école royale de Liverpool. In-8, prix, 6 sh. 6 d. Londres; Whittaker.

58r. Treatise on practical gacging. Traité de Jaugeage pratique, par $\Lambda$. Kusutт. In-19. avee 6 in 
exemples pratiques, et 180 grav. en bois. Prix : 8 sh. Londres, 1822; Longman.

582. Nicholson's nudiments of practical penspecTIVE. Plincipes de perspective pratique, par Nrcuolson, in-8., enrichi de 38 pl. gr. par Lowry. Prix : 14 sh. cart. Londres; Taylor.

583. A familiar treatise on perspective. Traité ćlémentaire de perspective, destiné aux dames, et it ceux qui ne sont pas versés dans les principes d'optique et de géométrie, par W. Dantel. 3e édit, in-12, avec $16 \mathrm{pl}$. demi-rel. Prix, 5 sh. Londres; Harwy et Daston.

584. Matuematic.1l Essays. Essais mathématiques, par feu W. S penck, esq. publiés par J. W. HersCHEL, escr. avec un court mémoire de l'auteur. In- . Prix, I 1. 16 sh. br. Edimburgh ; Oliver et Boyd. 585. Dans le Newcastle magaz. n. 14 . Fevr. 1823 , p. 109 , on propose de résoudre le problème dont voici l'énoncé : * trouver les dimensions d'une montagne de glace de forme w cubique, qui s'élève verticalement de 100 pieds au-dessus v de la surface de la mer, en partant de l'hypothèse que les - pesanteurs spécifiques respectives de l'eau de mer et de la "glace sont, 1,0623 et $0,9^{21}$ 4." Si l'on représente par $x$ le côté du cube cherché, on aura de suite, en faisant abstraction du poids de l'air que déplace la montagne $\mathbf{1}, 06_{2} 3(x-100)$ $=0,921 / 4 x$, d'où $x=753,9^{3}$ pieds.

3.

\section{MATHÉMATIQUES THANSCENDANTES.}

586. The Doctrine and aprlication of fluxions, etc. Ia doctrine et les applications des fluxions, contenant un gramrl nombre de problemes nonveanx et intéressans dans les diverses branches des mathémati'ues, par T. Simpon, nouv. édit., z vol. in-8. Iris, 2 I sh. Cart. Londres; Whittaker.

587 . Fifments of the theory of Mechanics. F́lémeirs de la théorie de la mécanique, par G. VEN trinol. prof. dir mathémat. al'université de Bologne, trad. 
par D. Coneswers, et augmenté d'un recueil choisi de problemes de mécanique. Londres; Whittaker. 588. An Elemevtant treatise, on the geometrical and algebraical investigation, etc. Traité ćlómentaires sur la Recherche géométrique et algébrique des Maxima et Minima formant un cours de lecons données conformément au testament de lady Saller; angmenté d'un choix de propositions, dérivées des clímens d'Euclide par D. Creswell, $2^{\mathrm{e}}$. édit. in-8. Prix, r 2 sh. Cart. Londres; Whittaker.

\section{IACHINES ET CONSTRUCTIONS.}

589 . Ax Histonicil and Descriptive account of the steam engine. Histoire et description de la machine: à vapeur ; comprenant un tableau général des divers modes d'employer la force élastique de la vapeur comme premier moteur en mécanicque avec un appendice sur les patentes et les actes parlementaires relatifs à ce sujet. Par Ch. Fr. Pintizgton, de l'institution de Londres. In-8., enrichi de 8 pl. ot d'autres fig. Prix, 18 sh. Cart. Londres; Taylor.

590.4 practical treatrse on propelling ressels by steam, etc., ou traité pratique sur la manjére de faire marcher les bateaux par lit vapeur, cte., par Brcuane, avec iz pl. $x$ vol. in-8. prix, 20 sh. Londres.

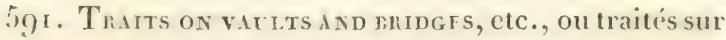
le's voutes et les ponts en briques, en pierres, en chaines, etc., contenant desobservations sur les différentes formes de voutes, sur le projat de démolix ct de rebatir le pont de Londres, et sur les principes des ares, expliqués par des tables étendues sur la construction des ponts, contenant anssi les principes des ponts susprendus relativement a liz cherche appliguée au pont te Menai, et un eximen théo- 
rétique de la cherche. In-8 royal, avec 20 pl. et io fig. grav. sur bois, prix, 20 sh. Cart. Londres; Longman.

592. Reponts, estimates, and treatises on canals, rivers, harbours, etc. Rapports, estimations et traités sur les canaux, les rivières, les ports, les digues, les ponts, les phares, les machines, les moulins, ete. par J. TAY Lon, 3 vol. in-4, avec 7 亿 pl. grav. par Lowry. Prix, 4 l. I/ sh. 6 d. Cart.Londres; Taylor.

593. Traité sur l'art de faire de bons mortiers, et notions pratiques pour en bien diriger l'emploi; précédé d'expériences faites sur les chaux de Provence et de Russie, par Ruccourt de Charleville; ancien élève de l'école polytechnique, ingénieur des ponts et chaussées de France; lientenant-colonel des voies de communication en Russie, et professeur de construction à l'institut de ce corps, etc., I vol. in- $\{$. xxxir, et 362 p. 4 pl. ct \& tabl. St.Pétersbourg, de l'imprimerie des voies de communication, i 822.

Cet ouvrage est divisé en trois parties. La première contient les principes de M. Vicat, sur lart de faire les mortiers; elle est empruntée toute entière aux Recherches expérimentales, publiées par cet ingénieur, en 1818 , sur les chaux, les bétons et les mortiers; ce sout les mèmes faits, les mèmes principes exposés synthétiquement en retranchant le développement des preuves, qui n'était pas nécessaire dans un Traité pratique. "Je ne puis trop répéter, dit MI. Raucourt, que tous les principes qui servent de bise it la premiere partie apparticnnent à M. Vicat ; cu les propageant, cn montrant 'qu'ils peuvent être partout d'un fructueux et facile emploi, je me fais un devoir d'en publier le mérite, de lui en laisser tout l'honncur.,

La seconde partie contient les expéricnces de l'autenr. Les premieres ont étí faites at Toulon, en 18 Ig et 1820 ; Mr. Rancourt assure qu'il a réussi, en suivant les procédés cinseigné dans l'ouvrage de M. Vicat, à transformer les chaux grasses de 
Provence en chaux maigres hydratiques; il at fait employer, dans le port de Toulon, le sable et les chaux hydrauliques factices aux fondations dans l'cau de plusicurs constructions importantes. Envoyé en Russie, M. Raucourt a entrepris à Saint-Pétersbourg, avec MII. Lamé et Clapeyron, anciens élèves de l'école polytechnique et inçénieurs des voics de communication, un long travail sur les chaux et les morticrs. Non-seulement il a obtenu les mèmes résultats que M. Vicat, mais il a ajouté à la science un grand nombre de faits et d'observations sur la cuisson des chaux et des pouzzolanes, sur l'emploi et la composition des chaux et des mortiers. La découverte la plus remarquable est peut-être celle d'une espèce de chaux éminemment liydraulique, comparable au roman cement d'Angleterre, qui s'est trouvée parmi les espèces de chaux qui avaient été envoyées à M. Raucourt pour ses expériences. En voyant que celte découverte a été attribuéc à MM. Lamé et Clapevron seulement, dans les Annales des Mines, tom. 7, pag. 483 , 3e. livraison de 1822 , nous pensons que l'ouvrage de $\mathrm{M}$. Raucourt n'était pas encore connu en France, à cette époque.

La troisième partie contient les procédés perfectionnés, dont l'auteur a fait usage pour fabriquer et employer les chaux et les mortiers, d'après les nouveaux principes. Jes personnes qui veulent s'aider des lumières de lis science dans l'art des constructions y trouveront tous les détails dont la connaissance est nécessaire pour appliquer avec succès les nouvelle's méthodes. Mr. Raucourt décrit deux procédés pour fabriquer la chaux hydraulique factice : l'un, qu'il a pratiqué en grand et duquel il peut répondre; l'autre, plus simple et plus économique, qu'il propose d'après des essais faits en petit; il se sert, dans ces deux procédés, de chaux grasse préalablement éteinte. On voit qu'il ne connaissait pas la manière de fibriquer la chaux hydraulique en se servant de craic réduite en poudre par trituration. MI. Raucourt a surtout approfondi la théorie du meilleur emploi des sables et des pouzzolanes. Fn un mot, il parait avoir fait un travail ntile sur toutes les parties de l'art qu'il a traitc.

59). Nicnolson's carpenters new rrunf. Nouveau guide: du charpentier, par Nicnor.son, in-f. 8 f pl.. fi. édit. Prix, r 1. I sh. relié. Londres; Tilylor. 
595 . Nichorson's campenter and jolner assistant, etc. Le Guide du charpentier et du menuisier, par $\mathrm{Nr}_{\mathbf{H}}$ crolson. $4^{\circ}$ édit. In-4. $79 \mathrm{pl}$. Prix, y l. a sh. relić. Londres; Taylor.

596 . Pain's practical house carpenter. Le charpentier domestique pratique par $P_{A I N}$ in-4. x $48 \mathrm{pl}$. Prix, 18 sh. relié. Londres; Taylor.

597. Fredgold's elementary principles of carpentry.

Principes élémentaires de charpente par Fredgolo, in-f. avec $22 \mathrm{pl}$. Prix, I 1.4 sh. Cart. Londres; Taylor.

598. Nicholson's treatise on the construction of stair cases. Traité de la construction des escaliers, par Nicholson, in-4. 39 pl., prix , is sh. relié. Londres; Taylor.

599. The standard measurer, etc. Le Modèle des mesures contenant de nouvelles tables à l'usage des constructeurs, des marchands de bois, des tuiliers; ainsi que des calculs tout faits pour la valeur des bàtimens : avec des explications pour l'usage des tables, des observations sur la mesure du bois de charpente et une méthode pour mesurer le travail de l'ouvrier, par T. Scotrant, géomètre arpenteur. In-8. prix, 7 sh. 6 d. (9 liv.) Edinburgh; Oliver et Boyd.

6oo. Penfectionnement de la machine hessoise à élever l'eau. Par le docteur A. Léor Crelle de Berlin, premier architecte du Roi de Prusse, avec une fig. (Teclin. reposit., n". 14, févr. 1823, p. 99.)

Ia machine hessoise est celle qu'on appelle communément machine is force centrifuge. L'auteur donne une forme particulière an tube d'ascension; mais on ne voit pas que cette forme soit un perfectionnement. G.-C.

for. Description de la machine statique hydraulique inventée par feu M. Westgarth, par J. Smeaton. (Techn. reposit., n". 3 , janv. 1823 , p. 26.) 
(iv2. Represextatiox de Smatox, ingénieur, concernant la machine hydraulirgue inventée par M. WI. Westgarth de Colecleugh dans le Northumberland, tendant à élever l'eau par la force de l'eau. (T'echn. reposit., $\mathbf{n}^{\circ}$. 3 , janv. 1823, p. 24 .)

(io3. Sur ia micmixe nymavurge de Westgarth, telle qui clle a été décrite par le célèbre feu M. Smeaton. (Techln. repusit, n. I3, janv. 1833, p. 24. Transac. of the Soc. for the encourag. of arts, man. and com. vol. 5.)

(io.). Patente pour l'invention d'un piston hydrostatique déployant une force suffisante pour résister à la pression de certains fluides et glisser facilement dans un cylindre imparfait, délivrée à Ed. B. S s mer. (Lond., Journ. of Arts andSc.n'.26, fév. I 833,p. .8.) (ios. Patsite pour une nouvelle méthode de faire agir les machines sans avoir recours à la vapeur, ì l'eau, au vent, à lair niau feu. Délir rée à G. Lixtox. (Iond.,Journ.of arts andSe., n". 26 , fér. I $8.23, \mathrm{p} .-2$. .) Le mécanisme dont il s'agit consiste en une roue garnie de 32 bras on leviers placés à égale distance sur sa circonferrence où ils sont fixe's par une charnière. Ces bras sont formés chacun de plusieurs pièces moliles; d'un còté, ils s'appliginent sur la circonférence, et s'étendent de l'autre de toute leur lonkrizur, et agissent alors comme levier. F.n passant, ils frappent une détente qui laisse rouler des boulets jusqu'aux extremités des bras déployés, oi ils se legent dans des emboilemens destinés a cet effet, pour augnenterl'action destras sur la rone. M. Iinton, comme on voit, prétend avoir résolu le problême du mouvement perpétuel.

fiof. Macume a nrssinfr. L ne nouvelle méthode pour multiplier les traits desoljets naturels at été inventice par M. de Cuscmus prof. des aspirans de lat marine a Toulon. lar le moyen d'un instrument appelé hylaographe, les dessins sont tracissur un verre, et ceux-ci sont transportés sur te papier par une copice d'encre; ce procede peut étre répúté comme cela a lien dans la lithographie.

Ro. 
6o- . Ponts de suspension en fer aux Indes. (Asiutic Journal, $n .85, p .6$. . )

Le lieutenant Schalch a fait approuver par le gouvernement anglais un projet de pont suspendu de $\mathbf{2} 20$ pieds anglais de longueur, pour être construit dans l'Inde, sur le Tolly's Millah à Kully-Ghant. On trouve à l'article indiqué un résumé des principes généraux de constructionsapplicables aux ponts suspendus, et la description sommaire de celui qui doit étre construit dans l'Inde. Ce pont est conçu dans le systène de ceux qui sont déjà établis en Angleterre.

G.-C.

6o8. Ponts suspendes.-Les ponts suspendus (Suspension bridges ) sont très-communs en Amérique, où ils sont quelquefois construits avec des cordes. Un pont remarquable de cette espèce, appeléle Penipe, traverse le Chambo au Pérou; il est suspendu sur un ravin, Sooo pieds au-dessus du niveau de l'Océan; ce pont a environ 120 pieds de long, et 7 ou 8 de large. - Il y a un pont remarquable dans la province de Shansi, en Chine, au confluent de deux grandes rivieres; il est établi sur cent trente barques cnchainées entre elles, mais disposées de manière à ouvrir un passage aux vaisseaux après qu'ils ont payé un droit. Cette espècede ponts est trèscommune en Chine. (Supl. Gentl. Mag., nº 15 , déc. 1822 , p. 640.)

609. Patente accondée à II. R. Palmer, ingénieur civil pour des perfeetiomemens dans la construetion des chemins en fer, (Rail-TFays, tram-roads) et des voitures qui les parcourent. (Lond. J. of Arts and $S c$. fév. 1823 , vol. $5, \mathrm{p} .57$. )

$\Lambda u$ lieu de deux ornières en fer sur le sol, l'inventeur établit sur des bornes une piece de bois recouverte d'unc bande de fer. Une roue en fonte, ayant la forme d'une poulie, roule sur cette bande. A l'axe de cette roue unique, on suspend, de chaque còté, une caisse renfermant les marchandises. Ces caisses ćtant au-dessous du niveau de la bande de fer qui supporte la roue, le systeme se tronve avoir assez de stabiliti pour pouvoir marcher sans exiger une érgalité parfaite dans le chargenent des deux caisses. Un cheval traine le tout en tirant obliqguement comme dans le hallage d'un bateau. Iies- 
sai de re moyen de transport parat avoir bien réussi. On trouvera dans la notice annorcée le détiil du moyen que pro. pose linventeur, pour opérer la rencontre sur ces espéces de chemins, et pour traverser les autres routes. G. C.

Gio. Patente pour l'investion d'unc nouvelle méthode de jeter ou de construire les fondations de piles, murs, plafonds, ares, colonnes, piliers, moulures et autres ornemens des édifices, délivrée a James Frost, architecte. (Lond., Journ. of Slits and Sciences, $\mathrm{n}^{\circ} \cdot 26$, févr. 1823 , p. 66.)

(ii i. Description n'ux uun creux élevé dans le jardin du duc n'Anreau. Ce mur, de 9 po. d'épaisseur, est bâti avec de bounes briques d'égale dimension, placées de champ, les jonctions faites avec soin et bouchées avec le meilleur mortier. Les briques sont placées avec leurs faces et leurs extrémités alternativement tournées en dehors, de manière que celles qui ont leurs extrémités exposées servent d'attache; dans charpue assise les jonetions des briques sont placées sur le mlieu des briques inférieures. Ainsi il se forme un espace creux, de quatre pouces de largeur, au milieu du mur, et cet espace n'est interrompu que par les briques qui se croisent pour l'assujettir. Le haut du mur est couvert d'une assise de briques, sur lesquelles sélève un faite de pierre de Portland, ayant deux pouces de saillie, et qui est fortific de vingt pieds en vingt pieds par des piles de quatorze pouces de jetée, comblruites de la mime manipre. Le mur que nous venons de decrire est assez fort pour tous les usicges des jardins; il iparyne un bon tiers de la depense ordinaire, et il a l'avantage de devenir sec apres les longues pluies, beaucoup plus rapidement qu'un mur massif de la mème epaisseur nu même moins epais. (New-1Ionthl), Mug. no. 25 , janv. 1823 , supl. pag. 22.)

Gis. Patente accordéc à W. IL xnas, fondeur dansle 1) rvonshire pour certains perfectionnemeus daus la constructiondes toitures en fer. (The Lond. Journ. of Arts and $S_{c .,}$ janv. 1823, p. 14.)

i.c perfectionnement imagrine par le patenté consiste a fiser lies ardoises sur les peitures en fer ; pour celat, on me- 
nage une rainure au-dessus du clievronen fer, et des deux côtés on place l'extrémité de l'ardoise sur cette rainure. Pour maintenir l'ardoise, on pose sur le chevron une piéce de fer qui le recouvre dans toute son étenducet qu'on y fixe avec des boulons; cette pièce recourre le bord desardoises posérs dians l'encastrement roénagé sur le chevron, et forme ainsi le dessus de la rainure, où entre le bord de l'ardoise. On met du mastic dans cette rainure pour sceller l'ardoise. G.-C.

6i3. Patente pour me méthode perfectionnéed'empêcher le dépérissement prématuré du bois de construction, des substances métalliques et des toiles, méthode qui les garantit respectivement de la pouriture, des vers, des insectes, de la rouille, en leur assurant une plus longue durée; délivrée à John Oxfond. (Lond., Joum. of Arts and Sc., nº.26, fúv. I 823, P. $\left.6 g^{\circ}\right)$

6r4. Sur les avantages du ciment romain de

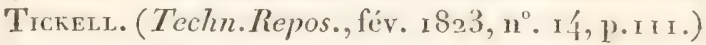

On peut, avec ce ciment, réunir 17 briques pesant chilcune sept livres et demie (poids anglais), et fixer ces briques réunies contre un mur. Le poids des 17 briques ne peut séparer la promicre du mur où elle est appliepuéc avec ce ciment. L'adhérence arrive à ce point dans l'espace de deux minutes.

G. C.

6i5. Rechenches str Les montiens, par L. J. Vicit,

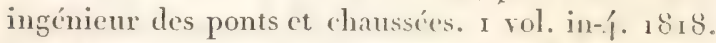
6.6. Mémone relatif à la fabrication des Pouzzolanes ardificielles, par le mème. (Ann. de chim., 1. I 5. p. 36j.) Extrait, par M. Connes, éleveing. des mines. (2sp.)

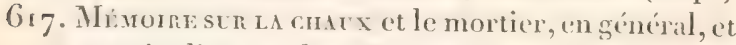
en particulicr sur la diférence entre les mortiers de chaux de: copquilles, ot de pierre calcaire, avec la théorie des mortiers. (Ouvrage couronné en Isis, par la soc. Hol. Jes se.) par M. le docteur Jonn, Extrait : ( 1 / p.)

Cirs. (Oeservations sur le mémoire de M. John. par M. Vicat. Extrait : (2 p.)

'Jonf. I. 
6rg. Axaryse de difierentes pieres a chanx, par M. Bentimel. (22 p.)

Gen. Onservatioxs sur lesmorticis, par le meme. (6jp.) Ann. des mines, $1822.3 c$. livr.

Cette série d'estraits et de mémoires, sur la chanx et le's morticrs, présente la réunion des résultats qui ont été obtenus par une foule d'expéciences, particulierement relatives anx chaux hydrauliques et aux bétons. MI. Vicat et M. Johu saccordent sur des fitits généraux d'une grande imprortance pour lart des constructions, mais ils different en queleques points qui sont également importans, ct M. Berthie's, après avoir comparé ces deux opinions, en éclairant sa dincussion par vingt-cinc analyses exactes de differentes espieres de picrres calcaires, conclut que la théorie des mortiers est encore très-imparfaite et qu'il est à desirer qu'on s'ittache it recueillir des observations nouvelles, propres à íclairer cette théorie. Voyez le $\mathbf{n}^{\circ} .593$.

BD.

62x. Cinsxt xivfrest. Pour obtenir ce ciment ajontez à une once de mastic, de l'esprit-de-vin tris-rectific, en quantilé suffisante pour le dissoulre. Trempez une once de colle de poisson dans l'eau jusqu'i ce qu'elle soit tout-a-fait amollic; dissolve 2 -la dans du rhum pur on dans de l'eau-de-vie pure, jusrua ce quielle forme une forte grelée, ajontez alors envijon un quart d'once de gomme ammoniarue bien pulvérisie. Exposez les deux mélanges cnsemble dans un vase de terre a une douce chaleur; lorsque les substances seront bien mélingees, versez dans me fiole qui duit rester bien bouchée. Pour faire usage du eiment, on met la fole dams de l'ean chande, et l'on ficit chanffier les objets en porcelaine on en verre que lon veut réunir aver le ciment. Il sera convenable

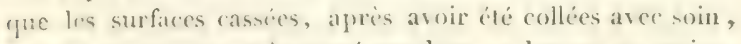
gesteut en contact tres-servé pendant 12 heures atu moins, jungrua ee que le ciment soit tris-assis. Apres cela la place cassie erra aussi solide que le reste du vase et a peine prer-

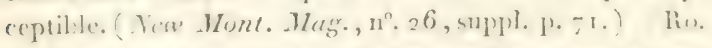

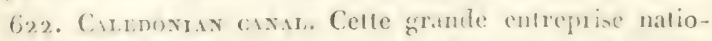

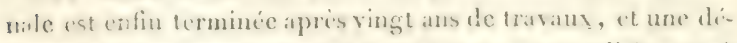

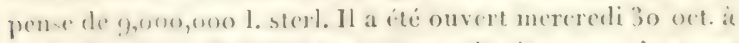
in h, ta matin. Il ctablit une commmication outre les mets 


\section{Topographie, Géodésie.}

oriculales et occilcntales; de petits vaisseaux peuvent passer le catral de Moray Writh, au fort William, arixiles, à Glasgow, a Liverpool et en Irlande (Investig. n". I x, janv. I8.3, p. I8.)

\section{TOPOGRAPHIE, GÉODESIE.}

623. On a dernièrement inventé, en $\Lambda$ utriche, des fusées qui montent is une hauteur si prodigicuse, qu'on dit les aroir rues à une distance de plus de fo lieues. Si ce fait est exact, ces fusées pourront être employées comme signaux, et servir avantagensement dans les opérations géodésiques. (Journ. gén. de la litt. étr., déc. 1822 .)

62.. On a appris par des lettres du fort Marlborough, que la commission nommée par le gouvernement de Madras pour se rendre at Sumatru, afin d'y faire les observations nécessaires pour déterminer la longueur du pendule a l'oquatcur, est arrivée à Bencoolen, à bord du Alorniner Stur. I.es instrumens sont en aussi bon état qu'en partant de Madras. Un vaisseau chargé de transporter la commicsion à l'equateur, et de lui fournir des provisions, une garde, des charpentiers, des maçons, et les matérianx nécessaires à la construction de la colonne sur laquelle doit étre établi le pendule, avait été équipé pour cet oljjet par le gouvernement de Bencoolen. (Asiutic Journ., $\mathrm{n}^{\mathrm{n}} .85$, janv. 18 23, p. 62.)

\section{ASTRONOMIE, COSMOGRAPHIE.}

625. ATrfatise on astronom theoretical and practical. Traité d'astronomie théorique et pratique, par R. Wooprouse, nour. édit. en 2 part. in-s'. prix, 30 sh. cart. Londres.

Gial. Astroxony explainen. L'astronomie expliquée d'après les principes de Newton, par J. Ferguson, arec des notes et chapitres supplémentaires, par D). Binewsten, 2 vol. in-8. avee pl. prix, 24 sh. carl. Londres; Whittalier.

(i)-. A NEW theORY OF THE HeIVENIY MOTIONS. Nouvelle théorie des mouvemens célestes, montrant que les principes admis par fiewton n'existent pas; in-8. prix, 3 sh. Londres. 
628. A complete systive or asthoxnmy. Systertio complet d'astronomice par S. Vixce, 3 vol. in-1. prix 5 l. xy sh. caut. Londres; Mawman.

629. The elentrass of astronomy. Émens d'astronomie, par J. Brinkley, in-8. prix, is sh. Londres.

63o. Érucuénes des distances des í planites, Vémus, Mars, Jupiter, et Saturue, ete., calleulés par les soins de MI. Scuvaracier, a vol. in-f. (.)penhague, 1822 .

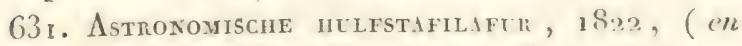
allemand.) Tables de secours pour l'astronomic cu 1822, in-8. prix, 7 sh. Londres.

632. Afnuaine prísexté au ror, par le burcau des longitudes, pour l'an $18_{2} 3$, 1 vol. in-r2. Prix, I fr. Paris, I 822 ; Bachelier.

Lespages pour chaque mois, dans cet annuaire cxtrait de la connaissance des temps publice par le bureau des longritudes, offrent les circonstances les plus remarquables du cours du soleil, de la lune et des planetes, et tout ce que l'on trouw dans les autres calendriers. Viennent ensuite des articles dont nous allons enoncer les titres. - Da temps, de sa mesure et du calendrier, extrait de l'Exposition du systime du monde. - Sur les plus grameles marées de chriue année, avec une table pour ${ }^{8} \delta_{2} 3$, jar M. Borvarin. - Calcul de Pheure de la pleine mer. - Table domant le temps dont lia haute mer doit avancer on relarder tous les jours, en raison de l'heure du passage de la lune au méridien. - Table des heures de la pleine mer dans les principaux ports des cótes de l'Turope, les jours de la nouvelle et de la pleinelune.Nouvelles mesures, extrait de l'Exposition du surtime du monde; avec plusieurs tables de réduction.-Viteur an pair des monnaies, et tableau de comparaison des momnates etrangeres avec les monnaies francaises, toutes supposes exactes de poids of de tilre, dapres les lois de fabrication. - Table de la mortalite a de lat population en France. - Consommation de la ville de Paris pendant 1821 . Monvement de 
l.t population du royaume de France pendant l'année 1820. P'opulation de chaque département suivant les recensemens faits en 1820 et les limites du traité de $181 \%$ - - Sur la littitude et la longitude terrestre, extrait de l'Erposition due systeme du monde. - Table des longitudes et des latitudes des principales villes du globe et de leurs plus courtes distances a Paris.-Hanteur des principales montagnes du globe et der fuelques lieux habités, au-dessus du niveau de l'Océan. - Hauteur de quel fues édilices. - Table des principaux élémens du systeme solaire. - Pesanteurs spécifiques des fluides élastirques, des liquides et des solides. - Termes de fusion de différens corps et d'ébullition de divers liquides. - Table des dilatiations linéaires qu'éprouvent différentes substances, depuis le terme de la congélation del'eau, jusqu'à celui de leur abullition, d'apres MM. de Laptace et Iavoismer. - De la quantité d'eau qui tombe annuellement dans quelques villes. - Table de la force du vent. - Époques des principales découvertes géographiques, observations astronomiques, et inventions d'instrumens d'astronomie et de marine. Table pour calculer les hauteurs des montagnes d'aprés les observations barométriques, par Oltmanss.-Températures moyennes d'un certain nombre des points du globe, extrait du dernier vol. de la Socicité d'-Arcueil. - Quelques observations météorologiques faites à Paris. - De linfluence de la lune sur les saisons, traduit d'un mémoire de M. OLners. - Rénultats des expuriences faites par ordre du bureau des longitudes pour la détermination de la vitesse du son. $B$.

633. $\Lambda_{\mathrm{N}}$ ej.ementary tratise on astronomy. Traité élementire d'astronomic, ou introduction facile i la connaissance du ciel, destiné à ceux qui ne soni pats versés clans les mathématiques; avec 4 cartes des constellations et une grav. contenant des fig. rxplicatives de l'ouviage, $2 \%$ édit. corr. et aug. pat le pév. $\Lambda$. Mrune. Edimburgh; Oliver et Boyd.

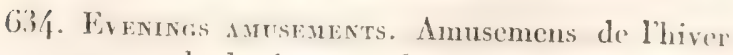
on spertacle des beautés des cieux, dans lepuel sont décrits plusicurs phemonimes rematquables

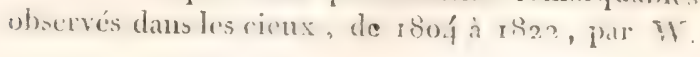


Frexn, 6 vol. in-12, prix, 30 sh. cart. Iondres: Mawman.

Citte suite de volumes est compliete, elle comprend ls skande période du cycle de Méton, avec larpulle saceortent prespue exactement les observations de la lune, jendant la mème suited'annies; les remarques que contient cet ourrage sur les mowvenens et les phénomenes cúlestes sont tris-utiles à ccux qui cultivent lastronomie. (slun. of philos.)

63j. Popllar parts of astroxomy. Parties d'astronomie à la portéc de tout le monde, compilation tiréc de Brixklex, Vrice, et autres, à l'usage de ceux yui ue sont point rersés dans les mathématiegues, in-S. aree des pl.Prix, $2 \frac{3}{4}$ sh. br. par J. l'ez јонк. Loudres; Longman.

636. Formele pratigen pour connaitre le quantiome de la semaine, cenx de l'année et du mois etant domés. - Cetie formule comvient parliculierement an 9 9. siecle, on plus exactement aux annes $x$ soo a $x \&(y)$; wais elle sajplique sauf une legiere modification, aux anmes ifoo a 1 figg et r 700 a 1799 . Elle consiste on denx opérations. () a ajoute ensemble, r'. le nombre indiquant le quantieme de l'amé 2". le fuart dix ce nombre (en ne ligeant la fraction) ; 30. ie nombere de jours coules depuis et compris le I't. janvier juscques a compris le jour donné. (On divise par - la somme qui en résulte, et le reste de la division indique le numéro du jone be las semaine, en commencant par le dimandie, c'est-at-dire dimandhe tant 1 , lumli 2 , ele., et samedi zero.

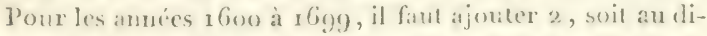
vidcule, soit au reste, of seulement I pour les amies r gon a 1-59. Si l'améc ent bissextile, il famt, au contraire, retranclier I dans tons les cas.

fizemple.-A quel jour de la semaine repontia le of and 1823? Les denx pormiers des nombes a ijouter somt: 182 ?

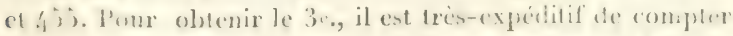
par nimestes, en olservant que le $x^{\mathrm{cr}}$. trimestede de lan-

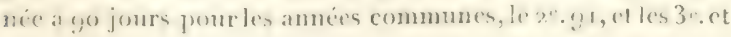
4. clacun $2^{2}$. Ainsi, du rer. janv. an 25 aoult inclus, il

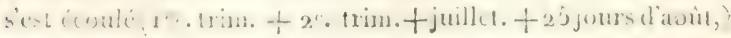


cirs-a-dire, $9^{0}+9 x+31+25$, ou 237 jours; 237 est done le troisieme numbre cherche. La somme à diviser par 7 étant $1823+455+237$ ou 2515 , on obticnt 2 prour reste de la division, qui indique que le 25 aoùl 1823 sera un lundi.

637. L'infatigable Brssel a commencé un ouvrage important que tous les analeurs d'astronomie doivent souhaiter de voir continuer arec succès. C'est me description générale des cieux divisés en zones; la première partic de l'ouvrage est. sous presse. (New Month. Mağ Scep. hist. p. 65.)

638. Ascemsion mooite vraie de 36 étoiles, pour claque jour de l'année 1823 , a leur passage au méridien de Greenwich, calculée daprés les tables de $x \delta_{20}$, de Bessel, par le

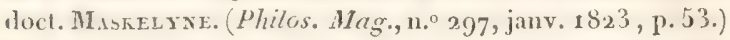

639. Le Prof. R ENwick a déluit la longitude de New-YonK. de l'éclipse desoleil du 27 août I 82 I, ctl'a trouvée de $7 / 4$ "s' I I " ou bien 4 h. $56^{\prime}{ }_{2} 3$ ", 4. O. de Greenwich. Les olsservations ont été faites à la coupole du collége de Colombia, par une latitude de 40 42' 45". (Edinburgh. phil. journ. 11". 15, janv. 1823, p. 182.) : B.

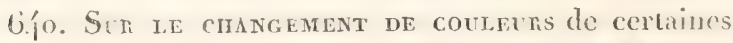
étoiles fixes par le doct. J. Fonster. (The Monlhl) Magaz., n. 25. p. 486).

6łr. Le docteur Ilocen de Minden a public dans le journal de celte ville un mémoire détaillé de son syslème d'après lequel il suppose que le noyau du soleil est d'or fondu. ( A K cu Month. Magaz., no. 26, fév. 1823, p. 68.)

6/2. MI. Arigo, vice-président de l'acalémie des sciences, a commmirqu, le ro mars, à ses collègues, une découverte aussi honorable quimpoctante pour l'astronomic moderne.

1. Charies hi man, astronome allemand altaché a l'ob. servatoire de Pirramallat, dans lit nouvelle Galle du sud, a refrous lat romete dite a courte période ou de $120 /$ joris, ol il a pul'observer pendant presige tout te mois de juin 1822 . 
Suivant Encke, directeur de l'observatoire de Seeberes, it Gotha, cette comète avait été observée quatre fois lans les années $1786,179^{5}, 1805$ et 1819 . Voyez à ce sujet le curieux mémoire qu'il a fait insérer dans l'Annuaire astronomique pour 1823 (astronomiches jahrbuch), et publié cn 1820 par M. Bode. Mr. Encke y donne les positions de la comete depuis le 22 février $1 \$ 22$ jusqu'au 27 juillet suivant, afin de fitciliter les moyens de la retrouver. On l'at vainement clierchée en Europe, où on désespérait de lia revoir. Le périhélie est au 24 mai.

Il risulte des observations de M. Rumker, que le passage réd de la comète au périhélie n'a différé que de trois heures du passage calculé par MI. Encke, accord presque inespéré en pareil cas.

Si nous remarquons que cette nouvelle acquisition scientifique nous vient d'une contrée naguère sauvage et labitée d'abord par le rebut de nos sociétés, quel triomphe pour la civilisation moderne!

B. $\mathbf{x}$.

\section{P II Y S Q U E.}

6ł3. Tire wonks of J. Playfirfo Otures de T. PlayFArr, ci-devant pofesseur d'histoire naturelle it l'umirersité d'Édimbourg. if rol. in-S. Prix: 21. ra sh. G d. cart. Londres; Strust. Robinson.

Il n'y a aucun ouvrage sur l'histuire des Seiences mathematiques et physiques dans notre langue, dit l'Edinlumshe levieu en annoncant cette publication, qui puisse è comparé pour l’éloquence philosophique a celui de M. Playfair.

(ifí. Coxversitions on natural philosophy. Conversations sur la pliysigue, dams lespuclle's le's démens de celle seienee sont expligués dine maniene

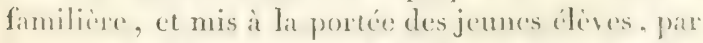
lantrue des Converstlions sur la rhimie, in-1". prix : tosh. 6 d. rel. cmichi de as grals. 30. éd. Londres; Longman.

6.5. A systru of mechanical philosophy ofe. Syslime de fhysiqu anćcanique, par J. Liobsasos, 
prof. de phil. naturelle, ou de plıysique, à l'université d'Éd. avec des notes, par D. Brewster, membre de la Soc. Iioy. de Londres, et secrét. de la Soc. Roy. d'Éd. 4 vol. in-S. avec r vol. de pl. prix: 4 1. Londres; 1822 ; Murray.

6 6 6. Le monde phrsigue, et le monde moral; ou lettres à $M$ me. de ${ }^{* * *}$, Ouvrage spécialement destinéaux persomnes qui veulent, sans le secours de la géométrie, étudier le monde physique, et le monde moral, et les rapports qui existent entre les lois qui gouvernent ces deux mondes; par $A$. Librs, $2^{e}$. édit. aug. du triple, 2 vol. in-8 de 4 I f. $\frac{t}{4}$, et $u$ pl. prix, io fr. Paris; 1822 ; chez l'auteur, rue Notre-Dame-Nazareth, n. 32 .

La première partie de cet ouvrage peut être considérée comme étant un cours élémentaire de physique, écrit avec clarté, sous forme de lettres, à la portée des personnes qui n’ont pas étudié la géométrie; la lecture en sera encore profitable aux personnes plus instruites, qui voudraient connaitre superficiellement les principes généraux, les principaux phénomènes, et les découvertes les plus récentes. Les différens sujets des lettres sur le monde physique sont: le système du monde, la doctrine de Newton sur la lumière et les couleurs, la polarisation de la lumière, les phénomènes de la chaleur, le thermométre métallique de M. Breguet, les propriétés de l'air, les expériences de MM. Chlandi et Oersted sur l'acoustique, les propriétés de l'ean, l'électricité, le magnétisme, les phénomènes électro-magnétiques, la météorologie, et l'explication nouvellement adınise du phénomène de la rosée.

Nous n'entrerons daus aucun détail sur les lettres de la deuxième partic, dont le sujet est ćtranger aux matières qui sont traitces dans le Piulletin. En divisant son ouvrage en deux partics, l'auteur a évité les rapprochemens plus ingénicux rqüinstructifs auxquels il aurait été conduit, s’il cùt mélé l'étude du monde moral à l'étude du monde phiysique. Une seule lettre à la fin de l'ouvrage est consacrée at ćtablir les rapports qui exislent entre les lois qui re gissent lex deux mondes. 
6.j. New rninciples of nalural plilusopliy. Nouveaux principes de philosophie naturelle ou de physigne, prix, ro sh. 6 d. cart. avec gras. Lond.; Richard Philips.

6.j8. Fisay oz mest, etc., Essai sur lit nature de lia chaleur, de la lumière, de l'électricité, par C. Bonpass, écuyer, in-8. Irix, 7 sh. Londres; Underwood.

6.jo. Prictical electnicity axd galvanism. B́lectricité el galvanisme praticjues; par J. Cutbentsos, in 8. prix : 2 sh. Londres.

6jo. As rssiy on magietic atritictexs, etc, Essili sur les attractions magnétiques, cl'e's lois du magnétisme terrestre, et de l'électro-minguélisme, contenant une suite d'expériences curieuses et intéressinntes sur ce dernier sirjet, et une méthode expérimentale et facile, prar corriger l'attraction locale: des vaisseaux sur lit boussole, dans toutes les parties clu monde; par P. Barlow, $2^{\prime \prime}$. él. cor. et aug. avec pl. in-8, 12 sh. cart. Londres; Mivman.

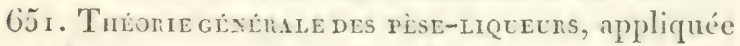
à la construction et à l'emploi de toutes sortes ditréometres, entierement comparables; avee des tables aréométriques très-étendues, domant les pesantemespécifiques, correspondant aux divers degrés de's pise-lirpueurs en usare, le titre de's catuxde-vie, des acides sulfuriques, ete., par T. M. N. Bratit ancien áleve de l'école polytednicue, ete., in-s. de 3 f. avec x pl. lithog. Prix, 3 fr. Paris; Barrois l'auné.

Lauteur propose denx nonvelles graduations de l'aréométre. Liune consiste à diviser en cent parties égales le volume de linstrument qui plonge dians l'eau distillée. Le muméro I étant place a la division infírieure, et les numéros allant en croissint a mesure qu'on séleve, le nombre roo correspond a latiliarement de leau et les nomberes plus

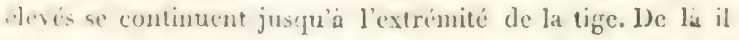




\section{Plysique.}

rénlte, I’ que si dans un liquide l'ariométre marque, par exemple, 59 degrés, la densité de ce ligunile est $\frac{m 0}{5 \%}$. Et , $2^{\circ}$. que le volume de $x$ hil. de ce même liquide est 5 y centimètres cubes.

Lautre geme de graduation consiste à diviser la tige de l'instrument en parties inégales, de telle sorte que la densité de l'eau étant représentée par roo, les divisions inférieures correspondent successivement aux densités I0 I, 102 , etc; et les smpérieures anx densités 99, g. , ite.

Ainsi le degré marqué par l'aréomètre est précisément la densité du liquide dans lequel il p̉longe.

L'un des moyens indiqués par l'auteur pour obtenir ces deux modes de gratuation a autant d'exactitude que le moyen gu'on emploie pour graduer l'aréometre de Beaumé; il est cependant un peu moins simple que celui-ci parce qu'il cxige trois pesées de plus. Mais il y a un autre moyen que l'auteur ne publie pas, et qui est fondé sur un instrument de son invention; il dispenserait des pesées, il rendrait les divisions plus faciles à faire, et mérne il poursait s'appliquer avec avantage à beaucoup d'autres modes de division.

L'anteur compare, par des formules trís-simples, les différentes espèces d'aréomètre. Il donne des tibles de comparaison. Il donne aussi une table des densités correspondantes aux différens degrés de laréomètre de Beaumé. Cette table est tres-bonne; si elle differe un pen des autres tables qui ont été données sur le mème sujet, soit avant la publication de son ouvrage, soit postérieurement, cela ne tient qu'a l'impossibilité absolue ou l'on est de faire des tables parfaitement exactes. Il suffit d'un centieme d'erreur sur une densité déterminée directement, ou de $\frac{\text { th }}{1-}$. d'erreur sur la mesure du ravon de la tige pour amener les différences qu'on observe entre les diverses tables. Or ces erreurs sont de celles dont on ne peut guere ripondre quelque soin qu'on apporte à faire les expériences.

Povillet.

6ion. Sir la manize de s'assurer de la densité des liquides, avec une description de l'aréometre ou de l'hydromitre, arec a pl. (Tech. Repusit., 11. I3, janv. 1823, p. r.)

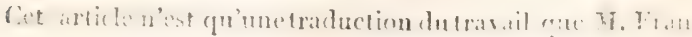


cocur a fait imprimer dans le dictionnaire lectunologengue', at: mot aréornètre. B.

653. Penfectonyentint ne microscont de M. IR. IIAL, pour le's oljets opaques. (Techn. lieposit., n. 13 , janv. 1823, p. 37.$)$

65\%. Penfectroynement des meroscopes solaires. (Techn. Réposit., n. 13, janv. I823, p. 36.)

655. Frúrances sur Le nemou et la propagation des ondes, par G. Brdone. (Hím. de l'acud. de Tiurin, t. 25. 1 820. )

Ce mémoire intéressant a deux objets. Le premier con. siste dans des expériences relatives aux remous qui s'érablissent dans un courant d'eau par l'effet d'un barrage; le second dans d'autres expériences sur la propagation des ondes.

Les premières expériences ont été faites sur des canaux rectangulaires, dans lesquels la section avait environ 2 pou. ces de profondeur sur 12 pouces de largeur, ct où l'eau prenait une vitesse moyenne de 4 à 6 pieds par seconde. La hanteur des barrages transversaux au-dessus du fond était de '́ à 9 pouces.

Les remous ont toujours offert une surfice partont convexe, qui, dans la presciue totalité de sa longueur, s'écartait fort peu de la ligne horizontale tangentea son sommet. Cette surfice s'ibaissait brusquement à quelque distance en amont du barrage, et à l'extrémité d'amont du remou, extrémité dont la position était parfaitement déterminée, la surface du remous et la surface naturelle du comrant n'étant point tangentes l'une a l'autre, mais séparées par une inflexion brusque.

Te point le plus élevi de la surface du remou était à pen de distance en amont du barrige. Ia longueur du remou thait tonjours beaucoup moindre que la distance a laguelle la ligne lorizontale, passant par ce point, allait rencontrer la surface naturelle du courant. La différence était d'environ un tiers. An dela de l'extrenite du remou, le niveau primitif du courant n'avait reer aucune alferation.

Lauteur essate d'tablir une formule an moyen de laquelle res phénomines puissentétre soumis an calcul. 
Tes expériences sur la propagation des ondes offrent dis résultats curicux, et qui paxissent féneralcment s'accorder i peu près avec ceux de la théoric dennéc par M. Poisson. L'auteur ayant plongé dans l'eau des ptismes à base carréce et triangulaire a observé que lés ondes formées en retirant bruscuement ces prismes offraient aussi des figures carrées ou rectangulaires, mais dont les angles et les cités correspondaient aux côtés et aux anğles des bases des prismes. L'n prisme à base elliptique donne des ondes qui s'élargissent rapidement dans le sens du petit axe, et deviennent très-promptement circulaires.

R.

656. Onswinations su les phénomènes de l'aiguille aimantée, par MI. Rovx. (B́ull. de la Soc. de Géog., n. $3, \mathrm{p} \cdot 99$. )

Ces observations intéressantes sont purement historiques.F. 657. Ser la fanteitron des aimans artificiels. (Wew Monthly Magaz., janv. I823.)

Le professcur Steinnauser a reconnu que si, d'aprés le procédé de Canton, on forme un rectangle avec deux barreaux dacier et denx barreaux de fer, il vaut mieux pour les aimanter opérer par la double touche progressivement, que par un mourement en avant et en arriere. Quand on veut aiminter des barscaux en fer à cheval, il convient de rapproclıer deux de ces barreaux par leurs pôles d'espéres diffürentes, de maniere à ce que le circuit magnétique en devienne complet, et que l'on puisse toucher circulairement avec les barreaux destinés à communiquer la faculté magnétique. Pour que les deux barreaux aimantés ne perdent presque rien de leur force, on applique, avant de les séparer, chaque armure sur son aimant. De cette manière, deux aimans pertvent ètre touchés dans le mème temps que le procédé ordinaire exige pour un seul.

C'est en se conformant à ces régles, et en placant les barreaux qui doivent étre aimantés sur d'autres quil'ont déja été, que le professeur Steinhauiser est parvenu à communiquer a des aimans une force extraordinaire et dans to moindre temps possible.

Ro.

658. M. Annensov de Perth vient d'inventer un nouvel мтмомитв. pour mesurer l'évaporation spontanée qui a 
lieu à la surfice de l'eau dans un temps domme; cet instrument est tout-ia-fait diffirent de celui décrit dans le $2^{\mathrm{e}}$. vol.

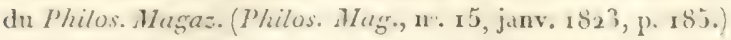
11 en sera rendu compte.

65y. Commotions électrieves produites par un

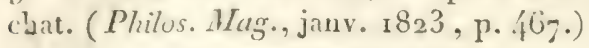

On sait que, par un temps froid, le chat, lorsqu'il est frotti, a la propriété de donner des étincelles électriques; mais il parait, d'après la note que nous avons sous les yeux, que cet. animal donne des commotions comme celles de la bouteille de Leyde, en passant une main sur son dos, et placant l'autre sone sa gorge; en pressant doucement l'os de l'épaule ou celui de la màchoire, le's bras et les épaules font une sorte de chaine électrique, et les commotions eprouvées sont trissensibles.

F.

\section{CHIMIE.}

66o. $\Lambda$ system of cremertry, etc. Systime de chimice par Th. Thomson, M. D., nouv. édit. entierement refaite, if gre vol. in-8". Prix : 3 1. sterl. Édimbourg; Blackwood.

G(ix. A Miscal of cnenistry, etc. Manuel de chimie: contenant les principaux faits de la scienee arrangés dans l'ordre des lecons données à linstiuntion royale; par WV. T. Braxde, secrét. de la Soc. Roy., profes. de chimic àl'institution royale, 2 . édit., enrichic de pl., de grav., de diagrammes, cte. 3 vol. in-8". Prix: 2 1. 5 sh. Londres; John Murray.

(i6). The ruminents of chenestry, etr. Élémens de: chimic, expliqués par des expérienees et des planrhes gravées représentant les appareils chimirgues; par Parkes, autrur du Citéchisme chimidur, dus lissais chimiques, etc., 3cédit. corrigéc avore soin.

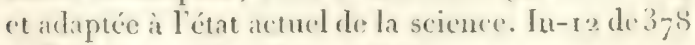
p. Prix : 7 sh. Londres; 1822 .

(ici). Av rotrove of chevistiy. Almege de chimic, 
dans lequel les principes de la science sont expliqués dans cent expériences instructives ct amusantes, qui peurent se faire sans le secours d'aucun apparcil excepté (quelques verres, nne houisille d'huile et un creuset, et sans le moindre danger; par J. Topnм, 2" élit. In-12., prix : 3 sh. 6 d. cart., Londres; Yhittaker.

66.1. Nunn.sy's chrmichi screxces. Élémens de chinie appliquéc aux arts, aux manufactures et aux phénoménes naturels; par J. BIunray, $2^{e}$. édit. avec des additions. In-8. Prix : 8 sh. Londres; Underwood. 665. The rSE or tue Blowripe in chemical analysis, etc. Usage du chalumeau dans l'analyse chimique et dans l'examen des minéraux, par J. J. Berizerus, de l'Acad. des Sc. de Stochholm, trad. du français de M. Fressel ; par J. G. Cimldnex, suivi d'une esquuisse du systeme de minéralogie de M. lierzelins et avec des notes et additions du traducteur. In-8". de $38_{2}$ p. Prix: 12 sh., Londres; 1822 ; Baldwin.

666. Une's nictioniry of chemestry. Dictionnaire de chimie, par Ure. In-S"., prix, a sh. Londresison. 66-. Rechenches mistorięres el obscrvations médiciles sur les caux thermales et minérales de Néris, (Allier); par M. P. Bonot Desservens, D. ML., inspect. de l'établissement thermal de Néris, ete. Paris, 1822, in-8.

Voici les résultats de l'analyse chimiqque que M. Boirot Desservicrs a fail de ces earx ; cent partics de résidu ont produit :

Carbonate de soude. . . . . . . . ${ }_{2} 3$

Sulfate de soude. .......... 17

Muriate de soude.......... 12

Carbonate de chaux. ......... r r

Silice. .............. 7

Eau. ................ 8

Mlatièrc animale et pâte. ....... $\frac{32}{100}$ 
Gige. Osstnratrons sur le sublimé corrosif, par J. D.w, M. D. (Journ. of Sc. litt. und the arts, 110.23, p. 384.)

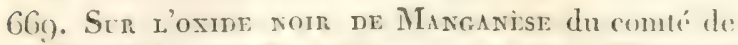
Warwick, par Sts. PAlikes, écuyer. (Truns. of

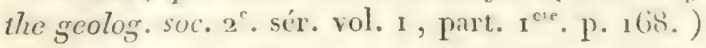
6jo. Sen tr. fer ex ster x'Acier; par Th. Gille. (Repert. of arts, manufact., etc. $n^{0} .{ }^{78}$, janr., I $823, \mathrm{p} \cdot 97$.)

fijr. Recherches sur da soude, le plomb) blame et le rert de Brunswick, par un correspondant. (Teclun.Magaz., n. г3, janv. 1833, p. 68.)

6j\%. Ser la derinire analise des substances végétales et animales, par $\Lambda$. URe, M. D. (Journ. of Sc. litt. and the arts, $\mathrm{n}^{\circ} .28, \mathrm{p} .388$. )

673. Axiluse de frett du baoban (Adansoniea) par

M. Varpredin (Mém. du Mhuscium d'hist. naturelle, t. VIII.)

Ce fruit contient de l'amidon et une matière sucrée dont la fermentation produit une liqueur alcoholique, qui devient promptement acide.

V. ADD.

(i-j. Rapponts de Mil. Lateert et Carentor, sur un

Mémoire intitulé, Nourel Essai sur la bile humaine, par .I. Chevalier. (Bull. de la Soc. Méd. d'Emulat., déc. 1822, p. $507^{\circ}$ )

Ces recherches confirment la découverte du Pycromel, dont la présence assignec aux animaux sculs, pirt M. The'nard, parait avoir été rencontréc par M. Chevalier, dans l'homme et dans les cadavres humains.

P. F,

G-jo. Axthys nes concrítoxs trouvées dans un taureau

affecté de plithisie tubereuleuse, par J. I. IAsssaene. (Rev. méd., janv. 1823.)

On sait que celte malidie affecte un graml nombre d'animanx, et que elle detruit lis plupart des hites a cornes dans 


\section{Géologie.}

piusicurs contrées. Il devenait intéressant d'étudier la nature des concrétions qu'on trouve dans divers organes. M. Lassaigne en a fait l'analyse, et il a constaté qu'elles étaient formées: $x^{\circ}$. de phosphate de chaux en grande quantité; $2^{\circ}$. de carbonate de chaux; 30 . de matière animale.

6-6. Maxilne de consumer la fumée dans les fonderies de fer ; par un correspondant, dans une leure à l'édit. (Techn. Rieposit., n'. x 3 , janv. 1823, p. 亿 I .) 677. Spremfication de la patente accordée à J. P. Londor, chimiste praticien, pour un moyen de détruire ou décomposer les vapeurs nuisibles qui s'exhalent par la distillation des matières animales ou végétales, datée du 25 janv. 1820 avec une grav. (Repert.of arts, $\mathrm{n}^{0} .249$, janv. I823, p. г32.)

\section{GÉOLOGIE.}

678. Lemrbuct der geolorie, etc., ou Cours de géologie, par S. Breislack, traduit sur la 2e. édit. française, refondue et comparée à la première italienne; par F. Ch. de Strowbeck, 3 vol. in-8, avec 7 pl. Prix : 8 rixd. Brunswick; 1822.

679. History of ture entiti and animated nature. Histoire de la terre et de la nature animée, par O. Goldsittr, 4 vol. $\frac{1}{2}$, in-8. avec 49 pl. culum. en I/ cah. de 3 sh. chacun. ( fr. 3. 75 .) édit. de Coxton. Londres; H. Fisher.

Parmi les objets intéressans de cet ouvrage, il faut remarquer les suivans : Escquisse de l'univers. - Contemplation succincte du globe. - Aperen de la surfice de la terre. Revuc des différentes théories de la terre. - Traité des fossiles. - Structure intéricure de la terre, cavités et passages souterrains. - Mlines, mouffetles et vapeurs minérales. Volcaus, tremblemens de terre et notice sur l'apparition et la disparution des iles. - Source des rivières. - Océan. Marées. - Fssai sur l'Ilistoire naturelle de l'air, des vents, des métiores; Histoire de l'houme, des monstres, des quadrupètes cn général, des insectes, des poissons.

Tомк I. 
680. A companative estimate of the mineral, cic, Comparaison de la géologie minérale, avec la géox logie de Moyse, par Granvilue pexa, a $10 l$. in-s; prix, 1 s sh. cart. Londres; Baldwiu.

68x. Vensteu ersen grogatostrschea Beschreibung von Oberschlesien, ete., Essai d'une description géonostique de la Haute-Silésie et des contries voisines, situées dans la Pologne, la Gallicie et la Silésie Autrichienne; par Ch. de Orvanarswa. Essen, r 822 , chez Bnedeker, grr. in-S. xxxry, et $47 \mathrm{p}$, avec unc carte géognostique en a $\mathrm{f}$.

Cet ourrage, bien imprimé, quoique dans une très-petite ville du pays cle Clèves, nous a été communiqué par MI. Beudant. On y trouve décrits en détail des terrains de presque toutes les formations connues; l'anteur a ipporté un soin particulier a faire bien connaitre les terrains honillers ( $p$. I 2 a 197 ) et les gites de minerai de plomb, de minerai de fer et de calamine, dans le calcaire $\{p, 203$ i 253$\}$. Les patges 331 a 360 sont consacrées a la description de quelques terrains de porphyre, de diabase et de basalte. C. M.

683. A Descrimtios of the Shetland islands, etr. Description des iles Shetland, consenant le tablean de leur grologie, paysages, antirquités et superstitions; par S. Hrbert, II. D. in- $\{$. avec une grande carte grinlogirue et des pl. Prix, 3 l. 3 sh. eart. Londres; Hurst, Robinson.

683. A Drscriptiox of the western islands of Scotland, rete, Description des iles occidentales de licosse, comprenant lile de Man; avee un tableau de leur structure géologitue, et des remarques sur leur agriculture, leurs paysages et lenrs antiquités; par J.M'tecleoch, ? vol.in-s, avee I vol. de pl. in-f. Prix, 31.3 sh. cart. Londres: Hurst, Robinson.

68. A A secence accorixt of the lime rocks of Plymouth, cte. Description succincte des roches calcai- 


\section{Geologie.}

res de Plymouth; sujet de plusierirs communications faites aux membres de la Société géologique de Londres, et dont quelques-unes ont cté imprimés dans leurs Transactions, avec so pl. lith.; par le: chev. R. Ilensun, in-8; Prix, 12 sh. Londres; Whittaker.

(is5. Grolorical essays, ete. Essais de géologie comprenant un apercu de l'ordre des couches du charbon de terre et des minéraux du district de l'A par J. Scteliffe, in-8; prix, 4 sh. Londers.

686. Némome str les carrines et les fours à plitre de Saint-Léger sur Dheme, (Saône-ct-Loire); par M. Levallors, aspirant des mines. (Ann. des Mines, 1822,3 . livr. 12 p.)

Ce múmoire est divisé en trois sections: la première traite de la constitution géologique des environs de Saint-Léger. ()"n y roit que la formation gypseuse repose sur le terrain houiller, et fqu'elle est recouverte par un calcaire à grryphites. La deuxième section fait connatre la méthode dexploitation du gypse de Saint-Légger, soit par galeries sculement, soit par puits et galcrics. Dans la troisième section, l'auteur indique les différens modes de cuisson du plittre de cette localité, dans des fours à calcination périotlique pour le plàtre destimé à la laatisse, et dans des fours à calcination continue, pour celui qui est destiné à servir d'engrais.

BD.

687. Sun l'origre des ossemens accumulés dans plusieurs grottes d'Angletere; par M. (G. Cenderi ind. (Ann. of Plilos., fév. 1823, p. 127.)

Il s'agit principalenent, daus ce const article, d'une cavité découverte dans la reche caleaire près de Plymouth, en ex. i)loitant des pierres pour les grands travaux de ce port. 1)ans cette cavité, qu'on peut dire avoir été fermée hermétinuement avant que les ouvriers y eussent pénétré, on a trouvé réunis des ossemens d'animatux herbivores et carnivores de différentes espèces, notammont un nombre inmense d'os de chieval. M. Cumberland attribue la réunion d'animanx de meurs aussi oppurécs à la nécessité ou ils se trouverent, a léporque 
du délnge, de chercher un asile sous des rochers qui s'affaim.sèrent ensuite, lorsque les eaux vinrent a se retirer.Il combat les autres explications quon a données d'un fait semblable observé dansles grottes de la vallée de Pichering en Yorhshire. C. $M$.

688. Esprisse de la constitution géolngique de la vallíe du Mississipi; par E. Jimss, (Journ. of the - lcud. of nat. sc. of Plitudelphia, $\mathrm{n}^{\circ} .1 \mathrm{I}, \mathrm{p} .326$. ) avec deux sections verticales de cette vallée, l'une sous le $/$ I $^{\circ}$, I'autre sous le $35 \%$ degré de latitude, dessinées par le major Lovg.

Ce mémoire, qui ne comprend que cinq pages in-S, a uniquement pour objet d'expliquer dans quel esprit ont été tracées les coupes de terrain qui l'accomparnent. Ce n'est qu'un premier apercu de cequ'on trouvera avee plus de détails dans la relation d'une expédition de découvertes qui doit ètre publiée incessamment a Philadelphie. Ces coupen se terminent à l'est aux monts Alleghany, et a l'ouent à la chaine des Rorly-Mountains. Une échelle des hateurs est placé à la matrese. Elle donne, pour l'élévation de cette derniere chaine, sous le 35 . parallele, environ 10,200 pierls anglatis, et sous le fie., 12,00o pieds. Pour la nature et la superposition des différente's couches et pour leur inclinaison, nous ne pouvons que renvoyer aux coupes mèmes, auxquelles on suppléerait mal par une description.

C. $M$.

Gig. Sixcruje dicouverte par l'excavationd'une citexue. (. Asiatic Journ., n. 86i, fév. Is:33, p. 155.)

Fu creusint a 60 pieds de profondeur, a l'angle de l'esplanole firs, avec le Dhurrumtolah a Calcutla, on a dicouvert den frones darbres massifs debout, dans leur position naturelle. (guoigue tris-altérés, il parait qu'ils ont crù la a une

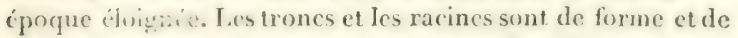
grandeur naturelles, ot s'étendent comme si les arbress'étaient pas morts ; les morceaux que nous avons examinés ressemblent a du vieus bois pouri.-On a aussi trouré, il y a quelques année, une inagre d'or, et la carcassed'un batean, en creusant une citerne dans le Garden reach. 
UISTOIRE NATURELLE GÉNÉRALE.

bogo. Natural mistory of Seldonim. Histoire naturelle de Selborne, par Gilben't IV IHte; augmentée? du Naturalist's calenner, le Calendrier du Naturaliste, mélange d'observations, et poëmes, nouv. édit. avec grav. 2 vol. in-8. Prix, I6. sh. eart. Londres; Hurst, Robinson.

Ggr. Usmel knowlenge, ete. Connaissances utiles, on tablean familier des diverses productions de la nature minćrales, végétales et animales, qui sont principalement à l'usage de l'homme, enriehi de nombreuses fig., et destiné à ètre un ouvrage d'instruciion et de consultation; par IV. BrokgLey, auteur de la biographic animale, 3". édit. 3 vol. in-12. Prix, 1 1. I sh. Londres; Baldwin.

6 g. IIstone el description du Muséum royal d'histoire naturelle, ouvrage rédigé d'après les ordres de l'administration du Muséum; par M. Des.ruze. ave trois plans et quatorze vues des jardins, des saleries et de la ménagerie, i vol. in-8. Paris, I 823. chez M. Royer, au Jardin du roi.

La premiere partie de cet ourrage est destinée a tracer l'histoire du Jardin du roi, depuis sa création en 1635 , jusqu'à nos jours. Des notices lingraphiques sur les hommes célébres qui l'ont illustré se lient à cctte partie historiq̨ue et en diversifient lintérèt. Ce tubleau se termine par la liste des principaux employés du Muséum en janvier 1822 , avec l'indicationde leurs ouvrages et unc comparaison de l'établissement tel qu'il était en 1789 , à ce quil est aujourd hui.

On voit que quoique lit dépense ne soit que de moins d'un tiers en sus de ce qu'elle était autrefois, l'étendue du jardin est presque double. Le nombre des bâtimens actuels est au nombre des anciens bàtimens dans le rapport de sept à tum. It n' y avait que trois chaires, il y en a treize aujourd hui. p'innieurs collections, maintcnant très-nombreuses, telles que celle des assemens fussiles of relle de la géologice, n'existaient prats 
alors. I.es autres ont éti en géníral portées à dix et rente. fois leur nombre primitif.

La seconde partic comprend la description des différentes divisions du jardin et des objets remarquables quielles prísentent. Ce n'est point un catalogue aride de tousces objets, catiblogue dont queldues parties ont été dejà publices. I'unteur s'attacle principalement à indiquer ce qui peut intéresser la curiositi des lecteurs de toutes les classes, en renvoyant aux ouvrages spéciaux pour les détails. Il décrit successivement de cette maniere l'ensemble du jardin, l'école de botanique, l'école des arbres fruitiers, l'école des plantes d'usige dans l'économic domestique et dans les arts, l'école de culture, le jardin des semis et celui de naturalisation, la - erre tempérée, les serres chaudes et les galeries de botanique.

Les planches qui accompagnent ce volume sont très-remarquables par la beauté de la gravure. Ces parcs, destinés aux animaux paisibles, forment de charmans paysages; dans lesquels les formes étrangères des dromadxires, de líléphant, du bison, des autruches, et de divers antres animaux, produisent l'effet le plus pittoresque.

Un second volume, maintenant sous presse, completerat l'histoire de l'établissemsent en décrivant les objets renfermés dans les galeries de minéralogie, de síologie, de zoologie et d'anatomic comparée, ainsi que les animanx vivans de la ménagerie. Cette partic a été rédigre d'après des notes fournie's par MIII. Valenciennes, Dufresne, Latreille, de la Fosse, Ered. Cuvier et Laurillard.

Cet ouvrage, traduit en anglais, se trouve également chez M. Royer, au Jardin du roi. de Basterot.

(ig3. Taxmensy or a complete treatise on the art of preparing, ete. Taxidermie, ou traité complet de lart de préparer, monter et conserver tous les oljots dhistoire naturelle, pour les muséums, 2 . élit. r vol. in-r2, avec pl. 7 sh. 6 d. cart. Londres; Longman.

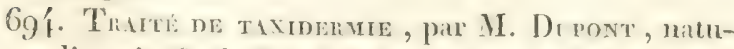
raliste, in-8. do \& fo cellemie, plus í pl. Prix, f́f.

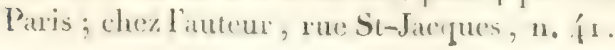


ZOOLOGIE.

695. The pullosomy of zoology. Philosophie de la zoologie, ou vues générales sur la structure, les fonctions et la classification des animaux, par $\mathbf{J}$. Fleming, 2 vol. in-8. arec grav. Prix, 1 l. io sh. cart. Londres; Hurst, Robinson.

6g6. De l'organisation des animaux, ou principes d'anatomie comparée; par M. H. M. Ducrotay de Blainville, prof. etc. 'Tom. I'r , contenant la morphologie et l'aisthésologie. In-8. de 574 p., plus ro tabl. de classification des animaux. Paris, 1822 ; Levrault.

Cet ouvrage, qui porte la date de 1822 , a réellement paru (-1) janvier 1323 . Cette fixation pouvant avoir quelque inportance pour des questions de priorité sur les découvertes des faits qu'il contient, nous croyons bon de l'établir ici, attendu que l'auteur, pour ne pas trop multiplier les eitations, a pris le parti de n'en pas faire; "réservant, dit-il, à l'historien impartial de la seience, si jamais il en existe un, de juger si j'ai eu l'ivantage de faire des découvertes plus ou moins importantes."

Dans son introduetion, M.de Blainville donne les curactères des corps organiques et inorganiques, les différences des deux grandes classes de corps organiiésé et définit ainsi l'unimal: $\mathbf{I}^{\circ}$. " une certaine combinaison d'organes, produisant certaines forces, et cntreautres une force digestive et une force locomotive, iffectant une forme déterminée, et agissant sur les circonstances ext trieures d'une manière également détermincéc. n Entrant ensuite en matière, il distingue six espèces d'anatomic, savoir : pittoresque, chirurgicale, médicale, physiologgique, zoologique, philosophique ou transcendante; celte dernière, dit-il, s'élevant des faits anx abstractions, c'est-a-dire du postériori au priori, et descendant de celui-ci à celui-li, est de beaucoup la plus difficile. Celte anatomic transcendante et l'matomic zoolognique font l'objet de la premiere partie de l'onsage, oin, grace a l'anteur, les diffecultés du snjet prarai. eront sans doute moinkives quil le dit. 
Après avoir analysé les tissus élémentaires, exposé la connbinaison de ces tissus pour former les oruanes et appareils, d'organes, donné la di-ision de ces appareils, l'anteur entre en matière par la description de l'enveloppe de l'animal, considérée comme établissant ses rapports extéricurs avec les corps environnans: concue comme déterminant la forme de lanimal, cette enveloppe offre le sujet de la morpholocic; conçue comme déterminant la perception des corps extérieurs au moyen de l'appareil des sens, elle devient celui de l'aisthésologie.

Linteur, dans tout le cours de ses démonstrations, a ressuscité une méthode de raisonner à priori tombée en désuétude depuis Bacon; mais il ne l'emploie qu'en concurrence avec la méthode à postériori. Les personnes assez heurouses pour avoir entendu comme nous l'ingénieux professeur exposer ses idées d'après ces denx méthodes, ne se préviendront pas contre son entreprise de réhabiliter la première. Ceux qui n'ont pas joui de cet avantage auraient tort de se prévenir contre lui a cause de cette innovation.

La morphologie est prícédée de dix tables synoptiques donnant les divisions et sous-divisions du rigne animal et de ses classes d'après les idées propres a l'auteirr; tables déja publiérs en i 8 r 6 dans le Bull. des Sc. par la société philomathique.

N. $P$.

(ig7. Deutscriands fauna. Faune d'Allemagne, par

Z. St: ru, in-12. Partic 6. Vers. Cah. 5. Nurembers; 1822.

698 . Faxie des médeciss, ou histoirc naturelle des animaux et de leurs produits, considérés sous le rapport de la bromatologie, et de l'hygiene en grénéral, de la thérapeutique, de la pharmacologie et do la wricologie, ouvrage entierement neuf, avere fir. par II. Chopeet, D. M. P. membre de l'acadimie rosale de médecine, ete., ton. I"r, in-s. de

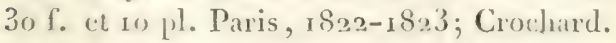

En liant intimenent la méslecine avec la zoolocrie, ect one vage parail avoir pour but d'étendre la seience des médica- 


\section{Zoologie.}

mens et de leur enploi, de la perfectionner, de la débarrasser de plusicurs de ces vieilles erreurs que le silence d'une longue suite d'années semble avoir consacrées, de les signaler afin qu'elles soient repoussées du domaine de lit thérapeutique et renversées plus facilement sous les efforts de l'expérience. Il offre l'histoire spéciale de chacun des animaux qui peuvent intéresser en quuclque point la santé des hommes, et il renferme d'es détails sur leur synonymie et mème sur l'étymologie de leur nom, sur l'ensemble de leurs caractères et des propriétés qui les distinguent et doivent servir à les faire reconnaitre, enfin sur les lieux qu'ils habitent, détails qui appartiennent à tous sans exception.

Mais l'anteur ajoute de nouvelles considérations propres à telle ou telle espece, suivant que, comme la sangsue, la cantharide, le musc, elle est consacrée au soulagement de nos maux; ou que, comme la vache, le porc, la poule, elle est destinée à assouvir notre faim et à réparer les pertes de notre organisation. Il appelle ici toutes les connaissances de l'hygiène à son aide.

D'autres traits encore signalent ces animaux qui, possesscurs d'un poison actif, répandent antour d'eux la douleur et la mort. M. II. C. indique au médecin appelé pour combattre la sorte d'empoisonnement : lacquelle ils donnent lieu, et les symptômes du roal, et les remèdes propres à le combattre. En suivant ce plan, il trouve occasion de développer avantagcusement une foule de notions itendues en thérapeutique, en physiologie, en chimie, cal histoire naturelle, et de tirer parti des nombreuses applications réciproques de ces différentes sciences.

Cet ouvrage sera publić en $3 o$ livraisons de six fuilles de texte $\left(9^{6}\right.$ pages $)$ in- $8^{\circ}$., et de deux planches.

Ic prix de chaque livraison est de deux francs pour les souscripterurs, et de 2 fancs $50 \mathrm{c}$. pour les livraisons qui ont déjà paru à l'époque ou l'on souscrit. En ajoutant un frane par livraison, on a un exemplaire avec les planches colorices et retouchées au pinceau avec soin.

Il a déja été publié neuflivraisons de la Faune des médecins. Les cinq premieres forment le $x^{e r}$. volume, dans lequel on remarcyue l'histoire des abeilles domestiques, amalthéc, unicolore, etc.; celle de l'ablette, qui a donné occasion à l'au- 
tcur de décrire mp procéde flharmaceutique nonvean et qqui lui appartient; celle des aciphlahocystes, oi l'on trouve la description d'une nowwelle espuce, l'acephalorgatis ractumosa; celle des actinies, de l'asping ou vipere rouge, de lasneau, de l'agouti, de l'aigrle-royal, de l'aigle de mer, de l'aignillat, de l'albumen, de l'ammodyte, de l'aloueite, des araiguées, de l'anguille, des anchois, de l'ambre gris, de l'algazel, etc. ete.

699 . Mrumilis of british Quancpedes, etc., ou mémoires sur les quadrupèdes anglais, avce zo grav.; par IIowiтн. Royal in-8. Prix, I 1. i 5 sh. cart.; sur pap. imp. et pl. enlum., 3 l. 13 sh. 6 d. Loudres; Harvey et Darton.

Il reste encore quelques exempliaires de cet ourraģe.

700. Ilfustrations of british onxithology, etc., ou explications de l'ornithologie britannique. Prem . part. Oiseaux du pays, par P. J. Selby. In-fol. Prix, I 1. I s sh. 6 d. grav. en noir, on 5 l. 5 slı. grav. color. avec soin, d'après nature; $5 \mathrm{n}^{\prime \prime}$. avec $\mathrm{r}$ a gr. pl. Londres, Hurst, Rolinson.

zoi. Essirs entomorogieres, ñ. I. Queleques observations sur la blaite germaniegue (blutta germaniea, Fabricius), par A. D. Hzwarl (de GothemJours), cher. de St.-Wladimir, ete. Pr. in-s. de Is p., en langue francaise. St.-Pétersbourg, I $S_{2}$ I.

J'antenr y trate particulièrement de la ponte de eet insrete, qui n'est que trop commun en liussie dans les maisons des pauvres et des paysans.

703. Fssirs fatomodorieres, n'. 2 , sur les insectes de Saint-Pétersbourg, peudant l'été sëza. Lettee a la Société des naturalistes de Noscou, par le meime. $1822 ; 30$ p. mime format, igalement on francais. St.-Pétersbourg.

L.es liusses ont fitt les plus grands progrès en entomologie, ils sont au courant des onvragen lesplus nouvenux. Dans celui-ci les insectes dont il est question et qui somt au nombre dinviron 3an, lont plus de 250 eolioptizes, sont classís el 


\section{Zoologie.}

nommés d'après les dernicrs ouvrages de 1 I Latreille et le catalogue de Mr. Dejean pour les coléoptères. L'auteur décrit deux espéces qui lui ont paru nouvelles sous les noms de $\mathrm{Ca}$ tops punctuto-striatus et Bembidium fockii.

703. Observations norvelles sur l'organisation extéricure et générale des animaux articulés, et à pieds articulés, et application de ces commaissances à la nomenclature des principales parties des mémes animaux; par M. LAtreille. (lickn. du Mus. d'hisl. natur. p. 169. )

L'autenr propose de substituer le mot de Condilope, formé de deux mots grees, necud et pied, à ce nom ou plutôt à cette phrase descriptive : animaux articulés à pieds articules, sous laquelle on comprend les Insectes, les Arachnides et les Crustacis. Il traite ensuite des divers appendices du corps dans chacune de ces classes. V. Avv.

704. On trouve dans le $2^{\circ}$. cahier de la Bibliothéque indienne de AT. Schlegel (jndische Bibliothek), publicie a Vienne, un article sur l'histoire de l'éléplkant, où ce savant a réuni avec grand soin toutes les notions à l'aide desquelles il était possible de compléter et d'éclairer non-seulement lhistoire naturelle de l'élépliant, mais encore ce que l'on jourrait appeler l'histoire mythologique, civile et militaire de ce puissant quadrupède. Ces notions sont coordonnées entre elles de manière à présenter des apercus d'un grand intérêt pour l'histoire générale de la civilisation humaine. F.

705. Stri plesictis espices nouverues de poissons et de crustncés, obserrées par M. Mrarov ne Procé, correspondant de la soc. phil. ( $\mathrm{Bul}$. des sc. par la soc. phil. sept. I 822.$)$

Ce ménoire renferme les phrases caractéristiques de vingt espèces de poissons, et de six crustacés nouveaux dont les noms suivent, et qui ont été recueillis par. MI. Marion de Procé, dans un voyaģe qu'il a fitit à Manille cn I8rg et 1820.

Porssuns. ro. Squalus indicus, voisin du squate Nez, mais remarquable par une carine pinniforme sur les côtés de la (quene; $2^{\circ}$. Titraodon manillensis; 30 . Tetr. nigroviridis, petit poisson longer d'un ponec et demi, lisse, vert, et marque de 
faclies noires et rondes sur les flanes, observé dans une mare d'eau douce, sur la côte Y.E. de Sumatra; 4. Tetr. compressus; 5*. Balistes rotundatus; 60. Bal. punitutus; $7^{\circ}$. Bul. marmoratus; So. Bul. cinereus; $9^{n}$. Bal. ornutus; $10^{\circ}$. Saurus depressus, tres-déprimé, à dents mobiles, à ligne latéme carénéc; poision pèclué en grande abondance à Manille, où sa chatir "ut estimée; I I ${ }^{\circ}$. Sternoptyx cyanea; r $\mathbf{2}^{\circ}$. Clupea manillensis, sorte de sarline a bouche peu fendue, ayant le. dos blentitre, l'abdomen et les flanes argentés, très-répandue dans la baie de Manillo, ou elle fournit une nourriture abondante et de bon goût; $13 \circ$. Gobius rufus; 140 . Labrus bacchatus; 15\%. T)entex elongatus; $16^{\circ}$. Holocentrus Zelora; 17․ Treniunotus minutus; 8 . Mullus manillensis; 19'. Curanx scutatus; $20^{\circ}$. Amphacanthus oratus.

Crustacis. $1^{\circ}$. Portunus tropicalis, fauve faclie de blane, a six dentelures frontales et neuf laterales, trouví sur le furus nutans, dans le sud des Acores; 2 . Port. denticulatus de Manille; 3". Port. unteserifruns de Manille; 4". inarhus bifidus; 5 . Inach. inflexus; 6 . Poneus monillensis, ayant le rostre pourvu de huit dents en dessus et de trois en dessons, le test et la queue velus, et le sixieme segment caréné: cette espèce, longue de 7 à 8 pouces, fournit un excellant aliment aux hitbitans de MIanille.

DEsn.

706. Tínorre str on nouvelu geñe de poissons, nommé Alépocéphale, vivant dans les grandes profondenrs de la mer de Nice; par A. Risso. ( Mém. de l'acad. desse. de Turin, vol. 25. p. 270.)

M. Risso reconnat que ce poisson nouveau appartient a I'ordre des Malacopterygicns ablominaux de M. Cuvier, el que, selon la méthode ichthyologgique de re naturaliste, il doit Are placé, dans la familie des Esoces, entre le grenre Microstome et le genre Stomias.

Outre les caractires de la fitmille à laquelle il se rapporte, l'Aripocípuste, Alepoccphalus rostratus, offe les suivitns : museau avancé, arrondi; grueule ample; michoires et palatius garnis de dents tres-fines et aigui ; langue lisse; veux tres-grrandis; onies bien fendues; corps oblongr, aplati; une seule dorsale opposic a l'anale, situce pres de la queue;

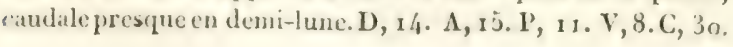




\section{Zoologie.}

MIB , 8. Les écailles sont grandes, peu adhérentes, d'un bleu violàtre, et liserées de noir; la tête est nue et d'un noir luisant. La longucur totale du corps est de o, m.286.

Ce poisson fait son séjour habituel dans les profondeurs de la mer, et il n’a encore été pêché que dans les mois dejuillet et de novembre.

Une figure au trait, peu détaillée, accompagne ce mémoire. (Desm.)

707. Mémoine sur deux nouvelles espèces de poissons du genre Scopèle, observées dans la mer de Nice, par A. Risso, avec fig. (Mém. de l'Acad. roy. des sc. de Turin, vol. XXV, p. 262.)

Le genre Scopéle, de l'ordre des malacoptérygiens abdominaux et de la famille des Salmones, a été créé par M. Cuvier pour placer deux poissons que M. Risso avait décrits dans son ichthyologie de Nice, parmi les Serpes, tout en indiquant qu'ils devaient être séparés de ce genre.

Les Scopèles présentent, outre les traits de conformation qui sont communs à tous les poissons de la famille des Salmones, les caractères suivans : museau très-court, obtus; mâchoires garnies de dents aiguës; gueule ct ouies très-fendues; neuf à dix rayons à la membrane branchiostége; la langue et le palais lisses; deux nageoires dorsales.

Les deux espéces anciennement connues sont : $\mathrm{r}$. Scoprots Croconilus. S. corpore compresso, ore amplo, dentibus valdè temuibus. P, 18. V, 8. D, I4-4. A, 20. C,22. MB , 10.

$2^{\circ}$. Scopelus IIvmionto. S. corpore subcompresso, ore mediocre; dentilus acutis. P, 18. V, 8. D, 14-4. A, 20. C, 2. M. MB, Iо.

Les deux espèces nouvelles sont: $3^{\circ}$. Scopelus angtstiDENs. S. corpore subcompresso, ore mediocre, dentibus parvis acutissimis. P, 12. Y, 8. D, 22, 4. A. 20.C. 22. MP. 10.

40 Scopelfen Balbo. S. corpore compresso punctulato, ore amplo, dentibus longissimis. $\mathrm{P}, 10, \mathrm{~V}, 8 . \mathrm{D}, \times 2.3 . \Lambda$, 34. C, 48. MB. 8.

Les $x^{\text {re }}$, $2^{\circ}$. et $4^{\prime \prime}$. espèces sont figurées au trait, et d'une manière assez peu arrêtéc.

La 3e. reste continuellement cusevelie dans les profondeurs de la haute-mer et ne s'approche que fort rarement 
des rivages, dans les mois de mai et de juin. La derniere habite les moyennes profondeurs, nare avec beaucoup de rapidité, el apparait sur les côtes, aussi trés-rarement, dans le mois de décembre

(Desu.)

708. De t'onicrare et des phogrìs De L'entomologif, par M. Intrimle. (Mém. du Mus, d'hist. nalü, t. 8, p. 46 I.)

L'anteur partage l'histoire de l'entomologie en sept piriodes cubrassant les divers âges, et les parcourt successivement, en faisant conn uitre les principaux travaux des hommes qui se sont illustrés dans la science. $\quad$ V. Avo.

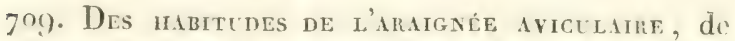
Linneus, par M.Litreilee. (Mém. du Lus. d'hist. natur., tom. 8 , p. 456.)

Les renseignemens quon avait obtenus jusqu'a présent. sur l'araignée aviculaire étaient fort incomplets. M. Tatreille ayant reçu de Ji. Goudot jeune, négociant voyageur, des notes exactes sur cette espèce d'araignée, accompasnós du nid de l'animal, s'est empressé d'exposer ces firits nouveaux, en les accompagnant de considérations fort curieuses.

\section{Avo.}

7ro. De L'origane arsicil des criquets et des truxales, et sa comparaison, avec celui des màles des cigales, par M. Litreille. (Mém. du Mus. d'hisl. nalur., p. 122.)

I. titre de ce mémoire indique suffisamment le but que l'auteur s'est proposé; il a comparé les organes particuliers situés sur le premier anneau du corps de labdomen des iruxales et des criruets, aver l'appareil du chant de ces cigales.

V. Aun.

711. Fichurcissemess relatifs à lopinion de M. Huber fils, sur l'origine et l'issue extérieure de lat cire; par M. Litrerlez. ( Mém. du Mus. dhist. nalur., t. 8, p. I 33.)

On a cru long-temps que le pollen des flenrs contenait la cire brute en nature, qui, apres s'itre transformce en cire vierge dans l'entomac de liabeille, sortait par sa bouche; 
tnais II. Iluber fils a depuis démontré que dans le miel résident exclusivement les principes élémentaires de la cire, et il a recomnu que cette cire était produite par une sécrétion qui a lieu intérieurement entre les anneaux de l'abdomen. M. Latreille confirme ces résultats par plusieurs considérations nouvelles. V. Aun

7i2. Essai str le vol des insectes, par M. J. Chibrisk, avec fig. (Ménz. clu Mus. d'hist. natur., p. 47 et 349.)

C'est une suite d'un travail imprimé dans les Mémoires de l'année précédente, et dans lequel l'auteur étudie anatomiquement le thorax des insectes, datus le but d'arriver à la démonstration d'une théorie nouvelle sur le rol en général.

$$
\text { V. Avo. }
$$

713. Monographe de gexre nint do, ou description des espèces de sangsues qui se trouvent, ou qui sont en usage en Pićmont, avec des observations sur la génération, et sur dautres points de lhistoire naturelle de cquelques-unes de ces espèces, par le prof. II. CAnevi, avec fig. d'après nature. ( $1 \mathrm{~cm}$. della reale Accad. dell. se. di Torino, t. XXV, p. 2.73.)

L'auteur fait remarquer que la sangsue employée à Turin (hirudo provincialis Carisa) ne se trouve point dans les caux du Piémont, mais qu'on l'apporte de Toulon et de Marseille. La sangsue que l'on trouve en Italie, et dont on fait usage en médecine, appartient à une espèce fort différente. M. Giorna observe que l'hirudo alpina du Dr. Dana (H/ $\mathrm{cm}$. della reale stcad. delle sciense di Torino, t. III, 1. 19g) et l'espèce désignée sous le mème nom par l'abbé Ray (Koologie universclee, in-40", Paris 1788 ), et par le docteur Titet (Traite de la sangsue médicinale, Paris 1859 ), n'est pas une sanghsue; mais qu'elle appartient au genre platnaire. Passant encuite à la monograplie du genre sangsue, M. Giorna en décrit dix espèces.

I". Hirudo medicinalis. Lrsw. Mur.t. Cette espece laibite les lacs de la vallée de Suze, et se trouve dans les eaux de toute la partio septentrionale du liumont, a puis Chivasso jusqu'à Aoste. 
$2^{n}$. Hir. provincialis. Canexa. Elle n'habite pas les eaux de Piémont, et se trouve aux environs de Toulon et de Marscille.

30. Mir. verbana. Canena. Elle a été observie dans le lac MIajeur.

4. Hir. sanguisuga. Lixs. Mür.. Très-commune aux environs de Turin.

5. Hir. vulgaris. MüLt. - Octoculata. Lrxy. Trouvée dans le lac de Viverone, près d'Ivrée. Cette espèce est ovipare.

6.. Hir. atomaria. Canena. Moins commune que la précédente.

7. Hir. complanata. Liva. Müll. Bergar. Dans les lacs d'Avigliana et du Canavais.

8o. Hir. cojhlalota. Canera. Cette espèce est vivipare. Elle a été rencontrée dans les mẻmes lieux que la précédente.

$9^{\circ}$. Hir. bioculata. Mijur. - Stagnalis. Lixv. Très-commune dans le lac de Viverone et dans celui de Bertignano, près d'Ivrée.

no . Hir. trioculata. Canexa. Espèce trís-rare, observie dans les lacs d'Avigliana.

11. Carena ajoute a la suite de la description de toutes ces espièces, des remarques curieuses sur difiérens points de leur listoire naturelle. Il a aussi tenté plusieurs expériences sur leur mutilation. Une sangsue dè l'espèce qu'il nomme provincialis ayant été coupée transversalement en 3 parties, et le fragment moyen ayant été conscrvé, il ne reproduisit pas de nouvelles parties; mais il vivait encore dix mois apres l'expérience.

V. AuD.

7 I 4. Exṕninence facile pour produire des entomostra-

cées, par Wiegman. (Isis $\mathbf{1}^{\mathrm{e}}$. call. $\mathbf{1}_{22}$, Lilerarischer anzeiger, p. 432.)

On inet un quart d'once de corail blanc ou rouge préparé (mudrepora oculata ou isis nobilis) dans 6 onces d'eau distillée; on place ce mélange au soleil, dans un verre assez grand; on le remue souvent chaque jour, et au bout du I ' an soleil.

Au bout de rá jours, on obtient une matiere verte dans 


\section{Zoologie.}

iarpuelle it se forme des conserves, d'oit se développent, au bout de 3 ou 4 mois, surtout pendint l'ité, des cy prides détecte. Lorsqu'on expose aux rayons ardens du soleil l'eau que l'on verse dans un cylindre hant et étroit, il s'y forme des espèces de Chrysalides où se développent, long-temps après, des Daphnice longispina.

M. Wiegman communiquera une foule d'autres expériences dans le $\mathrm{I}^{\circ}$. vol. des Nlémoires der Kaiserl. Leopoldinisch-Carolinischen ahademie der naturforscher.

7 i5. Mímonre sur la distrinution géographique des animaux vertébrés, moins les oiseaux, par ì. DesMovlins, D. A. (Bull. des sc. par la soc. phil. de Paris, p. $157 \cdot 1822$.

L'autcur s'attache à combatre l'opinion de quelques sitvans, qui, admettant pour les animaux un point central de création, pensent qu'ils se sont répandus de ce point sur toute la surface du globe. Mr. Desmoulins rappelle les travaux récens de MM.Cuvier, de Ifumboldt et Latreille, et invoque à l'appui de sa manière de voir plusieurs de leurs observations, et entre autres, celles qui ont été faites sur les poissons dans les Indes par H. deHumboldt, et dans les I'yrínées par M. Ramond. Ce dernier obscrvateur en conclut que dans l'état actuel de nos comnaissances on doit admettre comme préférable à toute autre, l'hypothèse de la création simultanée de plusieurs types ayant une organisation assortie à l'état plyysiqque de chaque localité; ce qui, pour peu que l'on réfléchisse, équivaut ì dire quil y a eu plusieurs points distincts de création. H. Desmoulins arrive égulement à ce résulat; mais il envisage son sujet sous un plus grand nom-bre de fices, et se prononce ouvertcment pour la plualité des centres de création, sans cependant en fixer le nombre. II arrive d'ailleurs par l'étude des faits à plusieurs résultuts, qu'il regrarde comme autant de propositions insuffisamment démontrées.

V. Avd.

7r6. Sta les nevolutrons que la géognosie indigue s'ètre opérées dans le règne animal, par le doct. Fleming. (Philos. Jour., n". 15 , janv. 1 823, p. I io.)

717 . Les sirixes ancifanes et modernes, et superstiTome I. 
Lion résultant de la croyance aux sirencs. ( 1 siat. Journ., nº.83, p. 49.)

7 7. Il a tré lu, a la séance publique de la société Linnécnne, du 28 décembre is 822 , une notice surdes aras lileus, mes en France ot acrlimatés dans le dipartement du P'ri-deC'alais, par M. Iamouroux, correspondant de latadénie. des sciences a Caten. Ne connaissant pas ce mómoire nous ne. pouvons entrer dans d'autres détails.

I.

\section{BOTANIQUE.}

7in. Timoncction to motaxy, etc, on introduction à In botanique, expliquéc par des renvois, sous clarque définition, aux plantes que l'on prut aisément se procurer, parlV. Brirs Ley in-i?, avec pl. prix, 4 sh. 6 d. rel. et avec les pl. color. 7 sh. $6 \mathrm{~d}$.

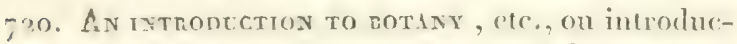
lion à la botanique, dans une suite de lottres familieres, avec des gravures explieatives, 8". édit. cor. prix, / sh. cart. ou $7 \div$ sh. avee des pl. culum. Londres; Harvey et Darton.

;?r. LFCtURES ON THE ELEMENTS OF bOTANx, etc., OH lecons cilémentaires de botanique, contenant l'anatomic descriptive des organes dont dépendent la crnissance et la conservation des végétaux, par A. F. Thomson, a vol. in-8. Prix r 1.8 slı, cart. avec pl. et grav. Londres; Longman.

722. A natemal arangament of British plants, ete., ou Classification naturelle des plantes, d'apro's Jussieu, Decandolle, Brown et autres; aree une inunduction à l'étude de la botanique, par 7. IP. Gray, 2 vol. in-8. Londres; 1822.

733. Potinicat. nilesthatons, ele., on illustralions botaniques des vingt-quatue dasse's du systime des vécétanx de Linnéc, avee des modiles elooisis parsii les planter les plus communes en Angletrore. 
Prix, $3 \frac{1}{2}$ sh. on 6 sh. culum. Londres; IIarrey et Darton.

72). Disenestro entstrons academiea de discrimine sexuali jam in seminibus plantarum dioieasum apparente. Pramio regio ornata. Additis quibusdam de sexu plantarum argumentis generalilus. Auct. F. Antenreter , \& cum tab. lithog. Tubingx; $182 x$.

Réponse à la question suivante de la faculté de Mrédecine de Tubingue.

Constat, is seminitus plantarum dioirarum, plantas wel masculas vel femineas nasei: interrogatur, an jum in seminilus harum planturum ipsis, vel in germinatione et erolutione errum, rel in positione corumdern in plantis aduiIis diversitate's reperiantur, quilus semina mascula it semet nilus femineis discerni possint. Les planches représentent des parties de Lychnis, Spiriaca et Cannabis.

725. Dei libil di Teofrasto efesto intorno alle piamte commentati da Gasp. Hofrana etc. Des livres de Theophraste Fphésius, sur les plantes, commentis par G. Hoffun, par G. Montesanto, in-fol. asee port. Padoue; 1822 ; Imp. de la Minerve.

-gh. Antonir Bertoloni , med. doct. ete., excerpla de re herbaria. Recueil d'observations botaniques. par $A$. Bertolon, prof. de hotan. a Bologme. 17 p. in-\{. avec I pl. gr. Bononice, 1820. ( Typis Aunesii de Nobilibus.)

Sous ce titre, M. Bertoloni a publić les descriptions de dixhuil especen de plantes dont onze appartiennent a la flore d'Italie et sept a la Flore al A mérique. La juplart de ces der

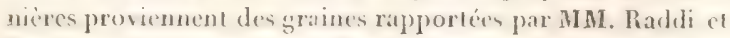

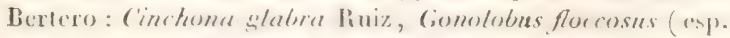

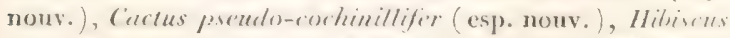

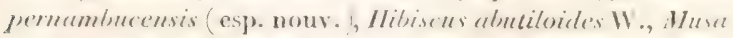

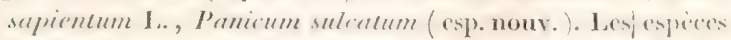

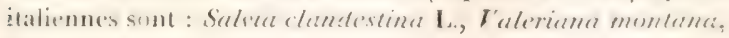
K., foliis incequaliter serratis, superioribus cuspidatis; 
Arena villosa (esp. nouv.), Fioka alpina Jacq., Allium roseum : Bert., Allium silleale i., Delphinium selutinum Bert. , Fucus sclaginoire's Wuli., Fucus discors I.. , Fucus abies $\beta$. Bert., Fucus serruculosus Bertol.

I'Avena villosa appartient au groupe des drena sempur rirens, sedernersis et sctacea; elle differe de toutes ces espices et peut-itre de toutes les graminées connues, par sun clianme velu.

T.a flanche annexée représente le Gonolozus floconsus, avec fleurs et fruiti, sans details grosis. J. Gir.

72,- Aztozin Bingolozi, méd. dect. cte, lucubrationes de ra lierieric: Teilles Jutamicues, par 1. Bentalori, pref. de botanione, à Belogne, Bunonice, 1822 . (Trpis Laresii lo Ricbilibus ). 4o p. in-4. arec I pl. $\mathrm{gI}^{2}$.

Ce n'st pas ici le lieu de rapporter l'histoire du Pamphyton siculaziz de Cupani. On la truatvera p. 6-I a de la Sicular. Fl. ccutur. I de bivona-liernardi Palerme, 1So6.) Il suffit de dire gu'il existe 2 élit. de cet ouvrage; une rre. qui fut interrompue par lit mort de l'atleur, en I $\mathbf{1}$ I ; et une $2^{\circ}$.

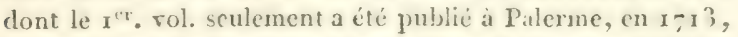
par Bonanni. Duelque rare que soit cette dernicre edition, Ia seule qui soit entrúe dans le commerce, elle existe dans quelques cullections et a été souvent cilée par les botanistes. Mais personne, jusqüi ce moment, n'avait entrepris de revoir la totalité des planches dont elle se compose $19^{\circ}$, selon Bivona; I68, selon Bertoloni) pour yappliçaer des noms linnéens. Ce travail a éte, en graude partic, exceute par M. bertoloni dans le Itemoire que nous ammoneous. $18 .$, plantes, figurées par lomanni, recoivent ici, guelopes-unes pour la premicre fois, des noms tirés des meilleurs auteurs. Cielle nomenclature forme la premiere section du Llemoire.

Dans la $2^{\circ}$. sect., intitulée De plantis minus notis, M. Fertoloni dierit on esp. de plantes; 2 indighenes, polonsmum flagellare, bortol. et Arnica focerse (esp. nouv. eres-voisine et comprenere du semecio Doromirumi; et \& exotiques, rapperties de l’ Imér. mirid. par Radli et lertero, sitvoir

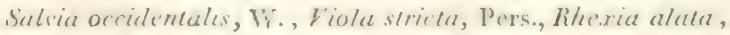
liadil., Jyjutis racemosa, Zureagn., ()domia tomentose 
(senre nouv.), Sebastiuniu heterophlylla (genre nouv.), Xanthium occidentale, Bertol., et Telephora pavonia, Bert.

La figure annexée représcnte l'Odonia iomentosa, espèce de léguminetse appartesant au groupe des Glycines, etc., que M. Bertoloni considère comine un genre noureau. Il est à regretter que ce genre, ainsi que le Scbasticnic (rerbesinut mutica, Linn.), aient été caractérisés par l’autcur avec un liaconisme tel, qu'il est impossible de juger en quoi ils diffèrent des genres voisins.

J. GAY.

728. Die kfinung der Pflanzen, te., ou la Germination des plantes, expliquée par 'zs ieseriptions et des figures des semences, etc., par Z. A. Titwmane, in- $\{$. avec $27 \mathrm{pl}$. color. contenant iro fig. Trix, 8 rixd. Dresde; $182 x$; Walter.

L'auteur a dessiné lui-même et gravé à l'eau-forte toutes les figures de plantes, en sorte que l'on peut compter sur une représentation fidèle.

739. Neue entdeckufigen im ganzón Un. rang der Pfanzenkunde, etc., on nouvelles ' couver'ces en botanique, par C. SPRERigel, tom. 3, in-3. FTix, 2 rixd. \& gr. Leipsic; 1822 ; Fleisc er.

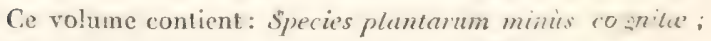
ot Traité des nouveaux genres suivans : Diplocalyrwand. Tetractys. - Sacara. - Theophr. - Keithic. - Stesdeliu. - Astropus. - Hornschuchia.

730. Plantes de la France ou naturalisées et cu'tivées гu France, décrites et peintes l'apris nu:ure,

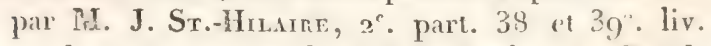
2 calı. in-8., cusemble de $\frac{1}{n}$ de f. ilus $22 ; \%$ col. -Idem, $40^{\circ}$. et $41^{\circ}$. liv. 2 cahiers in-S., ensemble de a f. plus 22 pl. Prix de la livr., 8 f: in-f. pap. vél. $15 \mathrm{fr} .4$ Paris, chez l'autcar, rue de Furstemberg, no. 3.

73r. Flora entuxerlensis, exhibens caracteres generum et specierum plantarum, circa Bruxellas crescentium, secundim Linnam disposita, cum syno- 


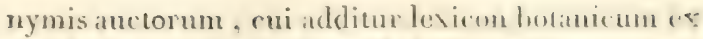
cjustem cuà, in-8. I'rix, 6 fr. Bruxellis.

73.). Froma manan, etc., ou Flore ilalieme, pat

Cistet. Siva, infol. Prix, r 3 liv. Pise; I $822 \%$ Capurre.

;33. Fond veroxexsis quam in prodromum florit Italix septentrionalis exhibet, Frres Portunus, in-8. Tom. I* arec pl. Térone; r8z2; soc. typ.

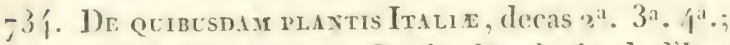

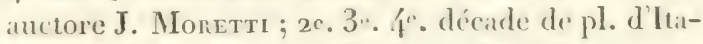
lir, par J. Moretre, prof. d'agricult, à Pavic.

M. Noretti travaille à une Flore d'Italie, ef il n'est aucun effort qui lui coute pour se préprarer à remplir dignement selte tiche. Déjia ses vovages en diverses parties de l'Italie, la comparatson des herbiers de Bertoloni, Temore, livona et Viviani, et les communications qu il sost ouvertes aver litranger, lui ont procure des renseignemens précieux sur les plantes qu'il est appelé a décrire et à classer. Il aurat pu les réserver pour la redaction de son grand ouvrage. Il at cru se rendre plus utile a la science en grénéral, et aux savans de son pays en particulier, en unetlant suceessivement. sous les venx du public le résultat de ses infatigables re- herches. De la sont nécs les Jicules que nous annoncons, dans lespuelles l'auteur décrit 30 esp. de plantes italiennes, nvec nne synonymie tres-soignce, fant ancienne (que moderne, et les observations nécessaires pour lever les doutes auxquels ces plantes ont pu donner licu.

Ia rw. déc, a été impriméc a Milan, en 1818 , dans le xir". tom. de la Bibliot. ituliuna, page 3 fig et suivantes. Nous ne l'avons pas sous les yeux.

Lat $2^{\prime \prime}$, lit 3". et la 4 ". déc. font partie du x"r., du 2". et du 1,e vol. du Journ. de Phys., de Chum. el d'Hive mat., qui s"imprime a Pavie. Les \& vol. dont il est ici question ont di publiés con ris2. Quetoges exemplaires des dicades ont che tirés a part et communiqués piar l'auteur à ses correyponelans.

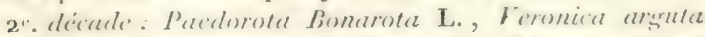

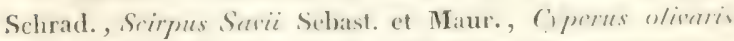

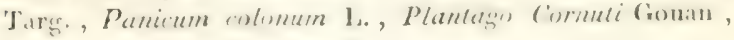


I'erlascum W'eldeni (esp. nouv.), Smyrnium rotundifolium Mill., Smyrnium perfolintum Mill. et Carduus Decandolit Moretti. - I I p. in- $4^{\circ}$.

3e. dicade: Salicornia amplexicaulis Vahl, Schexnus nigricans L., Cyperus fuscus L., Campanula glomeruta ò minor Morett., Campanula elatinoïdes (esp. nouv.), berliascum micronthum (esp. nouv.), Icrbascum heterophlyllum (esp. nonv .), Cnicus spathulatus ( esp. nouv.), Elichrysum frigidum W., Orchis coriophora L. $-8 \mathrm{p}$. in-4 $0^{\circ}$.

4. dicade: Cyperus difformis L., Saccharum Teneriffer Linn. fil., Arundo Plinii Turra, Arundo Pliraginites, L. Primula slaucescens (esp. nouv.), Alisma Plantago L., Saxi. fraga bulbifera L., Aquileyzia pyrenaïa Decand., Arabis lurida, Linn. fil., Inula hetrusca (esp. nouv.) - 15 p., in-4".

J. GAY.

735. Dentschlande flora in Abbildungen, etc., ou

Flore d'Allemagne, par Z. Sтепм, in-12, cahier/9?, avec 16 pl. color. Nuremberg; 1822 .

736. Fuona scotica, etc., ou Flore écossaisse, ou description des plantes de l'Écosse, arrangées d'aprés les méthodes naturelle et artificielle: par W. Jackson Ноокеr, en deux parties, in-8. Prix, I 1 sh. cart. Londres ; Robinson Hurst.

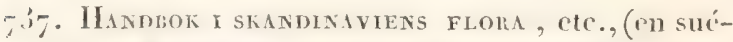
dois. ) Manuel de la Flore de Scandinavie, contenant les plantes de la Suede et de Norwège, y compris les mousses, avec une introduction qui traito des principes de la botaniepe, in-8. avec tig. Prix, 3 rixd. I6 gr. Stockholm; $1820 ;$ Haggstrom.

bintroluction remplit 62 pages et explique les termes fechniques-botariques, notamment ceux en langue suédoise. T.es synonymes sont joints aux deseriptions des plintes des 23 clases, dont la dermiere comprend les cryptogames.

7,38. Deitera phagenses, historiam naturalem spectantes, edite A. J. Swatopleco, prest. et C. lonwoco, pres. in-s. \& gr. Prix, a rixd. Pragn: ; 1822 ; Calve. 
Les deux auteurs de cet ouvrage sont conms par leur Flo. ra cechica. Contenu: Plantarum rariorum Sicilice aliarum. que minus cognnitarum diugnoses et descriptiones. - Plantarum nowarum Brasilice presertion filicum Linna i diugnoses et descriptiones. - Addiumenta ad fuunam protosicam, sistens descriptione's aliquas animalium in succino inclusorum. - Mantisset prima al floran ceshicam, sistens diasnoses et descriptiones plantarum is nowarum Cerhice indigenurum. - Deseripliones duaruin plantarum cryptogamarum Cechia indigenarum.

739 . Plaritz nova, quas in itinere jussu et auspiciis, Max. Jos. Bav. Aegis a. I $17-18 \approx 0, p e r$ Buasiliam suscepto, obscrvarit et depingi curast C.rv. DF HAnTIAs.

La monographie des palmiers contiendra roo planches lithogr, in-fol. grand format; elle sera composée de 4 livr. cha." eme de 25 pl. en noir, du prix de $5-$ f. $7^{5}$ c. et colorices, 103 f. 10 c. Le reste des nouvelles plantes paratrat en liv. de 12 pl. in-1" Prix de chaque ró f. 65 c. fig. on noir et 30 f. -j 5 c. Ag. col. On souscrit chez Lindaner librare a aimich, et chez Treutlel et Wurtz i Paris, wondres et Strisuourg. F.

7\%o. Exotic flona, elc., ou i'lore exolique, contenant les fige et la description de plantes exctiques nouvelles, rares ou aurement intéressantes, spérialenent pour celles qui méri'ent äètre cultivées dirns nos jardius, aree des remaryans sur leur cavartère générique et spécifiqur, lear or lre nat urel, Leur histoire, leur culture ed le tern:'s de leur flomisum. Par IV. Jeckson Hoorra, dort. en droit, aree so grar prix, 8 sh. - lil. cmlum. 15 id. Edimlungh; PV. Blachwood : Londes; Cadell.

7.́. C. Chllogle n'une collection de plantes, nerurillic daus la Jloride orientale, durant les mois

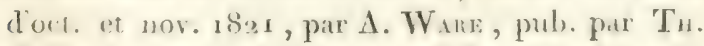
Derrus. (American Journ. of Sc. alld - Lits; ly Silliman., vol. V., no.2. juillet $\mathrm{x} 822$. )

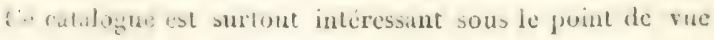


ráographique en nous faisant connaittre, d'une manière trèsincomplete, il est rai, les plantes de la partie la plus méridionale des Etats-Lnis. - Ie nombre des especes qui y sont indiquées est de 2ł. I. Ja plup̧art sont des plantes déjà observées daus la Caroline ou la Géorgie. On y remarque cependant quelques gुenres de l'Lmérique équinoxiale qui n’ont pas été obseryés plus au nord; telles sont lés espéces sui-

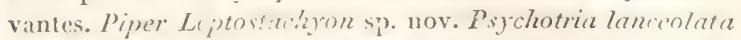
sp. nov. Chiococea racemosa Willd, Plusieurs especes de Tillandsia dont on n'avait encore observe qu'une seule espece dans la Caroline (Tillumblim usneroides), tumlis que cette collection quoique peu nombreuse en renferme 5 especes qqui, par leur port se rapprochent plus des e péces de l'Amérirpue innatoriale, Rhizoploora mangle L., Marunta arundinacea IVilld, ets.

Parmiles espères renfermées dans ce catalogue, il y en a 22 nouvelles dont lauteur at donné les caracteres. AD. B.

zí. Snumenatio puanteras horti regii botanici

Berclinensis altera, auct. K. F. Lrnk, in-S. Pars

2. Pris, 2 rix $\vdots .2$ gr. Berolini; 1822 ; Reiner.

Crlte sceonde partis comprend les plantes des íf dere

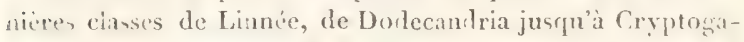
mia. Le jardin botanique de Berlin contient anjourd'hui io, 323 plantes, sans compter un grand nombre diautres qui ne fleurissaient pas encore.

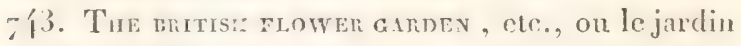

fleuriste britannique; ouvage destiné aux dames, anx messieurs, et aux cultivateurs, en génétal, contenant quatre figures col. des plantes les plus belles et les plus curieuses, qui peuvent ètre rultivées avec avantage sous le climat de la Cirande-

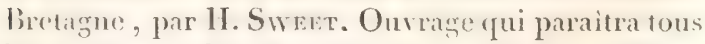
les mois, par liv. à compler du 1'r mars. Pris, 3 sh. par no.

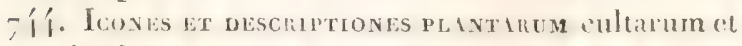
colendarum, additi colendi ratione. Auce. Th. Rercinexblek, fasc. 5 (4 6. Prix, a risd. chayue liv. Lipsix; 1822 ; Baumgartner. 
Ces denx livraisons conticunent: Eillardiera wa melem.Fillard mutabilis sulisb. - Muporum andrale. - Fiscat-

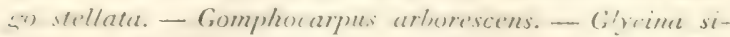
nensis. - P'soralea verrucosa. - Psor. aphylla. - Iris dichotoma. - Houn surnosa. - Puluenea retusa. - Monsonia filia. - Mons. pilosa. - Mons. speciosa.

z in der arzneihunde gebraublichen gewathse. Description et représentation fidele des plantes oflicinales, ete.; par F. G. Harxe, tom. 7. liv. 5 et 8 , et $t .8$; liv. I et f. in-f. aree pl. col. Berlin; 1822 ; chez l'auteur.

; 6 6. Die Vrasissmeavicut Artax Deutschlands, ou les esprees de myosotis ou germandries de IAllemagne, par J. L. Rexcmistcm, in-s, avec if pl. color. Prix, r fl. $55 \mathrm{kr}$. Nuremberes; ros?.

T.es espreces décrites et représentées sont les suivantes: Myosotis manna. Will. - Wyos. palustris. Witz.-.11. Lariflora. Redha. - M. Parviflora - M. Strigrulosa. Rehb-M. Repens. bonn. - M. Alpestris. Schm. - M. Silsatica Fhrh. - H. Cesprtosa. Schulz. - 11. Sparsitora. Mik. H. - Suneolens. Kil. - I. Lilhosyermifoliu. Hornem. -

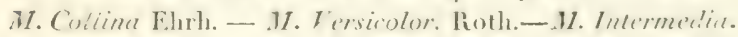
I ink. - W. Arvensis. Sibth. - M. Arvensis. Var. Multinunlis.

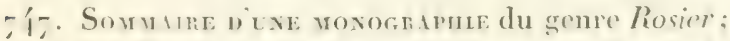
par .J. Procuras, membere de la socichédiagrieulture du dipartement de Srime-et-Oise, etc. in-s'. de 36 . Prix, a fr. 25 c. Paris; 11 ane. Inzard.

L'antene sest borné dans cette petite brochure a fire annater lesespreses qu'il croit devoir composer le sedure du

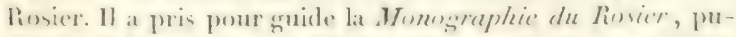
blice en 1820 par 31 . Linlley: son ouvrage mime peut itre considere eomme ertrait de celui-ci. It donne le tablean de

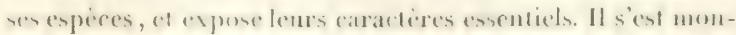

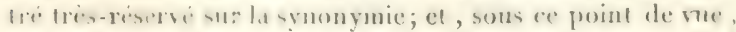
1) a degi asee protence : en effer, dans un gener ou il est st 
rifficile d'établir les espèces, sans leequel la nature a placé lerigritaux qui nous flattent le plus, et ru'elle semble avoir variés exprès pour angmenter nos jonissances; dans ce genere, disons-nous, la synonymie exigerait un travail long, pénible, et souvent méme inulite, s'il fallait la présenter complete. D'ailleurs, elle est trè-filendue dans l'ouvrą̧e de Lindley. L'auteur comprend dins le genre Rosier 69 espices; toutes ont été décrites atrant lui. Il les classe d'abord en denx sertions; dins la premier sont les especes dont les fruits sont globuleux et prespute ronds: (dle, sont partagées en cinc tribus, dont les caracteres n'étant pas pris sur les mèmes parties ne peuvent ètre propres a faciliter la détermination des especes; ce qui nous semble un défaut de classification. Lit denvieme section renferme les especes a fruits ovales ou d'aupres formes variahles; clles sont présentées en sir tribus. Cetle clissification est une cojie de celle de Lindley, excepté que ce naturaliste n'a pas jugé devoir signaler la division on deux grandes sections qui, chez lui, est tacite, et òte ainsi a Ia critique des armes pour combattre le naturaliste anghlais. A la fin sont notées quelques espèes douteuses, ou que l'autcur n'a pu rapporter à celles qu'il admet.

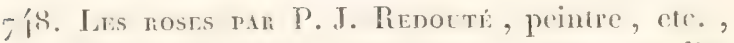

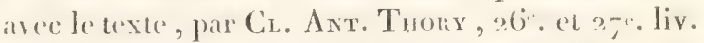
in-fol. de 3 f. et 6 pl. Prix, 35 f. la livr. Intu. de F. Didot, à Paris; 'Treuttel et Wurtz.

Cet ouvage, bien connu par la belle cxécution des planches, qui en font la plus grande partie, forme déjà 27 lisraisons in-folio, renfermant chacune six planches tircés en couleur :

Une tramle partie ne sont que des varibfís et des sousvarietés doubles produites par la culture, et dent, comme on sat, le nombre ent immense. Les epprees simples ot sanvigen y sont ausci liguries; mais on respelle que celles-cine soient

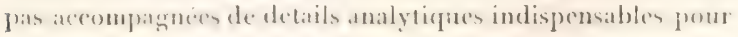
fers botanistes, tels gut la forme des fruits, des stylen, des ipines, la disposition exate des dentehres, tons caratcteres nevessieres pour bien fiver les especes, et dont liabence remel

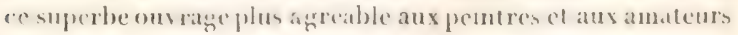
de flemrs quiutile anx butanistes proprement dits. 
Les deux biraisons que nous amoneons renferment: $1^{\circ}$. la Rose i cent feuilies prolifire, monstruositi asse fréquente ot tris-curicuse; 2". Rese collina, var. Monsoniunu; 3".

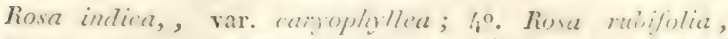
I'ursh, jolie espece at fleurs simples, des Etats-Cnis ; j". Fowe

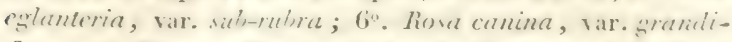

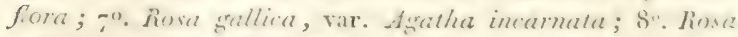
Ealliar, var. mateda; $g^{\circ}$. Rova reclimata is fene simple et double; 10 . Rosa hispiela, var. argentea; $11^{\prime \prime}$. Fiose sentematinne, espece à fleur double assez remarquable par sa forme.

A.r. B.

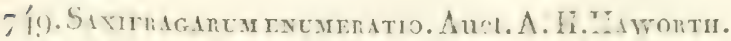

Accedrnt revisiones plantarum sucilentarum, in-8. Prix, ro sh.6. d. Londini; $182 x$.

750. Die Dretschey Broveerestratche(Rubi), ete., ou les Pinders -auvares le l'Allemagne, décrits et

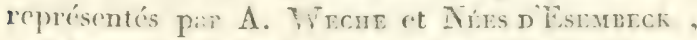
in-fol. arec 8 , l. et 19 f. de t xie en allemand et en latin. Bonn; 1822 .

Cet ourrage est imprimé anx frais des éditcurs qui n'ont trowvi que 55 souscripteurs. On n'a connu juscrüici en Allemache que deux espices de muricrs sauvares, le fiubus frutirnsus et le Cosius; les auteurs en font connaitre beaucoup dinutres, tols que: liubus plicatus, fustigialus, affinis, cortifolius, rhamnifolius, tomentosus.

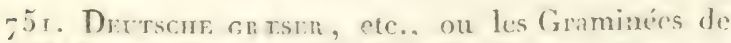
1.Allomatge, par 1. IVrane, in-8. liv. g. Lemgo:

1822 ; Meyer.

Cette livraicon intient: Lappueso mecmosa. - Rriza

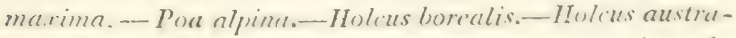

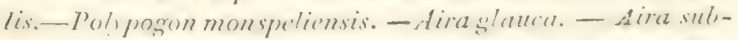
spicatra - Jestuca tenuifolia. - Festura primata. - Dromus relutimus. - Eromus asper. - Arumelo fiesuedena. -

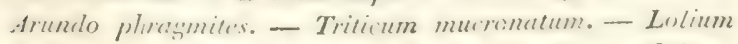
icmulentum. - Trimpleorum triquetrum. - Siripus fuituns -Juncus botruirus. - Luzule palleserns. - Triglochin maritimum. - Carex dasulliana.-Carex allue-Carex mmentosa. - Carex filiformis. 
752. Geschichte der merkwurdigsten Pilze, ele, ou llistoire des clinmpignons les plus remarquables, trad. de l'angl. de $\mathbb{Z}$. Bolton, par Ch. L. Windoenow ; conti"uéc par Núes d'Esembeck, in-8. tom. \{. avec lif pl. Col. Berlin.

Ce volume contient la description de $24 \mathrm{x}$ espreces de chatmpiognons des environs d'Ilalifax, représentés en goo fig. peintes, grav. et col. par l'auteur. 7. rixd. $12 \mathrm{gr}$.

753. Deetsche lichesica, ete., ou recueil des Lichens de lidíenragne, arec des remarques, par II. G. Fionke, in-fol. Livraisons, 7.-10. Rostock; I822; Stiller

Chaque lis raison est composée de ro pl. in-fol., ct d'une fcuille de texte, dont le prix est de 2. rixd. La classification des genres est adoptée par $\Lambda$ charius dans son $S_{j n o p s i s}$ Lichenum.

754. Critis's вотыnicil magizine, ete, ieprésentant

les plantes d'ornemens ćtrangères cultivées en pleine terre, en orangerie ou en serre chande, dessinées et colorices dapres nature, par J. Siss, MI. D. memb. de la soc. roy. et de la soc. Liun. de Londres. (n". 433, fév. $x 823$.)

Ce cahier contient les figures et les descriptions des espicces suivantes:

23-'s. Hedychium flasum, Wallich, in Roxb. flor, ind. r, p. 3 I.

2379. Schizopetalon Walkeri.

Noureau scenre de cruciferes encore incompletement connu, puisqu'on n'en a pas vu le fruit; il est cinsi caractérisé dans le Botanical magazine.

"Cas. cylindraceus basi cequalis, clausus.

"Petala orate inciso-pinnatifide. Stromata gillensa, "approximata."

Cette plante parait par son port se rapprocher des fingsmum. Elle croît an Chili.

2380. Astragalus Stipitatus. Don. Mss. labite le Nepat et la chaine des Himalaya. 
2351. Boltonia glastifolia. Mich.

2382. Dirodian ixioides.

le senre brodien a dé etabli par Smilh dans les Transictions Linncennes, vol. X, p. 2. Cette nouvelle espiece est du Chili.
2383.
Azalea pontica, var. alliflora.
An. B.

-55. The metrinea megistra, ete. chaque numélon renfermant huit fig. color. de plantes evotiepues dessincés dapres les plantes virantes, par Srovenur Eowards et autres. ( $n^{\circ} \cdot 9^{6}$, rer. fév. i 823.$)$

Cc numéro renferme les espèces suivantes :

983. Costus afer. var. A., glaber var. B. pubescens.

\section{hab. Sierra-Leone.}

684. Bidens procera

(Coreopsis angustifolia Pavon. Mss.)

hab. Mexico.

685. Globularia Lonsifolia. Wilh. Spece. Plant. hab. Madere.

686. Eulophia guineensis.

Ce nouveau genre de Ia famille des Orchidées avait él.

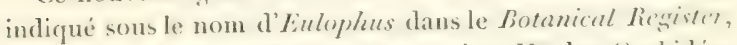

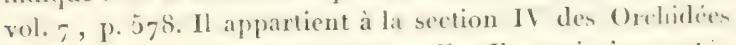
du Prodromus flora: nose Hollandice. Il est ansi caractirisé dans l'article que nous extrayons.

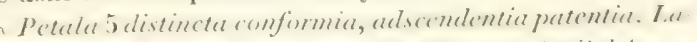

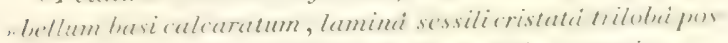

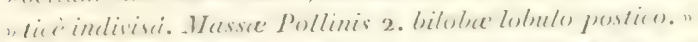

La plupart des enpeces de ce senre croisent atu calp de Jome-Lipirance.Celle figurée dans ce numero est de SierraLeone.

687. Salvia splendens.

Espiec nouvelle, voisine du Salstu tubifira. Elle habile le Brésil.

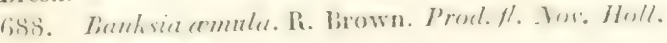

689. Aristolochia labiosa.

lcones aristolochia ringens. Link hort. Berdl. non. Vahl. symb. hab. Brésil.

1. alier rontient en onter un appendice renfermant des 


\section{Botaniqué.}

notes sur des articles des volumes précédens, et me table Mplabétique de toutes les espèces décrites et figurées dans l'ouvrage.

AD. B.

756. Remaneues sur la physiologie des fibres de la racine, par J. Menina. (Edimb. philos. Jour'n. n". I 5 , janv. I 823, p. 37 .)

L'anteur rapporte des exemples de plantes qui peuvent croitre long-tempss, quoique privées de racines, telles que les plantes grasses; d'autres qui n'absorbent que de l'eau ; ct il croit pouvoir en décluire que les racines ne sont pas les organes particuliers de l'absorption dins les plantes. $\Lambda$ d. B. 757. Comaentare surle Hontus malabitices, part. 1 $^{\text {re }}$; par F. Haxulton. (Trans. of the Linn. soc. Vol. XIII, pars 2, p. 474-560.)

Ce mémoire, comme son titre l'indique, est un recueil dobservations sur un grand nombre d'espéces figurécs daus l'Hortus malubaricus de Rheede, et particulièrement sur les erreurs desynonymie qu'on a commises en citant cet ouvralge. Un pareil mémoire est peu susceptible d'un extrait almége; nous nous contenterons de ciler quelques-uns des articles les plus importans. L'abteur pense que le MLarotti, Hort. malal, p. 65, fig. 36, qui n'a ćté cité par aucun auteur moderne, doit former un genre nouveau qu'il nomme Cunzoria diz nom de Clilmori qu'on lui donne au Bengrale. Lapocitionde ce genre parait encore douteuse; l'auteur en décrit deux espéces avec beaucoup de détails. Il donne une description intíressante de 3 espèces, dont deux nouvelles du genre Em. blica. A l'occasion du Cata Oitasa (Hort. mal. 97, espece de Mlorinda, il décrit 7 espéees nouvelles de ee genre, il dicrit aussi 4 espeices nouvelles de Galega en parlant du (o/nnil. (Hort. malab.) P. ro3, fig. 55.

A l'occasion du C'arue de l'Hort. malat., p. 107, fig. iqu'il rapporte an Laurus coessin, il donne lat description de 5 espèces nouvelles du genre Laurier.

En gémíral chacun de ces articles renferme des oberrsattions précieuses sur la botanique de l'Inde, sur la distinction des cespeces, sur leurs unages, etc. Les descriptions sont trim complètes et faites avec beaucoup de soin.

Av, B. 


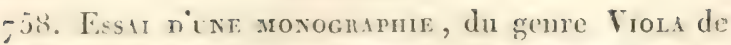
Limú, romprenant toutes les espéces observées jusqǚ présent en Amírique, par Lrwis, D. ne schweinitz. (Americ. Journ. of Sc. and Arts, by Sillinian. Vol. V, n' $\mathbf{n}^{\circ}$, juin, I822.)

Lauteur doshe dans celte monograghie la description de 30 espiers orrissint tontes dims l'Amérique septentrionale dont 5 son nomvelles; elles portent les noms de tiola

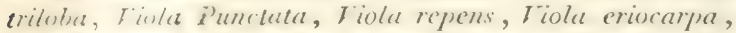
7 iolaminla, nous ficons remaxquer que cette derniere n'est pas la / inlastriuta de 11 illdenow et de la plupart des auteurs, et que l'auteur a donné le nom de l'iolu ochroleuca à l'esfive décrite sous le nom de $r^{r}$. striata par cesauteurs : nous ne sarons qu'est-ce qui a pu determiner l'auteur à faire ce changement.

Les descriptions et la synonymic sont faites avecbeancoup de soin et de détal; elles sont en latin et les observations sont en anglais.

$\Lambda \mathrm{D}$. B.

959. Moxographie de genme Sivifrare, par M. D. Don. (Tians. of the Linn. suc. Vol. XIII, pars 3, pag. $341-452$.)

L'auteur a cherché principalement dans cette Monowraplice à fixer exactement la limite des especes, et a donner une syonymic exacte et une distribution naturelledes especes si nombrenses de ce enenre; pour atteindre ee but il a sommis depuis plusicurs annes un grand nombre deeprees, a la enture, et il s'est assuré que plu-ieuro n'etatient que des varite's; c'est anni quil a diminué le nembre des especes, quoique en ayant ajouté plusieurs nouvelles.

Il a divise le genre Satiffage on 5 sections fondés sur des caracteres deduits des organes de la fructification, et subdivisées alle-mimes d'après la forme de's feuilles. L '́ctendue de cet article ne nous permet pas de rapporter le caractise de res sections qui paraissent en général tres-naturelles; nous riterons senlement quelques-unes des espices les plus connues qui s'y rapportent.

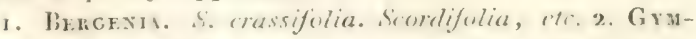
xopras. S. neum. S. mmlerosa. S. cuncifolin. S. stellaris, 


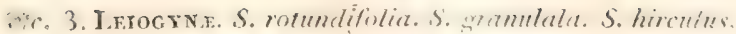

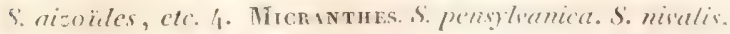
S. darurica, etc. 5. Simfrade vara. Celte demiere section renferme toutes les espèces à ovare infericur.

Le nombre total des espèces est de 104 . On remarque les suivantes qui n’avaien: pas encore élé décriles, on qui avaient élé confondues avec d'autres espèces.

S. spicata. (S. gram Purs.) - S. Nolsoniana, S. armernta, et $S$. nudicualis, des cùtes occidentales de l'Amérique seppntrionale. - S. liructeatu, Sibéric oricntale. - S. mutans, Unalaska. - S. myosotifolia, Sibérie. - S. brachypoda, Nepal. - S. hispidulu, Nepal. - S. cherleriö̈les, Kantschatka. - S. lonģiscapa, Sibérie - S. fumbriata, côle occidentale de l'Amérique sepe.-S. parnassifolia, Nepal.S. obtusifula, Espagne. - S. latifida, kispagne. - S. incurvifolia, Irlande. - S. denuduta, Écosse. - S. pulihella, Allemagrne. S. Andirola, Péron. - S. stellata, Pérou. S. Paronii, Pérou. - S. laetevirens, Ecosse,

Ce mémoire n'est accompagné d'aucune planche. $\Lambda$ n. B. 7o. Mlimorte sur le gexre Musa, et monographid: de ce genne, par L. Cols. (Mém, de l'acad. des sciences de Turin, tom. 25. 1820.)

L'antemr a donné un précis historique des divers antenrs 'pui ont parlé du Bananier, du nom qu'il porte dans divers pays: il fait dériver le nom de Bananier du nom Balı. quil porte dans l'Inde; et celui de Musa, du nom arabe: Mauz, dont Avicenne a fait le mot Musa.

Il décrit avec beaucoup de détail un individu du Muss: Sapientum, qui a fleuri dans le jardin de Turin; il rapporte: plusicurs faits curieux sur sa végétation, son developpencul et sa culture; enfin, passant à la description des espèces, il fait remarquer que, la plupart ne portibut pas de gratine, il est difficile de fixer si re sont des varietés ou des esperes: néanmoins adoptant lopinion la plus sénéralement rerme. que ce sont des espèces listinctes, il décrit les espéces snivanles :

$\$$ I. Spermophora on bananicrs dont les fruils renferment des graines.

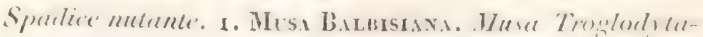
'I'ome I. ${ }_{2} 6$ 
ilum, var. B. Willel. 2. Mr-a lin !umph. 3. Musa Sxuffistris. Rumph.

sparlice erecto. 1. Husa unswoscopus. Rumph.

\$I. Asperme? ou Bananiers qui ne portent pas de semences.

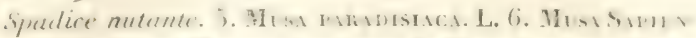

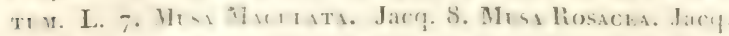
9. Musa cсmixata. Muse Simiarum. Rumph.

Spadice erecto. 10. Musa coccrive. Andr. Willd.

C. néncire, dont il a té tire des exemplaires à part, esi

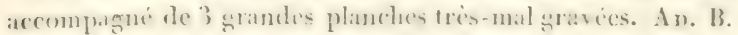

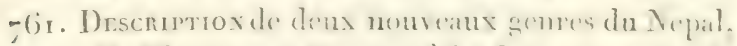
par N. Warich. ('Fims. of the bimn. suc. vol. 13. pars. 2, p. 6o8-6r4.)

Ces deux semresapparticnuent, le premier a la familledes Tabiées, le second a la famille des Scrophularinces ou perat-

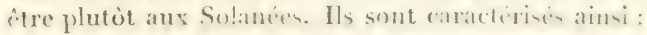

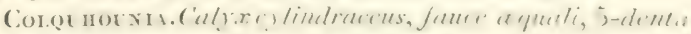

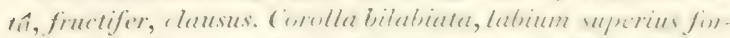

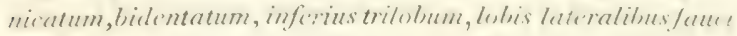
ampliate utringue insereis, intermedie minure, intespro.

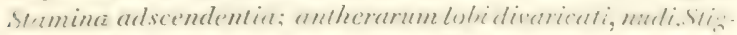

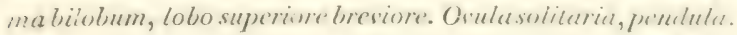
Arhenia maxima, alata; perispermum appiosum. Iimlerge erectus.

\section{Colquhounia cocciner.}

Asbrisseau grimpant, velu is fenilles opposede ovalen. avales, dentés; a fleurs axillaires prosque verlicilien. ilabite dans les montagnes du Nepal.

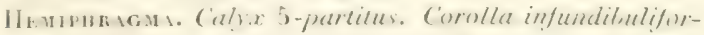

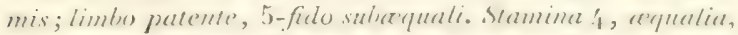

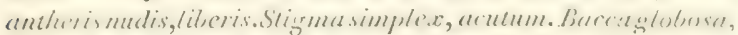
ersuce, incomplete litoculuris, polysperma, disst pimentes placentifero sursum fixo.

1. Hemiphragma heterophyllum.

Plantengète, rampante, velue, a feuilles de tensformes : le

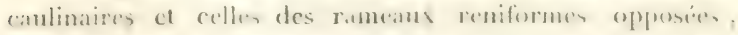

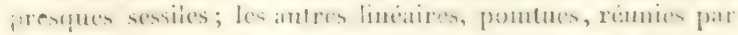
faiscraux. Fleurs solitaires, axillaires.

A. D. B. 
762. Centarnes espieces de Carduus et de Cnicus qui

paraissent ìtre diö̈ques; par Tr. Sмrтा. ( Trans. of the Linn. soc, vol. I3. pars 2, P. 5y2-6o3.)

L'auteur rappelle que les plantes dioiques, regrardées d'aloord comme cxtrêmement rares parmi les composées, ont été indiquées depuis par plusicurs autenrs dans différens senenres de cette fimille, tels que les Tussilngo, Gnaphalium, SerraIula, Baccharis, Molina, Petrolium, Erachylena. It a fait la meme observation sur plusieurs C'nicus et Carduus; ainsi les Chicus arvensis, pratensis, palustrisncaulis, erisithales, etc. les Carduus rivularis, chius, rigens, serratulö̈les,paniculatus, etc. paraissent constamment dioï ques ou polygames, présentans souvent des pieds hermaphrodites et d'autres uniquement fonelles; dans quelques - uns seulement on trouve des pieds qui ne portent que des fleurs máles. Les individus mâles diffèrent ordinairement parleurs formes extérieures des individus femelles.

AD. B.

763. Onservations sur le Chrysanthemum indicum de

Linnæus; par J. Sabine. (Trans. of the Linn. soc. vol. 13, pars 2, p. 56 r -5 j8.)

Ce mémoire est entièrement composé d'une discussion de synonymic sur les divers auteurs qui ont parlé de cette plinte maintenant si commune dans nos jardins, et rapportée alternativement aux genres Chrysanthemum, Anthemis et Matricariu, sous des noms spécifiques très-variables; mais l'auteur ne fixe pas dans lequel de ces trois genres cette espèce doit être rangée.

Av. B.

764. Remarnces sur l'hypmum recognitum, et sur plusieurs espèces nourclles de Roscoea dans une lettre de. J. Ed. Snitr, à V. G. MIntox. (Trens. of the Linn. soc. vol. 13. Pars 2, p. 459-464.)

Cette lettre renferme plusienrs observations sur les plantes cultivées dans le jardin de botanicque de Liverpool, el sur quelques plantes des environs de cette ville.

L'anteur a remarqué que plusienrs espéces de Curruma sécretent et retiennent dans l'aisselle de leurs bractés un fluide aqueux trés-abondant; il décrit 4 espèces nouvelles de Roscoede, genre de la famille des Amomies établi par le 


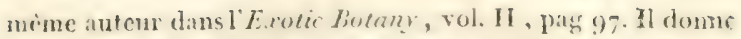
a ces quatre especes les noms de $R$. gracilis. - R. clatior.R. spicrla. - R. capientr. Teurs caractires sont diduite principalement de la forme du calice.

Le jardin de Liverpool renferme jhesieurs plantes remar'puables des Inder et du Nepat, et un srand nombre de Fou-

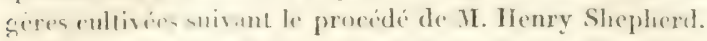

Cest anpries de la ville de Liverpool quon a observé pour la premiere fois l'/e) pmum recognitum décrit dansla Flore britamnica et dans l'Enghish Dotany. L'auteur domne plusieurs observations sur la synonymie et la distinction de cette espéce que MM. Ilooker et Taylor avaient réunic à l'hyjpmum proliferum.

AD. B.

765. M. D. Dox a lu à la société wernérienne d'lidimbourg, dans la séance du 16 novembre $\mathbf{8 2 2}$, un mémoire sur li's Meilastomées dans lequel il décrit i n nouveaux genres de cette famille. (Edimb. Philos. Journ., $n^{\circ}$. 15 , janv. 1823 , p. I8o.)

Ad. B.

966 . Le doct. Wallis, de Calcutta, connu dans le monde savant pour habile botaniste, est revenu dernierement it Cilcutta apres un royage d'un an qu'il a fait dans les montagnes de Népaul : il communiquera sous peu le résultat de re voyage qui promet d'augmenter amplement nos connais-

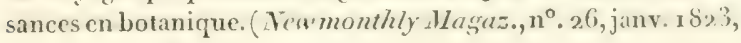
sup. hist., p. 68.)

\section{MINERALOGIE.}

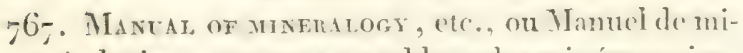
néralogie, contruant un tableau des minéraux simples, avec la deseription et Jarrangement des roches des montagnes, par R. Tiursoy, prof. dhist. natur. il l'université d'Fdimburgh. I gros vol. in-s. Pris, 15 sh. cart. Londres; llurst, Rolinson.

768. A ststex of vixeralogr, Systeme de mincialogir, par R. Junsox, 3". edit. considérabloment. augmenté, ares un grand nombre de pl. 3 rol. in-8. Prix, 2 $1.16 \mathrm{sh}$. eart. Iondres; Huxal, Robinson. 


\section{Minéralogie.}

Ces deux ouvrages d'un savant bien connu ne se trouvane pats a Paris, nous n'en pouvons procurer l'analyse.

76 . A treatise on the external, chemical, etc., ou

Traité sur les caractères extérieurs, chimiques et physiques des minéraux, par R. Junfeson, prof. 3c. édit. in-8. Prix, i 2. sh. Londres; Hurst, Robinson, etc.

$770 . \Lambda$ TREATISE on DHMONDS and precious stone's; Traité sur les diamans et les pierres précieuses, comprenant leur rapport avec l'histoire naturelle. et le commerce; un précis détaillé sur leur valeur artuelle, les règles de praticque pour apprécier les diamans bruts et polis, la manière de découvrir les imitations des picries précieuses, enfin celle de les tailler et de les polir; par J. Mawe. $2^{2}$. édit. in-s. avec des pl. enl. Prix, 5 sh. Londres; Longman.

77x. Ser la calaite ou Turquoise de Perse et le litzuli (Philos. Joum., no ${ }^{\circ} 5$, janv. 1823, p. 19?.)

Berzelius a trouvé que la calaite est composée de phom phate d'alumine, de phosphate de chaux, de silice, d'ovide de fer et d'oxide de cuivre. Le lazulite est un composé t! phosphate d'alumine, de phosphate de manganese, de phosphate de fer et d'oxide de fer.

7,2. M. Brens NeErchird a nouvellement communique it l'Institut un travitil intéressant, surtout pour la partie histerique de la Tourmuline'; son auteur y donne des éclaircissemens sur l'origine du nom de celle pierre, et prouse an'elle était connue en Lurope plusieurs années avant que $L^{-1}$ mery en cut parlé, quoiqu’il cùt été regardé comme la personne qui en avait donné la première connaissance. Messieur's les professeurs brongniart et Brochant ont été nommés rapporteurs.

7-3. Grotte ne Ilix,--L'académie de Bruxelles ayant d..siré atvoir des renseigriemens positifs sur la grotte de llan of le nouveau passage que l'on y a découvert de puis peu, a desigence pour aller examiner les lieux, et lui faire, a ce majet, un rappore circonstancie, deux de ses membere, MLit. Kirctix 
et Quetelet. La relation qu'ils ont publuce de latu woyage. offe des observations physiques, zoologidgues, folminges el minéralogiques, intéressantes.

Ces deux académiciens ont visité la carrière de Saint-lir-my, d'oì l'on tire ce beau marbre rouge veiné de blane, de vert et de bleu, gui est un des plus bean de l'Furope. La: carriere s'éleve a 6 is metres att-dessus du niveau de la petitc riviere de l'Omme, et a 27 I au-dessus du niveau de la mer. Malheureusement, disent les voyageurs, les moyens de transport sont trop difficiles et la carricre parait entierement abandonnée.

Cette montagne renferme, ainsi que celles de Rochefort, de la galene, du fer sulfuré et hydraté, dont on a commence: lextraction, mais qui est abandonnie aujourd'hui a cause des difficultés de transport et l'éloignement des usines.

On exploite à Vedrin, dépendant de la commune de Frisée, à une lieuc de Namur, une mine qui contient, comme celles de Saint-Remy et de Rochefort, du fer sulfuré et hydraté, de la blende et de la giléne. La découverte de cette. mine remonte à l'an 1619 . Elle a commencé à étre expluitie. en 1632 ; abandonnée en $179^{2}$, elle fut reprise en $x$ Sofi. Le minerai s'y trouve en grosses et petites boules, ou en srains plus ou moins fins, enveloppés d'une gangue de fer hydraté ocreux ou compacte, de fer sulfuré et d'irgile. Les bonles sont formées de galene à larges facettes : les grains constituent un mélange de plomb oxidé et carbunité. On méle ces minerais avec des schlames ou résidus des anciens travaux, dans des proportions telles, que le produit de charpue fonte, qui est de 3,000 livres, rapporte au moins 30 pour cent de. plomb. (Journ. d'agricult., etc., de's P'ars-Bis, tom. 1'), p. 55, janv. 1823.)

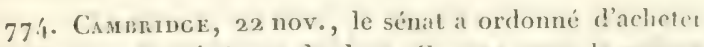
la collection de minéraux du doet. Chark. pour Ja somume de 1500 liv. ster.! Hew montl. Hagraz., n". 25 janv., p. 1 i.. 


\section{PALONTOGLAPHIE}

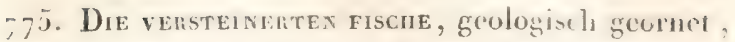
ou deseription géolugrique des poissons fossilus; traduit du fiangais de MI. de Bearvinte, aree des remargues ct une pélice, par J. F. Kruger; in-8. Quedlinbourg, 1822 . Basse.

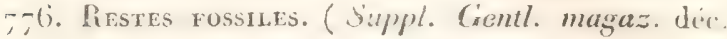

$$
\text { I322, pag. 627.) }
$$

Les ossemens fossiles d'inimaux appartenans au monte. primitif se trouvent en Suisse dans une quantité proportionnce à l'attention quion y porte; et il arrive souvent fu'on les jelte au feu avec les charbons de terre parmi lesque', ils se trouvent. Dans la réunion des naturalistes suisse' le selte année, M. de Lue de Creneve at présenté str ca sujet un Biémoire dans lequel il cherche à réfuter l'assertion: de Ir.. Cuvier, d'aprés laquelle il ne serait pas prouve que la température des climals du Nord ait changèe depuis t, inmpis où l'on y trouvait des animaux de l'espéce de lieli: phant, de l'hipunopotame, du rhinoceros, de l'élasmothercum ct du lapir. A prés quelques observations préliminaires, M. de Lue exprime son opinion, savoir : que la nature de l'atmo uphere a pu suive les changeraens qui se sont opérés sur terre ct sur mer. L'existence de l'éléphant, plus grand sur le sol qui sétemi depuis la sicile jusqu’a la Sibérie, pronverai aussi le changement du climat qui n'est plus ce qu'il état jaclis. L'auteur conclut, avec son illustre oncle, que "le d." luge a produit une srande révolution sur la terre, meine a l'égard de l'influence qu'exercent les ravons du soleil; que: la temperature itait autrefois moins vaxice dans les differens pays qu'elle ne l'est à présent, et gu'il en éfait de mène a l'ezard des saisons. C'est pourquoi les grands animanx ponvaient alors subsister dans les rlimats oi ils ne sanderent vir w daus l'époque actuelle.»

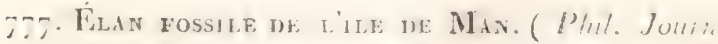
n. 15 , jauv. 1823, p. 198.$)$

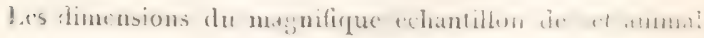




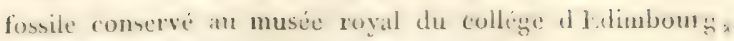
sont :

Hauteur juscru'a l'extremite de l'apophyse de lit premice vertèbre dorsale. ........6 6 pieds I pouce.

Hauteur jusqu'à l'angle antérieur supérieur de la scapule. ........ 5

Longueur depuis la première rertèbre dorsale jusqu'à l'extrémité de l’os coccyx.

Ilauteur jusqu'à l'extrémité de la corne druite............... y

Diamètre latéral ou horizontal du thorax à la partie la plus creuse, c'est-àdire à la onzième côte. dorsale jusqu'au sternum ì la jonction de la huitième côte. ...... 2

tne belle gravur: de cet échantillon, faile d'apries le di... sin de Lizars, paraitra daus le prochain volune du suppli. ment à l'Encyclopédie britannique. Ces restes, les plus pitrfaits que lon ait jimais connus, ont ete deterrés dans la paroisse de Kirk Balliff, et c'est le due d'lthole qui e-c parvenu i les procurer au muséc royal. Ils elaient enfoncis daus me marniere de coquilles mobile dams laquelle il ? avait une quantité de branches et de racines d'arbres inalement entoncées. Lat marnière était couverte d'une couche d. sable, et, pir dessus le sable, chait une eouche de tourlu compose principalement de petites hrancles et de feuille pouries. Il yavait, au-dessus de la tourbe, de la terre vigitale. ordinaire.

\section{PII YSIOLOGJE.}

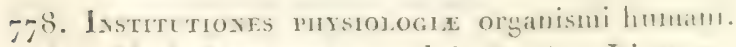

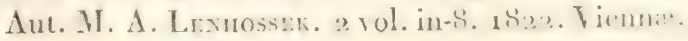

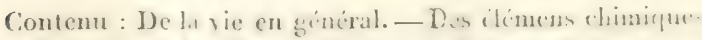

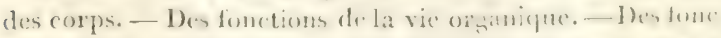

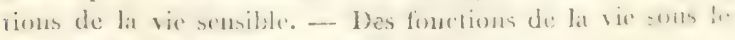
fapport de la génération: - Sur lat mort. 


\section{Pligsiologsie.}

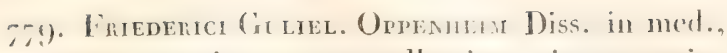
sistens experimenta nommlla circa vitam arteriarum et circuitum sangunis per visa collateralia, in-4. I,822. Manheimii.

- So. Fximen cxpérimental des lois des fonclioms vitiles, avec des olservations sur la nature et ip traitencut des malidlies internes, par A. 1'. W. Phlnp. M. D. reproduites en partic aver perminsion elu président de la socicté royale, d'aprés hes Transactions philosophiques de 1815 et 1817 , ance un rapport de linstitut national de france, sur les "spériences de M. (iallois, et les observations sur. ce mème rapport. $2^{\mathrm{e}}$. édit. in-8. Prix, in sle. ( r liv. 5o.) Londres, T. a (i. Lnderwood.

$78 \mathrm{I} . \Lambda$ view of the structure of the stomach. Apereu de la structure, des fonctions et des maladies de l'estomac, par T. H.1ve, in-8. i 2 sh.

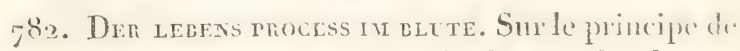

la vie dans le sang ; examen fondé sm des déconvertes microseppiques, par Cin. 1I. Scrutzz; in-s. avec fig. Berlin, 1822 , Reimer.

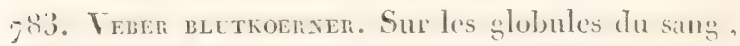
par J. Ch. Schnet, in-f. aree pl. Wertzbur fir, 1822 , Becker.

Recueil de tout ce qui a été écrit juscqu’ici str celte mintière.

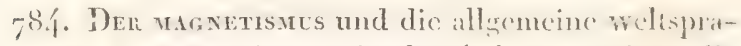
che. Le magnétisme animal et la langue miversedte. de: tons les peuples, par II. Westrinsx, in-i'. Creveld, I822, Funcke, x fl. 45 kx.

Ourage mystique, en faveur du mesmérisme. La langme universelle est la laugne du magnétisme ou celle des coprita. 785. An Ixet an into the mature and properties of the

Blood, cte. ou exitment de la nature el dis pro-

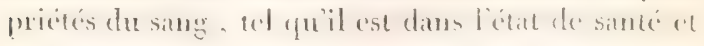




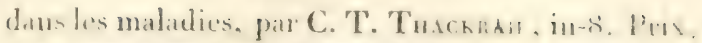
Es sh. cart. Lombles, Murst, Robiusmen, it compl.

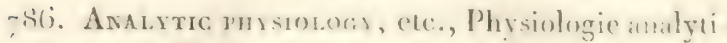
que, par S. Hoon, in-s. prix, 10 - sh. Jondure.

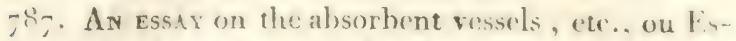
sai sur les raisseatux absorbaus, dimontrant qü. leur action nest point exposce it irre inllurncér. par le's agens artificiels employés ordinairement. par H. SElri.e, in-I?; prix, I sh. Londres.

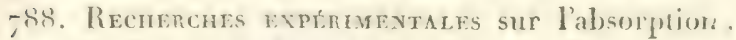

et l'exhalation, lues à l'institut de Frince pat M. Fonćn. (Journ, dephys siol. exp. 1. 3, 1". 1.)

Ce travail est encore inédit. M. Fodera a pour but de demontrer que l'exhalation qu'il appelle transsudation, et l'ab. sorption qu'il apprelle imbilition, ne sont qu'un mème phenomine, qui est dù a limbibition des differens vatsscaus opérans, dans le premier cas, de l'intrieur du vai ssean a les. rérienr; et dans le second, de l'extériens a limterient.

L'auteur rappelle que .1. Magendie a rrouve que labsorp

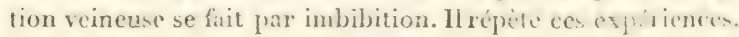
et en fait dans le sens inverse; par exemple, il injecte num substance vénéneuse, avec toutes les précautions convenablen, a l'intéricur d'une portion d'artere comprise entre deus ligi

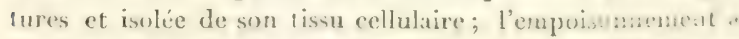
lexu : mème résultat en se servant d'une portion de wine wat

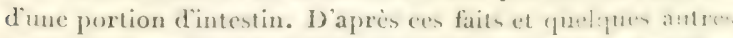
moins importans, M. Fodéra conclut que lextatation ai a quan phenomene de transsudation à travers les parois lanculires. Wautres expésiences servent il lauteur a chablir que l: double phénomine dexhalution et d'absorption peut so. pérer dans foutes les parties, et que les lirpuides, dont wilus s.

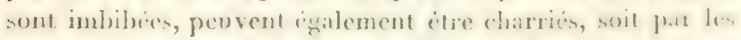
vaisscaux lymphatiques, soit par les vaisseaux artiriels el vei neux; mais lanteur ajoute qu'on ne doil point regardes ees

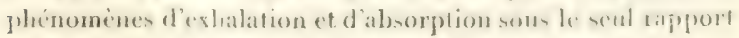

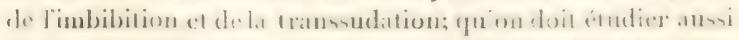

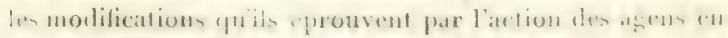

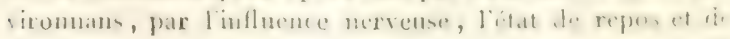


mouvement, l'énergie de la circulation, les affinités des substances avec les tissus, les dérangemens produits par les maladies, et l'elaboration que les flnides subiscent pendant que l'absorption et l'exhalation ont lieu. Mr. Fodéra a repris les expériences fitites en Angleterre par Darwin, et plus récemment par Wollaston, lirande et Marcet, qui tendiaient a prouver que diverses substances se trouvaient mélées à l'urine sans avoir passé par les vaisseaux lymphatiques ou sancuin., et il leur a fait subir une ingéniens: modification, qui pronr. l'extrème rapidité de l'absorption, et démontre aussi que l, prussiate de potasse injecté dans l'estomac, et trouvé danl'urine, y est conduit par les voies circulatoires ordinaires.

Ce mémoire, riche en faits nouveau, est propre à jeter le plus grand jour sur un des points les plus importans de la physiologie : c'est un beau supplément au travail de M. Magendie, sur l'absorption.

D. $F$.

789 . Note sur l'exhalation et l'alsorption de l'azowe dans la respiration, lue à l'acadómie des sciences. par Mi. Envaros, D). M. 'n déc. I \&̇es. (Journ. de physiol. exp. t. 3, no. $\mathrm{x}$. )

Les sivans, dit MI. Fdwards, ne sont d'accord dan.s l'acte de la respiration que sur deux points: $x^{\prime \prime}$. disparition d'une yortion de l'oxigène de lair; $2^{\circ}$. prexhuction d'acide carbonique. Sur tous les autres il y a divergence, d'abord, relativenent à la cruntité d'oxigrene qui disparait, comparée à celle de l'acide carbonique produit; les uns trouvent que ces quantite sont sensiblement ésates, les autres qu'elles different d'unc manière marquée.

$2^{n}$. Relativement a l'azote, mème différence de résultats; des savans ont constate l'absorption de l'azote dans la respiration des animaux vertébrés; d'autres l'exlaalation, c'est-adire l'anguentation de proportion de ce gaz daus l'air eyphí.

Pérsuadé que des résultats difierens el mème opposés ne s'excluent pas nécessitirement, M. Edwards s'est applicjue a varier tellement ses recherehes quil est parvenu, d'apréses expériences et celles d'Allen et l’epys, a prouver quil y a 'n mime temps absorplion et exhalation, ch que la quantite diage en plus ou en moins indique la differene d'arelivite de ces deux fonctions.

1) $\mathrm{f}$. 


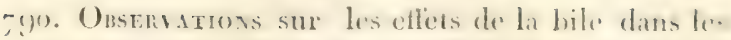
digestions, par li. C. Mhome, F. R. S. prof. dimal

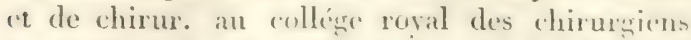
(A Joum. of se. litterat. and the arts, n. 25, 18.3.

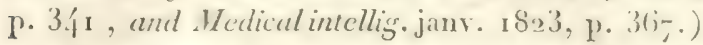

M. Brodie a fait les expériences suivantes, principalenent sur de jeunes chats.

Ine liwature applique au canal choledoque intercepte le: pitsage de la bile. La formation du chyme a lieu dans l'esfomar ; mais point de trace de chyle dins les intestins ou les visueaux lactes. Si on laissitt virre l'animal pendant quelyue jours, on recommaissat qu'il as ait la jaunisse par l'aspect de la conjonctive et de l'urine.

Au bout de $y$ à 8 jours, il arrivait souvent que le cours de la bile se rétablissait pax le moyen suivant:

Le canal choledoque était environné d'une masse d'albumine au-dessus et au-dessous de la ligrature. Lat ligature avait causé l'ulcération de la partie sans adhésion des parois, et se séparait. M. Brodie se propose de publier, dans la suite, des recherches expérimentales sur lat digestion. Les fitits pricédens font partie de ce travail.

E.

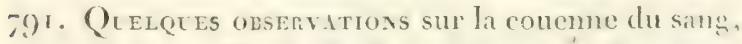
par J. Divr, M. D. F. R. S. (Trans. pluilos. de la

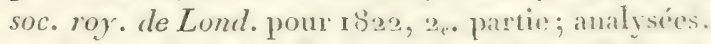
1 Journ. of sc. litt. and the arts, $15.23, n^{\prime \prime}$. 2S. Lond

\section{J. Murray.)}

1. La formation de lit coucnne a été attribuce par quselque un.s a une ténaciti morbide du sangr, par dautres a sat coit. falulation lente. La premiere opinion semble appuyed par le. fait que le sang se coagule anssi vite dans certaines maludien rue daus l'état de santé; cependant il se forme nue couenue mais elle est infirmée par cet atulre liait que la enravite sprecifigue du sings n'ent pas diminue dans len matadies inflammatoires.

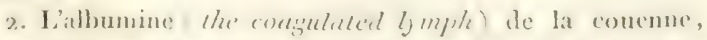

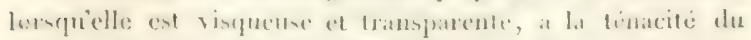

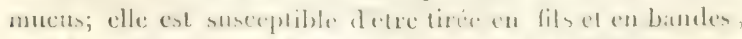

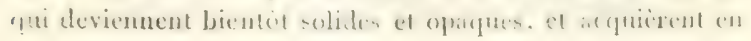


т.ro d'heures lenr maximum de ténacité. On ne sanrait jumer. "omme on le suppose, de l'ancienncté des brides cutre tes organes adhérens, par le degré de leur ténacité.

3. Il ne parait pas, d’après les expériences du D. Davy, qu'il y ait effusion đe sérum après la mort. E.

79?. Répétition n'une des expéniences supposíes concluantes de J. Hunter, pour déterminer la question des organes de l'absorption. (The Edinb. Med. and surg. journ. p. 154, $\mathbf{n}^{\circ}$. 44. Janv. 1823.)

L'expírience suivante a été faite pour savoir si les vaisscaux lactés penvent absorber autre chose que du clyyle, comme M. Magendie l'a avancé, en opposition anx opinions de Hunter. On a lié une anse d'intestin sur un chien, et on y a injecté de l'indigo et de l'amidon délayé dars l'eau. Le liquide coloré n’a pas pénétré dans les chylifères; mais, dit lauteur de cet article, on s'est apercu d'une illusion d'optique qui peut expliquer les conclusions de Inunter. P. V.

793. Dispositions partictuires dusysteme veineux chez un grand nombre d'animaux, par L. Jicosson. ( Edinb.Med. and surg. journ. p. 78. )

On sait que, chez les mammiferes, les veines des visceres abdominaux se réunissent pour formcr le système de la veine porte, mais que celles des parties postéricures et moyennes du corps se rendent directement au cœur par un tronc commun. Il n'en est pas de même chez les oiscaux, les reptiles et l's poissons. Les veines de ces partics présentent trois modifications principales dont M. Jacobson décrit ici les particularités; et il conclut, d'après ses xecherches anatomiques of physiologiques, que ces vaisseaux sont destinc's a porter le sing veinenx de ces parties du corps aux reins et au foie, ou bien aux reins seulement, et que c'est de ce systeme veincux que dépend la sécrétion de ces organes. II. E.

79'1 Des neras qui coordoment l'action des muse li's de la poitrine dans la respization, la parele et leespression ; servant de suite à un mémoire sur la strute. ture ct les fouctions des nerl's, par Ci. Bris. (()unt.

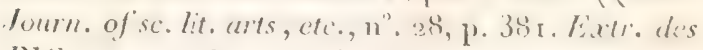
Philos. irans. 1 822, p. 2.) 
M. Bell dénontre par des experiences que bors de la sec. tion des differens nerfe quill arat préalablement désione roms le nom de respiratoires, les parties auxquelles ils se rendent, uagissent plus daus la respiration. L'auteur examime rnsuite les connexions qui existent entre l'upparcil re piritluire et les diverses parties avec lesqueiles il se combine pune produire l'expresion, la parole, ete. Il termine son 1 I'moire far des consictérations sur l'influence des émotions sur le. rour, et de cet oryane sur les muscles de l'expression par l'intermédiaire des nerfs respiratoircs.

II. E.

-g). Ripporit fait à l'acad. des se. sur un mémoire

de M. Finotrexs, intitulé : Détermination des pro-

prickis du systime nervenx, on recherches phisiynues sur linritabilité et la sensibilité, par M. C ver. (Noup. Giblioll. mid. janv. p. x3.)

Ce rapport contient l'exposí rijpitle des faits principaux élablis par le travail de BI. Flourens. Ies concincions yue M. Cuvier tire de cesexpériences ne sont pas identicues asee relles de l'auteur; mais il pense qu'elles suffisent pour prouver que la faculté de propager l'irritabilité et la douleur appartient exclusivement aux nerfs et à la moelle épinière, et cesse anx tubercules quadrijumaux; que c'est a cet endroit, an muins, que doivent arriver les sensations pour ềre persues, et que c'est de la que se transmet la volonté. Par l'exlirpation des lobes cérébraux, l'animal perd lat faculté de perceroir la plupart des sensations, ainsi que la ménoire, rufin la volonté ne se manifeste plur par des actes spintamis. Jiuntigrite du cervelet est nécessate a la régularité des monvemens de translation: l'extirpation de ces oryanes no fera perelre ni la volonté ni les sensations, mais bien le pouvoir de faire obéir les muscles à la volonté.

H. E.

7?6. Rappont fate a Licanime des sciences, sur un memoire de M. Drions, arant pour titre: Ire L I

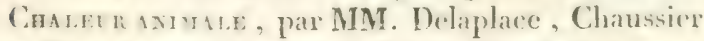

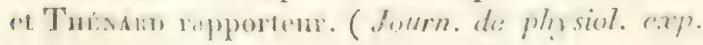
เ. 3 , n. 1.)

Damblatat de sante la fixation de loxigine absorbé pandant la repiration est-e.le suffisante pour reparer la perte 


\section{Physiologic.}

de chaleurque font tes animaux dam he conditions naturelle.' ou en d'antres termes: lá chaleur animale est-elle due tonte untiere a la combustion qui a lieu an sein des animanx dan, l'élat de la respiration?

Les expériences de M. Dulongr sont remarquables par lour cxactitude. La conséquence qu'il en tire est, que la chistrut animale est plus saralie que cethe qui est dégagée par la fixation de l'oxigène, et qu'il duit, par conséquent, exister une autre cause de calorification.

D. F.

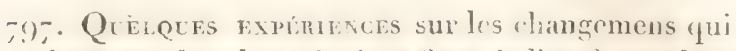
s'operent dans les principes fises de liente pendant

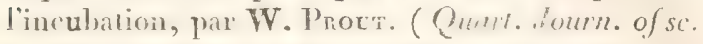
p. 385 , ot Ann. of phil.n". xi, p. 1 10 , Laxtr. des philos. Trans. I 822, p.2.)

L'anteur, aprés avoir indiqué la diminulum sucessive da proids de l'ouf, examine les changemens qui in y ejerent pendant lincubation. Il tronve (fu'à une próriode atrance. ie jaune de l'ouf perd une grande partie de son phosphore, wt que cette substance se retrouve à l'stat d'ateile, combinc: avec la clanx dans le squelette du foetus. Avant lincubation. l'ceuf ne contient pas de chaux; cette substance, dont l'origrine est incornue, ne s'y manifeste qu'après. II. E. 7gh. Recmmenfs sun r.onganisation et le développement de loreille externe chez quelques animax. par Predagze, interne à licipilal de la Charité. (Journ. de plyss.exp.t. $3, \mathbf{n}^{\circ}$. I.)

Dans ce mémoire, l'auteur démontre quäl existe, à la nasinsanee, une différence très-grande entre l'oreilie de l'homme it celle de quelugues animaux, et que diez le premier l'andition ne se fitt pas par défunt de developpenent, tandi, que chez les animaux ce sont des obstacles mécaniques yni s'y opposent.

D. F.

799. Ixnretions suy les rapports du cerveau aree

l'intelligence, par MI. Dession liss. (lees. meid. déc. 1822.$)$

Diapres un grand travail sur le systeme nervenx (its poin sons, ee médecin a cru décourrir que la supériorite de:

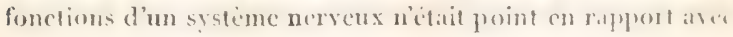


sem solume hybostatigne, mais asee l'atembe de sts sm

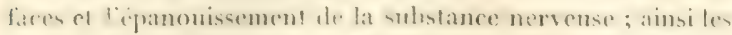

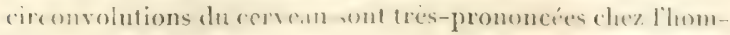
me principalement.

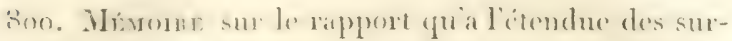
faces de la reline et du nerf optigue des oiscaus, avec linergie et la portée de leur ve, par M. $\Lambda$. Wissurutus, D. N. ; présente à l'académice des sriences de linstitut, le 23 déc. Issa. (journ. de physiol. exp.t. 3, $\mathrm{n}^{\circ}$. I.) D. F.

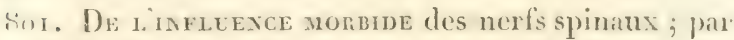

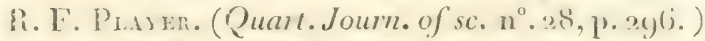

Cette note contient une séric de projositions, $I^{\circ}$. sur les mpports existans entre les maladies locales et les affections de. lorigine des nerfo qui se rendent à ces parties; $2^{\circ}$. sur les avantages quon peut retirer, dans or cas, de lat saignie locale, et de la vésication des parties qui correspoment à l'origine de ces nerfs. $\quad$ H. E.

Soz. Onsenvations michoscopiques sur les changrmens qu'épronve l'ouf de la poule, pendant l'incubation, accompagnées dedessins, par sir E. Hos s. ( Quart. Journ. of sc. no. 1 I , p. 383.)

Ce mémoire n'est, pour ainsi dire, 'qu'un texte explicatit des dessins qui l'accompagnent.

11. E.

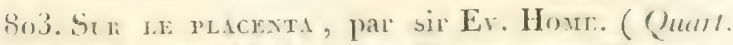

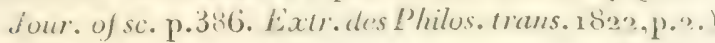

fes observations contenues dans ce mémoire sont dentinitis a servir de base a une nouvelle classification des animanx, dapres la structure de heur Placenta.

11. E.

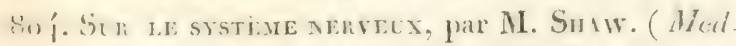
I ncllig. n. 3z, déc. 1822 , pag. 5 ү.)

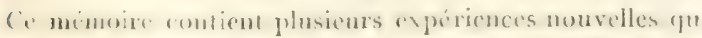

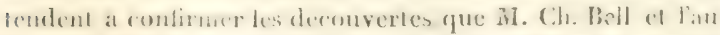

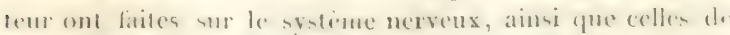
1I. Magendic. 1. V. 


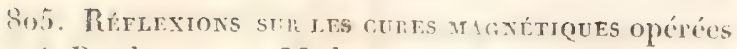
a Bamberg, par M. le prince Ilohenluhe, par le doct. Pfeufer. (Rev. méd., janv. i823.)

Des diverses relations qui ont été pullliécen sur ce sujet, celle du docterur Pfeufer est lia pius extcte. Il dimontre que cest en agissant sur les sentimene religieax que ce prince développait la confiance et la foree; of , con second lien, qu'il n'a oféré anteune cure miraculense ni radicale; mais senlemont il a diminué lés douleurs el momentanément fait disparatre certaines maladies nerveuses. Cest du magnetisme.

Solo. Rechumes sur les fonthind de la rate; par

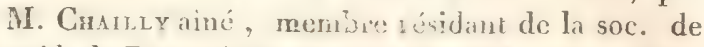

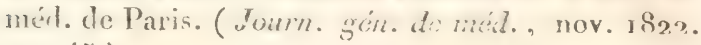
p. I 45.$)$

Lianteur déclare qu'il est loin de croire à l'utilité des exfríricuces sur les anmaux vivans, et qu'insi al rechercliera * simplement l'existence du secours dont est la rate, dans " sa nécessité, dans ses empếchemens, et dans ses consé" qquences.

il pense que la rate sert à contenir l'excédinnt du sange qui

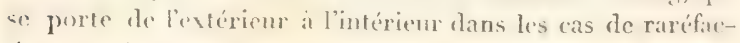
Limn considérables et subites de ec liquaide, ete. P'. V.

Soz. Honue mostrueux de Macao. Notice pulliée? avee mue fig. grav. Madrid, 18s2, Munne y Fildes,

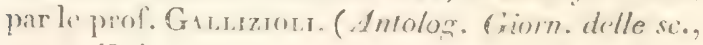
pag. 1 43.$)$

In Chinois, natif de ILacao, digé d'onviron 23 atns, et bien

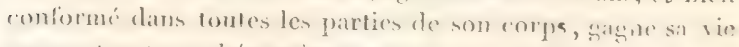

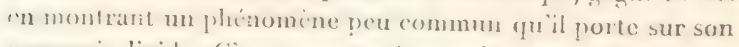

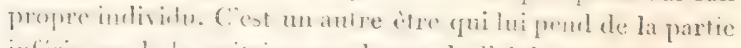

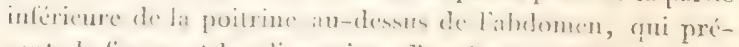

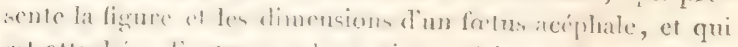

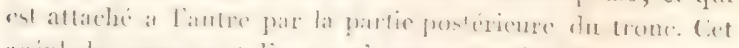

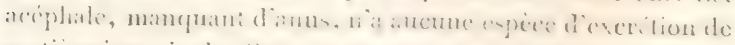

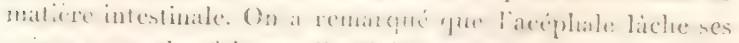

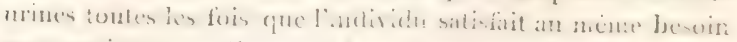

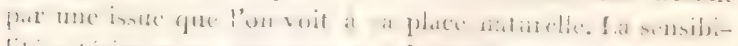

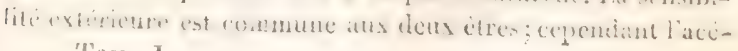
T'one 1. 
phale est moins sensible relatirement a l'individu; il parait depourvu de tout mourement volontaire, malgré la flexibilité de ses membres et l'ignilité de la chalcur qui leur est commune.

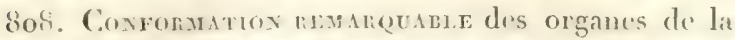
génération. (Frtrait d'une lettre du chev. Arbracut De Scuonmetí, M. D. demeurant à Naples, à l'édit. dujourn. allemand, Medecinisch und chinuryisile Zeitum, is, 5 aout, I822. (London, med. and plizs. Journ., fév. 1823, p. 1 73 .)

Une chère présentait le vagin, l'utérus et tous les orfrance féminius de la génćration, a l'exception des ovaires, et en même temps deux testicules sous les tégumens des plis de l'aine. Les organes urinaires offraient aussi des anomalies.

H. E.

Son. MarLaurface et Coates ont public dans le Journal de Philadelphie (aont 1822 ), une série d'expériences sur l'ab)sorption. Ils injecterent du sulfate de fer dans la cavité abdominale, et du prussiate de potasse dans le tissu cellulaire de ses parois. Bientôt le liquide contenu dans le canitl horacique se colora en bleu, ainsi que le serum du sang, les floumons et même lurine; mais ils ne purent recomatitre l'existence de cessels daus le sangr qu'aprés qu'ils furent parvenus à la partic supéricure du canal thoracique. (Lomdon Medic. Reposit., Janv. 1823, p. 15.)

II. E.

810. A Norfolk en Amérique, il existe un homme ígé de 60 ans, douc d'une intelligence rare, qui esi venu au monde noir, et qui a reste negre jusqu'al'ane de 95 ans; depuis ce tempe sat peatu a successivement changé de couleur, de sorte que les trois quarts de son corps sont devenus entierement blanes; ba pean des bras et des mains a acquis une finesse et une transparence qui ne peuvent etre surpassies par lat.fenme Ia phus délicate. Tillotson's philus. Magaz., sept. 1822.)

$\Lambda N \Lambda T O M I E$.

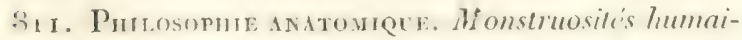
nes, our rage contenant une classification des monsmas : la description et la comparaison des puinci- 


\section{Anatomic.}

paux genres; une histoire raisomnée des phénomenes de la monotruesité et des litits primitifs qui la produisent; des vues nourelles truchant la nutrition du fixus et d'autres circonstances de son développement; et la détermination des diverses parties de l'organe sexuel, pour en démontrer l'unité de composition, non-seulement chez les monstres, ou l'altíration des formes rend cet organe méconniassable, mais dans les deux sexes, et, de plus, chez les oiseaux et chez le's mammifires, par M. le

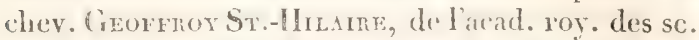
I vol. in- 8 . de $38 \mathrm{f}$. ; plus un atlas de $7 \mathrm{pl}$. in-4.

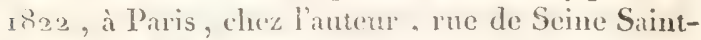
Vietor, n. 33, ct chez les principatux libraires pour l'anatomie. Prix, to fr. et fr. de port, in f.

Cet ouvrage fait suite à un premier ouvrage qui a paru

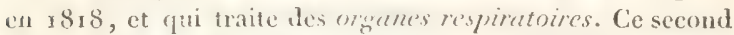
volume de philosophic anatomique est un Traite complet sur les Aonstruesile's humatnes. L'auteur y donne tous les principaux faits del'histoire pliysiologique du foetus; y discute, sous un nouveau point de vue, l'importante question de la préexistence des germes, ot y determine la cause des malais's de la :rossense, qu il attribue à des formations désordonncis non-prersévérante's ; lespuelles, au contraire, en cuts de persistance, ensendrent les monstruosités. Ainsi décrivant lous les tiwdbles de l'orranisation dont les productions, au moment de leur naisance, inspirent atu vulgaire un si vif sentiment de dignont et d'horreur, il présente, pour les avoir etudies plutot encore dius les icarts les plus monstrueux

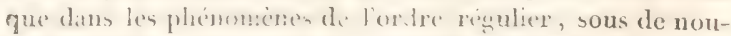

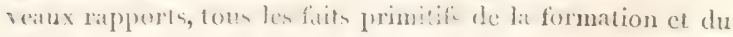
développenent dez êtres.

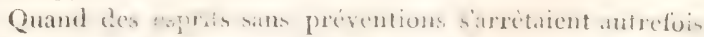

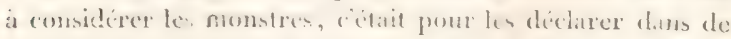

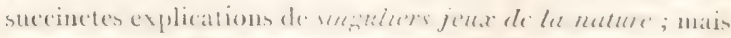

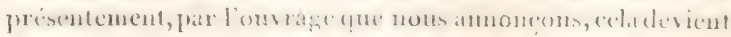

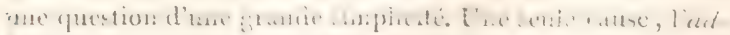

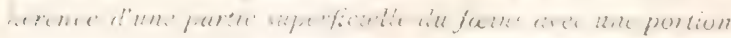




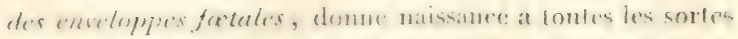

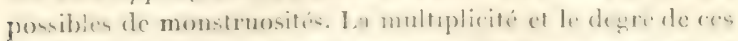

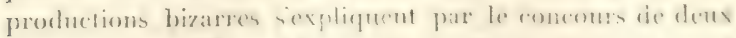

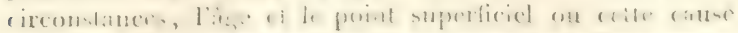

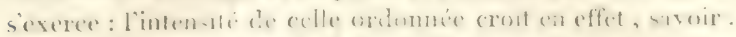

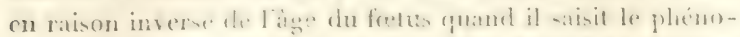
mene de la monstromire, et en raison directe de limpertance des orgranes qui sont atteints.

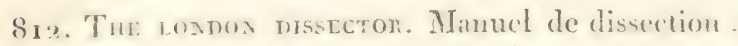
in-I2. Prix, 5 sh. Londres.

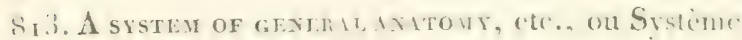

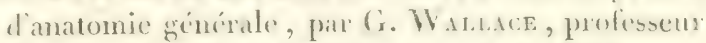
dimatomie et de chinumie, etc, in-s. P'is, Londres; Underwood.

C.t ourrage comprend tom re quil y at de plis interes-

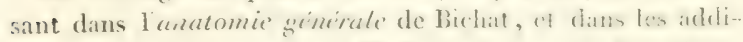
lions an mème ouvrage, par Mr. Béchard, avere les fatsobervés dans le pays, etc.

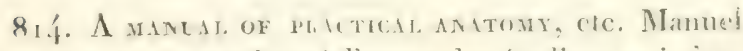
dianatomie pratique a lusage des cumbians yui sap-

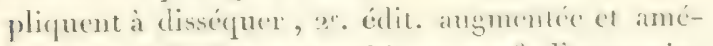

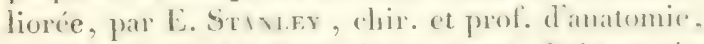
a lhopital de St.-liathedemi, in-re. l'ris. g sh. Londres; Undersolı.

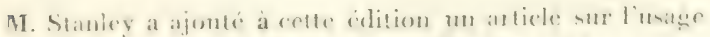

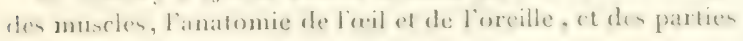
affectées de hernic.

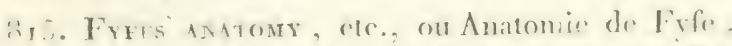

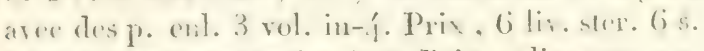

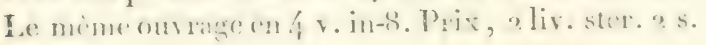
J.ondres.

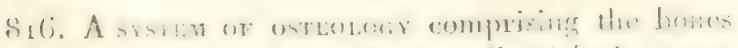

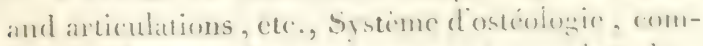

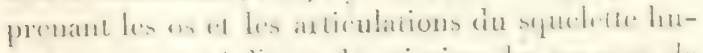

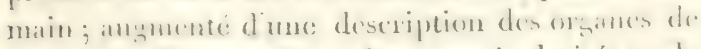

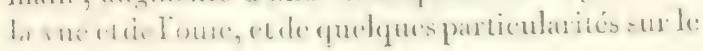


Sertus; destince à servir d'appendice an Lundon dissector, pour suppléer anx défauts de cetotrrage et le compléter. In-1\%, Londres, $182 \%$. Burgess.

les descriptions sont clitires et exactes, et l'ouvrage remyhit parfitement le but que son auteur anonyme s's propore. P. $Y$.

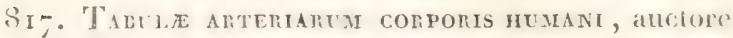
F. Tredrann, Carlsruhæ, $\imath$ 822. Muller.

Cet onvarge se composera de 36 planches in-folio; la 110 . livraison a paru et contient 9 planches.

\$is. Lecte niks on the structure and phissiology of the mile urinary, ele., ou Lecons faites au collége royal de chirurgie, sur la strurdure et la physiologie des organes de la génćration et des conduits urinaires de lhomme, ainsi que sur la nature et le traitement de leurs maladies, par feu J. Wr won, M. D. Prix, 14 slt.

Sig. Mémorres sur quelques sujets intéressans d'anatomic et de physiologie, traduit du Hollandais, d. M. G. Vroj.ik, par M. Fallot, D. M. in-í. arre 13 pl. Prix, 14 fr. Amsterdam, L. Vam lis.

Soro. Tabila anatomico-patholosice, modos omnes, quibus partium corporis hum. omnium forma externa atyue interna i normi recedit, exhibenu's. Auct. Z. F. МекеL, in-fol. fisc. 3. systemil digestionis. Cum 9 tab. an. Prix, 7 rixd. Lipsie: Gleditsch.

:231. De Lisitonie patrofogieve du cerveau et de ses membialues, par Craige, M. D. (Lidiul. med.Journ., p.63.)

Dans cette partie de son travail, l'auteur examine les rapports dui existent entre les lisions organifues din cervean el

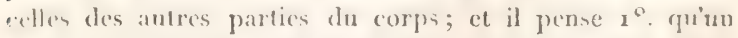

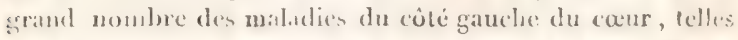
que l'ossification des alsules, atce, tendent at se termine r par 
la production d'un cpanchement dans le cervean, qui ditenmine lapoplexie et l.t mort; $2^{\circ}$. que, quniquil y ait de exemples de développement spontami des maladies du cerveau et de ses membranes, il n'est pas rare de soir ces affections produites par le trouble quioccasionent, diens la rirculation, les maladies du cocur; $3^{\circ}$. que ces phenominen s'expliquent acsez facilement par la distension des veines ecrebrales, qui doi prodnire les obstacles au cours du sans dans le thorax.

I. C.

822. Rechrenes rinatome patrolorigue, démontrant le rapport qui existe entre lirritation de la membrane muepueuse du canal intestinal et celle: de la méningine du cerveau; par Scoctetten, D. M. (Journ. unis. desse. méd., tom. 28, p. $2.5 \%$.)

L'auteur avance que, d'appes lesouvertures d'ungrand nom bre de cadavres, il s'est assuré qu'il existait une liaison fellement étroite entre le canal intestinalet la méningrine, qque quand le premies est irrite d'une maniere aiguè et chronique, la membrane du cerveau participe toujours aux memes nuances d'irritation. Il ajoute erpendant que toutes les parties du canal intestinal ne sympathisent pas ćpalement avec la meningine; que l'estomice of les intestins grêles paraisent avoir avec elle des rapports bien plis étroits que le gros inteslin. $\mathrm{\Lambda u}$ surplus, c'est sur lit membrane mucuense que l'on remarque plus spécialement cette corrélation.

Apres avoir expons l'atat de la meningine dams les cat d'inflammation aigrä de lintestin, il convidere len athirations de cette membrane, apres loinfammation cloronitue des organes de la digestion.

En poursuivant lis meme idec, l'auteur est ronduit à conclure que l'apoplexie n'est pas une maladie instantanice, comme on le croit grenciralement; qu'clle est, nu contraire. préparce de loneuc-rnita par lirritation concomitante deorganes de la dignestion et de la mésing ine du cerveau ; d ou

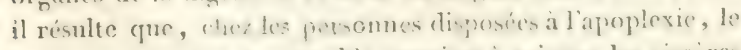
traitement le plus comvenable combiste a snigner les viscriress

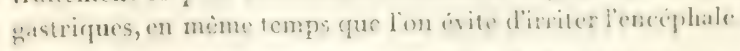

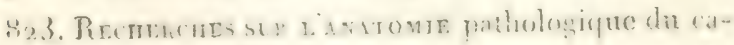




\section{Anatomie.}

nal digestif, par M. Arnest fils. (Nouv. journ. de méd. et de chir., niov. 1822, p. 193.)

Dans un premier article, l'auteur décrit les caractères anaiomiques de linflammation du canal digestif, et entre dans des détails très - mimutieux sur les ulcérations, lés exanthếmes, les perforations dont ce canal peut être le siéze. Il établit des comparaisons entre ces diverses altérations, tant sons le rapport de leur frécquence absolue que sous celui de leur développement varrié, suivant la région qu'elles occupent.

Dans un second article, il est traité des tissus accidentels qui se forment dans les intestins, comme les tubercules, les inmeurs sqquirreuse's, les mélanoses, les kystes séreux; il y est question aussi de l'œedème des intestins.

Ces maladies ont été long-temps regardées conme indépendantes de l'inflammation du canal alimentaire; les nombreuses observations recueillies par l'auteur démontrent la vérité de cette opinion, et lui font admettre, dans bien des cas, le caractère atonique de ces affections, ce qui modifie singulièrement leur traitement.

P.

834. Cesenvatrons sur la structure des tendons, par

le prof. Isenfiame. (Archiv. goén. de méd. Janv. 1823, p. 131.)

825. Doutes suti le systexine des vaisseaux dans l'écrevisse fluviatile, par un anonyme, avec fig. (Isis, II ${ }^{e}$. cah. 1822 , p. I 230.)

L'auteur, après avoir observé que M. Cuvier, dans ses lecons d'anatomie comparée, n’a décrit le système vasculaire des écrevisses que par quelques traits détachés, el que ce système n'a pas encore été décrit d'une manicre complète, en donne une description tres-détaillée, avec deux figures, dont l'une représente le creur de l'érevisie, vu par le dos avec les raisseaux qui s'y rendent ; et l'autre, une vue de profil du mème cour aver les vaisseaux adhérens. Il résulte de sa description que le cour d'une écrevisse a six branches de vaisseaux artiriels, trois devant, deux dessons, et une derriime; qu'outre ces six branches, il y en a enrore une septieme, au-dessoun de la naissance de lartere de derriere, ordinairement a sat droite; cette septieme hrenche, la seule 


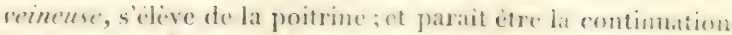
de la branche artericlle du milien. Cille-ci, ditns son conr: a travers les os de la poitrine, prend de laceroissement, serevant de clodgue cote des ramilications quin partent des branclaies, ef se surossit de plus en plus on dercendant, et vient enlin se terminer, comme on lia dit, vers da partie posterienee du ecour. $\Lambda$ cette brancle vient se réunir une ramilication comsidérable qui part de la quene, et ra se confundre avec la veine de la poitrine; ce valissean inférierer do la quene pourrait bien etre celui dont M. Cuvier parle dans la squillamomlis. L'anteur prétend, d'aprés plasienrs observations, que ce vaissean n'aboutit point anx branchies, mais a lavine de la poitrine qui monte an corur. II eroit ne puint devoir adopter l'opinion de M. Cuvier, ni celle de Suchow, et pense qu'il y a eneore beancoup) de rloses à constater et à éclaircir à ce sujet.

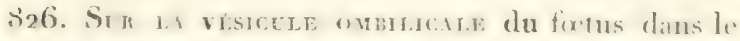

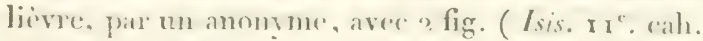
× 822. p. 1228.$)$

Il cxinte, dit lantere, une opinion donfeuse sur la vésisule ombilicale du lievre et du lupin, yui n'a pas encore ché :claircic d'une maniexe satisfissante; savoir, si la viniente ombilirale ent réllement me vésicule ou mo simple enve-

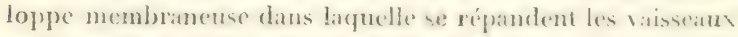

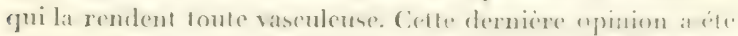

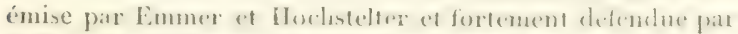
J. F. Mehel. Ohen n'y a point cru. MII. Cusiog el Doutreehet ont pretendu le contraire, en sipluy

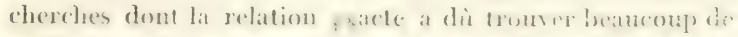

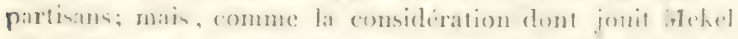
peut laisser, a e smjef, plusienes persomese daus le doute,

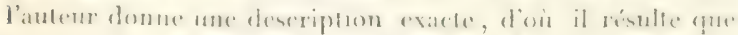

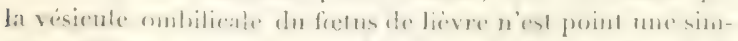

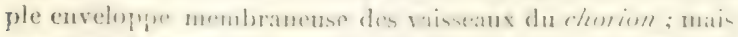

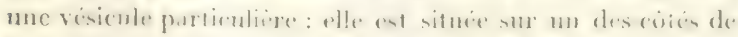

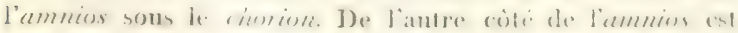

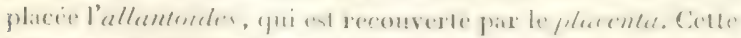

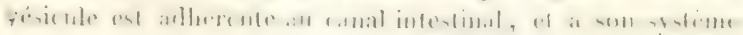




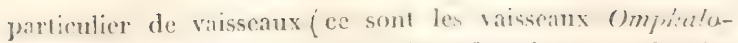
miternicriques), el remplit les niemes fonctions que clez les antres animaux. Cependant le sinus terminalis de cette vésicule s'inastomone en phosieurs endroits avec les ritisseax du placentu, ainsi (1) 'avec le systeme des vaisseaux ombilicaux. Cette communicition des deux especes de vaisseans est bien réelle; elle n'est point en contradiction avec l'usage des vaisseaux Omphalo-mésentériques.

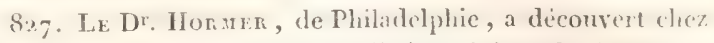
l'homme un nouveau muscle de l'oil, qui satlache a l'os ungnis, et de là se porte cu avant et se divise en deux portions, qui se terminent dans les paupières près des points lacrymaux. En se confractant, ce muscle applique les panpieres sur Io globe de l'ceil, et tourne les points lacrymanx vers le nez. (London med. and f,lys. Journ., jan., $1 \$_{2} 3$, p. 2.)

\section{H. E.}

828. M. A. Coopen conscrve la préparation anatomique d'un onfant chez lequel l'uesophage manduait; le pharynx se terminait en cul-de-sac et l'estomac ne présentitî pas d'orifice corliaque. Cet enfant récut huit jours: la dighlution était impossible; mais le's ivacuations alvines et urinaires se faisiatent comme at l'ordinaire. (Lond. med. and pleys. Repository, jan. 1823 , p. то.)

\section{MÉDECINE.}

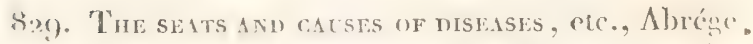
des recherehes anatomiques sul le siege el leng canses des matadies, ete. par J. B. Morgagni, et:.. arec des notes, pare su. Cookr, membre du coll.

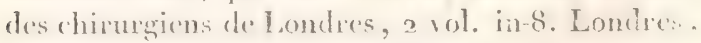
I 822 .

Celte traduction du bal onvage du professenr de l'akme ent fite dims un exeellsut enprit ; lantemr avat tout le talcai niecssatire pour distinguere ce qu'il atait utile de conserser en

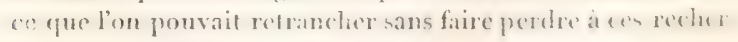
ches Ieur conleur originale.

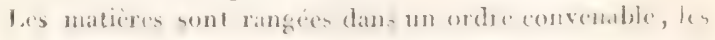


notes, abondantes en faits, metlent au courant des travaux modernes. M. Cooke a divise sa traduction e't cing chapitres. dont les deux premiers correspondent aux deux juemiers livres dn Trate orierinal, qui contiennent les altirations pathologrigues de lit lite ot de la poitrine. Ines divisions des clappitres correspondent ans lettres de Morsigni. M. le doct. Cooke a termine dracm des denx premiers clatpirses par les plaies et les blessures de la téte et de la poitrine, qui faisaient partie du í. livre de Morgagni, rapprochement qu'il était convenable de faire.

Le 3". chap. de cette traduction comprend le 3 . livre de MIor-

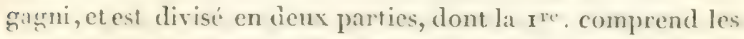
maladies des organes de la digestion, et, dans nue section siplaré, les lésions et les plates de l'abdomen; le f". chatp. contient les maladies des voies urinaires, et des parties crénitales. Le dernier chap. contient des maladies trie-diffirentes, quisont, a peupres, toutes celles qui nom pu trouver place dans les précédens; ainsi, ce sont des recherelus sur les fievres, les tumeurs, les maladies des os et des articulitions, la stranģulation et l'empoisonnement ; cette traduction, atma dépouillée de tout ce quil y a d'inutile dans l'ouvaše original, est fort propre a rendre plus fréruente la lecture des ouvres de Mormagni, et a faire sentir anx praticiens, combien sont précieux les résultats quoftre l'anatomie patholosique, pour perfectionner le diagnostic melical. I). IV.

830. OFuvies complitis ne Cabanis, nembre du sinat, de l'institut, elc., accomplosne's d'une norice sur sa vic et ses onurases. Tom. I 1.t s, ? vel. in-S. ensemble de 7 I. - Imp. de F. Didor. a l'aris. A Paris, chez Rossange freres, el chez J. Didut. Prix, I4 fr.; pap. vélin, $28 \mathrm{fr}$.

Rëlition anrat 7 vol.; le tome rer. conticnt : Revolutione et reforme de ta mederine; rapport fait an eonseil des fingCents sur l'orenanination des écoles de médecine; du degre de certitude de la médecine.

Le lome 2, jonrn.1 de lit maludie et de la mort de Mira-

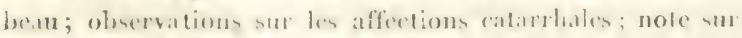

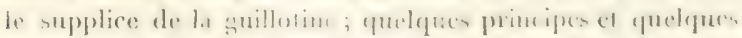

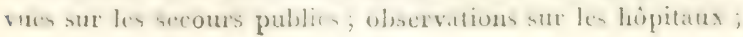


travail sur l'éducation publique; note sur un ogenre pirtirulier d'apoplexic.

$83 \mathrm{r}$. Histonicar sketer respecting the varietics and the sceondary oceurrence of small pox, etc., Acconvt of tur vartolom epmevie, lately previled in Edinburgh, ete. In. Esquisse historigure des opinions émises sur les variétés et les accidens sccondaires de la protite vérole, avee des observalions sur la nature et la certitude de la vaccine. $2^{0}$. Rielation de l'épidémic variolique qui a eu lien dernièrement à Edimbourg, et dans d'autres parties de TF́cosse, ete.; Tottres adressies a M. Urrigor, directeur général du départ. méd. 'le lanmér, ctc., par J. Thonson, D. M. Londers: Lengman.

L'auteur cherche a prouver, d'aprè des faits, lintentite de la petite vérole qui parait après la vaccination, avec la varicelle; il s'appuie sur un rapport fait en I8o3.

Secondement, dapres des observations faites on 1817 , le doct. Thomson pense que lit varcine n'est en tidfaut gue lor'sque l'on n'a pas suivi la vraice méthode die varcination.

D’après l'épidémie de varioles quil a obstrvée en $1.8 \mathrm{~s}$, l'anteur pense que, dans ce eas, on ne doit pas regarder lit vaccine comme un entier préservatif; mais comme un sù moyen d'affaiblir cette maladie, et de rendre, sans aurun danger, l'especedéruption qui suit la vaccination. D. F.

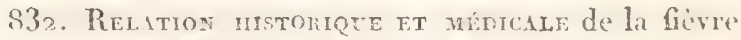
jaune qui a régné à Bareclone en x $\delta_{3} \mathrm{x}$; jar M. At docann, médecin des hoppitaux militaires de Paris, offecier de la kégion d'honneur, cheralier de

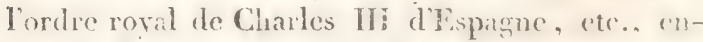
veyé à Barcelone par $\mathbf{S}$. Exe. le ministre de lit gluerese r vol. in-s. de 5 fo pases. Prix, 7 f. 50 c. à Paris, chez Méqquignon-Marvis.

Cet ouvarge contiont, non-sculement l:- docmundn histo-

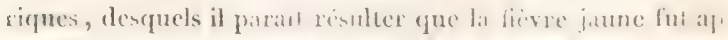

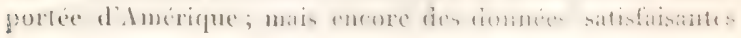




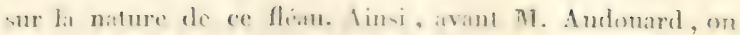

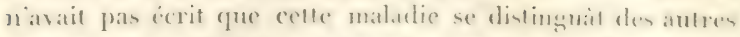

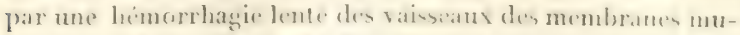

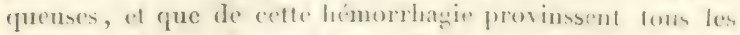
antres accidens ef la mort meme. Ced anteur estime, romtre:

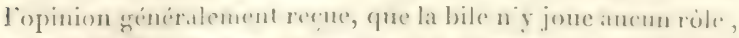

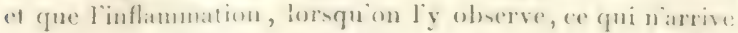

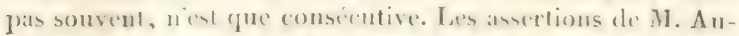

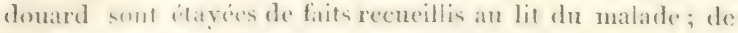

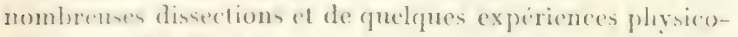
chimienes. Toila done, sur la mature de la maladie, une "pinion nouvelle qui, si elle ent confirmée par d'antres ma. decim olservateurs, ne peut manquer de conduire a de anuveaux moyens curatifs.

Lat question fe la contagion étant le second objet que le:

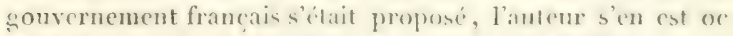

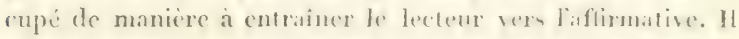

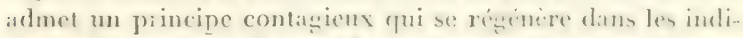
vidus atteinte, qui est soms forme de gaz, qui reghe antunt de's malares, dont les effets molsiliery et les redemens se chatrserte qui pent pasiel d'un appartement ou d'une maison dans une antre a l'aide de's comrans d'air peu prolonsés, es qui se detruit dans un gुrand espace, on far me grimberen. filation. Il parait que les cataves sont moins prepres a com-

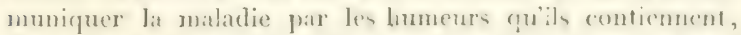
fue par les gaz qu'on fromse dame les inlestims. Hu vivant

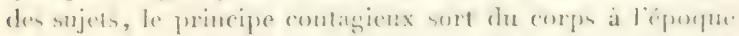

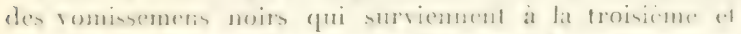

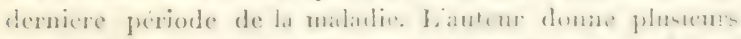

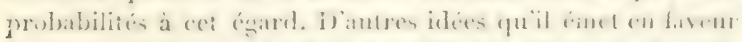

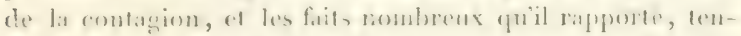

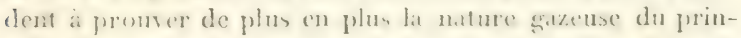

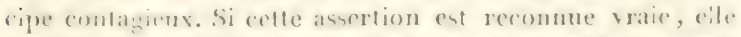

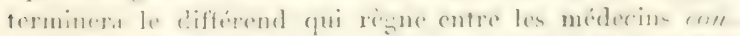

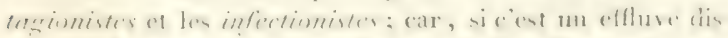

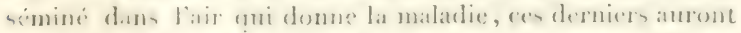

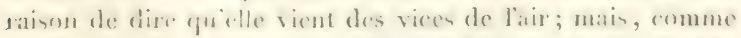

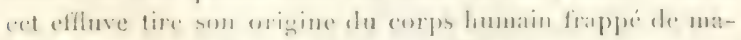

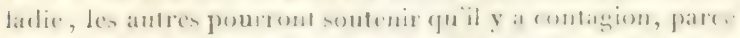

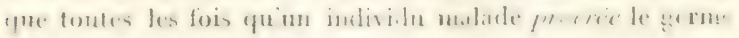




\section{Médecine.}

de la maladie d'un antre individu, il donme lien a une rentiagion, niniporte le moyen par lequel elle s'opère.

Il resute done da live rue 31. Andouard vient de publies, deux domeses nonvelles et fort importantes pour arriver a connaitre la fieve j:une. L'unc, que cette maladic est caractériséc par une hémorrhagie des membranes muquemes's la seconte, yp'elle est transminsible par l'intermediaire de l'air, i une courte distance, aussi-bien que par le contact.

Linteme se montre pen rigourenx sur les mesures que lon doit prendre pour privenir ou pour arrèter la propa-

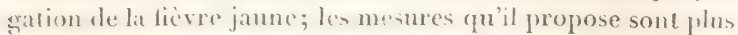
simples que celles qui sont prescrites par les lois. A son avis, lanir el l'eau sont les seuls mosens de désinfection prour les personnes comme peur les objets; cependant it traite des

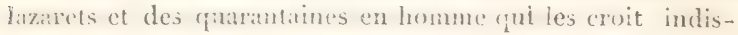
pensables.

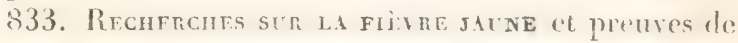
sa non-contagion dans les Antilles, par J. A. Rocuorx, D. M. Taris, $18 \% 2,1$ wol. intr. de fise p.

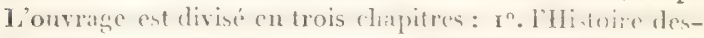
criptive de la Fièvre jaune; $2^{\circ}$. ses causes; $3^{\circ}$. son traite-

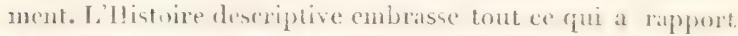
at lit maliadie elle-mème, à se's complications et and afiecrimats qui peuvent la simuler.

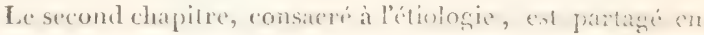
trois sertions. La premiere tatite des cantes envisanges dan-

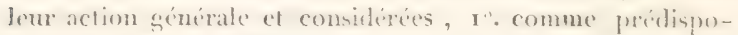
santes, e". comme efticientis. Fa seconde section fait con-

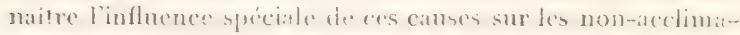

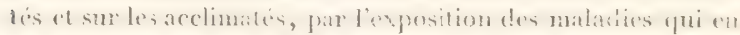

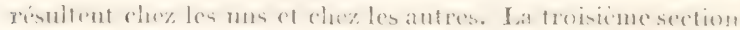

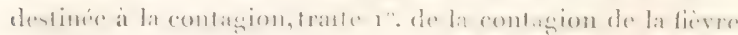
jaune entre les tropiçues; 20, de la contagion dans les régions tempérées. Dans le troisième chapitre, consacré au

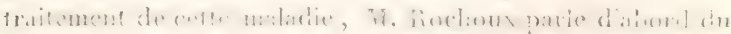

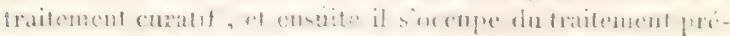
servatif.

1.

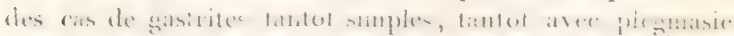
des reins, the la vessie, ou de l'arachnoide. 


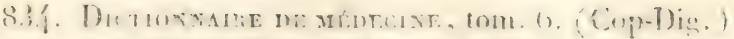

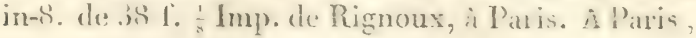
chez Béchet jeune.

NT. Beslard a fourni a ce volume le's articles crine, cuisse, dent, dentilion. - 11. Biett, l'article couperose. - M. Bres-

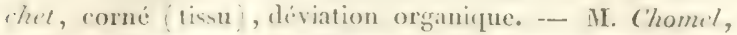
erachat, diagnustic, diaphragnite, diarrhée, diathise. M. Cloquet, corail, coralline, corne de cerf, cousin, crinon. - 1. J. Clopuet, conteau, cuissart, cyclotome, dibridement, détersif, dieplurasme. - M. Contuncent, crise, "vele. - I. Desormeaux, couches, crochet, delivance.-

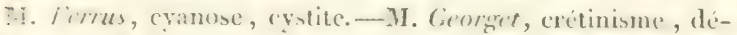

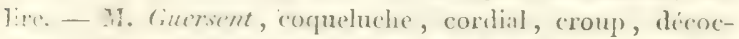
ton, ditivan, demi-bain, dentition pathol. , derivatif. diritation, diffusible. - M. Lagnesu, cor, crabe, cristalline, depilaton, depilatoire - N. Haw, deception,

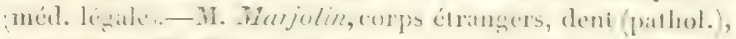

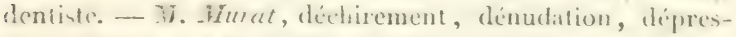
sion, dhimene - nî. Orfíd, cuive, ryanure - H. Pellelier, daphnine, daturine, delplinine, dazsede, diarrhodon.

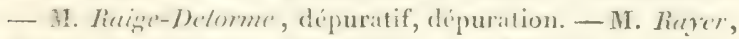
dartre, desquamation, désulation.-M. Richarel, cucurbitatrés, cynogloses, cynormbolon, dentelates, diaphorese, diat-

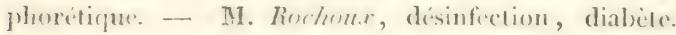

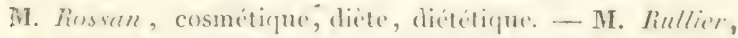
eri, doglutition. - Col ouv rage est evidemment fitt dans un enpit plus conforme an but d'un dietionnaire, drue celui de

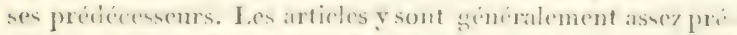
cis, et res-amblent mosins a des memoires anxquels on a semsent dimme une dendue demesure, depuis que les encyclopédies en ont donné l'exemple.

Fdr.

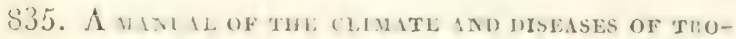

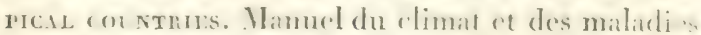

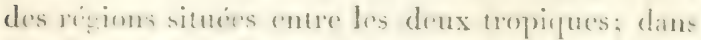

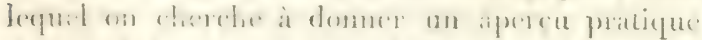

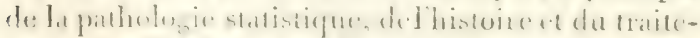

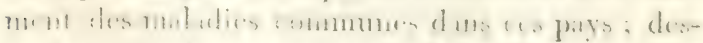

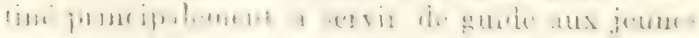


médecins en arrivant dans ces contróes, par C. Chisnolar, D. Mi. in-8. Prix, 9 sh. Londres; Burgess and Hill.

Dans cet ourrage, presque entierement pratique, M. Chisholm examine $1^{\text {n. }}$ la statistique pathologique des Indes occidentales, et les causes morbifiques les plus générales dins ce pays, ainsi que les meilleurs moyens d'y remédier; 2". les malladtes particulieres anx rémions íruatoriales ou qui s'y mamfestent ainsi que dans les antres parties du globe. II. E. 836. On rnsinity. Remarques sur la manie, tendant à expliquer les symptòmes pliysiques et le traitement de cette maladie, par Th. Mlı́, D. M. in-8. Londres; Underwood.

837. $\Lambda$ conprencris e treatise upon the symptoms, consequences, nature and treatement of venereal or syphilitic diseases. Traité concis sur les symptòmes, les conséfuences, la nature et le traitement des maladies vénériennes ou syphilitiques, traduit de la 5 e édit. française de F. Swedrurr, M. D. 2 v. en un seul, in-8. Prix, 9 sh. Londres; Burgess and Hill.

838. Rappont et consultations de médecine légale, recucillis par J. Risteluisebin, 4 rolumes in-8. Strasbourg.

Cet ouvrige, fort intéressint pour les médecins légistes, présente lit solution d'une question fort difficile : savoir, si un individu qui a fait un contrat, et qui est mort peu de temps aprés, était affecté de cette maladie avant de contracter, auquel cas la loi annulle l'acte.

839. Flámens ninguiane, ou de l'influence des choses physiques et morales sur l'homme, et des moyens de conserver la santé; par E. Tourtelle, $f^{e}$. édit. corr. et aug. de notes et additions; par J. ThischeTrav, D. H. P. a vol. in-S. ensemble de Gy fiuil. Prix, $12 \mathrm{fr}$. à Paris, cliez Rémont.

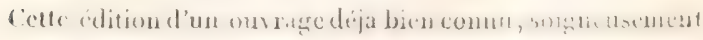




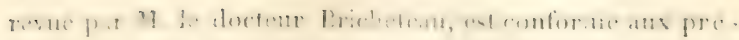
cédentes, excepté que l'éditene on a fait disparailre des

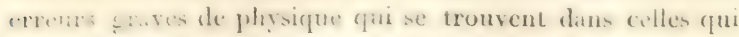
liont précédée.

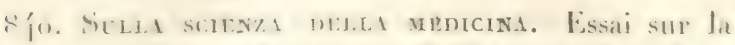

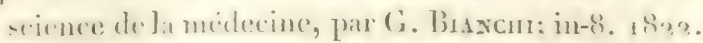

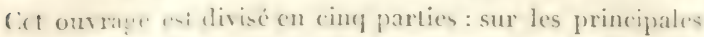
canses des nalarlies. - Sur les inflammations. - Sur la veritable cance de la sucuison des maladies. - I)e la cure des mataii - attribuecs ats remeles. - Arantages de la medecine hy fucratique sur les deux systemes de brown, et du controstimulus.

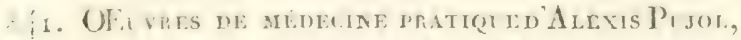
comlenant des mémoires sur les inflammations dhronifunes des viscrees, les nualadies lymphatiques, lart dexeider ou de modioner la fienre, les maladies de la jean, les malarlies hérédilaires, le vice serrfuleux, le: megnélismo mincial, la ficremilivere épidémigue, la ragere, le rachitis, la fienre puerpirale, la coligue hípatiegue, elce, avee uus motice sur la vie el less trasuax de lautem, ed des alditions, par F. C. Horssen, D. N. P. i wot. in-s. Prix, Jr. i 6 fr. à l'aris, cher J. B. Baillicere.

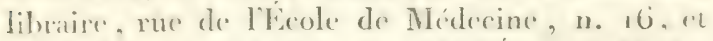

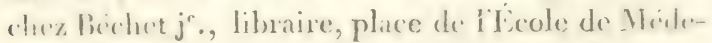
cine, 11.4 .

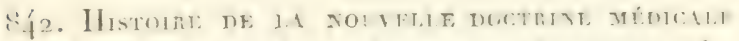

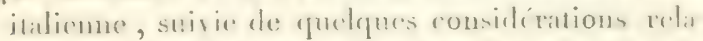

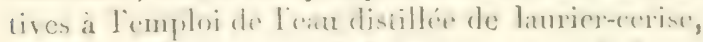
dans les inflammations de la poinioe, par J. Sir 1 -

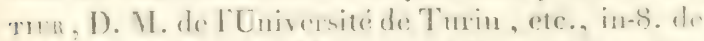

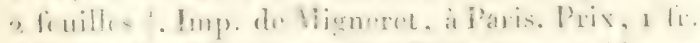

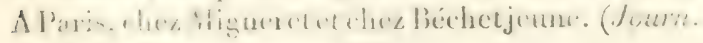
de Mél., déc. 1822, p. 365.)

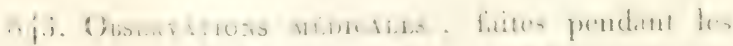

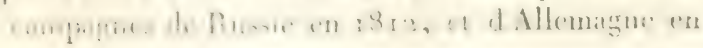


* 8 r 3 , on llistoire des maladies observées à la srande arméc francaise, lors de ces mémorables rampagnes; par II. J. Rox. L. de Kпгскиоғғ, D. M. s"édit. rev et angmentée, in- 9 . Prix, $5 \mathrm{fr}$. 50 c. Utrecht ; J. van Schoonhoven.

s作. Les oevres d'Hppocrite, traduites sur le texte grece, d'aprés l'édition de Foës; précédées d'une. notice sur la vie et less ouvrages attribués à Hiprocrate, par A. M. Dorisrele, de Bourg en Bresse: in-8. d'un quart de feuille. Imp. de Constant Chantpie, à Paris; à Paris, chez lanteur, rue Michel le Conte, n. 20 ; chez C. Chantpie, Dclaunay et Béchet jeune.

L'édition formera deux volumes in-8'. Les souscripteurs, avant le $\mathrm{I}^{\mathrm{er}}$. mai, paieront chaque volume $6 \mathrm{fr}$; ; les autres, $7 \mathrm{fr}$.

8,5. On niseases of the cinst, etc. Des maladies de la poitrine, par Laennec; traduit par le doct. Forbes, ayce une grav. ; in-8. Prix, 14 sh. Londres.

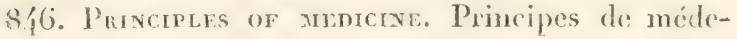
cine sur le plan de la philosophie de Bacon, V. r, sur les maladies fóbriles et inflammatoires, par $\boldsymbol{R}$. ). Llinuton; in-S. Prix, 9 sh. Londres; Cinderwood.

847. Ox rever. La pathologie de la fievre, comme sujed d'une lecronde Julston, par J. R. Park, in-8. Prix, 5 sh. Londres; Underwood.

\$.8. Researches nespectixg the Medicll towers of curorexw. Recherches sur les propriétés médicales du chlore, surtout dans les malidies de la rate, avec la description du nouvean mode d'employer red agent, pour modifier son inlluence sur l'éronoznic animale, par W. Wratsce; in-8. Prix, (ish. Londres; 1823.

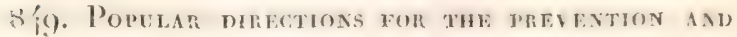
Tour. I. 


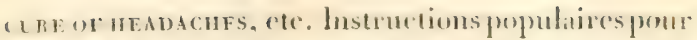
prévenir et guérir les maux de tere, le's rhumes de les indigestions, ave indication des ordonnances: et de dillérens cas prot 111 praticien expérimentó; in-18. Prix, $2 \frac{1}{2}$ sh. Londres.

850. Uiber nus fendir, ete., Théorie de la fienre, par C.n. Met ти ; in-S. Mayence, г822. Kupfuberg.

851. Prorrinua, qun nova pulmonum docimasia liydrostratica proponitur. Auct. J. Benst ; in-š. сии fig. Viennx, 1822 ; gevold. I fl. r $5 \mathrm{kr}$.

I. a traduction allemande est en regard du texte latin.

853. Unen scuernton, oder die Gefaluren dis friihen Begrabens. Sur la mort apparente, ou les daugers d'un enterrement précipité: par CH. L. K.Issk; in-8. avec pl. Francfort; 1822 ; Jö̈ger.

853. Dis kieler seEB.in dargestellt und rerghichen mit andern Secboïlern and der Ostsee und Vordsee. Les bains de mer de Kiel, décrits et comparés aver: ceux de la Baltieque et de la mer du Nord, pare Praff, prof. de méd. et de chimie à l'universití de Kiel; in-8. Kiel ; 1822.

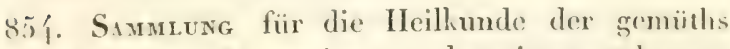
hrankhriten. Répertoire pour le traitement de's mat ladies del'ime, par Max. Jacobr, in-\$., tom. I avee: pl. lith. Prix, 2 rixl. Fhorfeld; rses; Sohonian.

Cet ouvrage est divisé en deux parties : Ia premiere contient une description de l'hòpital des fons à l'usage des quatkers près d'York, par Sam. Tuke. La seconde est un traté sur la manic, par Esquirol. Exirait du Dict. des sciences médicales.

855. Systev ner nistologie. Systeme d'histologie, par C. Fr. Hersixcren, in-á., cah. i ; prix, r rul. Eisenac; 1822 ; Barecke.

Cet ouvrage sera composé de 3 parties ou de 13 cahiers; 
In re. contiendra l'histographie; la $2^{\circ}$. l'histogénie; et la 3 e. I'histonomie. (Journ. gén. de la Lit. étr., déc. 1822.)

856. Medizinisch-practische Vorlesungen über die Natur und Heilung der Contagion. Conrs de médecine pratique sur la nature et le traitement des contagions; par Val AL. Brera, trad. de l'italien, avec des remarques, par $\Lambda$. Fr. Bцocu, in-8. Prix, I rixd. I6 gr'. Halberstadt; I822; Vogler.

857. Kimelne medizinische schriften, etc., Recueil d'opuscules de médecine du prof. G. Guill. HuFeLAND, in-8. avec pl. Tome I. Berlin; i 822.

Ce recueil contient les opuscules anciens et nouveaux du célèbre Infeland, insérés dans plusieurs journaux; il est revu et publié par l'sutenr lui-même. On remarqque, dans ce premier volume, les traités particuliers suivans: Dissertatio sistens usum vis electrice in asplyxid, experimentis illustratum. - Observations sur les fièvres de lait des femmes en couches. - De arsenico deglutito et sale alcalino segetabili, egregio ejus antideto. - Sur la contagion de la petite vérole. - Histoire de la maladie vénérienne. - Des effets de l'électricité sur la plante Hedysarum gy rans. - Sur l'incertitude de la mort.

858. Medizinisch-practische adverisarien. Observations médico-pratiques, recueillies au lit des malades, par P. J. Scuneider, in-8. liv. I. Tubingue, I822; Laupp.

Cette première livraison est divisée en 20 chapitres, dont les principaux sont : Sur les convulsions des enfans et leur traitement. - La stranguric. - Douze cas de mélancolie et d'alínation mentale. - Cas de monstre par excès. - Cas de fungus hocmatodes. - Cas de trismus des nouveau-nés. Observations sur le ver solititire. - Cas de catarrhexis vera. - Cure d'une diplopic. - Sur les effets de plusieurs remèdes nouveaux, tels que l'acide prussique, la racine de ratanhit, ete. 859. Bericht ïbr die taubsumien, Anstilt zu Konigsberg. Ripport sur linstitut royal des sourds 


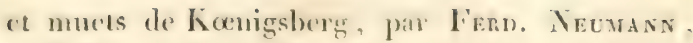
in-8. Koenigsberg. 1822. Unzer.

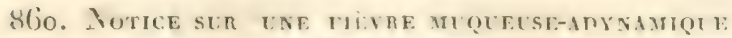
qui rigna pendant lié et l'automme is 822 , et fit périr environ un dixieme des habitans, dans lacommune de Tillechétive, eanton de Corisiers, chíp. de I'Yomne; par P. L. Dupró, offeier de santé à Cerisiors. (Yonne.) (Journ. de plyssiol. exp., t. 3, n. I.)

Celte maladie parait reconnaitre pour cause principale les émanations de plusieurs réservoirs d'eaux dormantes, ainsi que l'usace interne de ces mimes eaux pendant les chalents de l'été.

Les observations de M. Dupré sont dignes d'intérêl, parter qquel'épidémie qu’il a observée se lie à des expériences trésremarquables sur l'introduction des matières putrides dans lit circulation, faites par M. Magnendie; aussi cet habile experimentateur a-t-il fait suivre de quelques expériences les olservations de $\mathrm{MI}$. Dupré.

SGr. Remingues sur la notice préctidente aver quelques expériences sur les ellets des substances on putréfaction, par M. Magende.

On trouve dans ces expériences des détails curieux sur les accidens produits par les matieres putréfies introduites dins lat circulation; et l'on est frappé de l'analogie qui existe cutre les accidens qu'éprouvent les animaux sounis à ces épreuves, et quelques-uns des symptómes de la fievre janne. I). F. SGo. Méuecine piatique, par MI. A. Destris, D). M. I vol. in-8.

Cet ouvrage contient un recueil d'observations propres it l'auteur, et qui servent de texte à quelques discussions. $A$ u reste, cet ouvrage n'est nullement complet, et mérite peu de fixer l'attention des médecins. (liev. méd., jans. 18\%3). 863. Mranora sobre el contagio de la fiever amarilla.

Mémoire sur la contaģion de la frive jaune, par Rumono Romens-Verasecez; public par ordede de la société royale de médecine; in-8. Matrid; risos. 


\section{Médecine.}

86if. O) the yolow fever. Remarques sur la fièrre jaune épidtémique rui a paru par intervalle sur la cote méridionale de l'Espagne, depuis 1800 ; par Juckson, in-8. Prix, \& sh. Londres; Underwood.

865. A theatregen syuptomitic fevens, etc. Traité sur les fièves symptomatiqnes, comprenant les hémorThagies inflammatoires en les sécrétions muqueuses, par A. P. WV. Phulres. féd. in-8. Prix, rs sh. br. Londres.

866. Select dissentations on sevenal subjects of mentc la science. Disscrtations choisies sur difléjens sujets de la science médicale, par sir Gr. Blanc, médecin du roi, rassemblées maintenant pour la prenière fois, avee des changemens, des additions renfermant plusicurs articles originaux ct nouveaux; in-8. Prix, 12 sh. br. Londre's; Th. et (. Underwood.

S6- 7 . On the mantcal efficicy of electlicity on nervous and chronic disorders. De l'eflicacié de l'électricité dans les maladies nervenses et chroniques, par Labeavae. Prix ; io sh. Londres.

868. Onservations on some of the general principles, niture and treatment of inflammation. Observattions sur quelques-uns des principes généraux, la nature et le traitement de l'inflammation; ouvrage yui a remporté le prix fondé par Jackson, pour Isis; par J. Juxss; in-S. Prix, ro sh. 6 d. cart. Londres; Underwood.

869 . IIstony and nothod of eure of the various species of epilepsy. Llistoire des differentes esperees d'épilepsie et maniere de les guérir, formant le a". vol. d'un traité sur l's maladies nerveuse's, par J. Cooke, M. D. Londres; Longman.

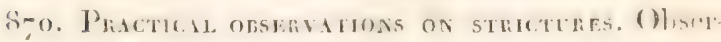


vations pratiques sur les rétréciec mons du canal de l'uretre, par C. Conntxay. Prix, $2 \frac{1}{3}$ sh. Londres.

87r. Dottrina der momo patechite, efe., Doctrime: théorique et pratique de la fievre pétéchiale, avese de nouvelles recherehes sur l'origine, les progres et le traitement de cetle maladie en particulier, et d'antres maladies contagieuses; par E. Aceribi in-8. Milan; 1822 ; Pirotta.

S-2. Ad icct et chnoxic.x splentums in humililus prasertim Italia locis considerata, ridenque tendentium morborum historicas animalversiones. Auct. St. Grottanelli, 8 fl. is 2. Piati.

873. Delma maxiera di fosidre, dirigere et conservare un instituto balneo-sanitario, etc. Sur la manière d'établir, de diriger et de conserver desbains médicamenteux, avec des observations cliniques sur les maladies auxquelles ils sont propres; par P. PAganini ; in-8. Turin ; I822; Imp. roy.

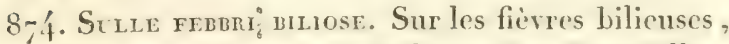
par Domen. Meli ; in-8. Milan; i 822 ; Brambilla.

8-5. Historre de la fì̀ne pítŕchile de Gímes, pendant les années r 7 !9 et 1800 ; par J. Rasori, proto-médecin près le ministèe de l'intérieur du roxaume dIalie, professeur de clinique au granel hôpital civil et à l'hipital militaire de Mlilan; traduit de l'italien, par I. Pн. Foxtaxerias, D. M. jn-8. Paris; is 3. (Journ. univ. de's sc. méc!. tom. 28, p. 152.)

La ville de Gênes, en 1799 , fut affliscée d'une épidémic qui, ayant commencé arant que le blocus de cette phace fit complet, dura aussi lons-lemps que le siéne, et se fit encore ressentir après qu'il cut élé levé.

Il est remarquable que, primitivement, les grens aisés et cenx d'une forte constilution furent plus particulicrement atteints de cette maladic, dont l'encrgic diminua lorspue lat 
famine ent commencé ses ravages, el qu'ensuite elle reprit ane nonvelle furcur' 'puand l'abondance reparut.

M. Worbe, dans l'analyse qu'il donne de l'ourrage de M. Rasori, indique les symptômes caractéristiques des diverses périodes de cette maladie, et éest dans la seconde que se développèrent le plus souvent les pétéchies ou autres exantliemes gui ont fait adopter a MI. Rasori la dénomination de fierre pétéchiale. Ce médecin croit que cette maladic a ité observée longr-temps avant 1515 , époque où on avait crut. pouvoir placer son origine.

La description et le traitement de cette maladic ne forment qu'une portion de l'ouvrage de M. Rasori. Il s'efforce d'en rechercher la cause, et arrive à cetle conséquence remarquable: Il y a indubitablement une matière étrangire rècue dans le systeme général, et dont l'action constitue la 'raie essence de la maladie; et celle-ci est sujelle il parrourir une période qui dépend tellement de l'cssence de le cause, qu'il n'est au pouroir de la médecine ni de la alitruire, ni méme de l'interrompre.

Enfin, examinant si cette fievre est contagieuse, M. liasori ne balance point à se prononcer pour l'affirmative, et a regarder conme erronce et pernicieuse l'opinion qui attribue son développenent à des causes locales. On sent que l'im portince de cette conclusion est telle que l'on doit isi se borner a rapporter et non a discuter l'opinion de l'auteur. \$-g. Onselivation d'une dégénération tuberculeuse du foie, avec accroissement de la rate, des alucis rénaux et des calculs, par Robinson. (Ediml. med. and surg. Journ., janv., p. 33.)

Pendant long-temps le malade éprouva des douleurs profondément situées dans l'hypocondre droit; tumeur cons:dérable du foie, etc. l’usage din mercure rétablit sa santé pendant un certain temps, mais les premiers symptòmes reparurent de nouveau et furent bientot compliqués de gratvelle, de rétention d'urine, cte. Aprés la mort an trouva l'épiploon réuni en une seule masse, le foie trés-velumincas, sa surface présentail un grand nombre de tubercules, d'alliérences, et les débris de la poche d'un abces : sal substance etait conpletentent désorganise. Lit rale etatil tris- 
volumineuse, ainsi que les reins. Les deux ureleren etaitent complétenent obstrués par des calculs ; la substance corticale des reins présentait des abcès, etc.

H. E.

87\%. Quxeges nians sur lintroduction de la varcine parmi les habitans de lintérient de: Ceylan. et sur une petite vérole épidémigues qui régnat dans les provinees de: Kandye, en 18 is g, par II. Marsum.l.(Edimb. med. and surg. Juarn., p. 7 1.)

Il parait que jusqu'ici les efforts du gouvernement jour favoriver la propagation de la vaccine n'ont été suisis que de peu de succés. Les habitans de ce pays regrirdent lit jetite vérole comme causée par la déesse Patima, dont ih craignent d'attaquer les prérogatires. Ils croient aussi que li vaccination est un signe indélébile de leur dépendance du gouvernement anglais.

H. E.

ö. S. Notices extraites des procés-rerlanx des rél-

nious de la sociétć pour la propagation de la mithode d'enseign. mutucl. (Antologia, janv. 1833.)

La Société, à la suite d'une lettre qui lui fut adresséc par le gronfalonier de la commune d'Lmpoli en Toscane, sur le: ravages de la petite vérole et les progrés de la vaccine dan cette commune, a décidé qu'à l'avenir aucun enfant ne serai admis dans les écoles gratuites d'enseignement mutuel, s"il n'avait cu la petite vérole, ou bien s̈il n'avait été vaccinse. Cette sage mesure, prise dans une ville aussi perplep que Florence, aura une grande influence sur la propatgation de lat vaccine qui, en Italie, n'a pas recu des gouvernemens les mêmes encouragemens qu'en France.

b̈-9. Revineues sur les signes dommés par les anteurs de médecine légale, eomme properes at faime commitre si le corjes d'une persome trouvic pertdue, Ja été apres la mort, on pendant qui ille wi-

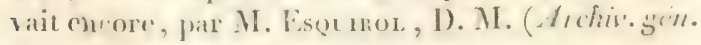
de. méd., janv. $\left.1823, \mathrm{p} . x_{\text {. }}\right)$

Il est un cas de méderine ligale oir la dercision du mede. cin peut entraner ou écuter les soupeons len plas giasen 
c'est lorsqu'il s'agit de déteminer si un individu trouvi pendu l'a été avant ou après la mort.

M. Esquirol, voulant éclairer par des observations nouvelles ce point important, rapporte quatre faits qui prouvent que les phénomines attribués a la stransgulation pendari la vie sont loin d'ètre aussi constans que les mélecins lénistes l'assurent. Des observations qu'il cite, et des consilerations anxquelles elles donnent lieu, l'auteur tire les consífuences suivantes :

Les signes de lividití, et surtout l'ecchymose du cou, ne sont pas des symptômes constans, et doivent être reçardín comme des indices trés-équivoques de la suspension avaint lat mort.

Lorsqu'un médecin est appelé pour faire la visite d'un cadlave trouvé pendu, il doit tenir compte de l'heure it laquelle la mort a eu lieu, et du temps que le lien a été maintenu autour du cou; ces deux circonstances molifiant beaucoup les phénomènes cadavériques, lesquels servent de lase au jugement qu'il doit porter. Il suffit d'indiquer les ohservations et les réflexions de l'auteur, pour en faire sentir toute l'importance.

Pinel fils.

88́o. Note sur l'emploi des préparations d'or en mó. decine. ( Bull. de la soc. philom. de Paris, nuv., p. 184.$)$

M. le professeur Lallemand, de Montpellier, vient de pu hier un travail sur l'emploi des préparations d'or, dans las pratique médicale. It a, en particulier, obtenu des suecien anssi prompts que durables du nuriate d'or et de sonde. chez plusieurs individns affectés presque en méme temps de maladies vénériennes invétérées, contre lescuelles les prépa rations mercurielles avaient échoué.

Ce praticien distingué préfere le sel d'or au mercure, foutes les fois qu'un premier traitentent mercuriel a été infouctueus, et, a plus forte raison, apres un second et un troisituse.

Il dit aussi lavoir employé avec autant de succés dians les affections récentes que dans les plus invétérées.

Il conscille de l'administrer 'n frictions, sur la langue, stu les gencives ou à l'intérienr des joues.

ba dose est d'abord d'un quinziene ou d'ua scizieme di 
grain par jour, et l'on passe ensuite sucecssivement a un puatorzicme, a un dousicme, ete. jusqu'i un sixieme do rrain.

Sept à huit grains suffisent ordinairement.

Pendant l'usage duremede il ne survient aucun changenent remarquable en mal daus l'état de santé; les greneives nien sont point affectées, et les caractères extéricurs de la malatie. disparaissent prompteınent.

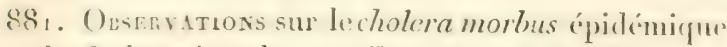
de's ludes orientale's, par Rixken, M. D. (Ëd.med. and sur's. Journ., janv. 1823, p. 1.)

Cetle épidintic, observic dins un camp situe prés de Jey. prars, dans le Bengrale, renfermant plus de 1500 hommen, natifs du pays, et une compagnie d'Européens, commena le 12 sept. 1818 . Dans lintervalle du i sept. an 2 oct., époque a larquelle la mortalité cessa, le nombre des maldelen admis dans les hôpitaux s'ileva à $\mathbf{1 5 1 4}$, et celui des morts à x leur violence de ceux du cliolera-morbus des antenrs. Les causes paraissaient ètre la déviation des saisons de lenx cours ordinaire, de vicissitudes extrèmes de température, une population tris-nombreuse, une nourriture insuffisante el malsaine, et enfin tout ce qui produit souvent les fievres intermittentes. L'auteur trouve une grande analogrie cntre les phénomènes et les suites de cette affection, et l'effet de certains poisons. I.es remedes les plus efficaces sont, t'apries lui, les stimulans diffusibles, et surfout la teinfure d'opium a trés-haute dose, et l'application de la chaleur a la surfare des corps et aux extrémités. Cent goultes de teinture d'opium administrécs au début des vomisiemenssuffisent souvent pour arréter la maladie. Taut que l'opium est rejetéinmediatement par le vomissement, il fant en renouveler la dose; s'il reute dans l'estomac sans produire de soulagement, on peut en domner troi, domes dans une henre. Employó en petite quan. lite ce médicament est inutile; les proratifs et la sinisnée paraissent dangereux.

11. E.

S82. Ie duct. Arems, midecin anericain, declare positi-

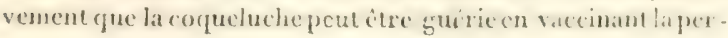


sonne malade pendant la seconde on la troisieme semaine depuis le commencement de la maladie; il est à désirer qu'on fasse de nonvelles épreuves de ce moyen, puisqu'elles sont sans danger. (Europ. Magazine, $\mathbf{n}^{\circ} .49^{3}$, févr. 1823, p. 77.)

$$
\text { D. F. }
$$

883. M. Peter A nuersov, de Sudermanie, propose pour la guérison des maladies syphilitiques les plus invétérées des fumigations. Le collére de médecine de Stockholm, voulan examiner ce mode de traitement et ses résultats, l'a cngagé à entreprendre la cure de plusieurs individus attaqués de cette maladie; huit d'entr'cux, sur lesquels le mercure et le régime n'avaient produit aucun effet, ont été complètemeñ guéris en deux, trois ou cinq semaines. M. II eigel, président du collége, satisfat de ces résultats, a fait a l'auteur du remède une gratification de 366 rixdallers, et lui en a promis encore autant si dans l'espace de deux ans les iudividus guéris n'éprouvaient aucun effet de leur ancienne maladie. (Europ. Magazine, févr, 1823, p. 79.)

\section{CHI I UR GIE.}

88 f. Défense de la littérature espagnolé en général, et en particulier de la chirurgic militaire, par don Manuel Ronngurz, chirur. en chef de l'hôpital de Mahon, in-\$. Madrid; 1822 . (Journ. gén. de la litt. étr., déc. I822.)

Cette brochure est dirigée contre le dictionnaire des Sciences Médicales, publié à Paris.

885. A treatise on dislocations, and on fractures of the joints. Traité des luxations et des fractures des articulations, par Sir Astrey Cooper, chirurgien du roi ; in-f. p. 562 , avee pl. Londres; 1832 ; che\% Longman.

Les essais chirurgicaux de sir $\Lambda$. Cooperet de M. Travers contenaient des mémoires sur les luxations, qui ont fait vivement désirer à tous les chirurgiens que leur auteur trititit, ex-professo, cette branche de l'art, sur quelques joints de liequelle il avait deja ripandu tant ele lumicre. Lionvrase 


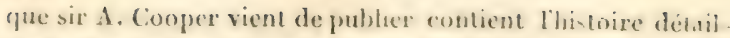
liede chaque luxation, ainsi que eclle de fiactures des estre.

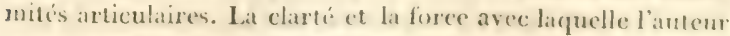
Hace le tableau des suites immodiales ou eloignées de coes accidens, et lesolsservations nombreuses qu'il rapporte poiar servir d'exemple on de preuve de tous les faits qüil avances, rendent cet ouvrage egalement utile a celui qui éudie, et it colui qui exere la chirurgie. Les méthodes de traitement dicrites ici ditfirent sombent de celles cmployées en France; nous ne pourons discuter ici leurs avantages respectifs. II nous suffira de dire que, quels que soient les modes de réduclion employis, les olservations et les sacres préceptes de sir A. Copoper ne peuvent manquer de fournir ume instruc: tion solide aux jeunes chirureriens, et de fixer lattention de ceux qui exercent depuis long-temps la méme profession.

IE luxe typosaphique de ce livre le rapproche des plus belles iditions de Didol. Il contiont 30 plandes qui ne: laissent rien a désirer pour le soin et le gout avec leguel elles sont exécutées.

H. E.

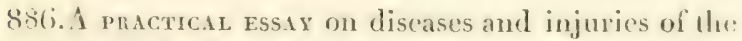
bladder, etc., on essai pratique sur les maladic's, v1 les blessures de la vessie; ontragequi a rempurto l:" pris fonde par Jachson, au collége royal des chi-

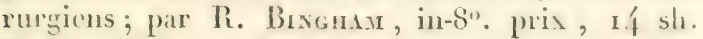
Londres; I822.

Cet ourage de M. Bingliam est tres-interessant: l'auteur distingne, dans les maladies de la vessie, celles qui affectent i. membrane muqueuse, de celles que prósente la unembranemus. culaire : lirritation de cet orane est un symptône tres-fre quent dans les maladies des or zyanes éloignés; par exemple, le derangrement des intestins. Cotte irritation donne licu a lat serétion d'nue matii re muqueuse qui s'écoube avec l'urime. L'inflammation de la vessie peut se terminer pats la sécrelion dine fanso membrane du pus, ou meme par la grangrence :

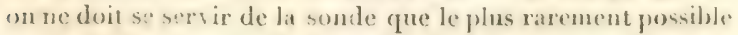

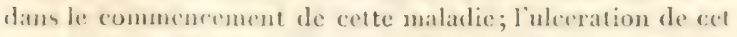

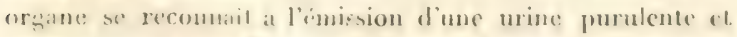

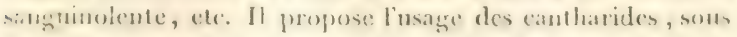

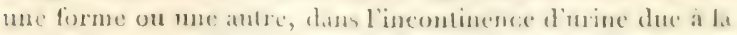


faiblesse de la vessie. Il croit que les calculs se forment souvent dans la vessic par une sécrétion propre de la membrane. muqueuse : voici la preave qu'il en domne: "la membrane. muçueuse est souvent si chargée de matiere terrense, qu'elle parait blanche, et quion peut, au toucher, sentir les particules salines, etc.

P. V.

ถู่า. Nouvelles néuonstrations d'accouchemensave: des pl. en taille douce, accompagnées d'un texte? raisonné propre à en ficiliter l'application, par .J. P. Matgrier. D. M.

L'art des accouchemens est une des parties de la médecin qui peuvent le mieux ètre éclairries par la représentation mème des objets. C'est un mécanisme animé dont les diverses pièces doivent être bien comuues, et dont les puissances penvent. Are utilement dirigées par l'art. M. Maigrier a joint à ces planches, qui sont très-bien gravées et tris-exactes, nu lexte qui expose les divers usiges de ces parties, et leur importance dans l'accouchement.--Cet ouvr. parait par livr. A. I).

sis. Handet ch der meedmien-kunst, ou mamud de l'art des aecouchemens, par Tu. Lenerer, in-s. avec 8 pl. Prix, 3 fl. Vienne; 1822 ; Armbruster.

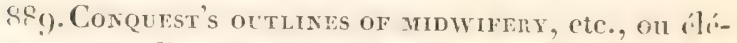
mons de lart de l'accoucheur; ou les principes at la praticgue en sont développés de manière à servir de live classique pour les étudians, et de répertoire nux jeunes praticiens, par J. T. Coneuest, D. M. membre du col. roy. des méd. de Londres, cle. "r. éd. fig. in-r 2. Prix, 7 sh. Londres, J. Anderson.

\&go. Symopsis of tue varous kinds of difficult parturition, ete., ou apercu des diflérentes espéers d'accouchemens difticiles, avec des observations pratiques sur les manoeuves à cmployer, avec un appendice contenant des cas particuliers, et des tables, par Samnel Mennzus, M. 1). 3". édie. in-8. considérablement amélioréce; Prix, in sh. Londres, Longman. 
¿gr. Mevoria seconna sopra il metodo d'estrarre la pielra pex via dell' intestino retto; ou second mémoire sur la méthode d'extritire la pierre par le rectum, par Benlragren, in-8. Pise; 1822 ; \istri.

¿g?. Cooper's srfigrcir. Dictionary, ete, ou dictiommaice de: chirurgic pratique, contenant un exposé complet de l'état actucl de la chirurgie théorique et pratique, par S. Cooper, fédit. in-S. Prix, r liv. sterl. Londres; Underwood.

8()3. The first lines of the prictise of surgerr, etc., ou premieres lignes sur la chirurgie praticuc, 2 vol. in-8. avec des pl. par S. Cooper, $3 e$. édit. Londres.

Sóf. De oferative meelkinde (en Ilollandais), ou la médecine opératoire, par A. G. Taxoxsexoont, tom. I in-8. avec fig. Prix, 6 fr. 50 c. Amsterdim; r822; Sulpke.

Syj. Uiber dex brecu des Schlüsselbeines, etc., ou sur la rupture de la clavicule, et les difúrentes méthodes de la guérir, par Ph. WiLnes, in-s'. avec $\mathrm{pl}$. lith. Würzbourg; 1822 .

896 . A rReatise on the radical cure of the hernia, etc, ou traité sur le traitement radical de lit hernie, par W. Dufour; prix, 5 sh.

Bg-. Lecturs ox tue opendtrive surgery of the eye, ele., ou lecons sur les opérations chirurexicales de l'xil, on extrait des lecons sur les principes et la pratique de la chirurgie en général, relativement aux maladies de cet organe, par C.J. Gutume, écuyer, in-8. Londres ; J. Souter.

Cet ouvrage, qui est sous presse, va paraitre incessamment.

Sige. Ronus ox caxeen, ete, ou Traité pratique sur le cancer du sein chez les femmes, par J. Ronvis, M. D. 2 édit. in-8. prix, 8 sh. Londers; Underwood. 
Beg. An essay on cenvatenes and distortions of the spine, etc. Fssai sur les courbures et le's déviations de l'épine dorsale, et sur quelques autres dérangemens morbides auxquels elle est sujette, par R. W. Banprese, l'un des chimurgiens de linfirmerie royale de la métropole. (The London med. and pliys. Journ., fév. I 823, p. 96.)

90o. Note sur une nourrlle espèce d'opération chirurgicale, la staplizlorapluie, par M. Roux, (Bull. dessc. par la soc. philom. de Paris, sept., pag. I fa.)

Dans le courant du mois de juin 1822 , MI le professeur Roux, de Paris, a présenté a l'A cadémie Royale de Médecine un jeune homme, qu'une division congéniale du voile du palais, avait privé de la faculté de parler. Par une opération assez analogue à celle du bec de lievre, cet habile chirurgien est parvenu à rapprocher les deux lambeaux et à les 'maintenir dans une parfaite adhérence.

\section{PIIARMACIE.}

gor. Vegatable materil menica. Matière mélicale végétale des États-Unis, on botanique médicale, contenant lhistoire botanique, génćrale et médicale des plantes indigènes aux États-Unis, ornér de gravures cnlum. avec des dessins faits d'aprèsnature, par W. P. C. Bнитол, M. D. prof. de botanique a l'université de la Pensylvanie, 2 vol. in-4. Prix, 1.6. 6. London; Robinson.

903. Note sur vane nésine nouvelde présentéc par M. Mazjean, et rapport fait à la société de pharmacie de Paris, par MII. Raymond et Viney, sur cette nouvelle résine. (Bull. res travaux de la soc. de pharm. de Paris.)

En I82 I, M. Maujean a cxaminé pour la société linnéenne, une substance résineuse qui n’est pas la résine élémi de l'amyris elemifera, et qui a néanmoins donné les mèmes ré- 


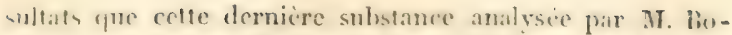
nastre, dont le travail est insciré dans le Pulletio de phermasie, 19\%2, p. 388. Ia résine, qui fait l’objet de la nose de 11. Manjean, apportée de Manilde par MI. Perroltet, déconle diun arbre, que les naturels appellent arbol a brea, arlire a brai). Il exuste deux pieds de ce végétal dans les serres du jardin du roi, et l'on pense qu'il appartient a la classe des foumineuses a fenilles pennées et à slipules. Io difuat de flems of de fruits empéche d'assigner d'une maniere exacte le caractère botanique.

Citte resine est d'un aspect verditre cendré, tres-pen framparente, mollasse, adherant facilement aux doiçts, se dessichant al'intérieur, et d'une odeur de térébnthine melice a colle de fenomil, surtout quand on lit chanffe. A l'asalrse, elle prisente deux résines bien distincles, l'une soluble: danslaticohol froid, l'autre seulement dans l'alcoliol honillan, raracteres communs aux deux résines trouvées dans lat résime élimi, par M. Bonastre.

D. I.

\section{ART VETERINAIRE.}

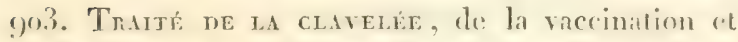
rlavelisation des bètes à laine, avec des notices historingues et physigues sur l'espéece bovine, sur la clavelé; beaucoup d'observations pratipues; drs histoires particulieres, et une histoire générale de fimtopsie des bites a laine dar leuses; lexposition flun grand nombre de faits rolatifs a lat vacrintion ut it la clatelisation; un parallite de la clasclese avere la variole; des experenees de clavelisalion humaine; des considéralions relatires a la darchere sur diverses especes d'animats, efe., etc., par

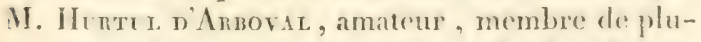
siemes socictés savantes, I vol. in-8. de plus de 300 p. Prix, $6 \mathrm{fr}$, et par la poste, 7 f. $25 \mathrm{r}$. Amicus: s'sas; de limp. de Madane veuse Iluzard, it Paris.

I.es grandes pertes qui sont si souvent la snite de linvasion de la clavele dans les troupeanx de biles a baine, rendent la 
connaissance de la nature de cette maladie, ainsi que les moyens de la próvenir et de la guérir, de première importance pour les propriétaires et les fermiers. Nous possédons quantité de bons éerits sur ces objets, notamment ceux de Tessier, Voisin, Barbançois, Clabert, Bumirat, Gilbert, etc.; mais ils sont la pinpart on difficiles à trouver cn collection dans la librairie, ou font partic de recueils volumineux, principalement des Annales d'agriculture.

M. Hurtul ci'Arboval a done rendu un éminent service à la science vétérinaire et à l'économie rurale, en réunissant ce qu'il a olscervé lui-même à tout ce qui avait été écrit précédemment sur ce sujet. Il se prononce, comme on devait sy attendre, en faveur de la clavelisation artificielle que quelques proprictaires, induits en erreur par des expériences mal faites, ont mal à propos repoussée.

Rendre un compte raisonné de l'ouvrage de cet amateur serait en composer un autre; car il cst si rempli de faits, qu'il faudrait presque le copier pour satisfaire le lecteur. $\mathrm{J}_{\mathrm{C}}$ me contenterai done d'annoncer qu'il est précédé d'une introduction très-sagement pensée, et très-bien écrite, sur rétat actuel de ce point de la science vétérinaire, et divisé en quatre parties, chacune subdivisée en chapitres, lesquels sont subdivisés en articles, et quelquefois les articles on sections. (Annaics de l'agric. franc., févr. $\mathbf{x} \mathrm{S}_{2} 3$.)

gof. Notice sur l'inocilation de la clavelée pratiquéc сn 1820,1821 , $182 \%$, par P. Mipuel, artiste vétérinaire ì beziers, et M. Thomizes, artiste vétérinaire, ì Nissan; in-8. d'une fenille. A Paris, au bureau de la Gazette de santé, rue Bergère, 11. ig.

gо5. Коте sur la pratique muisible de tailler, rogner et couper la corme de la fourchette des chevaux; par M. Bracr-Clareke, chirurgien-vétérinaire, membre de la Société Limmécnne de Londres. (Journ. de plyy., de chimie et d'hist. nat., sept. 1822.) 


\section{A GRICULTURE.}

go6. Tue present staxe of Figland, ou cill présent de l'Angleterre, sous le rappore de l'anticulture, du commerce et des finances, par M. Jos. Lowe, in-s. de 566 p. Londres; I 822 ; Lomgman.

On trouve damples extraits de cet ouvrage, dans le Jlon-

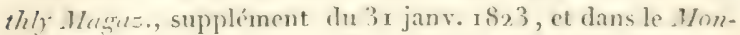
thly Consor du meme mois. Nons nemprunterons à ces journanx que quelques fatits statintiques, sans suivre l'auteur dans Je- conclusions politiques quil cherche à en tirer.

L'Angleterre, qui, de 1765 a i 8 i a eut constamment besnin d'importer des blés, en recueille depuis cette époque an delà de ce qu'exige sa consommation, quoique sa population se soit accrue des sept dixiemes. Ainsi, les moyens de sulpsistance ont augmenté plus rapidement encore que le nombre des habitans : ce qui semble répondre victoricusement aux argumens de M. Malthus.

M. Lowe trouve dans laccroissement des villes la cause principale de l'amélioration du sort des hommes. C'est dans les villes, dit-il, que se réunissent les arantaģes suivans: des marchés abondamment fournis, la division et la perfection du travail, des occupations appropriées aux diffírens degrés de force, d'intelligence et de talens.

It fait voir qu'en réduisant en mesures de blé froment les salaires des ouvriers de la campagne a différentes ípopues, et en les comparant à la valeur vénale du blé, le prix des journées, quoique presque double en apparenee, était réellement plus faible de moitie dans les premieres années de. ce siècle, qu’au milieu du siècle dernier.

Voici son tableau :

\begin{tabular}{|c|c|c|c|}
\hline Epoques. & $\begin{array}{c}\text { Prix des Journces } \\
\text { par senaine } \\
\text { en argent. }\end{array}$ & $\begin{array}{l}\text { Prix } \\
\text { du quarter } \\
\text { de ble. }\end{array}$ & $\begin{array}{l}\text { Journées } \\
\text { évaluies en } \\
\text { blé }\end{array}$ \\
\hline 1742.21752 & $6 \mathrm{sh}$. & $30 \mathrm{sh}$. & I02 pintes. \\
\hline$x-6 x$ à $177 x$ & 7 ct $\frac{x}{x}$ & 126 & 90 \\
\hline I780 a $179^{\circ}$ & 8 & $6{ }_{12}$ & 30 \\
\hline т795 а 1799 & 9 & 708 & 65 \\
\hline 2800 a $\times 808$ & II & 868 & go \\
\hline
\end{tabular}


Suivant un tableau détaillé donné par l'auteur, les dépenses nécessaires pour la culture de roo acres de terre, en Angleterre, s'élevaient en $159^{\circ}$, à 411 liv. st. 16 sh.; $\mathrm{cn}$ x 803 , à 547,11 ; en 1813 , à 771 , 16 .

M. Lowe prétend que les sommes qui sont entrées dans le (résor pullic de la Grande-Bretagne, soit par les impóts soit par les emprunts depuis le commencoment de $\mathbf{x} 79^{3}$, jusiqu'a la fin de 1815 , se sont élevées à $1,564,000,000$ de liv. sterl. Fin diéluisant de cette somme 46 i millions st. pour les dépenses qui auraient eu lien de méme en temps de paix, il reste pour les dépenses occisionées par 22 ou 23 anmées de guerre, onze cent millions sterl.

C. M.

907. A letter on the rresext state, etc, ou lettre sur l'état actuel et l'état futur de l'agriculture, par W. Whitmone, écuyer. Prix, 2: sh.

9o8. The farmer's directory and gutde, etc., ou guide du fermier et du maréchal ferrant, herbageur et cultivateur, suivi de l'Instituteur domestique, contenant un système d'économic agricole, et offrant les diflérentes manières d'exploitation des terres, avec fig. par M. L. Towne, r vol. in-f. Prix, I l. ro sh. Londres; Henry lisher.

909. Arsicuten von der Behandlung der Eedrinde, ou traité du sol et de la éulture de la terre, par C. G. Krifes, in-8, arec pl. Prix, is gr. Drescle; I822: Arnold.

910. An enctcloptan of gindenixe, etc., ou encyelopédie du jardinage, comprenant la théric et la pratique de: la culure des jardins, des fleur's, des arbres et des jardins de plaisane, ainsi you les derniers perfectionnemens; une histoire géucivile du jardinage dans tous les pays, et un aperecu stittistique de son état actuel, arec des vue's prour se's progrès futurs dans les iles britannicues, par J. C. LONDON, I gros vol. int-8. avec 600 grav. sur bois; prix, 2 1. 10 sh. Londres; Longmann. 
9tx. Joc nase of a hordiculumal tour throuerh fianders, Holland, ete. Jommal diun vorage welit-

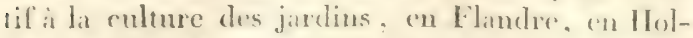

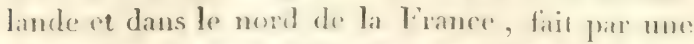
diputation de la socicté pour la culmue des jardins d'Écosse, avec pl. in-8. Prix, 16 sh.

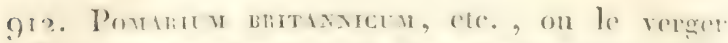
britumique, description historique et botamigne: des linits, par II. Phulles, 3r. édit. aus. de plusimus additions savantes, ainsi que d'ancedofes historirpues, étymologigues el botanipues, atres les

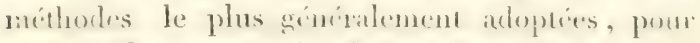
retarder la maturití des fruits, de maniere a prolongrer la jouissance de ces delicionses productions.

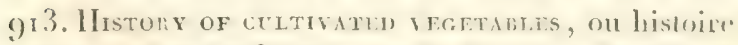
des vétanx cultivés, par II. Pmure, $2^{e}$.élit." vol. in-8. Prix, 2 1. Londres; Colburn.

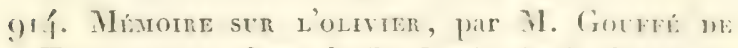
Thorsvilles, chev. de St.-Lonis; in-8. de (io p). couromé en rosa, par l'acadímie de Marseille. Marseille; Achard.

Une grelée de 11 à 12 derrés a frappé de mort, dans la muit du 12 janvier $x 820$, la plus grande partie des oliviers de France, qui avaient résisté aux précédentes.

Ce erand desastre, qui semble se renowiveler plus souscme depus un sicele, et qui a ruine un mombre comiderable de jropriétiares, a excité la sullicitude du gouvernement, ainsi que celle de plusieurs sociétés savantes.

Le prenier a fait imprimes chez madame Huzard, a Paris, une collection de memoires sar les moyens de diminur an-

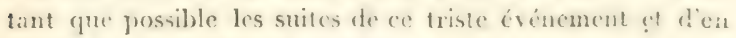
éloigner, s'il se pent, les retours.

Parmi les secondes, devait si montrer an premier rans, comme plus directement interesnie, et comme ayant deja provorué lesmeilleursouvrages sur lit culture de Folivier, l' l catdémie royale des Sciences, Letlres et Arts de Marscille; anui a-t-elle proposé un prix en 1821 , sur le's moy ine de rigurer 


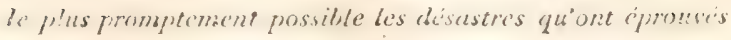
les oliviers par la gelie précitée.

Le mémoire de Ri. Couffe de Troisvilles a cité couronné, al il le méritait sans donte, comparativement anx trois autres envoyés aver lui an concours; mais, s'il est bien rédigré, il n'a pas répondu rigoureusement, à mon avis, à la question. Ën effet, au lieu d'indipuer exclusivement les moyens de répaver les dommages causés aux oliviers par la gelée, il s'occupe di lenr remplacement par rcjeions, par boutures, par éclits, par troncons, par ramérs, par bourgeons, par crossettes, par plancons et par greffe. Il par!e longuement, et hien, sur la taille, sur l'ébourgeonnement, sur les lalonrs, sur les engrais, sur les pépinières, tous objets qui devaient étre pris en convidcration, mais aprè̀s atroir indiqué les moyens de ríparer les oliviers dont les branches ont été dégradées par la gelf́e, dont les trones en ont eté frappés de mort, objets dont l'autenr annonce devoir s'occuper, mais dont it ne parle pas. Il semble qu'une partic de son manmserit se soi! perdue à l'imprimerie. Il y a trop peu de remarques critiques a fiare atu reste sur les details de cet ouvrage, Jour qu'il ne mérite pas d'tere recommand's aiex cultivateurs des difartemens ou croit l'olivier'; aussi je les invite à le lire. lionc.

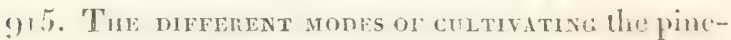
apple, etc., ou les diflérentes méthodes prour cultiver l'anamas, depuis son introduction en Burop", jusquanx derniers perfectionnemens de T. $\Lambda$.

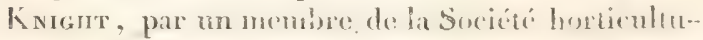
rale, in-8. avec 7 ́ grav. en bois. Prix, 9 sh. Londres; 1822 ; Longman.

gri. A concise a no practent treatise on the geowth,

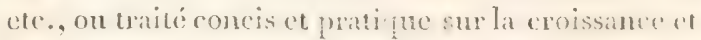
la culure de levillet carmé, de l'uxillet violet, de lat tnlipe, et autres fleurs, $2^{\circ}$. édit. aug. par 'Ти.

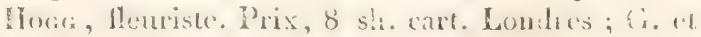
W. B. Whittaker.

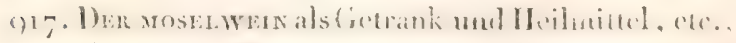

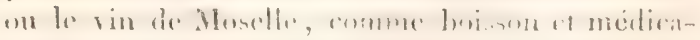
ment, avec un traté sar le conmerce du vin aux 


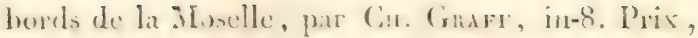
$54 \mathrm{kr}$. Bonn; 1822; Marcus.

9r8. Extuse des travaux die la société centrale diasriculture du département de la Seine-Infériemer, $8^{\mathrm{e}}$. cahier, in-s. de af. $\frac{1}{2}$, Rouen; imp. de Périaux père.

Rien n intigne le prix de l'abomnement ni le mode de publication.

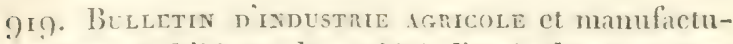
riire, pulblié par la société d'agriculture, arts et commeree de la Loire, section de l'arrondissement de St.-Étienne, ${ }^{\text {re }}$. et $2^{\prime \prime}$ livo oct. et déc. $18 \%$, 2 cahiers in-8. de 6 f. et I pl. grav. St.-Étienne; imp. de Boyer.

Il y aura 6 livraisons par an, a partir du 15 octobere 882 . -On s'abonne à St.-Etienue, chez le directeur de lis poste. Prix, par an, 8 fr.

920. De léconome peblique et rur.lle des Arabes et des Juifs, par L. Rerrere, in-8. imprimé à Lansanne. Paris et Genève; J. J. Paschoud.

Cette publication, suivant M. Reynier, n'est qu'une nonvelle livraison de son grand ouvrage, qui comprendra tout ee que l'on sait de l'économie publique ct rurale des anciens peuples; les deux livraisons précédentes ont traité des Coltes et des Germains, des P'erses et des Phéniciens. La nouvelle: est consacrée aux Arahes et aux Juifs.

L'oriz̧ine des Arabes, leur distribution en tribus nomades et sédentaires, la comparaison de l'arabe ancien et moderne; le pen de faits qui nous ont ćté transmis par les historiens, sur leurs mours, sur leur caractere ; l'influence qu'a ene sur l'ensemble de lenr existence politicue, lintroduction de l'islamisme, sont les divers points historiques qui, dans cette dissertition, précedent ce qu'il veut nous apprendre de leur acriculture et de leur commerce. Sur cette dernière partie, l'anteur emploie une srudition immense pour nous faire connaitre quelques faits isolés d'un bien faible intérêt.

Il est plus heureux en parlant des Juifs; il y a de la claleur 


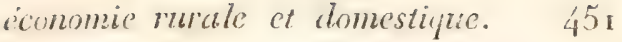

Aans ba narration; leur origine est présentée d’apres les divers sytimes qui les montrent ou.comme venant de l'A frique ou comme sortant de l'Asie, ou enfin comme une nation mélangéè nue éporn. reculée. Les nombreuses aliérations qu’a subies le gonvernement théocratique des Juifs sont assez bien décrites. Le chapitre oì est exposéc leur situation financiere, est une prenve des vastes connaissances de l'auteur'; on y retronve lorigine et la forme des impositions modernes; mais si les calculs de l'adieur élaient admis, les Juifs auraicnt paye plus le 43 " cais revenu, ec qui, ajoulé à la cessation des travaux pendant l'année sabbalique, aurait été pour la reproducfion des causes débilitantes tellement actives qu'elles l'au= mient, pour ainsi dire, anéantie.

Exeeple le temps du rigne de Salomon, jamais la Indée lia été une contrée commerçante; aussi est-ce comme peuple "rrricole que les Juifs sont ici représentés. Les rois, comme dins les premiers temps de $\mathrm{l}_{\mathrm{a}}$ Grèce, étaient pasteurs, et les fils des rois gardaient les trompeanx. Le culte des lois, les moxur, les institutions, tout est relatif aux champs el a leurs produits. Presfue tontes les bonmes móthodes de culture se retrouvent chez les Juifs; la clóture des propriélés, lirriģation pour un grand nombre de prothetions, la greffe, la eaprification, la multiplication des engrais naturels et artificiels, tout y est pratiqué avec une supériorité qui permettait, outre la nourriture de douze millions d'habitins, une immense exportation. Le froment de la Palestine nourrissait Rome, et chatit sa principale ressource; la culture des plantes utiles et de colles d'agrément y érait portéc au dernier degré de perfection; les jardiniers de cette nation étaient à Rome les plus estimés. Si les préceptes minutienx d'une loi positive n'avaient arrêté leurs progrés, ils eussent été cucore braucoup plus étendus. Nons renvoyons au livre mème les dítitils pleins d'intérét sur les bestianx et leur culture, sur celle des prairies, des poiagers et des jardins d'agrément. Cet ourage, débarrassé de quelques dissertations oiseuses et sumont phus chatié sous le rapport du style, nous paraitrait susceplible de fare partic obligée d'un: bibliothégue agricole, el d'etre le luxe drune bibiotheque écomonique.

BI RILIX.

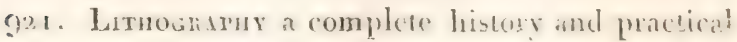


rourse, rte., ou cours complet de litlographio pratigue, depuis son orisine jusqu à présent; demiin-f. Prix, i 1. G sh. Londres; Ackermann.

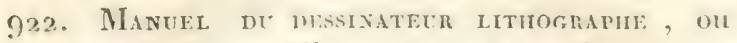
description des mullewrs noyens à employer pour fitire des dexins sur pierres, dans tous les grmes commus, suivie diune instruetion sur Je noureau

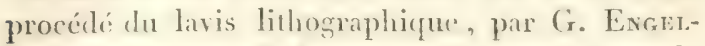
maxs, directem de la sociét lithographique: de: Mulhouse, in-s. de 5 f. $\frac{\dot{a}}{2}$ et 15 pl. Prix, $6 \mathrm{f}$. it Pinis, chez liauteur, rue Louis-le-!rmul, 11.27.

Cét ouvage contient un exposé des procédés à suivre pour exécuter sur pierre tous les dessins qu'il est possible de xeproduire avec le crayou et l'encre lithographiques; toils les détails nécessaires pour vaincre les nonbloreuse's difficultés que cet art présente, obtenir un travail satisfaisant sur lit pierre et assurer le succès de l'impression.

Il explique en outre, l’usage du tampon, proposé par .T. Engelman, pour imiter la gravure a l'atua-tintı, faire dans les paysages des ciels vaporeux, et donner aux teintes plus d'effet et d'harmonic. II. S.

923. Acclis practell treitise on chs-Light, ou

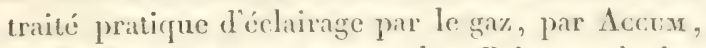
in-s. 4e. él. avec 7 grav. color. Prix, a sh. lur. Londres; Ackermann.

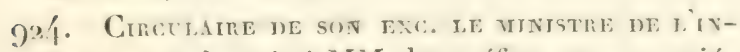

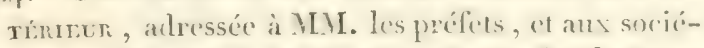
tés savantes du royaune, it r ponses de? plusicurs

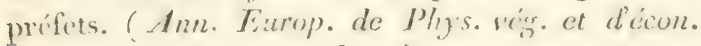
publ. To. III, $9^{\mathrm{e}}$. et $\mathrm{ro}^{\mathrm{e}}$. livr.)

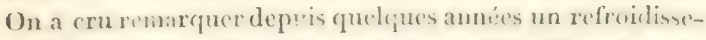
ment dans latmonphiere, des varialions subiten dans len satisons, des onrasias et des inondalions extranrdinaires de plus en plus frequens. On l'attribue en portic au déboisement des montanges, anx defrichemens, elc. I,e minstre a voulu constater, par lobservation et la rimion des faits pour ou contre, ec quil puuvait y avoir de fomdé dans celle: 
opinion, et les mesures lérislatives qu'il conviendatil demployer pour arrêter les dégrâts occasionés par les travanx des hommes. Tel est lobjet de cette circulaire du plus hant intirèt, et dans laquelle les autorités sont invitées à répondre aux cinq questions suivantes :

I". Quelles forêts existaient dans votre dípartement il y a trente ans? dans quelle zone et à quelle élévation élaientellos placées? quelles élaient leur étendue et l'espèce d'arbres dont elles étaient formées?

2०. Quels étaient les propriétaires?

$3^{\circ}$. Quelles sont celles qui existent encore et celles qui ont été abattues?

/". ()uelle influence a-t-on remar qué quela différence d'arbres exeruit sur le systéme météorologique du département? Les rivieres ont-elles eu des caux plus ou moins abondanies? Les inondations, les pluies, ont-elles cté plus on moins fitequenIfs? Y a-t-il cu plus souvent de la neige ou de la grièle, et, dans les pays de montagnes, s'est-on aperen que les glaces descendissent à de plus basses régions, repoussant et refoulant la végétation vers les plaines et les vallées?

5\%. Lesvents ont-ils été vlus violens, plus malfaisans, plus variables, et a-t-on remarqué que ceux du sud ou du nord cxercassent tout-a-coup, et par de sondains changenons, de plus grands ravages que dans le siecle dernier, et lorsque $\mathbf{l}_{a}$ France enfin était mieux boisće?

Les riponses a ces questions sont transmises par le ninisure à l'académie des sciences qui doit, aprés les avoir toutes cxaminces, faire un rapport sur l'encemble des fails recurillis et sur les moyens d'annélioration proposés. Yous fermas connaitre ce rapport d'un liaut intérét, lorsque l'acadéniés én sera occupéc.

Les departemens dont les réponses sont insérécs avec: les remarques incéressantes de Mi. Janch, dans les ge. et yo".

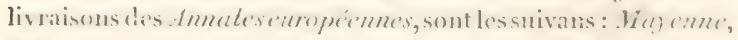

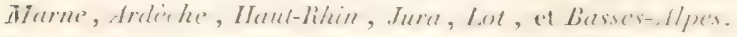

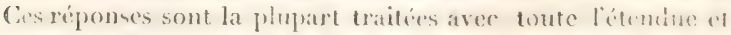
lo talent désirable's; mais on est fuché de voir gaim pelut nombre d'entre dles semblent avoir éte fities par lo premier (')mmis venu, et comme on rijondrat a la circulaire: lat pluinsignifiantc. 


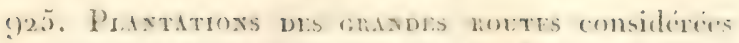
comme montumens publics. (Amn. I: urop) de ple) s. végét. etc., '1. 3, p. 39o:)

L'anteur de ce travail propose d'employer a cet unatere, le tilleul, le marronuier, le chitaignier, l'orme, le saule, le noyer, le penplier, et surtont le murier noir ou blane, celui d'Espagne et celui de Virginie.

DE CII.

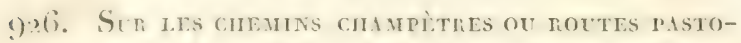
nitris. ( Ann. Fiurop. de pliys. vizét. et d'écon. publ. T. 2, p. for.)

Vin calculant que chacune des quaxante mille communes de. France pos-irle ('une dans l'autre), environ trois lienes de chemins vicinand oi peut passer une voitme, on tronve min lonsueur totale de cent vingt mille lienes, susceptiblen de recevoir deux cent soixante milliers de pieds d'arbes fruitérs équivalant à un million d'upens de bois at fruts. nf. (is.

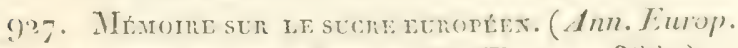

$$
\begin{aligned}
& \text { de plins. vescit. el d'écon. publ. T. 2, p. 36it.) }
\end{aligned}
$$

L'inteur de ce mémoire estime la consonmation de sucre pour l'Europe à quatre cent cinquante millions de livres par an, qui coùtent environ six cents millions en écus, et pense qu'il serait extrèmement fucile d'afranchir le continent de cet impôt inorme, si tons lés penples rui lhabitent s'entendaient. pour se plus se servir que de sucre de betteraves. Propric. taire Ini-sueme d'une fabrique de ce sucre, il indicue, dins differens chapilres de son mómoire, le choix a fire de l.t plante, la maniere de la semer, le temps dusemis et de la récolte, atusi que celui de commencer et de finir la fabrication.

Quant a l'espiec de betterave, il se lécide pour colle a pean blanche, dont la pulpe est d'une couleur semblable, et pour celle aussi à peau blanche veinćc de jaune.

On sime a la main, du xer an 1 ómars; on récolte cusepternlre, et la fibrication commencéc dans a mois, doit se termincr au 5 janvicr.

In etablissement travaillan sur quatre millions de lieres de befteraves, peut raisonmabliment compler faire, outre yma. ante mille pintes d'excellente can-de-vic, deur cents millies- 


\section{conomic mate et domestipue. 455}

de sucre brut, à raison de cinc livres pour cent de racines.

Sil'on obtient, comme on ne peut en douter, selon l'auteur, le millier de betteraves a so fi., la live de sucre brut pourra revenir à cing ou six sous, et le pain de sncre raffiné it dix ou douze sous la livre.

ve CiI.

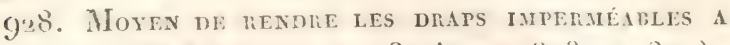

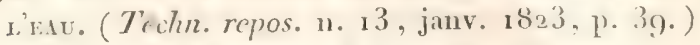

Il y a quelques années rue M. ACK ER ux , dans le Strand, commenca a s'occuper de ec procédé, dont la partie essentielle consiste dans l'immersion, pendant un certain temps, du drap, dans une dissolution de parties égales d'acétate de plomb et d'alun.

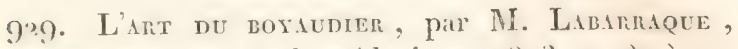
(Archiv. gén. de méd., janv. xง.s3, p. s̀g.)

MI. Labarraque vient de publier sous ce titre un mémoire couronné par la société d'encouragement. Parmi les oljets quilil renferme, il en est qui intéressent lit médecine; voiei les principaux: M. Labarraque a trouvé que le moven le plus eflicace, le plus prompt et le moins dispendieux d'enlever la membrane mucueuse des intestins traités dans les boyauderies, sans employer la macération et en s'opposint a lit puiréfaction, état de meitre les boyaux dans un mélange d'une prartie de chlorure de soude dans deux parties d'eau; l'odenr est détruite instintanément, et au bout de quelques heures la membrane muqueuse se détache tris-facilement.

Cé moyen peut être d'une grande utilité dans los amphithéâtres, soit pour conserver les cadavres, soit pour laver les tables, le sol, etc.

Pinel fils.

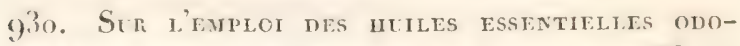
listre, pour prévenir la moisissure, par M. J. Mic Curtocn, D. M. (Edinb. philos. joum. janv. I 823 . p. 33.)

On sait depuis long-temps que le grirofle, mis dans de l'encre, empèche qu'clle ne se moisisse. On fout froduire be nóme effet avee une trés-pelite quantite dhuile de lavande, on de toute antre ensence. Les cuirs peusout ditre priservés de la moisinsure de la méme maniere. La colle de frime, qui moisit si aisement, mime lorstgion $y$ a mis de 


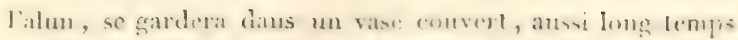

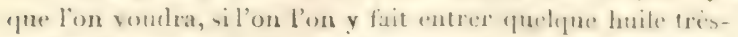
parfune, enmme celles de lavame, de menthe puivrie, d'anis, de bergamote. Ell: acenerra, en sechant, la consistance de la corne, mats nu pourra toujours s'en scrvit au besoin, après l'avoir liumectée.

A cette occasion, l'auteur comseille d'ajonter à colle espere: de colle, du sure brut pour la rendre flexible et cmperder quelle ne se ditiche des surfaces lisses on s'icaillant. II y mel ausi un pen de sublimé corrosif, soit pour écarter les insetes, soit pour empecher que cette coile ne fermente. C. M.

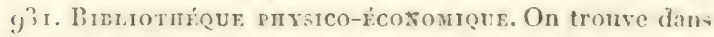
le calier d'oct. 1322 , I". me description de prunier, dil robe de sergent, et une instruction sur la culture de cet arbe, un des plus grands et des plus robustes parmi now muniers, à la suite de larjuelle l'auteur, M. uf Ru:vre, d'Agen, socerupe des malaties du prunier et des insecter qui lui sont le plus nuisibles; 2 . lit description el lat fisure d'un instrument pour remplir les tonneaux sans refonler daun le liquide le vin grité qui se trouve à sa surfice, par Al. Gras.

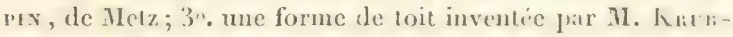
sucrso, de Sire, et destinte particulierement aux construe tions rurales; f". un procídé pour empiecher l'encre de se

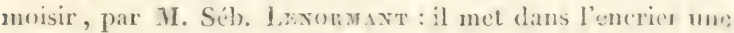
petite quantité de dentoxide de mereure on précipté ronge; b". la méthode employée en IIongrie, pour fite liv vermoult ou extrat d'absythe; $(j$. une instruction sur les puemier. soins a donner aux personues asphyxiés par les vapeurs du vin ou de lat biere, ete; 7 . lanalyse de menf sortes de farme de blé, par M. HeNrI; etc., ete.

Le caldier de novembere raferme, ". la manere de pro-

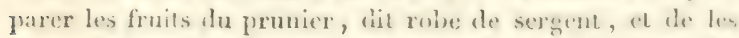

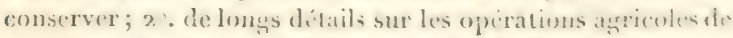

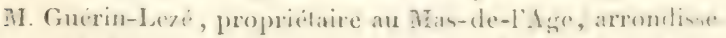

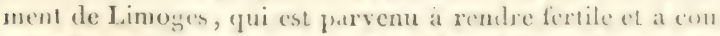

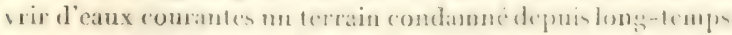

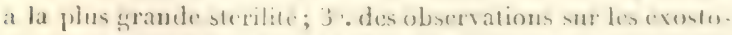

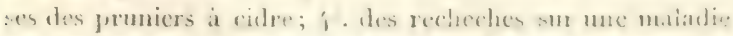

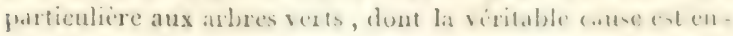


ore inconnue, et sur laquelle le riflacteur applelle l'atlention des pépiniéristes et des forestiers; 5. le résume des travaux de la societé linnéenne de Paris, pendant le mois d'octolure; 6u. une note de MI. Poiteau, sur les remerles employus a la riuyane contre la morsure des serpens venimoux; le plus remar([uable est nue infusion de la racine du cotonier, sossyfimm arboreum, I., ; ;". la description d'une nouvelle lialance-romaine pour peser les écheveaux de coton, invention

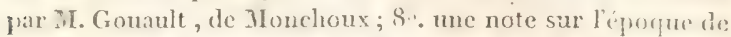
limportation du nérinos en lisplgne, d'apres M. Iinnams de Varsas; cet animal provient de l'Insteterre, tandis qu'il est ivident par deux passages de Columelle qu'il a été apportio de I'A frique.

I.c calice de décembre offre: I". une instruction sur la culture, les propriélís, les usages et l'histoire du tournesol amumel, par le rédactenr, M. Thiébant de Berncaud; 20. des procédís employés pour s'opposer aux ravaşes du campasnol; 30. de ronveaux faits sur lat maturité des grains ct sut les récoltes précoces; $4^{\prime \prime}$. le résumé dés travaux de la société linnéenne, pendant le mois de novembre. Dans un article intilule: Variété, on lit plusieurs ancedotes agricoles bonnes à conserver. Ce calier, qui termine le XII . volume d. Lit redaction de M. Thiebaut de liérneand, offe en ontre, comme les précédens, le tableau météorologigne raisonné des six derniers mois de I $\$_{22}$, et la table systématique des matières contenues dans ce volume.

932. Le chiconum ixtrints, ou clicorée, est indigene on Angleterre ou elle est trés-commune daus les terrains calcaires; on n'y fait aucune attention it la varieté de cefle? plante que l'on a améliorée en France par la culture. Les vaches qui s'en nourrissent domment nn tier, de plus de lati ; on arrache les racines a l'approche de l'hiver, et on les cmminatge dius une cave par couches séparces, avec du sable ou

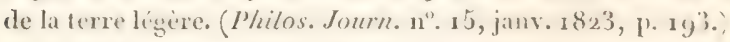

933. Une manufacture De papier oE parise vicnt de sélablir a Okainon, pres de $V$ arsovie; et son sucés fera, a co qu'on espere, batsses le prix du papier. Cette mamularIure se bornera pour le moment an carton et au zuros pajpiev. I.c propriélide, M. Asir. Mronack, se propose de propla- 
rer, dapris une invention a lui, une espece de papier pro pre a fitire des toits qui seront il l'épreuve de l'ean et du feu. (Investig., n'. $\mathrm{n}^{\circ}$, janv. 1823, p. 180. )

GEOGRAPHIF.

93. Biglayn's systeur of geography, etc., ou Syistime de géographie à l'usage des écoles, sur un nouveau plan exact et facile, et dans lequel les limites des états curopéens sont indiquées, ainsi qu’on l's a établies par le traité de paix de Paris, en nov. ISI5, ave 7 cart. grav. par Brglas, 5". edit. Prix, $2 \frac{x}{2}$ sh. rel. Londres; G. Cowle.

Le Monthly-reriesv, de mai 1817 , s'exprime ainsi : Yous pouvons recommander ce petit volume pour les écoles, vu rque, d'après notre opinion, il convient micux pour l'enseignement qu'aucun traité semblable que nous ayons vu.

935. A citecuism of geograpur, etc., ou catéchism: de géographie, comprenant les découvertes les plus récentes, et arrangées conformément aux derniers partages de territoire faits sur le continent; précédé diun court extrait des principes de créographic phy̧sique et mathématique, in-I 8 , avec $11 n$ frontispice. Prix, g den. br. Edimburg; Oliver and Boyd's.

936. A xry giomipucil Dictioniry, ete., on nouveau dictionnaire gégraphique, contenut ume description de tous les empires, royammes, étits et provines, avec leurs villes, montanes, eaps, mers. ports, rivieres, lass, etc., avee une notice exactede: Vetendue, des limites et des productions naturellen: Irenowrernement, les moxurs at la religion des hitbilans, lene commeree, lours manufactures; la loncitude de toutes les villes capitales et autres, l'nu hauteur et leur distance des antres villes remarquables, et les événemens remaryuables arrivés 


\section{Géographie.}

dans l'une ou dans l'antre, avec l'état de la population des comtés, villes capitales et autres, en $\Lambda_{n-}$ gleterre, Écosse et Galles, d'après le dernier reeensement fait par ordre du parlement, en ris r, ave cartes, grav. et plans, et une liste des villes les plus célébres dont il est question dans la géngraphie ancieme, et auxquelles on a ajouté leurs noms ar-

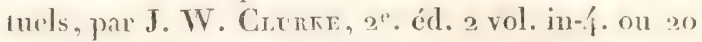
cah. à 5 sh. Londres; Lofisher.

937. Definition of some of the terms made use of in geograplyy, etc., on définition de queleques termes employés dans la géographie et dans l'astronomie. Ce livre (à l'instar de l'Esçuisse de la géographie de l'Angleterre), est destiné à itre transcrit par les étudians, pour en graver les mots dans leur mémoire, par M. Ilongkin, 2x. édit. Prix, 4 sh. Londres; Harvey et Darton.

938. A ceografical present, etc., ou cadean de géographie, ou description des principaux pays du monde, par M. $\Lambda$. Vennise, arec bo grav. représentant les diféerens habitans dans leurs costumes respectifs, trés-bien enlum. Prix, to $\div$ sh. demirel. on 8 sh. en noir : Londres; Ilarvey et Darton.

939. A Sketch of ture groghaphy of Engrand, ou esquisse de la géographie de l'Angleterre, 3".éd. Prix, a sch. Londres; Harvey et Dartou.

94o. Recherches mastonipues et gliographieres sur les médailles des nomes ou préfectures de lÉspte, par J. I'. Tochon-n' $\Lambda_{\mathrm{Nnec}}$, membre de linstitut de France, ete. I vol, in- $\{$. Paris, imp. roy. chez. A. A. Renoutard.

Cette production ne se reconmande pas seulement commo un ouvrage purement numismatique; flle est d'une trisgrande utilité pour la géographic ancienne.

L.

gúx. Catiey's americas atlas, elc., ou atlas amciri- 
cain, complet, historique, chronologigue et géocratphique, pour servir de guide a l'histuire des deur Amériques, avec un précis exact de leur dérouverte, leur populatione et des progrés des dibiorens roraumes, étits et provinces du nouveau munde; parlant atussi des ennerres, batailles célibres et é.

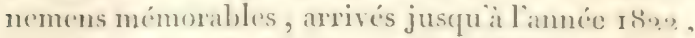
d'apres le plan de Lesage e et pour fatre permlant ì cet ous rase élebre amélioré par LArorsxa. Pris, 1. (i. (i. Londres; John Miller. Philatelphie; Catrey et Lea.

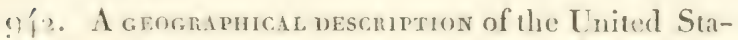
tes, ou description géographique des Fitats-Unis, par M. J. Мelisa, pour accompagner sa cartedes mèmes pays, nouv. édit. fort augmentée; in-s. de 49 r pag. Philadelphie. 1822.

Une nouvelle édition d'un ouvragre sur la gréograplie der litats-L nis doit être considérée sous un autre point de vne que celle de quelque production littraire que ce soit, a raison de la rapidité avec laquelle s'accroissent dans cet leureux pays le territoire, lat population, limiustrie, le commeree al toutes les ressources intéricures. MI. MI lish avait publit sil description en $\mathrm{I} 8 \mathrm{r} 6$; depuis cette époque, la Florite a iti cédé par l'Espagne. Au moyen de cetle acquisition, l'étendue des litats - Lnis, que termine an nord le qu" parallele, se

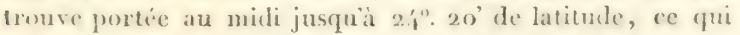
Jeur donne une longueur de press de 25 degrés (62.5 lienes communes ) lu nord au sud. Par-lit anssi, la surface des itats-l nis se trouve être mintentunt de 2,076 , fro milles d'Angleterre carrés, ou x ,328,9n2, 400 acres.

Ce qui aeroit surtout l'intérèt de cette nourelle élition, c'est que lauteur y a fitt usage des renseignemens que le recensment executé en 3820 a donnts sur la population des Etals-Lnis en fiencral, et de chacun des etats et comtes on districts, en praticuler, ainsi que sur colle de plusiones villes.

L'espace qui nous est accordi ne nou permet que de donner le réultat grinéral de ce recensement. Il s'cleve, sins 1 a Floride, à $10,037,19^{5}$ individus, savoir : 


\section{Géographie.}

Population blanche.

Hommes de couleur, libres. . . . . 233,557

Iisclaves.

I

Indigènes. (Intlians).

398,969

On a, compté à New - York.

$12,3,706 \%$.

A Philadelphic. : . . . . . II 1 , I IO

A Baltinore. . . . . . . 62,738

A Boston. . . . . . . . 43,940

A la Nouvelle-Orléans. . . . . 27, 27,5

$\Lambda$ Charleston.

$24,-80$

Nous avone recu par le méme cuxol de linérique les ouvrages suirans relatifs à la Floride.

93. Skrтches nistonical and toposraphical of the Fioridas, ou Fsani historigne et topographingue sur les denx Florides, particulierement sur la Floride orientale; par I. Gndxт Forвrs, in-8. de 228 p. New-York; 182 I; Van Winckle.

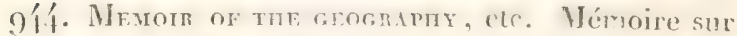
la géographie, ethistoire natrable et rivile de la

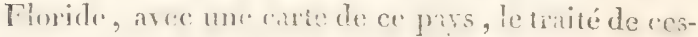
sion et d'autres piéres; par ( $x$. Unisr. inl. in-8. de $92 \mathrm{p}$. Philadelphie; 182 r; Palmer.

9) 15 . Notrers of Eist Fromol. Nolicesur la Floride: orientale, et str la nation des Śminoles, par un voyageur qui a visiti: réremment celte provinec; in 8 . de 105 p. Charleston; 1822 .

Ces differens ourages ajoulent aux connaissances rui avaient eti donnécs sur la Iloride par Cateshy, Bartram, Raynal, Pursh, of particulicement par le copiatine Bernard Romans, dans un E. sai su Thistoire particuliere d. (") pays. New-York, $177^{5}$.

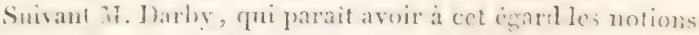

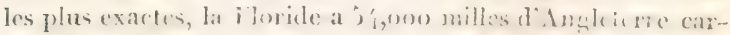

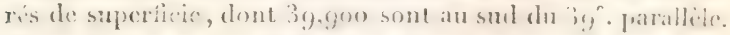
Cependant on y compte a peine 12,000 habitans, non compris les ancicns iadligenes, dont le nombre niest pas indieque, mais ne samrait etre considerahle. Ia population deit saumTOYe I. 
menter sapidement, eomme il ent arrive pome lis autres par

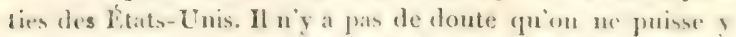
cubiver avee sucres lom les wigetanx du midi de la france, -t probablement ceux des contrées ancore plus chandes, notamment le riz, le colon, le cacá, l'indigo, mais surtont le sucre.

C. M.

9.jo. Mronone dams lepuel on prowe lidentité des Ossiefes peuplacke du Caucate, atree les Alains du moren ige, par J. Kenrotri lu a la socicté asia-

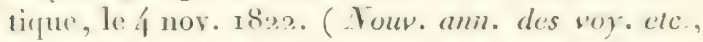
nov. 1822. )

Ies indices historiques sont ronfirmés par la langue ossite, sur larquelle l'atuteur a firt un travail particulier. On trousc dans ce mémoire quelques exemples de ress:mblance de la langue ossite avec d'autres qui apparticnnent i la mime souche.

9íg. Décocventes dans lintériour de l'Afingur.

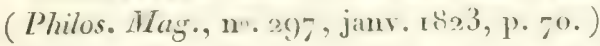

I.e capitaine I.sryg qui avait été envoyé de Sierra Leome, le 16 avril 1822 , a Falaba, capitale du royaume de Soulimana, était de retour le 2 g) octobre suivant, accompagné de plusieurs individus de la nation des Sonlimas, de celle des Kourankos, et de celle des Sangaras. Il y a dans cette derniere nation beaucoup de voyageurs ot de marthands, anxquels les deux autres nations ne permettaient pats de s'approcher de la côte et de trater directement avee les buropéens. M. Jaing parait ètre parvenu at lever ces obstacles.

Ce voyageur a reconnu lat source de la riviere Rohelle, siluée a $9^{\circ} 45^{\circ}$ de latitude nord, et a s $^{\prime \prime} 5^{\prime}$ de latitude ouest. Flle devient considérable aprés un cours de 30 milles anglats et serait encore navigable i partir de ce point, sans les rochers ipars sur lesquels elle coule. M. Laing rapporte que de deux éminences différentes, il a apereu la montagne de Lomba, ou est la source dn Viger, et que cette montisne

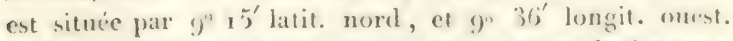
Sous le nom de Lembie, le Niger séparele pays de Sangara, situé sur sa rive droite ou orientale, du pays de Soulimana, qृui occupe la rive opposće. 


\section{Stutistique.}

465

I. pays de Kouranko est traversé de l'est a l'onest par la riviere Camaranca, dont la sonrce est à deux journées de celle du Niger. Le capitaine Laing l'a vu sur plusicurs points. Il parle aussi d'une grande rivière nommée Mungo, inconnue jusqu'à présent anx luropéens, et qui, après avoir recu le Kabba, se jette dans l'()céan par la même embonchure que le Scarcies. Le Labba prend naissance à environ 20 milles au sud de Timbo, eapitale du pays des Foulahs, située à $10 " 52$ latit.nord, et $10^{\circ} 34^{\prime}$ 'longit, oriest: C. M.

\section{STATISTIQUE.}

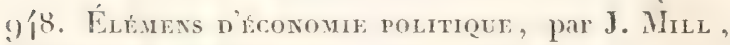
anteur de lhistoire de l'Tude, traduits de l'anglais; par J. Т. РАпısот, in-8. de so f. et demie. Prix, 5 f. 50 c. Paris ; Bossange frères:

Sous ce titre, M. Nill a non-seulement reproduit en fiveur du systime de Smith et de quelques autres écrivains de la mème école la plupart des argumens qui s'y rencontrent en faveur des principes abstraits à l'aide desquels on veut soutenir la doctrine de l'économiste anglais; mais, ayant le courage d'avoir: quelques idćes qui fussent a lui scul, l'auteur dont nous nous occupons les a consignées dans cet ouvrage.

Ce traité est clair, mćthodique, substantiel; et je ne crois pas que dans un cadre aussi resserré on puisse rencontrer plus de choses. La division est simple : il traite de la production en grénéral, de la distribution des richesses, des échanges, de la consonmation, et des circonstances qui la penvent affecter. Ces diverses parties sont présentées dans autant de chapitres qui sont eux-mèmes partagés en sections.

Au commencement de son live nous avons reneontre une idée nouvelle qui nous a frappé d'étonnement par sat fécondité, et cest avec regret que nous avons recounu que l'auteur n'avait fait que l'ébancher sans l'approfondir. Il atvait fait pressentir que la production ne pouvait ètre gue le rísultat du mouvement excité par le travail entretenu, varie $\mathrm{ct}$ combiné d'après les becoins socianx. Cette manire d'envisa. fer la question étail un point de vue nouvean, dont le de- 
seppement n'erit pas éte sans intétet. Il ctait facile de léwonde it la distribution der richeres; les cichanges cus

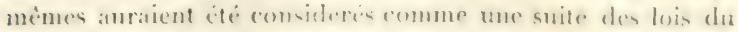
mouvement, et la consomunation pronvail domer been at de semblables applications. I. iconomie f gnamt a lat theorie

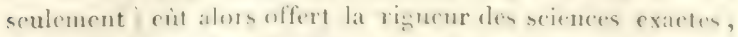

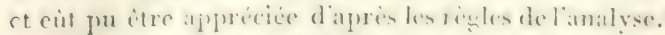

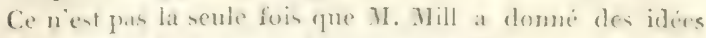
ingénicusen et des apercus fins: il noun suffira, pour appuyer motre assertion, de citer les promes quil établit pour mon-

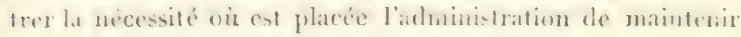
non epuilibre constant entre la tondance de lat population et we des captanx a sareroitre indefiniment. Si celle loi dar eroisement etait la mene daus les deux ras, la diffeultés so-

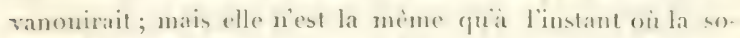
ciété sorganise. Bientot rompu, le xapport varie, el a mesure que lat rivilistion se perfectionne, le? termes de comparai-

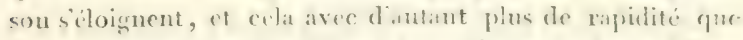
la prosperité nationale séteve. La moindre teudance a lar croissement est celle des capitaux. Le mourement de la population est multijle, tandis que relui des capitaux ne ve que par aceroissement additif. Aimsi te moyen anviliaire des changes perut senal créer une consommation fictive, et mainrenir le prix du trivail an fanx ou l'ouvrier pourra alfeintre le moyen de satisfaire ses besoins ( $I$ ).

Te chapitre des erhanges ne seol pas montri a noms arer le mime degre denteril que les pricidens; la theorie a pro

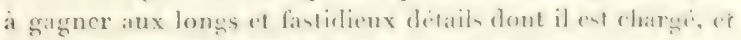
ron voit, par quelgues indications diune spplication farsse. que M. Mill nest que theoricion. Cimet dapre's lexpérience de la pratigue que nous domnetions, par exemple, des cunseils opposes pour mubiplier les dedanteses, on, en d'antres termes, pour havoriser laction du commeree exterieur. II y a loin du monvement rounlies et routinu qui asurerat an rommeree des chances a peu pres uniformes doprotions, a ce prohibitisme abolu qui isole les commeree des nations, a ectle liberti imlifinie proposie dans son ons rage.

Moins corore adopterions-nous la maxime paradovale

(1) La série de ces idécs n'est qu'entrevue dans l'onrrage. 


\section{Statistique.}

quili énonec comme un axione, sans la démontrer (I), qu'une aration ne gagne qu'en raison de's tenries qu'elle reevit; qu'ainsi plus les importatiors, sont élevies, et plus sa balance est favorable; mais nous nous reucontrons avec iui quand il appuie sur la nécessité d’augronenter les capitaux de circulation, pour diminuer en proportion le taux de l'intérêt, lorsyu'il conseille de se créer,par le perfectionnement de lat maind'eurre, des articles spéciaux d'échanges qui établisernt un commerce privatif. lin général, ce traité mérite d'ètre lu par cenx mèmes qui ont suivi les publications antérieures sur la mème matiere. La traduction est bien écrite, et, à l'exception de ruelques idiotismes, de quelqques tours embarrassés, elle nous a paru claire et précise.

Bertitevin.

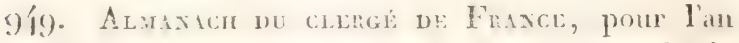

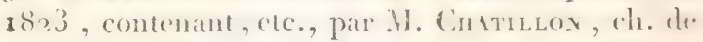
la Idigion-d'llomene, chef du bureau des aflitires reclésiastiques an ministere de lintérieur. Paris; I823; M. P. Guyot.

Les documens officicls, d'apries lesquels l'autene a rédigr: cet almanach lui ont permis de donner it cette prublication un interct plus grand que celui ordinairement offert par ces sorles de recueils. L'ourrage de M. C.hatillon renferme, outre tous les documens relatifo an persomel of a la législation ecclésiastirue, un relevé général de l'évaluation des lews firits anx établissemens religi us depuis $180 \%$ el quelques résultats ruricux de statistique que nutus eroyons devoir consigner ici.

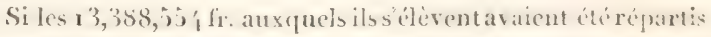
cutre le's 30,000 ctablissemens, chatenn d'eux anrait rewn ume

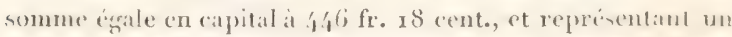
intérèt de 22 fr. 3 t cent. qui, à cinse des valeurs mobilieter qui entrent dans l'estimation, seratent passbles d'une re.

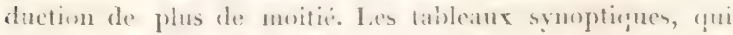
ierminent l'ourage, nous permettent d'en extraire les resullats suivans :

(1) L'Angleterre d'aprés ses restrictions, d'aprés le mode de solde

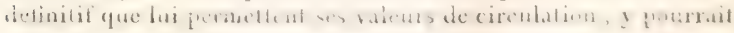
scule trouver de l'avantage, tandis que le pays, où le solde est effectif, ne rencontrerait que ruines, que pertes. 


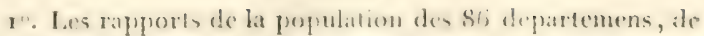
lenrs superfices avee les cures, domment les chiffressuivan:

La superficie de la france ent de 27,428 lienes carries, sur linguelle se repand une population de $30,951,1$ g h habitans. I.e nombre des communes s'éleve a 38,359 ; celui des cantons est de 2,8 ;o. 11 y a 2,915 cures et 26,350 succursale.

2". Ia tolalite de la dépense pour le cleréé, a ćté, pour les annces 1902 ot 3823 , de $29,520,000$ fr. ainsi distribues. Budget général pour traitemens. . . . 24,300,000 Pensions ecclésiastiques. . . . . . . 3,700,000 Travanx extraordinaires. . . . . 7 700,000 Dépenses ordinaires d'entretien des édifies.

\section{Sarnme égale,}

3". Ln etat sénéral du personuel du clergé qui présente 35,6-6ecelésiastiques, en activite, dont i5, oy6 sont agcis de plus de 60 ans. Il faudrait, pour subrenir aux enplois vacans, 15,267 prêtres de plus.

Berthevis.

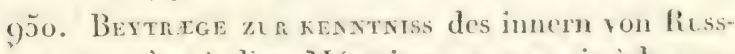
Lann, cest-a-dire, Mémoires pour seerir it la conmaissance de lintérieur de la Russic, par J. IF. Fuduax, D. M. et prof. à l'université de Dorpat, prem. part. Riga et Dorpat, in-8. v et 34́ pas. r8ss; avec un grand plan lithographic de la ville de Casan.

1)ans ce $\mathbf{I}^{\mathrm{Fr}}$. volume lauteur donne sous le titre de topographie médicale une description de la ville de Casan qüil a dabitie pendant sept ans, et de la province dont elle est la capitale. C"est une véritable stationtique ou il n'a oublic' ni le nombre des hibitans, ni leurs mocurs, ni leur industrie, et qui merite, suivant la Gazette littiraire de Italle, d'étre platcée a cóté de la Toposaphie de Pétersbourg, par M. Altenhofen.

Le gonvernement de Casan, partie de l'ancion xoyaume des Buldraren conquis vers le milieu dn $x$ z". siecle par le czan

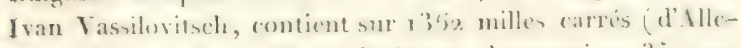

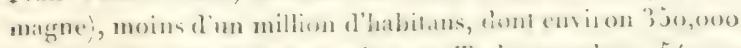

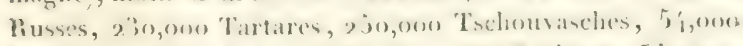

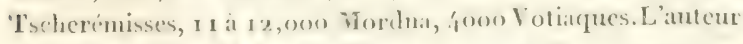
décrit les mours de ces differens peruples. Il ne peruse pras. 
quion all des donsiees suffisantes pour atsseoir un juärement rertain sur la population générale de la Russie el sur la proportion cntre les naissances et les décès dans cet empirce. It paraitrait, d'après un tableau de mortiliti des individus màles du gouvernement de Casan pour une année, tablean qui se trouve à la page $1 / 42$, et où les individus sont rangés par âges, que la mortalité des deux premières amnées de la vie est protigiense. Celle de la première amée culeve seule plus du tiers de tous les cnfans qui naissent, et à la fin de la cincquième année la mort a moissonné plus do la moitic d'une génération entière. Cette rapide et effrayante destruction tient a la pauvreté de la plus grande partie du peuple et à toutes les erreurs de régime qui se commettent. à l'égard des enfans.

La population de la ville de Casan est évaluce à 50,900 àmes.

La principale industrie s'exeree sur les peanx d'auinanx. Le's fabriques de ce genre, au nombre de 53 , fourni sent. plus de 200,000 peanx de coukur, et flus de $1 / 4,000$ coirs forts. Il se prépare, année commune, x fio,ooo pouds de satvon dent une partie se fait avee du beurre et des jaunes d'ocuf, an lieu d'huile.

Liuniversité de Casan, dont louverture solennelle a cu lieu en I814, n'avait encore en dernier lien que 6 professeurs ordinaires, 3 professeurs extraordinaires, et 6 atljoints.

L'ouvrage est terminé par de's pièces justificatives et autre's, ati nombre de 14 , parmi lesquelles on remarifue une courte description de l'agriculture de ce pays, et plusieurs tableaux relatifs à la population.

C. II.

Nous avons cru devoir reproluire ici sous son rai titre cel ouvrage, et une analyse qui présente les fitts slatistiques les plus importans, omis au $\mathbf{n}^{\mathrm{n}}, \mathbf{1} 48$.

F.

951. Lrs Antrlefs pramerses, particulièrement la Guadeloupe, depuis leur denourerte juspuan re".

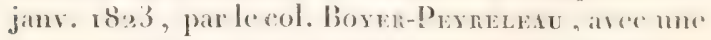
carte nouvedle de la (Buadeloupe et rílab. statise. iom. $\mathrm{I}^{\mathrm{er}}$. in-8. Paris; Brissot-'Thivars.

Cet ouvrage doit se composer de trois volumes, dont le

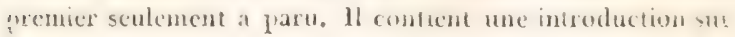


lietat physique et moral des Antillen, quatre livres divises en chapilres relatits à la Guatelonfere et mue carte lithographice des denx iles, Guadeloupe jorpuement dite, et Crande-Terre.

L'introduction traite:

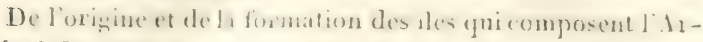
chipel des Antilles;

De leur situation géograplaique;

De leurs limat et wanpiature, et des ctiets qui en résultent sur l'économie animale et sur la végétation;

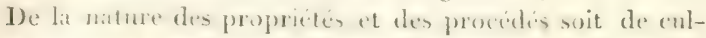
ture, soit de preparation de chacon des vigetaux dont les frotuits sont miles a la subsintance der hommes et des animaux, à la médecine, aux arłs et au commerce ;

Des ducrses esprees dinimanx q qualrupides, oiseaux, poissons, reptiles, insectes, mollusques);

De la population aborigène;

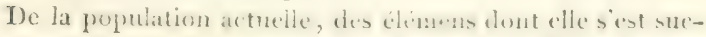
cesivement composece de coux gui la constntuent anjonrd'hui;

Du regime colonial das possessions francatises et des établissemens anglais aux Antilles;

De la traite des noirs depuis son origine;

Enfin des vues de l'Angletere sur be contin'nt d'I frique.

Le live premier trate de la statlistigue de lit cuadelonge proprement dite.

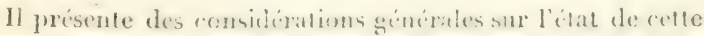

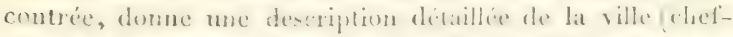

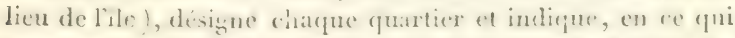

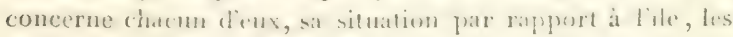

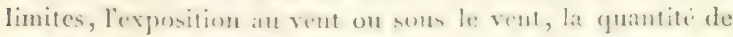

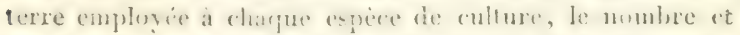
lespece des propritites ruales, lat quantite de bestiaux, les élifices publies, iglises, batteries et moyens di difense militaire quelconques.

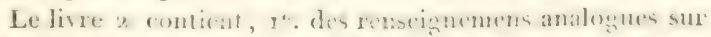

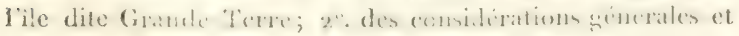

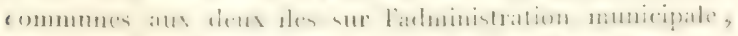

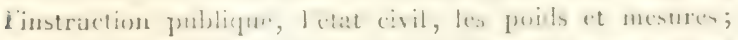

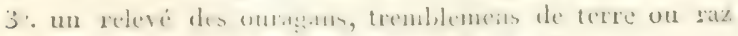

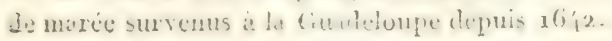


Le livre 3 renferme des apercus sommaires sur l'histoire et la statistique de chacune des dépemlances de lat Guate-

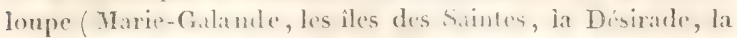
partic francaise de Sitint-Martin); sur lit partic hollandaise de Saint-Martin, lite anghlaise de l'Anguille, et la colonie suédoise de Saint-Barthélemi.

Le livre 4 traite du systime colonial des Antilles framcaises ot de ses vitriations. Il ficit comaitre l'organisation et les formes anciennes of actudles de ladministration et de lit justice, signale des abus dans l'ume et l'autre partie, notamment dans l'ordre judiciare, indlipue les moyens d'amelioration el contient des renscignemens et des vues concernant les dettes des colons et le système hypothécaire.

Iat carte géographique qui est annexéc à ce volume est remarquable par sun exactitude et par le mérite de son exécution.

JoLIV.

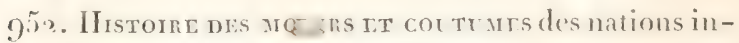

diennes qui hahitaient auterois la Pensylianie ot

les atats voisins, par J. Hzckewredir, missionnaire morave; r vol. gr. in-8. de 5- r p. trad. de l'angl. par MI. le clev. De Poncent. Paris; 1832 ; L. de Bure.

Cet ouvrage contient des détails très-intéressans sur la statistipue de cette partie de l'Imérique. L'anteur a vieu pendant 10 ans parmi les Indiens; il rapporte leurs traditions, il peint lene caractire, decrit leur gुouvernement, leursmorurs, leurs coutumes. Il parle de leurs hiéroglyphes; il dome une idé de leur mythologie, de leur maniere de compter le temps et de leurs connaissances en abtronomic et en gíographie, etc.

Lat partie la plus eurieuse est le chappifre ix où l'auteur cherche a prouser que dans toute la contrice comprise entre l'Atlantique, le Miscissipi et la baie d'lludson, il ny a que

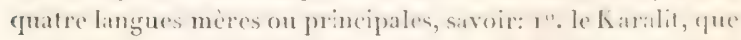
parlent les naturels du Groenland of les Indiens listuimatu;

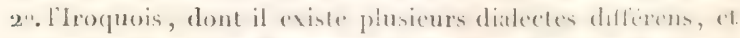
qui est parlé par les Mingoués on Indiens des Sis Dations; les 11 yandots ou llurons, les Ifolowessies, les Assinijoc.. tuhs, nommes par les Frangais Assiniboils, Issinipuils ot. 
Sum et par d'antres tribus tabitant an deth du flowe St.-

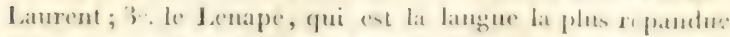
de touten celles que l'on parte a l'est du Mississipi : elle demine dans le Canadat, et depuis la cole du Labrarlor jus-

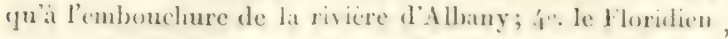
que parlent les nations indiennes habitant les parties méridionales des États-Unis et les Florides.

Il est a regretter que le trabucteur de cet olvrage nait pas ajoute les obervations si intiressantes, publices par M. 1)u l'onceau, jurisconsulte habile et littérateur distingué de Philandelphice. (On voit dans ces observations, que ces langues sont ires-compliquées a cause des inllexions et des termimatisons des mots, mais qu'elles n'en sont pas moins regulières et philosophiques.

W.

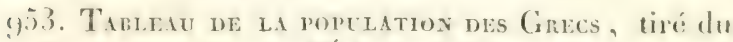
Scotsmann, journ. d'idimb). (Rev. encyclop., jans. I 823, p. 182.$)$

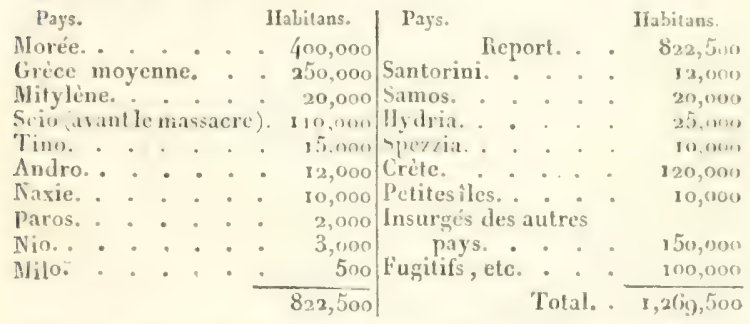

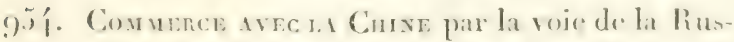
sie. (lixt. d'une lethe it R. R. eiun er it Londres, en $\mathbf{1} 82 \mathrm{I}$.

- Il parait que la politique des Russes est aujourdlani de: repousser tant qu'ils peuvent l’importation des marchantise du dehors, surtout de celles qui provioment de l'imdustice

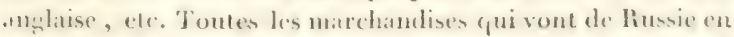
Cline par lat voie de Kiathlit (I) ne dépassent pass, annce

(1) Kiakira et non fiatchta, est un hourg considérable et surinut fort imporlant, comme étant presque le seul point de rén-

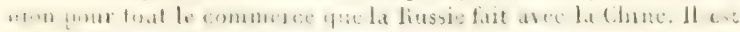


tommune, lat vilener d'un million sterling. Jes frais que supportent les marchandises avant d'arriver it hiathlit, se montent presque au triple du premier cont. Le voyage de Pétersboury a Kiakhta prend pries de cind mois, mène quand lo: transport a lieu directement; si les marchandises changent de mantre a la foire de Wishney-Novogorod, ce qui atrive presque toujour's, il faut près d'un an prour que leur vovage soit accompli.

Les marchandises de la Chine, obtenues en échange, ne perdent pas moins de temps pour arriver aux marchés ou elles se débitent : ainsi, une opération entre Pétersbouror el KS ialkiltit prend une période de plus de deux ansavant d'étre liquidée.

La foire de Wishney-Novogorod, où les marchandises destinées à Kiakhta clangent communément de propriétaire, se tient ai mois d'aoùt. De la elles sont portées sur des chars ou traineaux à Kiakitua, ou les marchands chinois les achitent en échange des leurs. Sur ce point, la demande commence en novembre, et se prolonge jusqu'ilu commencement de mars. Le marché ticnt, il est vrai, toute l'année; mais il n'a quelque importance qu'a l'époque indiquéc. Kiakhta n'est pas le seul point de la frontière de la Tartarie russe où vennent les marchands chinois; il se fait un commerce considérable dans la ville de D'haimatschin, sur la frontière de la Mengoulie. Je ne sais pas quelle est l'étendue de ce tralic; mais j'ai appris que, depuis quelque temps, il a beaucoup diminué, Kiakhta ayant la préférence.

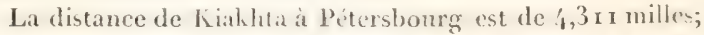
de Kiaklıta à Péhin, ror/4 : en tont, rle Pétersbourg à P'éhin,

sifuc dans le gouvernemont d'Irhoutsk ( $50^{\circ}$ r5' lat.) sur la frontiere de la Cline. Un pont sert de limite; vis-ì-vis est un autre

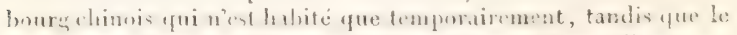

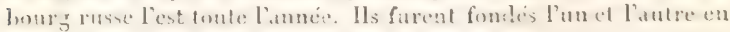
${ }^{7}-25$, à la suite du trité de commerce conclu par les liusses avec les Chinois. La principale foire s'y tient eu décembre. Ce commerce, 'qui vivifie presque toute la Sibćrie, est prurement d'échange avec les Chinois. Los rappoxts sont souvent infermmpus entre les deux peuples; mais un mamdarin et un commissaire russe

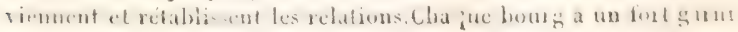
l'arlillerie. 
sio, milles anglais. Presque tout est rould de charroi par terre.

Lit quantite anmude de this importés par Kiakhta est d. 4o,000 a jo, noo caises, dent me moitie se vend ordinaire-

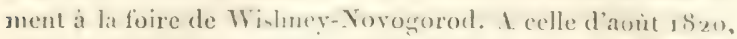
on $y$ a vendu 27,000 cairues de the noir, sans compter le: vert et le thé en pains, appelé en liussie ziegeltha.

Le noir se paic \$20 roubles la cainse; le vert, 500 a 600 roubles; le thé en pains, 17 a a 230 roubles; cotte dernière qualité n’est pas consommée en $\Lambda$ ng glelerre.

Les lainages de la Saxe, et des tat- du roi de Prosese ont un

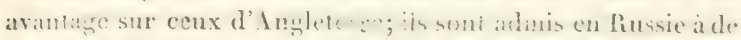
muistres droil que cenx-ci. ett ritution de droil fut ac. cordie pendant le systeme continental de Bonaparte, et a

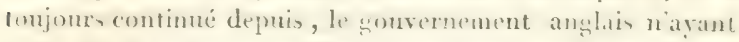
joint fait de réclamation. Ce lainages jonissent du transit par Kiakhta, moyennant un droit de 3 pour 100.

Les Russes sont tres-jatoma de lent commeres de fiontiere avec les Chinois ; ce n'est qu'um naturets de l'empire, pourvus de la patente de premiere classe, quil est peraitio de commercer a Kiakhta. Les Chinois, de leur coté, ont sommis ce commere an monopole d'une comprasnie privilenie de nationanx. Enfin, les marrhandises anglitises qui ront à Liahlita sont presque tontes apportios en controbarde, puingue le transit leur est refus: tandis que ce privilife es accordi aux marchationes de P'russe, et quil l'ent exclusivement.

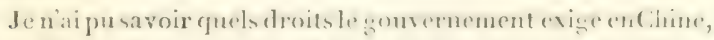

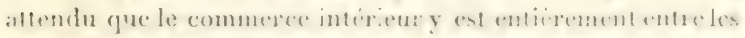

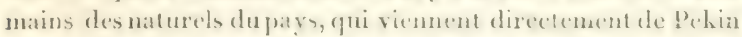

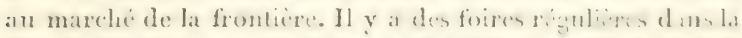

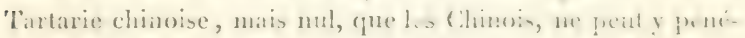
tree : je snis lows d'itat de vous dunner antun ditail sur

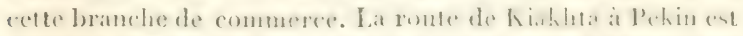

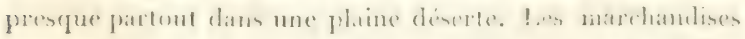

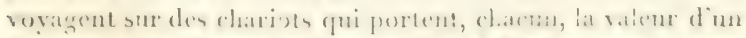

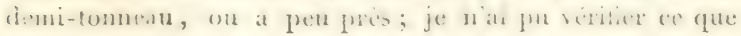
coute ce trinsport.

En juin dernier, il arxiva de l'intérieur a la Chine a

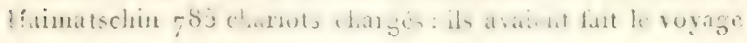




\section{Statistique.}

cie Pikin en cimpuante-un jours; ifs purtaient 94.3 ratises de thic, 589 balles de nankin, outre des étoffes de soie, dus suere canti, ete. Presque en meme temps il arrisiat de lat Riussie a Kiakhat des marchandises pour me valeur a pere près égale; les trois huitiemes environ étainut d'articles anghais, prineipalement cn velours te Minnchester, et queicytes monsidines ; le remie du convoi consistait en fourures onir-

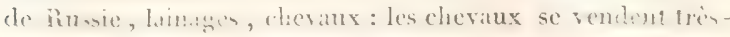
bien dans les provinces septentrionales de la Chine.

Mon unique objet, en vous donnant ces détails, est de rous pronver glie certitins articles de nos manufietures con virment a la consommation de ees provinces chinoises, et. In'en dépia de tons les obstales, ils savent y parvenir en

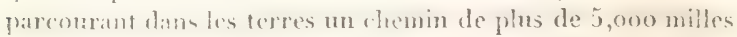
a traters des regione dont une partic nia ni chemins ni habitans.

F.

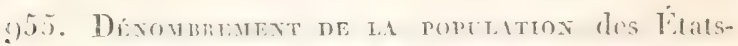

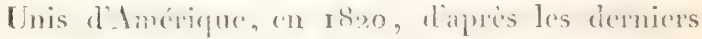
recensemens faits en vertu des actes du congrès, communique at la socété de scosraplate, par

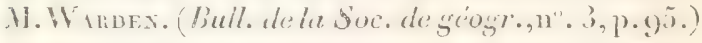

Ce denombrement détiallé foumit les résultits suivans:

IIommes blancs. . . . . . . 3,995,053

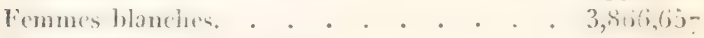

Hommes de coulcur libres. . . . . I I $2,7,70$

Femmes ilem. . . . . . . . . . x20,-rin

Toutes autres personnes libres que des Indiens, mon tixis. . . . . . . 4, fix Esclaves mailes. . . . . . . 788,028

Id. femelles.

$$
\frac{750,100}{9,637,999}
$$

On voit dans un 2e. tablcan lit poprlation, par état, com-

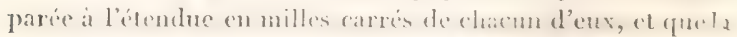

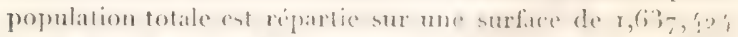

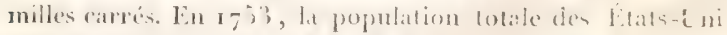

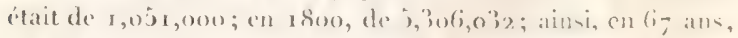

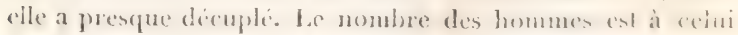

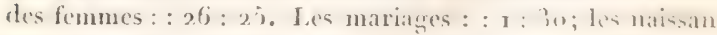
ces : : $1: 20$; les décès : : $1: 40$ cnviron.

F. 
474 Plans, cart. topog. et géogra.

\section{PLANS, CARTFS TOPOGRAPIIOUES ET GEOGRAPHIQUES.}

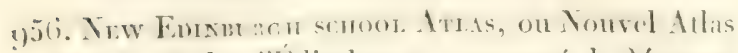
pomr les écoles dtidlimbourer, composé de 3́ cartes des dillérems empire's, royammes et états du momdr, gravés aree le plus grand som par Lrzas, diupres les resins faits esperés pour ret ourrage, conforméne'nt aux autorités les plus réeentes, et adaptés aux livesécrits par les géographes molemes les plus célibres. In- $\hat{f}^{\circ}$, jolie demi-rel. Prix, 2 I sh. enlum. Londres; Gev. Cowic.

957. Cenrk's New mos of all the states and hingdoms, ete., on Nouvelles Cartes de tous les etats et de tous les royaumes du monde; par Cr.ani ; arrangées d'aprés les autorités les plus récentes et les plus respectables. Prix de chatue caste enlnminée, 6 den., ou 63 centimes. Ces cartes sont an nombre de 30 , dont les 4 dernieres resudent la géograplie ancienne, sacrée et profane. Londres; J. Souter.

95. A thas pontatuf et complet du royaume de France, contenant les 86 cartes des départemens, ele. Par X. Cinden, ex-géographe des postes, et Riocir: ancé, anteurs proprictaires. In-8. de 1 / f. plus lespl. Prix, 24 fr. Paris; Dondey-Dupré.

Cet ouvrage, annoncé sous le n". 220 , vient de paraitre. 959 . Atras comacia ne ra Pranee, 20\%. division militaire; par Gsanss. Paris; Dandale, rue du Cherche-Midi, ${ }^{\circ}$. 40 .

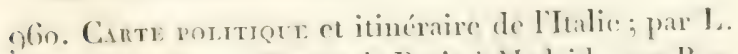

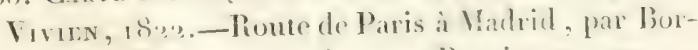
deatux et Bayome, ou Lyon ot Perpignan, cte. Rente de: Machid a toutes les principales villen maritimes d'Espagne et de Portugal. - Catte des 
royaumes d'Espagne et de Portugal pour 18.3. of., revues et ang., gravées par Coldrx. Paris.

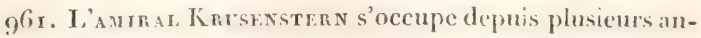
nées de l'atlas de lit mer du Sud, qui est pries d'étre achevé. Cet atlas qui contiendra une trentaine de cartes, est public: anx frais de l'empereur de Russic.

\section{ART NAUTIQUE.}

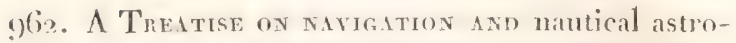
nomy, etc., ou Traité sur la navigation ct l'astronomic nautique adaptée is liz pratique ol servant is l'instruction élémentaire. Par F. Runne, institutour à l'école supérieure Royul naval asylum, it Greenwich.

963. Minitume grognaphy and statisties on descrijption, ete., on Géngraphic et statistique maritimes, ou Description de l'Océan ct de ses côtes, du commeree maritime, de la navigation, ete.; par J. F. Trekey. In-8. Prix, 2 1. 16 sh. 6 d. Londres.

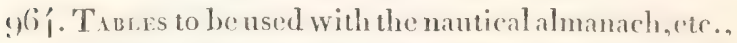
ou Tables dont on peut se servir arec l'almanach nautique pour trouver la longitude et la latitude. "n pleine mer; par le rév. WV. Lix, astronome royal, in-8. io sh. Londres.

9) 65. Nocvan moven de sauver les matelots naufragés.

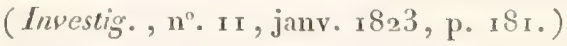

Ce moyen consiste à lancer du rivage an vaissean une fusce imaginée a cet effet par M. Hracin r, protedinicion du roi. Cotte fusce entraine avec elle une corde a l'une des extrémités de laquelle elle est attachéce de sorte que l'antre extrémité étant nouée avec un cáble, ce dernicr peut ensnite être tendu entre le vaisscalu et le rivatge. Alors on fail monvoir le long du cable et à l'aide de poulies une espiece de chilise, dins lacpuelle on emmene successivement it terre lous les hommes de l'équipage. 


\section{ART MILITAIRE.}

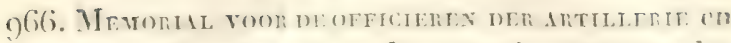
sernie, nitsgater tome de magazin meesters dex arlillerie, de platroclike commandanten, plats majors. etr. "Memorial pour les ofleders d'atillerie et du génie, et pour les rommindans de

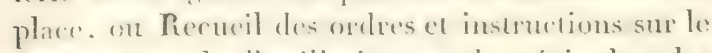
service tant de latelillerie que du senie dans les Pars-lias; public par le col. Doonimas. T. $(i$. Prix, $6 \mathrm{fx} .50$ c. La Haye; I 822 ; Kloots.

Le mémorial militaire hollandais contient les urdonnances relatives a l'armé, principalement celles relatives a lartillerie et an ginie, les instructions des inspecterse gineranx de ces armes, el queleques mimoires sur la seirine ou de l'artillerie ou de longeniens. Il a paru une androe detaillée des cinrf premiers tomes de cet ourrage, publits depuis sisti juspulau commencement de $1 \$ 20$, dins le Miliuirisclur Blatler de Duisbonrs, 3 . cahier, année $\times \$ 22$, page 23.4 . Voici, parmi les matiores qui sont contenues dans ces tomes, celles qui peuvent avoir le plus d'intérêt:

Ordonnances sur lintroduction d'un nourcau systime de poids et mesures.

Reglemens sur le serviere de l'infanterie, sur l'emploi des troupes aux travaux de fortification.

Instuctions tres-ifendues sur le servier des officiers du

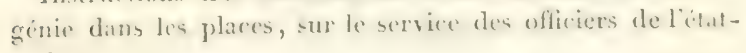
major.

Ordres et instructions sutr lemploi du ciment artifucie! d'Ansterdam. (On fiblerique ce ciment, on tratse arlificiel, alse le limon quion retire de la mer devant Imstertim. I oyez le Cours de construction de M. Sganzin, page 31.

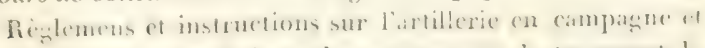

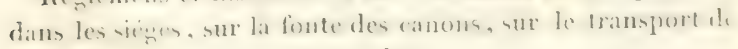
la poudre, sur les armes portatives.

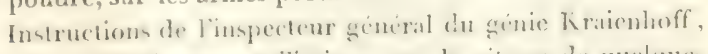

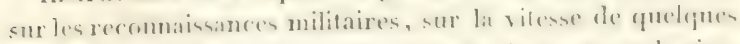

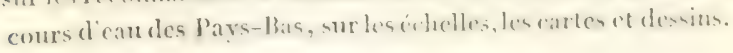


instruction sur l'ordre à suivre daus les mémoires deseriphifí des places fortes. Le rédacteur du Militairischer Bhiler fiit rquelques observations critiques très-justes sur cette insiruction, qui n'est pas de l'inspecteur général.

Mémoires: sur le tir à ricochet, sur les boulets ronges, sur les paratonnerres, sur les pièces d'artillerie en fer epronvées à Liége, etc.

A.

$96 \%$. De la Fontification penmanente; par G. II.

Defoun, lieutenant-colonel du génie, membre de la Légion-d'Homeur : in-4". xxrx et 455 pages, avec un atlas de 34 pl. Genive; 1822 ; Pasehoud.

L'auteur a cu pour but de faire commaitre les progrès de la fortification permanente, depuis Vaubinjusqua nos jours. Il traite dans le $\mathbf{1}^{\text {cr. }}$. chapitre du systeme de Vauban, du système de Cormontaiugne, et du système dit moderne, tel qu'il était enseigné dans les écoles françaises ou l'auteur a puisé son instruction, lorsque Genève, sa patrie, était réuni à la France. Il propose, dans le $2^{e}$. chap., quel ques corrections ou améliorations au systène modern:, parmi lesquelles nous ferons remarquer celle d'élever anx saillans des demilunes, pour garantir du ricochet les faces de ces ouvrages, des bonnettes de $S$ metres de hauteur au-dessus du parapet. Le 3e. chap. a pour objet les moyens d'augmenter la force des places; le $4^{\circ}$, les camps retranchés, et les citidelles, que lauteur regarde comme étant de toute inntilité; le 5e, les manourres d'eau et les mines, mais l'antenr n'a tratí avee détail que des mines; le 6e. chap., De la fortificalion plice au terrain, du défilement et du relief; ct le 7 ', Détails de construction sur les rampes, les revètemens, les ponts, etc., sont les chapitres les plus importans de l'ouvrage; ils contiennent toute lis partie de la fortification qui est fondée sur la géométrie. L'auteur les a rúdigies daprés ses rahiers de l'école de Metz, snivant l'exemple donn: par Bousmard en Prusse, et par le capilaine d'arillerie flerel, comte de Fatkland en Russie. Les planches relatives au S. (-halp., Des forts en pays de montagnes, présentent quelyues modrir; ingénieux de ces sortes de forts. Le 9". chap. a pour objut. les systemes de Cohorn et irs systemes à tours bastionnies; le $10 \%$ et dernier, la défense des places. Liourrage 'омх: 1. 
rest pricide d'une introduction ur liutilite des placesest termine pru des notes de calcul. les planches sont diun dessin correct et pur, et graves avee soin.

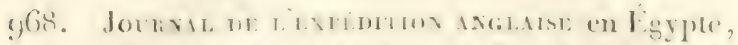
dams liamme soo, traduit de lang!nis du rapitaine Th. WAlls, par M. A. $\mathbf{T}^{* * *}$, avec des

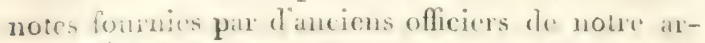
méc disype ; m appendice contenant des pieces

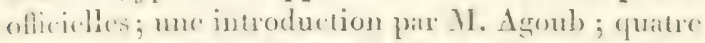
plans de batailles et quatre fisures roloriés ; in-s. (1e) 25 f., plus les pl. Prix, 3 fr. in e.: paip. wel. if fr. Paris; Ansclin et Pochard.

Ce jourtul cat divisé en 2 í chapitres qui suivent à peu par iorde des événemens; il n'est pas simplement militaire, il renferme des renseignemens geverraphiques et historigues sur Gibraltar, Minorque, Malte th Marmorite en Asie minewre. A la virité, la description de ces monillinges oil statimma da flotte anglaise, noffe rien de bien maxquant. Jantenr n'a vu ces lienx célibres ni en pliblosophe, ni en militire, at ses observations ne portent point le cachet de loriginalite el de linstruction si caracteristipue des houmes de sa nation. Il ne commante lintérêt qu'au chapitre IIII, ou il decrit le debarquement des Anglais as ity ple, re leurs preniers combats contre l'armée francaise. Hais alors mème il confirme, on quelque sorte, ce que nous as at appris le

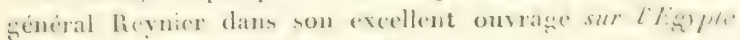
apreis la balnille d Melingrolis. Liateur, sibuphe oflicies dans

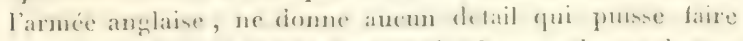

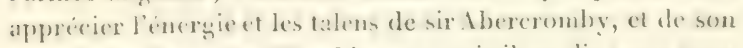

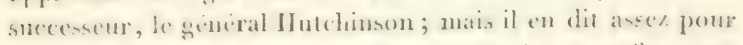

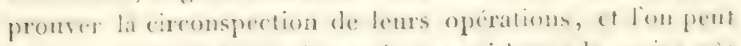

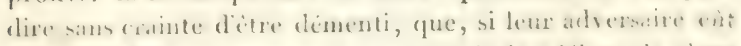

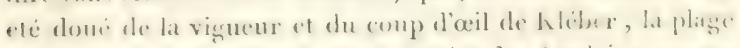

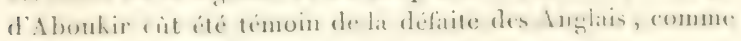

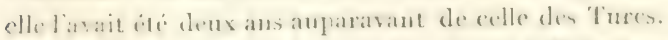

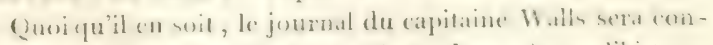

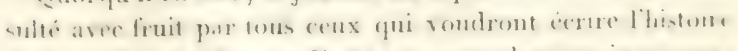

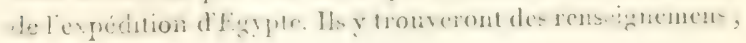




\section{Art militaire.}

gui, combinés avec les relations françaises, mettront dans leur verritalile jour des opérations sur lesquelles l'opinion des militaires restait encore indécise.

La traduction de cet ouvrage est claire et correcte. Elle cst accompagnée de notes puisées dans les ouvrages francais, qui redressent en partic les erreurs du texte, et d'une introduction, ou un refugré ligyptien esçuisse à grands traits les vicissitudes de sit patrie, et ee ru'clle deviat gagner sous la domination francaise.

K.

g(6). Hemor of the operitions of the allies arnies, cte. ou Mémoire dess opérations des armées allices sous le prince de Selhwarzemberg et le maréchal Blucher pendint les elernicers mois de 18 s 3

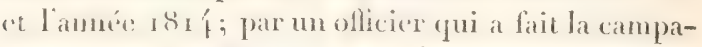
gne, auten des liémoires sur les premières rampagnes du duc de Wellington, avec plusicurs cartes enlum., in-8”. Prix, 2 I sh. Londres.

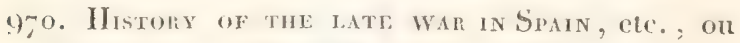
Histoire de la demiere guerre en Espagne et en Portugal; par R. Soctny y vol. in- $\}^{\circ}$. Prix, 2 liv. i2 s. 6 d.

gri ixmention de quelques ouvrages, plus ou moins inconnus en france, sur la géggraphic militaire de l'Allemagne.

Introuluction a la géographe militaire de la suisse; par Homerer. Breslau, 1305.

Minnel de stratégie, awe des exemples sur des terrains counus. Schleswig, $\mathbf{x} 800$. Il renferme la géograplie militaire de la Westphalie, par Venturini.

Grosmpaphe militaire des provinces orientales du Rhin, 2 parties, Leipzig, I802; par le mème.

Comaassance du terrain; par Muller. Berhun, 1807.

Idem, par G'omez. Vienne, 1818 .

P'rimcipes de stratégie, expliqués pror la campanne de $1799^{6}$, avec cartes et plans. Vicnne, $181 \%$.

La première partie contient une introduction it la des-

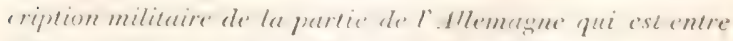

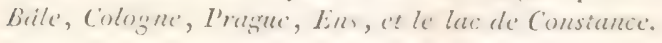


Histoire de la eampangune de 1799 , ared dixcellentes cartes et des plans. Vienne, 18 ig.

Obsersations militiares et politiques sur la défense de la Frane ; Frankfort-sur-le-Mein, $179^{5}$; avec une description de l'Alsace.

Mamuel de la grograplic moderne des états de l'Autriche: troisieme gartic, par Lichtenstern. Vienne: 18818.

Description de la Suisse prar Fussli et Komer, 1971 et $x$ So5.

In-truction pour royiger en Suisse, par Ebel, 4 vol. Zurich, 1810 .

Description des pays d'Anspateh et de Bayreuth, par I.eonardi. Halle, 1797 .

Description des provinces saxones, par le nême. I.eipzig, 1820 .

Description du Fichtelsebirg, par Geldfuss et bischof. Nuremberg, 1817 .

Deseriplion de la forct de la Thuringe, par ILof el Jakolss. Gotha, 1807 .

Idem, par IIeim. Weimar, 1796 .

Minuel pour royager dins le Harz, par Gottschalk. Magr debourg, $\times 8 \times 9 \cdot$

Instruction pour voyager dans le Ricsengebirge ( Non lagnes des géans, en Silésie); par Hoser. I.

\section{VOYAGES.}

973. Chank's tork of Errope, ele. Vingage me liurope, par Cinank aver des ratt. et des grat. Ahregé à l'usage des écoles. Prix, s sh. Lomdres l. Souler.

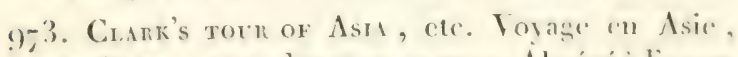

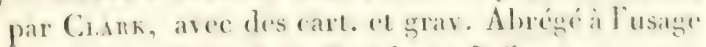
des écoles. Prix, \& sh. Londres; J. Sonter.

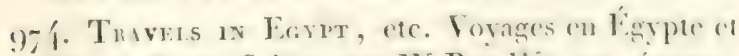
dans la Trere-Sainte, par WV.Ras: Wusox, couyer, in-s. ornes de fig. Prix, is sh. Idondres; Longman.

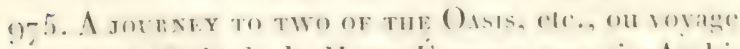

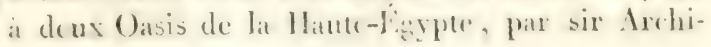




\section{Voyages.}

bald Enmonstonf, avee grav. in-s. Prix, to sh. Londres.

$97-4$ naritive of the expenrtion to Dongola and Senuar, etc. Narration de l'expédition de Denesolat et de Semnatr, sous les ordes d'Ismael l'arthal, par un Amćricain au service de . Yehemed $\Lambda$ li Parha.

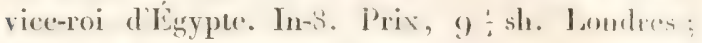
1822 ; J. Murray.

977. The toer of Africa, ete. Le tour de lifirgur, contenant un précis de tous les pays de cetce partie du monde, qui ont été visités par des Européens ; avere les moxurs et les usages des halitans: rédigé et arrangé d’après les meilleurs anteurs, par Catherine Hetron. 3 vol. in-8. avec des cartes. Prix, x l. 16 sh. Londres; Baldevin.

978. Reize nar Bengalen en teruz reize naar Europa, etc. (en hollandais), ou Voyage au lingate

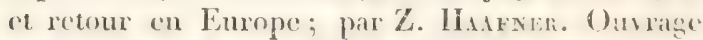
posthume. In-s. avec fig. Prix, (i fl. Amsterdaun; 1822; Van-der-huy.

9) 4 voxage of niscoverr. Voyage de découverles dans lis mer du sud et le détroit de behring, pour trouver un passige an $Y-()$, entrepris en 1855 , 16,17 et 18 , sur le navire le Ruric, commandé par Otто пе Котzве 3 rol. in-s, aree un grand nomber de pl. et de cartes. Pric ; 21.5 sh : cart. Londres; Longman.

Cers volumes contiennent divers fitits nomveanx ct intiressank, relatif's inx iles des mers du Sul; at les mimoires de M. de: Cinnsisso, naturaliste de l'expédtition, présentent une

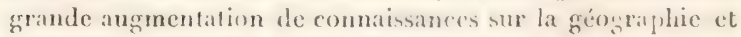
l'histoire naturelle du Grand-Océan.

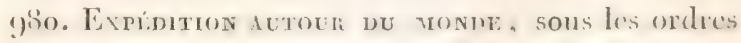
du capitaine Pòltel, par F. IV. Sieber.

liexpédition comprosed des deux vasstaus autehiens, 
sous les ordres du capitame Pôltel, partie du port de Trieste, le 20 octobre 1820 avee la dentination de Canton, at en grande. partie manqué le but 'fu'elle sétait proposé. Waborl elle: perdit desson arriviesons la ligne le baron Sehimmel penmings, augunel l'expédition atait eonfiece. Le capitaine P’ultel, sallcontredit le plus hiebile des eapitaines de vaiseans aulrichicns, arrivat presque mort at Rio-Janeiro. Le chotera morlus se mit dans liegnipacre, et, avant que les deus navires fussnt arrives a Jara, l'épuipage était presque deja réduil at moitic. Parmi les morts se iroura le butaniste Bolnma; ses collections furent jeties à la mer, et, malgré tout ce que l'ou sitait promis de cette expédition, les résultats ont ite de peu d'utilite pour les sciences et le commerce. (Isis, $1 \$_{22}$, no. I 1, p. I 167. )

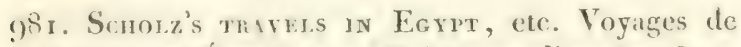

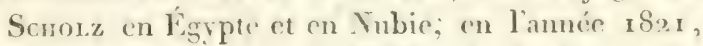
formant la fe part. du VIII". vol. du Journ. des Voyages. Prix, $3 \frac{1}{2}$ sh.

9) S\%. Precis n'in vosige au eap de Bonne-Espriance.

fait par ordre du gouvernement, par MI. Delalande.

(Mém. du mus. ihist. natur. de P'aris. 1. 8.)

MI. Delalinde rend eompte, dans ce precis, des differens voyages qu'il a fait antour de lit ville du Cap, pour se pro. eurer les nombreuses collections qu'il a rapportés an muséum.

V. Aun.

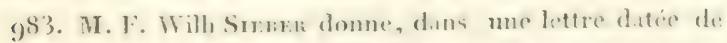

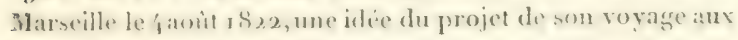
îles de France et de Bourbon.

AI. Hilsenberg se tromvait alors a llle-de-France depuis le: 3 juillet I 89 . Il avait daja fate an bout des frois premiers Iuois de an sijomr dius celle ille un envoi a Marscille; lequal rentermatis des plantes, des semenees, des insecten, des oiseams, des (e)quilles, ete. Il devait, ansstrit apren l'arrivé de 71 . Sir ber, se mondre au eap) de lionne-Espriance, y former un jardin holanique, et y lester $3-4$ ans. Son com-

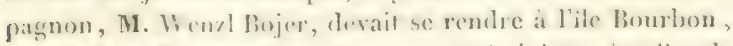
jour y faire de: mom dles collertioms, qui, jointes a celles de.

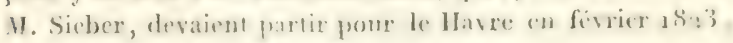




\section{Voyages.}

el dire transportées par IIambourg dans le comte de Nomchloss, pour le comte Vineent de Kaunitz.

Francois Rohant, jardinier, qui était revenu à Marseilleon sept. 1828 , après ćtre resté un an et demi à lia Martinifiur, d’où il avait rapporté beaucoup de choses rares, venait de partir le 5 mai avec son compagnon, Joseph Schnicedt, pour le Sénégal. On attendait déja un envoi de lai pour la fin d'octobre. Il était recommandé au gouverneur par M. J. Gay, botaniste distingué et secrétaire de la chambre des pairs de France. Du Sénégal il devait aller à Cayenne. Quelques jours aprés l'arrivée de M. Sieber a Marseille, il y trouva M. Frangois Wrka, jardinier natif de Butwitz en Moravie. Après avoir cherché inutilement à l'employer à Toulon ou à Montpellier, et connaissant la protection partieuliere dont l'honore M. le comte Maximilien de Wallis, M. Sieber lui remit une partie de la somme destinée pour son voyage, et l'envoya provisoirement à Cayente par la Guadeloupe, pour y attendre M. F. Rohaut. L'habileté et le zèle reconnus de M. Wrka font espérer des résultats intéressans sur les productions de ce pays. Son premier envoi devail ètre fait a Prague, et il devait lui-mème accompagner le second, composé de plantes vivantes et d’animaux vivans.

M. Sieber devait partir pour l'Ile-de-France le rós aout, où il espérait arriver à la mi-novembre, et se rendre de là a l'ile de Bourbon où il devait rester un an. Il compiait ensuite aller en découverte à Madagascar, en profitant de la saison favorable; entrer de suite dans les montagnes, oì l'air est plus sain que dans les plaines, en se faisant accompagner par un indigène qui aurait appris le francais, soit à Bourbon on al l'ile de France. Il espérait, s'il avait le bonheur de rénssir dans ces deux premieres entreprises, d'aller ensuite visiter Ceylan, les Moluques et la Nouvelle-IIollande. (Isis, 1 \$ 22, no. I1, p. I164.)

98 个. Stcone worage ne M. Mansney, missionnaire anglitis, a la Anuvelle-Zúlande. (dan. des 'or nov. I822.)

Cette relation contient des particularités singulites sur les mœurs et les usages des Zélandais.

L.

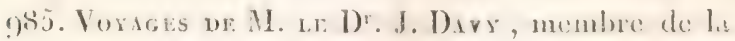


Sociele royale de Londres, dan lintériene de Ceylan, tratuit de fanglats. ( Evedes Yous. Ann. des Voyug., novembre 1822.)

toyage dans la prewine ditouwh. - L'analyse de ce voyage renferme beancoup d'observations barométriques et themométriques, et elle offre des détails intéressans pour la géographie plhysique.

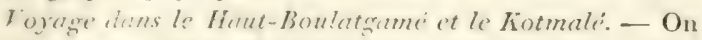
trouve encore, dans cette relation, des documens utiles pour lat grogrraphie physique et la description des temples de Dombouloa creusés dans le ronc. Ces temples sont les plus frinds de l'ile, les plus parfaits dans teur genre, les plus anciens et les mirux conservés.

Lacs sulés de Muluegram-Pattou. - Ces laess sont situes dans la partie méridionale de lile. L'évaporation ćtant trèsrapide, ils se de s'r hent, et le sel se cristallise. C'est principalement de cre lies que lile tire sat provision de sel. Ce seul aljet prodnit au gouvernement un revenu annisel de 1 0,000 l. st.

L.

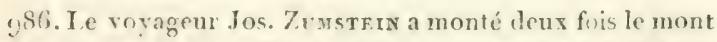
Resa, aux mois de juillet et aoùt, et est parverm a la hauteur d'environ 14,000 pieds. Il a recueilli des plantes et des minéraux, el a fait des observations barométriçues. ( Journ. gećn. de lat lit. étr., nov, 1822.)

g9-7. M. J. BengGnas, aumònier de la légntion suédoise a Constantinople, est arrivé dans cette ville le 2.4 avril der. nier, apris aroir termine un grand voyage dim, l'Orient. (Ann. des tioy., 1822.)

g8s. Le I)r. John Nichol pr. Fonase, aceompagné d'un M. Black, a traversé liz Cordiliere des Andes par Menduzit, -t estarrivé a San-Iago de (hili. Anne des foy, nov. 18.2.)

(59). Le's vaisseaux Golensmin of finrumen, envovers par la comparnice de l'I merique ruse ponr fire des diconvertes sur la cote N.-1), de cette prartie du monde, en sont resemus s:ius et saufs. Ih ont non-seulement examine plus allentive ment la cote $\gamma_{.}-()$), mais ils ont an $\rightarrow$ d dicousert une belle et

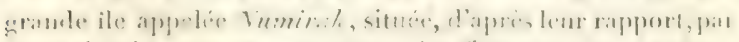
$590^{\circ} 34^{\prime} 57^{\prime \prime}$ de lat. N., et $193^{\circ} \times 7^{\prime} 12^{\prime \prime}$ de long. L. (Philos. magraz. and journ., janv, $1823.1 \% 469$. 
009

\section{DEUXIEME SECTION.}

\section{TRAVAUX DES SOCIÉTÉS SAVANTES.}

\section{SE A N CES.}

990. Panıs. - Institut de France. - Séance du I 7 fivrier 1823.- M. Paulet présente un mémoire manuserit intitulé: : Homonymie et Synonrmie des plantes de Theophraste et de' Linne'-- M. Magendie lit, au nom d'une commission, un rapport sur une note de M. le docteur Edwards, relative a l'exhatation et l'alosorption de l'azote dans la respiration. Iat commission propose d'approuver la note de M. Edwaris. L'acalémie approuve le rapport, et en adopte les conclusions. -Ondonne lecture de deux let tres écrites de l'Inde: l'une datée d'Hydevabat, le 9 juin 1822 ; l'autre de Pondichéry, le 25 juillet mème annéc. Dans la première, M. le colonel Cambton fait comaitre les nouveaux progrès et les rísultats actuels dela gorande opération géodésique qu'il a entreprise dans l'Indoustan, par ordre du grouvernement britamnique, et qui a pour objet de mesurer avec précision un are de méridien dont l'útendue est d'environ 15 degrés. La seconde lettre est de M. Warren, qui réside dans l'Inde, et y cuitive les sciences, et spécialement l'astronomie. Elle contient diverse's observations, et notamment celles qui font connaitre l'itat ordinaire de l'atmosphere, et la diffeulti d'observer les astres it l'horizon.

Sírnce du 2/ ferrier 1 823.- MM. Poisson et Cancliy funt un xapport sur le mémoire de M. Walsh, relalif au binome.

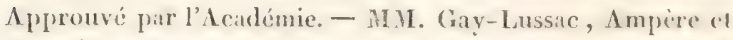
Fourrier, rapporteurs, font un rapport sur le mémoire de. M. Savary, qui a pour objet de determiner, par le caleul, divers effets des courans déletrigues. Approuvé par l'acadé-

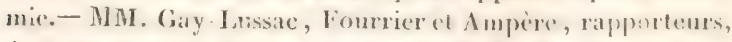
font un rapport sur le mimoire de M. de Bontforant, re-

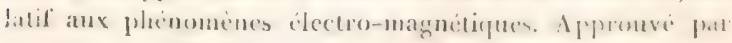
l'academice - 11. Jupin présute, de la part de M. Barlow,

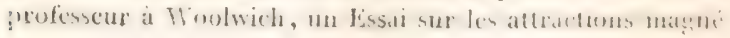


tiques et sur les lois du mignetisme, ol de lidertriciti ferroster. - M11. Duméril et Cham iex fout un rapport sur un mimoire de M. le doctenr Carteron, de Troyes, relatif is des hylatides acephalocystes. Approuvi par iateademie. M. Jowelich adresse de Madere une Flore de cette ile. MM. Desfontaines ch Civier font un rapport sur le Commenmentaire de MI. l'aulet sur les plantes et les animaux nommés par Virgile. Approuvé par l'académie.

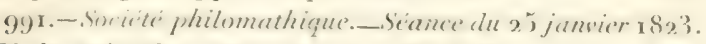
-M.dul'etit-Thouarsanonce que M.de Saint-dmans at fit savoir a l'acudémie des inscriptions et belles-lethes qu'on at tronvé récemment, a Saint-Agens, des huitres calcinees semblables en tout a celles qui ont etc observiens aux environs de Saintes, et dont M. Brongniart a parlé à la societe.

Séance du is férier. - M. Climent donne verballoment quelques details sur le résultat de l'explosion de la machine a vapeur a haute pression de M. Ferret, a Essonne. I ne portion de la claudiere, pesant 3000 kilogrammes, a dé lincée, à ro ou 12 pieds, contre un pilice tries-solide du biifiment, et l'at endommagé. L’autre partie de la chandiere a été brisée sur place. Les bouilleurs ont été brisés et les fourneaux renverse's. Au moment de l'explosion, la machine exergait une pression de 65 livres par pouce carre, ou de 4 ', atmosphices, et les soupapes de subrété fonctionnaient. La chandiere avait $1 / 2$ pouces de dametre. Ses parois on fonte étaient epaisses de $\mathrm{Io}$ a $\mathrm{I} \&$ lignes : la fonte itait un peu aigre, et il parait que les emboitures n'aviaient pas assez de: solidité.

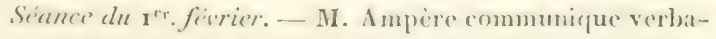
Iement à la société le résultat de quelgues experiencés de plyy sique faites a Muniel. - I n opticien de celle ville a fait des prismes tres-parfaits, an moyen despuels le ypectre coloré n'est pas contiun, mais formé d'un grand nombre de: handes iorizontales, sépares par des traits noirs; w qui permet de diterminer exactement et de comparer les refrac:tions des differentes trintes. - M. Sebeh at recomm par de nouvelles experienes l'influence de la chalenr pour exciter les contacts électrigues.- On amonee ansi quon est par . venu nouvellement, par he moyen d'instrumens triverlelicat., a rendre sensible lo changement de formen qu' ípourent le. 


\section{Séances.}

corps les plus durs it la simple pression du duigt, ou lit diviation qu'on operere dans la position des murs d'un batiment, en tirant une simple ficelle. - M. du Petit-Thonars communique verbalement le résultat de quetques nouvelles expériences quili a faites sur la moelle des végétaux ligneux, desquelles il résulterait que cette substance contient plus d'huzinidité dans les temps de gelée sèche, que dans les temps pluvieux. - MI. André fait lecture d'un mémoire quu'il avait communiquéprécédemment à la société d'listoire naturelle,sur la silice pure, nouvellement découverte par lui aux environs de Vierzon, département du Cher.

Séance du 8 férrier:- M. Desprets annonce qu'en mettant en contact avec du chlore, le graz produit par la distillation de l'huile, il a obtenu une liqueur hinileuse particuliere, d'une odeur agréable, et qui parait différente de la liqueur des Hollandais.

992. Societé d'histoire naturelle.-S Siance du I 7 jansier.Mr. Pelletier de Saint-Furgesu lit une note sur une monographie des insectes liyménopteres de la famille des Tenthrédines. - Mr. Desnoyers donne lecture d'une notice sur une ammonite comprise dans une pierre de la cathédrale de Baycux.

Sécunce du 3r janvier. - - M. André lit un mémoire sur la silice native qu'il at trous ćè Vierzon, département du Cher.M. Guérin communiqque une note topographique sur quelques insectes coléoptires, suivie de la description de deus especes des genres Badiater et Bembiclion. - M. Brongniart fils lit un mémoire sur les plantes fossiles de la famille des Algues.

Siance du I/4 fistrier. - M. Dounere lit une notice sur quelques arachindes, qui comprend, $1^{\circ}$. la rectification de la symonymie de deux espreces de Cludions; $2^{\circ}$. la descrip-

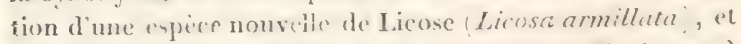
de deux espices de Pteridions. ( $P$. anomalum et $P$. (inctum.)

993. Société de géographie. - Séance dú 7 férrier. MT. Ronx, secrétaire de lit section de puldication, donne lecture d'un rapport sur \& manuserit de Mareo-Paulo, exisrant à lat Tibliothéque du Roi, dont la publication a été proposie a la socinté. la commission centrale arrête que ce mamuscrit scra public anx frais de la socitio. - Le baron 
Coquebert de Montbret donne un aperen du recensement officicl de la population de la Grande-Bretagne el de l'Irlande.

Sicunce du a férier. - M. Roux présente de nouveanx diveloppemens sur les divers manuserits de Marco-Paulo... M. Cassini, entre plusieurs onvrages imprimés intéressans sur les sciences, offre à la société 16 cartes manuserites de la triangulation par laquelle ont été déterminés les cours des cinq rivieres suivantes, savoir : la Seine en $8 \mathrm{f}$. - l'Oise en $3 f$. - la Marne en $2 f$. l'Yonne en $2 f$. l'Aube en I f. - Il offre aussi 13 cartes des vues des còtes, prises pendant le voyage en Californie de M. Chappe.

\section{PRIX PROPOSÉS.}

994. Paris. - L'acadimie royale de médecine, section de chirurgie, propose de déterminer par l'obervation, lexpi. rience et le raisonnement, quelle est la méthode priférable dans le traitement des plaies pénétrantes de la poitrine?

Le prix sera décerné dans la séance publique de 1924 . Le's mérnoires, écrits en français ou en latin, seront adressés, sous Ie convert de S. E. Ie ministre de l'intérieur, a M. Hielerand, secrétaire de l'acabienie royale de médecine, soction de chirurgic. Les concurrens attacheront a leur memoire leur nom écrit, avec l'épigraphe, dans un hillet cacheté. Is prix eonsiste en une médaille de ha saleur de mille frames. I.es membres honomires et titulaires de l'acadimie sont seuls exclus du concours.

995. Suite del'analyse du programme des prix proposés jats

la socirté dencouragement pour l'industrie mulionale.

8".Prix pour l'applicutione de la presse ronnue dan: les ants sous le mom de preswe halrauligue, in l'extraction des huiles a' dusin, et ens surmal dessu sdes fruits.-Ce prix le 2oouf., fondé par feu M. Ratton, Portugais, sera adjugé a celui qui aura construit la prese hydraulinue la plus simple, la plus solide, la plus farcite a mancinver, et la mons condeuse at

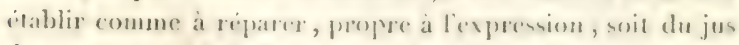

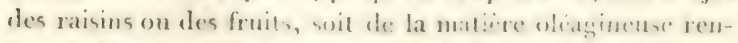

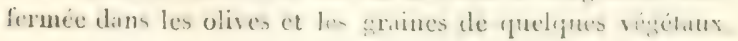


Ontre les traités de mícanique conmus, on peut consulter le le Bulletin de la société, années 10, ix $, 12,13,14,15,16$ et 17. On remarquera sans doute, dans les élémens de la presse hydraulique, les garnitures de piston employées par Bramal à Londres. Les concurrens sont tenus d'envoyer à la société, avant le $1^{\mathrm{er}}$ mai 1823 , une presse exécutéc en grand.

$9^{\circ}$. Prixpour la construction d'une machine propre ì raser les poils des peaux cmployés dans la chapellerie.--. La société décernera ce prix de rooo fr. à celui qui présentera une machine simple deconstruction, d'un service prompt. et facile, peu dispendicuse, et susceptible de raser ou tondre toutes sortes de peaux propres a la clapellerie, après que les poils en ont été sécrétés ou touchés par la dissolution mercurielle; propriétés dont ne jouissent pas les machines anglaises destinées à cet objet. La machine derra raser au moins 12 livres de poils par jour, de manière à séparer facilement les diverses qualités, et offrir sur le travail à la main un bénéfice net de 50 pour xoo au moins. Les concurrens adresseront, avant le 1 er. mai 1823 , un mémoire descriptif de leurs machines, accompagné d'un dessin sur échelle ou d'un modèle, et de certificatsauthentiques des autorités locales, constitant qu'elles sont inontíes en grand, et qu'clles fonctionnent habituellement.

10. Prix pour la fabrication du fil d'utier propre it faire des aiguilles it condre. - Aucune des tréfileries de France ne fabrique ce fil, qui doit étre uni, de même grosseur d'un bout à l'autre dans chaque degré de finesse, d'un frain fin, homogìne, susceptible de prendre la forme d'aisuille sans se briser, supporter l'opération du recuit sins perdre la qualité acceuse, et prendre a la trempe la dureté convenable. La societi accordera un prix de fooo fr. a celui qui sera parvenu a fabriquer des fils dacier dans fous les degrés de finesse, ayant les gualités reopuises pour la fabricaition des aiguilles; qui pronvera en meme tompss quil prut les livrer aux mémes prix que les fabricans étrangers, "t quil a forme un établissement permanent, capalble de fournir du fil dacier a lom les besoiun du commeres. Des certificats des autorités lociles constateront liexistence de lat fit- 


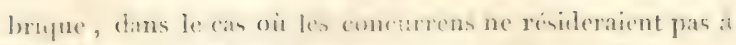
Paris. le concours reste onvert jusqu'an r's. mai r S.j.3.

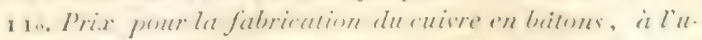
stace des tiresurs d'or. - las socite propose un prix de 2000

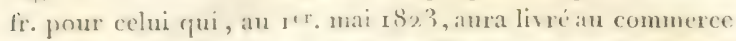
la plus grande quantiti de cuivre affimi, tel que les tireurs dor limploient pour la tilbrication de la dorure appelée mifine, et qui amxi en méme trmps présenté au mombre des produits néssates aux besoins de la passementerie, l'alliane emplové pour la dorme en four. L'espece de cuivre demander, doit avoir ete preparie en France aver des cuivres brut pouvant provenir de l'etranger; outre qu'elle doit itre Iris-pure, il fant qu'elle soit surtout parfaitement ductile. feredintillons de cuivre en biton, aree les certificats aullontiques constatant la quantité fourne aux lireurs d'or, doivent être envoyés avant le ${ }_{1}{ }^{\mathrm{er}}$. mai 1823 .

12". Prix relatif aux laines propmes it faire de's chaperax

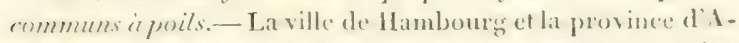
ragon nous foumisent a hatut prix une espere de laine. dont les clappeliers se servent pour fitbriguer des chapeaus converts de poils qui flattent le consommateur, et destinc's partienlierement ans militaires. Ia seciete propose un prix de foo fr. a celui qui aura conslaté par des réériences rignoureuses, 1". quelle est la cause de la difference qu'offre ke

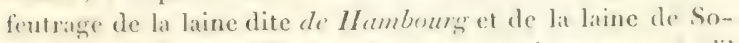
logne, gui forme un fentre tris-serré et tonjours ras; "s sil se trours en France nne race de brehis dont la latine jonime: de la proprite de celle dite de Inamboures. Les eoneurrens sont invités a porter leurs recherches sur les protites races des borels de la mer; ils pourront sedirer des observations de

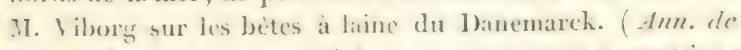
lestit. frameaise, tom. 10.) I.e concours restera onvert jusqu'all $x^{\text {er. mai } 1823 .}$

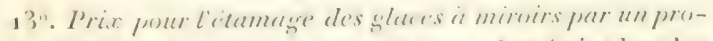

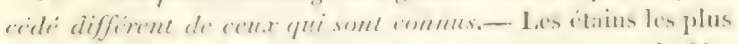

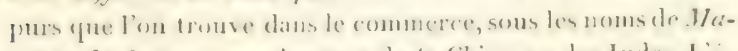
lace et de binca, proviennent de la Chine on des Indes. Jie-

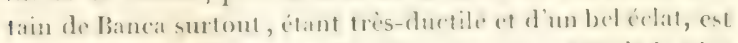
recherehe pour l'elamatge des grlaces par lie midhode ba plus ancirnne et la plus noitée, qui consiste dans lemploi de l'é- 
fain en fenilles unies an mercure, opreation quise fait a froid ou du moins à une température pen élevée. On connait cependant deux autres manieres d'étamer les verres. La Ire.est due à M. Véréa, qui imatgina en ISIa de se servir, par un procédé analogue au clichage, de plomb et d'étain fondas enscmble. (Bull. de la Soc. d'encourag. n". cix, p. 188 .) La 2". maniere est celle usitie particnlièrement pour l'étamage de l'interivur des vaisseaux soufflés en cylindres ou en ghlobes. Lianaleame, dunt on se sert alors, se compose de mercure, d'etain, de bismuth et de plomb; il est appliqué a chaud. C'est co procédé d'étamage que la société voudrait voir approprier anx surfaces planes. Elle propose done un prix de 2quofr. á celui qui aura trouvé un moyen économique d'éfamer les glaces a miroir, d'apres le procéde indiqué ci-dessus (1), ou par tout autre moven analogue. 1.es concurrens adresseront avant le $\mathbf{I}^{\mathrm{er}}$. mai $\mathbf{1}_{2} 3$, denx glaces étamées, l'une de 30 pouces sur 20 , l'autre de 40 pouces sur 30 , accompagnées de procés verbaux des autorités locales, constatant que les glaces on été passées an tain d'après les procédés énoncés, et décrits en détail dans le mémoire de

(1) Pour obtenir I'am.lgame on fut fomblre d'abord ensemble dans uncreunel r partie d'ctain avee I partie de plomb; on ajoute ensuite I parlie de bismuth écrasé en petils morceaux, et quand he tout est foudu, on met a parties de mercure puritic; on hrasse bies le mélange avec une baguelte de fer, on l'ćcume et on le laisse refroidir sulisamment pour pouroir être employé, en le fisisant conler successivement of lentement sur tontes les parties de la surface intérieure des vaiseaux, laruclle drit itre bien nette, bien sèche et un peu échaufée, pour que le verre ne soit pas étonné. Voici quelques indications au sujet du prix proposé : $\mathbf{I}^{\circ}$. tenir l'amalgame à une chaleur telle, 'quil roussise legrement un papior plomgé dans le hain ; $2^{\circ}$. placer le fouracan destiné i le chauffor le plar prés powible des glares a étamer; 30 disposer la table: de lapprareil qui portera les ghlaces, de manire a recevair les iu-

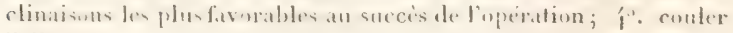
l'alliage, sous forme de nappes, sur la largeur de la glace; 50. garantir les côtés de la gla 'e par des bordures susceptibles de s'opposer à la fuite de l'amalgane, et de le conduire vers le pied de

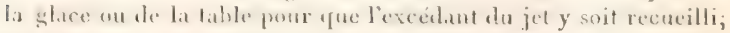
$6^{\circ}$. donner à cette table la faculté de se mouvoir sous le jet;

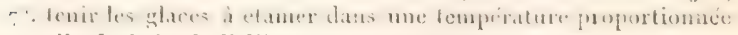
à celle du bain de l'alliage, au moment de le couler. 
l'auteur. Cemimoire dievra ètre accompagni de dessins sur fichelle, représentant les plan, coupe, profil et chlisation tant des fourneaux, tables a étamer, que des étuves, ontils, ete. nécessaires au succés de l'opération.

Ia socite d'encouragement n'ayant pas mis assez d'intervalle entre la publication de ses prix pour 1823 , et l'́porque de lin cloture des concours (le $x^{\mathrm{er}}$. mai), nous sommes forcís, par le peu d'expace dont nous pouvons disposer ici, a nous borner an simple énoncé des sept autres prix qui seront décernés, comme les précédens, dans la séance générale du mois de juillet $18_{2} 3$.

Ín". Prix pour le perfectionnement de's matriaux emplojés dans la grasure in taille doure. - Valeur, $1500 \mathrm{fr}$.

I5". Prix pour la dirouserte d'un mital ou alliage moins oridable que le fer et l'arier, propere a tere cmplone dans les machines is divise'r le's suldstance's molle's alimentaires. Valeur, 3000 fr.

16. Prix pour la fabrication de la colle de poisson. - V Vleur, $2000 \mathrm{fr}$.

$17^{\circ}$. prix pour la construction d'un moulin is lwas, propre à écraser les légumes secs. - Valcur, rooo fr.

I $8^{n}$. Prix pour la dicouverte d'une mative se moulanl comme le platre, et capable de résister it l'air autant yu" la pierre.-Valeur, $2000 \mathrm{fr}$.

19 . Prix pour un meimoire sur les avantages de liclevedes moutons à laine superfinc de race d' Espagne, et sur lo croisement des moutons indigines de France. - Valcur fournie par M. Ternaux, une médaille d'or de $3 o o f r$.

20". Prix pour la constimtion d'un moulin propme it nettojer le sarrasin. - Valeur, 6oo fr.

Nous contimuerons dins le $\mathrm{n}^{\prime \prime}$. suivant l'analyse du prosamme, pour les prix a dicerner en 1824,1825 ct 1830.13.

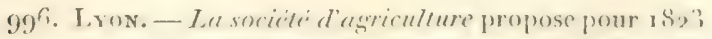
un prix de roo f., plus une medaille d'aregent a l'elfuge de fozier, au rultivateur du départenent du hlóne qui anra linit avec le plus de sucres des essaims artificiels d'apués la methode de B. Lombard. Elle aceordera une seconde srande mélaille d'argent i celui qui, par des procédés quelconenues, anra cleve la plus grande quantite d'abeilles. 


\section{Vente d'objets scientifiques.}

Les concurrens présenteront, à l'appui de leurs mémoires, des certificats constatant leurs opérations et leurs succès.

Le concours sera clos le $\mathrm{i}^{\mathrm{er}}$. décembre $\mathbf{1} 823$.

\section{TROISIEME SECTION.}

\section{ANNONCES DIVERSES.}

\section{VENTE D'OBJETS SCIENTIFIQUES.}

On trouve, au magasin des livres rares et des manuscrits précieux, rue du Pont de Lodi, $\mathrm{n}^{\circ} .7$, des manuscrits qui ont précédé l'époque de l'imprimerie, très-importans par leur sujet, ou par leurs ornemens en lettres d'or, miniatures et arabesques ou brillent les plus vives couleurs, et qui attestent les premiers progrès de l'art en France. Parmi ccux qui constatent l'état des sciences au moyen âge, on distingue un recueil unique qui renferme a la fois 3 ouvrages du sénateur romain Boécz, célèbre par son excellent Traité de la consolation de la philosophie, souvent réimprimé, en latin. et en français; ceux-ci, qui n'ont pas été connus par l'impression, sont: $\mathrm{I}^{\circ}$. l'Astronomie des Arabes au 8e. siecle; $2^{\prime \prime}$. la Science des nombres; $3^{\circ}$. l'Histoire de la Musique des Anciens; plusieurs savans et bibliographes, qui l'ont examiné, ont assuré qu'il n'en existe dans nos grandes bibliothéques, ni de plus beau, ni de plus complet, ou qui fasse si bien connaître la science musicale de l'antiquité; c'est un grand volume in-fol'. de la plus belle écriture, sur beau vélin, à 2 rolonnes, de I 4 feuillets enrichis de miniatures magnificques; il est estimé $1500 \mathrm{fr}$.

Noтice d'une Bibliothéque de mathématiques, de philoso-

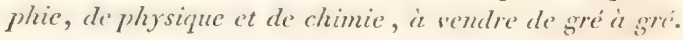

Cette Bibliothéque, de 1500 à I 600 volumes, a été formée par feu M. Arbagast, membre de l'Institut. Sous le rapport des mathématiques, il y a peu de bibliotheques damateur plus completes; elle pent fournir de quoi faire l'histoire de la science, et contient beaucoup d'ouvrages rares. Outre les collections académiques de Paris, de Berlin, de Pitersbourg et de Turin, elle contient les autcurs

ToM: I. 


\section{i94 Tente dobjets scicntifiques.}

originaux depuis la naissance des mathématiques jusquau commencement de re siecle, tels que Euclide, Archimede, Diaphante, Apollonius, Pappus, Cardau, Viele, Tartaglia, Recriomontanus, Purbach, Bachet de Méziriac, Descartes, II ersenne, Fermat, Pascal, lioberval, Plusius, van Schooten, Watlis, Neper, les Gregori, Barrow, Huygens; Newton, Leibnitz, les Bernouilli, Mermann, Colligis, Cran, Raphlson, Stirling, Mrivre, Montmort, Còtes, Taylor, Varignon, de lllospital, Fontenelle, Lagny, de La Hire, Clairaut, Maupertuis, d'Alembert, Euler, Cramer, Mac-Laurin, Emerson, Simpson, Landen, Rohins, Hutton, W aring, Boscowich, Bougainville, fontaine, Condorcet, Lagrange, Taplace, Legendre, Lacroix, Prony, L'lluilier, Cuusin, Monge, Carnot, Lambert, Kiestner, Hintenburg, Pfaff, Arbogast, Paoli, Casseli, les journaux de l'école polytechnique et ceux de l'écoie normale, l'Histoirc dies mathématiques de Montucla, et celle de Bossut, etc.

En astronomic, il y a Aristarque, Copernic, Keppler, Gassendi, Cassini, Lacaille, les Mayer, Lalande, Schubert, Kostner, Hennert, Schroter, etc.

En philosophie, Aristote, Bacon, Descartes, Malebranche, Locke, Condillac, Dégérando, Kant, Montiigne, Montesquieu, Huart, Filangièri.

En physique, Boyle, Keil, Rohault, Mariotte, S'Gravesande, Muschenbroek, Sigaud de la Fond, Brisson, Malpighi, Tournefort, Duhamel, Needham, Pricotley, Deluc, van Schwinden, Pott, Saussure, Sennebier, Pictet, Macko, Kirsten, Chladni, Crawford, Spalanzani, Voita, etc.

En chimic : les 40 premiers tomes des $\mathrm{A}$ nuales de Climic, avec la table des matières; huit années des Annales de Chimie de Crell, Sthall, Kirwann, Lavoisier, Morvean, Berthollet, Chaptal, Fourcroy, Bergmann, Scheel, IIermstadt, Hildebrand, Westrumb.

Outre ces ouvrages imprimés, il y a, en manuserits inćdits, la correspondance de Jean Iernouilli avec Varignon, le marguis de l'Hospital, Moivre et Minpertuis; les lettres d'luler a Lagrange, de 7755 jusquion $17-5$; celles de d'Alembert a Iargrange, de $1-5 \%$ a 17,2 ; celles de Charpit a Arbograst; ainsi que trois Mimoires d'Arbogast et un de Charpil.

On desirerait la vemlre en bloc, ou du moins en parties 
assez considérables. On donnerait la totalité pour la somme de 4,000 fr. S'adresser, pour plus amples renseignemens, ou la communication du catalogue détaillé, à M. Français, professeur à l'École Royale de l'Artillerie et du Génie, à Metz.

Liste des Journaux Français qui ont paru pendant le mois de Mars.

Archives des découvertes pour 1822. (I)

Annales de chimie et de phys., oct., et nov. I 822 . (7)

Gazette de santé, $\mathrm{n}^{\circ} \cdot 7$ à 9. (ro)

Journal univ. des sciences méd., n. 85,86 , janv et fév.(13)

Journ.compl.du dict.des sc. méd., $\mathrm{n}^{0} .55$ et 56 , janv.et fév.(11)

Archives génér. de médecine , $\mathrm{n}^{\circ}$. 2. févr. (12)

Nouvelle bibl. médic., $\mathrm{n}^{0}$. 2 et 3 , févr. et mars. (15)

Annales de la médecine physiologique, janv. (I 7 )

Journal de pharmacie, février. (19)

Annales de l'agriculture française, mars. (23)

Journal des voyages , février. (26)

Nouvelles Annales des voyages, février. (27)

Journal des savans, février. (29)

Galignani's litterary gaz, $n^{0}, 248$ ct $249 \cdot(34)$

Galignani's monthly review, janv. (35)

The Paris monthly review, janv. (33)

Annales de l'industrie, février. (36)

Annales françaises des arts, tom. XI, no. 6,7 et $8 .(37)$

Bibliographic de la France, $\mathrm{n}^{\circ} .9$ a 13 , mars. (39)

Journal général de la littér., nov. et déc. (40)

Journal général de la littér. étrang., janv. (4 I $)$

Bulletin des sciences de la société philom., janv. (4i)

Journal général de médecine, février. (47)

Journal asiatique, mars. $(54)$

Bulletin de la soc. de géographie., no. 4. (52)

Journal polymathique de Montpellier, $\mathrm{n}^{\mathrm{o}}$. I, janv.

Annales de mathématiques, $n^{\circ} .6$ à 8 , janv., févr.

Bulletin de la soc. d'agric. de l'Héraut, déc.

Journal d'agriculture du Tarn, $n^{\circ} \cdot 2$

Lycée armoricain, $n^{\circ} .2$ et 3.

Tablettes de la Drôme, $\mathrm{n}^{\circ}$. x à 3 , janv., férr.

Annales de la soc. roy. des sc. d'Orléans, $60 m 5, n$. 1 . 


\section{TABLE DES MATIERES.}

\section{CONTENUES DANS CE VOLUME.}

\section{PREMIERE SECTION.}

Annonce des Ouvragres, Extrails des Journaux et Nouvelles Scientifiques.

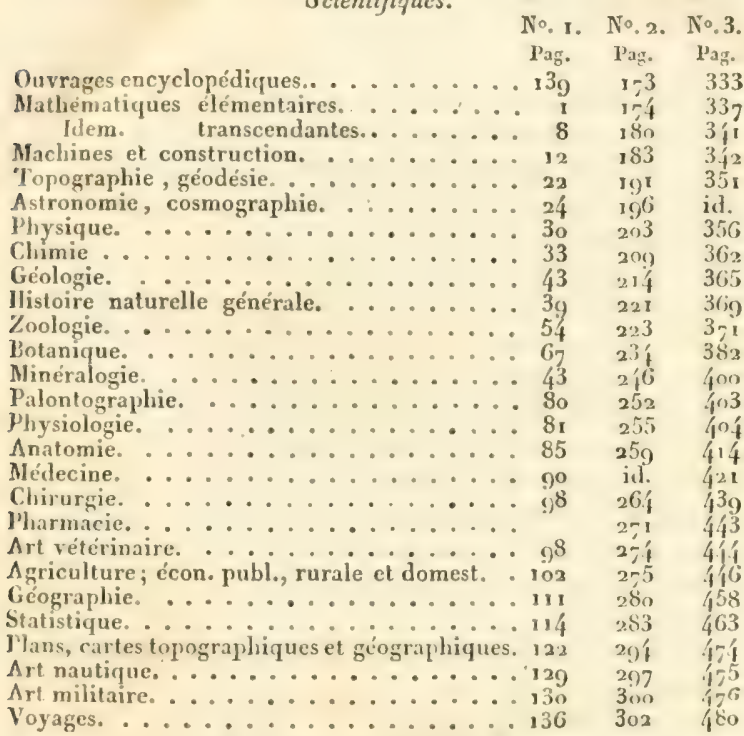

DEUXIEME SECTION. Travaux des Sociétés savantes.

Séances $\cdots \cdots$ Prix proposés. .......................... 154 Nominations, ćloges. ............... 159

TROISIE IIE SECTIC.

Annonces diverses.

Entreprises scientifiques. ......... 161

Indications scientifiques. .......... 163

Vente d'objets scientifiques. ....... I64

Nićcrologie. 


